\title{
Face Recognition Vendor Test (FRVT)
}

Performance of Face Identification Algorithms

\author{
NIST Interagency Report 8009
}

Patrick Grother Mei Ngan

\section{Information Access Division \\ National Institute of Standards and Technology}

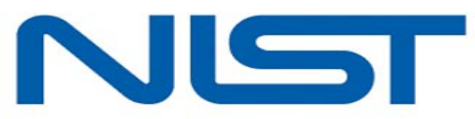

May 26, 2014 


\section{Acknowledgements}

The authors would like to thank the sponsors of this activity. These are the Criminal Justice Information Systems (CJIS) division and the Biometric Center of Excellence (BCOE) of the Federal Bureau of Investigation, the Science and Technology (S\&T) Directorate in the Department of Homeland Security (DHS), and the Office of Biometric Identity Management (OBIM) office, also in DHS.

\section{Disclaimer}

Specific hardware and software products identified in this report were used in order to perform the evaluations described in this document. In no case does identification of any commercial product, trade name, or vendor, imply recommendation or endorsement by the National Institute of Standards and Technology, nor does it imply that the products and equipment identified are necessarily the best available for the purpose. 


\section{Executive Summary}

Purpose and scope: This report documents the performance of one-to-many face recognition algorithms, and compares it with that measured in 2010. Performance in this context refers to recognition accuracy and computational resource usage as measured by executing those algorithms on massive sequestered datasets. These are: reasonable quality law enforcement mugshot images; poor quality webcam images collected in similar detention operations; and moderate quality visa application images. The mugshot and visa images are used to approximate performance obtainable using high quality ISO standardized images collected in passport, visa and driving license duplicate detection operations. These applications constitute the largest revenue segment in the face recognition marketplace. The webcam images are included to show how recognition accuracy degrades in non-ideal poorly-controlled situations - results will mimic recognition in adverse e.g. surveillance situations to the extent that those applications produce images similar to the webcam set.

Out of scope: Not within the scope of this report are: performance of live transactional systems like automated border control gates; human recognition accuracy as used in forensic applications; and recognition of persons in video sequences (which NIST is evaluating separately and will report on later). Some of those applications are likely to share technologies that are tested in this report.

Participation: The report includes performance figures for prototype algorithms from the research laboratories of many of the major commercial suppliers of face recognition technologies. It thereby facilitates robust comparative evaluation. However, while participation in the test was open to any organization worldwide, neither social media companies nor most academic institutions elected to submit algorithms, and this report therefore does not capture their technical capabilities except to the extent that those technologies have been adopted or licensed by FRVT participants.

Background: Face recognition error rates have declined massively in the two decades since initial commercialization of the various technologies. NIST has tracked that improvement and its conduct of regular independent, free, open, and public evaluations has fostered improvements in the state of the art. One-to-many face identification systems are mostly used in conjunction with trained human facial reviewers. The systems are configured to operate in two regimes: first, with a low threshold that necessitates adjudication of many false positive candidates by a reviewer specifically employed to do so; second, with a high threshold, in which false positive outcomes are rare and human intervention is only needed infrequently. Low false positive rates are accompanied by higher false negative rates - this report includes extensive quantification of this tradeoff.

Results summary: Since NIST's last evaluation was published in August 2010, the algorithms from NEC remain the most accurate followed by those of Morpho which merged its algorithms with those acquired from L1 Identity Solutions in 2011. Thereafter Toshiba, Cognitec Systems, and 3M/Cogent constitute the leading commercial suppliers. Algorithms with lesser levels of capability are those from Neurotechnology, Zhuhai Yisheng, HP, Decatur, and Ayonix. Importantly, however, performance is not single-faceted and any ranking of performance across algorithms must be weighed by applicationspecific requirements. For example, some algorithms are more suited to recognition of difficult webcam images; and the search speed of some algorithms increases only slowly with enrolled population size.

The headline results follow in the Technical Summary.

\begin{tabular}{|c|c|c|c|c|c|c|}
\hline $\begin{array}{l}\mathrm{A}=3 \mathrm{M} / \text { Cogent } \\
\mathrm{G}=\text { Hisign } \\
\mathrm{P}=\text { Zhuhai-Yisheng }\end{array}$ & $\begin{array}{l}\mathrm{B}=\text { Cognitec } \\
\mathrm{H}=\text { CAS-IA } \\
\mathrm{Q}=\text { JunYu }\end{array}$ & $\begin{array}{l}\mathrm{C}=\text { Neurotechnology } \\
\mathrm{I}=\text { CAS-ICT } \\
\mathrm{S}=\text { Decatur }\end{array}$ & $\begin{array}{l}\mathrm{D}=\text { Safran Morpho } \\
\mathrm{J}=\text { Toshiba } \\
\mathrm{T}=\text { Ayonix }\end{array}$ & $\begin{array}{l}\mathrm{E}=\mathrm{NEC} \\
\mathrm{L}=\text { Tsinghua U. II }\end{array}$ & $\begin{array}{l}\mathrm{F}=\text { Tsinghua } \mathrm{U} \\
\mathrm{M}=\mathrm{HP}\end{array}$ & $\begin{array}{l}\text { FNIR(N,R,T,L) “Miss rate" } \\
\text { FPIR(N,T,L) "False alarm rate" }\end{array}$ \\
\hline
\end{tabular}




\section{Technical Summary}

Absolute accuracy: When mugshot images from 1.6 million individuals are enrolled by the most accurate algorithm, $4.1 \%$ of subsequent mated searches fail to yield the correct mate in rank one position. Practically this result assumes that a human reviewer will be employed to adjudicate the candidate identities. If, on the other hand, a threshold is elevated to limit false positive outcomes to only 1 in 500 searches $(0.2 \%)$, the failure to find the mate rises to $7.5 \%$. Using poorly constrained webcam images, which exhibit serious departures from most quality-related clauses of published image standards, identification miss rates are typically between two and five times higher such that the correct mate is not found at rank one in $20-60 \%$ of searches. Exceptionally, however, the most accurate algorithm fails in only $11.3 \%$ of searches. This latter result is notable in that it indicates that face recognition can work on nonideal images. Thus, while the webcam images were collected from nominally cooperative subjects, the use of inferior equipment and procedures means, for example, that images from non-cooperative bank ATM machine and surveillance camera deployments will be recognizable in a useful number of cases.

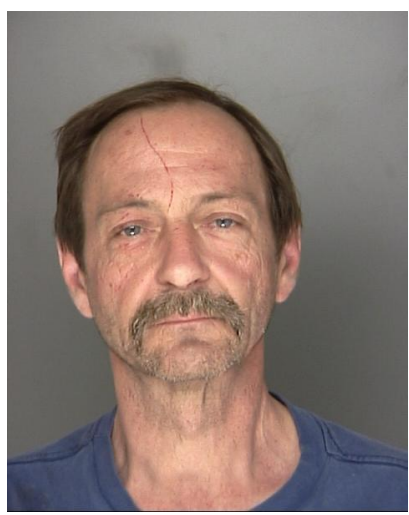

(a) Good quality mugshot

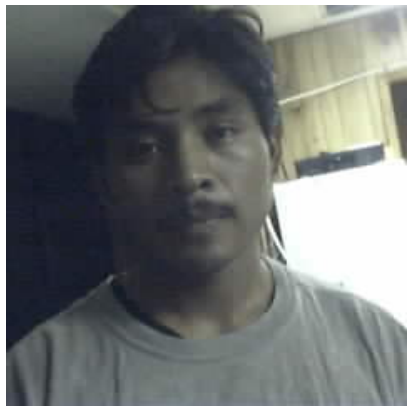

(b) Poor quality webcam

Figure 1: Examples of images used in this report. Such outcomes have been reported operationally [19] and in the laboratory [17].

Accuracy across commercial providers: Recognition accuracy is very strongly dependent on the algorithm and, more specifically, on the developer of the algorithm. Recognition error rates in a particular scenario range from a few percent up to beyond fifty percent. Among the most accurate developers, the rank one miss rates for recognition in a population size of 1.6 million are $4.1 \%$ (NEC), 9.1\% (Morpho), 10.7\% (Toshiba), 13.6\% (Cognitec), 17.2\% (3M) and 20.5\% (Neurotechnology). For webcam images, this sequence is $11.3 \%$ (NEC), $23.7 \%$ (Toshiba), $29.8 \%$ (Morpho), 36.4\% (3M), 57.6\% (Cognitec) and $66.9 \%$ (Neurotechnology). While results for up to six algorithms from each developer are reported here, the intraprovider accuracy variations are usually smaller than the inter-provider variations. That said, some developers submitted different, less accurate but computationally lightweight algorithms.

Accuracy under increasing population size: As more identities are enrolled into a biometric system, the possibility of a false positive increases due to lookalike faces that yield extreme values in the tail of the nonmate score distribution. However these scores are lower than most mate scores such that when an identification is configured with a threshold of zero, and where human adjudication is always necessary, rank-one identification miss rates scale very favorably with population size, $\mathrm{N}$, growing approximately as a power law, $a N^{b}$. Depending on the algorithm, the exponent $b$ for mugshot searches is low, on the range $[0.08,0.16]$ meaning that a large, 10-fold, increase in $\mathrm{N}$, yields only a 1.2 to 1.4 fold increase in the rate at which mated searches do not yield the correct mate. Thus the rank one mugshot miss rates, for search into a database of just 160,000, are only modestly improved over those at 1.6 million: $4.1 \%$ to $3.4 \%$ (NEC), 9.1\% to $7.6 \%$ (Morpho), $10.7 \%$ to $7.9 \%$ (Toshiba), $13.6 \%$ to $10.9 \%$ (Cognitec), $17.2 \%$ to $13.3 \%$ (3M) and $20.5 \%$ to $16.9 \%$ (Neurotechnology). While extrapolation to larger populations is technically problematic, face identification systems will be useful, if imperfect, in nation-state population sizes.

Utility of adjudicating long candidate lists: In the regime where a system is configured with a threshold of zero, and where human adjudication is always necessary, the reviewer will find mates on candidate lists at ranks far from one. The accuracy benefits of traversing such lists are usually substantial: For example, in a population of 1.6 million, the rank one and rank fifty miss rates are $4.1 \%$ to $2.6 \%$ (NEC), $9.1 \%$ to $7.1 \%$ (Morpho), $10.7 \%$ to $5.7 \%$ (Toshiba), $13.6 \%$ to $8.4 \%$ (Cognitec), $17.2 \%$ to $9.8 \%(3 \mathrm{M})$ and $20.5 \%$ to $13.6 \%$ (Neurotechnology). These are diminishing returns, however, with miss rates for some algorithms growing as power-law, $a R^{c}$, in the number of candidates a reviewer is willing to consider, $R$. The exponent $c$ for mugshot searches is typically on the range $[-0.2,-0.1]$ indicating that rank 1 miss rates are reduced by from $20 \%$ to $40 \%$ if 10 candidates are available for inspection, but only by $30 \%$ to $50 \%$ if 50 are considered.

Human adjudication workload: Human reviewers will typically only need to search the first few (highest scoring) candidates returned in a search. The expected number of comparisons constitutes a workload measure which can be used to both compare algorithms and to inform operational labor requirements. If a score threshold is applied to reduce the

\begin{tabular}{|c|c|c|c|c|c|c|}
\hline $\begin{array}{l}\mathrm{A}=3 \mathrm{M} / \text { Cogent } \\
\mathrm{G}=\text { Hisign } \\
\mathrm{P}=\text { Zhuhai-Yisheng }\end{array}$ & $\begin{array}{l}B=\text { Cognitec } \\
H=\text { CAS-IA } \\
Q=\text { JunYu }\end{array}$ & $\begin{array}{l}\mathrm{C}=\text { Neurotechnology } \\
\mathrm{I}=\text { CAS-ICT } \\
\mathrm{S}=\text { Decatur }\end{array}$ & $\begin{array}{l}\mathrm{D}=\text { Safran Morpho } \\
\mathrm{J}=\text { Toshiba } \\
\mathrm{T}=\text { Ayonix }\end{array}$ & $\begin{array}{l}\mathrm{E}=\mathrm{NEC} \\
\mathrm{L}=\text { Tsinghua U. II }\end{array}$ & $\begin{array}{l}\mathrm{F}=\text { Tsinghua } \mathrm{U} \\
\mathrm{M}=\mathrm{HP}\end{array}$ & $\begin{array}{l}\text { FNIR(N,R,T,L) “Miss rate" } \\
\text { FPIR(N,T,L) “False alarm rate" }\end{array}$ \\
\hline
\end{tabular}


length of candidate lists, substantial reviewer workload reductions can be realized but at the expense of increased miss rates. In the best case, application of a threshold to candidates from a Morpho algorithm reduced workload by $60 \%$ with only a factor of 1.05 more misses than without a threshold.

Accuracy by age group: Identification accuracy is strongly dependent on subject age. For all algorithms, older individuals are both easier to recognize as themselves, and easy to tell apart from each other. The opposite is true in children: both false negative and false positive rates are much higher, with infants being very hard to identify. Moreover, the trends are progressive throughout adulthood, with young adults being identified with worse accuracy than older. These results are derived over images taken, on average, 2-3 years apart so the effects of ageing (craniofacial shape change) are influential only on the results for younger individuals.

Accuracy gains 2010-2013: For the four developers who submitted algorithms to NIST in 2010 and 2013, accuracy has improved in all cases. Rank one miss rates have reduced by about $10 \%$ for Cognitec, Neurotechnology, and Morpho, and by about $28 \%$ for NEC (from $8.9 \%$ to $6.4 \%$ ). More substantial reductions have been realized when a threshold is applied to permit false positive outcomes in only 1 in 500 nonmate searches: There Morpho realizes a $21 \%$ reduction in misses (from $24.8 \%$ to $19.5 \%)$, while for NEC, the figure is $60 \%(26.9 \%$ to $10.8 \%)$. Finally the accuracy gains when reviewers examine up to 50 candidates are more modest, ranging from 3\% (Cognitec) to $12 \%$ (Neurotechnology).

Sketch recognition: By searching a non-operational set of sketch images against photographs seeded into a population of 640,000 nonmated mugshots, the most accurate algorithms produce the mated photograph only infrequently: The mate is not among the top 50 candidates at the following rates: $73.3 \%$ (3M/Cogent), $73.8 \%$ (NEC), 78.5\% (Toshiba), $80.3 \%$ (Morpho), and $81.5 \%$ (Neurotechnology). While these error rates are very high, they are neverthess valuable in developing investigative leads in cases which are otherwise cold. An important caveat is that sketch-identification was never declared to be part of FRVT and better algorithms may be available from the providers. That said, face recognition algorithms are being used to recognize sketches operationally. Further accuracy will clearly be dependent on eye-witness recall, artist interpretation if any, and software interfaces.

Conclusions: As with other biometrics, accuracy of facial recognition implementations varies greatly across the industry. Absent other performance or economic parameters, users should prefer the most accurate algorithm. Note, however, that the results of this section are entirely rank-based befitting use of face recognition in the investigational mode in which an reviewer is willing to traverse candidate lists looking for mates. Subsequent investigations in this report consider threshold-based metrics appropriate for identification mode applications. Note that the absolute values of identification accuracy will always depend on the dataset used, specifically to the properties of the images in use. In particular, the main dataset used here includes some images that are not perfectly frontal, such that conformance to the appearance-related requirements of the ISO/IEC 19794-5 "gold" standard is imperfect.

Improving face recognition: On the basis of the results in this report, we identify the following drivers of overall recognition accuracy, and quantify their relative influence relative on false negative miss rates.

$\triangleright$ Quality: Improvement of image quality is the largest contributing factor to recognition accuracy. Results in this report note a four fold reduction in miss rates using mugshots vs. webcam images. Further improvements in accuracy can be obtained by enhancing conformance to the ISO/IEC 19794-5 "gold" standard [26], particularly using two complementary approaches: by design via improved optical and photographic aspects, and by careful application of compression algorithms; and by detection of non-conformant (for example, blurred or non-frontal) images at the time they are collected. A new program has been established to evaluate algorithms capable of detecting defective images $[9,10]$. The best practice should be to collect and retain forensic quality photographs (i.e. subject acquisition profile 50/51 instances of the ANSI/NIST Type 10 standard [27]), from which ISO/IEC 19794-5 images should be prepared for automated recognition.

$\triangleright$ Human adjudication of candidate lists: Recognizing that a human is often involved in examining candidates produced in an automated one-to-many search, it is imperative that the enrolled reference "exemplar" image be of high quality. Given ready availablity of high resolution digital cameras, the ANSI/NIST standard advocates collection of forensic quality images for which the interocular distance is around 800 pixels. Such images are not used directly for automated face recognition, so the default guidance is to collect and retain the forensic image, and to prepare from it the ISO standard image (120 pixels interocular distance) for automated face recognition.

Accuracy can be improved by supplementing automated facial identification with human adjudication of the can-

\begin{tabular}{|c|c|c|c|c|c|}
\hline $\begin{array}{l}\mathrm{A}=3 \mathrm{M} / \text { Cogent } \\
\mathrm{G}=\text { Hisign } \\
\mathrm{P}=\text { Zhuhai-Yisheng }\end{array}$ & $\begin{array}{l}\mathrm{B}=\text { Cognitec } \\
\mathrm{H}=\text { CAS-IA } \\
\mathrm{Q}=\text { JunYu }\end{array}$ & $\begin{array}{l}\mathrm{C}=\text { Neurotechnology } \\
\mathrm{I}=\mathrm{CAS}-\mathrm{ICT} \\
\mathrm{S}=\text { Decatur }\end{array}$ & $\begin{array}{l}\mathrm{D}=\text { Safran Morpho } \\
\mathrm{J}=\text { Toshiba } \\
\mathrm{T}=\text { Ayonix }\end{array}$ & $\begin{array}{l}\mathrm{E}=\mathrm{NEC} \\
\mathrm{L}=\text { Tsinghua U. II }\end{array}$ & $\begin{array}{l}\mathrm{F}=\text { Tsinghua } \mathrm{U} \\
\mathrm{M}=\mathrm{HP}\end{array}$ \\
\hline
\end{tabular}


didate photographs. Particularly by traversing long candidate lists (for example, up to length 50) miss rates can readily be reduced by a factor of two (i.e. 50\%) An important caveat here is that the accuracy with which human reviewers can reliably adjudicate the most-similar faces returned in a large-population one-to-many search remains poorly quantified.

$\triangleright$ Algorithm selection: Algorithm performance varies substantially - even among the leading providers a factor of two reduction in identification miss rates can be realized by replacing one algorithm with a better one. Such a step should be undertaken only while paying due attention to other performance related factors, such as computational expense and impostor distribution stability.

\begin{tabular}{|c|c|c|c|c|c|c|}
\hline $\begin{array}{l}A=3 \mathrm{M} / \text { Cogent } \\
G=\text { Hisign } \\
P=\text { Zhuhai-Yisheng }\end{array}$ & $\begin{array}{l}B=\text { Cognitec } \\
H=\text { CAS-IA } \\
Q=\text { JunYu }\end{array}$ & $\begin{array}{l}\mathrm{C}=\text { Neurotechnology } \\
\mathrm{I}=\text { CAS-ICT } \\
\mathrm{S}=\text { Decatur }\end{array}$ & $\begin{array}{l}\mathrm{D}=\text { Safran Morpho } \\
\mathrm{J}=\text { Toshiba } \\
\mathrm{T}=\text { Ayonix }\end{array}$ & $\begin{array}{l}\mathrm{E}=\mathrm{NEC} \\
\mathrm{L}=\text { Tsinghua U. II }\end{array}$ & $\begin{array}{l}\mathrm{F}=\text { Tsinghua } \mathrm{U} \\
\mathrm{M}=\mathrm{HP}\end{array}$ & $\begin{array}{l}\text { FNIR(N,R,T,L) “Miss rate" } \\
\text { FPIR(N,T,L) “False alarm rate" }\end{array}$ \\
\hline
\end{tabular}




\section{Release Notes}

$\triangle$ FRVT Tracks: NIST initiated FRVT in the second half of 2012. We invited participation in five tracks.

$\rightarrow$ Class A: Accuracy of algorithms executing one-to-one verification comparisons to determine if two samples originate from the same person or not.

$\rightarrow$ Class B: Accuracy of algorithms executing one-to-one verification but with an enrollment database present. This track was discontinued after the 2010 evaluation. Accuracy gains over class A are available.

$\rightarrow$ Class C: Accuracy of algorithms executing one-to-many identification searches to determine either that the person is not enrolled, or to determine the identity of the person.

$\rightarrow$ Class D: Accuracy of algorithms tasked with determining the sex or age of a person in one or more input images. A separate class D track tasked algorithms to determine whether a face in an image is frontal to the camera or not.

$\rightarrow$ Class F: Effectiveness of algorithms that take one or more (non-frontal) input images of a person, and produce one (or more) frontally posed images of that person.

$\rightarrow$ Class V: Effectiveness of algorithms that execute one-to-many identification of persons whose faces appear in frames extracted from video surveillance sequences.

This report details results only for class $\mathrm{C}$ algorithms.

$\triangleright$ FRVT Reports: The results of the FRVT appear as a series of NIST Interagency Reports. The reports were developed separately and released on different schedules. In prior years NIST has mostly reported FRVT results as a single report; this had the disadvantage that results from completed sub-studies were not published until all other studies were complete.

All reports are linked from http: //face.nist.gov/frvt and its sub-pages.

$\triangleright$ Appendices: This report is accompanied by a number of appendices which present exhaustive results on a peralgorithm basis. These are machine-generated and are included because the authors believe that visualization of such data is broadly informative and vital to understanding the context of the report.

$\triangleright$ Typesetting: Virtually all of the tabulated content in this report was produced automatically. This involved the use of scripting tools to generate directly type-settable $\mathrm{HT}_{\mathrm{E}} \mathrm{X}$ content. This improves timeliness, flexibility, maintainability, and reduces transcription errors.

$\triangleright$ Graphics: Many of the Figures in this report were produced using both Deepayan Sarkar's Lattice package [22] and Hadley Wickham's ggplot2 package running under R, the capabilities of which extend beyond those evident in this document.

$\triangleright$ Contact: Correspondence regarding this report should be directed to PGROTHER at NIST dot GOV.

\begin{tabular}{|c|c|c|c|c|c|c|}
\hline $\begin{array}{l}\mathrm{A}=3 \mathrm{M} / \text { Cogent } \\
\mathrm{G}=\text { Hisign } \\
\mathrm{P}=\text { Zhuhai-Yisheng }\end{array}$ & $\begin{array}{l}\mathrm{B}=\text { Cognitec } \\
\mathrm{H}=\text { CAS-IA } \\
\mathrm{Q}=\text { JunYu }\end{array}$ & $\begin{array}{l}\mathrm{C}=\text { Neurotechnology } \\
\mathrm{I}=\text { CAS-ICT } \\
\mathrm{S}=\text { Decatur }\end{array}$ & $\begin{array}{l}\mathrm{D}=\text { Safran Morpho } \\
\mathrm{J}=\text { Toshiba } \\
\mathrm{T}=\text { Ayonix }\end{array}$ & $\begin{array}{l}\mathrm{E}=\mathrm{NEC} \\
\mathrm{L}=\text { Tsinghua U. II }\end{array}$ & $\begin{array}{l}\mathrm{F}=\text { Tsinghua } \mathrm{U} \\
\mathrm{M}=\mathrm{HP}\end{array}$ & $\begin{array}{l}\text { FNIR(N,R,T,L) “Miss rate" } \\
\text { FPIR(N,T,L) “False alarm rate" }\end{array}$ \\
\hline
\end{tabular}




\section{Contents}

ACKNOWLEDGEMENTS 1

DisClAimer

EXECUTIVE SUMMARY 2

TECHNiCAL SUMMARY 3

Release Notes $\quad 6$

1 INTRODUCTION 11

2 PARTicipation 11

3 EVAluATiON DATASETS 12

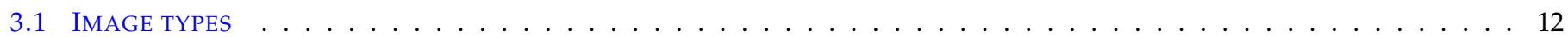

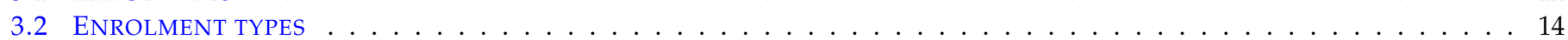

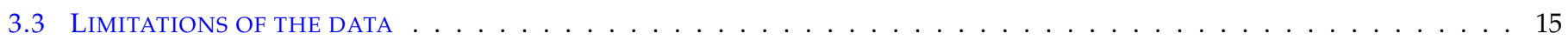

4 Performance metrics 16

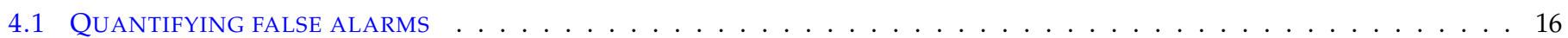

4.2 QUANTIFYING HITS AND MISSES . . . . . . . . . . . . . . . . . . . . . . . . . 17

4.3 Best PRACTICE TESTING REQUiRES EXECUtion Of SEARCHES With AND Without MATES . . . . . . . . . . . . . . . 18

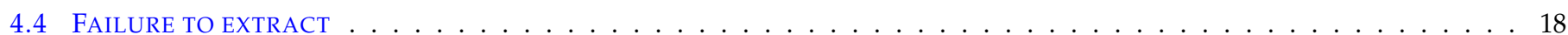

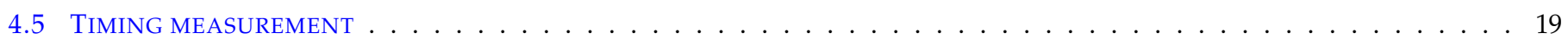

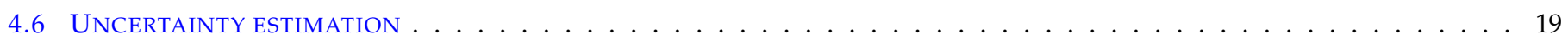

5 RESUltS 20

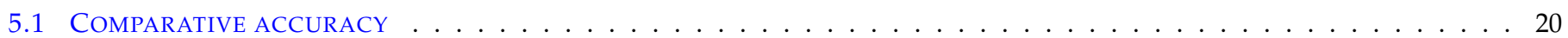

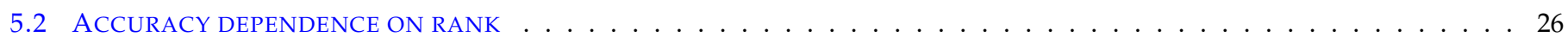

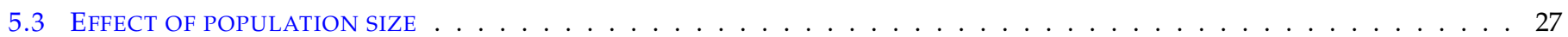

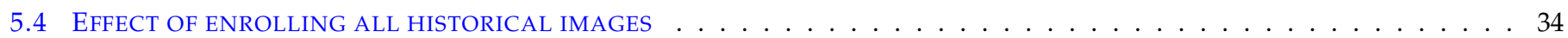

5.5 ACCURACY DEPENDENCE ON SUBJECT AGE . . . . . . . . . . . . . . . . . . . . . . . 36

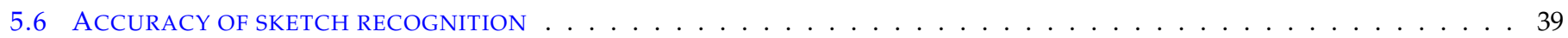

5.7 HUMAN WORKLOAD FOR CANDIDATE LIST ADJUDICATION . . . . . . . . . . . . . . . . . . . . . . 41

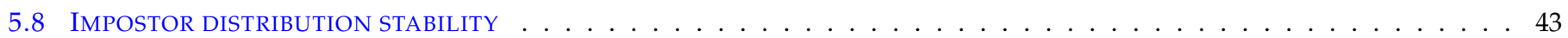

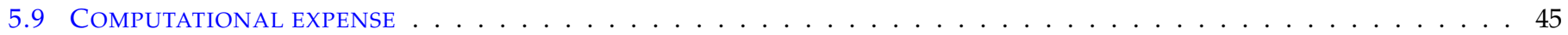

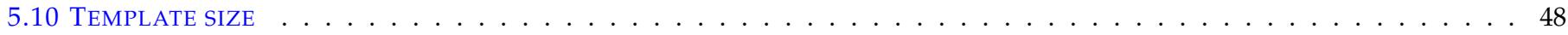

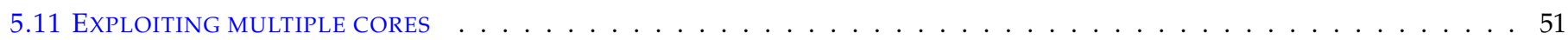

A Biometric ERror Rate Tradeoff Characteristics 54

A Algorithm report cards 58

A Algorithm accuracy by age group 98

\section{List of Tables}

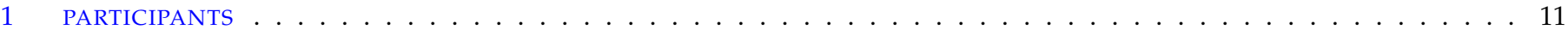

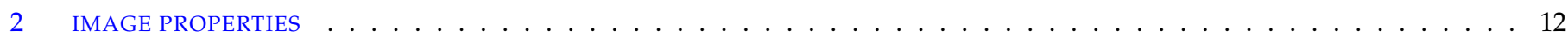

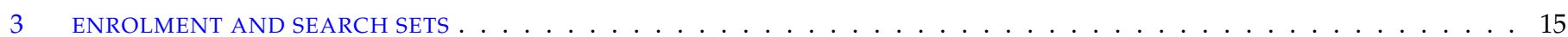

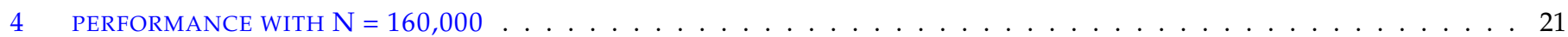

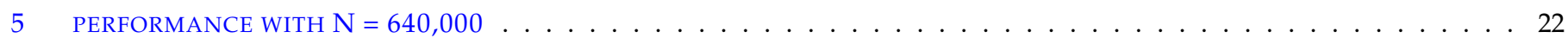

6 PERFORMANCE WITH $\mathrm{N}=1,600,000 \ldots \ldots \ldots \ldots$

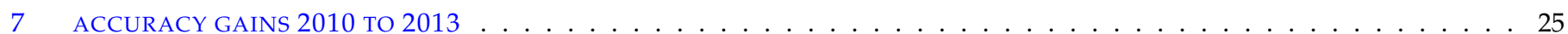

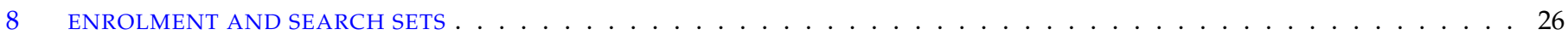

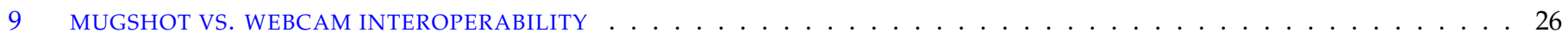

\begin{tabular}{|c|c|c|c|c|c|c|}
\hline $\begin{array}{l}\mathrm{A}=3 \mathrm{M} / \text { Cogent } \\
\mathrm{G}=\text { Hisign } \\
\mathrm{P}=\text { Zhuhai-Yisheng }\end{array}$ & $\begin{array}{l}B=\text { Cognitec } \\
H=\text { CAS-IA } \\
Q=\text { JunYu }\end{array}$ & $\begin{array}{l}\mathrm{C}=\text { Neurotechnology } \\
\mathrm{I}=\text { CAS-ICT } \\
\mathrm{S}=\text { Decatur }\end{array}$ & $\begin{array}{l}\mathrm{D}=\text { Safran Morpho } \\
\mathrm{J}=\text { Toshiba } \\
\mathrm{T}=\text { Ayonix }\end{array}$ & $\begin{array}{l}\mathrm{E}=\mathrm{NEC} \\
\mathrm{L}=\text { Tsinghua U. II }\end{array}$ & $\begin{array}{l}\mathrm{F}=\text { Tsinghua } \mathrm{U} \\
\mathrm{M}=\mathrm{HP}\end{array}$ & $\begin{array}{l}\text { FNIR(N,R,T,L) “Miss rate" } \\
\text { FPIR(N,T,L) “False alarm rate" }\end{array}$ \\
\hline
\end{tabular}


10 MISS RATE VS. POPULATION SIZE AT RANK $1 \ldots \ldots \ldots$

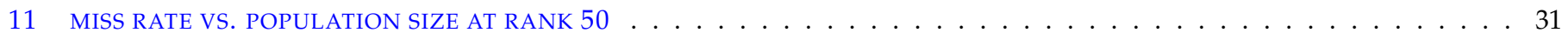

12 POWER-LAW MODELS OF CUMULATIVE MATCH CHARACTERISTICS . . . . . . . . . . . . . . . . . . . . 35

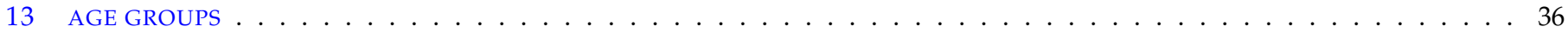

14 SKETCH VS. PHOTO IDENTIFICATION ACCURACY . . . . . . . . . . . . . . . . . . . . . . . . 40

15 EFFECT OF POPULATION SIZE . . . . . . . . . . . . . . . . . . . . . . . . . . . . . . . 46

\begin{tabular}{|c|c|c|c|c|c|c|}
\hline $\begin{array}{l}\mathrm{A}=3 \mathrm{M} / \text { Cogent } \\
\mathrm{G}=\text { Hisign } \\
\mathrm{P}=\text { Zhuhai-Yisheng }\end{array}$ & $\begin{array}{l}\mathrm{B}=\text { Cognitec } \\
\mathrm{H}=\text { CAS-IA } \\
\mathrm{Q}=\text { JunYu }\end{array}$ & $\begin{array}{l}\mathrm{C}=\text { Neurotechnology } \\
\mathrm{I}=\text { CAS-ICT } \\
\mathrm{S}=\text { Decatur }\end{array}$ & $\begin{array}{l}\mathrm{D}=\text { Safran Morpho } \\
\mathrm{J}=\text { Toshiba } \\
\mathrm{T}=\text { Ayonix }\end{array}$ & $\begin{array}{l}\mathrm{E}=\mathrm{NEC} \\
\mathrm{L}=\text { Tsinghua U. II }\end{array}$ & $\begin{array}{l}\mathrm{F}=\text { Tsinghua } \mathrm{U} . \\
\mathrm{M}=\mathrm{HP}\end{array}$ & $\begin{array}{l}\text { FNIR(N,R,T,L) “Miss rate" } \\
\text { FPIR(N,T,L) "False alarm rate" }\end{array}$ \\
\hline
\end{tabular}




\section{List of Figures}

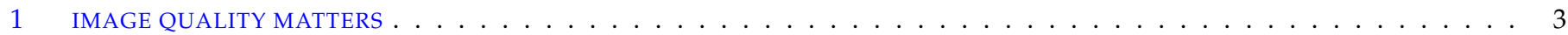

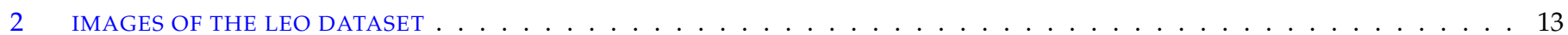

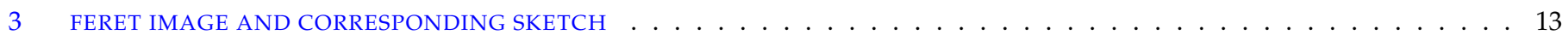

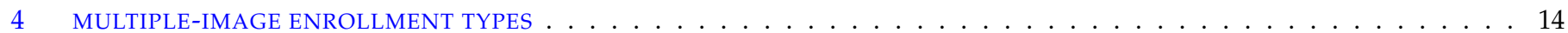

5 SELECTIVITY VS. FALSE POSITIVE IDENTIFICATION RATE . . . . . . . . . . . . . . . . . . . . . . . 17

6 ALGORITHM COMPARISON: DET CHARACTERISTICS . . . . . . . . . . . . . . . . . . . . . . . 24

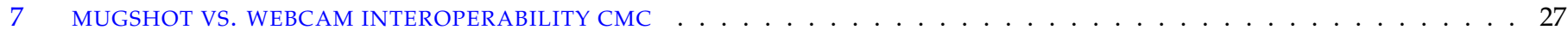

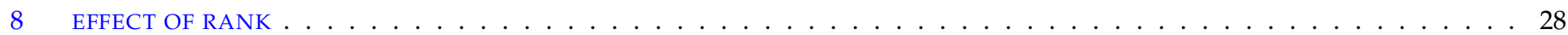

9 MISS RATES VS. POPULATION SIZE . . . . . . . . . . . . . . . . . . . . . . . . . . . . . . . 29

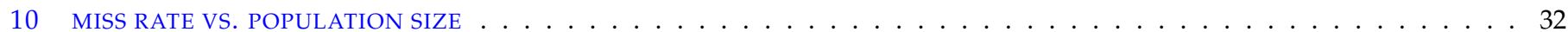

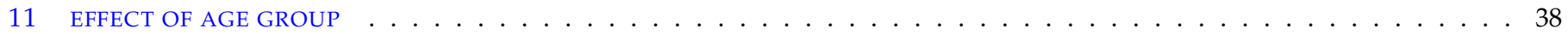

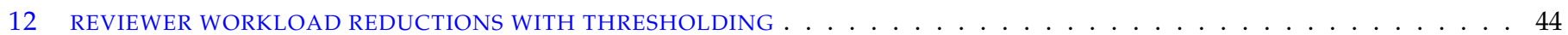

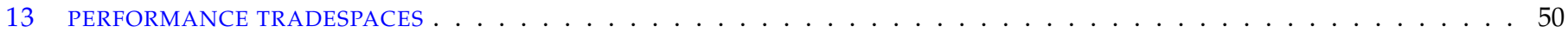

14 DET PROPERTIES AND INTERPRETATION : : ERROR RATES ． . . . . . . . . . . . . . . . . . . . . 55

15 DET PROPERTIES AND INTERPRETATION : NON-IDEAL TESTS . . . . . . . . . . . . . . . . . . . . 56

16 DET PROPERTIES AND INTERPRETATION :: ALGORITHMS USED IN COMBINATION . . . . . . . . . . . . . . . . . . . 57

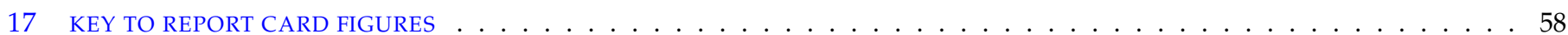

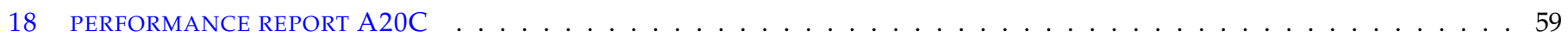

19 PERFORMANCE REPORT A30C PERFORMANCE REPORT A31C

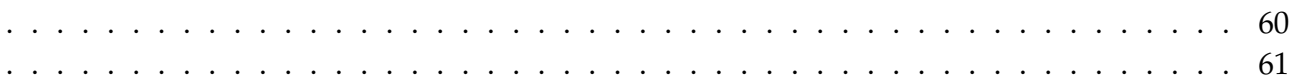

PERFORMANCE REPORT A32C

PERFORMANCE REPORT B30C

PERFORMANCE REPORT B31C

PERFORMANCE REPORT B32C

PERFORMANCE REPORT B33C

PERFORMANCE REPORT C20C

PERFORMANCE REPORT C30C

PERFormanCE REPORT C31C

PERFORMANCE REPORT C32C

PERFORMANCE REPORT D20C

PERFORMANCE REPORT D30C

PERFORMANCE REPORT D31C

PERFORMANCE REPORT D32C

PERFormance REPORT D33C

PERFORMANCE REPORT D34C

PERFORMANCE REPORT E20C

PERFORMANCE REPORT E21C

PERFORMANCE REPORT E30C

PERFORMANCE REPORT E31C

PERFORMANCE REPORT F20C

PERFORMANCE REPORT F30C

PERFORMANCE REPORT F31C

PERFORMANCE REPORT G30C

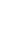

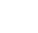

(7)

7

9




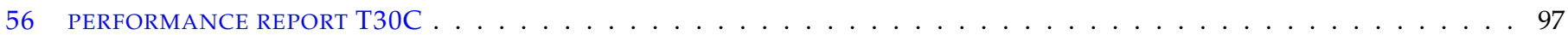

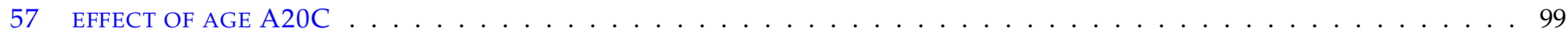

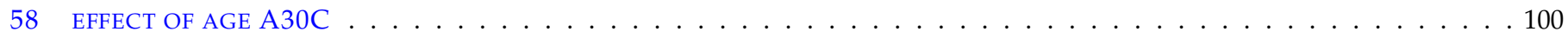

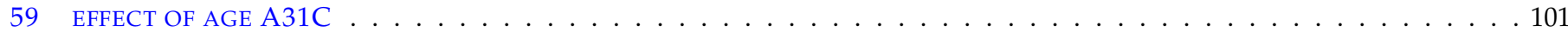

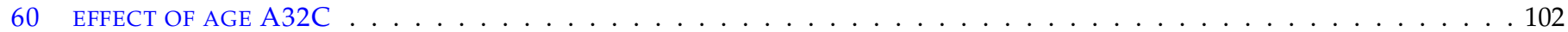

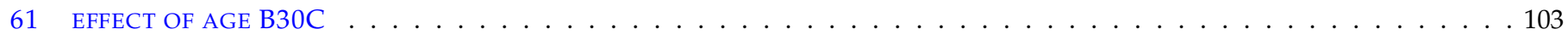

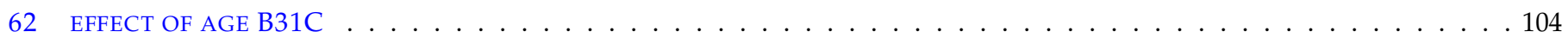

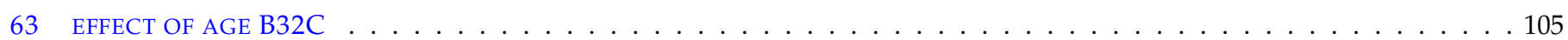

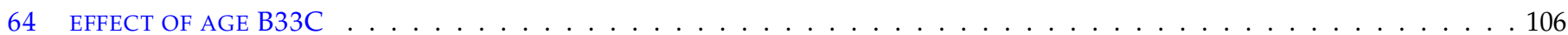

65 EFFECT OF AGE C20C

66 EFFECT OF AGE C30C

67 EFFECT OF AGE C31C

68 EFFECT OF AGE C32C

69 EFFECT OF AGE D20C

70 EFFECT OF AGE D30C

71 EFFECT OF AGE D31C

EFFECT OF AGE D32C EFFECT OF AGE D33C EFFECT OF AGE D34C EFFECT OF AGE E20C EFFECT OF AGE E21C EFFECT OF AGE E30C

EFFECT OF AGE E31C EFFECT OF AGE F20C EFFECT OF AGE F30C EFFECT OF AGE F31C EFFECT OF AGE G30C EFFECT OF AGE G31C EFFECT OF AGE H30C EFFECT OF AGE J20C EFFECT OF AGE J30C EFFECT OF AGE J31C

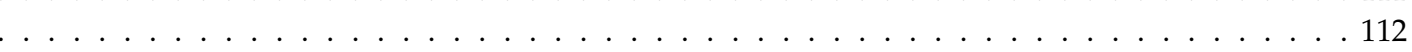

EFFECT OF AGE T30C

\begin{tabular}{|c|c|c|c|c|c|c|}
\hline $\begin{array}{l}\mathrm{A}=3 \mathrm{M} / \text { Cogent } \\
\mathrm{G}=\text { Hisign } \\
\mathrm{P}=\text { Zhuhai-Yisheng }\end{array}$ & $\begin{array}{l}\mathrm{B}=\text { Cognitec } \\
\mathrm{H}=\mathrm{CAS}-\mathrm{IA} \\
\mathrm{Q}=\text { JunYu }\end{array}$ & $\begin{array}{l}\mathrm{C}=\text { Neurotechnology } \\
\mathrm{I}=\text { CAS-ICT } \\
\mathrm{S}=\text { Decatur }\end{array}$ & $\begin{array}{l}\mathrm{D}=\text { Safran Morpho } \\
\mathrm{J}=\text { Toshiba } \\
\mathrm{T}=\text { Ayonix }\end{array}$ & $\begin{array}{l}\mathrm{E}=\mathrm{NEC} \\
\mathrm{L}=\text { Tsinghua U. II }\end{array}$ & $\begin{array}{l}\mathrm{F}=\text { Tsinghua } \mathrm{U} \\
\mathrm{M}=\mathrm{HP}\end{array}$ & $\begin{array}{l}\text { FNIR(N,R,T,L) “Miss rate" } \\
\text { FPIR(N,T,L) "False alarm rate" }\end{array}$ \\
\hline
\end{tabular}




\begin{tabular}{|c|c|c|c|c|c|}
\hline Provider & Letter & \multicolumn{4}{|c|}{ Number of algorithms submitted } \\
\hline Name & Code & Phase 1 & Phase 2 & Phase 3 & Total \\
\hline 3M/Cogent & A & 3 & 1 & 3 & 7 \\
\hline Cognitec & $\mathrm{B}$ & 1 & 0 & 4 & 5 \\
\hline Neurotechnology & $\mathrm{C}$ & 3 & 1 & 3 & 7 \\
\hline Safran Morpho & $\mathrm{D}$ & 1 & 1 & 5 & 7 \\
\hline NEC & $\mathrm{E}$ & 1 & 2 & 2 & 5 \\
\hline Tsinghua University (EE - Prof. Wen) & $\mathrm{F}$ & 2 & 3 & 2 & 7 \\
\hline Beijing Ivsign Technology Co. Ltd. & G & 2 & 1 & 2 & 5 \\
\hline Chinese Academy of Sci. - Inst. Automation (Prof. Liu) & $\mathrm{H}$ & 1 & 0 & 1 & 2 \\
\hline Chinese Academy of Sci - Inst. Computing Technology (Prof. Shan) & I & 2 & 1 & 0 & 3 \\
\hline Toshiba Corporation & $\mathrm{J}$ & 2 & 2 & 3 & 7 \\
\hline Tsinghua University (EE - Prof. Su) & $\mathrm{L}$ & 3 & 2 & 2 & 7 \\
\hline HP / Virage & M & 1 & 2 & 1 & 4 \\
\hline Zhuhai Yisheng Electronics Tech. Co. Ltd. & $\mathrm{P}$ & 2 & 0 & 1 & 3 \\
\hline JunYu Technology Co. Ltd. & Q & 3 & 1 & 1 & 5 \\
\hline Decatur Industries Inc. & $\mathrm{S}$ & 0 & 1 & 0 & 1 \\
\hline Ayonix Inc. (JP) & $\mathrm{T}$ & 0 & 0 & 1 & 1 \\
\hline
\end{tabular}

Table 1: Algorithm submissions by provider The number of different algorithms (i.e. libraries and ancillary files) submitted to FRVT by Phase, and in total. Phase 1 closed in October 2012, Phase 2 in March 2013, and Phase 3 in October 2013. Algorithms are identified in this report by the letter code in column 2, the phase it was submitted, and a sequence number starting from zero. This report does not include results for Phase 1 algorithms. NIST limited the total number of algorithms to 7 .

\section{Introduction}

One-to-many identification represents the largest market for face recognition technology. Algorithms are used across the world in a diverse range of biometric applications: detection of duplicates in databases, detection of fraudulent applications for credentials such as passports and driving licenses, token-less access control, surveillance, social media tagging, lookalike discovery, criminal investigation, and forensic clustering.

FRVT tested only open-set identification algorithms because real-world applications are almost always "open-set". This means that some searches have an enrolled mate, but some do not. For example, some subjects have truly not been issued a visa or drivers license before; some law enforcement searches are from first-time arrestees ${ }^{1}$. In an "open-set" application, algorithms make no prior assumption about whether or not to return a high-scoring result, and for a mated search, the ideal behaviour is that the search produces the correct mate at high score and first rank. For a nonmate search, the ideal behavior is that the search produces zero high-scoring candidates.

In most applications, the core accuracy of a facial recognition algorithm is the most important performance variable. Resource consumption will be important also as it drives the amount of hardware, power, and cooling necessary to accomodate workflows. Algorithms consume processing time, they require computer memory, and their static template data requires storage space. This report documents all of these variables.

\section{Participation}

The organizations listed in Table 1 elected to submit algorithm to the FRVT evaluation.

\footnotetext{
${ }^{1}$ Operationally closed-set applications are rare because it is usually not the case that all searches have an enrolled mate. One counter-example, however, is a cruise ship in which all passengers are enrolled and all searches should produce one, and only one, identity. Another example is forensic identification of dental records from an aircraft crash.
}

\begin{tabular}{|c|c|c|c|c|c|c|}
\hline $\begin{array}{l}\mathrm{A}=3 \mathrm{M} / \text { Cogent } \\
\mathrm{G}=\text { Hisign } \\
\mathrm{P}=\text { Zhuhai-Yisheng }\end{array}$ & $\begin{array}{l}\mathrm{B}=\text { Cognitec } \\
\mathrm{H}=\mathrm{CAS}-\mathrm{IA} \\
\mathrm{Q}=\text { JunYu }\end{array}$ & $\begin{array}{l}\mathrm{C}=\text { Neurotechnology } \\
\mathrm{I}=\text { CAS-ICT } \\
\mathrm{S}=\text { Decatur }\end{array}$ & $\begin{array}{l}\mathrm{D}=\text { Safran Morpho } \\
\mathrm{J}=\text { Toshiba } \\
\mathrm{T}=\text { Ayonix }\end{array}$ & $\begin{array}{l}\mathrm{E}=\mathrm{NEC} \\
\mathrm{L}=\text { Tsinghua U. II }\end{array}$ & $\begin{array}{l}\mathrm{F}=\text { Tsinghua } \mathrm{U} \\
\mathrm{M}=\mathrm{HP}\end{array}$ & $\begin{array}{l}\text { FNIR(N,R,T,L) “Miss rate" } \\
\text { FPIR(N,T,L) “False alarm rate" }\end{array}$ \\
\hline
\end{tabular}




\begin{tabular}{|c|c|c|c|}
\hline Property & LEO/Mugshot & LEO/Webcam & VISA \\
\hline Collection Environment & Law enforcement booking & Border detainee booking & Visa application process \\
\hline Collection Era & $\sim 1960 \mathrm{~s}-2008$ & 2006-2010 & 2006-2010 \\
\hline Camera & Digital, live & Webcam, live & Scanned paper, some live \\
\hline Documentation & NIST Special Db 32 Vol 1 & NIST Special Db 32 Vol 1 & http://travel.state.gov/visa/visa_1750.html \\
\hline Image dimensions & $\begin{array}{l}\text { Various, } 480 \times 640,240 \times 240, \\
768 \times 960\end{array}$ & $240 \times 240$ & Mostly 252x300 \\
\hline Compression & JPEG 20:1, mean-size: $48 \mathrm{kB}$ & JPEG, mean size: $5.7 \mathrm{kB}$ & JPEG, mean size: $9.2 \mathrm{kB}$ \\
\hline Eye to eye distance & Mean $=107$ pixels, $\mathrm{SD}=40$ pixels & Mean $=45$ pixels, $\mathrm{SD}=12$ & Mean $=67$ pixels, $\mathrm{SD}=6$ \\
\hline Frontal pose & Moderate control & Poor control & Well controlled \\
\hline Full frontal geometry & $\begin{array}{l}\text { Mostly, but varying torso } \\
\text { visibility }\end{array}$ & $\begin{array}{l}\text { Rarely, cluttered, large, bright } \\
\text { backgrounds }\end{array}$ & $\begin{array}{l}\text { Good but cropped more closely than ISO } \\
\text { full-frontal }\end{array}$ \\
\hline Parent & Operational data & Operational data & Operational data \\
\hline Population & US, adult & Central America, adult & Global, adult + children \\
\hline
\end{tabular}

Table 2: Image sources and properties. The table summarizes the key characteristics of the images used in this study.

\section{Evaluation datasets}

\subsection{Image types}

LEO images: As in our last evaluation, this report primarily reports results for an operational dataset of law enforcement images, referred to as LEO. Additionally it employs a new dataset of VISA images. The properties of these sets is summarized in Table 2. As shown in Figure 2, the LEO set contains images of two distinct types:

$\triangleright$ Mugshots: Comprising about $86 \%$ of the LEO database, are mugshots having reasonable compliance with the ANSI / NIST ITL 1-2011 Type 10 standard's subject acquisition profiles levels 10-20 for frontal images [27]. The major departure from the standard's requirements is the presence of mild pose variations around frontal - the images of Figure 2 are typical. The images vary in size, with many being 480x600 pixels with JPEG compression applied to produce filesizes of between 18 and 36KB with many images outside this range, implying that about 1.25 bits are being encoded per color pixel.

$\triangleright$ Webcam images: The remaining $14 \%$ of the images were collected using an inexpensive webcam attached to a flexible operator-directed mount. These images are all of size 240x240 pixels, that are in considerable violation of most quality-related clauses of all face recognition standards. As evident in the figure, the most common defects are non-frontal pose (associated with the rotational degrees of freedom of the camera mount), low contrast (due to varying and intense background lights), and poor spatial resolution (due to inexpensive camera optics). The images are overly JPEG compressed, to between 4 and 7KB, implying that only 0.5 to 1 bits are being encoded per color pixel.

In our 2010 report [12], accuracy was only stated for the aggregrate LEO dataset. Here, for the first time, we report accuracy separately for the mugshots and the webcams images.

Visa images: In addition, we utilize a smaller visa database that has not been used in prior NIST evaluations. It consists of very well controlled frontal photographs of adults and children born worldwide. However, some of the images are scanned from paper photographs and therefore exhibit a reduced optical resolution. Further, as noted in Table 2, the images have a low mean interocular distances (IOD) of 67 pixels and compressed sizes of 9.2KB. These values are below the preferred minimums for the "Token" image format indicated in the ISO/IEC 19794-5:2005 standard for modern credentials, namely 120 pixels interocular distance, filesize of at least 15KB, and an image size of 480x640 pixels.

FERET Sketch images: The FERET database was collected in the 1990s and has been very widely studied. As such it is not used for evaluation here. However the City University of Hong Kong employed an artist to produce, for each person,

\begin{tabular}{|c|c|c|c|c|c|c|}
\hline $\begin{array}{l}A=3 \mathrm{M} / \text { Cogent } \\
G=\text { Hisign } \\
P=\text { Zhuhai-Yisheng }\end{array}$ & $\begin{array}{l}\mathrm{B}=\text { Cognitec } \\
\mathrm{H}=\text { CAS-IA } \\
\mathrm{Q}=\text { JunYu }\end{array}$ & $\begin{array}{l}\mathrm{C}=\text { Neurotechnology } \\
\mathrm{I}=\text { CAS-ICT } \\
\mathrm{S}=\text { Decatur }\end{array}$ & $\begin{array}{l}\mathrm{D}=\text { Safran Morpho } \\
\mathrm{J}=\text { Toshiba } \\
\mathrm{T}=\text { Ayonix }\end{array}$ & $\begin{array}{l}\mathrm{E}=\mathrm{NEC} \\
\mathrm{L}=\text { Tsinghua U. II }\end{array}$ & $\begin{array}{l}\mathrm{F}=\text { Tsinghua } \mathrm{U} \\
\mathrm{M}=\mathrm{HP}\end{array}$ & $\begin{array}{l}\text { FNIR(N,R,T,L) "Miss rate" } \\
\text { FPIR(N,T,L) “False alarm rate" }\end{array}$ \\
\hline
\end{tabular}




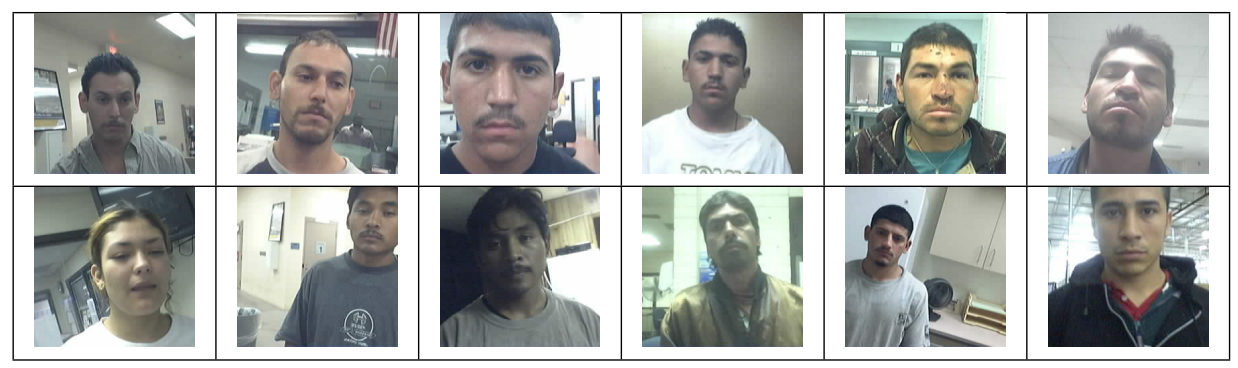

(a) Webcam

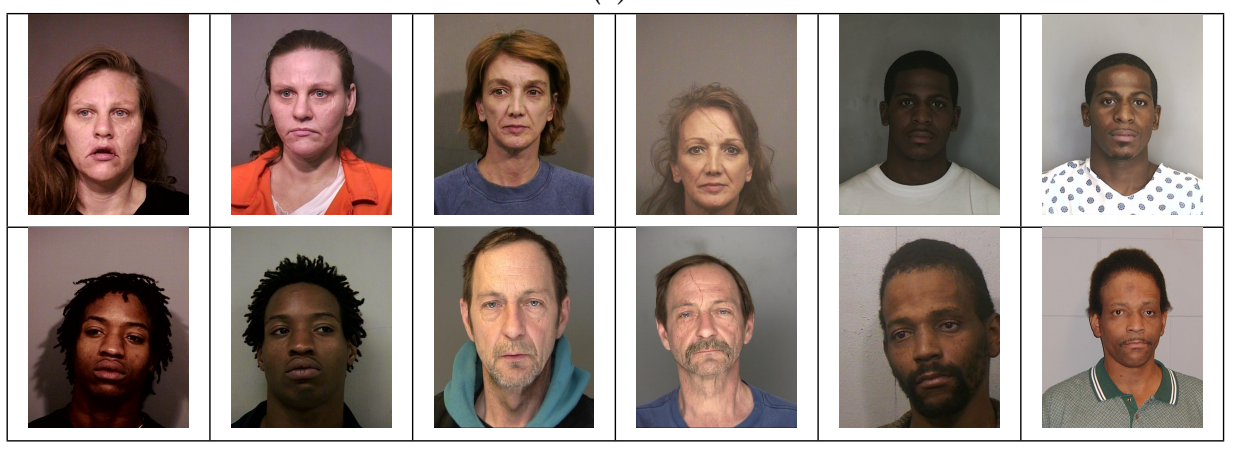

(b) Mugshot

Figure 2: Examples of the two kinds of image that comprise the LEO database.

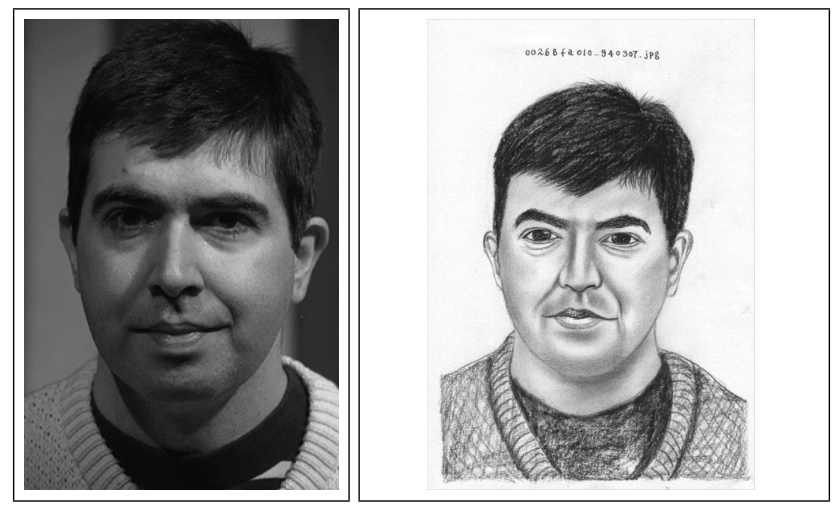

Figure 3: Sketch realism: A pair of images of an individual in the FERET database. At left is the grayscale version of the original 1994 frontal image. At right is the artists sketch of the grayscale image. This production of a sketch is atypical operationally: it is unusual for an artist to have access to an image of the individual.

\begin{tabular}{|c|c|c|c|c|c|c|}
\hline $\begin{array}{l}\mathrm{A}=3 \mathrm{M} / \text { Cogent } \\
\mathrm{G}=\text { Hisign } \\
\mathrm{P}=\text { Zhuhai-Yisheng }\end{array}$ & $\begin{array}{l}\mathrm{B}=\text { Cognitec } \\
\mathrm{H}=\text { CAS-IA } \\
\mathrm{Q}=\text { Jun } \mathrm{Yu}\end{array}$ & $\begin{array}{l}\mathrm{C}=\text { Neurotechnology } \\
\mathrm{I}=\mathrm{CAS}-\mathrm{ICT} \\
\mathrm{S}=\text { Decatur }\end{array}$ & $\begin{array}{l}\mathrm{D}=\text { Safran Morpho } \\
\mathrm{J}=\text { Toshiba } \\
\mathrm{T}=\text { Ayonix }\end{array}$ & $\begin{array}{l}\mathrm{E}=\mathrm{NEC} \\
\mathrm{L}=\text { Tsinghua U. II }\end{array}$ & $\begin{array}{l}\mathrm{F}=\text { Tsinghua } \mathrm{U} . \\
\mathrm{M}=\mathrm{HP}\end{array}$ & $\begin{array}{l}\text { FNIR(N,R,T,L) “Miss rate" } \\
\text { FPIR(N,T,L) “False alarm rate" }\end{array}$ \\
\hline
\end{tabular}




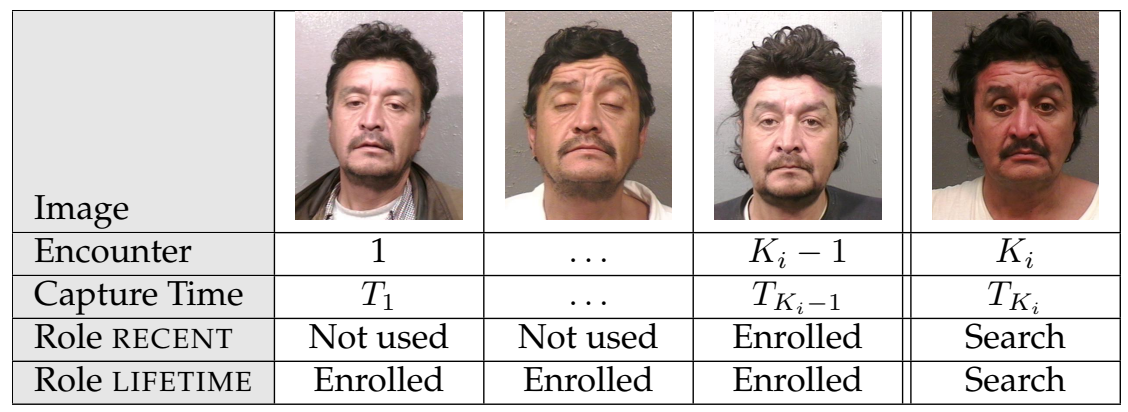

Figure 4: Depiction of the "recent" and "lifetime" enrollment types.

"a face photo with lighting variation and a sketch with shape exaggeration drawn by an artist when viewing this photo." The data was collected to support research into synthesis and recognition of sketches [25,28]. An example is shown in Figure 3.

\subsection{Enrolment types}

Many operational applications include collection and enrolment of biometric data from subjects on more than one occasion. This might be done on a regular basis, as might occur in credential (re-)issuance, or irregularly, as might happen in a criminal recidivist situation [3]. The number of images per person will depend on the application area: In civil identity credentialing (e.g. passports, driving licenses), the images will be acquired approximately uniformly over time (e.g. ten years for a German passport). While the distribution of dates for such images of a person might be assumed uniform, a number of factors might undermine this assumption ${ }^{2}$. In criminal applications, the number of images would depend on the number of arrests. The distribution of dates for arrest records for a person (i.e. the recidivism distribution) has been modeled using the exponential distribution but is recognized to be more complicated ${ }^{3}$.

In any case, the 2010 NIST evaluation of face recognition showed that considerable accuracy benefits accrue with retention and use of all historical images [12].

To this end, the FRVT API document provides $K \geq 1$ images of an individual to the enrolment software. The software is tasked with producing a single proprietary undocumented "black-box" template ${ }^{4}$ from the $K$ images. This affords the algorithm an ability to generate a model of the individual, rather than to simply extract features from each image on a sequential basis.

As depicted in Figure 4, the $i$-th individual in the LEO dataset has $K_{i}$ images. These are labelled $x_{k}$ for $k=1 \ldots K_{i}$. To measure the utility of having multiple enrolment images, this report evaluates two kinds of enrolment:

$\triangleright$ Recent: Only the second most recent image, $x_{K_{i}-1}$ is enrolled. This type of enrolment mimics the operational policy of retaining the imagery from the most recent encounter. This might be done operationally to ameliorate the effects of face ageing.

$\triangleright$ Lifetime: All except the last image are enrolled, $x_{1} \ldots x_{K_{i}-1}$. This strategy might be adopted if quality variations exist where an older image might be more suitable for matching, despite ageing.

\footnotetext{
${ }^{2}$ For example, a person might skip applying for a passport for one cycle, letting it expire. In addition, a person might submit identical images (from the same photography session) to consecutive passport applications at five year intervals.

${ }^{3}$ A number of distributions have been considered to model recidivism, see for example [2].

${ }^{4}$ There are no formal face template standards. Template standards only exist for fingerprint minutiae - see ISO/IEC 19794-2:2011.
}

\begin{tabular}{|c|c|c|c|c|c|c|}
\hline $\begin{array}{l}\mathrm{A}=3 \mathrm{M} / \text { Cogent } \\
\mathrm{G}=\text { Hisign } \\
\mathrm{P}=\text { Zhuhai-Yisheng }\end{array}$ & $\begin{array}{l}\mathrm{B}=\text { Cognitec } \\
\mathrm{H}=\text { CAS-IA } \\
\mathrm{Q}=\text { JunYu }\end{array}$ & $\begin{array}{l}\mathrm{C}=\text { Neurotechnology } \\
\mathrm{I}=\mathrm{CAS}-\mathrm{ICT} \\
\mathrm{S}=\text { Decatur }\end{array}$ & $\begin{array}{l}\mathrm{D}=\text { Safran Morpho } \\
\mathrm{J}=\text { Toshiba } \\
\mathrm{T}=\text { Ayonix }\end{array}$ & $\begin{array}{l}\mathrm{E}=\mathrm{NEC} \\
\mathrm{L}=\text { Tsinghua U. II }\end{array}$ & $\begin{array}{l}\mathrm{F}=\text { Tsinghua } \mathrm{U} \\
\mathrm{M}=\mathrm{HP}\end{array}$ & $\begin{array}{l}\text { FNIR(N,R,T,L) “Miss rate" } \\
\text { FPIR(N,T,L) “False alarm rate" }\end{array}$ \\
\hline
\end{tabular}




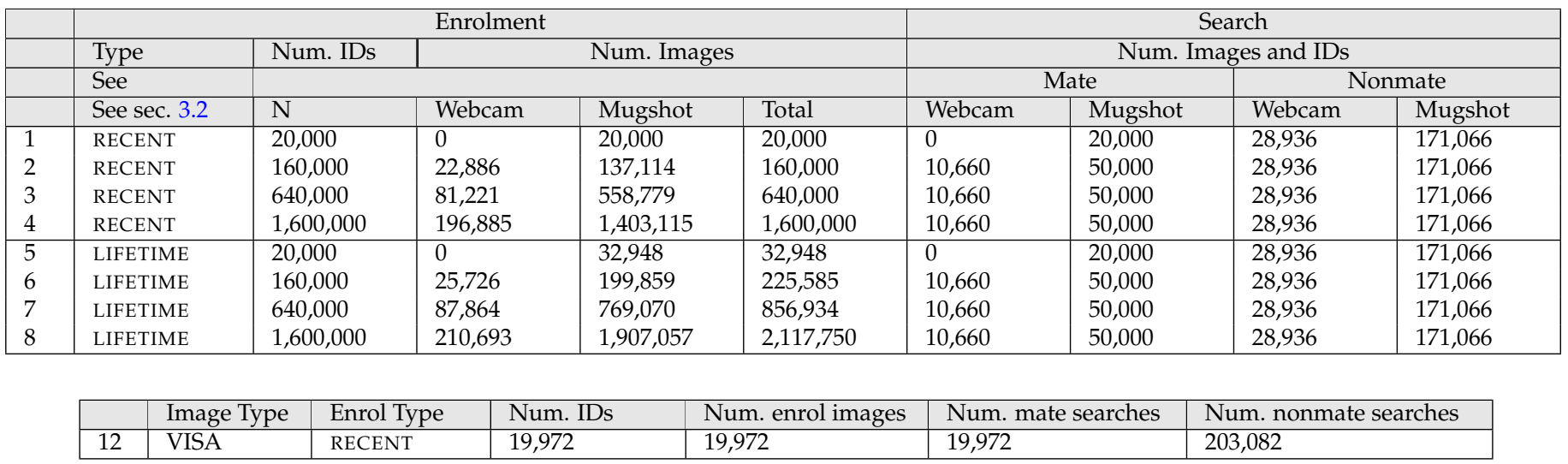

Table 3: Enrolment and search sets. Each row summarizes one identification trial. The upper table concerns use of the LEO images; the lower, the VISA images. The column labeled "Num. IDs" gives the number of enrolled identities. This precedes the numbers of images, and then the number of mate, and nonmate, searches. Rows 1-8 describe trials in which webcam and mugshot images are enrolled in the natural proportions. Row 12 refers to the visa database that, uniquely, contains images of children.

In all cases, the most recent image, $x_{K_{i}}$, is reserved as the search image. For the 1.6 million subject enrolment parition of the LEO data, $1 \leq K_{i} \leq 33$ with $K_{i}=1$ in $80.1 \%$ of the individuals, $K_{i}=2$ in $13.4 \%, K_{i}=3$ in $3.7 \%, K_{i}=4$ in $1.4 \%$, $K_{i}=5$ in $0.6 \%, K_{i}=6$ in $0.3 \%$, and $K_{i}>6$ is $0.2 \%$ for everyone else. This distribution is substantially dependent on United States recidivism rates.

We did not evaluate the case of retaining only the highest quality image, since automated quality assessment is out of scope for this report. We do not anticipate that such strategies will prove beneficial when the quality assessment apparatus is imperfect and unvalidated.

Finally, we did not evaluate the case where $K_{i}>1$ images from the same person are enrolled under different identifiers. This very common circumstance arises in so-called "event-based" applications where no attempt is made to consolidate images of a person into a single identity. Searches against such systems are likely to yield more than one image of a person in the top ranks. We do not test this kind of enrolment. Instead, we use our consolidated identity design to: a) realize accuracy gains by affording the algorithm the opportunity to build a holistic model of identity (as is common in speaker recognition systems); and b) to simplify accuracy measurement.

\subsection{Limitations of the data}

Neither the mughots nor the visa images have ideal properties. The former, particularly, has too much pose variation, and the latter is degraded by its acquistion process (scan-from-paper) and too much JPEG compression. A prior NIST report [21] documents the dependence of recognition accuracy on compression and spatial sampling rate (eye-to-eye distance). While results for the LEO/Mugshot images are the best indicators of the accuracy that can be expected in contemporary deployments of one-to-many identification, it should be understood that accuracy increases can be realized by improving conformance to the appearance-related requirements of the ISO/IEC 19794-5 standard [11] ${ }^{5}$. That said, conformance to that standard requires both deliberate system design (capture equipment, capture environment, image preparation processes), and capture-time monitoring (to check for aberrant subject behavior). This latter aspect will in turn require conformance checks, either automated or human, even if the photographic design is perfect. Commercial quality-conformance checking tools are available, and recently have become subject to independent testing $[9,10]$. Human-

5 The ISO/IEC 19794-5:2005 Face Image standard [11], was amended in 2007 to guide photography [6] and, in 2009, to add 3D data. These additions and and other improvements resulted in a second edition in 2011 [26]. Passport guidelines(e.g. [24]) are derived from this standard.

\begin{tabular}{|c|c|c|c|c|c|c|}
\hline $\begin{array}{l}\mathrm{A}=3 \mathrm{M} / \text { Cogent } \\
\mathrm{G}=\text { Hisign } \\
\mathrm{P}=\text { Zhuhai-Yisheng }\end{array}$ & $\begin{array}{l}\mathrm{B}=\text { Cognitec } \\
\mathrm{H}=\text { CAS-IA } \\
\mathrm{Q}=\text { JunYu }\end{array}$ & $\begin{array}{l}\mathrm{C}=\text { Neurotechnology } \\
\mathrm{I}=\text { CAS-ICT } \\
\mathrm{S}=\text { Decatur }\end{array}$ & $\begin{array}{l}\mathrm{D}=\text { Safran Morpho } \\
\mathrm{J}=\text { Toshiba } \\
\mathrm{T}=\text { Ayonix }\end{array}$ & $\begin{array}{l}\mathrm{E}=\mathrm{NEC} \\
\mathrm{L}=\text { Tsinghua } \mathrm{U} . \mathrm{II}\end{array}$ & $\begin{array}{l}\mathrm{F}=\text { Tsinghua } \mathrm{U} \\
\mathrm{M}=\mathrm{HP}\end{array}$ & $\begin{array}{l}\text { FNIR(N,R,T,L) “Miss rate" } \\
\text { FPIR(N,T,L) “False alarm rate" }\end{array}$ \\
\hline
\end{tabular}


mediated checks on image quality are common in passport and visa issuance procedures where photographers and, later, immigration officials, check adherence to published quality requirements.

Results for the webcam partition have some applicability to cases where image quality is significantly degraded e.g. bank ATM machine, surveillance, although the data is acquired from (mostly) cooperating subjects.

\section{Performance metrics}

This section gives specific definitions for accuracy and timing metrics. Tests of open-set biometric algorithms must quantify frequency of two error conditions:

$\triangleright$ False alarms: Type I errors occur when search data from a person who has never been seen before is incorrectly associated with one or more enrollees' data.

$\triangleright$ Misses: Type II errors arise when a search of an enrolled person's biometric does not return the correct identity.

Many practitioners prefer to talk about "hit rates" instead of "miss rates" - the first is simply one minus the other as detailed below. Sections 4.1 and 4.2 define metrics for the Type I and Type II performance variables.

Additionally, because recognition algorithms sometimes fail to produce a template from an image, or fail to execute a oneto-many search, the occurrence of such events must be recorded. Further because algorithms might elect to not produce a template from, for example, a poor quality image, these failure rates must be combined with the recognition error rates to support algorithm comparison. This is addressed in section 4.4 .

Finally, section 4.5 discusses measurement of computation duration, and section 4.6 addresses the uncertainty associated with various measurements. Template size measurement is included with the results.

\subsection{Quantifying false alarms}

It is typical for a search to be conducted into an enrolled population of $N$ identities, and for the algorithm to be configured to return the closest $L$ candidate identities. These candidates are ranked by their score, in descending order. A human analyst might examine either all $L$ candidates, or just the top $R \leq L$ identities, or only those with score greater than threshold, $T$. The workload associated with such examination is discussed later, in 5.7.

False alarm performance is quantified in two related ways. These express how many searches produces false positives, and then, how many false positives are produced in a search.

False positive identification rate: The first quantity, FPIR, is the proportion of nonmate searches that produce an adverse outcome:

$$
\operatorname{FPIR}(N, T, L)=\frac{\text { Num. nonmate searches where one or more enrolled candidates are returned at or above threshold, } \mathrm{T}}{\text { Num. nonmate searches attempted. }}
$$

Under this definition, FPIR can be computed from the highest nonmate candidate produced in a search - it is not necessary to consider candidates at rank 2 and above. FPIR is the primary measure of Type I errors in this report.

Selectivity: However, note that in any given search, more than one nonmate may be returned above threshold. In order to quantify such events, a second quantity, selectivity (SEL), is defined as the number of nonmates returned on a candidate

\begin{tabular}{|c|c|c|c|c|c|c|}
\hline $\begin{array}{l}\mathrm{A}=3 \mathrm{M} / \text { Cogent } \\
\mathrm{G}=\text { Hisign } \\
\mathrm{P}=\text { Zhuhai-Yisheng }\end{array}$ & $\begin{array}{l}\mathrm{B}=\text { Cognitec } \\
\mathrm{H}=\text { CAS-IA } \\
\mathrm{Q}=\text { Jun } \mathrm{Yu}\end{array}$ & $\begin{array}{l}\mathrm{C}=\text { Neurotechnology } \\
\mathrm{I}=\mathrm{CAS}-\mathrm{ICT} \\
\mathrm{S}=\text { Decatur }\end{array}$ & $\begin{array}{l}\mathrm{D}=\text { Safran Morpho } \\
\mathrm{J}=\text { Toshiba } \\
\mathrm{T}=\text { Ayonix }\end{array}$ & $\begin{array}{l}\mathrm{E}=\mathrm{NEC} \\
\mathrm{L}=\text { Tsinghua U. II }\end{array}$ & $\begin{array}{l}\mathrm{F}=\text { Tsinghua } \mathrm{U} \\
\mathrm{M}=\mathrm{HP}\end{array}$ & $\begin{array}{l}\text { FNIR(N,R,T,L) “Miss rate" } \\
\text { FPIR(N,T,L) “False alarm rate" }\end{array}$ \\
\hline
\end{tabular}




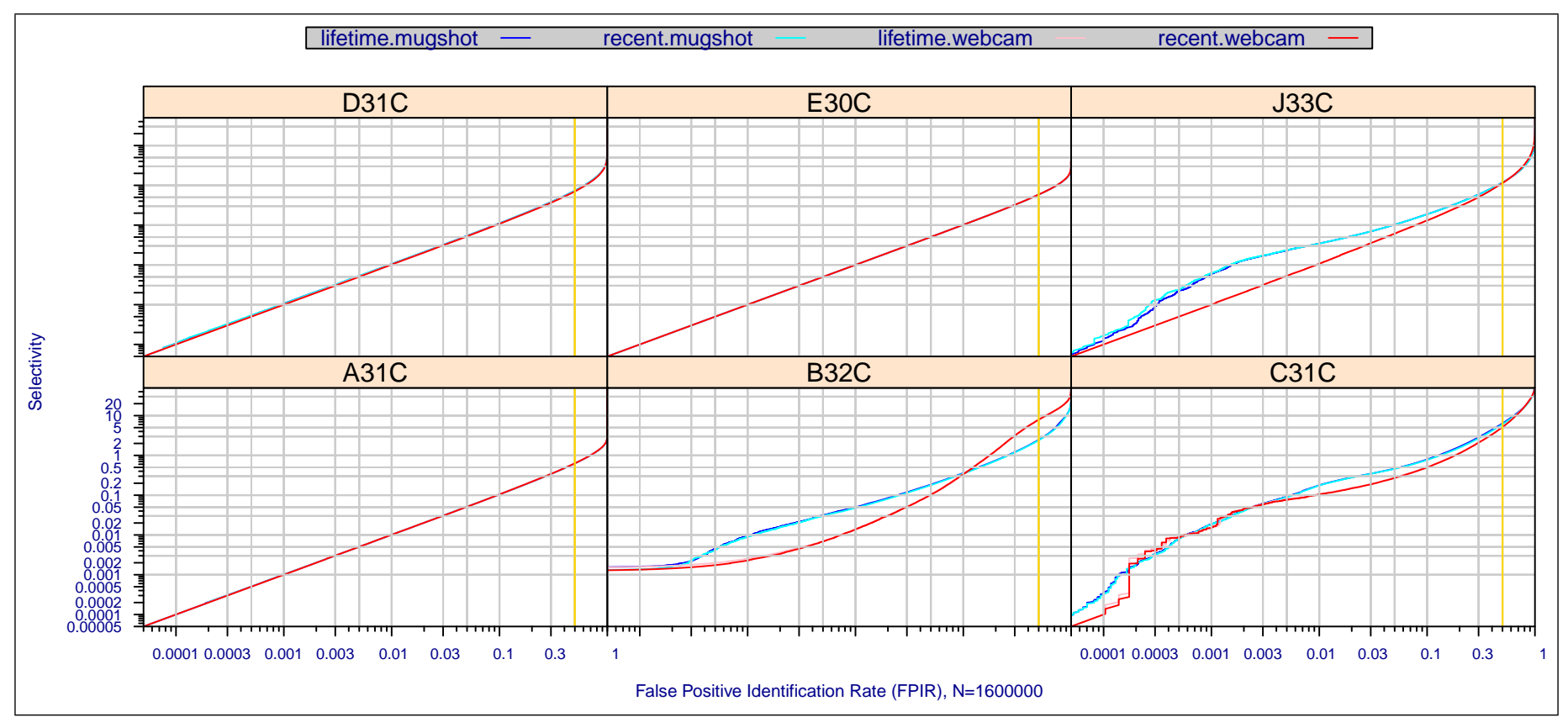

Figure 5: Relationship of SEL and FPIR. For six algorithms from the six most accurate suppliers, the figures plot SEL(N, T, L) vs. FPIR(N, T, L) parametrically with threshold, T. Selectivity is always greater than or equal to FPIR. The two are not equal when false positives are concentrated in candidate lists rather than being distributed across searches. The population size is $N=1,600,000$. Algorithms were tasked with reporting $L=50$ candidates. Each panel corresponds to one algorithm. The red lines correspond to searching poor quality webcam images. The blue lines correspond to searching better quality mugshot images. Analogous plots for other algorithms appear in the "report cards" of Appendix A.

list, averaged over all searches.

$$
\operatorname{SEL}(N, T, L)=\frac{\text { Num. nonmate enrolled candidates returned at or above threshold, } \mathrm{T}}{\text { Num. nonmate searches attempted. }}
$$

Both of these metrics are useful operationally. FPIR is useful for targeting how often an adverse false positive outcome can occur, while SEL as a number is related to workload associated with adjudicating candidate lists. The relationship between the two quantities is complicated - it depends on whether an algorithm concentrates the false alarms in the results of a few searches or whether it disburses them across many. Figure 5 plots $\operatorname{SEL}(T)$ vs. $\operatorname{FPIR}(T)$ for several algorithms. Note that SEL $\geq$ FPIR, by definition. It is clear that some algorithms exhibit an identity relationship, SEL = FPIR, except at high values, but that some algorithms give selectivity values with complicated dependence on FPIR. It is not clear that the linear behavior is more, or less, desirable. On the one hand, the concentration of false matches in particular searches would lead to reduced adjudication labor in the long run. But on the other hand, the presence of several false matches on a candidate list is peculiar in that it is unlikely that several individuals would be biometrically similar to a search image. Such occurrences must be due to some property common to the pair of the images (for example, similar pose, the presence of moles, or heavy-framed glasses) and of algorithm sensitivity to it.

\subsection{Quantifying hits and misses}

If $L$ candidates are returned in a search, a shorter candidate list can be prepared by taking the top $R \leq L$ candidates for which $T \geq 0$. This reduction of the candidate list is done because thresholds may be applied, and only short lists might be reviewed (according to policy or labor availability, for example). It is useful then to state accuracy in terms of $R$ and $T$, so

\begin{tabular}{|c|c|c|c|c|c|c|}
\hline $\begin{array}{l}\mathrm{A}=3 \mathrm{M} / \text { Cogent } \\
\mathrm{G}=\text { Hisign } \\
\mathrm{P}=\text { Zhuhai-Yisheng }\end{array}$ & $\begin{array}{l}\mathrm{B}=\text { Cognitec } \\
\mathrm{H}=\text { CAS-IA } \\
\mathrm{Q}=\text { JunYu }\end{array}$ & $\begin{array}{l}\mathrm{C}=\text { Neurotechnology } \\
\mathrm{I}=\text { CAS-ICT } \\
\mathrm{S}=\text { Decatur }\end{array}$ & $\begin{array}{l}\mathrm{D}=\text { Safran Morpho } \\
\mathrm{J}=\text { Toshiba } \\
\mathrm{T}=\text { Ayonix }\end{array}$ & $\begin{array}{l}\mathrm{E}=\mathrm{NEC} \\
\mathrm{L}=\text { Tsinghua U. II }\end{array}$ & $\begin{array}{l}\mathrm{F}=\text { Tsinghua } \mathrm{U} \\
\mathrm{M}=\mathrm{HP}\end{array}$ & $\begin{array}{l}\text { FNIR(N,R,T,L) “Miss rate" } \\
\text { FPIR(N,T,L) “False alarm rate" }\end{array}$ \\
\hline
\end{tabular}


we define a "miss rate" with the general name false negative identification rate (FNIR), as follows:

$$
\operatorname{FNIR}(N, R, T, L)=\frac{\text { Num. mate searches with enrolled mate found outside top R ranks or score below threshold, } \mathrm{T}}{\text { Num. mate searches attempted. }}
$$

This formulation is simple for evaluation in that it does not distinguish between causes of misses. Thus a mate that is not reported on a candidate list is treated the the same as a miss arising from face finding failure, algorithm intolerance of poor quality, or software crashes. Thus if the algorithm fails to produce a candidate list, either because the search failed, or because a search template was not made, the result is regarded as miss, adding to FNIR.

Hit rates, and true positive identification rates: While FNIR states the "miss rate" as how often the correct candidate is either not above threshold or not at good rank, many communities prefer to talk of "hit rates". This is simply the true positive identification rate(TPIR) which is the complement of FNIR giving a positive statement of how often mated searches are successful:

$$
\operatorname{TPIR}(N, R, T, L)=1-\operatorname{FNIR}(N, R, T, L)
$$

Reliability and sensitivity are corresponding terms, typically being identical to TPIR. This quantity is often cited in automated fingerprint identification system (AFIS) evaluations.

An important special case is the cumulative match characteristic(CMC) which summarizes accuracy of mated-searches only. It ignores similarity scores by relaxing the threshold requirement, and just reports the fraction of mated searches returning the mate at rank $\mathrm{R}$ or better.

$$
\operatorname{CMC}(N, R, L)=1-\operatorname{FNIR}(N, R, 0, L)
$$

We primarily cite the complement of this quantity $1-\mathrm{FNIR}$, the fraction of mates not in the top R ranks.

The rank one hit rate is the fraction of mated searches yielding the correct candidate at best rank, i.e. CMC (N, 1, L). While this quantity is the most common summary indicator of an algorithms' efficacy, it is not dependent on similarity scores, so it does not distinguish between strong (high scoring) and weak hits. It also ignores that an adjudicating reviewer is often willing to look at many candidates.

\subsection{Best practice testing requires execution of searches with and without mates}

FRVT embedded 1:N searches of two kinds: Those for which there is an enrolled mate, and those for which there is not. The respective numbers for these types of searches appear in Table 3. However, it is common to conduct only mated searches. This is bad practice because if the information that a mate always exists is revealed to a test participant, or can be reasonably assumed, then unrealistic gaming of the test is possible. The cumulative match characteristic is computed from candidate lists produced in mated searches. Even if the CMC is the only metric of interest, the actual trials executed in a test should nevertheless include searches for which no mate exists. As detailed in Table 3 the FRVT reserved disjoint populations of subjects for executing true nonmate searches.

\subsection{Failure to extract}

Template generation can fail either during enrolment, or ahead of one-to-many search. This report quantifies only failure-

\begin{tabular}{|c|c|c|c|c|c|c|}
\hline $\begin{array}{l}\mathrm{A}=3 \mathrm{M} / \text { Cogent } \\
\mathrm{G}=\text { Hisign } \\
\mathrm{P}=\text { Zhuhai-Yisheng }\end{array}$ & $\begin{array}{l}\mathrm{B}=\text { Cognitec } \\
\mathrm{H}=\text { CAS-IA } \\
\mathrm{Q}=\text { JunYu }\end{array}$ & $\begin{array}{l}\mathrm{C}=\text { Neurotechnology } \\
\mathrm{I}=\text { CAS-ICT } \\
\mathrm{S}=\text { Decatur }\end{array}$ & $\begin{array}{l}\mathrm{D}=\text { Safran Morpho } \\
\mathrm{J}=\text { Toshiba } \\
\mathrm{T}=\text { Ayonix }\end{array}$ & $\begin{array}{l}\mathrm{E}=\mathrm{NEC} \\
\mathrm{L}=\text { Tsinghua U. II }\end{array}$ & $\begin{array}{l}\mathrm{F}=\text { Tsinghua } \mathrm{U} \\
\mathrm{M}=\mathrm{HP}\end{array}$ & $\begin{array}{l}\text { FNIR(N,R,T,L) “Miss rate" } \\
\text { FPIR(N,T,L) “False alarm rate" }\end{array}$ \\
\hline
\end{tabular}
to-enrol. This quantity is denoted FTE. It is computed as the proportion of images that do not produce an output template. The corresponding search-phase number, FTX, is not reported because we consider the FTE number adequate. This is 
supported by a) the observed error rates being very low, usually zero; b) we assume, that the same underlying algorithm is used ${ }^{6}$; and c) the enrolment sets are much larger.

Failure to extract rates are incorporated into FNIR and FPIR measurements as follows.

$\triangleright$ Enrolment templates: Any failed enrolment is regarded as producing a zero length template. Algorithms are required by the API [14] to transparently process zero length templates. The effect of template generation failure on search accuracy depends on whether subsequent searches are mated, or nonmated: Mated searches will fail giving elevated FNIR; Nonmated searches will not produce false positives, so FPIR will be reduced by (to first order) a factor of 1-FTE.

$\triangleright$ Search templates and 1:N search: In cases where the algorithm fails to produce a search template from input imagery, the result is taken to be a candidate list whose entries have no hypothesized identities and zero score. The effect of template generation failure on search accuracy depends on whether searches are mated, or nonmated: Mated searches will fail giving elevated FNIR; Nonmated searches will not produce false positives, so FPIR will be reduced.

$$
\begin{gathered}
\mathrm{FNIR}^{\dagger}=\mathrm{FTX}+(1-\mathrm{FTX}) \mathrm{FNIR} \\
\mathrm{FPIR}^{\dagger}=(1-\mathrm{FTX}) \mathrm{FPIR}
\end{gathered}
$$

This approach is the correct treatment for positive-identification applications such as access control where cooperative users are enrolled and make attempts at recognition. This approach is not appropriate to negative identification applications, such as visa fraud detection, in which hostile individuals may attempt to evade detection by submitting poor quality samples. In those cases, template generation failure should be investigated as though a false alarm had occurred.

\subsection{Timing measurement}

Algorithms were submitted to NIST as implementations of the application programming interface(API) specified by NIST in the Evaluation Plan [14]. The API includes functions for initialization, template generation, finalization, and search. Two template generation functions are required, one for the preparation of an enrolment template, and one for a search template.

In NIST's test harness, all functions were wrapped by calls to the function get_time_of_day() which enables duration measurements with microsecond resolution. Timing was measured on a dedicated computer equipped with 192GB of main memory. The computer was not running any other processes except those back-grounded as part of the operating system. Timing measurements do not include disk access unless the algorithm under test elected to access enrolment or configuration data during a search (something that is not necessary because the API supported initialization prior to searching).

The FRVT test plan formally stated the durations limits on the core elemental functions of the algorithms. The times were stated as 90 -th percentiles.

\subsection{Uncertainty estimation}

This study leverages operational datasets for measurement of recognition error rates. This affords several advantages. First, large numbers of searches are conducted (see Table 3) giving precision to the measurements. Moreover, these do

${ }^{6}$ While this assumption is violated for at least Morpho's algorithms which use larger search templates than enrolment templates, the failure to make a

\begin{tabular}{|c|c|c|c|c|c|c|}
\hline $\begin{array}{l}\mathrm{A}=3 \mathrm{M} / \text { Cogent } \\
\mathrm{G}=\text { Hisign } \\
\mathrm{P}=\text { Zhuhai-Yisheng }\end{array}$ & $\begin{array}{l}\mathrm{B}=\text { Cognitec } \\
\mathrm{H}=\text { CAS-IA } \\
\mathrm{Q}=\text { JunYu }\end{array}$ & $\begin{array}{l}\mathrm{C}=\text { Neurotechnology } \\
\mathrm{I}=\mathrm{CAS}-\mathrm{ICT} \\
\mathrm{S}=\text { Decatur }\end{array}$ & $\begin{array}{l}\mathrm{D}=\text { Safran Morpho } \\
\mathrm{J}=\text { Toshiba } \\
\mathrm{T}=\text { Ayonix }\end{array}$ & $\begin{array}{l}\mathrm{E}=\mathrm{NEC} \\
\mathrm{L}=\text { Tsinghua U. II }\end{array}$ & $\begin{array}{l}\mathrm{F}=\text { Tsinghua } \mathrm{U} . \\
\mathrm{M}=\mathrm{HP}\end{array}$ & $\begin{array}{l}\text { FNIR(N,R,T,L) "Miss rate" } \\
\text { FPIR(N,T,L) “False alarm rate" }\end{array}$ \\
\hline
\end{tabular}
template rates are very close to zero. 
not involve reuse of individuals, and thus, binomial statistics can be expected to apply to recognition error counts. In that case, an observed count of a particular recognition outcome (i.e. a false negative or false positive) in $M$ trials will sustain $95 \%$ confidence that the actual error rate is no larger than some value. As an example, the minimum number of searches conducted in this report is $M=10,660$, and the observed FNIR is never below 0.01 so the measurement supports a conclusion that the actual FNIR is no higher than 0.0113 at $95 \%$ confidence level, and 0.0133 at the $99.9 \%$ level. On the false positive side, we tabulate FNIR at FPIR values as low as 0.002. Given estimates based on 171,066 nonmate trials, the actual FPIR values will be below 0.00214 (at 95\%) and 0.00235 (at 99.9\% confidence). The point is that large scale evaluation, without reuse of subjects, supports tight uncertainty bounds on the measured error rates.

Bootstrapping is an empirical method of measuring the variability of a statistic, often employed when the variability cannot be determined analytically. In the context of this evaluation, bootstrapping is sometimes used to measure the distribution of error statistics (i.e. FNIR or FPIR) at a fixed threshold. Each bootstrap iteration samples with replacement from the original set of comparisons. The statistic of interest is then computed over the sampled data. This process is repeated for a large number of bootstrap iterations to produce a distribution of the measured statistic. Bootstrapping relies on several assumptions, including that the sample data is independent and identically distributed. However, when different comparisons involve the same individual, the comparisons are likely to be correlated due to the existence of Doddington's zoo [7]. Thus, the independence assumption is violated. Determining the effect this has on the bootstrapped distributions is beyond the scope of this evaluation, but the likely result is an underestimation of the variability of FNIR and/or FPIR in some cases. The experimental design here avoids this issue.

\section{Results}

This section details performance of the algorithms submitted to the Class-C identification algorithm track of the FRVT evaluation. Performance metrics were described in section 4 . The following subsections address specific aspects of performance. Appendix A includes a report for each algorithm, where multiple plots are assembled side-by-side.

\subsection{Comparative accuracy}

Methods: LEO/Mughots and LEO/Webcam images are enrolled in their natural distributions using both the "lifetime" and "recent" enrolment types of section 3.2. Four different population sizes are used. The numbers of enrolled individuals are $20000,160000,640000$, and 1600000 . For the recent enrolment type, the number of images is identical to the number of individuals. For the lifetime enrolment type, the numbers of enrolled images are: 32948, 225585, 856934, and 2117750 as documented in Table 3. Each successive enrolled population includes the subjects and images of the smaller population.

Two nonmate search sets are used, one containing 10660 webcam images, and another containing 171066 mugshots.

Results: Table 4 presents FNIR values for all algorithms submitted to FRVT in $2013^{7}$ applied to an enrolled population size of $\mathrm{N}=160,000$. Table 5 presents the same data for $\mathrm{N}=640,000$ but it excludes algorithms that did not demonstrate high accuracy in the $\mathrm{N}=160 \mathrm{~K}$ trial. Table 6 likewise presents the same data for $\mathrm{N}=1.6$ million enrolled identities.

Figure 6 shows detection error tradeoff (DET) characteristics for algorithms from the most capable algorithms. These summarize the tradeoff of FNIR for FPIR for searches conducted into enrolled galleries containing 1.6 million people.

Discussion: As in the 2010 test, the algorithms from the NEC corporation give broadly the lowest error rates on all

${ }^{7}$ The table excludes entries that did not execute to completion or that did not meet minimum speed criteria. It additionally excludes algorithms submitted to phase 1 of the FRVT in August 2012.

$\mathrm{A}=3 \mathrm{M} /$ Cogent

$\mathrm{G}=$ Hisign

$\mathrm{P}=$ Zhuhai-Yisheng
$\mathrm{B}=$ Cognitec

\begin{tabular}{l|l}
$\mathrm{B}=$ Cognitec & $\mathrm{C}=$ Neurotechnology \\
$\mathrm{H}=$ CAS-IA & $\mathrm{I}=$ CAS-ICT
\end{tabular}

$\mathrm{Q}=\mathrm{Jun} \mathrm{Yu}$
$\mathrm{S}=$ Decatur
$\mathrm{E}=\mathrm{NEC}$

$\mathrm{L}=$ Tsinghua $\mathrm{U} . \mathrm{II}$

$\mathrm{D}=$ Safran Morpho

$\mathrm{J}=$ Toshiba
$\mathrm{T}=$ Ayonix
$\mathrm{F}=$ Tsinghua $\mathrm{U}$. $\mathrm{M}=\mathrm{HP}$
FNIR(N,R,T,L) “Miss rate"

FPIR(N,T,L) "False alarm rate" 


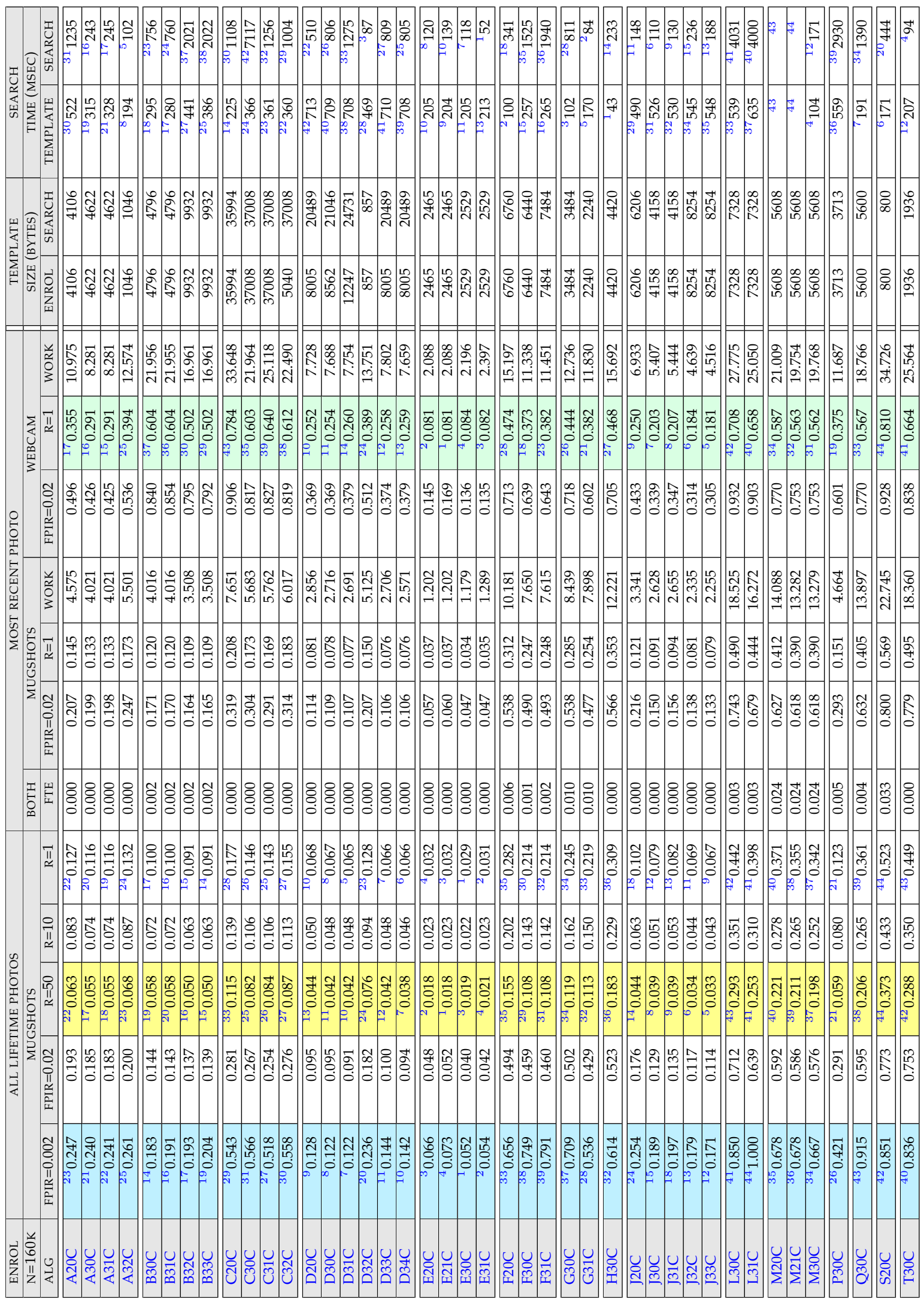

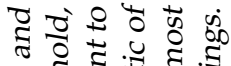
\& की के

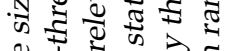

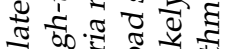
ส. छั क ष 3 है

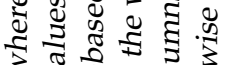

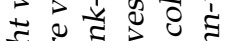

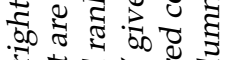

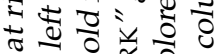
평 ष्य ช ฮิ \&

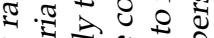

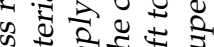
है क्षेत्य क ¿

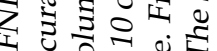

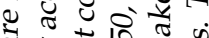
ส

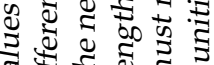

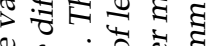
ฐ

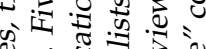
$\infty$ \&

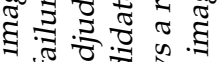
ฐ ปี ฮี ฮี ธี ฮี ฮี ฉ ¿ उ ปี ส 중 ส क छ

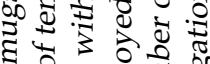
है ठै क्ष है

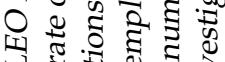

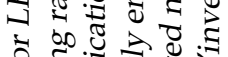

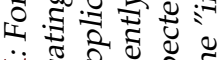
ㅂ. 워 ₹ 의 है ㄱ.

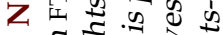

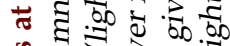
क ज के 80.5

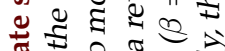
존 등 ส ब हो उ च है क क क के

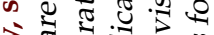
包 ฐ

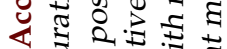
- 0 \%

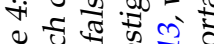
๙ิ

\begin{tabular}{|c|c|c|c|c|c|c|}
\hline $\begin{array}{l}\mathrm{A}=3 \mathrm{M} / \text { Cogent } \\
\mathrm{G}=\text { Hisign } \\
\mathrm{P}=\text { Zhuhai-Yisheng }\end{array}$ & $\begin{array}{l}B=\text { Cognitec } \\
H=\text { CAS-IA } \\
Q=\text { JunYu }\end{array}$ & $\begin{array}{l}\mathrm{C}=\text { Neurotechnology } \\
\mathrm{I}=\text { CAS-ICT } \\
\mathrm{S}=\text { Decatur }\end{array}$ & $\begin{array}{l}\mathrm{D}=\text { Safran Morpho } \\
\mathrm{J}=\text { Toshiba } \\
\mathrm{T}=\text { Ayonix }\end{array}$ & $\begin{array}{l}\mathrm{E}=\mathrm{NEC} \\
\mathrm{L}=\text { Tsinghua } \mathrm{U} . \mathrm{II}\end{array}$ & $\begin{array}{l}\mathrm{F}=\text { Tsinghua } \mathrm{U} \\
\mathrm{M}=\mathrm{HP}\end{array}$ & $\begin{array}{l}\text { FNIR(N,R,T,L) “Miss rate" } \\
\text { FPIR(N,T,L) “False alarm rate" }\end{array}$ \\
\hline
\end{tabular}




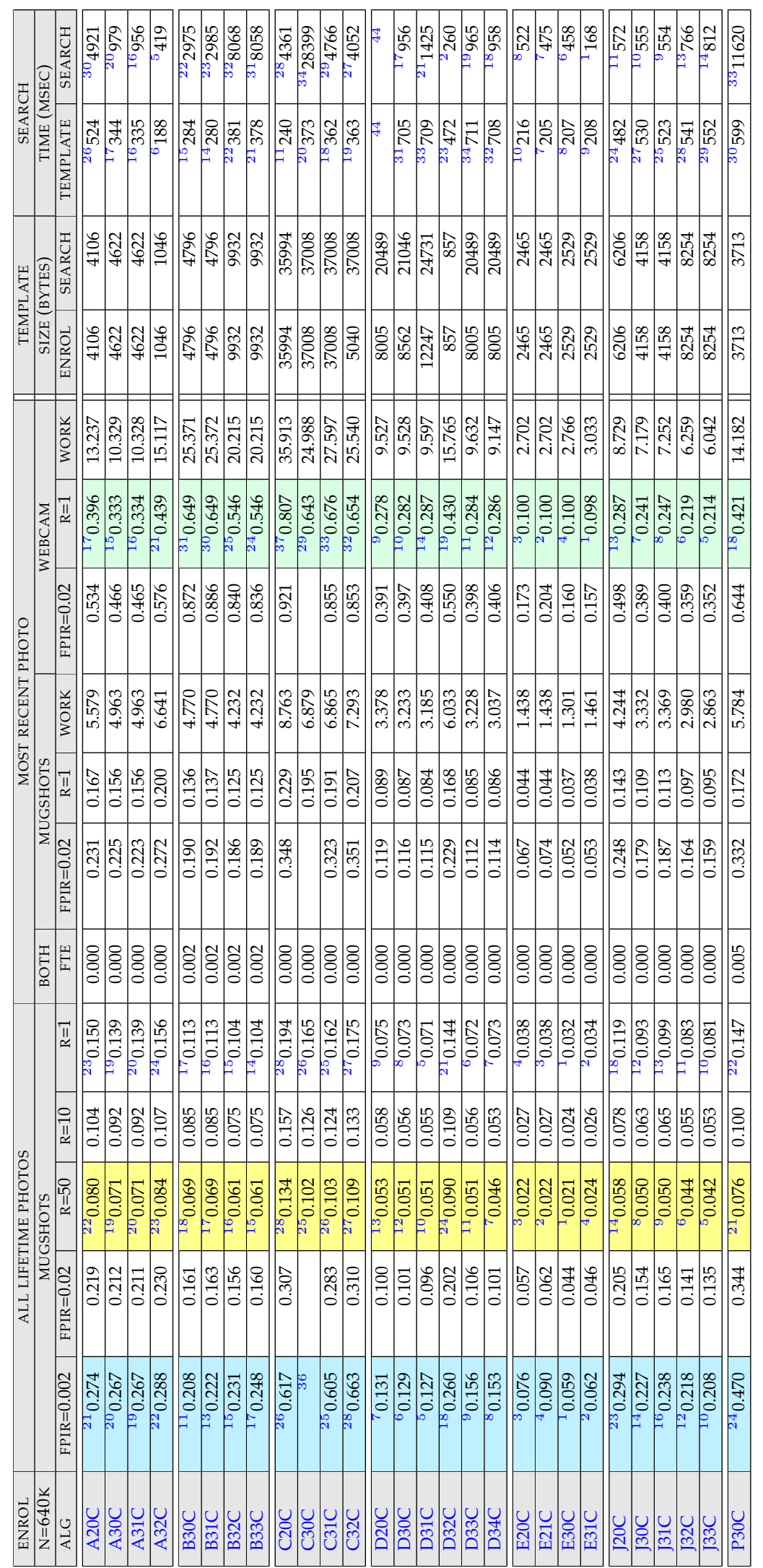

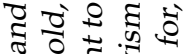

कू की

के च ¿

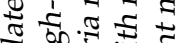

क्षे

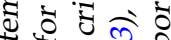

क व $\approx$ च

$\frac{3}{\pi} \pi \cdot \frac{\pi}{4}$

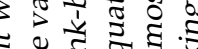

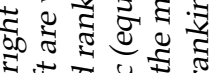

$\exists \pm \tau . \bar{\tau} \pm$

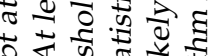

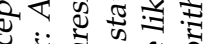

वें $\approx \approx \approx 0$

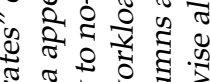

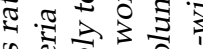

की

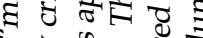

ঊ

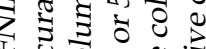

뤄워

व

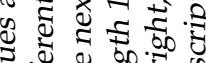

ฐ

ฮี

ฐ ฐ

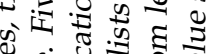

$\infty$ ن

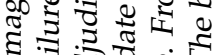

ฐ สีत

สี ฐี สี สี

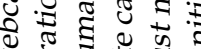

3 ป స

ฐ

ब $\frac{\pi}{\pi}$ :

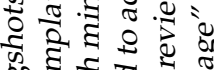

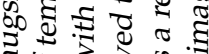

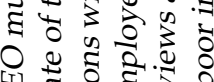

可

ఫิ

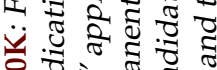

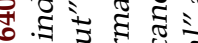

II

乙最它 0

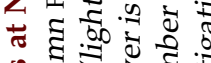

岃 :

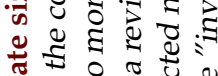

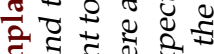

สี สิ

हो 3 ज

屯े

ठั

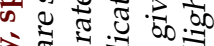

包

: क क 0

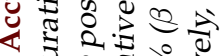

เิ เก ส

$\mathrm{A}=3 \mathrm{M} /$ Cogent

$\mathrm{G}=$ Hisign

$\mathrm{P}=$ Zhuhai-Yisheng \begin{tabular}{l|l}
$\mathrm{B}=$ Cognitec & $\mathrm{C}=$ Neurotechnology
\end{tabular} $\mathrm{H}=$ CAS-IA $\mathrm{I}=\mathrm{CAS}-\mathrm{ICT}$ $\mathrm{S}=$ Decatur
D = Safran Morpho $\mathrm{J}=$ Toshiba $\mathrm{T}=$ Ayonix
$\mathrm{E}=\mathrm{NEC}$ $\mathrm{L}=$ Tsinghua $\mathrm{U} . \mathrm{II}$

$\mathrm{M}$

$\mathrm{F}=$ Tsingh

$\mathrm{M}=\mathrm{HP}$
FNIR(N,R,T,L) “Miss rate"

FPIR(N,T,L) "False alarm rate" 


\begin{tabular}{|c|c|c|c|c|c|c|c|c|c|c|c|c|c|c|c|c|c|c|c|c|c|c|c|c|c|c|}
\hline & 它 & 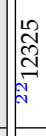 & 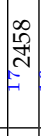 & 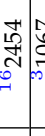 & & 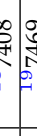 & & 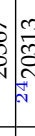 & & & & $\mid \begin{array}{l}0 \\
0 \\
0 \\
0 \\
0 \\
0\end{array}$ & & & & & & & & & 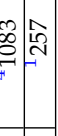 & & & & & \\
\hline 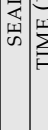 & & 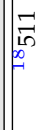 & & & & N & & $\begin{array}{l}0 \\
\substack{1 \\
-\infty}\end{array}$ & & & & & $F$ & & & & & 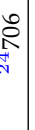 & & & సి & & & & & b. \\
\hline & & $\mid \frac{8}{7}$ & & 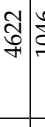 & & 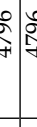 & की & $\tilde{\sigma}$ & 㕝 & & $\left|\begin{array}{l}\infty \\
0 \\
0 \\
m \\
m\end{array}\right|$ & 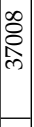 & ঠ্ণ & & $\begin{array}{l}\vec{N} \\
\text { Aै } \\
\text { N }\end{array}$ & 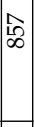 & & 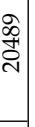 & 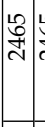 & & 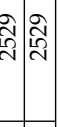 & 赵 & $\begin{array}{lll}\infty & \infty \\
10 \\
7 \\
7\end{array}$ & 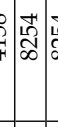 & & \\
\hline$\frac{1}{2}$ & 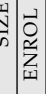 & $\mid \begin{array}{l}8 \\
7 \\
7\end{array}$ & $\begin{array}{l}\text { ה̃ } \\
0 \\
+1\end{array}$ & 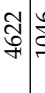 & & 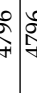 & की & $\hat{\delta}$ & 索 & & \begin{tabular}{|l|}
$\infty$ \\
0 \\
0 \\
m.
\end{tabular} & $\begin{array}{l}\text { 울 } \\
\text { in }\end{array}$ & $\begin{array}{l}\mathscr{L} \\
0 \\
\infty \\
\infty\end{array}$ & $\begin{array}{l}\text { W } \\
\infty \\
\infty\end{array}$ & Iิ & 会 & 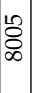 & $\begin{array}{l}\stackrel{2}{0} \\
\varnothing \\
\infty\end{array}$ & 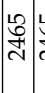 & & 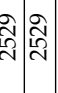 & $\mid$\begin{tabular}{l}
0 \\
\multirow{0}{0}{}
\end{tabular} & \begin{tabular}{l|l}
$\infty$ \\
$\infty$ \\
$F$ \\
$F$
\end{tabular} & 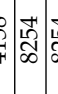 & & \\
\hline & & $\mid$\begin{tabular}{l}
10 \\
$\varnothing$ \\
\hdashline \\
\hdashline \\
-1
\end{tabular} & $\mid$\begin{tabular}{l}
$R$ \\
$\infty$ \\
\hdashline \\
$=$
\end{tabular} & 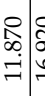 & & 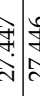 & 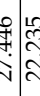 & 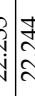 & 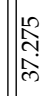 & $\begin{array}{l}\infty \\
\infty \\
\infty \\
\\
\end{array}$ & 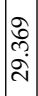 & $\mid \begin{array}{l}\vec{\Xi} \\
\vec{u} \\
\vec{a} \\
\end{array}$ & $\begin{array}{l}\text { ले } \\
\infty \\
0 \\
0 \\
\dot{a}\end{array}$ & $\begin{array}{l}\hat{A} \\
\hat{⿵} \\
0 \\
-\end{array}$ & 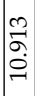 & 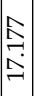 & & 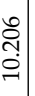 & 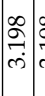 & $\begin{array}{c}\infty \\
\frac{2}{m} \\
m\end{array}$ & 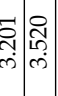 & $\mid$\begin{tabular}{c}
1 \\
6 \\
\hdashline \\
\hdashline \\
\hdashline
\end{tabular} & & 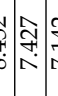 & & $\begin{array}{l}8 \\
8 \\
0 \\
\dot{8} \\
10\end{array}$ \\
\hline 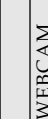 & 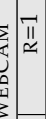 & సิ & 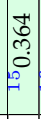 & 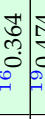 & & 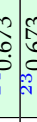 & 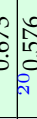 & 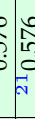 & $\mid \begin{array}{l}\tilde{N} \\
\infty \\
0 \\
0 \\
0\end{array}$ & $\begin{array}{l}2 \\
0 \\
0 \\
0 \\
0 \\
0\end{array}$ & 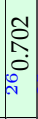 & $\mid \begin{array}{l}\mathbb{2} \\
0 \\
0 \\
0 \\
0 \\
0 \\
0\end{array}$ & 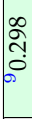 & $\begin{array}{l}\text { के } \\
\text { के }\end{array}$ & $\begin{array}{l}\hat{0} \\
\hat{0} \\
0 \\
0 \\
0\end{array}$ & $\begin{array}{l}\mathbb{2} \\
0 \\
0 \\
0 \\
0 \\
0\end{array}$ & 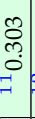 & 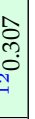 & & & 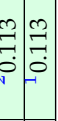 & $\mid \begin{array}{l}\infty \\
\infty \\
0 \\
0 \\
0\end{array}$ & & 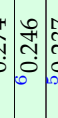 & & b \\
\hline & 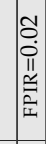 & | & & $\begin{array}{l}1 \\
\vdots \\
\vdots \\
0\end{array}$ & & 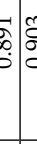 & 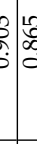 & 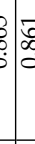 & 玟 & & $\left|\begin{array}{c}\mathbb{N} \\
0 \\
0 \\
0\end{array}\right|$ & $\mid \begin{array}{c}0 \\
0 \\
0 \\
0 \\
0\end{array}$ & 各 & $\begin{array}{l}\infty \\
\forall \\
\forall\end{array}$ & 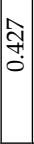 & $\begin{array}{l}0 \\
\infty \\
10 \\
0 \\
0\end{array}$ & $\begin{array}{l}\infty \\
7 \\
7 \\
0\end{array}$ & 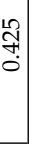 & $\mid \begin{array}{l}\alpha \\
\vdots \\
\sigma \\
\delta\end{array}$ & & 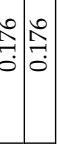 & $\mid \begin{array}{c}+ \\
\hat{n} \\
\substack{n \\
0}\end{array}$ & 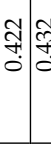 & 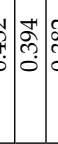 & & \\
\hline $\begin{array}{l}z \\
y \\
y \\
y\end{array}$ & & 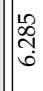 & & 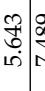 & & 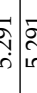 & $\stackrel{7}{7}$ & 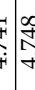 & 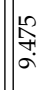 & 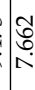 & 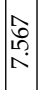 & $\mid \begin{array}{l}\hat{\alpha} \\
\dot{0} \\
\infty\end{array}$ & 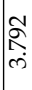 & & $\begin{array}{l}\text { त్ర్ } \\
\text { ले }\end{array}$ & 帒 & $\mid \begin{array}{l}\mathfrak{R} \\
\hat{6} \\
\dot{n}\end{array}$ & 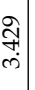 & 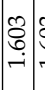 & 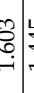 & 趈 & $\mid$\begin{tabular}{c}
$q$ \\
\hdashline \\
$\dot{+}$
\end{tabular} & 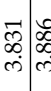 & 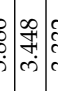 & & $\begin{array}{l}\dot{8} \\
\dot{0} \\
\dot{B} \\
\text {. }\end{array}$ \\
\hline & & $\mid \begin{array}{l}\infty \\
\infty \\
0 \\
0\end{array}$ & $\begin{array}{c}N \\
N \\
0 \\
0\end{array}$ & 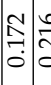 & & 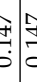 & $\stackrel{0}{0}$ & 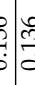 & 㞼 & $\stackrel{\vec{N}}{\vec{I}}$ & 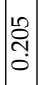 & 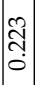 & ठ̊. & סू & סू & $\mid \begin{array}{l}\infty \\
\infty \\
0 \\
0 \\
0\end{array}$ & $\mid \begin{array}{l}-\overrightarrow{0} \\
0 \\
0\end{array}$ & $\begin{array}{l}2 \\
\delta \\
0\end{array}$ & $\begin{array}{l}9 \\
0 \\
0\end{array}$ & 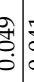 & 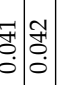 & $\mid \begin{array}{c}\overrightarrow{6} \\
-3 \\
0\end{array}$ & \begin{tabular}{c}
\multirow{2}{*}{} \\
0 \\
0
\end{tabular} & 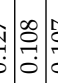 & $\begin{array}{l}0 \\
\stackrel{0}{0} \\
\vdots \\
0\end{array}$ & \\
\hline & 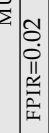 & 空 & \begin{tabular}{l}
0 \\
\multirow{2}{*}{} \\
$\vdots$ \\
0
\end{tabular} & స్తి & & 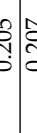 & & & 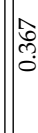 & & & 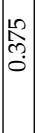 & $\begin{array}{l}\text { İ } \\
\text { - }\end{array}$ & ज̂ & & 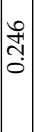 & \begin{tabular}{l}
0 \\
\hdashline \\
$\vdots$ \\
0
\end{tabular} & $\begin{array}{c}\overrightarrow{7} \\
\vdots \\
0\end{array}$ & 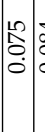 & & $\begin{array}{l}\hat{b} \\
\vdots \\
0 \\
0\end{array}$ & $\mid \begin{array}{c}1 \\
\hat{N} \\
\\
\delta\end{array}$ & 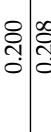 & 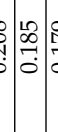 & & \\
\hline $\begin{array}{l}\mathrm{T} \\
0 \\
0\end{array}$ & $\begin{array}{c}\text { 可 } \\
\end{array}$ & $\| \stackrel{8}{\circ}$ & 足 & ¿े & & $\stackrel{s}{s}$ & $\stackrel{s}{s}$ & șa & $\underset{8}{8}$ & : & $\begin{array}{l}8 \\
8 \\
0 \\
0\end{array}$ & $\mid$\begin{tabular}{l}
0 \\
\hdashline \\
\hdashline \\
$\circ$
\end{tabular} & \begin{tabular}{l}
8 \\
\hdashline \\
0
\end{tabular} & ஜ & ஜ & \begin{tabular}{l}
8 \\
\hdashline \\
\hdashline \\
\hdashline
\end{tabular} & $\mid$ & $\begin{array}{c}\vec{s} \\
\dot{0} \\
\dot{0}\end{array}$ & ৪) & \begin{tabular}{c}
8 \\
$\varnothing$ \\
\hdashline
\end{tabular} & \begin{tabular}{l|l}
8 \\
\hdashline \\
\hdashline \\
\hdashline
\end{tabular} & 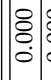 & ¿̊: & 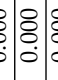 & \begin{tabular}{l}
8 \\
\hdashline \\
0 \\
0
\end{tabular} & \\
\hline & & $\mid$\begin{tabular}{l}
0 \\
0 \\
0 \\
0 \\
\hdashline \\
\hdashline
\end{tabular} & $\begin{array}{l}0 \\
10 \\
10 \\
0 \\
0\end{array}$ & 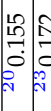 & & 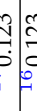 & 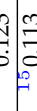 & 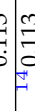 & స్ & 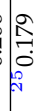 & 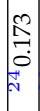 & $\begin{array}{l}0 \\
\vdots \\
0 \\
0 \\
0 \\
0\end{array}$ & $\begin{array}{l}\circ \\
: \\
0\end{array}$ & 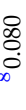 & $\begin{array}{l}\hat{1} \\
\hat{0} \\
0 \\
0 \\
0\end{array}$ & 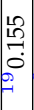 & $\begin{array}{l}\infty \\
0 \\
0 \\
0 \\
0\end{array}$ & مَّ人 & 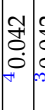 & 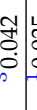 & $\begin{array}{ll}0 \\
0 \\
0 \\
0\end{array}$ & $\mid \begin{array}{c}\infty \\
0 \\
0 \\
0 \\
0\end{array}$ & & $\begin{array}{c}0 \\
0 \\
0 \\
0\end{array}$ & & 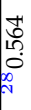 \\
\hline & & 光 & \begin{tabular}{l}
0 \\
$\stackrel{0}{0}$ \\
\hdashline
\end{tabular} & $\mid \begin{array}{l}1 \\
0 \\
0 \\
0 \\
0\end{array}$ & & '. & 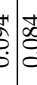 & : & 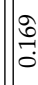 & वे & 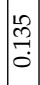 & 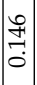 & 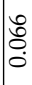 & $\begin{array}{l}\mathscr{8} \\
\ddot{0} \\
0\end{array}$ & $\mid \begin{array}{l}0 \\
0 \\
0 \\
0\end{array}$ & స్. & 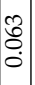 & ל̂. & $\left|\begin{array}{l}\varnothing \\
\delta \\
0 \\
0\end{array}\right|$ & 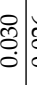 & 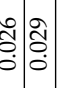 & $\mid \begin{array}{l}8 \\
0 \\
0 \\
0\end{array}$ & 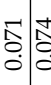 & 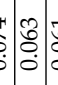 & \begin{tabular}{l|}
$\vec{b}$ \\
0 \\
0
\end{tabular} & 䅸 \\
\hline 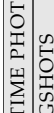 & 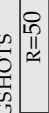 & $\mid \begin{array}{l}2 \\
0 \\
0 \\
0 \\
0\end{array}$ & $\begin{array}{l}\infty \\
0 \\
0 \\
0 \\
0\end{array}$ & 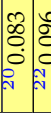 & & 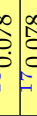 & 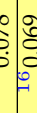 & 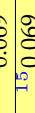 & 年 & 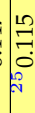 & $\begin{array}{l}7 \\
-7 \\
0 \\
0 \\
0\end{array}$ & 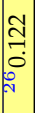 & $\begin{array}{l}\overline{8} \\
\vdots \\
0\end{array}$ & $\begin{array}{l}2 \\
\ddot{0} \\
0 \\
0\end{array}$ & $\begin{array}{l}0 \\
\text { ô. } \\
0 \\
0\end{array}$ & $\begin{array}{l}5 \\
0 \\
0 \\
0 \\
0 \\
0\end{array}$ & $\begin{array}{l}0 \\
0 \\
0 \\
0 \\
0 \\
=\end{array}$ & $\begin{array}{l}0+1 \\
\stackrel{2}{0} \\
0 \\
0\end{array}$ & 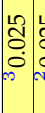 & $\begin{array}{l}\mathrm{L} \\
0 \\
0 \\
0\end{array}$ & $\begin{array}{ll}0 \\
0 \\
0 \\
0 \\
0 \\
0 \\
0 \\
0\end{array}$ & $\mid \begin{array}{ll}\infty \\
\infty \\
0 \\
0 \\
0 \\
0\end{array}$ & & $\begin{array}{l}0 \\
\vdots \\
0 \\
0 \\
0\end{array}$ & & 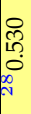 \\
\hline 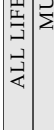 & 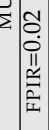 & & 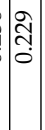 & 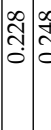 & & 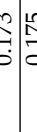 & $\frac{1}{2}$ & $\frac{1}{2}$ & 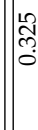 & & & $\mid \begin{array}{c}\infty \\
\tilde{m} \\
0 \\
0\end{array}$ & ? & $\stackrel{1}{0}$ & مै & $\mid \begin{array}{l}0 \\
\overline{\hat{~}} \\
0 \\
\end{array}$ & 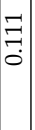 & 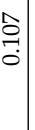 & ț & $\begin{array}{c}\widehat{T} \\
0 \\
0 \\
\vdots\end{array}$ & 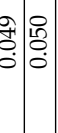 & 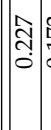 & 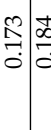 & 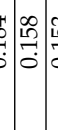 & & $\begin{array}{l}\text { బ̂ } \\
0 \\
0 \\
0\end{array}$ \\
\hline & 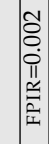 & 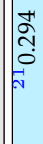 & $\begin{array}{l}\infty \\
0 \\
0 \\
0 \\
0 \\
0 \\
0\end{array}$ & 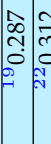 & & & & 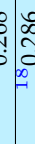 & $\mid \begin{array}{ll}1 \\
0 \\
0 \\
0 \\
0 \\
0 \\
0\end{array}$ & & 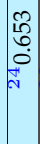 & 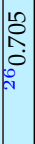 & & & & & $\begin{array}{l}\overrightarrow{0} \\
\overrightarrow{0} \\
0\end{array}$ & & 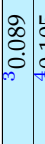 & & 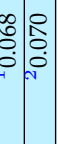 & 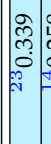 & & 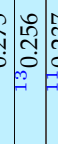 & & \\
\hline 至 & \begin{tabular}{l|l|l}
1 & 0 \\
\end{tabular} & & & $\vec{m}$ & & 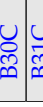 & 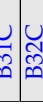 & & లె & & & & & & & & & . & 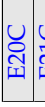 & & 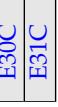 & 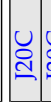 & 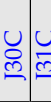 & 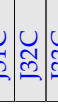 & & \\
\hline
\end{tabular}

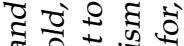

ฮิ कี

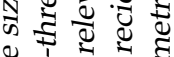

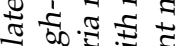

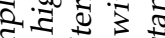

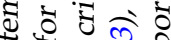

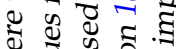

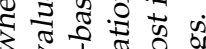

$3 \vec{z}$ है.

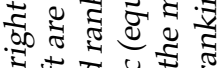

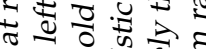

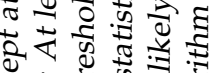

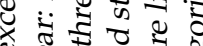

ช

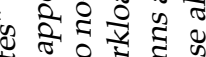

ब

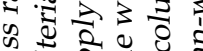

हाँ ट्वाँ

๙

خ

额

वิ

\& ฮี ڤ

ฮี ฐี

ฐ ฐ

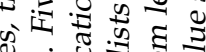

$\infty$ ن

ส 5 .

ฐี สี

ฐี ธี สี ฮี สี

\&

3 む.

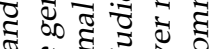

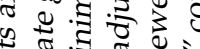

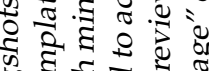

की

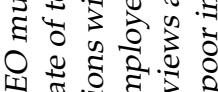

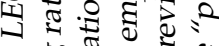

tั

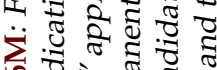

ํ. ปี สี

II

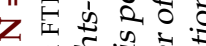

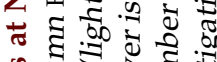

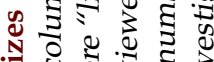

के है क

ฐ $\approx$ ह

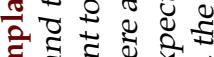

สี สี ชิ

हो 3 ज

के

के क्षे \&

क ज $\widetilde{\pi}: \overrightarrow{0}$

范

: क ० 0

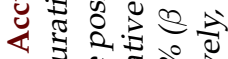
ช.

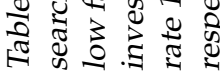

$\mathrm{A}=3 \mathrm{M} /$ Cogent

$\mathrm{G}=$ Hisign

$\mathrm{P}=$ Zhuhai-Yisheng \begin{tabular}{l|l}
$\mathrm{B}=$ Cognitec & $\mathrm{C}=$ Neurotechnology
\end{tabular} $\mathrm{H}=$ CAS-IA $\mathrm{I}=\mathrm{CAS}-\mathrm{ICT}$ $\mathrm{S}=$ Decatur
$\mathrm{D}=$ Safran Morpho $\mathrm{J}=$ Toshiba $\mathrm{T}=$ Ayonix
$\mathrm{E}=\mathrm{NEC}$ $\mathrm{L}=$ Tsinghua $\mathrm{U}$

$\mathrm{L}=\mathrm{Tsinght}$
$\mathrm{F}=$ Tsingh

$\mathrm{M}=\mathrm{HP}$
$\operatorname{FNIR}(\mathrm{N}, \mathrm{R}, \mathrm{T}, \mathrm{L})$ "Miss rate"

FPIR(N,T,L) “False alarm rate" 


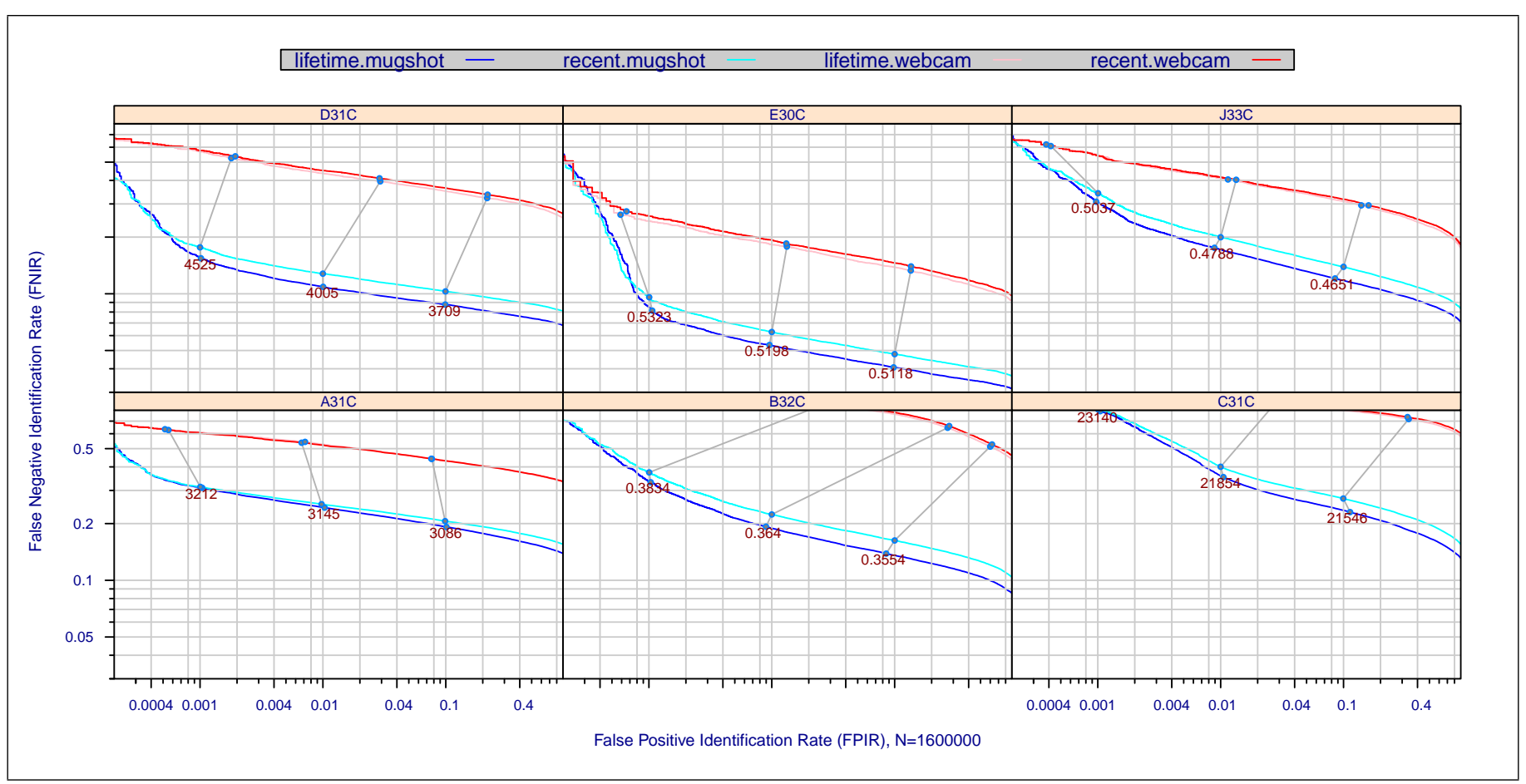

Figure 6: Identification error tradeoff for most accurate developers. For mugshot and webcam images searched against images of 1.6 million individuals enrolled in the "recent" and "lifetime" types of section 3.2, each panel plots FNIR (miss rate) vs. FPIR (false alarm rate) on log-log axes. Each panel presents data for the leading algorithm from the providers of the six most accurate algorithms. The (upper) red lines correspond to searching poor quality webcam images. The (lower) blue lines correspond to searching better quality mugshot images. The short grey lines link points of fixed threshold; they show how the error rates change with image and enrolment types. The threshold iitsel is shown in dark red. Non vertical lines indicate FPIR variation that a system operator would need to plan for. Performance data is tabulated in Tables 10 and 4- 6. DETs for other algorithms appear in the "report cards" of Appendix A.

datasets. This applies across image types, mugshots and webcam, and enrolment types (sec: 3.2). FNIR values for NEC are a factor of two or more lower than for the closest competitors, Morpho and Toshiba. For example, when 1.6 million individuals' lifetime mugshots are enrolled, the NEC's rank one miss rate is 0.035 vs. Morpho's 0.077. At rank 50, the NEC result is 0.023 with Toshiba at 0.049 and Morpho at 0.054 . Similarly the margin for webcam images is larger still: Table 6 shows rank one miss rate for NEC is 0.11 vs. 0.24 for Toshiba and 0.30 for Morpho. Indeed the NEC algorithms yield webcam recognition accuracy better than many algorithms' accuracy on mugshot images.

At the other end of the DET plot, however, the NEC algorithm exhibits rapid elevation in error rates at FPIR $<0.001$. There the lifetime mugshot DETs of Figure 6 show that Morpho's algorithm gains a modest advantage: at FPIR $=0.0003$, the NEC miss rate is 0.374 while the Morpho value is 0.311 . While the shape of the DETs is persuasive, this result is at the limits of statistical significant since the number of false positives, 51, supports a statement only that FPIR is, with $99.9 \%$ confidence, below 0.00044 and there, Morpho's error rate is virtually tied with NECs: 0.248 vs. 0.252 respectively. Refinement of this comparison would require more nonmate searches to improve FPIR estimates.

For mugshots, Toshiba's algorithms give slightly fewer misses than Cognitec's which, in turn, produce error rates below those of 3M/Cogent. Finally Neurotechnology's algorithms are beaten by those of Zhuhai Yisheng at $N<640,000$ but not for $N=1,600,000$ where the P30C algorithm fails substantially.

All algorithms are intolerant of webcam images, giving elevated miss rates, a factor of around three higher than for mugshots. This is particularly true for algorithms from Neurotechnology which give miss rates in excess of 0.6. As discussed earlier, results for webcam images have only niche operational relevance because most contemporary systems

\begin{tabular}{|c|c|c|c|c|c|c|}
\hline $\begin{array}{l}\mathrm{A}=3 \mathrm{M} / \text { Cogent } \\
\mathrm{G}=\text { Hisign } \\
\mathrm{P}=\text { Zhuhai-Yisheng }\end{array}$ & $\begin{array}{l}\mathrm{B}=\text { Cognitec } \\
\mathrm{H}=\text { CAS-IA } \\
\mathrm{Q}=\text { JunYu }\end{array}$ & $\begin{array}{l}\mathrm{C}=\text { Neurotechnology } \\
\mathrm{I}=\text { CAS-ICT } \\
\mathrm{S}=\text { Decatur }\end{array}$ & $\begin{array}{l}\mathrm{D}=\text { Safran Morpho } \\
\mathrm{J}=\text { Toshiba } \\
\mathrm{T}=\text { Ayonix }\end{array}$ & $\begin{array}{l}\mathrm{E}=\mathrm{NEC} \\
\mathrm{L}=\text { Tsinghua } \mathrm{U} . \mathrm{II}\end{array}$ & $\begin{array}{l}\mathrm{F}=\text { Tsinghua } \mathrm{U} \\
\mathrm{M}=\mathrm{HP}\end{array}$ & $\begin{array}{l}\text { FNIR(N,R,T,L) “Miss rate" } \\
\text { FPIR(N,T,L) “False alarm rate" }\end{array}$ \\
\hline
\end{tabular}




\begin{tabular}{|l|r|r|r|r|r|r|}
\hline ENROLLED & \multicolumn{7}{|c|}{ RANK ONE ACCURACY } \\
\hline N=1600000 & SDK & YYYY-MM & FNIR(R=1) & FNIR(R=10) & FNIR(R=50) & FNIR(FPIR=0.002) \\
\hline \hline Cognitec & X21 & $2010-02$ & 0.187 & 0.148 & 0.125 & 0.342 \\
\hline & B10C & $2012-08$ & 0.213 & 0.174 & 0.151 & 0.349 \\
\hline & B32C & $2013-10$ & 0.170 & 0.140 & 0.121 & 0.421 \\
\hline \hline Neurotechnology & Z22 & $2010-02$ & 0.258 & 0.219 & 0.194 & 0.537 \\
\hline & C11C & $2012-08$ & 0.268 & 0.232 & 0.208 & 0.952 \\
\hline & C20C & $2013-03$ & 0.267 & 0.229 & 0.206 & 0.684 \\
\hline & C31C & $2013-10$ & 0.231 & 0.193 & 0.170 & 0.685 \\
\hline \hline & W22 & $2010-02$ & 0.135 & 0.113 & 0.103 & 0.248 \\
\hline & D10C & $2012-08$ & 0.145 & 0.126 & 0.120 & 0.233 \\
\hline & D20C & $2013-03$ & 0.124 & 0.106 & 0.100 & 0.194 \\
\hline & D31C & $2013-10$ & 0.121 & 0.103 & 0.099 & 0.195 \\
\hline NEC & V21 & $2010-02$ & 0.089 & 0.063 & 0.050 & 0.269 \\
\hline & E10C & $2012-08$ & 0.164 & 0.121 & 0.098 & 0.410 \\
\hline & E20C & $2013-03$ & 0.072 & 0.055 & 0.047 & 0.132 \\
\hline & E30C & $2013-10$ & 0.064 & 0.051 & 0.046 & 0.108 \\
\hline
\end{tabular}

Table 7: Accuracy gains and losses 2010 to 2013: For the four providers whose algorithms successfully completed the largest identification trial in the $2010 \mathrm{MBE}$ evaluation [12], the table shows how accuracy has changed in the three and half years from early 2010 to late 2013. This is done for a fixed dataset of $N=1.6$ million individuals each of whom is enrolled with $K \geq 1$ historical images. The number of mated searches is 40,000. The number of nonmate searches is also 40,000. The images are a subset of the LEO dataset drawn randomly in their natural mixture of approximately of $86 \%$ mugshots and $14 \%$ webcams.

target the ISO/IEC 19794-5 standard's appearance requirements. That said the algorithm providers were aware that such images had been used in the 2010 test, and have access to images of this type in the MEDS Special Database 32 [5].

\subsubsection{Face accuracy 2013 vs. 2010}

Methods: Algorithms from providers who participated in both 2010 and 2013 were used to identify LEO images, repeating almost exactly the $\mathrm{N}=1.6$ million trial reported in MBE 2010 [12]. A small percentage of duplicate same-image pairs that were removed from the 2010 results were not removed in this study. The number of candidates was restricted to $L=50$.

Results: Table 7 shows modest reductions in miss rates from 2010 to 2013.

Discussion: At rank 1, there will be approximately 10\% fewer misses for the most accurate Cognitec, Neurotechnology and Morpho algorithms and nearly $30 \%$ fewer with NEC's. At rank 50, the gains are more modest, $10 \%$ for Neurotechnology and NEC, and below 4\% for Cognitec and Morpho. At the other end of the DET curve, where thresholds are applied to produce false positive outcomes in one in every 500 searches (FPIR $=0.002)$ the situation is mixed. While NEC realizes a full $60 \%$ reduction in miss rate, and Morpho a $20 \%$ reduction, both Neurotechnology and Cognitec appear to have worse accuracy. These observations ignore speed differences - particularly, the Cognitec algorithms in 2013 are considerably faster than those evaluated in 2010.

\subsubsection{Cross-acquisition recognition}

The prior subsection gave exhaustive tabulations of results for the general LEO population. There, mated pairs could be either mugshot-mugshot, webcam-webcam, or mugshot-webcam, and accuracy was only reported by whether the search image was a mugshot or a webcam. This section reports specifically on the accuracy of the various combinations. The experimental design is given in Table 8 and the results appear in Table 9.

For all the algorithms tested, the best recognition is obtained, as expected, by searching mugshots against enrolled mugshots. The worst recognition results, however, are not obtained by searching webcams against webcams, but instead from searching webcams against mugshots. This result is unfortunately contrary to the aspiration of frontal image

\begin{tabular}{|c|c|c|c|c|c|c|}
\hline $\begin{array}{l}A=3 \mathrm{M} / \text { Cogent } \\
G=\text { Hisign } \\
P=\text { Zhuhai-Yisheng }\end{array}$ & $\begin{array}{l}\mathrm{B}=\text { Cognitec } \\
\mathrm{H}=\text { CAS-IA } \\
\mathrm{Q}=\text { JunYu }\end{array}$ & $\begin{array}{l}\mathrm{C}=\text { Neurotechnology } \\
\mathrm{I}=\mathrm{CAS}-\mathrm{ICT} \\
\mathrm{S}=\text { Decatur }\end{array}$ & $\begin{array}{l}\mathrm{D}=\text { Safran Morpho } \\
\mathrm{J}=\text { Toshiba } \\
\mathrm{T}=\text { Ayonix }\end{array}$ & $\begin{array}{l}\mathrm{E}=\mathrm{NEC} \\
\mathrm{L}=\text { Tsinghua U. II }\end{array}$ & $\begin{array}{l}\mathrm{F}=\text { Tsinghua } \mathrm{U} \\
\mathrm{M}=\mathrm{HP}\end{array}$ & $\begin{array}{l}\text { FNIR(N,R,T,L) “Miss rate" } \\
\text { FPIR(N,T,L) “False alarm rate" }\end{array}$ \\
\hline
\end{tabular}




\begin{tabular}{|l|l|l|l|l|l|l|l|l|}
\hline & \multicolumn{3}{|c|}{ Enrolment } & \multicolumn{3}{c|}{ Search } \\
\hline & Type & Num. IDs & \multicolumn{3}{|c|}{ Num. Images } & \multicolumn{3}{|c|}{ Mum. Images and IDs } \\
\hline & See & \multicolumn{3}{|c|}{ Mate } & Webcam & Mugshot & Webcam & Mugshot \\
\hline & See sec. 3.2 & $\mathrm{N}$ & Webcam & Mugshot & Total & Wonmate & 171,066 \\
10 & RECENT & 0 & 10,660 & 0 & 10,660 & 10,660 & 0 & 28,936 \\
10 & RECENT & 10,660 & 0 & 10,660 & 10,660 & 0 & 10,660 & 28,936 \\
11 & RECENT & 10,660 & 0 & 10,660 & 10,660 & 10,660 & 0 & 171,066 \\
\hline
\end{tabular}

Table 8: Enrolment and search sets. Each row summarizes one identification trial. The column labeled "Num. IDs" gives the number of enrolled identities. This precedes the numbers of images, and then the number of mate, and nonmate, searches. Rows 9-11 are dedicated to mugshot-mugshot, webcam-webcam, and mugshot-webcam recognition - the number of individuals here is limited to 10660 which corresponds to the number of individuals who were captured with webcams and traditional mugshot cameras.

\begin{tabular}{|l|r|r|r|r|r|r|}
\hline & \multicolumn{3}{|c|}{ RANK 1 } & \multicolumn{3}{c|}{ RANK 50 } \\
\hline ENROL & \multicolumn{1}{|c|}{ MUGSHOT } & \multicolumn{1}{c|}{ MUGSHOT } & WEBCAM & MUGSHOT & MUGSHOT & WEBCAM \\
\hline SEARCH & MUGSHOT & WEBCAM & WEBCAM & MUGSHOT & WEBCAM & WEBCAM \\
\hline A31C & ${ }^{5} 0.092$ & ${ }^{3} 0.467$ & ${ }^{4} 0.264$ & ${ }^{5} 0.042$ & ${ }^{4} 0.187$ & ${ }^{4} 0.107$ \\
\hline B32C & ${ }^{4} 0.082$ & ${ }^{6} 0.591$ & ${ }^{8} 0.476$ & ${ }^{4} 0.040$ & ${ }^{7} 0.344$ & ${ }^{8} 0.240$ \\
\hline C31C & ${ }^{7} 0.125$ & ${ }^{9} 0.782$ & ${ }^{10} 0.617$ & ${ }^{7} 0.063$ & ${ }^{9} 0.472$ & ${ }^{11} 0.428$ \\
\hline D31C & ${ }^{3} 0.061$ & ${ }^{4} 0.489$ & ${ }^{3} 0.230$ & ${ }^{3} 0.036$ & ${ }^{3} 0.184$ & ${ }^{3} 0.105$ \\
\hline E30C & ${ }^{1} 0.025$ & ${ }^{1} 0.104$ & ${ }^{1} 0.066$ & ${ }^{1} 0.018$ & ${ }^{1} 0.031$ & ${ }^{1} 0.026$ \\
\hline G31C & ${ }^{8} 0.182$ & 12 & ${ }^{5} 0.343$ & ${ }^{8} 0.075$ & 12 & ${ }^{5} 0.153$ \\
\hline H30C & ${ }^{9} 0.271$ & ${ }^{8} 0.762$ & ${ }^{7} 0.428$ & ${ }^{9} 0.136$ & ${ }^{8} 0.389$ & ${ }^{7} 0.223$ \\
\hline J32C & ${ }^{2} 0.052$ & ${ }^{2} 0.236$ & ${ }^{2} 0.153$ & ${ }^{2} 0.023$ & ${ }^{2} 0.067$ & ${ }^{2} 0.051$ \\
\hline L31C & ${ }^{11} 0.358$ & ${ }^{10} 0.913$ & ${ }^{11} 0.629$ & ${ }^{11} 0.188$ & ${ }^{10} 0.714$ & ${ }^{10} 0.389$ \\
\hline M30C & ${ }^{10} 0.298$ & ${ }^{7} 0.670$ & ${ }^{9} 0.521$ & ${ }^{10} 0.142$ & ${ }^{6} 0.341$ & ${ }^{9} 0.280$ \\
\hline P30C & ${ }^{6} 0.107$ & ${ }^{5} 0.530$ & ${ }^{6} 0.344$ & ${ }^{6} 0.045$ & ${ }^{5} 0.242$ & ${ }^{6} 0.161$ \\
\hline S20C & ${ }^{12} 0.492$ & ${ }^{11} 0.968$ & ${ }^{12} 0.789$ & ${ }^{12} 0.306$ & ${ }^{11} 0.890$ & ${ }^{12} 0.604$ \\
\hline
\end{tabular}

Table 9: Mugshots, webcams, and interoperable recognition: The figures are miss rates, $F N I R(N, R, T, L)$ with $N=10,660, R=$ $\{1,50\}, L=50, T=0$, for enrolment using the image type given on row 2 and search image type given on row 3 . The blue superscripts indicate algorithm rankings, by column. Missing values are due to software crashes.

standards (i.e. ISO/IEC 19794-5), which aims to support best accuracy by establishing full-frontal geometry requirements for all images. Here recognition accuracy is better if either both images are full-frontal (i.e. mugshots) or both are not (i.e. webcams). As soon as there is heterogeneity (i.e. mugshot-webcam) recognition accuracy degrades. The specific cause of this result is that while the webcam images are inferior, any given pair can share the same non-frontal pose (typically an adverse pitch angle). Further study of this issue might quantify the relative roles of pose angle, optical resolution and illumination.

\subsection{Accuracy dependence on rank}

Identification algorithms yield candidate lists where the mate is sometimes not at rank 1 because it has a low score relative to some nonmates. This occurs typically because the face is of poor quality or has some difference to the enrolment image. While rank-1 performance is a primary indicator for comparison of algorithms, a more relevant metric for applications where a human reviewer is retained to adjudicate an $L$ element candidate list, is the frequency at which mates can be found at ranks up to rank $L$. In this report we quantify this as the miss rate $\operatorname{FNIR}(N, R, 0, L)$ for $R \rightarrow L$, and we fix $L=50$.

The workload associated with reviewer adjudication of candidate lists is discussed further in 5.7.

Method: The benefit of considering higher ranks is quantified by re-analyzing results from the same LEO images and enrolment and search sets given in rows 1-8 of Table 3 i.e. for $\mathrm{N}$ up to 1.6 million identities.

Results: Previously, Tables 4 - 6 had given some FNIR values for, respectively, N =160K,640K and 1.6M. A visualization of those results for $\mathrm{N}=640 \mathrm{~K}$ appears in Figure 8 which shows FNIR vs. $\mathrm{R}$ on log-log axes.

\begin{tabular}{|c|c|c|c|c|c|c|}
\hline $\begin{array}{l}A=3 \mathrm{M} / \text { Cogent } \\
G=\text { Hisign } \\
P=\text { Zhuhai-Yisheng }\end{array}$ & $\begin{array}{l}\mathrm{B}=\text { Cognitec } \\
\mathrm{H}=\text { CAS-IA } \\
\mathrm{Q}=\text { JunYu }\end{array}$ & $\begin{array}{l}\mathrm{C}=\text { Neurotechnology } \\
\mathrm{I}=\text { CAS-ICT } \\
\mathrm{S}=\text { Decatur }\end{array}$ & $\begin{array}{l}\mathrm{D}=\text { Safran Morpho } \\
\mathrm{J}=\text { Toshiba } \\
\mathrm{T}=\text { Ayonix }\end{array}$ & $\begin{array}{l}\mathrm{E}=\mathrm{NEC} \\
\mathrm{L}=\text { Tsinghua U. II }\end{array}$ & $\begin{array}{l}\mathrm{F}=\text { Tsinghua } \mathrm{U} \\
\mathrm{M}=\mathrm{HP}\end{array}$ & $\begin{array}{l}\text { FNIR(N,R,T,L) "Miss rate" } \\
\text { FPIR(N,T,L) “False alarm rate" }\end{array}$ \\
\hline
\end{tabular}




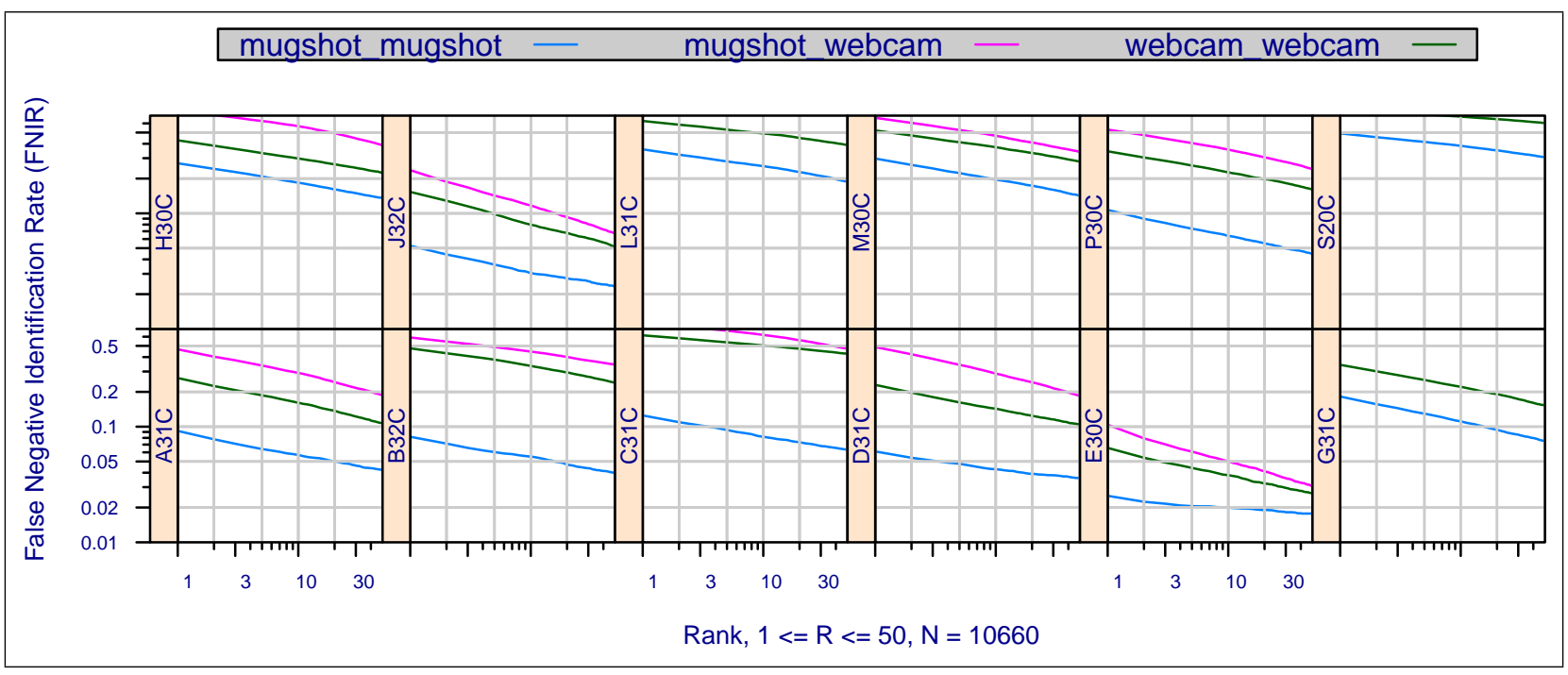

Figure 7: Identification accuracy by acquisition type. The figures plot miss rate vs. rank i.e FNIR $(N, R, 0, L)$ with $N=10,660$ and $L=50$. Each panel corresponds to one algorithm. The three traces correspond to the use of the identified acquisition type for the enrolment and search image respectively. Notably, cross-type recognition gives the worst accuracy.

Discussion: The following observations are notable.

$\triangleright$ Magnitude of accuracy gains: By definition, miss rates decrease monotonically with $R$, and the size of the effect can readily extend to a factor of two reduction in FNIR from $R=1$ to $R=50$.

$\triangle$ Differences across algorithms: For enrolment of $\mathrm{N}=640 \mathrm{~K}$ "lifetime" identities, the A31C algorithm gives a miss rate of 0.071 at rank 50 vs. 0.139 at rank 1 for a miss rate reduction factor of 2.0. For B32C, the values are 0.061 vs. 0.104 for a gain of 1.7. For $\mathrm{C} 31 \mathrm{C}$, the values are 0.103 vs. 0.162 implying a gain of 1.6. For D31C, the factor is 1.4 from 0.051 vs. 0.071 . For the most accurate algorithm overall E30C, FNIR reduces to 0.021 from 0.032 for a gain of 1.5. Finally for J33C, the gain is 1.9 as FNIR dropped to 0.042 from 0.081 . These trends are evident in Figure 8. These ratios broadly apply to other algorithms from the respective suppliers. The more accurate algorithms typically realize fewer gains at rank 50 because they place the mate at rank 1 more frequently. The result, however, is that the J33C algorithm is inferior to the D-series algorithms at rank 1, but superior at rank 50.

$\triangleright$ Power-law dependency: The observation that many of the plots in Figure 8 are straight lines is discussed later in section 5.3.1. Note the plots for the D-series and F-series algorithms are not straight.

\subsection{Effect of population size}

How algorithmic performance degrades with increasing population size is a primary challenge in any application where individuals are enrolled at a greater rate than they are un-enrolled. This scalability issue is examined in this section by re-analyzing results from the same LEO images and enrolment and search sets given in rows 1-8 of Table 3 i.e. for $\mathrm{N}$ up to 1.6 million identities.

The results are presented in four ways as follows. This includes results for both mugshot and webcam searches, and both recent and lifetime enrolment types.

\begin{tabular}{|c|c|c|c|c|c|c|}
\hline $\begin{array}{l}A=3 \mathrm{M} / \text { Cogent } \\
G=\text { Hisign } \\
P=\text { Zhuhai-Yisheng }\end{array}$ & $\begin{array}{l}\mathrm{B}=\text { Cognitec } \\
\mathrm{H}=\text { CAS-IA } \\
\mathrm{Q}=\text { JunYu }\end{array}$ & $\begin{array}{l}\mathrm{C}=\text { Neurotechnology } \\
\mathrm{I}=\mathrm{CAS}-\mathrm{ICT} \\
\mathrm{S}=\text { Decatur }\end{array}$ & $\begin{array}{l}\mathrm{D}=\text { Safran Morpho } \\
\mathrm{J}=\text { Toshiba } \\
\mathrm{T}=\text { Ayonix }\end{array}$ & $\begin{array}{l}\mathrm{E}=\mathrm{NEC} \\
\mathrm{L}=\text { Tsinghua U. II }\end{array}$ & $\begin{array}{l}\mathrm{F}=\text { Tsinghua } \mathrm{U} \\
\mathrm{M}=\mathrm{HP}\end{array}$ & $\begin{array}{l}\text { FNIR(N,R,T,L) “Miss rate" } \\
\text { FPIR(N,T,L) “False alarm rate" }\end{array}$ \\
\hline
\end{tabular}




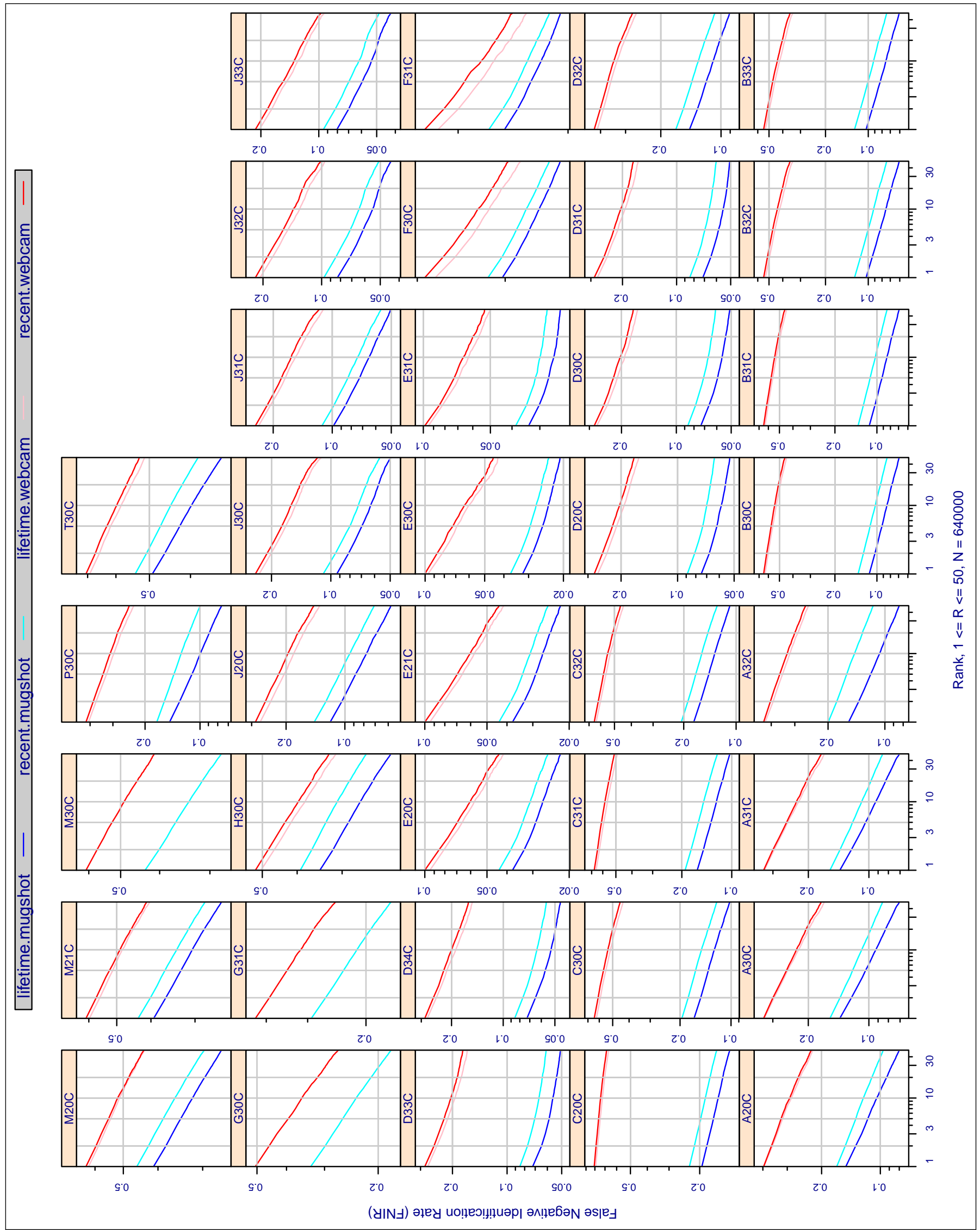

เก่ ฐี

11 เे

$\rightarrow$ के

สิ ฮี

ริ

ఫิ

II $: 3$

Z

事

㲾

คิ $\underset{\frac{\pi}{2}}{\frac{\pi}{2}}$

$\therefore \&$

之志

๘

近 吉

.-400

更

돈

का త్

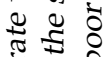

क

สี สี

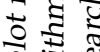

र. ज क

¿ 000 용

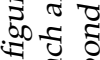

o 20

F อ

군

컹 ฮ

잉

․ㅠ ป

त

ఖ

(ี)

อั

त्

记

ส

ปี ส

픈

도ㅇㅝㅛ

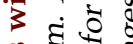

㞼

of o \&

귱

뜬

ปี ซ

¿

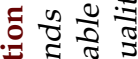

हี ส

की है

ปี 80

चَత

$\infty$ वิ

$\because 2 \pi$

วิธี \&

江舟:

\begin{tabular}{|c|c|c|c|c|c|c|}
\hline $\begin{array}{l}\mathrm{A}=3 \mathrm{M} / \text { Cogent } \\
\mathrm{G}=\text { Hisign } \\
\mathrm{P}=\text { Zhuhai-Yisheng }\end{array}$ & $\begin{array}{l}\mathrm{B}=\text { Cognitec } \\
\mathrm{H}=\mathrm{CAS}-\mathrm{IA} \\
\mathrm{Q}=\text { JunYu }\end{array}$ & $\begin{array}{l}\mathrm{C}=\text { Neurotechnology } \\
\mathrm{I}=\text { CAS-ICT } \\
\mathrm{S}=\text { Decatur }\end{array}$ & $\begin{array}{l}\mathrm{D}=\text { Safran Morpho } \\
\mathrm{J}=\text { Toshiba } \\
\mathrm{T}=\text { Ayonix }\end{array}$ & $\begin{array}{l}\mathrm{E}=\mathrm{NEC} \\
\mathrm{L}=\text { Tsinghua U. II }\end{array}$ & $\begin{array}{l}\mathrm{F}=\text { Tsinghua } \mathrm{U} \\
\mathrm{M}=\mathrm{HP}\end{array}$ & $\begin{array}{l}\text { FNIR(N,R,T,L) “Miss rate" } \\
\text { FPIR(N,T,L) “False alarm rate" }\end{array}$ \\
\hline
\end{tabular}



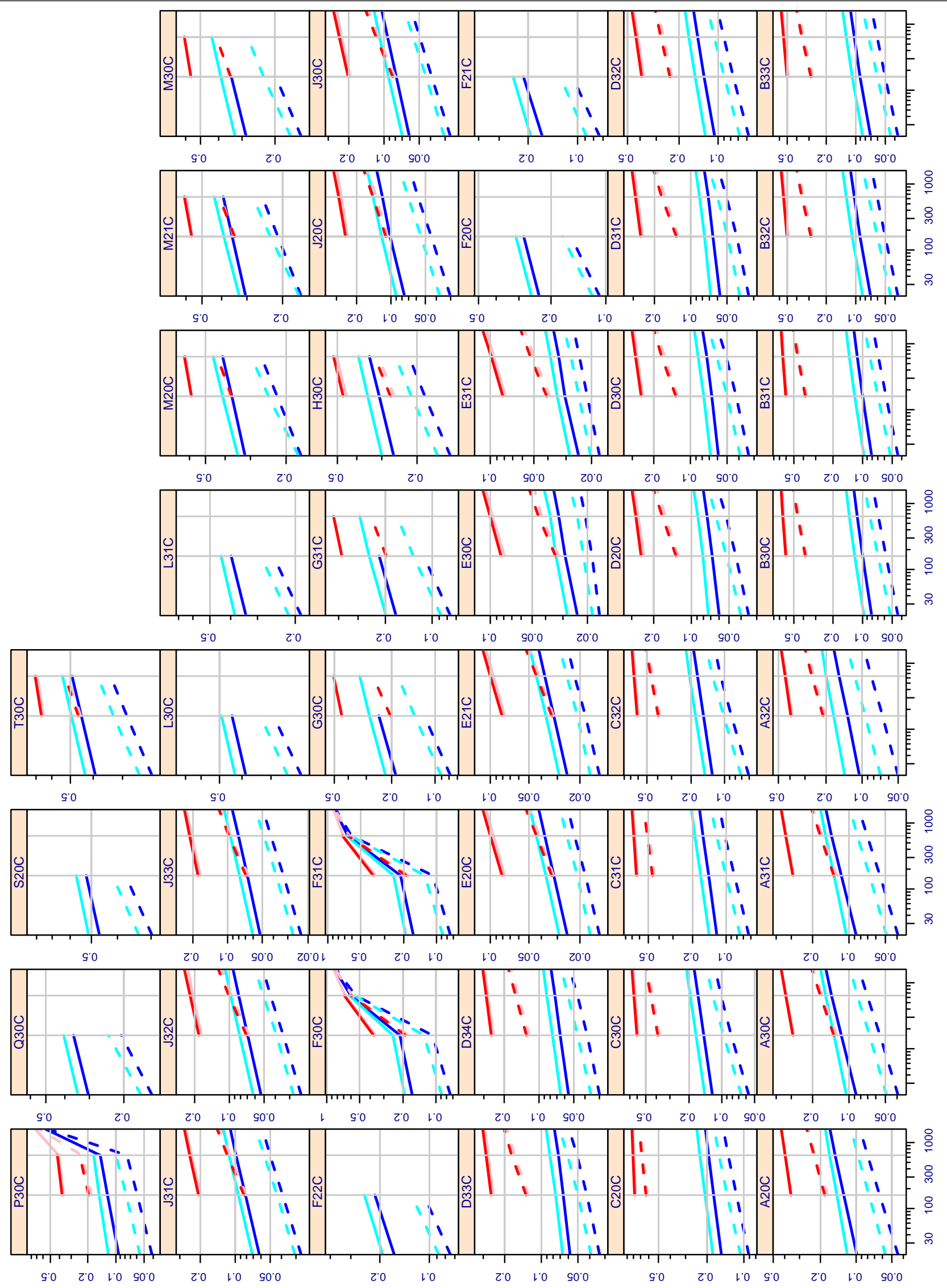

0 = pІочsәдчl '

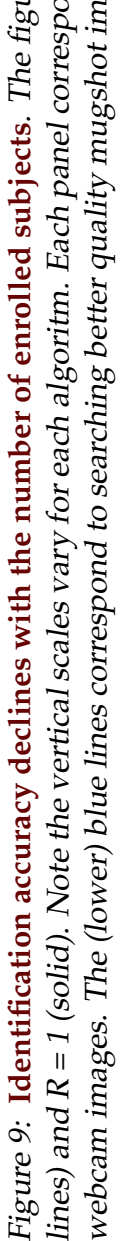




\begin{tabular}{|c|c|c|c|c|c|c|c|c|c|c|c|c|}
\hline \multirow{3}{*}{$\begin{array}{l}\text { ENROL } \\
\text { RANK=1 } \\
\text { ALG }\end{array}$} & \multicolumn{7}{|c|}{ ALL LIFETIME PHOTOS } & \multicolumn{5}{|c|}{ MOST RECENT PHOTO } \\
\hline & \multicolumn{3}{|c|}{ WEBCAM } & \multicolumn{4}{|c|}{ MUGSHOT } & \multirow{2}{*}{$\begin{array}{c}\text { VISA } \\
\mathrm{N}=20 \mathrm{~K}\end{array}$} & \multicolumn{4}{|c|}{ MUGSHOT } \\
\hline & $\mathrm{N}=160 \mathrm{~K}$ & $\mathrm{~N}=640 \mathrm{~K}$ & $\mathrm{~N}=1600 \mathrm{~K}$ & $\mathrm{~N}=20 \mathrm{~K}$ & $\mathrm{~N}=160 \mathrm{~K}$ & $\mathrm{~N}=640 \mathrm{~K}$ & $\mathrm{~N}=1600 \mathrm{~K}$ & & $\mathrm{~N}=20 \mathrm{~K}$ & $\mathrm{~N}=160 \mathrm{~K}$ & $\mathrm{~N}=640 \mathrm{~K}$ & $\mathrm{~N}=1600 \mathrm{~K}$ \\
\hline A20C & 0.351 & 0.395 & ${ }^{17} 0.429$ & 0.099 & 0.127 & 0.150 & ${ }^{22} 0.166$ & ${ }^{16} 0.101$ & 0.119 & 0.145 & 0.167 & ${ }^{22} 0.182$ \\
\hline $\mathrm{A} 30 \mathrm{C}$ & 0.286 & 0.331 & ${ }^{16} 0.362$ & 0.087 & 0.116 & 0.139 & ${ }^{21} 0.155$ & ${ }^{11} 0.084$ & 0.105 & 0.133 & 0.156 & ${ }^{20} 0.172$ \\
\hline A31C & 0.287 & 0.331 & ${ }^{15} 0.362$ & 0.087 & 0.116 & 0.139 & ${ }^{20} 0.155$ & ${ }^{12} 0.084$ & 0.105 & 0.133 & 0.156 & ${ }^{19} 0.172$ \\
\hline $\mathrm{A} 32 \mathrm{C}$ & 0.382 & 0.431 & ${ }^{19} 0.467$ & 0.105 & 0.132 & 0.156 & ${ }^{23} 0.172$ & ${ }^{24} 0.124$ & 0.140 & 0.173 & 0.200 & ${ }^{25} 0.216$ \\
\hline B30C & 0.592 & 0.634 & ${ }^{24} 0.658$ & 0.081 & 0.100 & 0.113 & ${ }^{17} 0.123$ & ${ }^{23} 0.123$ & 0.097 & 0.120 & 0.136 & ${ }^{17} 0.147$ \\
\hline B31C & 0.592 & 0.634 & ${ }^{23} 0.658$ & 0.081 & 0.100 & 0.113 & ${ }^{16} 0.123$ & ${ }^{22} 0.123$ & 0.097 & 0.120 & 0.137 & ${ }^{16} 0.147$ \\
\hline B32C & 0.488 & 0.531 & ${ }^{21} 0.560$ & 0.071 & 0.091 & 0.104 & ${ }^{15} 0.113$ & ${ }^{18} 0.106$ & 0.085 & 0.109 & 0.125 & ${ }^{14} 0.136$ \\
\hline B33C & 0.488 & 0.531 & ${ }^{20} 0.560$ & 0.071 & 0.091 & 0.104 & ${ }^{14} 0.113$ & ${ }^{17} 0.106$ & 0.085 & 0.109 & 0.125 & ${ }^{15} 0.136$ \\
\hline C20C & 0.768 & 0.793 & ${ }^{28} 0.810$ & 0.153 & 0.177 & 0.194 & ${ }^{27} 0.206$ & ${ }^{33} 0.244$ & 0.179 & 0.208 & 0.229 & ${ }^{27} 0.243$ \\
\hline C30C & 0.585 & 0.624 & ${ }^{22} 0.651$ & 0.122 & 0.146 & 0.165 & ${ }^{25} 0.179$ & ${ }^{26} 0.213$ & 0.143 & 0.173 & 0.195 & ${ }^{24} 0.211$ \\
\hline C31C & 0.624 & 0.659 & ${ }^{26} 0.685$ & 0.121 & 0.143 & 0.162 & ${ }^{24} 0.173$ & ${ }^{25} 0.200$ & 0.142 & 0.169 & 0.191 & ${ }^{23} 0.205$ \\
\hline C32C & 0.596 & 0.637 & ${ }^{25} 0.664$ & 0.129 & 0.155 & 0.175 & ${ }^{26} 0.190$ & ${ }^{28} 0.221$ & 0.150 & 0.183 & 0.207 & ${ }^{26} 0.223$ \\
\hline D20C & 0.239 & 0.267 & ${ }^{9} 0.287$ & 0.060 & 0.068 & 0.075 & ${ }^{9} 0.080$ & ${ }^{9} 0.066$ & 0.073 & 0.081 & 0.089 & ${ }^{9} 0.096$ \\
\hline D30C & 0.245 & 0.273 & ${ }^{10} 0.290$ & 0.059 & 0.067 & 0.073 & ${ }^{8} 0.080$ & ${ }^{5} 0.061$ & 0.068 & 0.078 & 0.087 & ${ }^{8} 0.093$ \\
\hline D32C & 0.376 & 0.415 & ${ }^{18} 0.445$ & 0.106 & 0.128 & 0.144 & ${ }^{19} 0.155$ & ${ }^{20} 0.115$ & 0.125 & 0.150 & 0.168 & ${ }^{21} 0.180$ \\
\hline D33C & 0.248 & 0.274 & ${ }^{11} 0.291$ & 0.058 & 0.066 & 0.072 & ${ }^{6} 0.078$ & ${ }^{6} 0.062$ & 0.067 & 0.076 & 0.085 & ${ }^{6} 0.091$ \\
\hline D34C & 0.248 & 0.277 & ${ }^{13} 0.296$ & 0.055 & 0.066 & 0.073 & ${ }^{7} 0.079$ & ${ }^{8} 0.064$ & 0.065 & 0.076 & 0.086 & ${ }^{7} 0.092$ \\
\hline E20C & 0.077 & 0.094 & ${ }^{4} 0.108$ & 0.025 & 0.032 & 0.038 & ${ }^{4} 0.042$ & ${ }^{4} 0.026$ & 0.029 & 0.037 & 0.044 & ${ }^{4} 0.049$ \\
\hline E21C & 0.077 & 0.094 & ${ }^{3} 0.108$ & 0.025 & 0.032 & 0.038 & ${ }^{3} 0.042$ & ${ }^{3} 0.026$ & 0.029 & 0.037 & 0.044 & ${ }^{3} 0.049$ \\
\hline E30C & 0.079 & 0.097 & ${ }^{2} 0.108$ & 0.024 & 0.029 & 0.032 & ${ }^{1} 0.035$ & ${ }^{1} 0.017$ & 0.028 & 0.034 & 0.037 & ${ }^{\mathrm{I}} 0.041$ \\
\hline E31C & 0.079 & 0.095 & ${ }^{I} 0.106$ & 0.025 & 0.031 & 0.034 & ${ }^{2} 0.037$ & ${ }^{2} 0.023$ & 0.028 & 0.035 & 0.038 & ${ }^{2} 0.042$ \\
\hline F20C & 0.465 & & 37 & 0.232 & 0.282 & & 37 & ${ }^{36} 0.278$ & 0.256 & 0.312 & & 36 \\
\hline F30C & 0.356 & 0.678 & ${ }^{29} 0.867$ & 0.165 & 0.214 & 0.603 & ${ }^{29} 0.843$ & ${ }^{29} 0.232$ & 0.194 & 0.247 & 0.618 & 34 \\
\hline F31C & 0.365 & 0.720 & ${ }^{30} 0.886$ & 0.163 & 0.214 & 0.656 & ${ }^{30} 0.864$ & ${ }^{32} 0.239$ & 0.192 & 0.248 & 0.670 & 43 \\
\hline G30C & 0.423 & & 44 & 0.187 & 0.245 & & 44 & ${ }^{39} 0.308$ & 0.220 & 0.285 & 0.333 & 44 \\
\hline G31C & 0.369 & & 38 & 0.171 & 0.219 & & 38 & ${ }^{27} 0.214$ & 0.198 & 0.254 & 0.293 & 37 \\
\hline $\mathrm{H} 30 \mathrm{C}$ & 0.451 & 0.504 & 35 & 0.262 & 0.309 & 0.346 & 35 & ${ }^{38} 0.285$ & 0.300 & 0.353 & 0.392 & 32 \\
\hline J20C & 0.240 & 0.272 & ${ }^{14} 0.297$ & 0.076 & 0.102 & 0.119 & ${ }^{18} 0.133$ & ${ }^{19} 0.114$ & 0.090 & 0.121 & 0.143 & ${ }^{18} 0.161$ \\
\hline $\mathrm{J} 30 \mathrm{C}$ & 0.194 & 0.231 & ${ }^{7} 0.253$ & 0.061 & 0.079 & 0.093 & ${ }^{12} 0.104$ & ${ }^{15} 0.097$ & 0.070 & 0.091 & 0.109 & ${ }^{12} 0.122$ \\
\hline J33C & 0.173 & 0.204 & ${ }^{6} 0.229$ & 0.052 & 0.067 & 0.081 & ${ }^{10} 0.091$ & ${ }^{10} 0.082$ & 0.060 & 0.079 & 0.095 & ${ }^{10} 0.107$ \\
\hline L30C & 0.691 & & 41 & 0.385 & 0.442 & & 41 & ${ }^{42} 0.348$ & 0.428 & 0.490 & & 40 \\
\hline L31C & 0.640 & & 40 & 0.339 & 0.398 & & 40 & ${ }^{41} 0.332$ & 0.382 & 0.444 & & 39 \\
\hline M20C & 0.586 & 0.625 & 32 & 0.319 & 0.371 & 0.412 & 32 & ${ }^{37} 0.279$ & 0.346 & 0.412 & 0.458 & 29 \\
\hline M21C & 0.561 & 0.598 & 39 & 0.304 & 0.355 & 0.393 & 39 & ${ }^{34} 0.273$ & 0.327 & 0.390 & 0.435 & 38 \\
\hline M30C & 0.547 & & 33 & 0.285 & 0.342 & & 33 & ${ }^{35} 0.273$ & 0.327 & 0.390 & 0.435 & 30 \\
\hline P30C & 0.361 & 0.408 & ${ }^{27} 0.728$ & 0.093 & 0.123 & 0.147 & ${ }^{28} 0.564$ & ${ }^{21} 0.117$ & 0.120 & 0.151 & 0.172 & 33 \\
\hline Q30C & 0.551 & & 42 & 0.303 & 0.361 & & 42 & ${ }^{40} 0.309$ & 0.343 & 0.405 & & 41 \\
\hline S20C & 0.796 & & 43 & 0.467 & 0.523 & & 43 & ${ }^{44} 0.420$ & 0.512 & 0.569 & & 42 \\
\hline T30C & 0.650 & 0.692 & 34 & 0.391 & 0.449 & 0.492 & 34 & ${ }^{43} 0.351$ & 0.432 & 0.495 & 0.540 & 31 \\
\hline
\end{tabular}

Table 10: Rank-1 miss rates: The table gives FNIR values for different enrolled population sizes, three different image types, and two enrolment strategies. At left are seven columns indicating accuracy for searches of legacy webcam images and mugshots; these results apply where all but the most recent image of an individual is enrolled, and the last image is searched. On the right side, only the penultimate image is enrolled, and the last image is searched (see section 3.2). The column in yellow is highlighted because it applies to well posed visa images of individuals including children. All other columns apply to images drawn from the LEO set. The blue superscripts are column-wise algorithm rankings. Empty cells indicate that the run was not attempted, or was unsuccessful.

\begin{tabular}{|c|c|c|c|c|c|c|}
\hline $\begin{array}{l}\mathrm{A}=3 \mathrm{M} / \text { Cogent } \\
\mathrm{G}=\text { Hisign } \\
\mathrm{P}=\text { Zhuhai-Yisheng }\end{array}$ & $\begin{array}{l}\mathrm{B}=\text { Cognitec } \\
\mathrm{H}=\text { CAS-IA } \\
\mathrm{Q}=\text { JunYu }\end{array}$ & $\begin{array}{l}\mathrm{C}=\text { Neurotechnology } \\
\mathrm{I}=\mathrm{CAS}-\mathrm{ICT} \\
\mathrm{S}=\text { Decatur }\end{array}$ & $\begin{array}{l}\mathrm{D}=\text { Safran Morpho } \\
\mathrm{J}=\text { Toshiba } \\
\mathrm{T}=\text { Ayonix }\end{array}$ & $\begin{array}{l}\mathrm{E}=\mathrm{NEC} \\
\mathrm{L}=\text { Tsinghua U. II }\end{array}$ & $\begin{array}{l}\mathrm{F}=\text { Tsinghua } \mathrm{U} \\
\mathrm{M}=\mathrm{HP}\end{array}$ & $\begin{array}{l}\text { FNIR(N,R,T,L) “Miss rate" } \\
\text { FPIR(N,T,L) “False alarm rate" }\end{array}$ \\
\hline
\end{tabular}




\begin{tabular}{|c|c|c|c|c|c|c|c|c|c|c|c|c|}
\hline \multirow{3}{*}{$\begin{array}{l}\text { ENROL } \\
\text { RANK=50 } \\
\text { ALG } \\
\end{array}$} & \multicolumn{7}{|c|}{ ALL LIFETIME PHOTOS } & \multicolumn{5}{|c|}{ MOST RECENT PHOTO } \\
\hline & \multicolumn{3}{|c|}{ WEBCAM } & \multicolumn{4}{|c|}{ MUGSHOT } & \multirow{2}{*}{$\begin{array}{c}\text { VISA } \\
\mathrm{N}=20 \mathrm{~K} \\
\end{array}$} & \multicolumn{4}{|c|}{ MUGSHOT } \\
\hline & $\mathrm{N}=160 \mathrm{~K}$ & $\mathrm{~N}=640 \mathrm{~K}$ & $\mathrm{~N}=1600 \mathrm{~K}$ & $\mathrm{~N}=20 \mathrm{~K}$ & $\mathrm{~N}=160 \mathrm{~K}$ & $\mathrm{~N}=640 \mathrm{~K}$ & $\mathrm{~N}=1600 \mathrm{~K}$ & & $\mathrm{~N}=20 \mathrm{~K}$ & $\mathrm{~N}=160 \mathrm{~K}$ & $\mathrm{~N}=640 \mathrm{~K}$ & $\mathrm{~N}=1600 \mathrm{~K}$ \\
\hline A20C & 0.174 & 0.222 & ${ }^{17} 0.255$ & $\overline{0.044}$ & 0.063 & 0.080 & ${ }^{21} 0.093$ & ${ }^{16} 0.033$ & 0.057 & 0.078 & 0.097 & ${ }^{21} 0.110$ \\
\hline A31C & 0.126 & 0.167 & ${ }^{16} 0.198$ & 0.039 & 0.055 & 0.071 & ${ }^{20} 0.083$ & ${ }^{6} 0.019$ & 0.049 & 0.067 & 0.086 & ${ }^{20} 0.098$ \\
\hline $\mathrm{A} 32 \mathrm{C}$ & 0.203 & 0.253 & ${ }^{18} 0.288$ & 0.050 & 0.068 & 0.084 & ${ }^{22} 0.096$ & ${ }^{18} 0.039$ & 0.070 & 0.094 & 0.115 & ${ }^{23} 0.131$ \\
\hline B30C & 0.371 & 0.443 & ${ }^{25} 0.491$ & 0.044 & 0.058 & 0.069 & ${ }^{18} 0.078$ & ${ }^{24} 0.053$ & 0.052 & 0.070 & 0.084 & ${ }^{18} 0.094$ \\
\hline B32C & 0.274 & 0.341 & ${ }^{21} 0.383$ & 0.037 & 0.050 & 0.061 & ${ }^{16} 0.069$ & ${ }^{20} 0.042$ & 0.044 & 0.060 & 0.074 & ${ }^{15} 0.084$ \\
\hline B33C & 0.274 & 0.341 & ${ }^{20} 0.383$ & 0.037 & 0.050 & 0.061 & ${ }^{15} 0.069$ & ${ }^{19} 0.042$ & 0.044 & 0.060 & 0.074 & ${ }^{16} 0.084$ \\
\hline$\overline{C 20 C}$ & 0.615 & 0.667 & ${ }^{28} 0.700$ & 0.091 & 0.115 & 0.134 & ${ }^{27} 0.147$ & ${ }^{39} 0.143$ & 0.107 & 0.137 & 0.160 & ${ }^{27} 0.174$ \\
\hline C30C & 0.373 & 0.433 & ${ }^{22} 0.478$ & 0.057 & 0.082 & 0.102 & ${ }^{25} 0.115$ & ${ }^{31} 0.106$ & 0.066 & 0.096 & 0.122 & ${ }^{25} 0.137$ \\
\hline C31C & 0.441 & 0.490 & ${ }^{26} 0.528$ & 0.063 & 0.084 & 0.103 & ${ }^{24} 0.114$ & ${ }^{30} 0.102$ & 0.075 & 0.100 & 0.122 & ${ }^{24} 0.136$ \\
\hline $\mathrm{C} 32 \mathrm{C}$ & 0.382 & 0.444 & ${ }^{23} 0.489$ & 0.060 & 0.087 & 0.109 & ${ }^{26} 0.122$ & ${ }^{33} 0.109$ & 0.071 & 0.102 & 0.128 & ${ }^{26} 0.145$ \\
\hline$\overline{\mathrm{D} 20 \mathrm{C}}$ & 0.127 & 0.161 & ${ }^{11} 0.188$ & 0.035 & 0.044 & 0.053 & ${ }^{13} 0.061$ & ${ }^{11} 0.021$ & 0.040 & 0.052 & 0.064 & 130.072 \\
\hline D30C & 0.127 & 0.163 & ${ }^{12} 0.189$ & 0.033 & 0.042 & 0.051 & ${ }^{12} 0.059$ & ${ }^{8} 0.020$ & 0.039 & 0.050 & 0.061 & ${ }^{11} 0.071$ \\
\hline D32C & 0.228 & 0.264 & ${ }^{19} 0.292$ & 0.058 & 0.076 & 0.090 & ${ }^{23} 0.101$ & 220.049 & 0.068 & 0.089 & 0.107 & ${ }^{22} 0.119$ \\
\hline D33C & 0.128 & 0.166 & ${ }^{13} 0.191$ & 0.033 & 0.042 & 0.051 & ${ }^{11} 0.059$ & ${ }^{9} 0.020$ & 0.039 & 0.050 & 0.061 & ${ }^{12} 0.071$ \\
\hline D34C & 0.121 & 0.151 & ${ }^{10} 0.174$ & 0.030 & 0.038 & 0.046 & ${ }^{7} 0.054$ & ${ }^{10} 0.020$ & 0.035 & 0.046 & 0.056 & ${ }^{7} 0.064$ \\
\hline E20C & 0.032 & 0.042 & ${ }^{3} 0.049$ & 0.014 & 0.018 & 0.022 & ${ }^{3} 0.025$ & ${ }^{3} 0.007$ & 0.017 & 0.021 & 0.025 & ${ }^{3} 0.028$ \\
\hline E21C & 0.032 & 0.042 & ${ }^{2} 0.049$ & 0.014 & 0.018 & 0.022 & ${ }^{2} 0.025$ & ${ }^{2} 0.007$ & 0.017 & 0.021 & 0.025 & ${ }^{2} 0.028$ \\
\hline E30C & 0.032 & 0.043 & ${ }^{\mathrm{I}} 0.048$ & 0.016 & 0.019 & 0.021 & ${ }^{\mathrm{I}} 0.023$ & ${ }^{1} 0.006$ & 0.018 & 0.021 & 0.024 & ${ }^{1} 0.026$ \\
\hline E31C & 0.039 & 0.050 & ${ }^{4} 0.058$ & 0.018 & 0.021 & 0.024 & ${ }^{4} 0.026$ & ${ }^{4} 0.007$ & 0.020 & 0.024 & 0.028 & ${ }^{4} 0.031$ \\
\hline F20C & 0.251 & & 37 & 0.108 & 0.155 & & 37 & ${ }^{37} 0.130$ & 0.118 & 0.173 & & 36 \\
\hline F30C & 0.180 & 0.584 & ${ }^{29} 0.831$ & 0.074 & 0.108 & 0.545 & ${ }^{29} 0.820$ & ${ }^{25} 0.082$ & 0.089 & 0.129 & 0.555 & 34 \\
\hline F31C & 0.180 & 0.636 & ${ }^{30} 0.855$ & 0.074 & 0.108 & 0.606 & ${ }^{30} 0.844$ & ${ }^{28} 0.085$ & 0.088 & 0.128 & 0.616 & 43 \\
\hline G30C & 0.196 & & 44 & 0.078 & 0.119 & & 44 & ${ }^{36} 0.123$ & 0.091 & 0.139 & 0.182 & 44 \\
\hline G31C & 0.187 & & 38 & 0.076 & 0.113 & & 38 & ${ }^{29} 0.087$ & 0.088 & 0.132 & 0.168 & 37 \\
\hline H30C & 0.260 & 0.312 & 35 & 0.136 & 0.183 & 0.219 & 35 & ${ }^{38} 0.132$ & 0.158 & 0.214 & 0.257 & 32 \\
\hline J20C & 0.107 & 0.134 & ${ }^{9} 0.160$ & 0.030 & 0.044 & 0.058 & ${ }^{14} 0.068$ & ${ }^{17} 0.034$ & 0.037 & 0.055 & 0.071 & ${ }^{14} 0.083$ \\
\hline J30C & 0.080 & 0.111 & ${ }^{7} 0.135$ & 0.027 & 0.039 & 0.050 & ${ }^{8} 0.058$ & ${ }^{15} 0.029$ & 0.031 & 0.044 & 0.056 & ${ }^{8} 0.067$ \\
\hline $\mathrm{J} 33 \mathrm{C}$ & 0.066 & 0.094 & ${ }^{6} 0.117$ & 0.023 & 0.033 & 0.042 & ${ }^{5} 0.049$ & ${ }^{12} 0.021$ & 0.027 & 0.038 & 0.048 & ${ }^{5} 0.057$ \\
\hline L30C & 0.489 & & 41 & 0.222 & 0.293 & & 41 & ${ }^{43} 0.173$ & 0.254 & 0.334 & & 40 \\
\hline L31C & 0.433 & & 40 & 0.188 & 0.253 & & 40 & ${ }^{41} 0.161$ & 0.214 & 0.290 & & 39 \\
\hline M20C & 0.377 & 0.436 & 32 & 0.169 & 0.221 & 0.266 & 32 & ${ }^{32} 0.108$ & 0.174 & 0.245 & 0.297 & 29 \\
\hline M21C & 0.349 & 0.403 & 39 & 0.162 & 0.211 & 0.252 & 39 & ${ }^{34} 0.109$ & 0.165 & 0.230 & 0.281 & 38 \\
\hline M30C & 0.333 & & 33 & 0.145 & 0.198 & & 33 & ${ }^{35} 0.109$ & 0.165 & 0.230 & 0.281 & 30 \\
\hline P30C & 0.182 & 0.230 & ${ }^{27} 0.663$ & 0.041 & 0.059 & 0.076 & ${ }^{28} 0.530$ & ${ }^{21} 0.043$ & 0.056 & 0.078 & 0.100 & 33 \\
\hline Q30C & 0.305 & & 42 & 0.144 & 0.206 & & 42 & ${ }^{40} 0.148$ & 0.164 & 0.240 & & 41 \\
\hline S20C & 0.639 & & 43 & 0.298 & 0.373 & & 43 & ${ }^{44} 0.237$ & 0.333 & 0.418 & & 42 \\
\hline T30C & 0.452 & 0.514 & 34 & 0.225 & 0.288 & 0.338 & 34 & ${ }^{42} 0.163$ & 0.254 & 0.328 & 0.384 & 31 \\
\hline
\end{tabular}

Table 11: Rank-50 miss rates: The table gives FNIR values for different enrolled population sizes, three different image types, and two enrolment strategies. At left are seven columns indicating accuracy for searches of legacy webcam images and mugshots; these results apply where all but the most recent image of an individual is enrolled, and the last image is searched. On the right side, only the penultimate image is enrolled, and the last image is searched (see section 3.2). The column in yellow is highlighted because it applies to well posed visa images of individuals including children. All other columns apply to images drawn from the LEO set. The blue superscripts are column-wise algorithm rankings. Empty cells indicate that the run was not attempted, or was unsuccessful.

\begin{tabular}{|c|c|c|c|c|c|c|}
\hline $\begin{array}{l}\mathrm{A}=3 \mathrm{M} / \text { Cogent } \\
\mathrm{G}=\text { Hisign } \\
\mathrm{P}=\text { Zhuhai-Yisheng }\end{array}$ & $\begin{array}{l}\mathrm{B}=\text { Cognitec } \\
\mathrm{H}=\text { CAS-IA } \\
\mathrm{Q}=\text { JunYu }\end{array}$ & $\begin{array}{l}\mathrm{C}=\text { Neurotechnology } \\
\mathrm{I}=\mathrm{CAS}-\mathrm{ICT} \\
\mathrm{S}=\text { Decatur }\end{array}$ & $\begin{array}{l}\mathrm{D}=\text { Safran Morpho } \\
\mathrm{J}=\text { Toshiba } \\
\mathrm{T}=\text { Ayonix }\end{array}$ & $\begin{array}{l}\mathrm{E}=\mathrm{NEC} \\
\mathrm{L}=\text { Tsinghua U. II }\end{array}$ & $\begin{array}{l}\mathrm{F}=\text { Tsinghua } \mathrm{U} \\
\mathrm{M}=\mathrm{HP}\end{array}$ & $\begin{array}{l}\text { FNIR(N,R,T,L) “Miss rate" } \\
\text { FPIR(N,T,L) "False alarm rate" }\end{array}$ \\
\hline
\end{tabular}




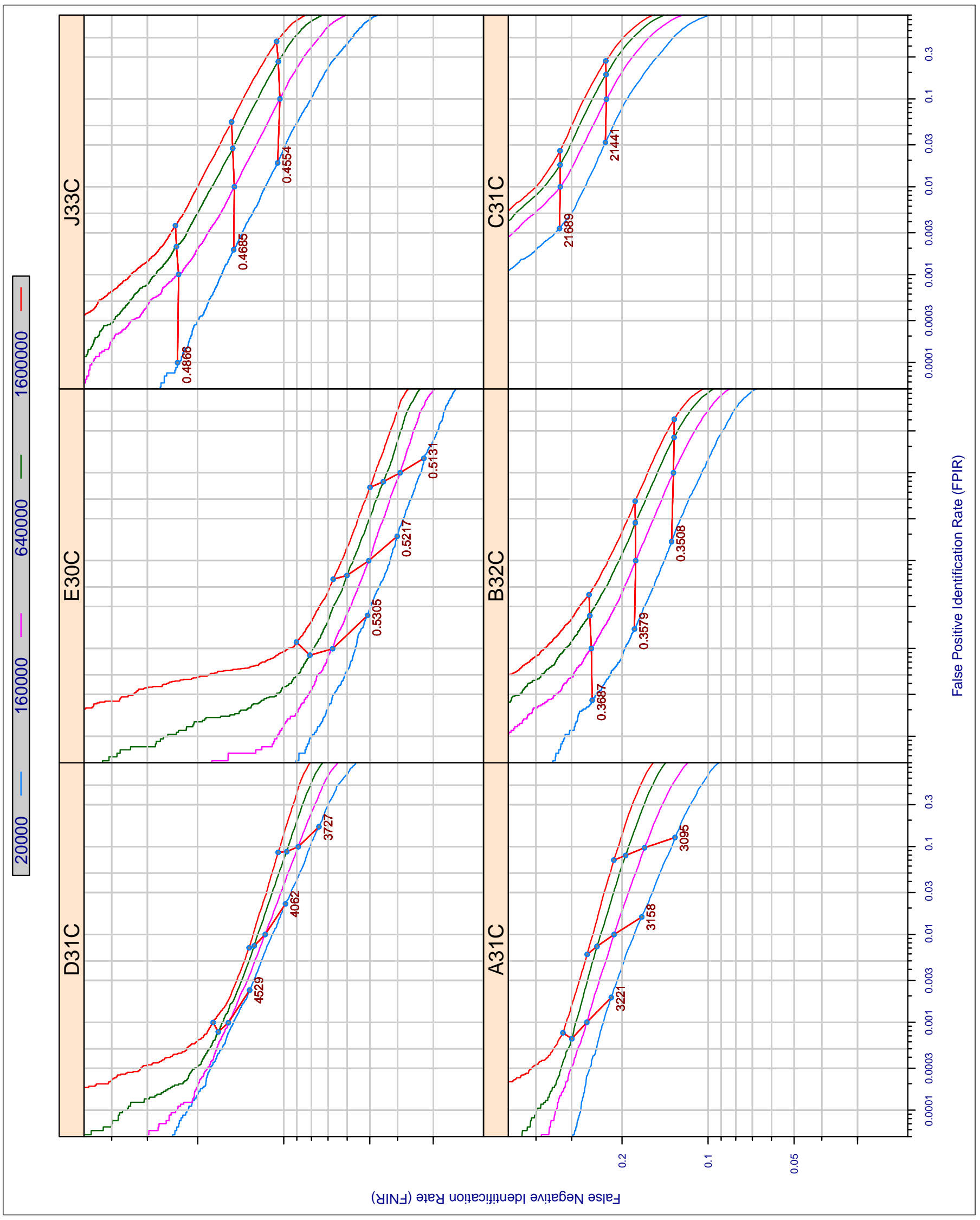

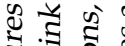

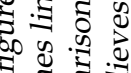

$\approx: \frac{\pi}{2}:$

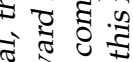

宽

둥

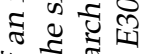

of

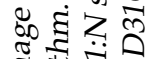

교 $\pi$ is

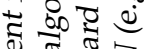

$\Xi$

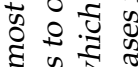

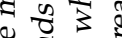

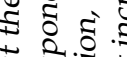

के क्षेत्र पे

: 8 : : :

$\widetilde{\Xi} \widetilde{\Xi}$

도원

ฐ ซ 5

क ज :

2 용

6. 용

घ

도윰

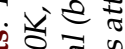

ษ్ శี

ㄴ. 임

क ฮ :

ग II $\overrightarrow{2}$

की

ब.

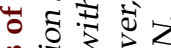

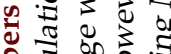

요 조

र

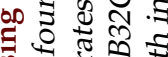

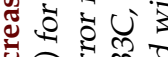

Ð

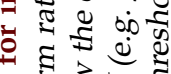

\# ส

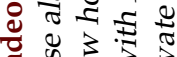

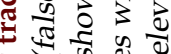

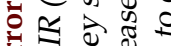

4 高志过

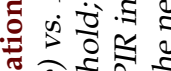

త

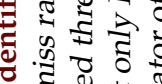

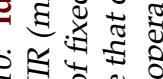

윽

: 近壳芯志

\begin{tabular}{|c|c|c|c|c|c|c|}
\hline $\begin{array}{l}A=3 \mathrm{M} / \text { Cogent } \\
\mathrm{G}=\text { Hisign } \\
\mathrm{P}=\text { Zhuhai-Yisheng }\end{array}$ & $\begin{array}{l}\mathrm{B}=\text { Cognitec } \\
\mathrm{H}=\mathrm{CAS}-\mathrm{IA} \\
\mathrm{Q}=\text { Jun } Y \mathrm{u}\end{array}$ & $\begin{array}{l}\mathrm{C}=\text { Neurotechnology } \\
\mathrm{I}=\text { CAS-ICT } \\
\mathrm{S}=\text { Decatur }\end{array}$ & $\begin{array}{l}\mathrm{D}=\text { Safran Morpho } \\
\mathrm{J}=\text { Toshiba } \\
\mathrm{T}=\text { Ayonix }\end{array}$ & $\begin{array}{l}\mathrm{E}=\mathrm{NEC} \\
\mathrm{L}=\text { Tsinghua U. II }\end{array}$ & $\begin{array}{l}\mathrm{F}=\text { Tsinghua } \mathrm{U} \\
\mathrm{M}=\mathrm{HP}\end{array}$ & $\begin{array}{l}\text { FNIR(N,R,T,L) “Miss rate" } \\
\text { FPIR(N,T,L) “False alarm rate" }\end{array}$ \\
\hline
\end{tabular}


$\triangleright$ Tabulation: $\operatorname{FNIR}(N, R, 0, L)$ is tabulated exhaustively for four population sizes and, for $R=1,50$, in Tables 10 and 11.

$\triangleright$ Graphs: This same data is plotted as $\operatorname{FNIR}(N, R, 0, L)$ against $\mathrm{N}$ in Figure 9.

$\triangle$ DETs: Detection error tradeoff characteristics appear for the more accurate algorithms in Figure 10. These plot $\operatorname{FNIR}(N, L, T, L)$ vs. $\operatorname{FPIR}(N, T, L)$ for the four population sizes, and all thresholds.

$\triangleright$ Models: The rank-based FNIR(N, R, 0, L) data is modeled using illustrative power-law models.

Discussion: It is clear from Figure 9 that mated search miss rates increase with enrolled population size and decrease with rank. Most importantly, miss rates usually only increase slowly with population size. While this aspect is common to other biometric modalities, it alone is largely responsible for the operational utility of face identification algorithms. Specifically, with a ten-fold population size increase, from 160,000 to $1,600,000$, the rank one miss rates only increase by small factors, 1.2 for E30C (0.029 to 0.035 , Table 10$)$, 1.1 for D31C (0.065 to 0.071), and 1.4 for J33C (0.067 to 0.091$)$. This result is modeled in the next section.

The $\mathrm{F}$ and $\mathrm{P}$ algorithms have problems at the largest population size; while this may be a property of the underlying mathematics, it may also be a (software) implementation issue.

When algorithms are configured with high thresholds to curtail false positives, the dependence on $\mathrm{N}$ is mostly similar. However, Figure 10 shows that, at FPIR $=0.002$, there is reduced sensitivity of FNIR on N. For example for the D31C algorithm, as population size increases from 160,000 to 1,600,000, FNIR increases from 0.122 to 0.134 , a factor of 1.1 (lifetime mugshots, Tables 4 and 6).

Figure 10 also shows lines connecting DETs corresponding to fixed thresholds. These reveal various dependencies of FPIR on $\mathrm{N}$; this is discussed further in section 5.3.2.

\subsubsection{Models of FNIR dependence on $\mathbf{N}$ and $\mathrm{R}$}

Many plots in Figure 9 are approximately linear on log-log axes. Moreover, Figure 8 shows a similar linear reduction in FNIR with rank, again on log-log axes. These observations motivate development here of FNIR models i.e. empirical formulae giving miss rate as a function of population size, $\mathrm{N}$, and rank, $\mathrm{R}$. The goal here is not to produce a definitive and usable model of accuracy but instead to highlight sublinear scalability effects, and to support reasoning about how accuracy varies.

For any given dataset, the coarse empirical observation is that $\log$ FNIR is approximately $\operatorname{linear}$ in $\log N$ and $\log R$. This corresponds to a power-law model:

$$
\operatorname{FNIR}(N, R, 0, L)=a N^{b} R^{c}
$$

where the coefficient $a$ is an implied recognition rate for $N=R=1, b$ is a scalability exponent, and $c$ quantifies the occurrence of mates at higher (poorer) rank. Note that this model applies to the investigational application where the threshold is relaxed $T=0^{8}$, and the requested number of candidates, $L$, is assumed to not affect the candidates appearing at $\operatorname{rank} R=1 \ldots L$.

This model does not exhibit the correct behavior for the case $N \rightarrow R$ and, more seriously, does not capture the behavior seen (e.g. for algorithm D20C) showing that searching to rank 50 is less worthwhile relative to rank 1 at large population

${ }^{8}$ More advanced models of how accuracy scales with $\mathrm{N}$ have been proposed. These include threshold as an independent variable - the goal being to assist in setting thresholds as the enrolled population increases. These models $[4,13,15,16,23]$ are beyond our purpose here.

\begin{tabular}{|c|c|c|c|c|c|c|}
\hline $\begin{array}{l}\mathrm{A}=3 \mathrm{M} / \text { Cogent } \\
\mathrm{G}=\text { Hisign } \\
\mathrm{P}=\text { Zhuhai-Yisheng }\end{array}$ & $\begin{array}{l}\mathrm{B}=\text { Cognitec } \\
\mathrm{H}=\mathrm{CAS}-\mathrm{IA} \\
\mathrm{Q}=\text { JunYu }\end{array}$ & $\begin{array}{l}\mathrm{C}=\text { Neurotechnology } \\
\mathrm{I}=\text { CAS-ICT } \\
\mathrm{S}=\text { Decatur }\end{array}$ & $\begin{array}{l}\mathrm{D}=\text { Safran Morpho } \\
\mathrm{J}=\text { Toshiba } \\
\mathrm{T}=\text { Ayonix }\end{array}$ & $\begin{array}{l}\mathrm{E}=\mathrm{NEC} \\
\mathrm{L}=\text { Tsinghua U. II }\end{array}$ & $\begin{array}{l}\mathrm{F}=\text { Tsinghua } \mathrm{U} \\
\mathrm{M}=\mathrm{HP}\end{array}$ & $\begin{array}{l}\text { FNIR(N,R,T,L) “Miss rate" } \\
\text { FPIR(N,T,L) “False alarm rate" }\end{array}$ \\
\hline
\end{tabular}


sizes. This implies that the scaling exponent $b$ should itself be dependent on $\mathrm{R}$.

$$
\operatorname{FNIR}(N, R, 0, L)=a(N-R)^{b-d \log R} R^{c}
$$

The models of equations 8 and 9 are fit to the available empirical FNIR data for each algorithm, for the "recent" enrolment type, and for mugshot and webcam acquisition types. The exponents of the fits are tabulated in Table 12. The result show the scalability exponents of equation 8 typically has values $0.08<b<0.16$ indicating very sublinear growth with $N$. The models also reveal that human adjudication of long candidate lists (to rank $\mathrm{R}$ ) offers diminishing returns - the exponent $c$ in eq. 8 has values $-0.18<c<-0.09$. We caution against using any of these values to compare algorithms since the models are inexact and lack theoretical support.

\subsubsection{Dependence of FPIR on $\mathrm{N}$}

Figure 10 shows lines connecting DETs corresponding to fixed thresholds. These reveal that algorithms exhibit one of two broad dependencies of FPIR on N:

$\triangleright$ Linear: Some algorithms exhibit an almost linear dependence of FPIR, while others have some invariance to population size. In classical biometric theory, $\operatorname{FPIR}(N, T)=N \operatorname{FMR}(T)$ where FMR is the one-to-one false match probability. This is an approximation to the Binomial model of independent failures FPIR $=1-(1-\mathrm{FMR})^{N}$ with small FMR. The linear dependence of FPIR on $\mathrm{N}$ approximately holds for algorithms from Cognitec, Neurotechnology and Toshiba. Implementations that implement 1:N search as $\mathrm{N}$ 1:1 comparisons would exhibit this behavior.

$\triangleright$ Constant: Other algorithms, including those from 3M/Cogent, Morpho and NEC, exhibit $\operatorname{FPIR}(N, T)$ having a complicated dependence on N, with FPIR being approximately constant (independent of N), or in some cases even reducing at larger population sizes. Such behavior can be achieved via score-normalization [15] and by implementing results from extreme value theory - the statistics of the largest of $\mathrm{N}$ random samples.

In any case, owners of systems that employ non-zero thresholds will need to understand the dependence of false positive rates on population size - the goal being to have predictable FPIR. Either approach is viable given adequate documentation and planning.

\subsection{Effect of enrolling all historical images}

Methods: The LIFETIME enrolment type is defined by enrolment of $K_{i}$ images per person as described in section 3.2. By executing the standard set of searches, FNIR can be computed over searches where the mate was enrolled with $K_{i}=$ $1,2, \ldots$ enrolled images.

Results: Substantially reduced miss rates are measured for all algorithms as functions of K.

Discussion: The overall FNIR values reported previously for the LIFETIME enrolments represent the maximum realizable accuracy given this data. The relevance of the results in this section is on system design policy: operators should plan to store, enrol, and use the full lifetime history of images from an individual. The results here do not, however, answer the question of how old an image should be before it is retired from use. While, the default guidance here is to retain all images regardless of capture date, an ageing study is indicated to quantify un-enrolment schedules. Such a study would need to quantify false positive consequences of retaining too many images.

\begin{tabular}{|c|c|c|c|c|c|c|}
\hline $\begin{array}{l}\mathrm{A}=3 \mathrm{M} / \text { Cogent } \\
\mathrm{G}=\text { Hisign } \\
\mathrm{P}=\text { Zhuhai-Yisheng }\end{array}$ & $\begin{array}{l}\mathrm{B}=\text { Cognitec } \\
\mathrm{H}=\mathrm{CAS}-\mathrm{IA} \\
\mathrm{Q}=\text { JunYu }\end{array}$ & $\begin{array}{l}\mathrm{C}=\text { Neurotechnology } \\
\mathrm{I}=\text { CAS-ICT } \\
\mathrm{S}=\text { Decatur }\end{array}$ & $\begin{array}{l}\mathrm{D}=\text { Safran Morpho } \\
\mathrm{J}=\text { Toshiba } \\
\mathrm{T}=\text { Ayonix }\end{array}$ & $\begin{array}{l}\mathrm{E}=\mathrm{NEC} \\
\mathrm{L}=\text { Tsinghua U. II }\end{array}$ & $\begin{array}{l}\mathrm{F}=\text { Tsinghua } \mathrm{U} \\
\mathrm{M}=\mathrm{HP}\end{array}$ & $\begin{array}{l}\text { FNIR(N,R,T,L) “Miss rate" } \\
\text { FPIR(N,T,L) “False alarm rate" }\end{array}$ \\
\hline
\end{tabular}




\begin{tabular}{|c|c|c|c|c|}
\hline & \multicolumn{2}{|c|}{ SIMPLE MODEL OF EQUATION 8} & \multicolumn{2}{|c|}{ EXTENDED MODEL OF EQUATION 9} \\
\hline ALGORITHM & RECENT MUGSHOT & RECENT WEBCAM & RECENT MUGSHOT & RECENT WEBCAM \\
\hline A20C & $0.034 N^{0.120} R^{-0.145}$ & $0.092 N^{0.109} R^{-0.140}$ & $0.044(N-R)^{0.099+0.014 \log R} R^{-0.330}$ & $0.137(N-R)^{0.080+0.019 \log R} R^{-0.392}$ \\
\hline $\mathrm{A} 30 \mathrm{C}$ & $0.027 N^{0.132} R^{-0.158}$ & $0.060 N^{0.129} R^{-0.165}$ & $0.034(N-R)^{0.114+0.012 \log R} R^{-0.315}$ & $0.090(N-R)^{0.098+0.021 \log R} R^{-0.445}$ \\
\hline A31C & $0.027 N^{0.132} R^{-0.158}$ & $0.060 N^{0.129} R^{-0.165}$ & $0.034(N-R)^{0.114+0.012 \log R} R^{-0.315}$ & $0.090(N-R)^{0.098+0.021 \log R} R^{-0.444}$ \\
\hline A32C & $0.041 N^{0.118} R^{-0.142}$ & $0.105 N^{0.107} R^{-0.130}$ & $0.052(N-R)^{0.101+0.011 \log R} R^{-0.290}$ & $0.152(N-R)^{0.080+0.017 \log R} R^{-0.362}$ \\
\hline B30C & $0.031 N^{0.110} R^{-0.126}$ & $0.237 N^{0.076} R^{-0.089}$ & $0.038(N-R)^{0.095+0.010 \log R} R^{-0.251}$ & $0.359(N-R)^{0.045+0.018 \log R} R^{-0.329}$ \\
\hline B31C & $0.031 N^{0.110} R^{-0.126}$ & $0.237 N^{0.076} R^{-0.089}$ & $0.038(N-R)^{0.095+0.010 \log R} R^{-0.251}$ & $0.359(N-R)^{0.045+0.018 \log R} R^{-0.329}$ \\
\hline B32C & $0.024 N^{0.122} R^{-0.137}$ & $0.164 N^{0.091} R^{-0.109}$ & $0.030(N-R)^{0.107+0.010 \log R} R^{-0.262}$ & $0.261(N-R)^{0.056+0.021 \log R} R^{-0.392}$ \\
\hline B33C & $0.024 N^{0.122} R^{-0.137}$ & $0.163 N^{0.091} R^{-0.109}$ & $0.030(N-R)^{0.107+0.010 \log R} R^{-0.263}$ & $0.261(N-R)^{0.056+0.021 \log R} R^{-0.393}$ \\
\hline C20C & $0.072 N^{0.088} R^{-0.105}$ & $0.504 N^{0.036} R^{-0.043}$ & $0.090(N-R)^{0.070+0.011 \log R} R^{-0.237}$ & $0.625(N-R)^{0.019+0.009 \log R} R^{-0.157}$ \\
\hline C30C & $0.042 N^{0.116} R^{-0.131}$ & $0.248 N^{0.072} R^{-0.090}$ & $0.061(N-R)^{0.087+0.019 \log R} R^{-0.377}$ & $0.358(N-R)^{0.044+0.016 \log R} R^{-0.302}$ \\
\hline C31C & $0.047 N^{0.106} R^{-0.121}$ & $0.319 N^{0.056} R^{-0.071}$ & $0.062(N-R)^{0.083+0.014 \log R} R^{-0.302}$ & $0.403(N-R)^{0.039+0.010 \log R} R^{-0.200}$ \\
\hline $\mathrm{C} 32 \mathrm{C}$ & $0.044 N^{0.116} R^{-0.130}$ & $0.255 N^{0.071} R^{-0.088}$ & $0.063(N-R)^{0.088+0.018 \log R} R^{-0.363}$ & $0.363(N-R)^{0.045+0.015 \log R} R^{-0.293}$ \\
\hline D20C & $0.025 N^{0.095} R^{-0.096}$ & $0.061 N^{0.113} R^{-0.127}$ & $0.038(N-R)^{0.063+0.019 \log R} R^{-0.340}$ & $0.107(N-R)^{0.071+0.027 \log R} R^{-0.485}$ \\
\hline $\mathrm{D} 30 \mathrm{C}$ & $0.023 N^{0.098} R^{-0.098}$ & $0.063 N^{0.110} R^{-0.130}$ & $0.033(N-R)^{0.069+0.017 \log R} R^{-0.319}$ & $0.112(N-R)^{0.068+0.027 \log R} R^{-0.493}$ \\
\hline D31C & $0.024 N^{0.095} R^{-0.096}$ & $0.064 N^{0.110} R^{-0.134}$ & $0.035(N-R)^{0.064+0.018 \log R} R^{-0.328}$ & $0.119(N-R)^{0.064+0.029 \log R} R^{-0.528}$ \\
\hline D32C & $0.042 N^{0.104} R^{-0.119}$ & $0.135 N^{0.087} R^{-0.110}$ & $0.053(N-R)^{0.085+0.012 \log R} R^{-0.268}$ & $0.162(N-R)^{0.074+0.008 \log R} R^{-0.220}$ \\
\hline D33C & $0.022 N^{0.100} R^{-0.092}$ & $0.067 N^{0.106} R^{-0.130}$ & $0.032(N-R)^{0.071+0.017 \log R} R^{-0.308}$ & $0.123(N-R)^{0.061+0.029 \log R} R^{-0.512}$ \\
\hline D34C & $0.022 N^{0.100} R^{-0.115}$ & $0.077 N^{0.098} R^{-0.152}$ & $0.030(N-R)^{0.077+0.015 \log R} R^{-0.303}$ & $0.113(N-R)^{0.069+0.019 \log R} R^{-0.408}$ \\
\hline E20C & $0.008 N^{0.124} R^{-0.140}$ & $0.010 N^{0.171} R^{-0.210}$ & $0.009(N-R)^{0.120+0.002 \log R} R^{-0.169}$ & $0.012(N-R)^{0.156+0.011 \log R} R^{-0.359}$ \\
\hline E21C & $0.008 N^{0.124} R^{-0.140}$ & $0.010 N^{0.171} R^{-0.210}$ & $0.009(N-R)^{0.120+0.002 \log R} R^{-0.169}$ & $0.012(N-R)^{0.156+0.011 \log R} R^{-0.359}$ \\
\hline E30C & $0.012 N^{0.083} R^{-0.112}$ & $0.014 N^{0.145} R^{-0.206}$ & $0.012(N-R)^{0.085+-0.001 \log R} R^{-0.094}$ & $0.018(N-R)^{0.128+0.013 \log R} R^{-0.377}$ \\
\hline E31C & $0.011 N^{0.089} R^{-0.083}$ & $0.013 N^{0.149} R^{-0.163}$ & $0.012(N-R)^{0.087+0.001 \log R} R^{-0.101}$ & $0.017(N-R)^{0.131+0.012 \log R} R^{-0.324}$ \\
\hline F20C & $0.069 N^{0.128} R^{-0.164}$ & - & $0.099(N-R)^{0.096+0.022 \log R} R^{-0.409}$ & - \\
\hline F21C & $0.046 N^{0.142} R^{-0.177}$ & - & $0.057(N-R)^{0.122+0.014 \log R} R^{-0.334}$ & - \\
\hline F22C & $0.046 N^{0.142} R^{-0.178}$ & - & $0.056(N-R)^{0.123+0.013 \log R} R^{-0.326}$ & - \\
\hline F30C & $0.000 N^{0.653} R^{-0.046}$ & $0.000 N^{0.614} R^{-0.058}$ & $0.001(N-R)^{0.453+0.116 \log R} R^{-1.574}$ & $0.002(N-R)^{0.445+0.096 \log R} R^{-1.321}$ \\
\hline F31C & $0.000 N^{0.733} R^{-0.037}$ & $0.000 N^{0.654} R^{-0.052}$ & $0.001(N-R)^{0.509+0.127 \log R} R^{-1.710}$ & $0.001(N-R)^{0.469+0.105 \log R} R^{-1.429}$ \\
\hline G30C & $0.048 N^{0.148} R^{-0.175}$ & $0.087 N^{0.133} R^{-0.172}$ & $0.068(N-R)^{0.119+0.020 \log R} R^{-0.427}$ & $0.139(N-R)^{0.097+0.025 \log R} R^{-0.495}$ \\
\hline G31C & $0.047 N^{0.139} R^{-0.160}$ & $0.092 N^{0.117} R^{-0.150}$ & $0.065(N-R)^{0.112+0.018 \log R} R^{-0.385}$ & $0.134(N-R)^{0.088+0.019 \log R} R^{-0.394}$ \\
\hline $\mathrm{H} 30 \mathrm{C}$ & $0.103 N^{0.102} R^{-0.125}$ & $0.138 N^{0.101} R^{-0.127}$ & $0.140(N-R)^{0.077+0.016 \log R} R^{-0.315}$ & $0.178(N-R)^{0.081+0.012 \log R} R^{-0.286}$ \\
\hline $\mathrm{J} 20 \mathrm{C}$ & $0.019 N^{0.151} R^{-0.184}$ & $0.046 N^{0.137} R^{-0.172}$ & $0.024(N-R)^{0.133+0.013 \log R} R^{-0.357}$ & $0.069(N-R)^{0.107+0.021 \log R} R^{-0.457}$ \\
\hline $\mathrm{J} 30 \mathrm{C}$ & $0.015 N^{0.147} R^{-0.172}$ & $0.031 N^{0.153} R^{-0.181}$ & $0.019(N-R)^{0.128+0.014 \log R} R^{-0.352}$ & $0.048(N-R)^{0.120+0.024 \log R} R^{-0.499}$ \\
\hline $\mathrm{J} 31 \mathrm{C}$ & $0.015 N^{0.151} R^{-0.180}$ & $0.032 N^{0.153} R^{-0.184}$ & $0.019(N-R)^{0.133+0.013 \log R} R^{-0.345}$ & $0.051(N-R)^{0.118+0.025 \log R} R^{-0.518}$ \\
\hline $\mathrm{J} 32 \mathrm{C}$ & $0.013 N^{0.147} R^{-0.169}$ & $0.026 N^{0.159} R^{-0.193}$ & $0.017(N-R)^{0.128+0.013 \log R} R^{-0.343}$ & $0.044(N-R)^{0.120+0.029 \log R} R^{-0.585}$ \\
\hline $\mathrm{J} 33 \mathrm{C}$ & $0.013 N^{0.149} R^{-0.174}$ & $0.027 N^{0.155} R^{-0.197}$ & $0.016(N-R)^{0.132+0.012 \log R} R^{-0.333}$ & $0.045(N-R)^{0.116+0.029 \log R} R^{-0.585}$ \\
\hline L30C & $0.169 N^{0.091} R^{-0.110}$ & - & $0.228(N-R)^{0.064+0.016 \log R} R^{-0.291}$ & - \\
\hline L31C & $0.140 N^{0.099} R^{-0.122}$ & - & $0.192(N-R)^{0.070+0.018 \log R} R^{-0.318}$ & - \\
\hline M20C & $0.112 N^{0.108} R^{-0.130}$ & $0.214 N^{0.083} R^{-0.104}$ & $0.156(N-R)^{0.081+0.018 \log R} R^{-0.343}$ & $0.299(N-R)^{0.057+0.016 \log R} R^{-0.305}$ \\
\hline M21C & $0.105 N^{0.109} R^{-0.131}$ & $0.198 N^{0.086} R^{-0.110}$ & $0.147(N-R)^{0.081+0.017 \log R} R^{-0.343}$ & $0.280(N-R)^{0.059+0.016 \log R} R^{-0.319}$ \\
\hline M30C & $0.105 N^{0.109} R^{-0.131}$ & $0.199 N^{0.085} R^{-0.110}$ & $0.147(N-R)^{0.081+0.017 \log R} R^{-0.343}$ & $0.283(N-R)^{0.058+0.017 \log R} R^{-0.322}$ \\
\hline P30C & $0.031 N^{0.131} R^{-0.159}$ & $0.096 N^{0.112} R^{-0.148}$ & $0.042(N-R)^{0.105+0.017 \log R} R^{-0.370}$ & $0.139(N-R)^{0.083+0.019 \log R} R^{-0.393}$ \\
\hline Q30C & $0.106 N^{0.115} R^{-0.151}$ & - & $0.161(N-R)^{0.078+0.025 \log R} R^{-0.430}$ & e \\
\hline S20C & $0.246 N^{0.072} R^{-0.090}$ & - & $0.321(N-R)^{0.048+0.014 \log R} R^{-0.244}$ & - \\
\hline T30C & $0.176 N^{0.086} R^{-0.103}$ & $0.302 N^{0.065} R^{-0.082}$ & $0.231(N-R)^{0.064+0.013 \log R} R^{-0.266}$ & $0.389(N-R)^{0.045+0.011 \log R} R^{-0.227}$ \\
\hline
\end{tabular}

Table 12: Approximate models of FNIR. For each algorithm, the table gives the power-law estimates (equations 8 and 9) as estimated using non-linear least squares. The formulae apply to LEO images searched against enrolment sets comprised of the most recent image of $N=20,000 \ldots 1,600,000$ individuals. The power-law functional form is chosen on the basis of the approximately straight lines observed in Figures 9 and 8. Caution: These formulae represent empirical models that lack theoretical support. It would be an abuse to evaluate these formulae at values $N>1.6$ million. In addition, the formulae only approximate the actual measured values.

\begin{tabular}{|c|c|c|c|c|c|c|}
\hline $\begin{array}{l}\mathrm{A}=3 \mathrm{M} / \text { Cogent } \\
\mathrm{G}=\text { Hisign } \\
\mathrm{P}=\text { Zhuhai-Yisheng }\end{array}$ & $\begin{array}{l}\mathrm{B}=\text { Cognitec } \\
\mathrm{H}=\text { CAS-IA } \\
\mathrm{Q}=\text { JunYu }\end{array}$ & $\begin{array}{l}\mathrm{C}=\text { Neurotechnology } \\
\mathrm{I}=\mathrm{CAS}-\mathrm{ICT} \\
\mathrm{S}=\text { Decatur }\end{array}$ & $\begin{array}{l}\mathrm{D}=\text { Safran Morpho } \\
\mathrm{J}=\text { Toshiba } \\
\mathrm{T}=\text { Ayonix }\end{array}$ & $\begin{array}{l}\mathrm{E}=\mathrm{NEC} \\
\mathrm{L}=\text { Tsinghua U. II }\end{array}$ & $\begin{array}{l}\mathrm{F}=\text { Tsinghua } \mathrm{U} \\
\mathrm{M}=\mathrm{HP}\end{array}$ & $\begin{array}{l}\text { FNIR(N,R,T,L) “Miss rate" } \\
\text { FPIR(N,T,L) "False alarm rate" }\end{array}$ \\
\hline
\end{tabular}




\subsection{Accuracy dependence on subject age}

Methods: The VISA dataset includes age information. As shown in the second entry of Table 3, one image of each of the $\mathrm{N}$ = 19972 individuals is enrolled. Thereafter, one mated search is conducted per person to allow FNIR estimation. Finally, 203,082 nonmated searches are run to support FPIR measurement.

We compute accuracy by age group. We define seven age groups corresponding to stages in life in which facial appearance is similar, and between which there is consensus that appearance is usually different ${ }^{9}$. We attach informal labels to these, as shown in Table 13. The age of the individual at the time the search image was collected is used to determine the age group bin. The enrolled image is acquired some time before that - statistics on the time elapsed between mated pairs appears in Table 13. FNIR is computed over those pairs via the normal definition of equation (3). The elapsed times are too short for the study to quantify longitudinal ageing effects. The results for young subjects are affected by ageing because the elapsed times, of 1-4 years are considerable in an infant.

\begin{tabular}{|l|l|r|r|r|r|}
\hline $\begin{array}{l}\text { Group } \\
\text { No. }\end{array}$ & $\begin{array}{l}\text { Group } \\
\text { Label }\end{array}$ & $\begin{array}{r}\text { Age } \\
\text { Range }\end{array}$ & $\begin{array}{r}\text { Search } \\
\text { Age Mean }\end{array}$ & $\begin{array}{r}\text { Mated Time } \\
\text { Lapse Mean }\end{array}$ & $\begin{array}{r}\text { Mated } \\
\text { Count }\end{array}$ \\
\hline 1 & baby & {$[0,3)$} & 2.3 & 1.6 & 57 \\
\hline 2 & kid & {$[3,8)$} & 5.7 & 2.8 & 340 \\
\hline 3 & pre & {$[8,13)$} & 10.7 & 3.7 & 533 \\
\hline 4 & teen & {$[13,19)$} & 17.0 & 2.5 & 1447 \\
\hline 5 & young & {$[19,30)$} & 25.4 & 2.0 & 5930 \\
\hline 6 & parents & {$[30,55)$} & 40.5 & 2.1 & 8293 \\
\hline 7 & older & {$[55,101)$} & 63.6 & 2.2 & 2709 \\
\hline
\end{tabular}

Nonmate searches produce top scoring enrolled candidates that vary by algorithm. FPIR is computed us-

Table 13: Age groups: Labels and sizes of age groups to which search images are assigned. Values in columns 3 to 5 are in years.

ing equation (1), applied for each search-image age group. The distribution of the age difference between the enrolment images and whatever non-mates are returned in the search is algorithm-specific, and is not documented in this report.

Results: The results appear in two places: For six, more accurate, algorithms, Figure 11 shows detection error tradeoff characteristics for the seven age groups. Appendix A shows identical graphs for all algorithms.

Discussion: Regarding the figures, the notable points are:

- Recognition is progressively easier with advancing age: All algorithms exhibit a strong dependence of FNIR on age. This effect is very large, spanning a factor of ten from infant to senior, and a factor of around five from teen to senior. Miss rates for older persons are very low: at a fixed FPIR of 0.005, the most accurate algorithm, E30C, gives FNIR of 0.008 for persons over age 55, 0.027 for young 20-somethings, and 0.057 for teenagers. For younger persons, the miss rates climb rapidly to 0.29 for pre-teens, 0.4 for kids, to 0.7 for babies. This progression is common to all algorithms.

- Young children are more difficult to recognize: Identification miss rates (FNIR) ascend rapidly for pre-teens, kids and the youngest individuals. For the baby group, 0 to about 3 years old, identification fails more often that it succeeds, i.e. FNIR is above $50 \%$. While the sample size is small (57 subjects), error rates are so high that the result remains significant. This result applies for image pairs collected on average 1.6 years apart (Table 13) and will be in considerable part due to the craniofacial shape change associated with rapid growth. The extent to which smooth "feature-less" skin texture affects FNIR is unknown. Likewise the pose variations inherent in photographing children have not been quantified.

- Young children are more difficult to discriminate: All of the algorithms exhibit higher false positive identification rates for younger subjects. The grey lines in Figure 11, which link points of equal threshold, slope upwards to the

${ }^{9}$ We are prevented from using Shakespeare's seven ages of man from the All The World's a Stage passage in As You Like It.

\begin{tabular}{|c|c|c|c|c|c|c|}
\hline $\begin{array}{l}A=3 \mathrm{M} / \text { Cogent } \\
G=\text { Hisign } \\
P=\text { Zhuhai-Yisheng }\end{array}$ & $\begin{array}{l}\mathrm{B}=\text { Cognitec } \\
\mathrm{H}=\text { CAS-IA } \\
\mathrm{Q}=\text { JunYu }\end{array}$ & $\begin{array}{l}\mathrm{C}=\text { Neurotechnology } \\
\mathrm{I}=\text { CAS-ICT } \\
\mathrm{S}=\text { Decatur }\end{array}$ & $\begin{array}{l}\mathrm{D}=\text { Safran Morpho } \\
\mathrm{J}=\text { Toshiba } \\
\mathrm{T}=\text { Ayonix }\end{array}$ & $\begin{array}{l}\mathrm{E}=\mathrm{NEC} \\
\mathrm{L}=\text { Tsinghua U. II }\end{array}$ & $\begin{array}{l}\mathrm{F}=\text { Tsinghua } \mathrm{U} \\
\mathrm{M}=\mathrm{HP}\end{array}$ & $\begin{array}{l}\text { FNIR(N,R,T,L) "Miss rate" } \\
\text { FPIR(N,T,L) “False alarm rate" }\end{array}$ \\
\hline
\end{tabular}


right, indicating simultaneously that younger subjects are less easy to recognize as themselves but also less easy to tell apart. This indicates that younger individuals are more difficult to discriminate from other individuals.

- False positive identification rate excursions: For algorithms C31C and J33C in particular, the reduction of FPIR at a fixed threshold continues progressively throughout adulthood. For example, using algorithm C31C configured with threshold 21539, FPIR reduces from 0.2 in infants, to 0.05 in teenagers, to below 0.002 in seniors.

\begin{tabular}{|c|c|c|c|c|c|c|}
\hline $\begin{array}{l}\mathrm{A}=3 \mathrm{M} / \text { Cogent } \\
\mathrm{G}=\text { Hisign } \\
\mathrm{P}=\text { Zhuhai-Yisheng }\end{array}$ & $\begin{array}{l}\mathrm{B}=\text { Cognitec } \\
\mathrm{H}=\text { CAS-IA } \\
\mathrm{Q}=\text { JunYu }\end{array}$ & $\begin{array}{l}\mathrm{C}=\text { Neurotechnology } \\
\mathrm{I}=\mathrm{CAS}-\mathrm{ICT} \\
\mathrm{S}=\text { Decatur }\end{array}$ & $\begin{array}{l}\mathrm{D}=\text { Safran Morpho } \\
\mathrm{J}=\text { Toshiba } \\
\mathrm{T}=\text { Ayonix }\end{array}$ & $\begin{array}{l}\mathrm{E}=\mathrm{NEC} \\
\mathrm{L}=\text { Tsinghua U. II }\end{array}$ & $\begin{array}{l}\mathrm{F}=\text { Tsinghua } \mathrm{U} \\
\mathrm{M}=\mathrm{HP}\end{array}$ & $\begin{array}{l}\text { FNIR(N,R,T,L) “Miss rate" } \\
\text { FPIR(N,T,L) “False alarm rate" }\end{array}$ \\
\hline
\end{tabular}



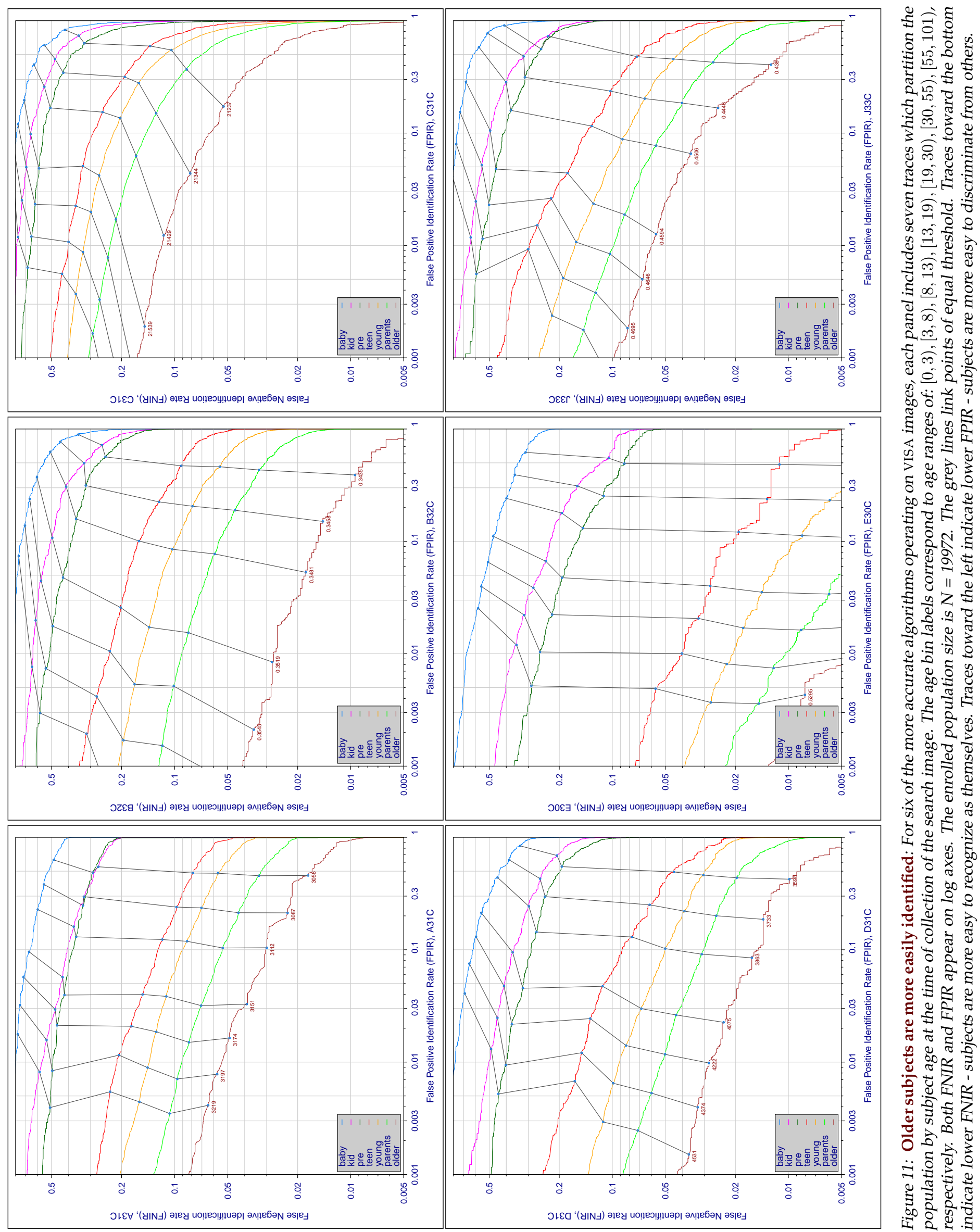

\begin{tabular}{|c|c|c|c|c|c|c|}
\hline $\begin{array}{l}A=3 \mathrm{M} / \text { Cogent } \\
G=\text { Hisign } \\
P=\text { Zhuhai-Yisheng }\end{array}$ & $\begin{array}{l}\mathrm{B}=\text { Cognitec } \\
\mathrm{H}=\mathrm{CAS}-\mathrm{IA} \\
\mathrm{Q}=\text { JunYu }\end{array}$ & $\begin{array}{l}\mathrm{C}=\text { Neurotechnology } \\
\mathrm{I}=\text { CAS-ICT } \\
\mathrm{S}=\text { Decatur }\end{array}$ & $\begin{array}{l}\mathrm{D}=\text { Safran Morpho } \\
\mathrm{J}=\text { Toshiba } \\
\mathrm{T}=\text { Ayonix }\end{array}$ & $\begin{array}{l}\mathrm{E}=\mathrm{NEC} \\
\mathrm{L}=\text { Tsinghua U. II }\end{array}$ & $\begin{array}{l}\mathrm{F}=\text { Tsinghua } \mathrm{U} \\
\mathrm{M}=\mathrm{HP}\end{array}$ & $\begin{array}{l}\text { FNIR(N,R,T,L) "Miss rate" } \\
\text { FPIR(N,T,L) “False alarm rate" }\end{array}$ \\
\hline
\end{tabular}




\subsection{Accuracy of sketch recognition}

Sketches have long been used in criminal investigations. Historically the most common occurrence is for a forensic artist to interview an eye-witness and, iteratively, produce a likeness of the individual recollected by the witness. The result, a forensic sketch, is essentially a pencil drawing. Nowadays, commercial software is typically used to the same ends, and this results in a composite sketch.

This section addresses whether such sketches can be matched, in one-to-many mode, against photographs resident in a mugshot database.

Methods: We leverage photos from the very commonly used FERET database and a set of sketches of those FERET images published by the City University of Hong Kong (CUHK). A pair is shown in Figure 3. Given photographs of $n=840$ sketched individuals and $N=640,000$ mugshots of different individuals (from the LEO dataset, we enrolled $n+N$ photographs. We then searched a) $n$ different frontal photographs from the FERET database; and b) the $n$ CUHK sketches of the enrolled FERET images. Searches produced $L=50$ candidates. This experimental design is similar to that used in prior algorithm evaluation of sketch identification algorithms [18]. That study enrolled $n=75$ plus $N=10,000$ mugshots and searched both forensic and composite sketches with $L=200$.

Results: Table 14 shows miss rates for the photo and sketch searches. The values are $\operatorname{FNIR}(n+N, R, 0, L)$ for $R=$ $\{1,10,50\}$, and $L=50$.

Discussion: Sketch identification accuracy is far inferior to that documented for mugshots elsewhere in this report, particularly Table 5 , for $\mathrm{N}=640 \mathrm{~K}$.

$\triangleright$ Use of non-sequestered, public-domain, images: The FERET images and sketches are all in the public domain so, in principle, the results could be manipulated either via training, or outright memorization. This is unlikely, however, since the algorithm providers had no prior reason to suspect that FERET images or sketches would be part of the FRVT evaluation.

$\triangleright$ Use of algorithms for tasks they were not designed: As sketch-identification was never declared to be part of the study, the algorithms are being used in a manner not expressly intended by the providers. Such "off label" usage ${ }^{10}$ is in fact operational reality - automated algorithms are being used to recognize sketches in many police departments.

$\triangleright$ High miss rates: The rank-1 identification miss rates are usually above $90 \%$. The most accurate result, for A20C from $3 \mathrm{M} /$ Cogent, misses the mate $89.7 \%$ of the time. At rank 50 , the best result, for that same algorithm, is $73.3 \%$ of mates are missed, i.e. only $26.7 \%$ are hit. Note the most accurate mugshot algorithms are not the most accurate sketch identification algorithms.

$\triangleright$ High miss rates may nevertheless be useful: The poor accuracy figures have to be compared with not having an automated search capability at all. In such cases, the miss rate is $100 \%$ and investigative leads have to be developed without automated face recognition. As such, the $26.7 \%$ hit rate represents a very useful resource in otherwise cold cases. The workload associated with human adjudication of long candidate lists is considered, for mugshots, in section 5.7

$\triangleright$ An upper bound on accuracy: The fact that the sketches were prepared by an artist viewing the exemplar photograph probably means that the accuracy measurements here represent a "best case" upper bound on accuracy. That said, the artist did not attempt to produce a photo-realistic image of the person but instead introduced a "shape

${ }^{10}$ The term "off label" is used in the medical community to indicate that a drug developed for one condition is prescribed by a doctor for a different, often unrelated, condition.

\begin{tabular}{|c|c|c|c|c|c|c|}
\hline $\begin{array}{l}\mathrm{A}=3 \mathrm{M} / \text { Cogent } \\
\mathrm{G}=\text { Hisign } \\
\mathrm{P}=\text { Zhuhai-Yisheng }\end{array}$ & $\begin{array}{l}\mathrm{B}=\text { Cognitec } \\
\mathrm{H}=\text { CAS-IA } \\
\mathrm{Q}=\text { JunYu }\end{array}$ & $\begin{array}{l}\mathrm{C}=\text { Neurotechnology } \\
\mathrm{I}=\mathrm{CAS}-\mathrm{ICT} \\
\mathrm{S}=\text { Decatur }\end{array}$ & $\begin{array}{l}\mathrm{D}=\text { Safran Morpho } \\
\mathrm{J}=\text { Toshiba } \\
\mathrm{T}=\text { Ayonix }\end{array}$ & $\begin{array}{l}\mathrm{E}=\mathrm{NEC} \\
\mathrm{L}=\text { Tsinghua U. II }\end{array}$ & $\begin{array}{l}\mathrm{F}=\text { Tsinghua } \mathrm{U} \\
\mathrm{M}=\mathrm{HP}\end{array}$ & $\begin{array}{l}\text { FNIR(N,R,T,L) “Miss rate" } \\
\text { FPIR(N,T,L) “False alarm rate" }\end{array}$ \\
\hline
\end{tabular}




\begin{tabular}{|c|c|c|c|c|c|c|}
\hline Enrolled & \multicolumn{6}{|c|}{ INVESTIGATION FNIR } \\
\hline$N=640000$ & \multicolumn{3}{|c|}{ PHOTOGRAPH } & \multicolumn{3}{|c|}{ SKETCH } \\
\hline ALG & RANK $=1$ & RANK $=10$ & RANK $=50$ & RANK $=1$ & RANK $=10$ & RANK $=50$ \\
\hline A20C & 0.014 & 0.001 & 0.001 & 0.897 & 0.803 & 0.729 \\
\hline $\mathrm{A} 30 \mathrm{C}$ & 0.014 & 0.001 & 0.001 & 0.900 & 0.821 & 0.750 \\
\hline A31C & 0.014 & 0.001 & 0.001 & 0.900 & 0.821 & 0.750 \\
\hline $\mathrm{A} 32 \mathrm{C}$ & 0.016 & 0.001 & 0.001 & 0.959 & 0.912 & 0.859 \\
\hline$\overline{\mathrm{B} 30 \mathrm{C}}$ & 0.017 & 0.005 & 0.001 & 0.994 & 0.983 & 0.964 \\
\hline B31C & 0.017 & 0.005 & 0.001 & 0.994 & 0.983 & 0.964 \\
\hline B32C & 0.017 & 0.002 & 0.001 & 0.987 & 0.967 & 0.948 \\
\hline $\mathrm{B} 33 \mathrm{C}$ & 0.017 & 0.002 & 0.001 & 0.987 & 0.967 & 0.948 \\
\hline C20C & 0.024 & 0.007 & 0.003 & 0.999 & 0.993 & 0.985 \\
\hline $\mathrm{C} 30 \mathrm{C}$ & 0.020 & 0.003 & 0.003 & 0.948 & 0.873 & 0.815 \\
\hline C31C & 0.021 & 0.005 & 0.002 & 0.964 & 0.922 & 0.890 \\
\hline C32C & 0.022 & 0.003 & 0.002 & 0.957 & 0.899 & 0.842 \\
\hline D20C & 0.014 & 0.001 & 0.001 & 0.950 & 0.883 & 0.808 \\
\hline D30C & 0.014 & 0.001 & 0.001 & 0.969 & 0.903 & 0.823 \\
\hline D31C & 0.014 & 0.001 & 0.001 & 0.952 & 0.879 & 0.806 \\
\hline D32C & 0.017 & 0.002 & 0.002 & 0.981 & 0.960 & 0.929 \\
\hline D33C & 0.014 & 0.001 & 0.001 & 0.963 & 0.899 & 0.831 \\
\hline D34C & 0.014 & 0.001 & 0.001 & 0.960 & 0.899 & 0.809 \\
\hline E20C & $\overline{0.014}$ & 0.001 & 0.001 & 0.903 & 0.823 & 0.738 \\
\hline E21C & 0.014 & 0.001 & 0.001 & 0.903 & 0.823 & 0.738 \\
\hline E30C & 0.015 & 0.001 & 0.001 & 0.923 & 0.855 & 0.763 \\
\hline E31C & 0.014 & 0.001 & 0.001 & 0.920 & 0.863 & 0.803 \\
\hline F20C & 0.506 & 0.494 & 0.494 & 0.953 & 0.902 & 0.845 \\
\hline F30C & 0.495 & 0.485 & 0.480 & 0.951 & 0.894 & 0.850 \\
\hline F31C & 0.563 & 0.555 & 0.550 & 0.957 & 0.901 & 0.858 \\
\hline$\overline{\mathrm{G} 20 \mathrm{C}}$ & 0.090 & 0.050 & 0.033 & $\overline{0.978}$ & 0.941 & 0.901 \\
\hline G30C & 0.051 & 0.016 & 0.009 & 0.973 & 0.935 & 0.852 \\
\hline G31C & 0.079 & 0.034 & 0.017 & 0.959 & 0.898 & 0.844 \\
\hline H30C & 0.070 & 0.041 & 0.027 & 0.963 & 0.907 & 0.833 \\
\hline $\mathrm{I} 20 \mathrm{C}$ & 0.022 & 0.008 & 0.005 & 0.942 & 0.892 & 0.820 \\
\hline$\overline{\mathrm{J} 20 \mathrm{C}}$ & $\overline{0.017}$ & 0.001 & 0.001 & 0.936 & 0.865 & $\overline{0.787}$ \\
\hline $\mathrm{J} 30 \mathrm{C}$ & 0.020 & 0.002 & 0.001 & 0.974 & 0.945 & 0.909 \\
\hline J31C & 0.020 & 0.002 & 0.001 & 0.977 & 0.948 & 0.909 \\
\hline $\mathrm{J} 32 \mathrm{C}$ & 0.016 & 0.002 & 0.001 & 0.957 & 0.907 & 0.865 \\
\hline $\mathrm{J} 33 \mathrm{C}$ & 0.016 & 0.002 & 0.001 & 0.957 & 0.907 & 0.865 \\
\hline M30C & 0.058 & 0.037 & 0.031 & 0.967 & 0.929 & 0.867 \\
\hline$\overline{\mathrm{P} 30 \mathrm{C}}$ & $\overline{0.019}$ & 0.006 & 0.005 & 0.957 & 0.921 & $\overline{0.872}$ \\
\hline Q20C & 0.466 & 0.087 & 0.049 & 1.000 & 0.993 & 0.973 \\
\hline S20C & 0.169 & 0.105 & 0.070 & 0.994 & 0.984 & 0.971 \\
\hline$\overline{\mathrm{T} 30 \mathrm{C}}$ & $\overline{0.088}$ & $\overline{0.056}$ & $\overline{0.051}$ & 0.979 & 0.937 & $\overline{0.888}$ \\
\hline
\end{tabular}

Table 14: Sketch searches mostly fail: FNIR "miss rates" for two kinds of searches made into an enrolled dataset of $N=640,864$ identities made up of 864 FERET photographs, and 640,000 background LEO mugshots. The first set is comprised of 864 searches of different frontal FERET photographs; the second set is of 864 CUHK sketches of those FERET photographs. The accuracy results for photo searches give very low error rates befitting good quality photographs. The sketch results are very poor - green shading is used to show miss rates below $90 \%, 85 \%$ and $80 \%$ at rank 1, 10 and 50 respectively. These thresholds are arbitrary.

\begin{tabular}{|c|c|c|c|c|c|c|}
\hline $\begin{array}{l}\mathrm{A}=3 \mathrm{M} / \text { Cogent } \\
\mathrm{G}=\text { Hisign } \\
\mathrm{P}=\text { Zhuhai-Yisheng }\end{array}$ & $\begin{array}{l}B=\text { Cognitec } \\
H=\text { CAS-IA } \\
Q=\text { JunYu }\end{array}$ & $\begin{array}{l}\mathrm{C}=\text { Neurotechnology } \\
\mathrm{I}=\mathrm{CAS}-\mathrm{ICT} \\
\mathrm{S}=\text { Decatur }\end{array}$ & $\begin{array}{l}\mathrm{D}=\text { Safran Morpho } \\
\mathrm{J}=\text { Toshiba } \\
\mathrm{T}=\text { Ayonix }\end{array}$ & $\begin{array}{l}\mathrm{E}=\mathrm{NEC} \\
\mathrm{L}=\text { Tsinghua U. II }\end{array}$ & $\begin{array}{l}\mathrm{F}=\text { Tsinghua } \mathrm{U} \\
\mathrm{M}=\mathrm{HP}\end{array}$ & $\begin{array}{l}\text { FNIR(N,R,T,L) “Miss rate" } \\
\text { FPIR(N,T,L) “False alarm rate" }\end{array}$ \\
\hline
\end{tabular}


exaggeration" to the face. While this does not go as far as a caricature artist would go, the change in appearance may or may not be representative of the situation in contemporary law enforcement processes where software is increasingly capable, but eye-witness recollections remain variable.

$\triangleright$ Algorithm comparison: Nevertheless, our use of this image set probably does reveal differences in algorithmic capability. Variation will in part depend on which facial information is represented. Prior work in this area [18] compared algorithms with and without landmark-based representations, the former outperforming the latter. Parties interested in establishing a sketch identification facility should inquire with their providers for algorithms specifically developed for sketch identification.

$\triangleright$ FERET photo recognition is very good: Many of the recognition algorithms give very low miss rates on the FERET photographs. This occurs because the FERET fa-fb frontal mated pairs are of good quality, better than that for the LEO images studied earlier in this report.

\subsection{Human workload for candidate list adjudication}

In a law enforcement scenario, for example, a human reviewer is usually employed to review the candidates returned from an identification search. Typically, the reviewer inspects the search image, and compares each candidate with that image, usually proceeding in the order of descending similarity score, and stopping when he is able to positively confirm a mate. The length of the candidate list may be fixed for all searches, or variable, depending on system configuration. The reviewer sometimes has the option to request a number of candidates, up to a certain limit. Variable length lists arise as a result of applying a threshold.

The following subsections advance models for the workload and costs associated with reviewer-led identification searches. Assumptions: This workload model assumes the following:

$\triangleright$ The candidates are reviewed serially, not all at once in a large screen GUI, for example.

$\triangleright$ The candidates are searched in decreasing order of similarity score, whether or not the reviewer is presented with the score.

$\triangleright$ Reviewers will stop after confirming a mate.

$\triangleright$ The database is correctly consolidated such that the number of mates is zero or one.

$\triangleright$ Reviewers always find a mate if it is present, and reviewers do not incorrectly associate a search with a nonmate candidate. Particularly, reviewer success is independent of the natural prior occurence of a mate. Effects of fatigue and boredom have been reported when this quantity is very low [20].

$\triangleright$ The time taken to confirm or exclude a candidate is independent of the rank of the candidate.

$\triangleright$ The time taken to confirm or exclude a candidate is independent of population size. This is potentially incorrect

\subsubsection{Fixed length candidate lists, threshold independent workload}

For now, assume also that the reviewer is not provided with, or ignores, similarity scores, and thresholds are not applied. Suppose an automated face identification algorithm returns $L$ candidates, and a human reviewer is retained to examine up to $\mathrm{R}$ candidates, where $R \leq L$ might be set by policy, preference or labor availability. Given the algorithm typically places

\begin{tabular}{|c|c|c|c|c|c|c|}
\hline $\begin{array}{l}\mathrm{A}=3 \mathrm{M} / \text { Cogent } \\
\mathrm{G}=\text { Hisign } \\
\mathrm{P}=\text { Zhuhai-Yisheng }\end{array}$ & $\begin{array}{l}\mathrm{B}=\text { Cognitec } \\
\mathrm{H}=\text { CAS-IA } \\
\mathrm{Q}=\text { Jun } \mathrm{Yu}\end{array}$ & $\begin{array}{l}\mathrm{C}=\text { Neurotechnology } \\
\mathrm{I}=\mathrm{CAS}-\mathrm{ICT} \\
\mathrm{S}=\text { Decatur }\end{array}$ & $\begin{array}{l}\mathrm{D}=\text { Safran Morpho } \\
\mathrm{J}=\text { Toshiba } \\
\mathrm{T}=\text { Ayonix }\end{array}$ & $\begin{array}{l}\mathrm{E}=\mathrm{NEC} \\
\mathrm{L}=\text { Tsinghua U. II }\end{array}$ & $\begin{array}{l}\mathrm{F}=\text { Tsinghua } \mathrm{U} \\
\mathrm{M}=\mathrm{HP}\end{array}$ & $\begin{array}{l}\text { FNIR(N,R,T,L) “Miss rate" } \\
\text { FPIR(N,T,L) “False alarm rate" }\end{array}$ \\
\hline
\end{tabular}


mates at low (good) ranks, the number of candidates a reviewer can be expected to review can be derived as follows. Note that the reviewer will:

$\triangleright$ Always inspect the first ranked image

Frac. reviewed $=1$

$\triangleright$ Then inspect those candidates where mate not confirmed at rank 1

Frac. reviewed $=1-\mathrm{CMC}(1)$

$\triangleright$ Then inspect those candidates where mate not confirmed at rank 1 or 2

Frac. reviewed $=1-\mathrm{CMC}(2)$

etc. Thus if the reviewer will stop at after a maximum of $\mathrm{R}$ candidates, the expected number of candidate reviews is

$$
\begin{aligned}
M(R) & =1+(1-C M C(1))+(1-C M C(2))+\ldots+(1-C M C(R-1)) \\
& =R-\sum_{r=1}^{R-1} C M C(r)
\end{aligned}
$$

A recognition algorithm that front-loads the cumulative match characteristic will offer reduced workload for the reviewer. This workload is defined only over the searches for which a mate exists. In the cases where there truly is no mate, the reviewer would review all $\mathrm{R}$ candidates. Thus, if the proportion of searches for which a mate does exist is $\beta$, which in the law enforcement context would be the recidivism rate [2], the full expression for workload becomes:

$$
\begin{aligned}
M(R) & =\beta\left(R-\sum_{r=1}^{R-1} C M C(r)\right)+(1-\beta) R \\
& =R-\beta \sum_{r=1}^{R-1} C M C(r)
\end{aligned}
$$

Results: Tables 4, 5 and 6 include values for this expression in columns labelled WORK.

Importantly, we restrict the analysis to the case of equation (11) where there is always a mate, i.e. $\beta=1$. This is done because the goal is to compare algorithms. Note that if $\beta<1$, reviewers will have to review more candidates than are plotted here. Indeed when $\beta=0$ all candidates will be reviewed regardless of which algorithm is used.

The tables show that if a reviewer is willing to review, $R=50$ candidates, then the expected number of candidates actually needing review will often be fewer than 10. For mugshot searches into an enrolled database of 1.6 million identities, enrolled with their most recent image, the NEC algorithms necessitate reviewers adjudicate around 1.6 candidates.

Cost implications: The above expressions for reviewer workload could be multiplied by suitable time and salary factors to estimate cost. Such a cost formulation should be extended to capture the cost of missing a mate altogether - this is a societal cost of failing to find a mate in the first $\mathrm{L}$ candidates.

\begin{tabular}{|c|c|c|c|c|c|c|}
\hline $\begin{array}{l}\mathrm{A}=3 \mathrm{M} / \text { Cogent } \\
\mathrm{G}=\text { Hisign } \\
\mathrm{P}=\text { Zhuhai-Yisheng }\end{array}$ & $\begin{array}{l}\mathrm{B}=\text { Cognitec } \\
\mathrm{H}=\text { CAS-IA } \\
\mathrm{Q}=\text { JunYu }\end{array}$ & $\begin{array}{l}\mathrm{C}=\text { Neurotechnology } \\
\mathrm{I}=\mathrm{CAS}-\mathrm{ICT} \\
\mathrm{S}=\text { Decatur }\end{array}$ & $\begin{array}{l}\mathrm{D}=\text { Safran Morpho } \\
\mathrm{J}=\text { Toshiba } \\
\mathrm{T}=\text { Ayonix }\end{array}$ & $\begin{array}{l}\mathrm{E}=\mathrm{NEC} \\
\mathrm{L}=\text { Tsinghua U. II }\end{array}$ & $\begin{array}{l}\mathrm{F}=\text { Tsinghua } \mathrm{U} \\
\mathrm{M}=\mathrm{HP}\end{array}$ & $\begin{array}{l}\text { FNIR(N,R,T,L) “Miss rate" } \\
\text { FPIR(N,T,L) “False alarm rate" }\end{array}$ \\
\hline
\end{tabular}

Conclusions: The use of more accurate face recognition algorithms implies decreased workload for human reviewers retained to adjudicate candidate lists. The expected number of candidates before a mate is found is a useful performance metric for identification systems. 


\subsubsection{Workload with thresholded variable length candidate lists}

In this section, the effect of thresholding candidate lists is considered. This has the potential to reduce workload further but at the expense of increased FNIR. If a score threshold is applied to a candidate list, either by the system or by the reviewer, the number of candidates that remain will be a random variable. In the previous section, that number was fixed at $R$; here it becomes $R_{i}(T)$ i.e. the number of candidates on the $i$-th candidate list that have score greater than or equal to $T$. The workload associated with adjudication of that list is given by equation 11 as $\mathrm{M}\left(R_{i}(T)\right)$. An accuracy loss arises, however, because low-scoring mates that are present on the full candidate list will not be available to the reviewer. This is stated by $\operatorname{FNIR}\left(N, R_{i}(T), T, L\right)$.

Results: Figure 12 shows this workload reduction, accuracy loss, tradeoff by plotting the two quantities parametrically with threshold, $\mathrm{T}$.

Discussion: The notable observations are:

$\triangleright$ Best result: For the D31C algorithm with an enrolled population of $\mathrm{N}=1.6$ million, workload can be reduced by $60 \%$ (to a factor of 0.4 times the baseline level) if a $5 \%$ increase in miss rates is tolerable.

$\triangleright$ Algorithm differences: For other algorithms the cost benefit position is worse. For the A31C, B32C, and C31C implementations the same $60 \%$ worklaod reduction gives around a $20 \%$ increase in FNIR. The position with E30C is more complicated: The algorithm is very accurate, and baseline workload is low, so the available benefits are limited.

$\triangleright$ Effect of population size: The tradeoff associated with thresholding is most pronounced at large population size. This occurs because the baseline workload is higher at large $\mathrm{N}$ (because mates are more likely to be displaced from rank-1 position when $\mathrm{N}$ is large). Thus for workload to be reduced to 0.4 times baseline, FNIR values are increased to as high as 1.4 times the baseline when $\mathrm{N}=20,000$.

$\triangleright$ Magnitude of the effect: The workload benefits are substantial - a reduction in workload by a factor of 0.5 corresponds to half the labor requirement, at least in applications where high volumes are sustained. The FNIR increases are relatively low, compared to the multipliers associated with a) the use of webcam images and b) the use of inferior recognition algorithms. They are comparable with the use of "recent" vs. "lifetime" enrolment types.

It it clear that candidate list reduction via thresholding can reduce the amount of work a reviewer does, but it will also reduce the number of hits found, and beneficially reduce the number of false alarms.

\subsection{Impostor distribution stability}

Section 4.1 defines false positive identification rate (FPIR) as the fraction of searches that yield one or more false matches. Likewise, selectivity (SEL) is defined as the expected number of false matches produced in a search. Both of these quantities are a function of threshold, with higher thresholds giving reduced values for FPIR and SEL.

As some systems are configured with thresholds that target a known (often low) FPIR [1], the operational question arises of how stable FPIR is with changes to the properties of images or individuals that are used with the system. This issue is determined by the stability of the nonmate distribution i.e. whether FPIR is independent of image quality, population demographics and ethnicity.

Most of the academic literature addresses improvement of Type 1 error rates such as better hit rates. The primary performance metrics are 1:1 FNMR at fixed FMR, and closed-set CMC. The importance of a stable impostor distribution has

\begin{tabular}{|c|c|c|c|c|c|c|}
\hline $\begin{array}{l}\mathrm{A}=3 \mathrm{M} / \text { Cogent } \\
\mathrm{G}=\text { Hisign } \\
\mathrm{P}=\text { Zhuhai-Yisheng }\end{array}$ & $\begin{array}{l}\mathrm{B}=\text { Cognitec } \\
\mathrm{H}=\mathrm{CAS}-\mathrm{IA} \\
\mathrm{Q}=\text { JunYu }\end{array}$ & $\begin{array}{l}\mathrm{C}=\text { Neurotechnology } \\
\mathrm{I}=\text { CAS-ICT } \\
\mathrm{S}=\text { Decatur }\end{array}$ & $\begin{array}{l}\mathrm{D}=\text { Safran Morpho } \\
\mathrm{J}=\text { Toshiba } \\
\mathrm{T}=\text { Ayonix }\end{array}$ & $\begin{array}{l}\mathrm{E}=\mathrm{NEC} \\
\mathrm{L}=\text { Tsinghua U. II }\end{array}$ & $\begin{array}{l}\mathrm{F}=\text { Tsinghua } \mathrm{U} \\
\mathrm{M}=\mathrm{HP}\end{array}$ & $\begin{array}{l}\text { FNIR(N,R,T,L) “Miss rate" } \\
\text { FPIR(N,T,L) “False alarm rate" }\end{array}$ \\
\hline
\end{tabular}




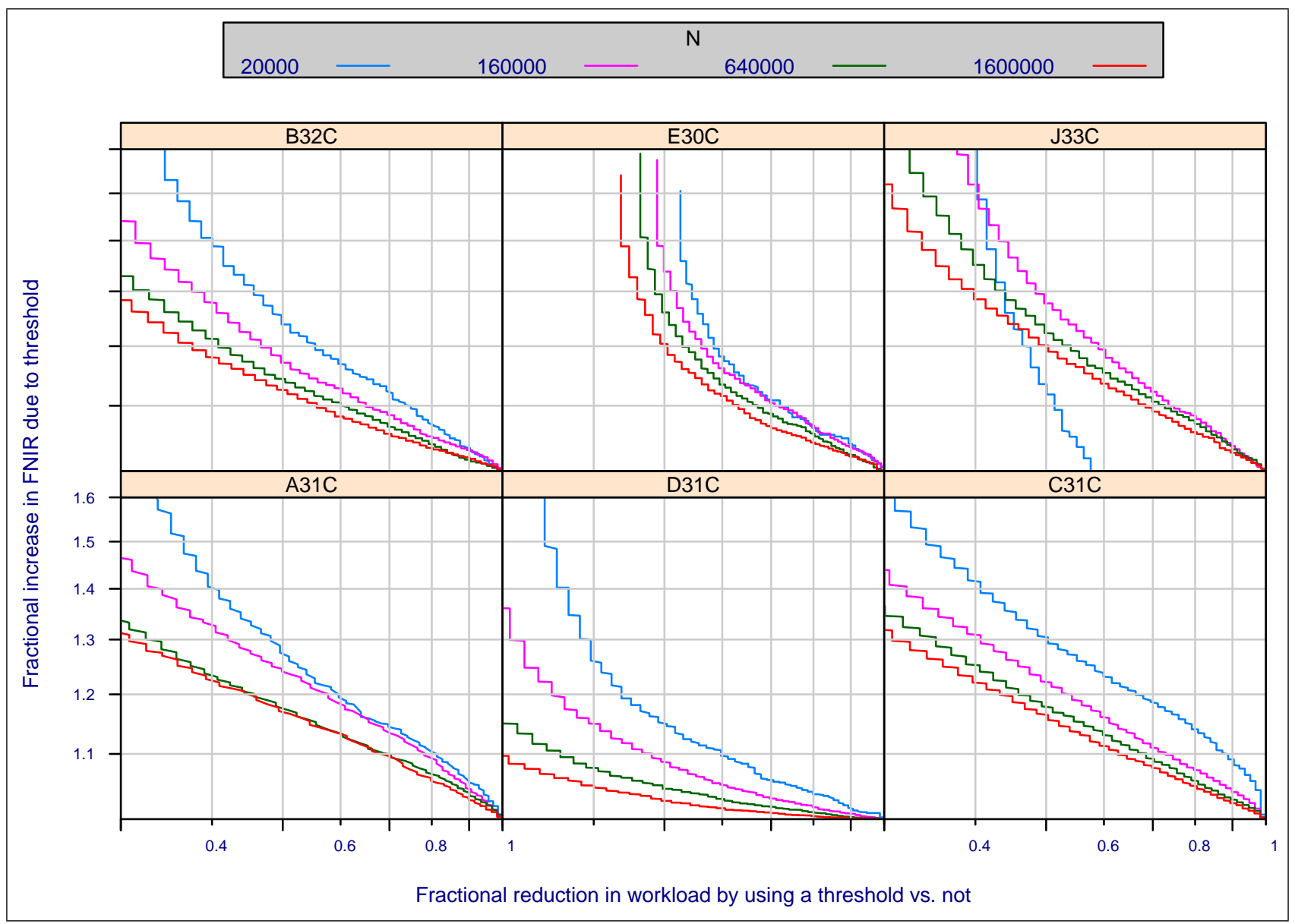

Figure 12: Reviewer workload reductions with thresholding: With recent LEO mugshots enrolled, the graphs plot on the $x$-axis the reduction in the number of candidates (workload eq. (11)) that would require human review if the candidate list were reduced in length by thresholding vs. if the full $L=50$ candidates were eligile for review. On the $y$-axis, is the reduction in accuracy i.e. the fractional increase in miss rates incurred by thresholding vs. considering all $L=50$.

\begin{tabular}{|c|c|c|c|c|c|c|}
\hline $\begin{array}{l}\mathrm{A}=3 \mathrm{M} / \text { Cogent } \\
\mathrm{G}=\text { Hisign } \\
\mathrm{P}=\text { Zhuhai-Yisheng }\end{array}$ & $\begin{array}{l}\mathrm{B}=\text { Cognitec } \\
\mathrm{H}=\text { CAS-IA } \\
\mathrm{Q}=\text { JunYu }\end{array}$ & $\begin{array}{l}\mathrm{C}=\text { Neurotechnology } \\
\mathrm{I}=\text { CAS-ICT } \\
\mathrm{S}=\text { Decatur }\end{array}$ & $\begin{array}{l}\mathrm{D}=\text { Safran Morpho } \\
\mathrm{J}=\text { Toshiba } \\
\mathrm{T}=\text { Ayonix }\end{array}$ & $\begin{array}{l}\mathrm{E}=\mathrm{NEC} \\
\mathrm{L}=\text { Tsinghua U. II }\end{array}$ & $\begin{array}{l}\mathrm{F}=\text { Tsinghua } \mathrm{U} \\
\mathrm{M}=\mathrm{HP}\end{array}$ & $\begin{array}{l}\text { FNIR(N,R,T,L) "Miss rate" } \\
\text { FPIR(N,T,L) “False alarm rate" }\end{array}$ \\
\hline
\end{tabular}


received little attention in the academic literature [15]. However it is critical to systems configured with a fixed threshold that are exposed to different kinds of images.

Methods: Webcam and mugshot images are used in 1:N searches, and scores from both mate and nonmate searches are retained and used in computation of the DET characteristics plotting FNIR vs. FPIR (see equations (3) and 1).

Section 5.5 details the effect on both FNIR(T) and FPIR(T) when individuals in different age groups are identified.

Results: Figure 6 shows DETs for six more accurate algorithms. Appendix A includes DETs for each algorithm for mugshots and webcam images. Similarly, Figure 11 gives DETs for identification by age group. In both cases, the plots include lines connecting points of fixed threshold.

Discussion: The horizontal displacement of the grey lines is an indicator that the impostor distribution is not stable under condition-change. The 3M/Cogent (A31C) and NEC (E30C) algorithms exhibit less than a factor of two change in FPIR when processing webcams vs. mugshots (Fig. 6), and when processing young persons vs. old (Fig. 11). The Neurotechnology (C31C) algorithm, on the other hand, gives more than a 100-fold increase in FPIR when processing infants vs. seniors. The Cognitec algorithm gives a roughly 20-fold increase in FPIR when processing webcam images. These results will manifest themselves operationally as longer candidate lists

\subsection{Computational expense}

\subsubsection{Search duration times}

Background: In most deployments, the enrolled population increases over time. This may be a continuous process or the result of merging separate datasets. If the database doubles in size, so does the search time. This has major implications for capital expenditure on computing hardware and ancillary equipment.

In our 2010 evaluation of face recognition algorithms [12], we showed that search speed scales sublinearly with enrolled population size. That aside, there is little documentation of search speed in operational biometric systems. There is a large and mature literature on fast search algorithms, although much of this is outside of the biometric arena. The term fast refers to algorithms for which average search time increases better-than-linearly with population size $\mathrm{N}$, for example as $\log \mathrm{N}$.

Methods: See section 4.5. For identification trials, the algorithm was permitted to use the available hardware as it saw fit. It could elect to start any number of threads $[1,16]$ and this could be varied dynamically and as a function of N. Only one provider, F, elected to use threading.

For timing measurement, both mate and nonmate searches were conducted in random order. However, the duration measurements given here are computed over only nonmate searches. Mate searches are not used because some algorithms implement so-called 1:FIRST semantics where the search is terminated once any sufficiently high scoring hit has been found. This may be operationally valuable, but it confuses measurement of underlying algorithmic efficiency.

The candidate list length was fixed at $\mathrm{L}=50$. Production of longer candidate lists may take longer - this is untested in FRVT. The estimates reported below are median values estimated over 2000 searches for which a mate exists, and 2000 searches for which a mate does not exist.

Results: Timing results are tabulated in Table 15. Graphs of search durations vs. N appear in the algorithm summaries of Appendix A.

There is wide variation in search speed. For $\mathrm{N}=1,600,000$, the most accurate algorithm (E30C) takes 1.093 seconds to

\begin{tabular}{|c|c|c|c|c|c|c|}
\hline $\begin{array}{l}\mathrm{A}=3 \mathrm{M} / \text { Cogent } \\
\mathrm{G}=\text { Hisign } \\
\mathrm{P}=\text { Zhuhai-Yisheng }\end{array}$ & $\begin{array}{l}\mathrm{B}=\text { Cognitec } \\
\mathrm{H}=\text { CAS-IA } \\
\mathrm{Q}=\text { Jun } \mathrm{Yu}\end{array}$ & $\begin{array}{l}\mathrm{C}=\text { Neurotechnology } \\
\mathrm{I}=\mathrm{CAS}-\mathrm{ICT} \\
\mathrm{S}=\text { Decatur }\end{array}$ & $\begin{array}{l}\mathrm{D}=\text { Safran Morpho } \\
\mathrm{J}=\text { Toshiba } \\
\mathrm{T}=\text { Ayonix }\end{array}$ & $\begin{array}{l}\mathrm{E}=\mathrm{NEC} \\
\mathrm{L}=\text { Tsinghua U. II }\end{array}$ & $\begin{array}{l}\mathrm{F}=\text { Tsinghua } \mathrm{U} \\
\mathrm{M}=\mathrm{HP}\end{array}$ & $\begin{array}{l}\text { FNIR(N,R,T,L) “Miss rate" } \\
\text { FPIR(N,T,L) “False alarm rate" }\end{array}$ \\
\hline
\end{tabular}




\begin{tabular}{|c|c|c|c|c|c|c|c|c|c|c|c|c|}
\hline & \multicolumn{2}{|c|}{ ENROLLMENT } & \multicolumn{3}{|c|}{ FINALIZATION } & \multicolumn{7}{|c|}{ SEARCH } \\
\hline ALG & ETIME & ESIZE & FEXPN & FTIME & FPOW & $\mathrm{S}_{\text {SIZE }}$ & STIME & $T_{s}(20) \mathrm{k}$ & $T_{s}(160) \mathrm{k}$ & $T_{s}(640) \mathrm{k}$ & $T_{s}(1600) \mathrm{k}$ & $T_{s}=a N^{b}$ \\
\hline A20C & 520 & 4106 & 1.0 & 1.8 & 1.7 & 4106 & 519 & $155 \pm 1$ & $1235 \pm 0$ & $4953 \pm 1$ & $12568 \pm 97$ & $0.0080 N^{1.00}$ \\
\hline $\mathrm{A} 30 \mathrm{C}$ & 327 & 4622 & 1.0 & 8.0 & 1.0 & 4622 & 324 & $29 \pm 0$ & $242 \pm 0$ & $979 \pm 1$ & $2454 \pm 0$ & $0.0018 N^{0.99}$ \\
\hline $\mathrm{A} 31 \mathrm{C}$ & 324 & 4622 & 1.0 & 12.2 & 1.6 & 4622 & 324 & $28 \pm 0$ & $243 \pm 0$ & $982 \pm 2$ & $2452 \pm 0$ & $0.0017 N^{0.99}$ \\
\hline A32C & 296 & 1046 & 1.0 & 0.6 & 1.6 & 1046 & 189 & $11 \pm 0$ & $102 \pm 0$ & $447 \pm 3$ & $1080 \pm 0$ & $0.0017 N^{0.94}$ \\
\hline B30C & 282 & 4796 & 1.0 & 3.6 & 1.0 & 4796 & 284 & $96 \pm 0$ & $756 \pm 0$ & $2972 \pm 0$ & $7494 \pm 18$ & $0.0059 N^{0.98}$ \\
\hline B31C & 287 & 4796 & 1.0 & 3.8 & 0.4 & 4796 & 282 & $95 \pm 0$ & $756 \pm 0$ & $2982 \pm 0$ & $7498 \pm 2$ & $0.0053 N^{0.99}$ \\
\hline B32C & 382 & 9932 & 1.0 & 25.3 & 1.1 & 9932 & 388 & $255 \pm 0$ & $2018 \pm 0$ & $8076 \pm 1$ & $20239 \pm 33$ & $0.0117 N^{1.01}$ \\
\hline $\mathrm{B} 33 \mathrm{C}$ & 385 & 9932 & 1.0 & 235.5 & 0.9 & 9932 & 382 & $255 \pm 0$ & $2019 \pm 0$ & $8064 \pm 1$ & $20285 \pm 4$ & $0.0119 N^{1.00}$ \\
\hline C20C & 263 & 35994 & 0.0 & 0.0 & 0.9 & 35994 & 222 & $164 \pm 3$ & $1143 \pm 15$ & $4518 \pm 65$ & $11149 \pm 170$ & $0.0079 N^{0.99}$ \\
\hline C30C & 363 & 37008 & 0.0 & 0.0 & 1.0 & 37008 & 362 & $991 \pm 2$ & $7157 \pm 20$ & $28652 \pm 79$ & $72246 \pm 195$ & $0.0382 N^{1.01}$ \\
\hline C31C & 361 & 37008 & 0.0 & 0.0 & 1.0 & 37008 & 361 & $179 \pm 2$ & $1279 \pm 17$ & $4986 \pm 65$ & $12575 \pm 154$ & $0.0056 N^{1.02}$ \\
\hline C32C & 236 & 5040 & 0.0 & 0.0 & 0.9 & 37008 & 360 & $135 \pm 0$ & $1014 \pm 1$ & $4032 \pm 6$ & $10136 \pm 14$ & $0.0067 N^{0.99}$ \\
\hline D20C & 714 & 8005 & 1.0 & 45.5 & 1.0 & 20489 & 716 & $443 \pm 0$ & $510 \pm 0$ & - & - & $228 N^{0.07}$ \\
\hline D30C & 754 & 8562 & 1.4 & 76.8 & 1.0 & 21046 & 709 & $722 \pm 0$ & $804 \pm 0$ & $958 \pm 0$ & $1263 \pm 1$ & $193 N^{0.13}$ \\
\hline D31C & 729 & 12247 & 1.2 & 88.1 & 1.0 & 24731 & 712 & $1218 \pm 0$ & $1278 \pm 0$ & $1422 \pm 0$ & $1761 \pm 0$ & $499 N^{0.08}$ \\
\hline D32C & 493 & 857 & 5.2 & 5.0 & 1.0 & 857 & 471 & $35 \pm 0$ & $87 \pm 1$ & $260 \pm 0$ & $605 \pm 14$ & $0.0026 N^{0.86}$ \\
\hline D33C & 733 & 8005 & 1.5 & 57.3 & 1.0 & 20489 & 712 & $736 \pm 0$ & $812 \pm 0$ & $954 \pm 0$ & $1271 \pm 0$ & $174 N^{0.13}$ \\
\hline D34C & 704 & 8005 & 1.3 & 46.7 & 1.0 & 20489 & 707 & $731 \pm 0$ & $813 \pm 0$ & $957 \pm 0$ & $1236 \pm 0$ & $182 N^{0.13}$ \\
\hline E20C & 216 & 2465 & 1.0 & 2.2 & 1.3 & 2465 & 204 & $15 \pm 0$ & $123 \pm 0$ & $513 \pm 1$ & $1143 \pm 0$ & $0.0058 N^{0.85}$ \\
\hline E21C & 196 & 2465 & 1.0 & 2.4 & 1.2 & 2465 & 204 & $17 \pm 0$ & $137 \pm 0$ & $481 \pm 0$ & $1245 \pm 2$ & $0.0004 N^{1.04}$ \\
\hline E30C & 229 & 2529 & 1.0 & 2.5 & 1.3 & 2529 & 208 & $22 \pm 0$ & $118 \pm 0$ & $450 \pm 0$ & $1093 \pm 0$ & $0.0021 N^{0.92}$ \\
\hline E31C & 205 & 2529 & 1.0 & 2.3 & 1.3 & 2529 & 210 & $13 \pm 0$ & $53 \pm 0$ & $168 \pm 0$ & $279 \pm 1$ & $0.0199 N^{0.67}$ \\
\hline F20C & 170 & 6760 & 1.0 & 3.2 & - & 6760 & 118 & - & $359 \pm 9$ & - & - & $0.0120 N^{0.87}$ \\
\hline F30C & 272 & 6440 & 1.0 & 3.0 & 1.4 & 6440 & 259 & $196 \pm 0$ & $1529 \pm 0$ & $3217 \pm 5$ & - & $1 N^{0.60}$ \\
\hline F31C & 262 & 7484 & 1.0 & 76.9 & 1.6 & 7484 & 263 & $242 \pm 0$ & $1901 \pm 1$ & $3510 \pm 1$ & - & $2 N^{0.54}$ \\
\hline G30C & 186 & 3484 & 1.0 & 1.3 & 1.7 & 3484 & 110 & $118 \pm 2$ & $843 \pm 0$ & $3376 \pm 9$ & - & $0.0043 N^{1.02}$ \\
\hline G31C & 145 & 2240 & 1.0 & 0.8 & 1.9 & 2240 & 192 & $14 \pm 0$ & $111 \pm 0$ & $418 \pm 12$ & - & $0.0003 N^{1.06}$ \\
\hline $\mathrm{H} 30 \mathrm{C}$ & 81 & 4420 & 1.0 & 5.9 & 1.9 & 4420 & 65 & $55 \pm 0$ & $231 \pm 2$ & $901 \pm 8$ & - & $0.0051 N^{0.91}$ \\
\hline J20C & 466 & 6206 & 1.0 & 444.5 & 1.7 & 6206 & 485 & $14 \pm 2$ & $117 \pm 0$ & $568 \pm 52$ & $1137 \pm 1$ & $0.0071 N^{0.84}$ \\
\hline $\mathrm{J} 30 \mathrm{C}$ & 532 & 4158 & 1.1 & 1167.7 & 0.9 & 4158 & 528 & $13 \pm 0$ & $134 \pm 0$ & $552 \pm 0$ & $1110 \pm 1$ & $0.0018 N^{0.94}$ \\
\hline J31C & 526 & 4158 & 1.1 & 4191.1 & 0.8 & 4158 & 527 & $13 \pm 0$ & $130 \pm 0$ & $454 \pm 47$ & $1194 \pm 2$ & $0.0012 N^{0.97}$ \\
\hline $\mathrm{J} 32 \mathrm{C}$ & 490 & 8254 & 1.0 & 70.5 & 1.0 & 8254 & 546 & $23 \pm 0$ & $244 \pm 1$ & $829 \pm 2$ & $2449 \pm 0$ & $0.0005 N^{1.07}$ \\
\hline $\mathrm{J} 33 \mathrm{C}$ & 550 & 8254 & 1.1 & 8403.1 & 0.6 & 8254 & 546 & $30 \pm 0$ & $191 \pm 0$ & $798 \pm 2$ & $2086 \pm 38$ & - \\
\hline L30C & 543 & 7328 & 1.0 & 151.7 & 1.0 & 7328 & 537 & $507 \pm 0$ & $4024 \pm 1$ & - & - & $0.0242 N^{1.00}$ \\
\hline L31C & 626 & 7328 & 1.0 & 9.2 & 0.8 & 7328 & 629 & $497 \pm 0$ & $3998 \pm 1$ & - & - & $0.0252 N^{1.00}$ \\
\hline M20C & 164 & 5608 & 1.0 & 0.1 & 0.0 & 5608 & - & - & - & - & - & - \\
\hline M21C & 164 & 5608 & 1.0 & 0.1 & 0.0 & 5608 & - & - & - & - & - & - \\
\hline M30C & 136 & 5608 & 1.0 & 0.1 & 0.9 & 5608 & 112 & $25 \pm 0$ & $179 \pm 1$ & $688 \pm 0$ & - & $0.0007 N^{1.03}$ \\
\hline P30C & 565 & 3713 & 1.0 & 11.0 & 1.0 & 3713 & 564 & $364 \pm 0$ & $2938 \pm 1$ & $11650 \pm 8$ & - & $0.0195 N^{0.99}$ \\
\hline Q30C & 170 & 5600 & 1.0 & 4.5 & - & 5600 & 170 & $78 \pm 1$ & $1417 \pm 5$ & - & - & - \\
\hline S20C & 411 & 800 & 1.0 & 0.3 & - & 800 & 172 & $55 \pm 0$ & $443 \pm 0$ & - & - & $0.0027 N^{1.00}$ \\
\hline $\mathrm{T} 30 \mathrm{C}$ & 283 & 1936 & 0.0 & 0.0 & 1.0 & 1936 & 189 & $12 \pm 0$ & $94 \pm 0$ & $348 \pm 0$ & - & $0.0022 N^{0.89}$ \\
\hline
\end{tabular}

Table 15: Resource consumption: From left to right: the duration of enrolment template generation; enrolment template size in bytes; the ratio of the size of post-finalized enrolment data on disk to $N$ times $E_{\text {SIZE }}$; the duration of the finalization call in seconds for $N=1.6$ million; the dependence of finalization time on $N$ i.e. $b$ in $N^{b}$; search template size in bytes; and its generation time; the time taken to execute searches of the given population, $N$; the power-law model of search duration; All times are medians, unless stated otherwise, and apply to the execution of API functions on a single core of a c. 2011 server-class PC-architecture processor. One exception to this is that the 1:N search for $F$ algorithms used up to 16 cores - those times have not been adjusted and are not therefore comparable.

\begin{tabular}{|c|c|c|c|c|c|c|}
\hline $\begin{array}{l}\mathrm{A}=3 \mathrm{M} / \text { Cogent } \\
\mathrm{G}=\text { Hisign } \\
\mathrm{P}=\text { Zhuhai-Yisheng }\end{array}$ & $\begin{array}{l}\mathrm{B}=\text { Cognitec } \\
\mathrm{H}=\text { CAS-IA } \\
\mathrm{Q}=\text { JunYu }\end{array}$ & $\begin{array}{l}\mathrm{C}=\text { Neurotechnology } \\
\mathrm{I}=\mathrm{CAS}-\mathrm{ICT} \\
\mathrm{S}=\text { Decatur }\end{array}$ & $\begin{array}{l}\mathrm{D}=\text { Safran Morpho } \\
\mathrm{J}=\text { Toshiba } \\
\mathrm{T}=\text { Ayonix }\end{array}$ & $\begin{array}{l}\mathrm{E}=\mathrm{NEC} \\
\mathrm{L}=\text { Tsinghua U. II }\end{array}$ & $\begin{array}{l}\mathrm{F}=\text { Tsinghua } \mathrm{U} \\
\mathrm{M}=\mathrm{HP}\end{array}$ & $\begin{array}{l}\text { FNIR(N,R,T,L) “Miss rate" } \\
\text { FPIR(N,T,L) "False alarm rate" }\end{array}$ \\
\hline
\end{tabular}


execute a search. The slowest algorithm by far, C30C, takes 72.2 seconds.

As shown in Appendix A, most search durations increase linearly on a log-log plot.

$$
\log T=a \log N+b
$$

This observation corresponds to a power-law form

$$
T=c N^{a}
$$

where the constant $b=\log c$ determines the intercept on the observed plot, and the constant $a$ is the slope. The parameters were estimated using nonlinear least squares regression without log transformation. Uncertainty estimates are from bootstrapping.

Discussion: By referencing the $a$ exponents in Table 15, it is evident that with three notable exceptions, all providers' algorithms exhibit a linear dependence of search duration on the enrolled population size. The exceptions are Morpho, where the exponent $a$ is typically around 0.1. This implies that a 10 fold increase in $\mathrm{N}$ gives a 1.25 fold increase in search duration. While this is an attractive proposition for very large deployments (several United States' driving license databases have sizes above $10^{7}$ ), the practical relevance of this will depend on the transaction volume demands and the number of available computers. The second exception is NEC, where the exponent $a$ varies from 0.92(E30C) down to 0.67(E31C) for which a ten fold increase in $\mathrm{N}$ leads to a 4.6 fold increase in duration. Finally the Tsinghua (F) algorithms exhibit sublinear speed.

Note that if $K$ processing cores are available on a computer, $K$ searches cannot typically be conducted simultaneously without some loss of speed due to memory bus bandwidth constraints.

Conclusions: Search durations scale approximately as a power of the database size. The exponents are dependent on the algorithm. There is approximately an order of magnitude difference in the search durations measured for the four most accurate algorithm providers. The most accurate algorithms are among the fastest.

\subsubsection{Template creation times}

Background: How long does it take to extract features from an image and make a template? Does this depend on the width and height of the input image? Does it depend on whether the template is used for enrolment, verification, or identification?

Drivers: Template generation time is often a large component of a 1:N identification search, obviously depending on N. If multiple images are to be searched, e.g. frames from a video sequence, then template generation time can be important, and can dominate an overall transaction time. Additionally, template generation time will be important if an existing image corpus is going to be re-enrolled by a new provider. For example, re-enrolment of an $18 \mathrm{M}$ person driving license database takes $1 \times 18 \times 106 / 64 / 3600=156$ hours if a one second template generation were sustained on a 32 core blade installation. This does not include de-duplication searches.

Methods: See section 4.5.

Results: Timing results are tabulated in Table 15. Template generation durations are graphed alongside search duration in the algorithm summaries of Appendix A.

Discussion: Template generation times start below 100 milliseconds (H30C, Q30C) and range from 200-750 milliseconds for the most accurate algorithms. There is some industry-wide tradeoff of template generation speed with accuracy:

\begin{tabular}{|c|c|c|c|c|c|c|}
\hline $\begin{array}{l}A=3 \mathrm{M} / \text { Cogent } \\
G=\text { Hisign } \\
P=\text { Zhuhai-Yisheng }\end{array}$ & $\begin{array}{l}\mathrm{B}=\text { Cognitec } \\
\mathrm{H}=\text { CAS-IA } \\
\mathrm{Q}=\text { JunYu }\end{array}$ & $\begin{array}{l}\mathrm{C}=\text { Neurotechnology } \\
\mathrm{I}=\mathrm{CAS}-\mathrm{ICT} \\
\mathrm{S}=\text { Decatur }\end{array}$ & $\begin{array}{l}\mathrm{D}=\text { Safran Morpho } \\
\mathrm{J}=\text { Toshiba } \\
\mathrm{T}=\text { Ayonix }\end{array}$ & $\begin{array}{l}\mathrm{E}=\mathrm{NEC} \\
\mathrm{L}=\text { Tsinghua U. II }\end{array}$ & $\begin{array}{l}\mathrm{F}=\text { Tsinghua } \mathrm{U} \\
\mathrm{M}=\mathrm{HP}\end{array}$ & $\begin{array}{l}\text { FNIR(N,R,T,L) “Miss rate" } \\
\text { FPIR(N,T,L) “False alarm rate" }\end{array}$ \\
\hline
\end{tabular}
Several of the more inaccurate algorithms are efficient in their production of templates (algorithms from Q, G, M, durations 
below $200 \mathrm{msec}$ ) whereas the providers of the second and third most accurate algorithms prepare templates more slowly, J in approximately $500 \mathrm{msec}$ and $\mathrm{D}$ in around 700 milliseconds. This implication is contradicted however by the production of provider $\mathrm{E}$ of templates in less than 250 milliseconds.

Conclusions: Template creation times are independent of the target population size, suggesting that developers did not tailor their algorithmic representation to the size of the identification search.

\subsection{Template size}

Each implementation encodes information derived from the face image in a proprietary representation of the feature data. This information is generally a trade secret. It encodes one or more mathematical representations of the face shape, structure, or texture but could also, in principle, encode anything else (e.g. non-tradtional information such as hair color, style, eye color).

Demand driver: Templates contain the mathematical representation of one or more images of a person. Biometric templates are proprietary, non-standard, and their content is protected as a trade-secret.

The size of the feature data is an important system-design parameter in most biometric applications. Template size is clearly influential on storage requirements, both on-disk and in-memory, on network transmission bandwidth requirements, and on machine throughput. In addition, a large template may be associated with computational complexity and computational expense of the matching algorithm.

Methods: The FRVT Evaluation Plan and API [14] explicitly supported measurement and reporting of facial recognition template size. Two direct measurements of template size are made: one for enrolment templates, and one for search templates. The API supports passage of $K \geq 1$ images to the template generation function under test. $K B$ bytes is preallocated, where maximum template size, B, was returned by an initialization function. For any given input, the exact template size was returned and used to save the template to disk.

Results: Table 15 shows template sizes, in bytes. The values also appear alongside the accuracy results in Tables 4 - 6 .

\section{Discussion:}

$\triangleright$ Between-provider size variation: Across all providers, template sizes vary from 0.8 to 37 kilobytes, with the more accurate algorithms having sizes between 2 and 10KB. Some providers submitted algorithms with notably small "lightweight" templates.

$\triangleright$ Within-provider size variation: Some providers submitted algorithms with varying template sizes. Larger templates are assumed to contain richer, more disriminative, features that should afford better accuracy.

3M/Cogent's most and least accurate algorithms used templates of size 4622 and 1046 bytes respectively. This more than four-fold size difference gave rank one miss rates of 0.133 and 0.173 respectively (Table 4, RECENT enrolment). This loss is less pronounced at low FPIR and for LIFETIME enrolment.

Cognitec submitted algorithms with sizes of 9932 versus 4796 bytes. This approximately two-fold variation gave rise to rank one miss rates of 0.109 vs. 0.120 respectively (Table 4, RECENT enrolment).

Morpho pushed this tradeoff still further by submitting one algorithm, D32C, with a template size of 857 bytes. The best algorithm, D31C, used enrolment templates of size 12247 bytes and search templates of about twice that size, 24731 bytes. This algorithm offers very small accuracy improvements over their second most accurate algorithm used enrolment templates of size 8005 bytes and search templates of size 20489 bytes. Thus comparing the smallest

\begin{tabular}{|c|c|c|c|c|c|c|}
\hline $\begin{array}{l}\mathrm{A}=3 \mathrm{M} / \text { Cogent } \\
\mathrm{G}=\text { Hisign } \\
\mathrm{P}=\text { Zhuhai-Yisheng }\end{array}$ & $\begin{array}{l}\mathrm{B}=\text { Cognitec } \\
\mathrm{H}=\text { CAS-IA } \\
\mathrm{Q}=\text { Jun } \mathrm{Yu}\end{array}$ & $\begin{array}{l}\mathrm{C}=\text { Neurotechnology } \\
\mathrm{I}=\mathrm{CAS}-\mathrm{ICT} \\
\mathrm{S}=\text { Decatur }\end{array}$ & $\begin{array}{l}\mathrm{D}=\text { Safran Morpho } \\
\mathrm{J}=\text { Toshiba } \\
\mathrm{T}=\text { Ayonix }\end{array}$ & $\begin{array}{l}\mathrm{E}=\mathrm{NEC} \\
\mathrm{L}=\text { Tsinghua U. II }\end{array}$ & $\begin{array}{l}\mathrm{F}=\text { Tsinghua } \mathrm{U} \\
\mathrm{M}=\mathrm{HP}\end{array}$ & $\begin{array}{l}\text { FNIR(N,R,T,L) “Miss rate" } \\
\text { FPIR(N,T,L) “False alarm rate" }\end{array}$ \\
\hline
\end{tabular}


template (D32C) with a second tier algorithm (D34C), the error rate approximately halves from 0.150 to 0.076 (Table 4, RECENT enrolment).

Toshiba explored this variation also submitting templates of size 4158 and 8254 bytes. These afforded error rates of 0.079 (J33C) and 0.091 (J30C).

In conclusion, the ability to reduce template size may be useful operationally, especially for example in speed and bandwidth sensitive applications. Error rate increases are often modest, but increase substantially with very small templates. Within-provider accuracy variations are usually smaller than between-provider - this is evident in the algorithm rankings of Tables $4-6$.

$\triangleright$ No dependence on $\mathbf{N}$ : In all cases, the size of a single enrolment template is independent of the size of the enrolled population. This shows that developers did not exploit the provision, via the API initialization call, of the integer number of subjects about to be enrolled. This information would have allowed the implementation to use larger, richer templates for larger $\mathrm{N}$.

$\triangleright$ Linear in K: Template sizes vary with the number of images input to the template generation function - see section 3.2. The API sends $K \geq 1$ images to the template generation function. For all algorithms, the size of the enrolment template grows linearly with the number of images that went into its creation. This indicates that algorithms are not integrating facial information across images.

$\triangle$ Some asymmetric templates: The API supported asymmetric or role-specific templates. This allows a template to be used only for enrolment, or only for search, but not vice-versa. Many template sizes are independent of role. The exceptions are algorithm C32C and all the D algorithms except D32C. Operationally, a verification template is usually not stored permanently as it exists only for the duration of a recognition transaction.

$\triangleright$ Within-memory representation: Further when $N$ images are enrolled, each producing a template of size, $x$, the notional enrolment database size will be $N x$. This collection of templates is sent to a one-time finalization function provided by the algorithm. This prepares the data for subsequent searches. Specifically, it takes the $N x$ bytes of input and writes $F$ bytes to disk. This size is recorded and used to compute the expansion factor $F / N x$. Some algorithms simply copy the input data, whence the expansion factor is 1 . Other algorithms re-arrange the input data for efficient in-memory search.

\subsubsection{Finalization times}

Background: Finalization is a processing step that is applied over a set of $\mathrm{N}$ templates from $\mathrm{N}$ individuals. It is executed once, before any searches are executed. It is included in the FRVT execution pipeline to allow algorithms to derive valuable information from the entirety of the data, i.e. information that cannot be derived during the image-to-template feature extraction operation.

Results: Finalization times are tabulated in Table 15.

Discussion: Most implementations execute finalization very quickly indicating only trivial data copying or re-arrangement. However, some algorithms execute more slowly, particularly those of Toshiba, indicating that the finalization step is a non-trivial mathematical operation. It is not known whether such processing could be executed in an operational context, where it is necessary to add and delete entries from the enrolment database on an ongoing basis. The assumption is that finalization is an operationally realistic process that could either be executed periodically during the lifetime of a biometric system, or on-demand after a batch enrolment.

\begin{tabular}{|c|c|c|c|c|c|c|}
\hline $\begin{array}{l}A=3 \mathrm{M} / \text { Cogent } \\
G=\text { Hisign } \\
P=\text { Zhuhai-Yisheng }\end{array}$ & $\begin{array}{l}\mathrm{B}=\text { Cognitec } \\
\mathrm{H}=\text { CAS-IA } \\
\mathrm{Q}=\text { Jun } Y \mathrm{u}\end{array}$ & $\begin{array}{l}\mathrm{C}=\text { Neurotechnology } \\
\mathrm{I}=\mathrm{CAS}-\mathrm{ICT} \\
\mathrm{S}=\text { Decatur }\end{array}$ & $\begin{array}{l}\mathrm{D}=\text { Safran Morpho } \\
\mathrm{J}=\text { Toshiba } \\
\mathrm{T}=\text { Ayonix }\end{array}$ & $\begin{array}{l}\mathrm{E}=\mathrm{NEC} \\
\mathrm{L}=\text { Tsinghua U. II }\end{array}$ & $\begin{array}{l}\mathrm{F}=\text { Tsinghua } \mathrm{U} . \\
\mathrm{M}=\mathrm{HP}\end{array}$ & $\begin{array}{l}\text { FNIR(N,R,T,L) "Miss rate" } \\
\text { FPIR(N,T,L) “False alarm rate" }\end{array}$ \\
\hline
\end{tabular}




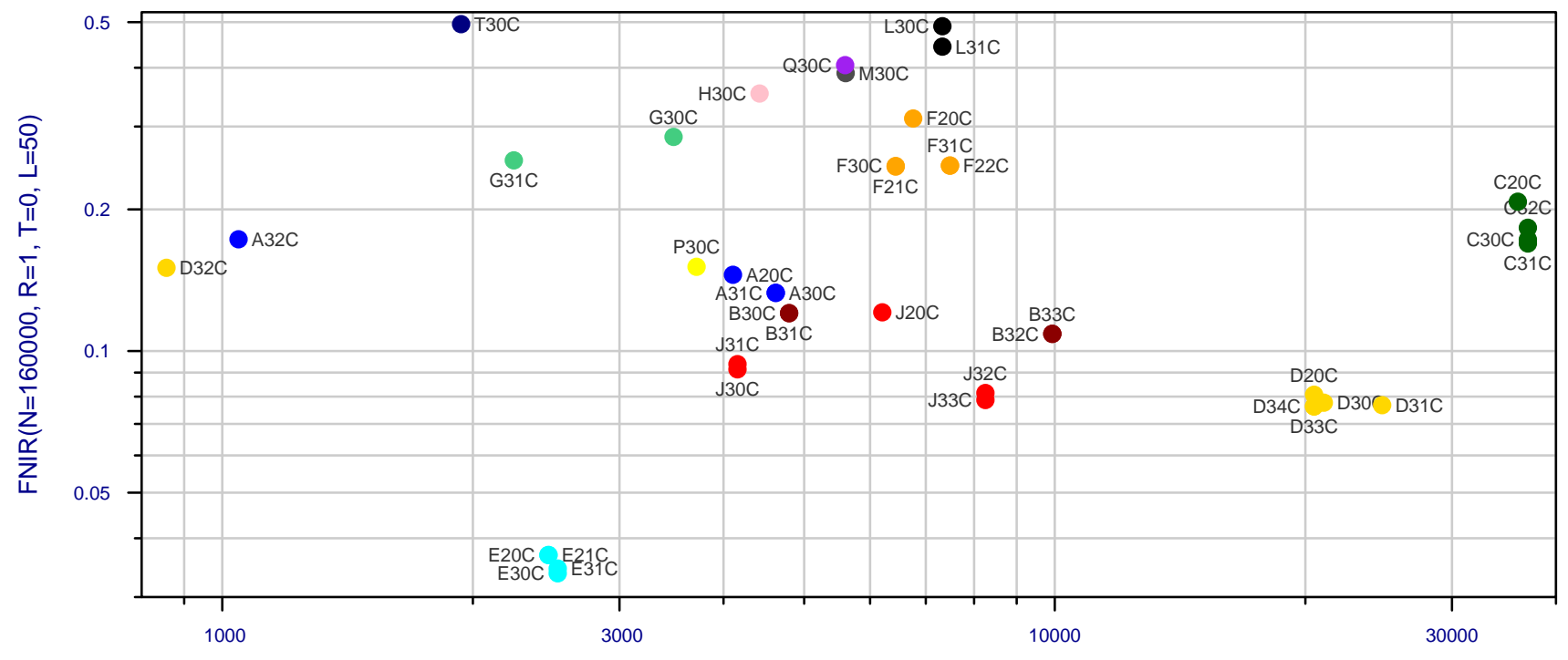

Median Template Size (bytes)

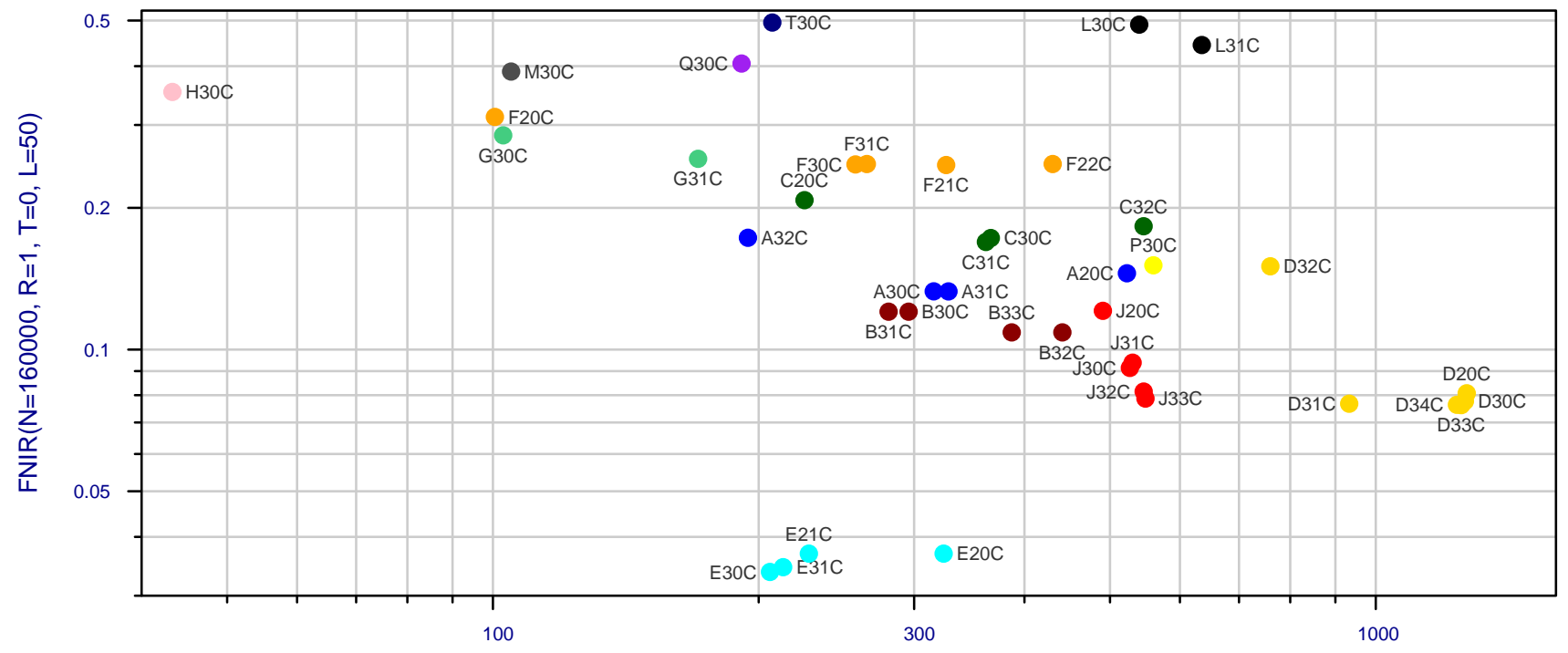

Median Template Generation Duration (msec)

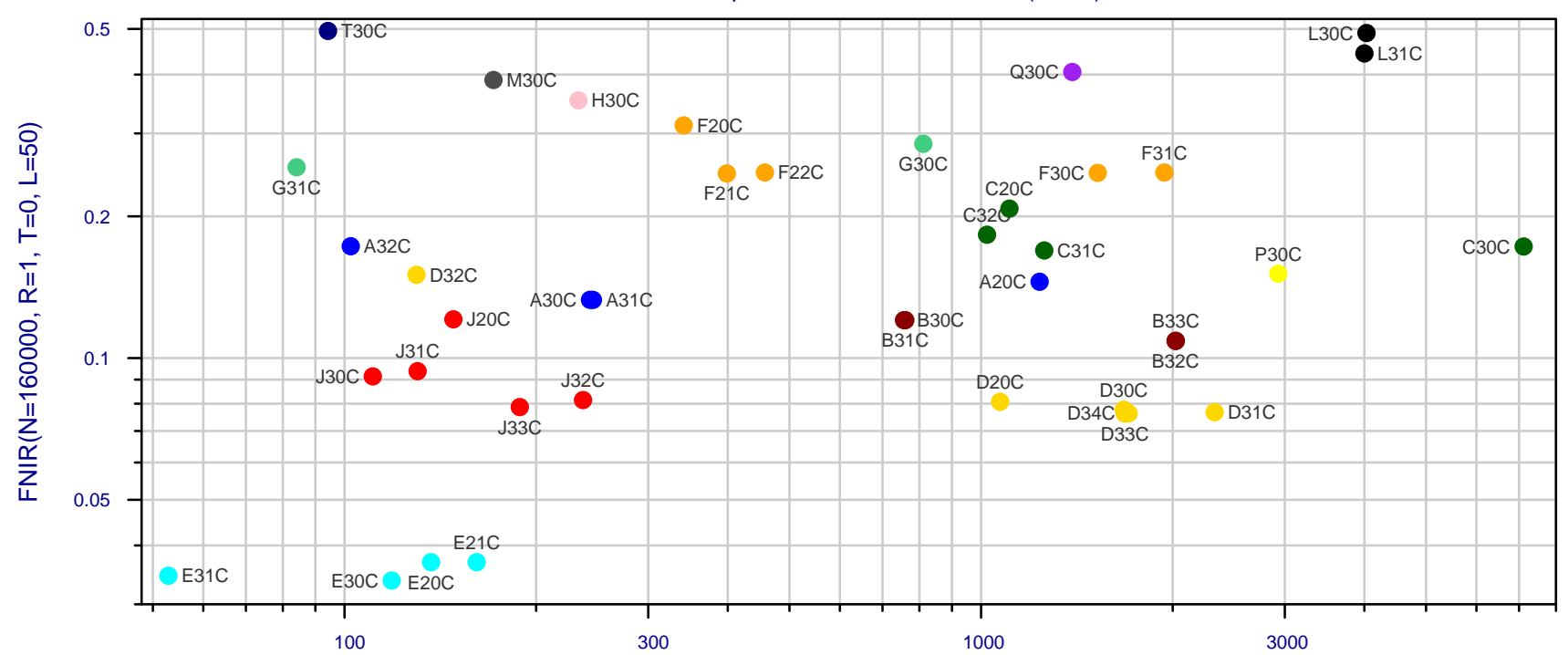

Median 1:160000 Search Duration (msec)

Figure 13: Performance tradespaces: The plots show rank one miss rates against three measures of resource consumption: feature size, feature extraction time, and nonmated search duration.

\begin{tabular}{|l|l|l|l|l|l|l}
\hline $\mathrm{A}=3 \mathrm{M} /$ Cogent & $\mathrm{B}=$ Cognitec & $\mathrm{C}=$ Neurotechnology & $\mathrm{D}=$ Safran Morpho & $\mathrm{E}=\mathrm{NEC}$ & $\mathrm{F}=$ Tsinghua U. & FNIR(N,R,T,L) "Miss rate" \\
$\mathrm{G}=$ Hisign & $\mathrm{H}=\mathrm{CAS}-\mathrm{IA}$ & $\mathrm{I}=\mathrm{CAS}-\mathrm{ICT}$ & $\mathrm{J}=$ Toshiba & $\mathrm{T}=$ Tsinghua U. II & $\mathrm{M}=\mathrm{HP}$ & FPIR(N,T,L) "False alarm rate" \\
$\mathrm{P}=$ Zhuhai-Yisheng & $\mathrm{Q}=$ JunYu & $\mathrm{S}=$ Decatur & $\mathrm{T}=$ Ayonix & &
\end{tabular}




\subsection{Exploiting multiple cores}

The FRVT Evaluation Plan and API document [14] did not support execution of a search across $B>1$ blades because there was no need. The reasons are as follows:

$\triangleright$ In all cases, for all population sizes, the entire enrolment database is small enough to fit in main memory.

$\triangleright$ A blade equipped with $C>1$ cores was fully utlized by running $C$ searches simultaneously as separate processes. This facility was not used for threaded implementations. (Timing measurements were made with $C=1$ process).

$\triangleright$ When searching an enrolment database of size $E$ on a blade with memory $M$, the number of copies of the enrolment data that can be made and kept in memory is $c=\lfloor M / E\rfloor$. This supports execution of $\min (c, C)$ completely independent processes, each running separate searches.

$\triangleright$ However, we can avoid this memory limit by making only $c=1$ copies of the enrolment database by using the LINUX fork() system call $C$ times. While this spawns $C$ entirely separate processes, the LINUX implementation of fork() uses copy-on-write semantics, which means that the enrolment data is not copied because, as a read-only element, it does not change.

D = Safran Morpho $\mathrm{J}=$ Toshiba $\mathrm{T}=$ Ayonix
FNIR(N,R,T,L) "Miss rate" FPIR(N,T,L) "False alarm rate" 


\section{References}

[1] Face recognition as a search tool foto-fahndung. Technical report, Bundeskriminamt (BKA), Thaerstrasse 11, 65193, Wiesbaden, Germany, February 2007.

[2] Blumstein, Cohen, Roth, and Visher, editors. Random parameter stochastic models of criminal careers. National Academy of Sciences Press, 1986.

[3] Thomas P. Bonczar and Lauren E. Glaze. Probation and parole in the united statesm 2007, statistical tables. Technical report, Bureau of Justice Statistics, December 2008.

[4] M. Brauckmann and C. Busch. Large scale database search. In Anil K. Jain and Stan Z. Li, editors, Handbook of Face Recognition, pages 639-654. Springer-Verlag New York, Inc., Secaucus, NJ, USA, 2011.

[5] S. Curry, D. Founds, J. Marques, N. Orlans (Mitre), and C. Watson (NIST). Meds - multiple encounter deceased subject face database - nist special database 32. NIST Interagency Report 7679, National Institute of Standards and Technology, 2011. http://www.nist.gov/itl/iad/ig/sd32.cfm.

[6] Working Group 3. Ed. D. D'Amato. ISO/IEC 19794-5 Amendment 1 - Biometric data interchange formats - Part 5: Face image data Conditions for taking photographs for face image data. JTC1 :: SC37, 1 edition, 12 2007. http:/ / webstore.ansi.org.

[7] G. Doddington, W. Liggett, A. Martin, M. Przybocki, and D. Reynolds. Sheep, goats, lambs and wolves: A statistical analysis of speaker performance in the nist 1998 speaker recognition evaluation. In Proceedings of 5th International Conference of Spoken Language Processing, ICSLP 98, Sydney, Australia, 1998. Paper 608 on CD-ROM.

[8] J. Egan. Signal Detection Theory and Analysis, pages 46-48. Academic Press, 1975.

[9] Ferrara et al. Face image iso compliance verification. Technical report, Università di Bologna, 2 2014. https:/ / biolab.csr.unibo.it/fvcongoing/UI/Form/BenchmarkAreas/BenchmarkAreaFICV.aspx.

[10] Matteo Ferrara, Annalisa Franco, Dario Maio, and Davide Maltoni. Face image conformance to iso/icao standards in machine readable travel documents. IEEE Transactions on Information Forensics and Security, 7(4):1204-1213, 2012.

[11] Working Group 3. Ed. P. Griffin. ISO/IEC 19794-5 Information Technology - Biometric Data Interchange Formats - Part 5: Face image data. JTC1 :: SC37, 1 edition, 2005. http://webstore.ansi.org.

[12] P. Grother, G. W. Quinn, and P. J. Phillips. Evaluation of 2d still-image face recognition algorithms. NIST Interagency Report 7709, National Institute of Standards and Technology, 8 2010. http:/ / face.nist.gov/mbe as MBE2010 FRVT2010.

[13] P. J. Grother and P. J. Phillips. Models of large population recognition performance. In IEEE Proceedings of the Computer Vision and Pattern Recognition (CVPR), June 2004. Main conference.

[14] Patrick Grother, George W. Quinn, and Mei Ngan. Face recognition vendor test - still face image and video concept, evaluation plan and api. Technical report, National Institute of Standards and Technology, 72013. http:/ / biometrics.nist.gov/cs_links/face/frvt/frvt2012/NIST_FRVT2012_api_Aug15.pdf.

[15] J.P. Hube. Using biometric verification to estimate identification performance. In Biometric Consortium Conference, 2006 Biometrics Symposium: Special Session on Research at the, pages 1-6, 92006.

[16] Herve Jarosz and Jean-Christophe Fondeur. Large scale identification system design. In Wayman, editor, Biometric Systems, chapter 9. Springer, 2005.

[17] J. Klontz and A. Jain. A case study of automated face recognition: The boston marathon bombings suspects. IEEE Computer Magazine, 11 2013. http:/ / openbiometrics.org/publications/KlontzJain_IEEEComputerNov2013.pdf.

[18] Scott Klum, Hu Han, Anil K. Jain, and Brendan Klare. Sketch based face recognition: Forensic vs. composite sketches. In Proc. 6th IAPR International Conference on Biometrics (ICB), June 2013. Madrid, Spain.

[19] Frank Main. Chicago police go high-tech with facial recognition software. Chicago Sun-Times, 72013. www.suntimes.com/21268770-761/chicago-police-go-high-tech-with-facial-recognition-software.html.

\begin{tabular}{|c|c|c|c|c|c|c|}
\hline $\begin{array}{l}A=3 \mathrm{M} / \text { Cogent } \\
G=\text { Hisign } \\
P=\text { Zhuhai-Yisheng }\end{array}$ & $\begin{array}{l}\mathrm{B}=\text { Cognitec } \\
\mathrm{H}=\text { CAS-IA } \\
\mathrm{Q}=\text { JunYu }\end{array}$ & $\begin{array}{l}\mathrm{C}=\text { Neurotechnology } \\
\mathrm{I}=\mathrm{CAS}-\mathrm{ICT} \\
\mathrm{S}=\text { Decatur }\end{array}$ & $\begin{array}{l}\mathrm{D}=\text { Safran Morpho } \\
\mathrm{J}=\text { Toshiba } \\
\mathrm{T}=\text { Ayonix }\end{array}$ & $\begin{array}{l}E=\text { NEC } \\
L=\text { Tsinghua U. II }\end{array}$ & $\begin{array}{l}\mathrm{F}=\text { Tsinghua } \mathrm{U} \\
\mathrm{M}=\mathrm{HP}\end{array}$ & $\begin{array}{l}\text { FNIR(N,R,T,L) “Miss rate" } \\
\text { FPIR(N,T,L) “False alarm rate" }\end{array}$ \\
\hline
\end{tabular}


[20] Expert Working Group on Human Factors in Latent Print Analysis. Latent print examination and human factors: Improving the practice through a systems approach. Technical report, U.S. Department of Commerce, National Institute of Standards and Technology, 22012.

[21] G. W. Quinn and P. Grother. Performance of face recognition algorithms on compressed images. NIST Interagency Report 7830, National Institute of Standards and Technology, 12 2011. http://face.nist.gov/mbe as MBE2010 FRVT2010.

[22] Deepayan Sarkar. Lattice: Multivariate Data Visualization with R. Springer, New York, 2008. ISBN 978-0-387-75968-5.

[23] Jamie Sherrah. False alarm rate: a critical performance measure for face recognition. In FGR, pages 189-194. IEEE Computer Society, 2004.

[24] United States. Passport photo guidelines. Department of State, 2011. http://travel.state.gov/content/passports/english/passports/photos/ph examples.html.

[25] X. Wang and X. Tang. Face photo-sketch synthesis and recognition. IEEE Transactions on Pattern Analysis and Machine Intelligence (PAMI), 31(11):1955-1967, 2009.

[26] Working Group 3. Ed. M. Werner. ISO/IEC 19794-5 Information Technology - Biometric Data Interchange Formats - Part 5: Face image data. JTC1 :: SC37, 2 edition, 2011. http:/ / webstore.ansi.org.

[27] Bradford Wing and R. Michael McCabe. Nist special publication 500-271: American national standard for information systems data format for the interchange of fingerprint, facial, and other biometric information part 1. Technical report, September 2011. ANSI/NIST ITL 1-2011.

[28] W. Zhang, X. Wang, and X. Tang. Coupled information-theoretic encoding for face photo-sketch recognition. In Proceedings of IEEE Conference on Computer Vision and Pattern Recognition (CVPR), 2011.

\section{$\mathrm{A}=3 \mathrm{M} /$ Cogent}

$\mathrm{G}=$ Hisign

$\mathrm{P}=$ Zhuhai-Yisheng
$\mathrm{D}=$ Safran Morpho $\mathrm{J}=$ Toshiba $\mathrm{T}=$ Ayonix
$\mathrm{E}=\mathrm{NEC}$ $\mathrm{L}=$ Tsinghua U. II
$\mathrm{F}=$ Tsinghua $\mathrm{U}$. $\mathrm{M}=\mathrm{HP}$
FNIR(N,R,T,L) “Miss rate" FPIR(N,T,L) "False alarm rate" 


\title{
A Biometric Error Rate Tradeoff Characteristics
}

This Appendix is intended to give a biometric identification-specific overview of the Detection Error Tradeoff characteristic DET. More general and detailed information is given in the Egan's class book [8].

\section{Accuracy Terms + Definitions}

\begin{abstract}
A detection error tradeoff (DET) characteristic represents the tradeoff between Type II and Type I classification errors. A receiver operating characteristic (ROC) is usually equivalent and the terms are synonymous. In biometrics, Type II errors occur when two samples of one person do not match - this is called a false negative. Correspondingly, Type I errors occur when samples from two persons do match - this is called a false positive. Matches are declared by a biometric system when the native comparison score from the recognition algorithm meets some threshold. Comparison scores can be either similarity scores, in which case higher values indicate that the samples are more likely to come from the same person, or dissimilarity scores, in which case higher values indicate different people. Similarity scores are traditionally computed by fingerprint and face recognition algorithms, while dissimilarities are used in iris recognition. In some cases, the dissimilarity score is a distance; this applies only when metric properties are obeyed. In any case, scores can be either mate scores, coming from a comparison of one person's samples, or nonmate scores, coming from comparison of different persons' samples. The words genuine or authentic are synonyms for mate, and the word impostor is used a synonym for nonmate. The words mate and nonmate are traditionally used in identification applications (such as law enforcement search, or background checks) while genuine and impostor are used in verification applications (such as access control).
\end{abstract}

For iris recognition, mate comparisons yielding dissimilarities greater than a threshold are false negatives. In identification these are called misses and contribute to the false negative identification rate (FNIR). Nonmate comparisons at or below a threshold are false positives; in identification these are sometime called false alarms, and they contribute to false positive identification rate (FPIR). The threshold can take on any real value, and it is conventional in biometrics testing to examine error rates as a function of the threshold. In many systems, the threshold can be varied continuously, while in other (production) systems, it may only take on a few settings.

Returning to the DET, it plots a function of FNIR against a function of FPIR. Here and in many other reports, the function is the logarithm function (log axes). However, a DET might also plot the hit rate, and the true positive identification rate, TPIR $=1-$ FNIR is plotted on a linear scale; this is often referred to as a ROC. More rarely, the function might be the inverse Gaussian function.

More detail and generality is provided in formal biometrics testing standards, see the various parts of ISO/IEC 19795 Biometrics Testing and Reporting. More terms, including and beyond those to do with accuracy, see ISO/IEC 2382-37 Information technology -Vocabulary -- Part 37: Harmonized biometric vocabulary

FNIR = False Negative Identification Rate

$F N I R=F N I R(N, T, L, R)$

FNIR is computed by executing mate searches into an enrolled population of size N. It is the proportion of mate searches for which the mate is

- EITHER not returned as any of $L$ candidates,

- OR is present but has dissimilarity above threshold T

- OR is present at rank greater than $R$.

In IREX III, the rank criterion is not used for DET computations, i.e. $R \rightarrow \infty$, so FNIR is solely a function of population size, $\mathrm{N}$ and threshold, T. FNIR(N, T).

FPIR = False Positive Identification Rate

$F P I R=\operatorname{FPIR}(\mathrm{N}, \mathrm{T}, \mathrm{L})$

FPIR is computed by executing nonmate searches into an enrolled population of size $\mathrm{N}$. It is the proportion of returned candidates which have dissimilarity at or below threshold $\mathrm{T}$. If $\mathrm{S}$ searches are conducted, $\mathrm{S} \times \mathrm{L}$ candidates will be returned, and FPIR is the number at or below threshold, divided by $(S \times L)$.

\begin{tabular}{|c|c|c|c|c|c|c|}
\hline $\begin{array}{l}\mathrm{A}=3 \mathrm{M} / \text { Cogent } \\
\mathrm{G}=\text { Hisign } \\
\mathrm{P}=\text { Zhuhai-Yisheng }\end{array}$ & $\begin{array}{l}\mathrm{B}=\text { Cognitec } \\
\mathrm{H}=\text { CAS-IA } \\
\mathrm{Q}=\text { Jun } \mathrm{Yu}\end{array}$ & $\begin{array}{l}\mathrm{C}=\text { Neurotechnology } \\
\mathrm{I}=\mathrm{CAS}-\mathrm{ICT} \\
\mathrm{S}=\text { Decatur }\end{array}$ & $\begin{array}{l}\mathrm{D}=\text { Safran Morpho } \\
\mathrm{J}=\text { Toshiba } \\
\mathrm{T}=\text { Ayonix }\end{array}$ & $\begin{array}{l}\mathrm{E}=\mathrm{NEC} \\
\mathrm{L}=\text { Tsinghua U. II }\end{array}$ & $\begin{array}{l}\mathrm{F}=\text { Tsinghua } \mathrm{U} \\
\mathrm{M}=\mathrm{HP}\end{array}$ & $\begin{array}{l}\text { FNIR(N,R,T,L) “Miss rate" } \\
\text { FPIR(N,T,L) “False alarm rate" }\end{array}$ \\
\hline
\end{tabular}



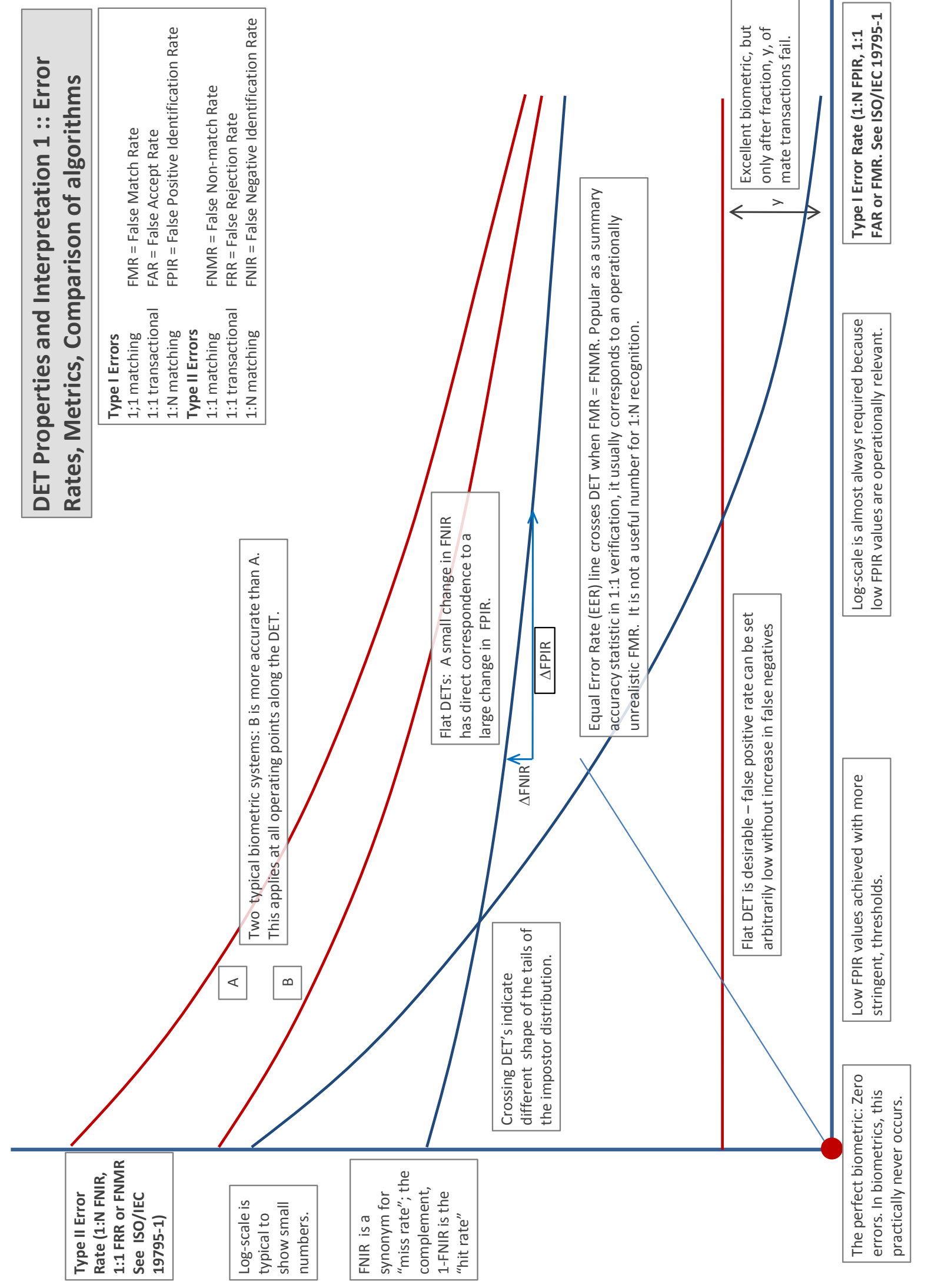

\begin{tabular}{|c|c|c|c|c|c|c|}
\hline $\begin{array}{l}\mathrm{A}=3 \mathrm{M} / \text { Cogent } \\
\mathrm{G}=\text { Hisign } \\
\mathrm{P}=\text { Zhuhai-Yisheng }\end{array}$ & $\begin{array}{l}\mathrm{B}=\text { Cognitec } \\
\mathrm{H}=\text { CAS-IA } \\
\mathrm{Q}=\text { Jun } \mathrm{Yu}\end{array}$ & $\begin{array}{l}\mathrm{C}=\text { Neurotechnology } \\
\mathrm{I}=\mathrm{CAS}-\mathrm{ICT} \\
\mathrm{S}=\text { Decatur }\end{array}$ & $\begin{array}{l}\mathrm{D}=\text { Safran Morpho } \\
\mathrm{J}=\text { Toshiba } \\
\mathrm{T}=\text { Ayonix }\end{array}$ & $\begin{array}{l}\mathrm{E}=\mathrm{NEC} \\
\mathrm{L}=\text { Tsinghua } \mathrm{U} . \mathrm{II}\end{array}$ & $\begin{array}{l}\mathrm{F}=\text { Tsinghua } \mathrm{U} \\
\mathrm{M}=\mathrm{HP}\end{array}$ & $\begin{array}{l}\text { FNIR(N,R,T,L) "Miss rate" } \\
\text { FPIR(N,T,L) “False alarm rate" }\end{array}$ \\
\hline
\end{tabular}




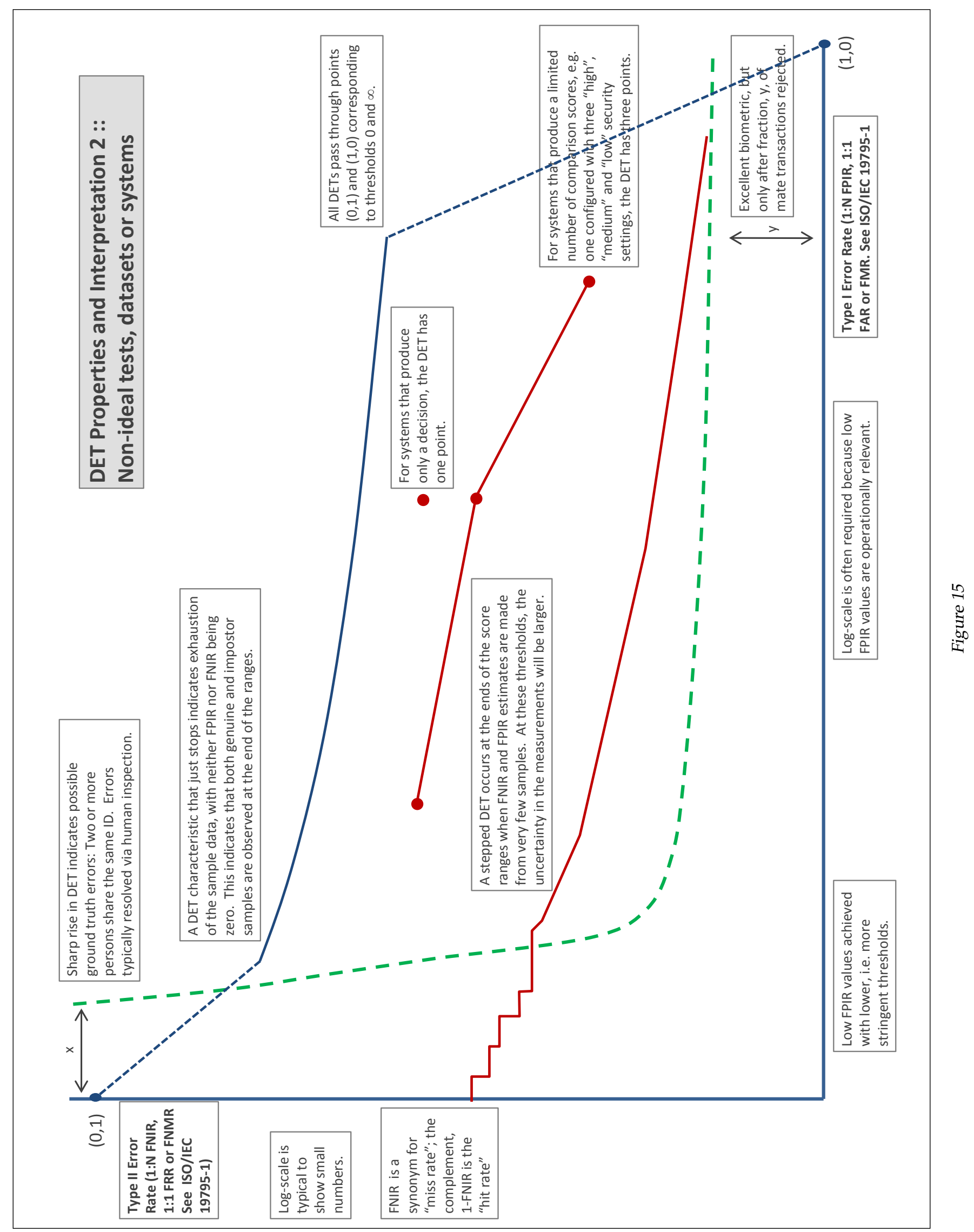

\begin{tabular}{|c|c|c|c|c|c|c|}
\hline $\begin{array}{l}\mathrm{A}=3 \mathrm{M} / \text { Cogent } \\
\mathrm{G}=\text { Hisign } \\
\mathrm{P}=\text { Zhuhai-Yisheng }\end{array}$ & $\begin{array}{l}\mathrm{B}=\text { Cognitec } \\
\mathrm{H}=\mathrm{CAS}-\mathrm{IA} \\
\mathrm{Q}=\text { JunYu }\end{array}$ & $\begin{array}{l}\mathrm{C}=\text { Neurotechnology } \\
\mathrm{I}=\text { CAS-ICT } \\
\mathrm{S}=\text { Decatur }\end{array}$ & $\begin{array}{l}D=\text { Safran Morpho } \\
J=\text { Toshiba } \\
T=\text { Ayonix }\end{array}$ & $\begin{array}{l}\mathrm{E}=\mathrm{NEC} \\
\mathrm{L}=\text { Tsinghua U. II }\end{array}$ & $\begin{array}{l}\mathrm{F}=\text { Tsinghua } \mathrm{U} \\
\mathrm{M}=\mathrm{HP}\end{array}$ & $\begin{array}{l}\text { FNIR(N,R,T,L) “Miss rate" } \\
\text { FPIR(N,T,L) "False alarm rate" }\end{array}$ \\
\hline
\end{tabular}



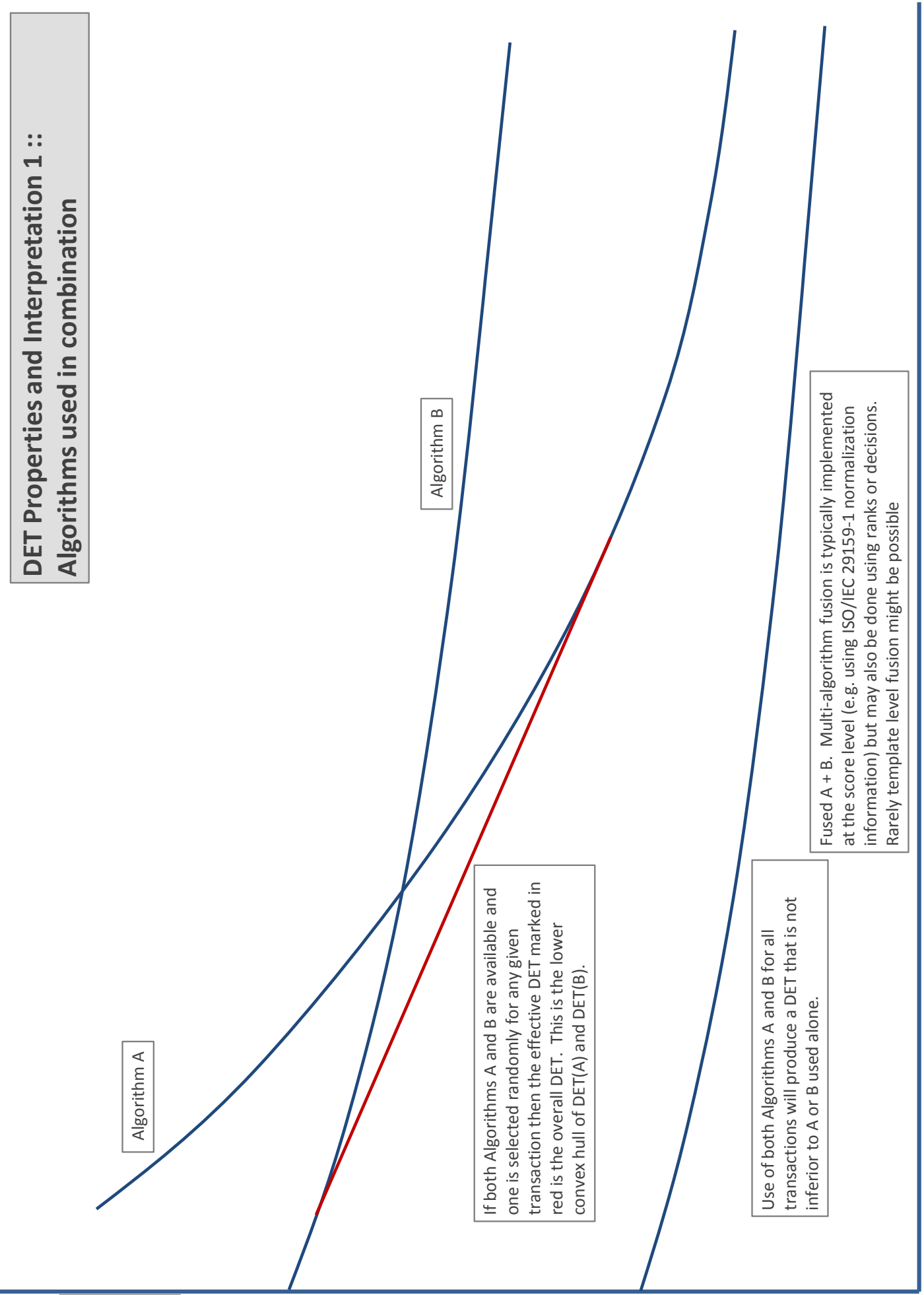

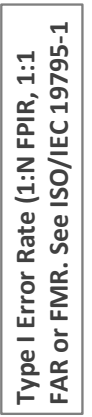

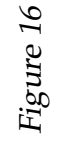

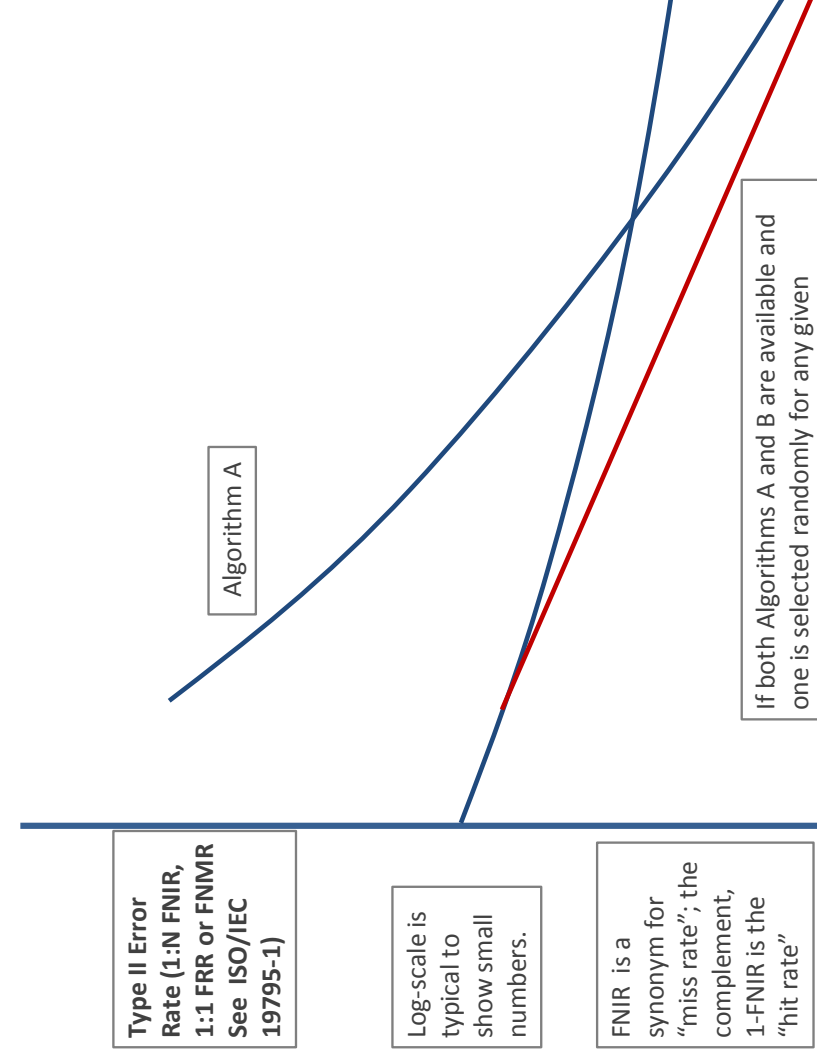

\begin{tabular}{|c|c|c|c|c|c|c|}
\hline $\begin{array}{l}\mathrm{A}=3 \mathrm{M} / \text { Cogent } \\
\mathrm{G}=\text { Hisign } \\
\mathrm{P}=\text { Zhuhai-Yisheng }\end{array}$ & $\begin{array}{l}\mathrm{B}=\text { Cognitec } \\
\mathrm{H}=\mathrm{CAS}-\mathrm{IA} \\
\mathrm{Q}=\mathrm{Jun} Y \mathrm{u}\end{array}$ & $\begin{array}{l}\mathrm{C}=\text { Neurotechnology } \\
\mathrm{I}=\text { CAS-ICT } \\
\mathrm{S}=\text { Decatur }\end{array}$ & $\begin{array}{l}\mathrm{D}=\text { Safran Morpho } \\
\mathrm{J}=\text { Toshiba } \\
\mathrm{T}=\text { Ayonix }\end{array}$ & $\begin{array}{l}\mathrm{E}=\mathrm{NEC} \\
\mathrm{L}=\text { Tsinghua U. II }\end{array}$ & $\begin{array}{l}\mathrm{F}=\text { Tsinghua } \mathrm{U} \\
\mathrm{M}=\mathrm{HP}\end{array}$ & $\begin{array}{l}\text { FNIR(N,R,T,L) “Miss rate" } \\
\text { FPIR(N,T,L) “False alarm rate" }\end{array}$ \\
\hline
\end{tabular}




\section{A Algorithm report cards}

This section details individual algorithm performance by including eight graphs on a single page. Figure 17 is a key for those graphs, giving captions applicable to each report.

Error tradeoff by camera, enrolment type: The figure shows the
tradeoff between false negative and false positives by plotting
FNIR(T) vs. FPIR(T). This is the detection error tradeoff charac-
teristic (DET). The plots are plotted parametrically on threshold,
$T$, which is swept across the entire range of genuine scores pro-
duced by the algorithm. Four traces are shown, one each of the
two enrolment types, and one each for the two capture processes.
The mostly vertical grey lines connect points of fixed threshold:
vertical lines show that only FNIR changes across those four con-
ditions; horizontal lines show that FPIR is affected. The grey text
identifies the threshold values.

Error tradeoff by population size: The figure shows the tradeoff between false negative and false positives by plotting FNIR(T) vs. FPIR(T). This is the detection error tradeoff characteristic (DET). The plots are plotted parametrically on threshold, $T$, which is swept across the entire range of genuine scores produced by the algorithm. Four traces are shown, one each population size. The mostly vertical grey lines connect points of fixed threshold: horizontal lines show that FPIR grows linearly with population size, as expected from classical binomial models of identification. The grey text identifies the threshold values.

Miss rates increase with enrolled population sizes: The figure shows the growth of miss rates with $\mathrm{N}$ for two enrolment types, two cameras, and two rank values, 1 and 50. The threshold is set to zero, and the values are simply the proportion of mated searches that do not yield the mate in the top $\mathrm{R}$ ranks. The traces are typically straight lines on a log-log plot consistent with a power-law behavior (see 5.3.1.

Value of enrolling historical images: The figure shows the reduction in miss rates with the number of available enrolment images, for four population sizes. As presented in section 3.2 when images from all historical encounters are retained and enrolled, accuracy can be improved vs. the case where only one image is retained. The error bars indicate confidence intervals from bootstrapping applied over searches (see section 4.6). The relevance of this result is discussed further in section 5.4.

Figure 17: Key to report card figures: The boxed text of this figure describe the graphs that appear in the report cards of this Appendix. Each report card contains 8 graphs, with a one-to-one spatial correspondence with this Figure.
Effect of rank: The figure shows the effect of considering long candidate lists by plotting miss rate against rank, i.e. FNIR(R). This is closely related to a cumulative match characteristic, which conventionally plots 1 - FNIR. Four traces are shown, one each of the two enrolment types, and one each for the two capture processes.

Workload savings: The figure plots two quantities parametrically with threshold. On the x-axis is the factor by which workload is reduced when a threshold is applied to shorten candidate lists vs. when when all $L=50$ candidates are retained for possible inspection. On the y-axis is the factor by which thresholding candidate lists increases miss rate over the case of a full candidate list, i.e. $\operatorname{FNIR}(N, R, T, L) / \operatorname{FNIR}(N, L, 0, L)$.

Processing time: The figure shows three traces: a horizontal line indicating the time taken to produce a template from an image prior to search; an ascending line indicating the time taken to compare a template with data from $\mathrm{N}$ enrolled subjects; and the sum of these two durations - the total search time. The curves cross where $\mathrm{N}$ is large enough such that the 1 : N search time exceeds the template generation time. All durations apply to processing on a single core of a c. 2011 server-class processor. All templates are resident in memory.

Selectivity vs. FPIR: As presented in section 4.1, false alarm rates can be quantified by FPIR - the fraction of nonmate searches that produce any candidates at or above threshold - or by selectivity the expected number of candidates at or above threshold. This figure shows SEL(T) vs. FPIR(T) plotted paramterically on threshold T. Selectivity is always greater than or equal to FPIR. The two are not equal when false positives are concentrated in candidate lists rather than being distributed across searches.

\begin{tabular}{|c|c|c|c|c|c|c|}
\hline $\begin{array}{l}\mathrm{A}=3 \mathrm{M} / \text { Cogent } \\
\mathrm{G}=\text { Hisign } \\
\mathrm{P}=\text { Zhuhai-Yisheng }\end{array}$ & $\begin{array}{l}\mathrm{B}=\text { Cognitec } \\
\mathrm{H}=\text { CAS-IA } \\
\mathrm{Q}=\text { JunYu }\end{array}$ & $\begin{array}{l}\mathrm{C}=\text { Neurotechnology } \\
\mathrm{I}=\text { CAS-ICT } \\
\mathrm{S}=\text { Decatur }\end{array}$ & $\begin{array}{l}\mathrm{D}=\text { Safran Morpho } \\
\mathrm{J}=\text { Toshiba } \\
\mathrm{T}=\text { Ayonix }\end{array}$ & $\begin{array}{l}\mathrm{E}=\mathrm{NEC} \\
\mathrm{L}=\text { Tsinghua U. II }\end{array}$ & $\begin{array}{l}\mathrm{F}=\text { Tsinghua } \mathrm{U} \\
\mathrm{M}=\mathrm{HP}\end{array}$ & $\begin{array}{l}\text { FNIR(N,R,T,L) “Miss rate" } \\
\text { FPIR(N,T,L) "False alarm rate" }\end{array}$ \\
\hline
\end{tabular}




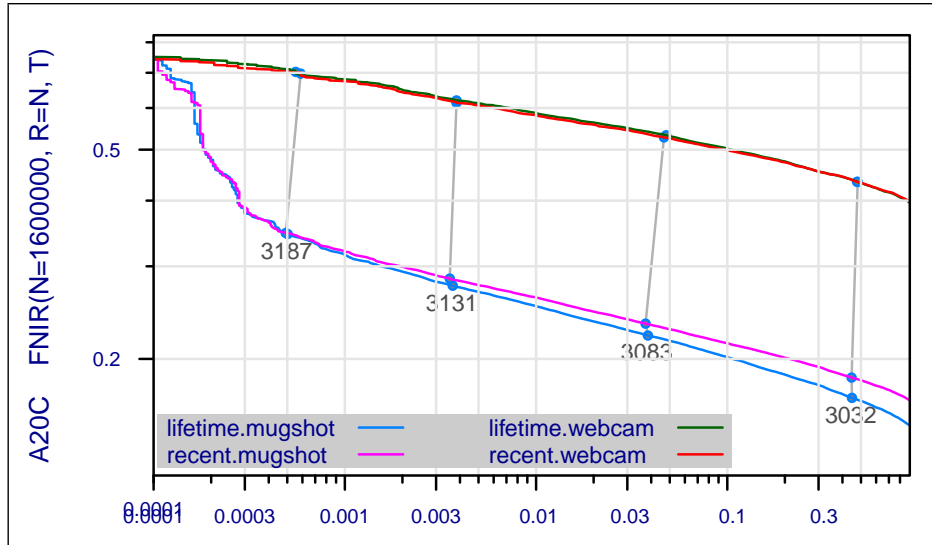

$\operatorname{FPIR}(\mathrm{N}=1600000)$
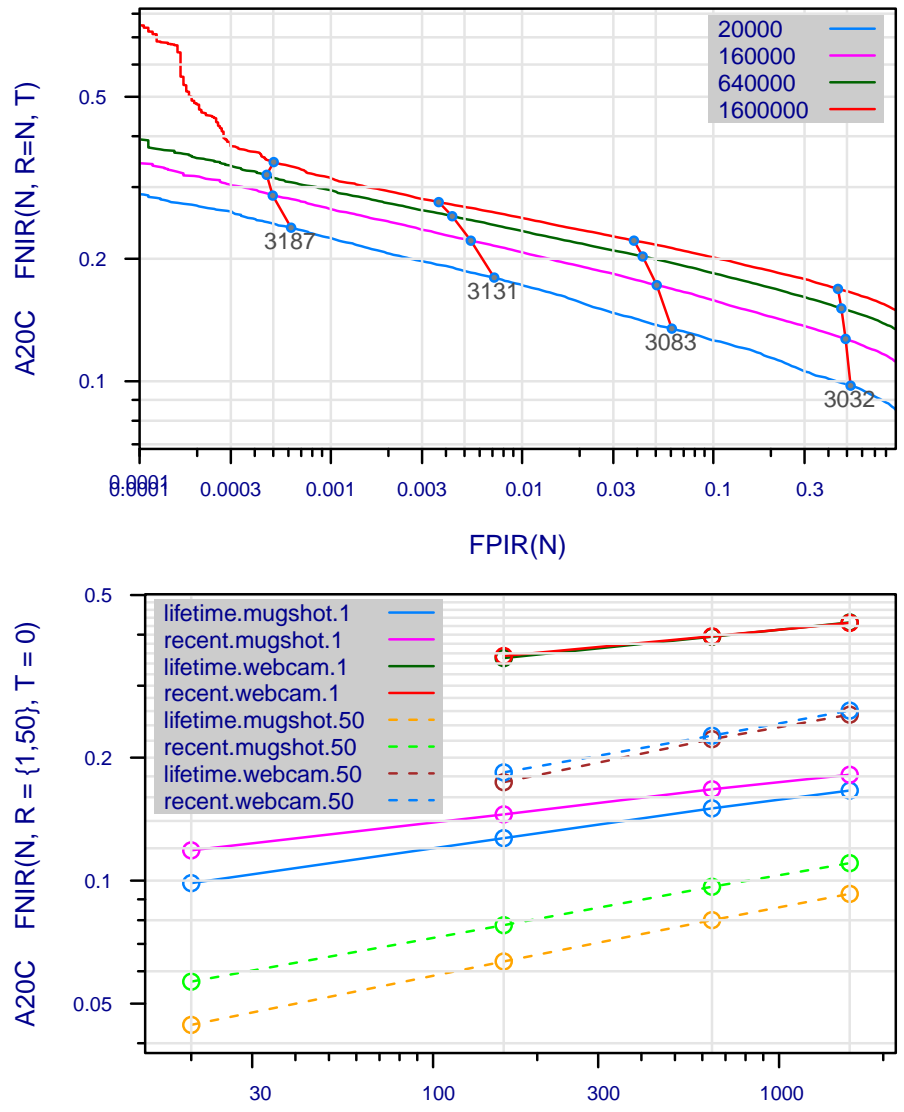

Num. enrolled identities, $\mathrm{N}$ (thousands)

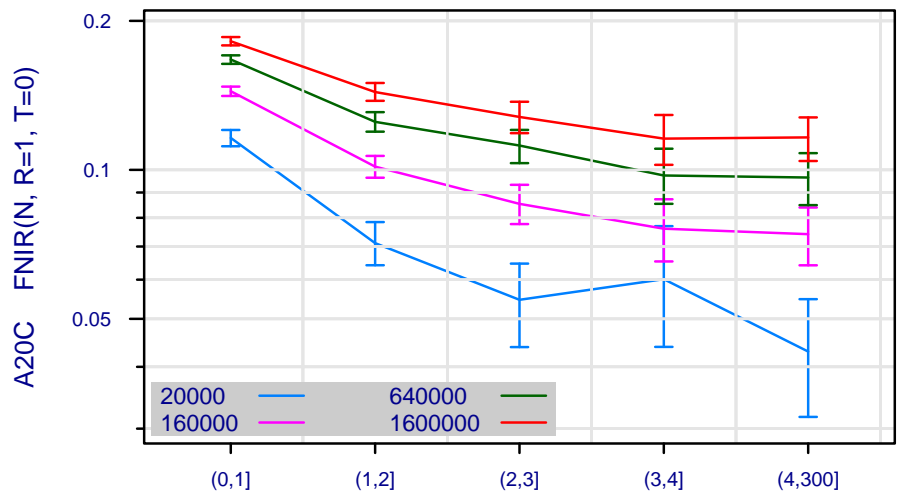

Num. enrolled images per identity

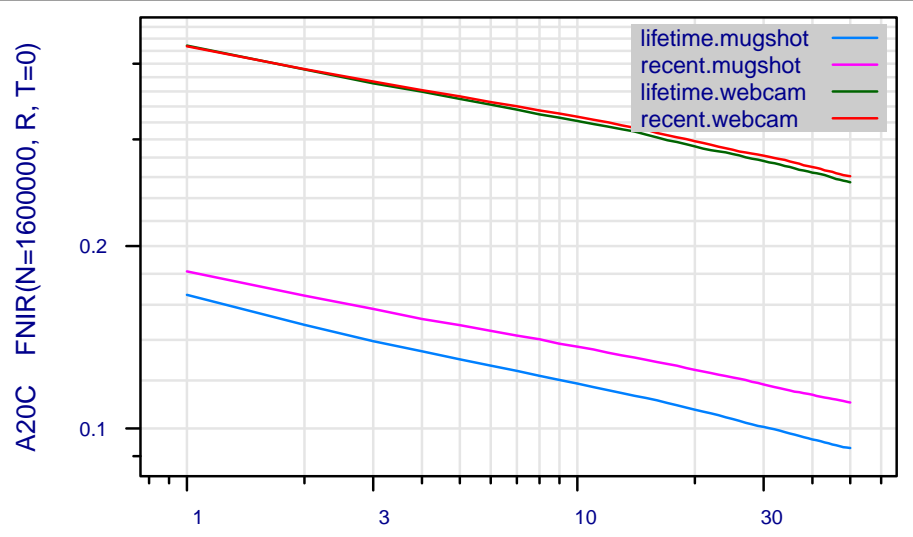

Rank, $\mathrm{R}$

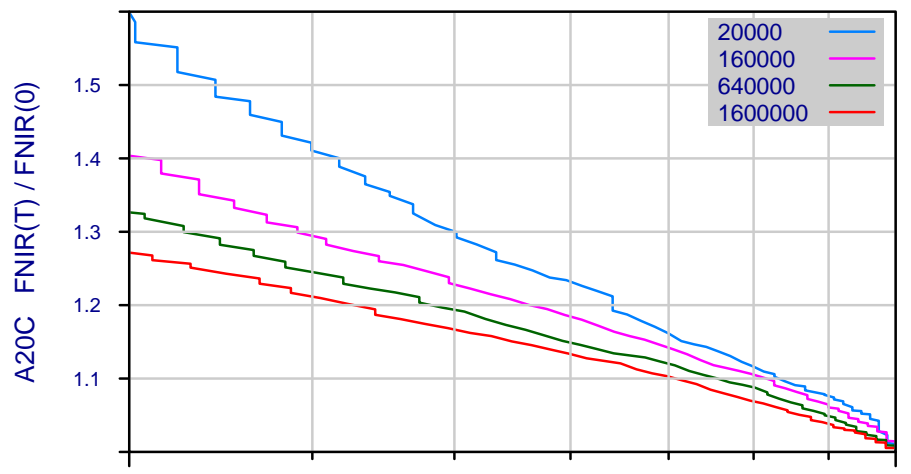

Frac. reduction in workload by using a threshold vs. not

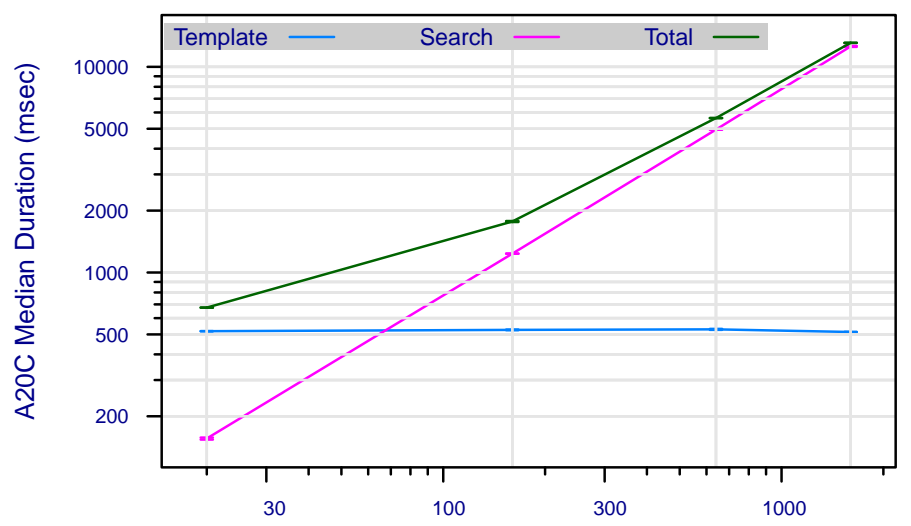

Num. enrolled identities, $\mathrm{N}$ (thousands)

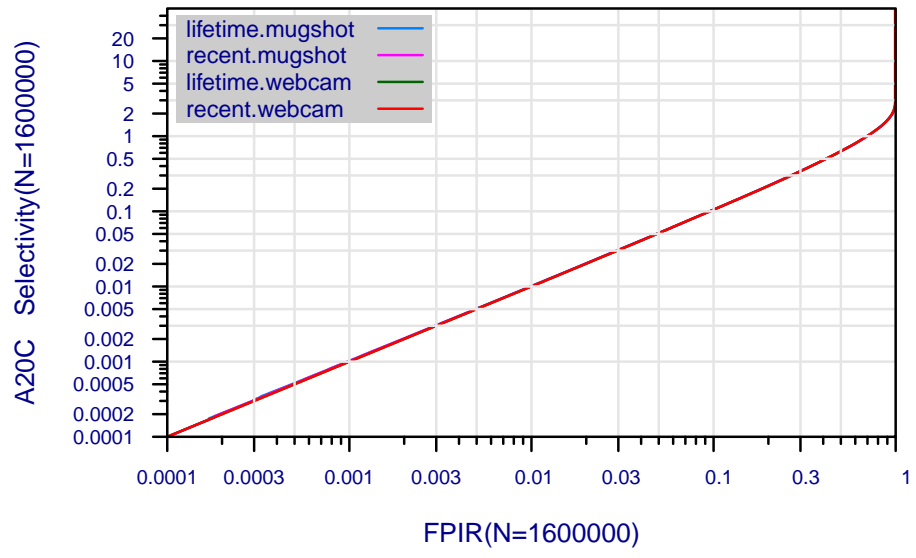

Figure 18: Collected performance reports for algorithm A20C. The figures are described at the beginning of this Appendix.

\begin{tabular}{|c|c|c|c|c|c|c|}
\hline $\begin{array}{l}\mathrm{A}=3 \mathrm{M} / \text { Cogent } \\
\mathrm{G}=\text { Hisign } \\
\mathrm{P}=\text { Zhuhai-Yisheng }\end{array}$ & $\begin{array}{l}\mathrm{B}=\text { Cognitec } \\
\mathrm{H}=\text { CAS-IA } \\
\mathrm{Q}=\text { JunYu }\end{array}$ & $\begin{array}{l}\mathrm{C}=\text { Neurotechnology } \\
\mathrm{I}=\mathrm{CAS}-\mathrm{ICT} \\
\mathrm{S}=\text { Decatur }\end{array}$ & $\begin{array}{l}\mathrm{D}=\text { Safran Morpho } \\
\mathrm{J}=\text { Toshiba } \\
\mathrm{T}=\text { Ayonix }\end{array}$ & $\begin{array}{l}\mathrm{E}=\mathrm{NEC} \\
\mathrm{L}=\text { Tsinghua U. II }\end{array}$ & $\begin{array}{l}\mathrm{F}=\text { Tsinghua } \mathrm{U} . \\
\mathrm{M}=\mathrm{HP}\end{array}$ & $\begin{array}{l}\text { FNIR(N,R,T,L) “Miss rate" } \\
\text { FPIR(N,T,L) “False alarm rate" }\end{array}$ \\
\hline
\end{tabular}




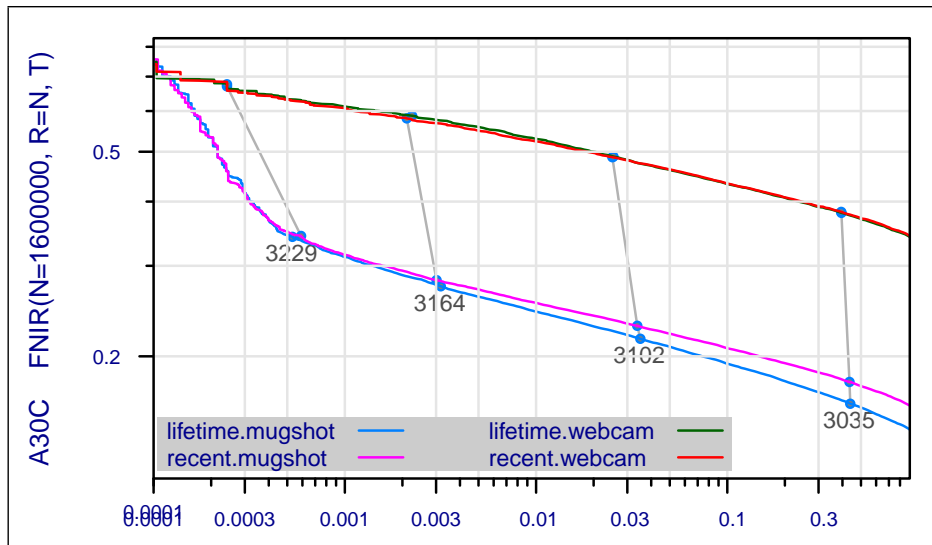

$\operatorname{FPIR}(\mathrm{N}=1600000)$
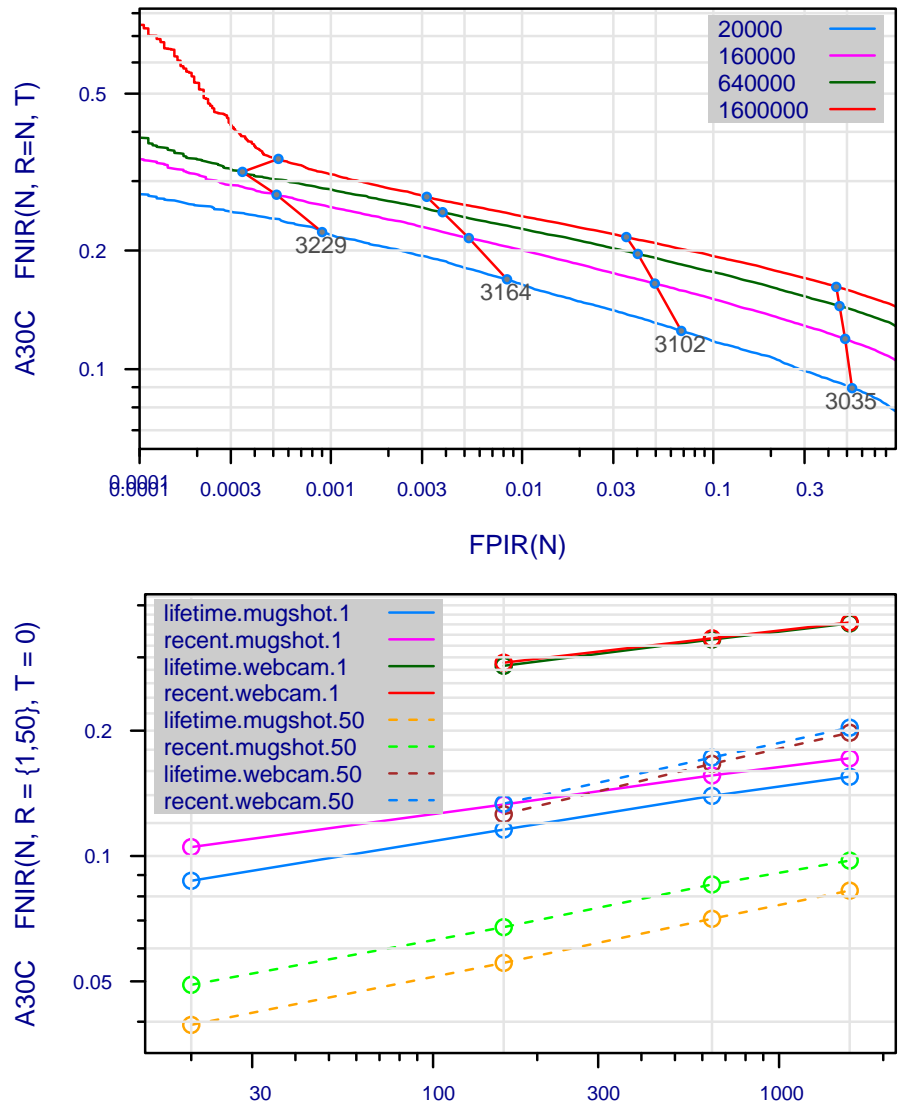

Num. enrolled identities, $\mathrm{N}$ (thousands)

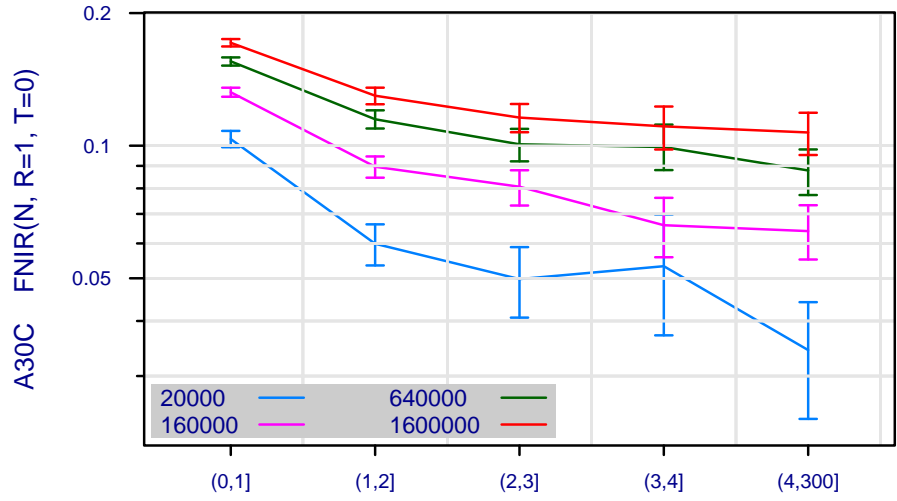

Num. enrolled images per identity

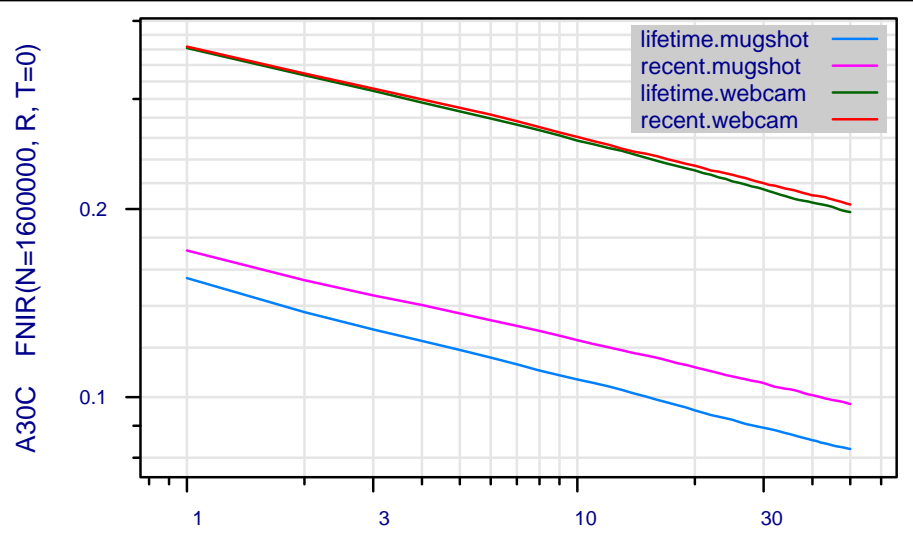

Rank, $\mathrm{R}$

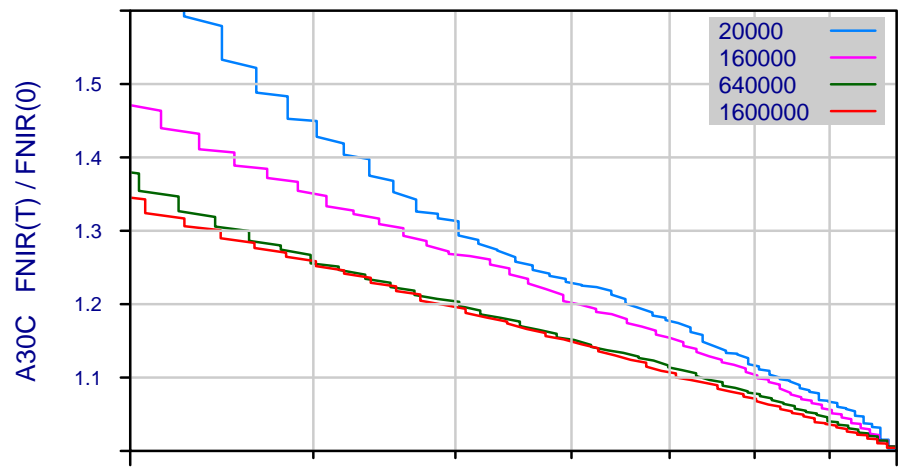

Frac. reduction in workload by using a threshold vs. not

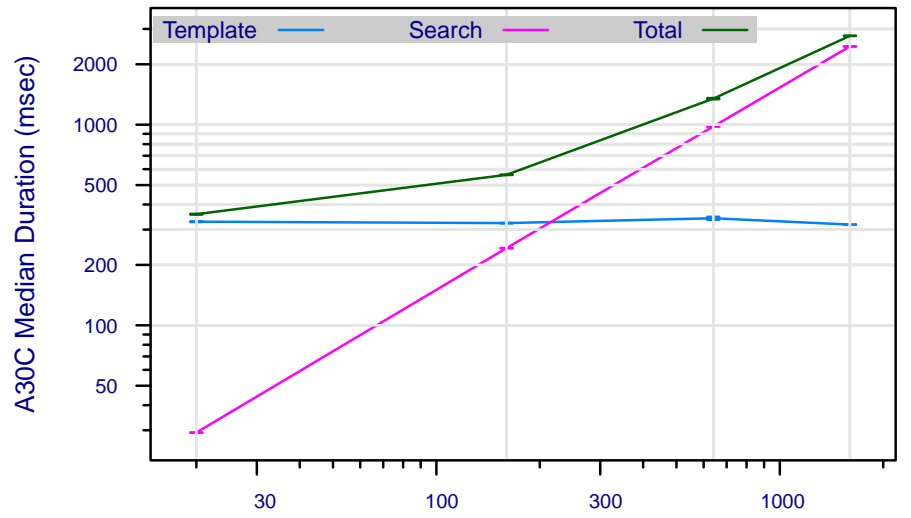

Num. enrolled identities, $\mathrm{N}$ (thousands)

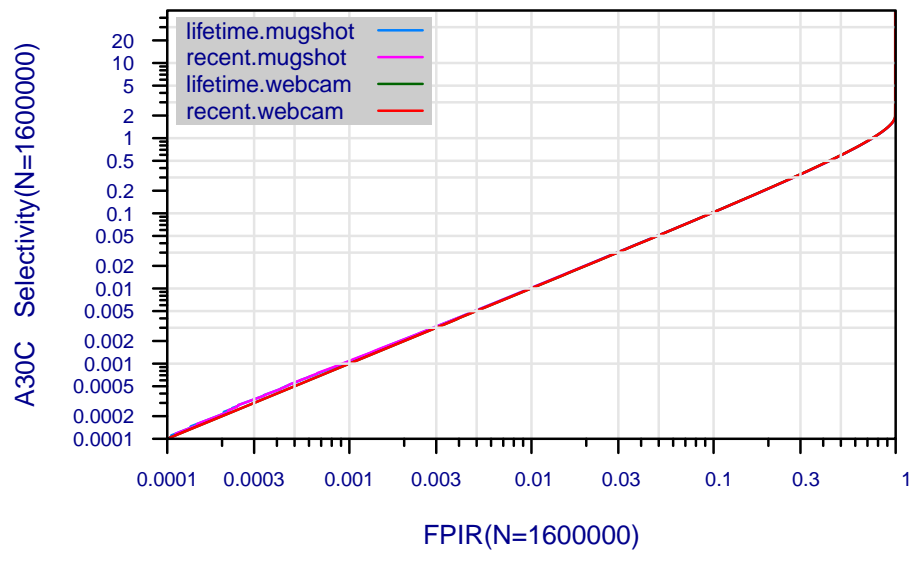

Figure 19: Collected performance reports for algorithm A30C. The figures are described at the beginning of this Appendix.

\begin{tabular}{|c|c|c|c|c|c|c|}
\hline $\begin{array}{l}\mathrm{A}=3 \mathrm{M} / \text { Cogent } \\
\mathrm{G}=\text { Hisign } \\
\mathrm{P}=\text { Zhuhai-Yisheng }\end{array}$ & $\begin{array}{l}\mathrm{B}=\text { Cognitec } \\
\mathrm{H}=\text { CAS-IA } \\
\mathrm{Q}=\text { JunYu }\end{array}$ & $\begin{array}{l}\mathrm{C}=\text { Neurotechnology } \\
\mathrm{I}=\mathrm{CAS}-\mathrm{ICT} \\
\mathrm{S}=\text { Decatur }\end{array}$ & $\begin{array}{l}\mathrm{D}=\text { Safran Morpho } \\
\mathrm{J}=\text { Toshiba } \\
\mathrm{T}=\text { Ayonix }\end{array}$ & $\begin{array}{l}\mathrm{E}=\mathrm{NEC} \\
\mathrm{L}=\text { Tsinghua U. II }\end{array}$ & $\begin{array}{l}\mathrm{F}=\text { Tsinghua } \mathrm{U} . \\
\mathrm{M}=\mathrm{HP}\end{array}$ & $\begin{array}{l}\text { FNIR(N,R,T,L) “Miss rate" } \\
\text { FPIR(N,T,L) “False alarm rate" }\end{array}$ \\
\hline
\end{tabular}




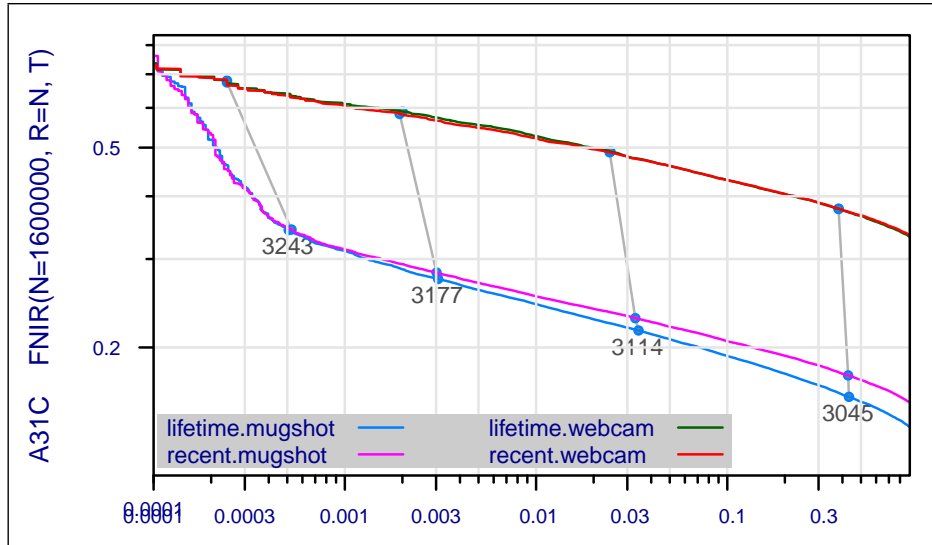

$\operatorname{FPIR}(\mathrm{N}=1600000)$
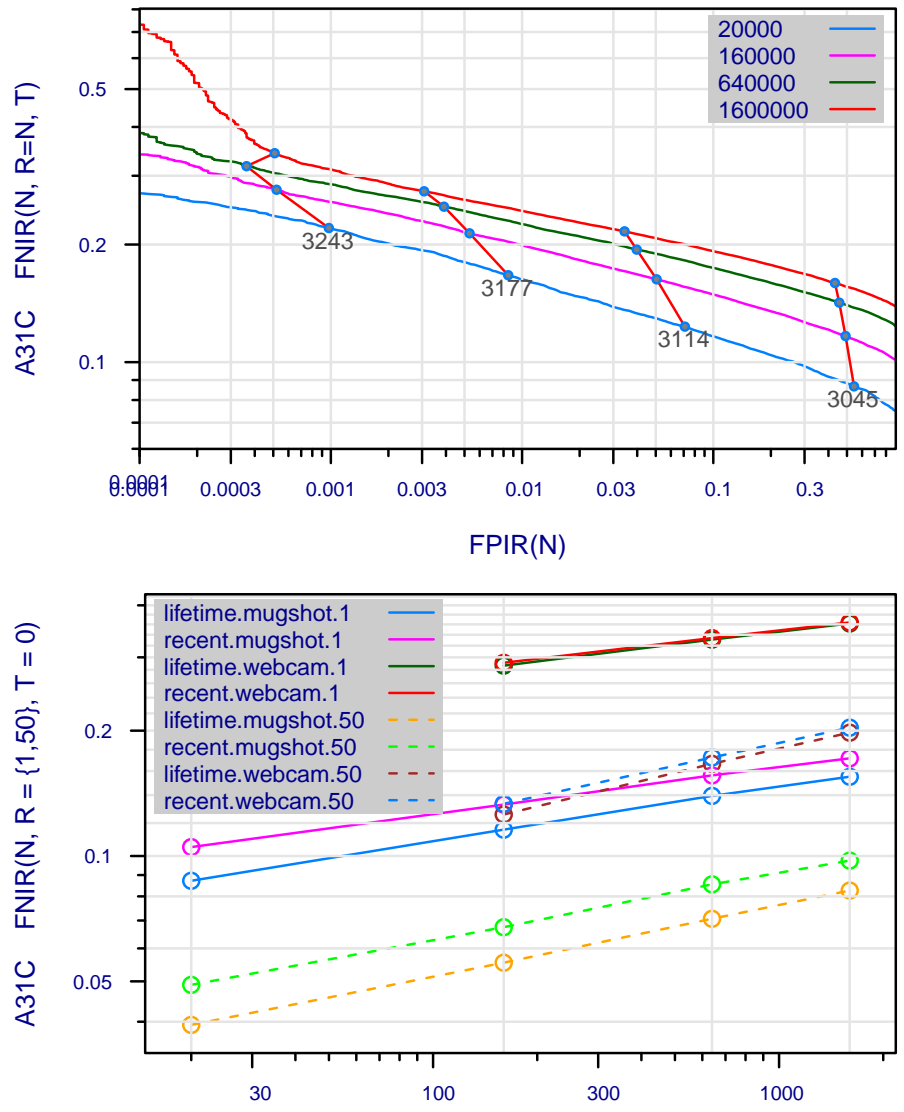

Num. enrolled identities, $\mathrm{N}$ (thousands)

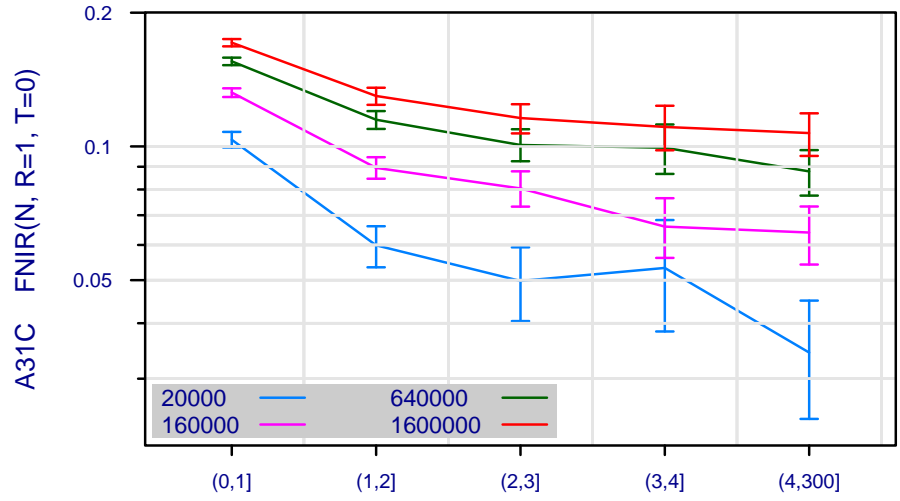

Num. enrolled images per identity

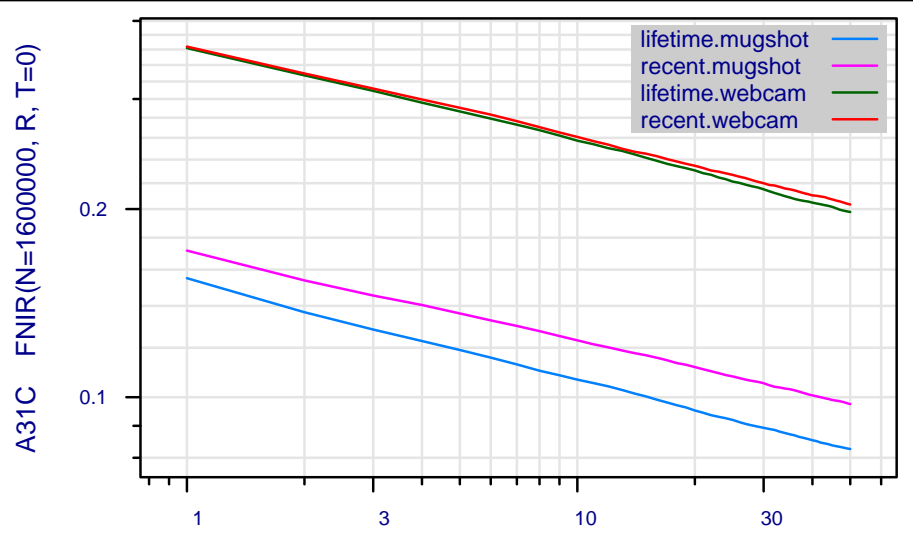

Rank, $\mathrm{R}$

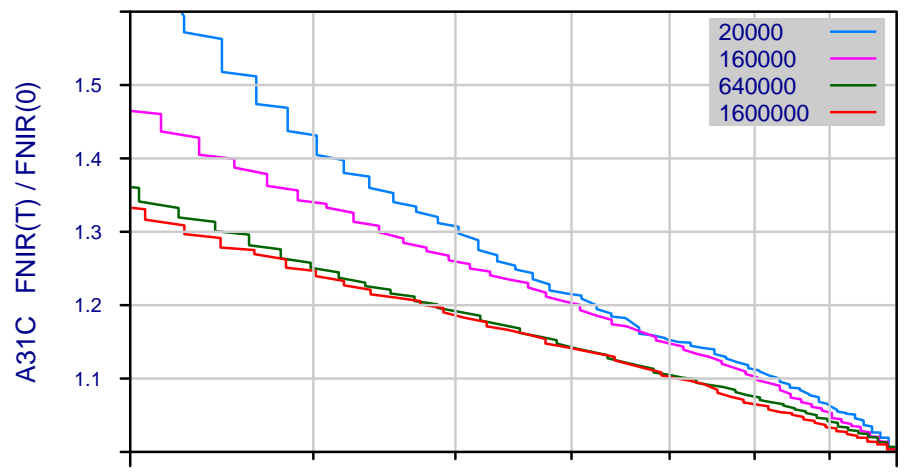

Frac. reduction in workload by using a threshold vs. not

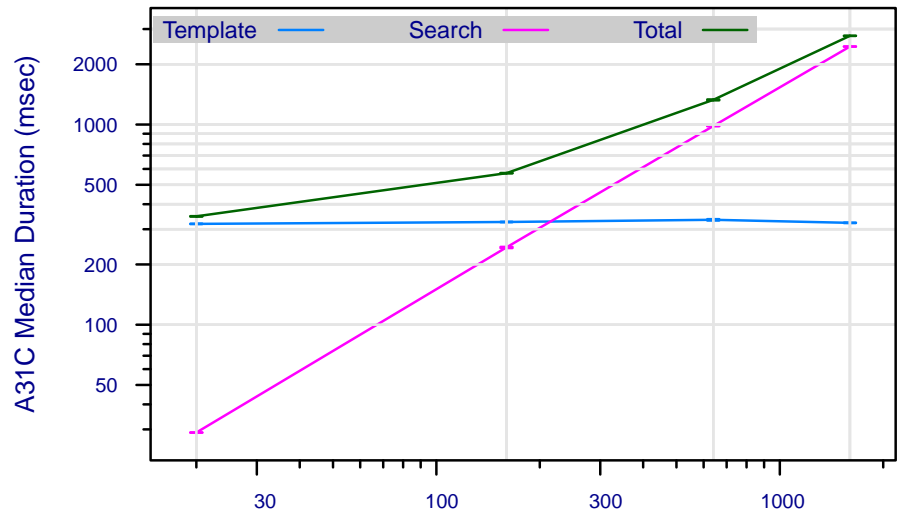

Num. enrolled identities, $\mathrm{N}$ (thousands)

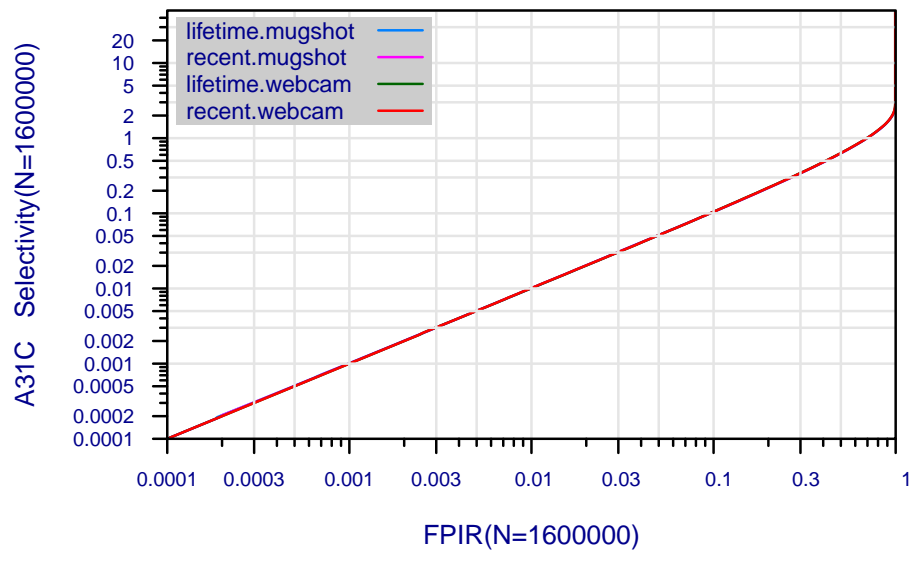

Figure 20: Collected performance reports for algorithm A31C. The figures are described at the beginning of this Appendix.

\begin{tabular}{|c|c|c|c|c|c|c|}
\hline $\begin{array}{l}\mathrm{A}=3 \mathrm{M} / \text { Cogent } \\
\mathrm{G}=\text { Hisign } \\
\mathrm{P}=\text { Zhuhai-Yisheng }\end{array}$ & $\begin{array}{l}\mathrm{B}=\text { Cognitec } \\
\mathrm{H}=\text { CAS-IA } \\
\mathrm{Q}=\text { JunYu }\end{array}$ & $\begin{array}{l}\mathrm{C}=\text { Neurotechnology } \\
\mathrm{I}=\mathrm{CAS}-\mathrm{ICT} \\
\mathrm{S}=\text { Decatur }\end{array}$ & $\begin{array}{l}\mathrm{D}=\text { Safran Morpho } \\
\mathrm{J}=\text { Toshiba } \\
\mathrm{T}=\text { Ayonix }\end{array}$ & $\begin{array}{l}\mathrm{E}=\mathrm{NEC} \\
\mathrm{L}=\text { Tsinghua U. II }\end{array}$ & $\begin{array}{l}\mathrm{F}=\text { Tsinghua } \mathrm{U} . \\
\mathrm{M}=\mathrm{HP}\end{array}$ & $\begin{array}{l}\text { FNIR(N,R,T,L) “Miss rate" } \\
\text { FPIR(N,T,L) “False alarm rate" }\end{array}$ \\
\hline
\end{tabular}



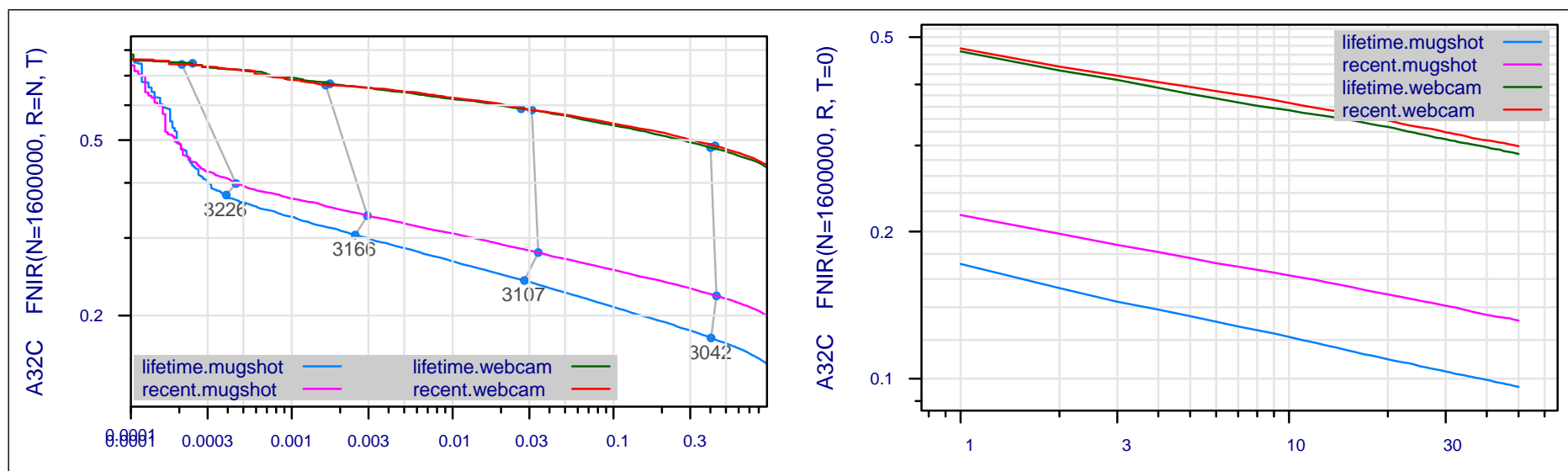

$\operatorname{FPIR}(\mathrm{N}=1600000)$
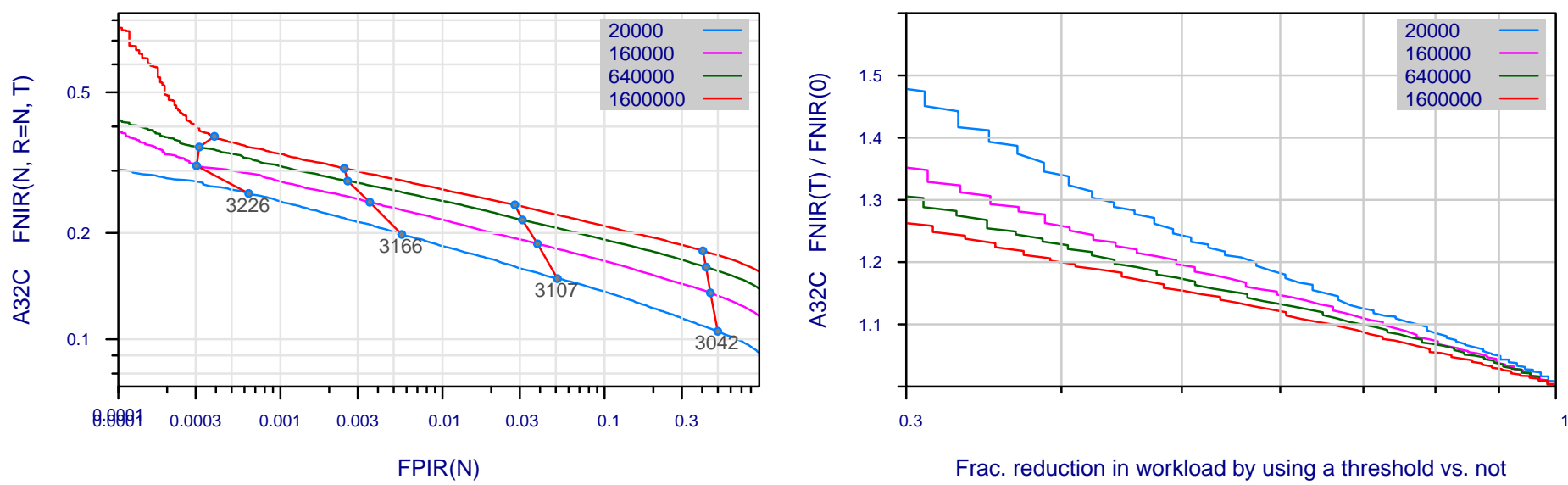

0.3

Frac. reduction in workload by using a threshold vs. not
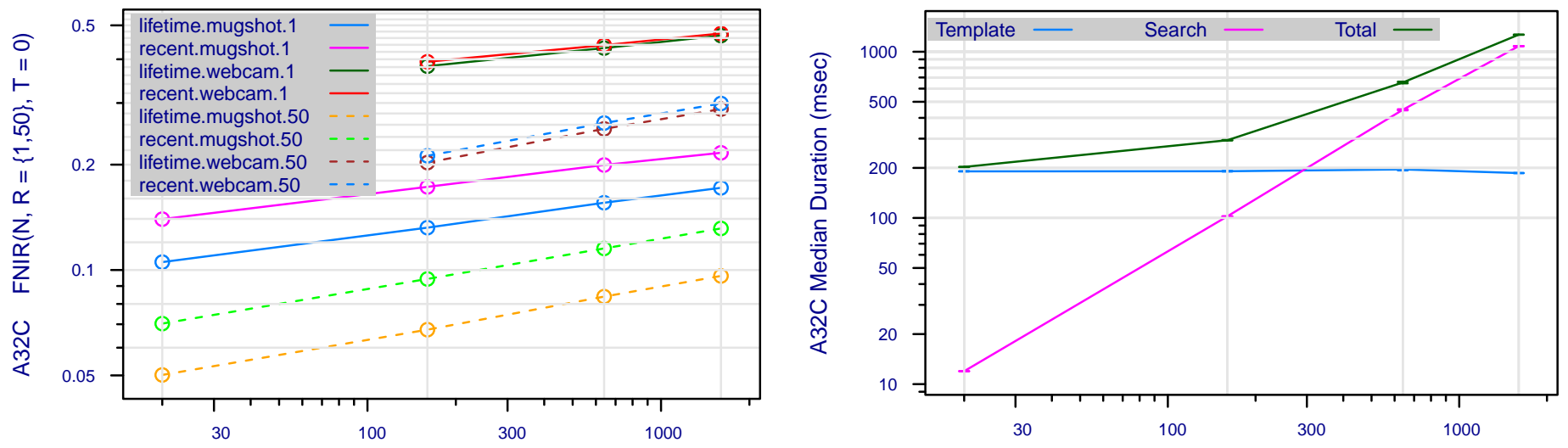

Num. enrolled identities, $\mathrm{N}$ (thousands)

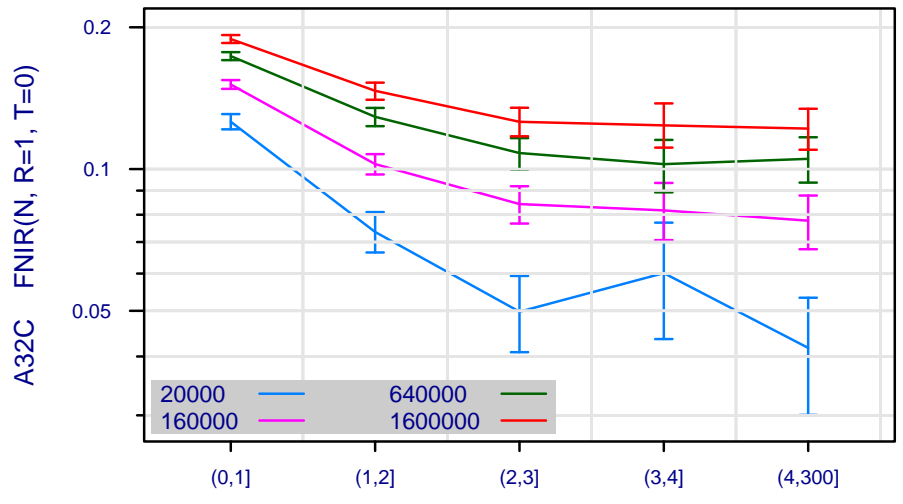

Num. enrolled images per identity

Num. enrolled identities, $\mathrm{N}$ (thousands)

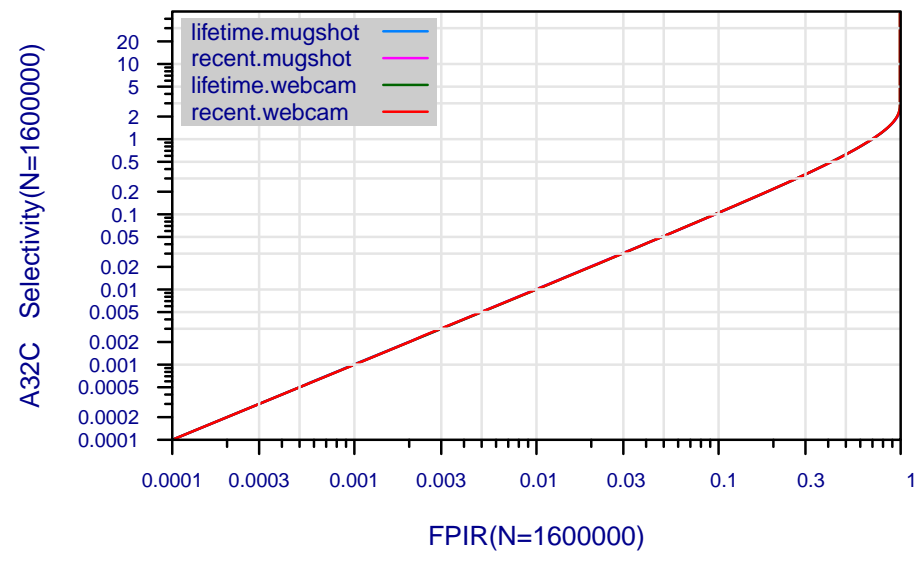

Figure 21: Collected performance reports for algorithm A32C. The figures are described at the beginning of this Appendix.

\begin{tabular}{|c|c|c|c|c|c|c|}
\hline $\begin{array}{l}\mathrm{A}=3 \mathrm{M} / \text { Cogent } \\
\mathrm{G}=\text { Hisign } \\
\mathrm{P}=\text { Zhuhai-Yisheng }\end{array}$ & $\begin{array}{l}B=\text { Cognitec } \\
H=\text { CAS-IA } \\
Q=\text { JunYu }\end{array}$ & $\begin{array}{l}\mathrm{C}=\text { Neurotechnology } \\
\mathrm{I}=\text { CAS-ICT } \\
\mathrm{S}=\text { Decatur }\end{array}$ & $\begin{array}{l}\mathrm{D}=\text { Safran Morpho } \\
\mathrm{J}=\text { Toshiba } \\
\mathrm{T}=\text { Ayonix }\end{array}$ & $\begin{array}{l}\mathrm{E}=\mathrm{NEC} \\
\mathrm{L}=\text { Tsinghua U. II }\end{array}$ & $\begin{array}{l}\mathrm{F}=\text { Tsinghua } \mathrm{U} \\
\mathrm{M}=\mathrm{HP}\end{array}$ & $\begin{array}{l}\text { FNIR(N,R,T,L) “Miss rate" } \\
\operatorname{FPIR}(\mathrm{N}, \mathrm{T}, \mathrm{L}) \text { “False alarm rate" }\end{array}$ \\
\hline
\end{tabular}




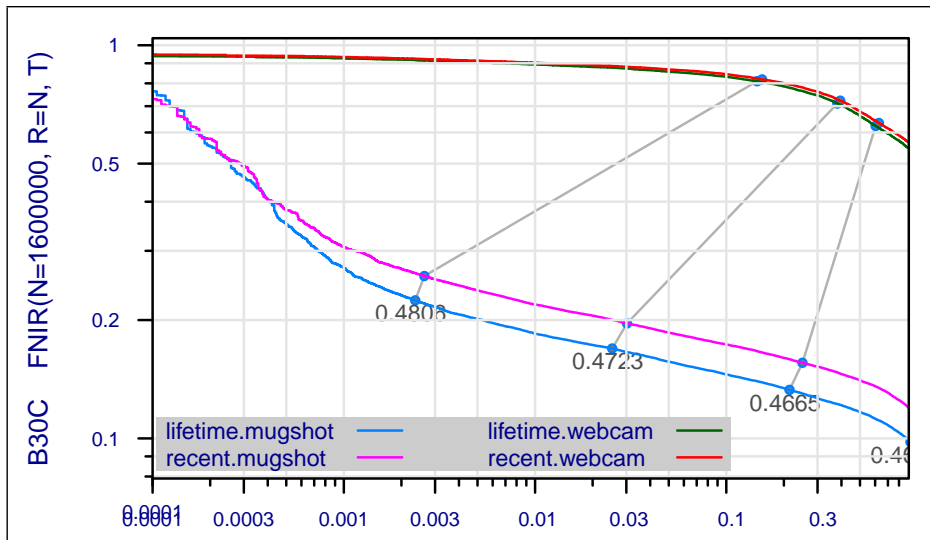

$\operatorname{FPIR}(\mathrm{N}=1600000)$
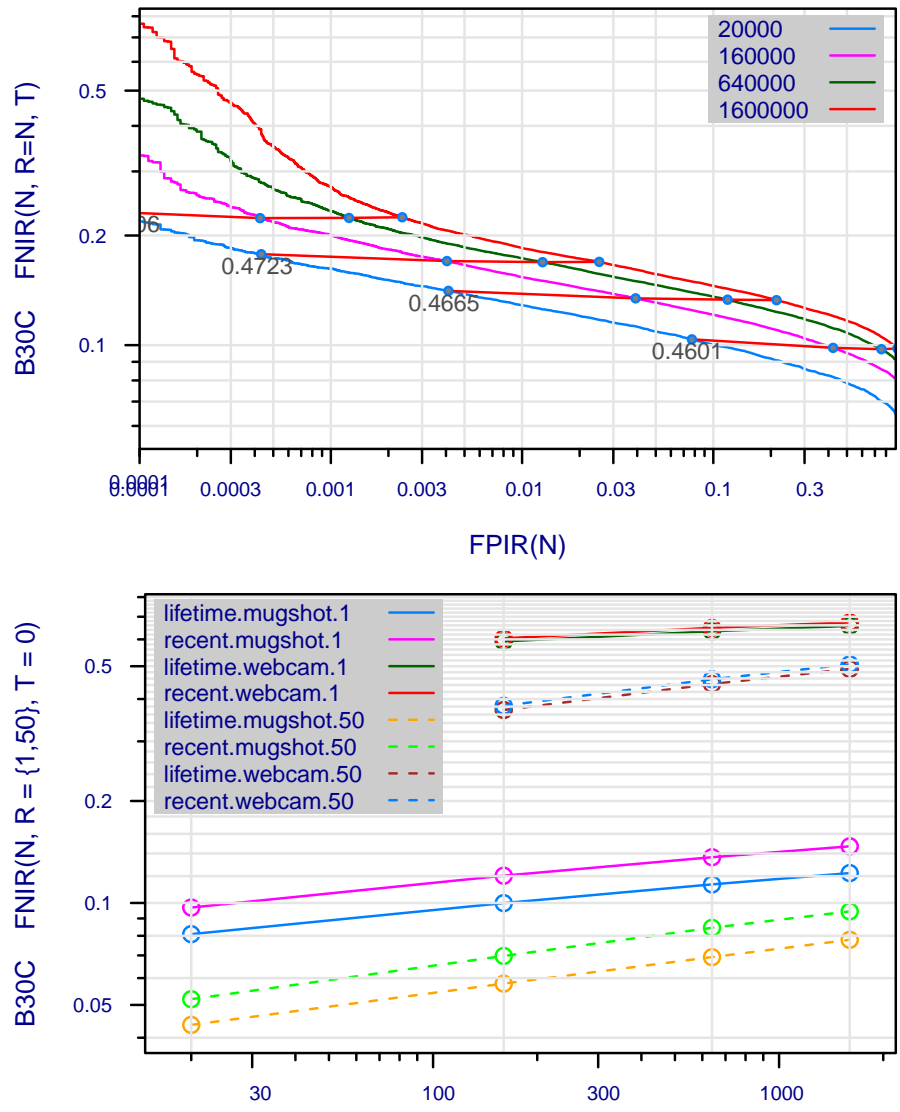

Num. enrolled identities, $\mathrm{N}$ (thousands)

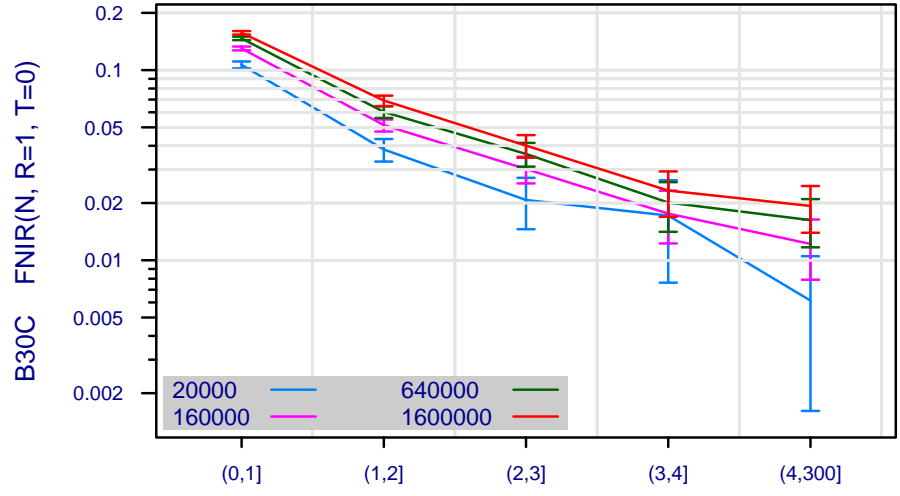

Num. enrolled images per identity

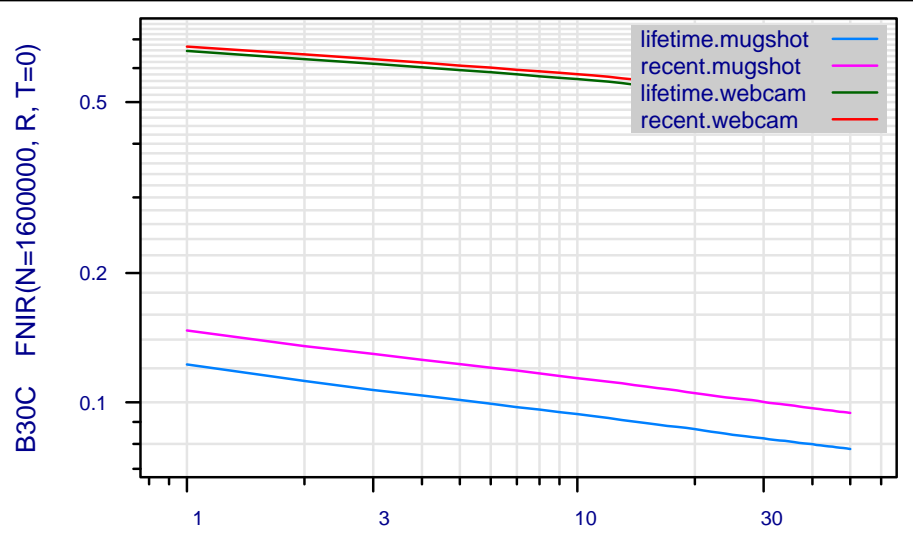

Rank, $\mathrm{R}$

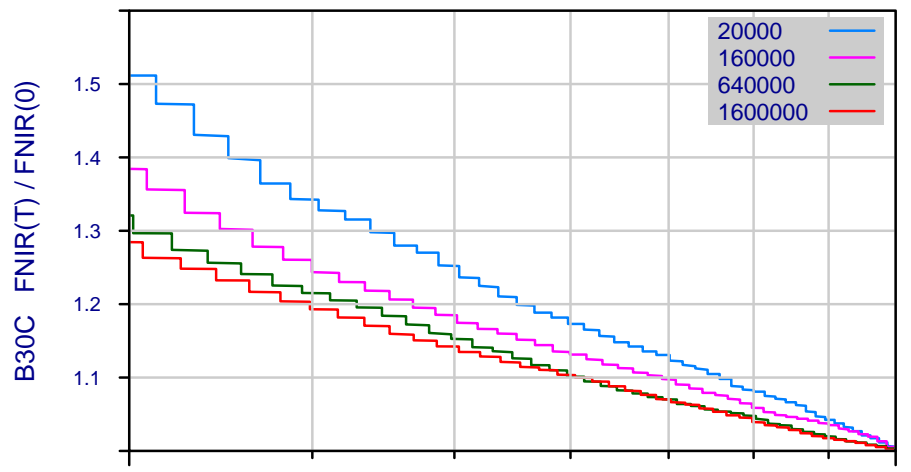

Frac. reduction in workload by using a threshold vs. not

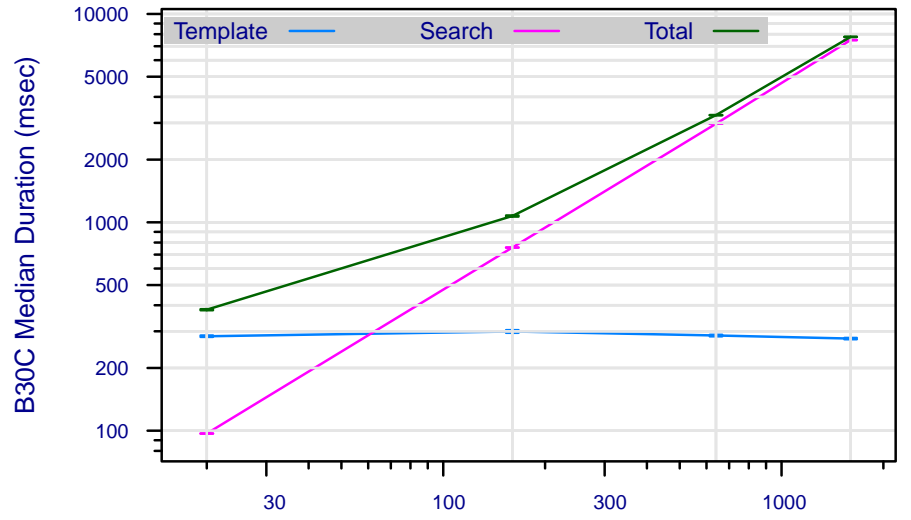

Num. enrolled identities, $\mathrm{N}$ (thousands)

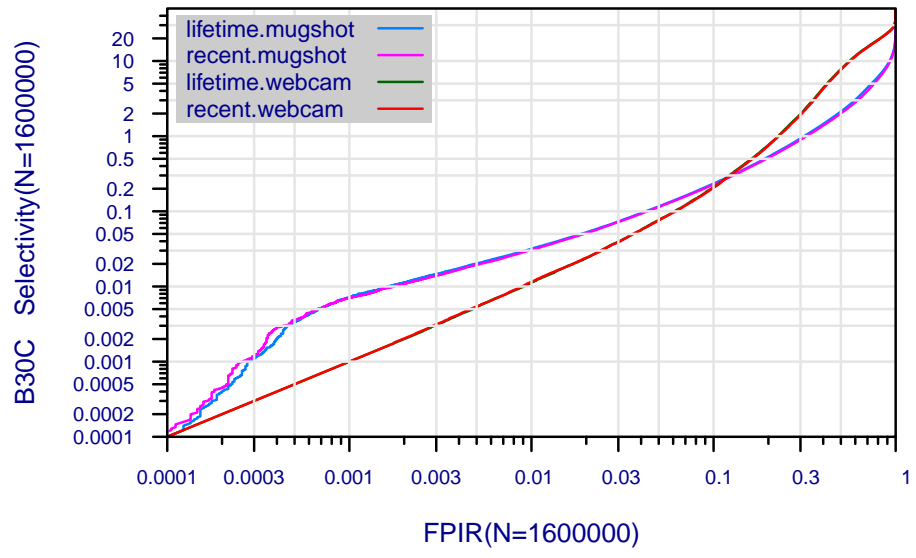

Figure 22: Collected performance reports for algorithm B30C. The figures are described at the beginning of this Appendix.

\begin{tabular}{|c|c|c|c|c|c|c|}
\hline $\begin{array}{l}\mathrm{A}=3 \mathrm{M} / \text { Cogent } \\
\mathrm{G}=\text { Hisign } \\
\mathrm{P}=\text { Zhuhai-Yisheng }\end{array}$ & $\begin{array}{l}\mathrm{B}=\text { Cognitec } \\
\mathrm{H}=\text { CAS-IA } \\
\mathrm{Q}=\text { JunYu }\end{array}$ & $\begin{array}{l}\mathrm{C}=\text { Neurotechnology } \\
\mathrm{I}=\mathrm{CAS}-\mathrm{ICT} \\
\mathrm{S}=\text { Decatur }\end{array}$ & $\begin{array}{l}\mathrm{D}=\text { Safran Morpho } \\
\mathrm{J}=\text { Toshiba } \\
\mathrm{T}=\text { Ayonix }\end{array}$ & $\begin{array}{l}\mathrm{E}=\mathrm{NEC} \\
\mathrm{L}=\text { Tsinghua U. II }\end{array}$ & $\begin{array}{l}\mathrm{F}=\text { Tsinghua } \mathrm{U} . \\
\mathrm{M}=\mathrm{HP}\end{array}$ & $\begin{array}{l}\text { FNIR(N,R,T,L) “Miss rate" } \\
\text { FPIR(N,T,L) “False alarm rate" }\end{array}$ \\
\hline
\end{tabular}



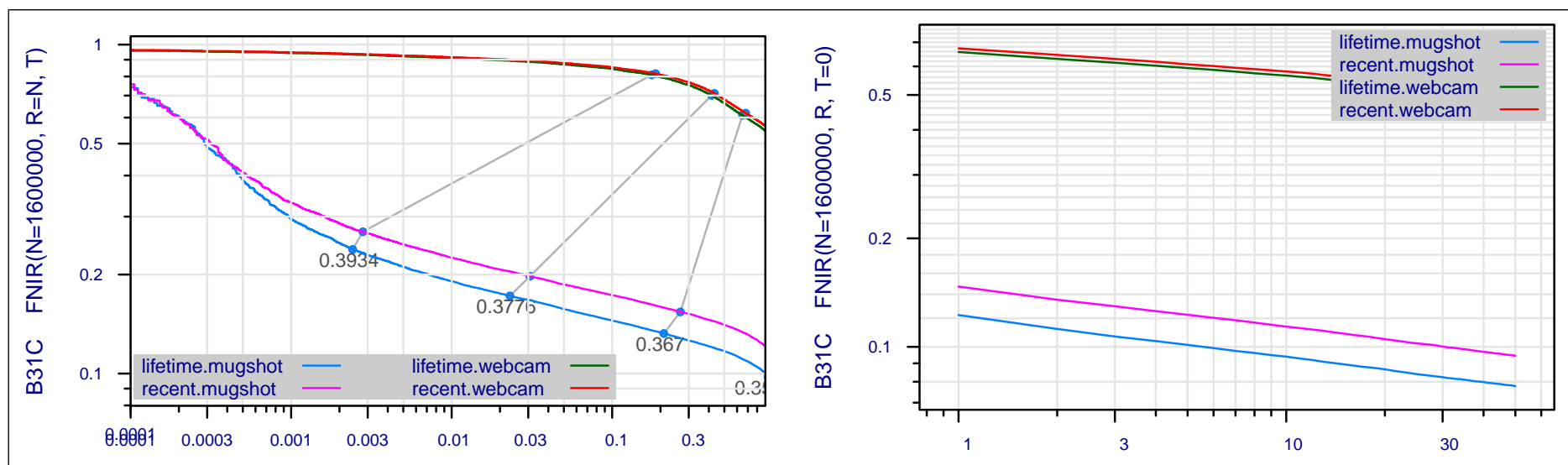

$\operatorname{FPIR}(\mathrm{N}=1600000)$
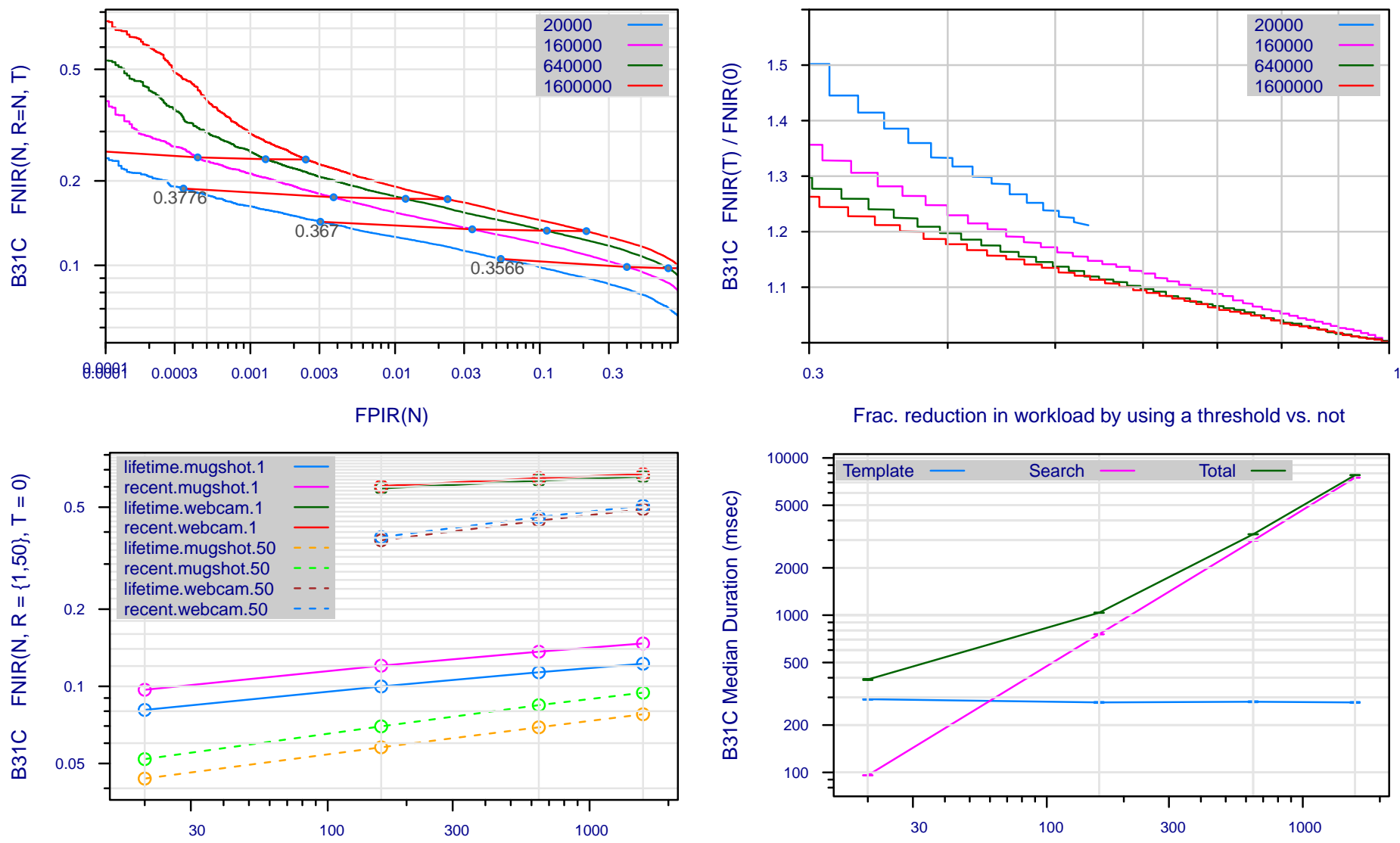

Num. enrolled identities, $\mathrm{N}$ (thousands)
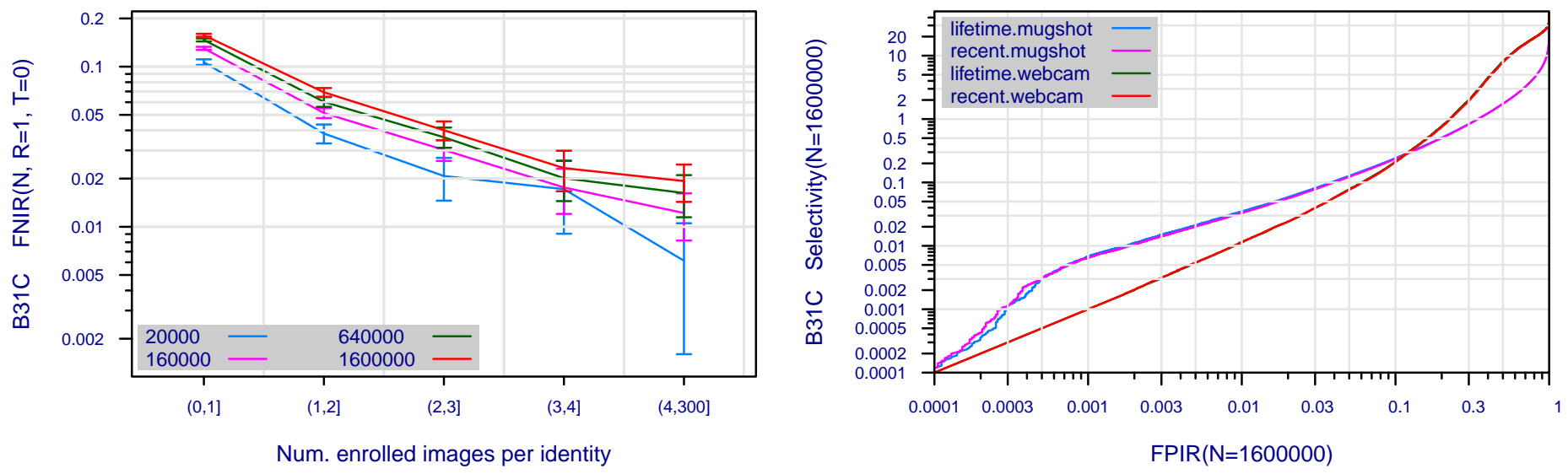

Figure 23: Collected performance reports for algorithm B31C. The figures are described at the beginning of this Appendix.

\begin{tabular}{|c|c|c|c|c|c|c|}
\hline $\begin{array}{l}\mathrm{A}=3 \mathrm{M} / \text { Cogent } \\
\mathrm{G}=\text { Hisign } \\
\mathrm{P}=\text { Zhuhai-Yisheng }\end{array}$ & $\begin{array}{l}\mathrm{B}=\text { Cognitec } \\
\mathrm{H}=\text { CAS-IA } \\
\mathrm{Q}=\text { JunYu }\end{array}$ & $\begin{array}{l}\mathrm{C}=\text { Neurotechnology } \\
\mathrm{I}=\mathrm{CAS}-\mathrm{ICT} \\
\mathrm{S}=\text { Decatur }\end{array}$ & $\begin{array}{l}\mathrm{D}=\text { Safran Morpho } \\
\mathrm{J}=\text { Toshiba } \\
\mathrm{T}=\text { Ayonix }\end{array}$ & $\begin{array}{l}\mathrm{E}=\mathrm{NEC} \\
\mathrm{L}=\text { Tsinghua U. II }\end{array}$ & $\begin{array}{l}\mathrm{F}=\text { Tsinghua } \mathrm{U} . \\
\mathrm{M}=\mathrm{HP}\end{array}$ & $\begin{array}{l}\text { FNIR(N,R,T,L) “Miss rate" } \\
\text { FPIR(N,T,L) “False alarm rate" }\end{array}$ \\
\hline
\end{tabular}




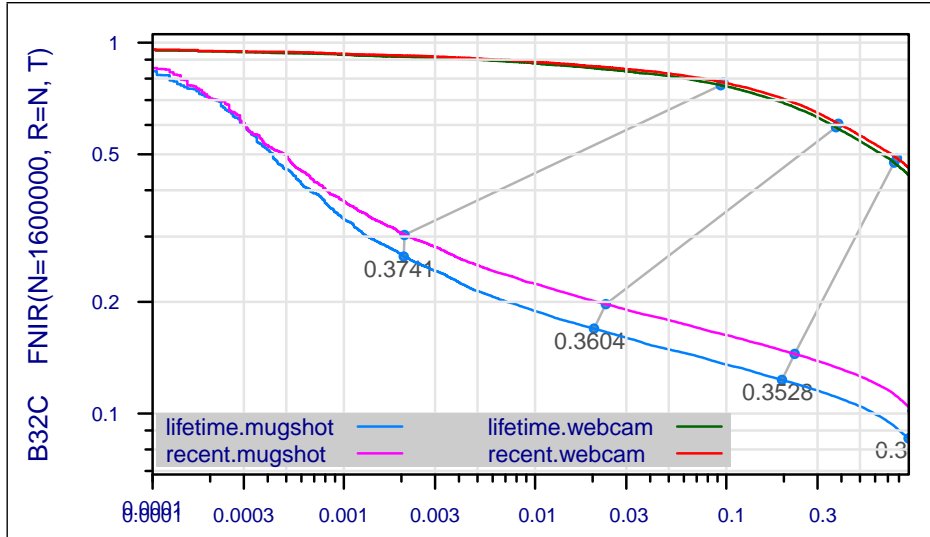

$\operatorname{FPIR}(\mathrm{N}=1600000)$
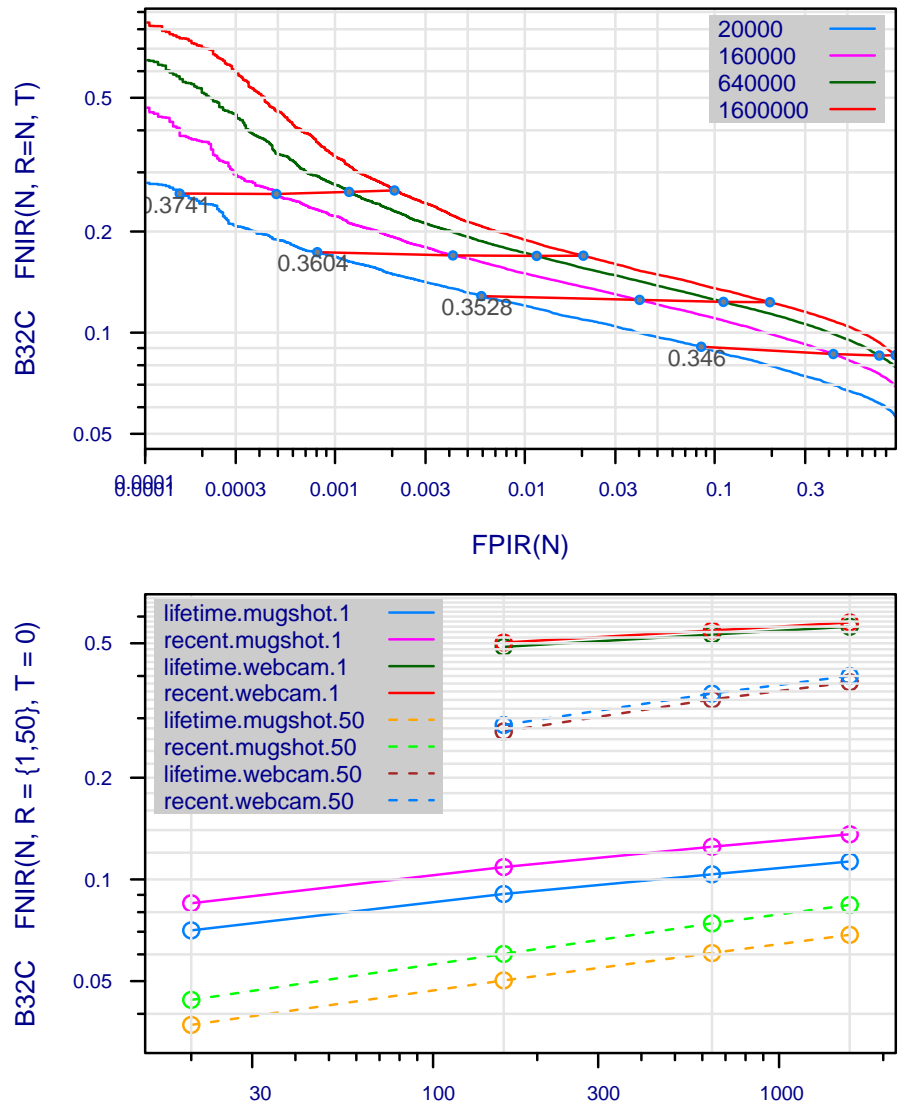

Num. enrolled identities, $\mathrm{N}$ (thousands)

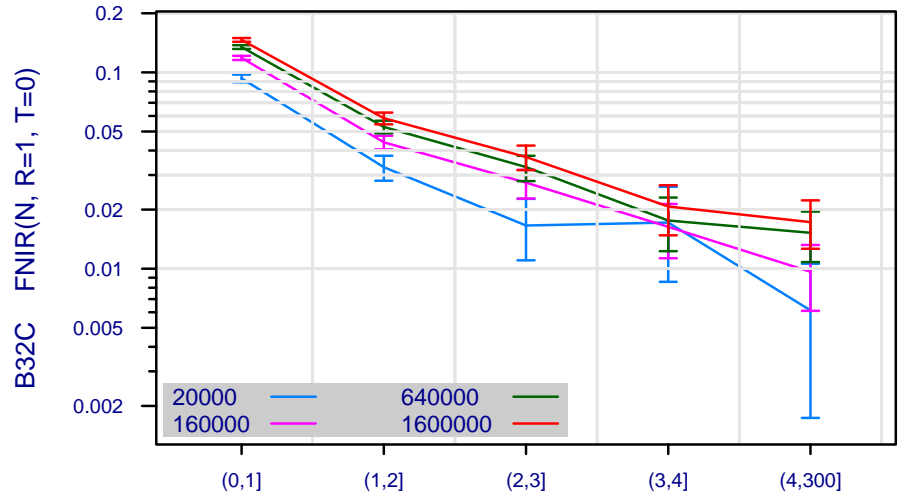

Num. enrolled images per identity

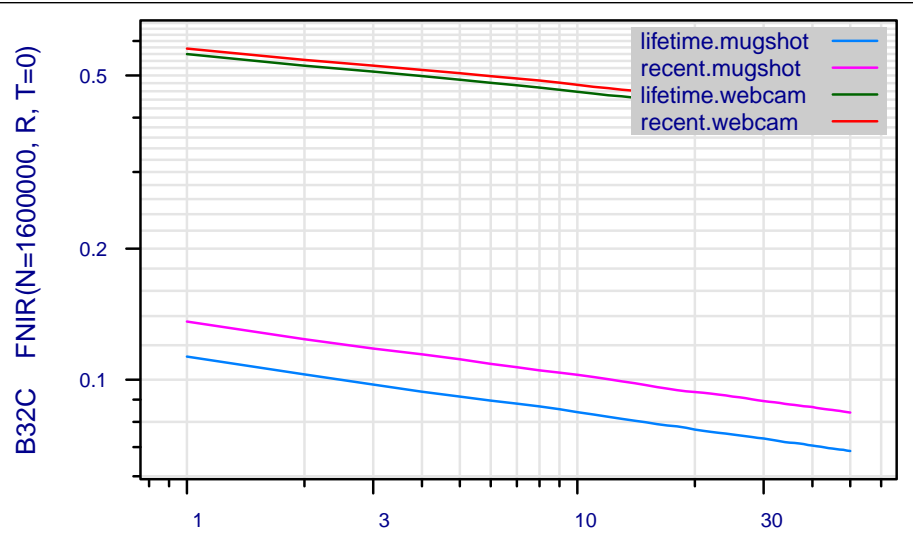

Rank, $\mathrm{R}$

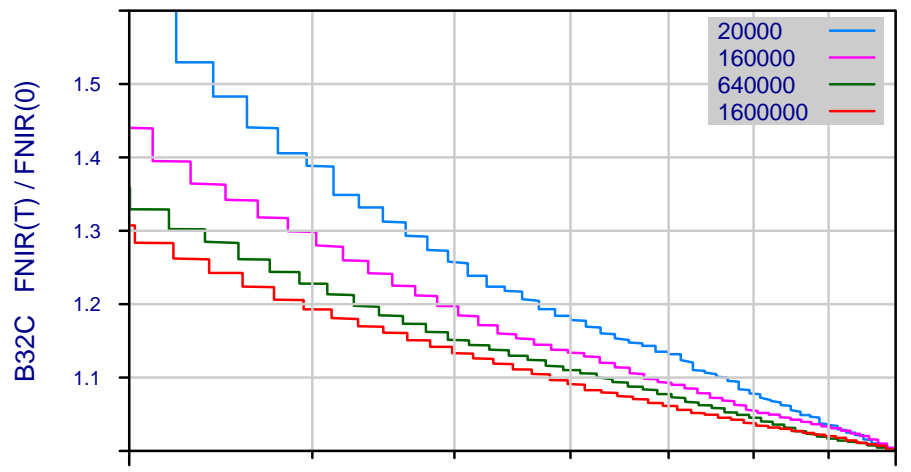

Frac. reduction in workload by using a threshold vs. not

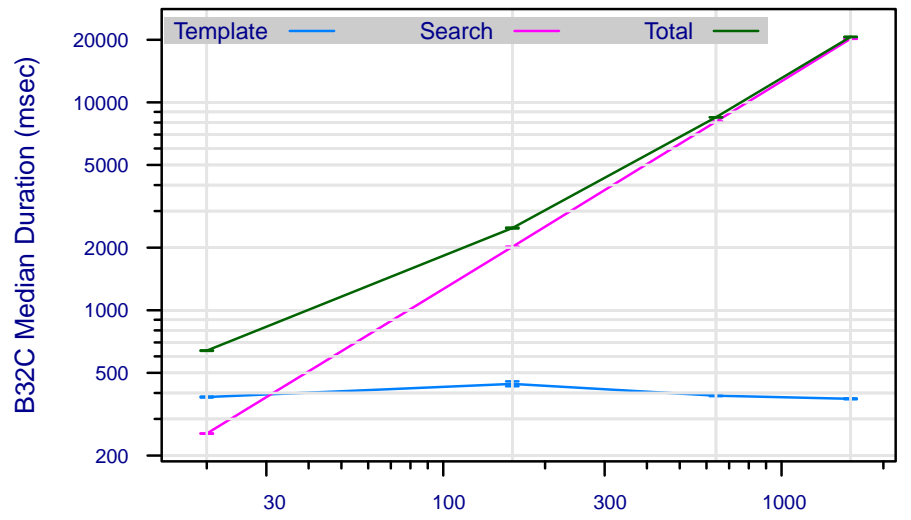

Num. enrolled identities, $\mathrm{N}$ (thousands)

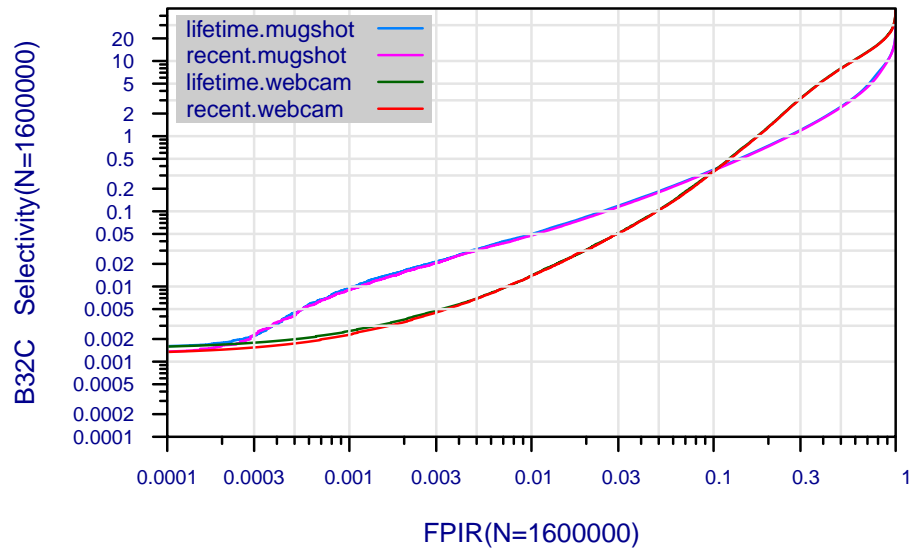

Figure 24: Collected performance reports for algorithm B32C. The figures are described at the beginning of this Appendix.

\begin{tabular}{|c|c|c|c|c|c|c|}
\hline $\begin{array}{l}\mathrm{A}=3 \mathrm{M} / \text { Cogent } \\
\mathrm{G}=\text { Hisign } \\
\mathrm{P}=\text { Zhuhai-Yisheng }\end{array}$ & $\begin{array}{l}\mathrm{B}=\text { Cognitec } \\
\mathrm{H}=\mathrm{CAS}-\mathrm{IA} \\
\mathrm{Q}=\text { JunYu }\end{array}$ & $\begin{array}{l}\mathrm{C}=\text { Neurotechnology } \\
\mathrm{I}=\text { CAS-ICT } \\
\mathrm{S}=\text { Decatur }\end{array}$ & $\begin{array}{l}\mathrm{D}=\text { Safran Morpho } \\
\mathrm{J}=\text { Toshiba } \\
\mathrm{T}=\text { Ayonix }\end{array}$ & $\begin{array}{l}\mathrm{E}=\mathrm{NEC} \\
\mathrm{L}=\text { Tsinghua U. II }\end{array}$ & $\begin{array}{l}\mathrm{F}=\text { Tsinghua } \mathrm{U} \\
\mathrm{M}=\mathrm{HP}\end{array}$ & $\begin{array}{l}\text { FNIR(N,R,T,L) “Miss rate" } \\
\text { FPIR(N,T,L) “False alarm rate" }\end{array}$ \\
\hline
\end{tabular}




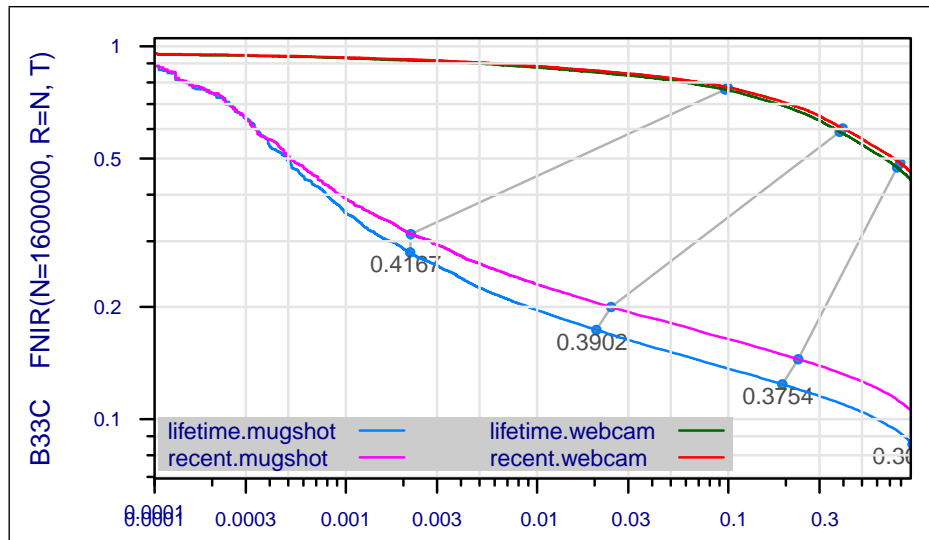

$\operatorname{FPIR}(\mathrm{N}=1600000)$
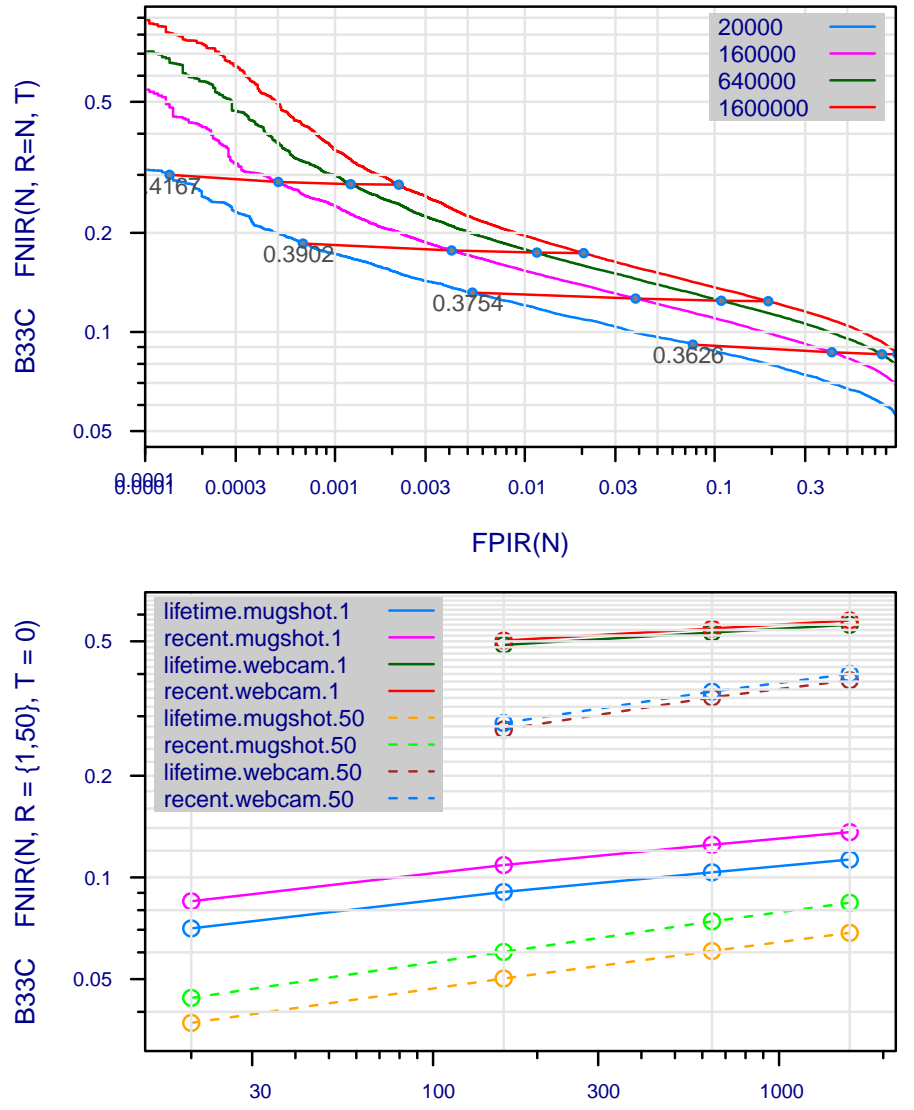

Num. enrolled identities, $\mathrm{N}$ (thousands)

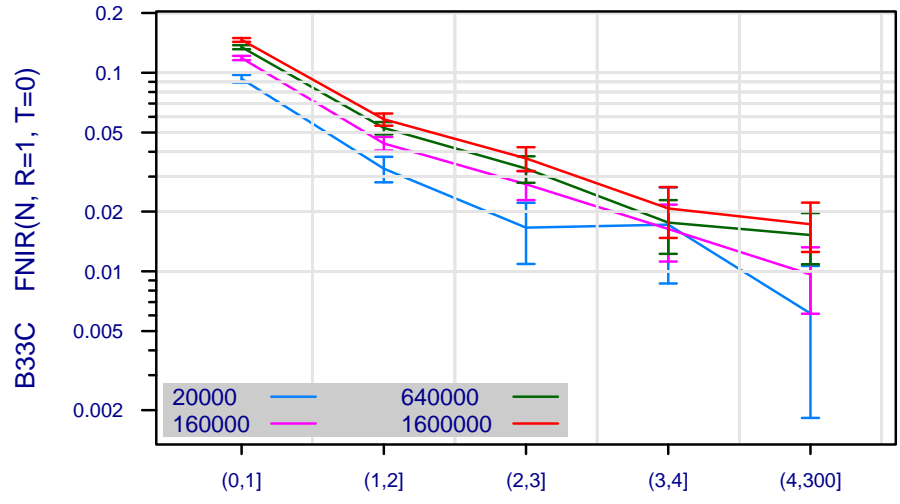

Num. enrolled images per identity

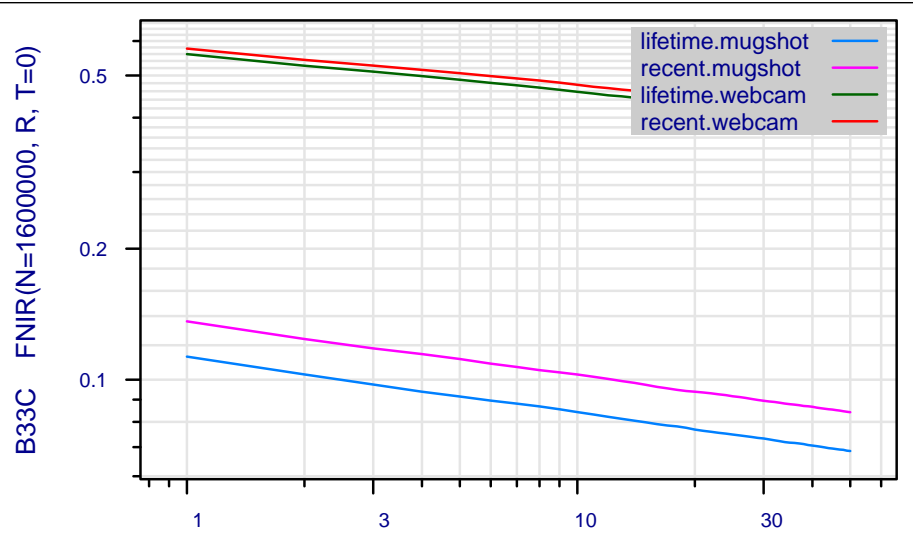

Rank, $\mathrm{R}$

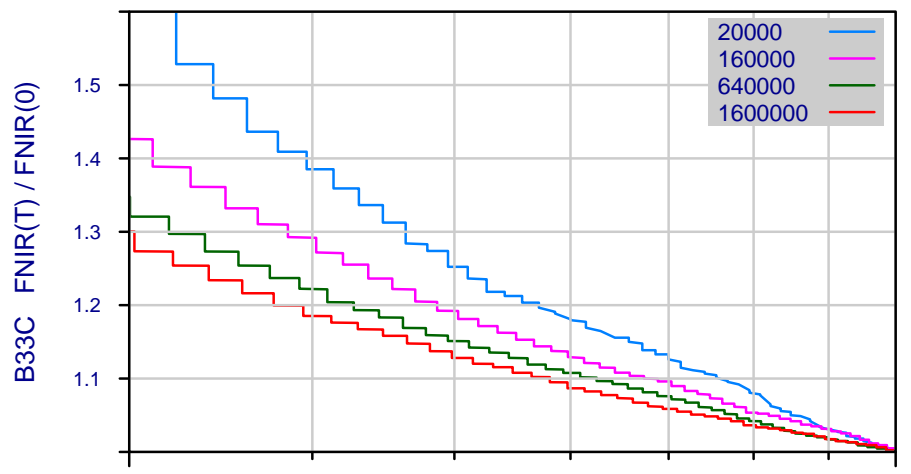

Frac. reduction in workload by using a threshold vs. not

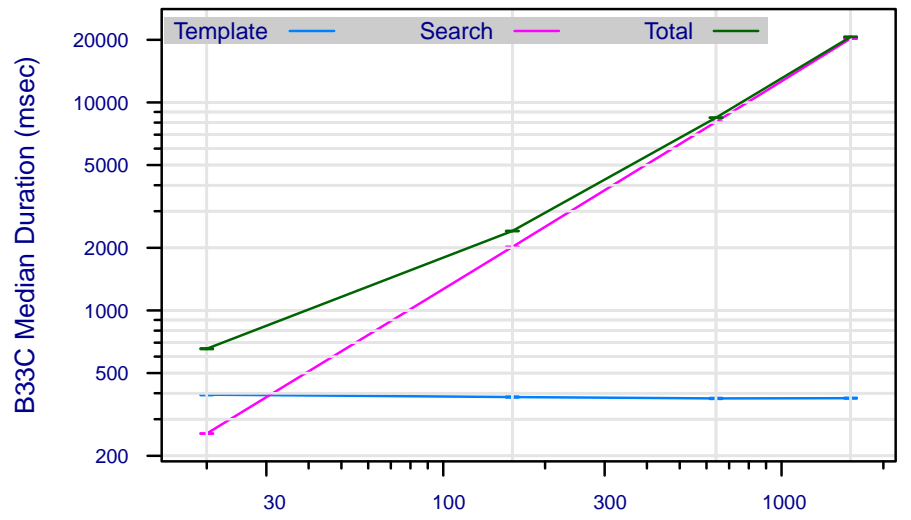

Num. enrolled identities, $\mathrm{N}$ (thousands)

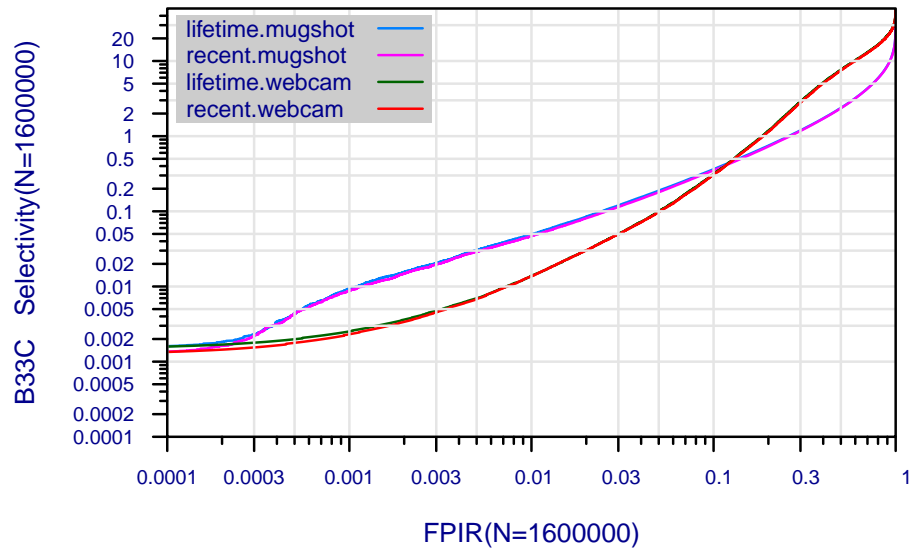

Figure 25: Collected performance reports for algorithm B33C. The figures are described at the beginning of this Appendix.

\begin{tabular}{|c|c|c|c|c|c|c|}
\hline $\begin{array}{l}\mathrm{A}=3 \mathrm{M} / \text { Cogent } \\
\mathrm{G}=\text { Hisign } \\
\mathrm{P}=\text { Zhuhai-Yisheng }\end{array}$ & $\begin{array}{l}\mathrm{B}=\text { Cognitec } \\
\mathrm{H}=\text { CAS-IA } \\
\mathrm{Q}=\text { JunYu }\end{array}$ & $\begin{array}{l}\mathrm{C}=\text { Neurotechnology } \\
\mathrm{I}=\mathrm{CAS}-\mathrm{ICT} \\
\mathrm{S}=\text { Decatur }\end{array}$ & $\begin{array}{l}\mathrm{D}=\text { Safran Morpho } \\
\mathrm{J}=\text { Toshiba } \\
\mathrm{T}=\text { Ayonix }\end{array}$ & $\begin{array}{l}\mathrm{E}=\mathrm{NEC} \\
\mathrm{L}=\text { Tsinghua U. II }\end{array}$ & $\begin{array}{l}\mathrm{F}=\text { Tsinghua } \mathrm{U} . \\
\mathrm{M}=\mathrm{HP}\end{array}$ & $\begin{array}{l}\text { FNIR(N,R,T,L) “Miss rate" } \\
\text { FPIR(N,T,L) “False alarm rate" }\end{array}$ \\
\hline
\end{tabular}



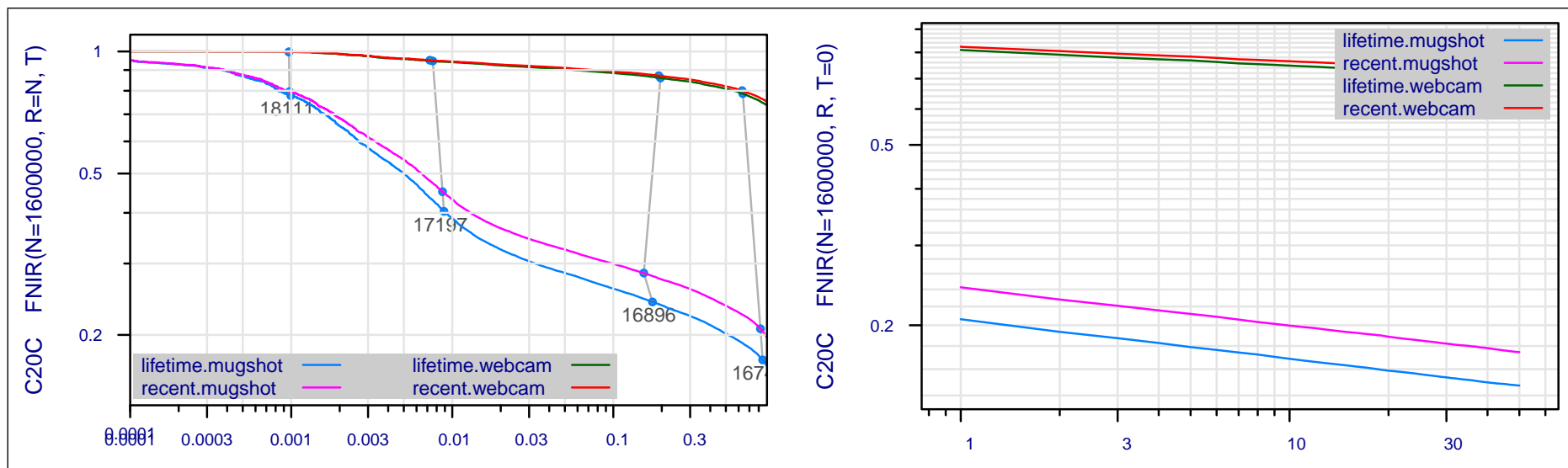

$\operatorname{FPIR}(\mathrm{N}=1600000)$
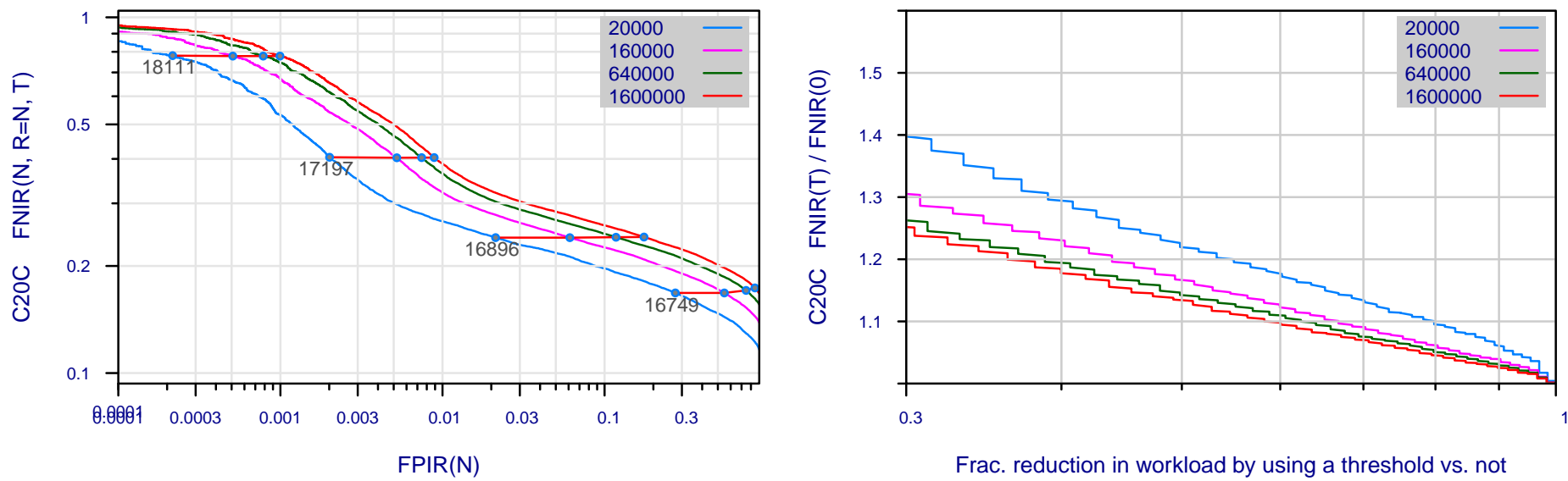

0.3

Frac. reduction in workload by using a threshold vs. not
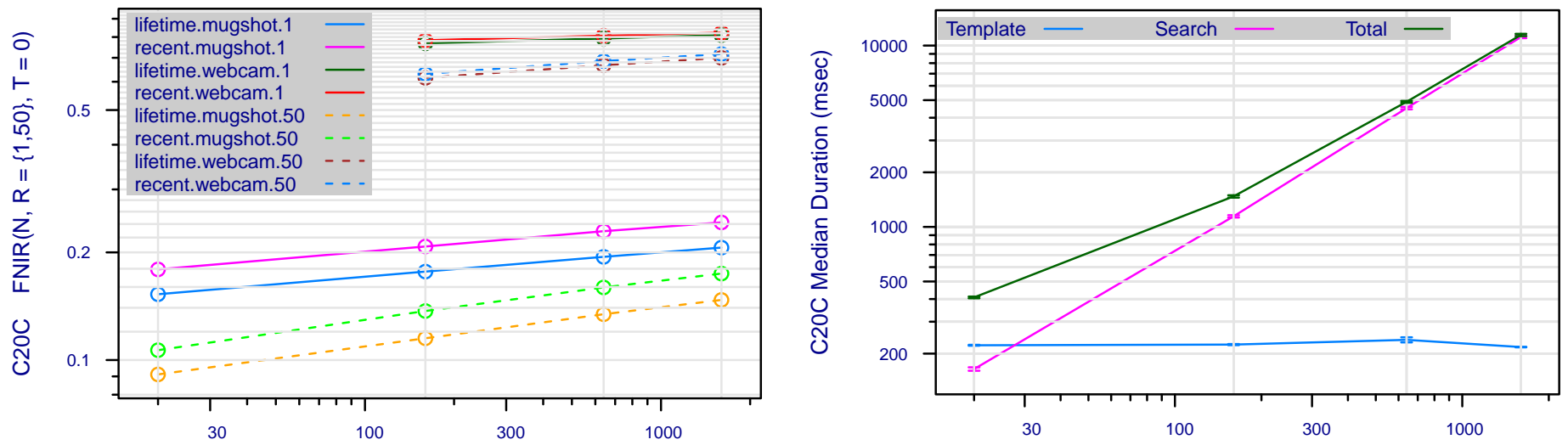

Num. enrolled identities, $\mathrm{N}$ (thousands)

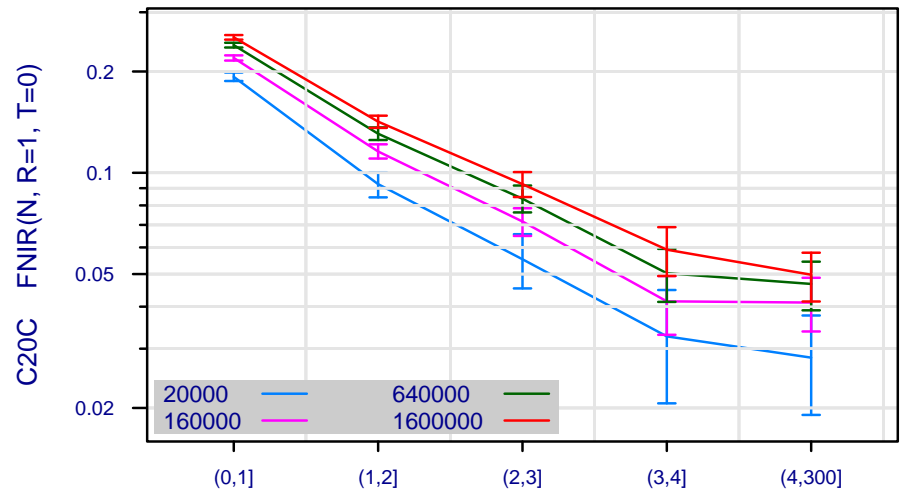

Num. enrolled images per identity

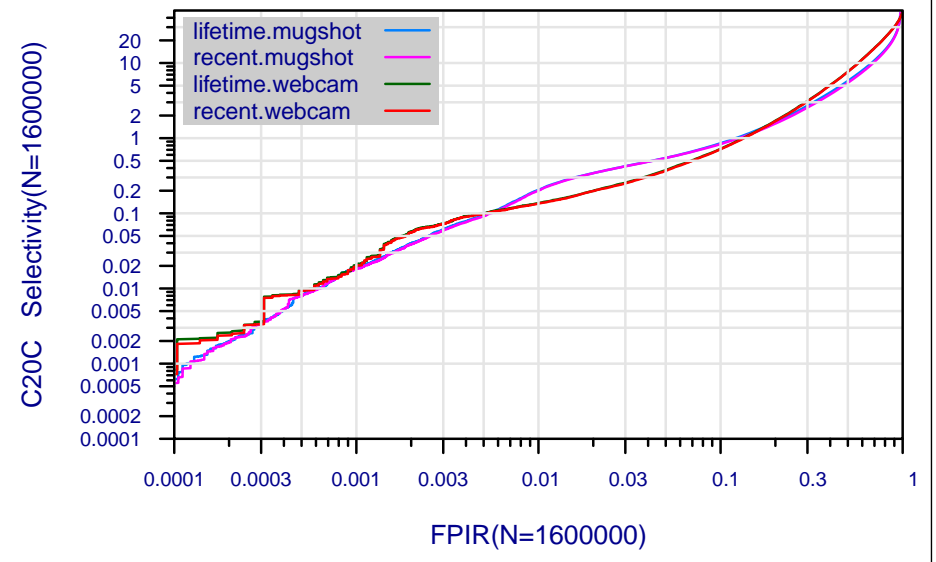

Figure 26: Collected performance reports for algorithm C20C. The figures are described at the beginning of this Appendix.

\begin{tabular}{|c|c|c|c|c|c|c|}
\hline $\begin{array}{l}\mathrm{A}=3 \mathrm{M} / \text { Cogent } \\
\mathrm{G}=\text { Hisign } \\
\mathrm{P}=\text { Zhuhai-Yisheng }\end{array}$ & $\begin{array}{l}B=\text { Cognitec } \\
H=\text { CAS-IA } \\
Q=\text { JunYu }\end{array}$ & $\begin{array}{l}\mathrm{C}=\text { Neurotechnology } \\
\mathrm{I}=\text { CAS-ICT } \\
\mathrm{S}=\text { Decatur }\end{array}$ & $\begin{array}{l}\mathrm{D}=\text { Safran Morpho } \\
\mathrm{J}=\text { Toshiba } \\
\mathrm{T}=\text { Ayonix }\end{array}$ & $\begin{array}{l}\mathrm{E}=\mathrm{NEC} \\
\mathrm{L}=\text { Tsinghua U. II }\end{array}$ & $\begin{array}{l}\mathrm{F}=\text { Tsinghua } \mathrm{U} \\
\mathrm{M}=\mathrm{HP}\end{array}$ & $\begin{array}{l}\text { FNIR(N,R,T,L) “Miss rate" } \\
\operatorname{FPIR}(\mathrm{N}, \mathrm{T}, \mathrm{L}) \text { “False alarm rate" }\end{array}$ \\
\hline
\end{tabular}




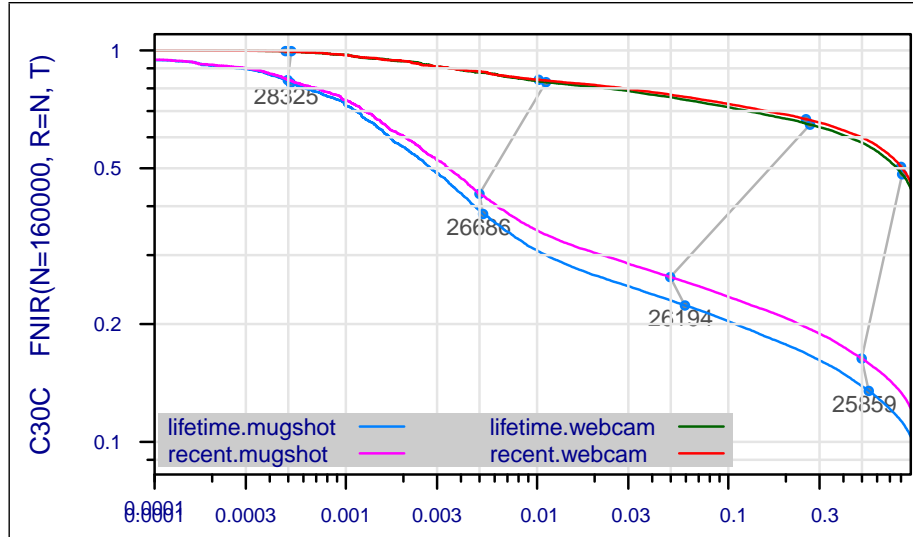

$\operatorname{FPIR}(\mathrm{N}=160000)$
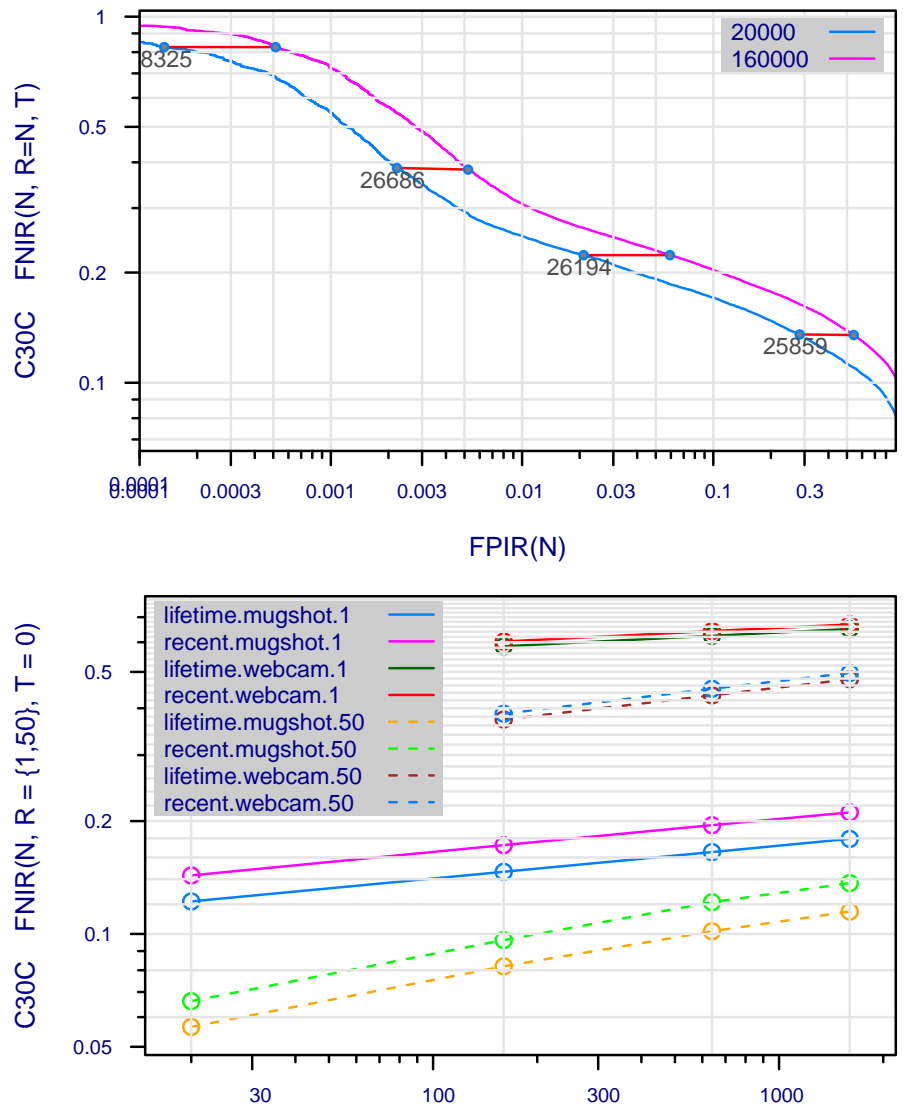

Num. enrolled identities, $\mathrm{N}$ (thousands)

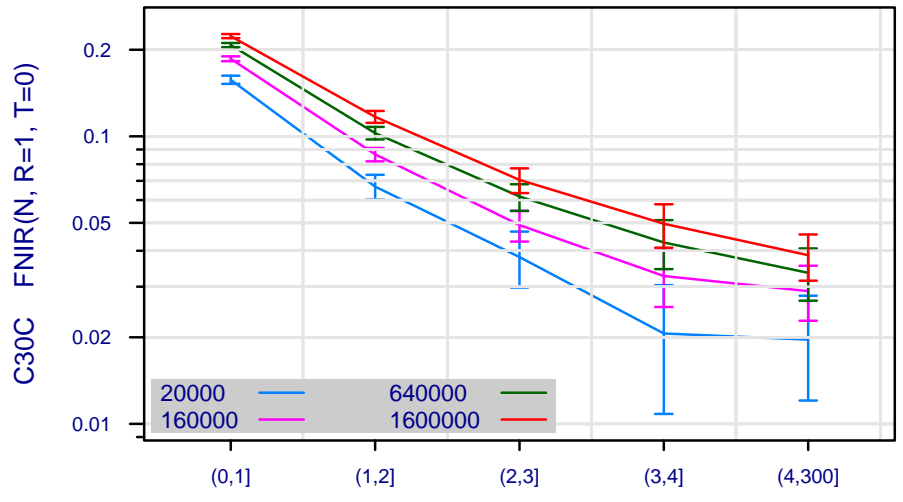

Num. enrolled images per identity

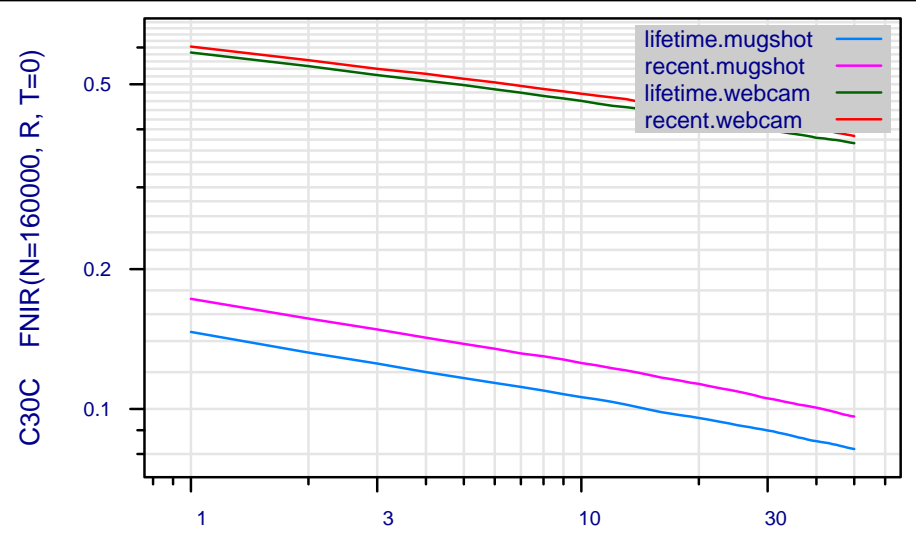

Rank, $\mathrm{R}$

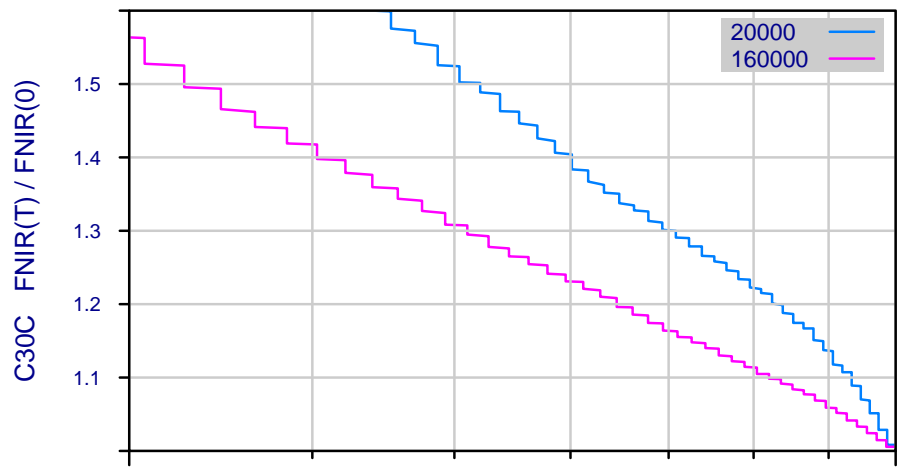

Frac. reduction in workload by using a threshold vs. not

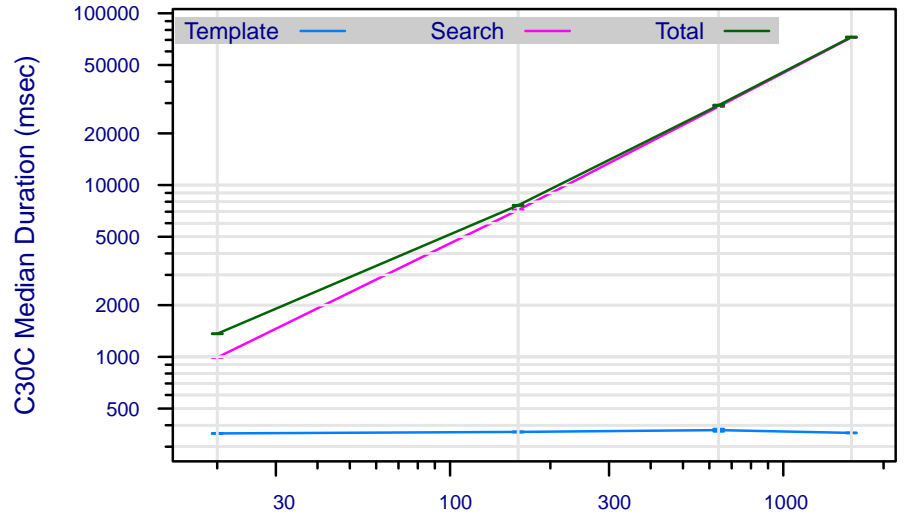

Num. enrolled identities, $\mathrm{N}$ (thousands)

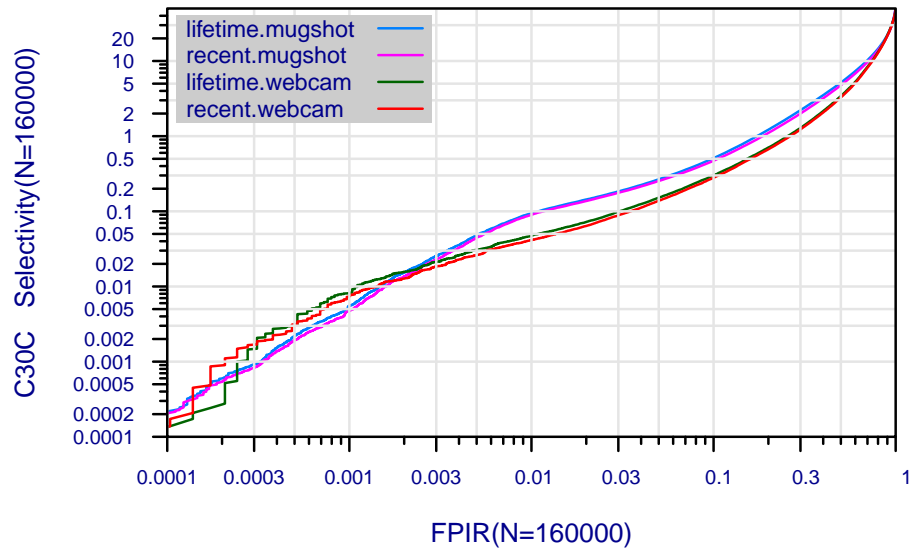

Figure 27: Collected performance reports for algorithm C30C. The figures are described at the beginning of this Appendix.

\begin{tabular}{|c|c|c|c|c|c|c|}
\hline $\begin{array}{l}A=3 \mathrm{M} / \text { Cogent } \\
G=\text { Hisign } \\
P=\text { Zhuhai-Yisheng }\end{array}$ & $\begin{array}{l}\mathrm{B}=\text { Cognitec } \\
\mathrm{H}=\text { CAS-IA } \\
\mathrm{Q}=\text { JunYu }\end{array}$ & $\begin{array}{l}\mathrm{C}=\text { Neurotechnology } \\
\mathrm{I}=\text { CAS-ICT } \\
\mathrm{S}=\text { Decatur }\end{array}$ & $\begin{array}{l}\mathrm{D}=\text { Safran Morpho } \\
\mathrm{J}=\text { Toshiba } \\
\mathrm{T}=\text { Ayonix }\end{array}$ & $\begin{array}{l}\mathrm{E}=\mathrm{NEC} \\
\mathrm{L}=\text { Tsinghua U. II }\end{array}$ & $\begin{array}{l}\mathrm{F}=\text { Tsinghua } \mathrm{U} \\
\mathrm{M}=\mathrm{HP}\end{array}$ & $\begin{array}{l}\text { FNIR(N,R,T,L) "Miss rate" } \\
\text { FPIR(N,T,L) “False alarm rate" }\end{array}$ \\
\hline
\end{tabular}



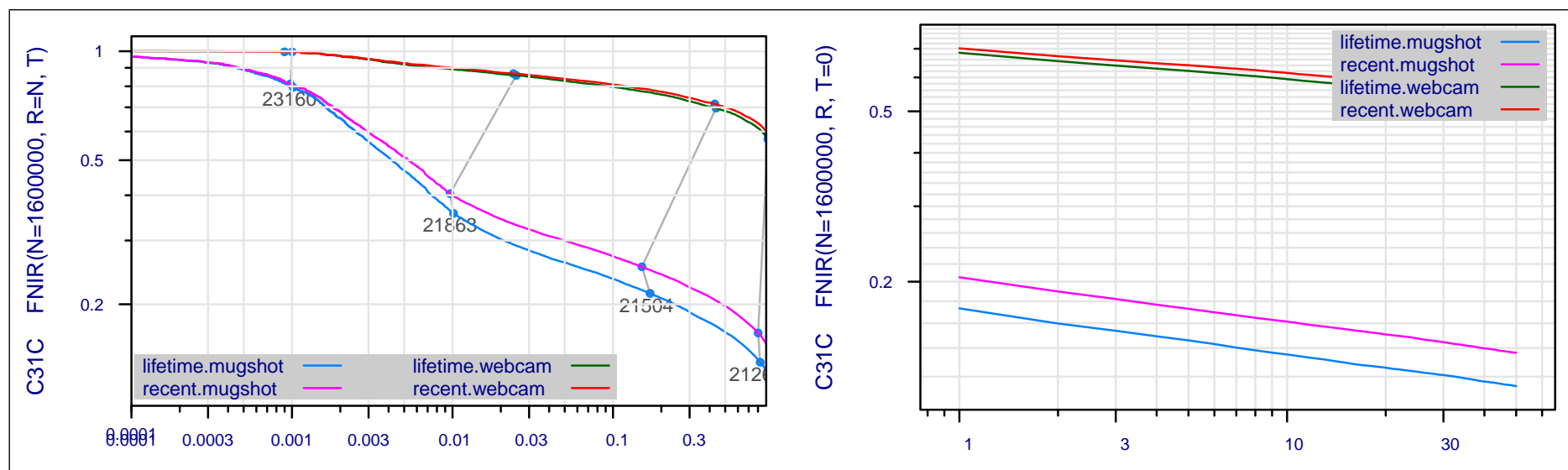

$\operatorname{FPIR}(\mathrm{N}=1600000)$
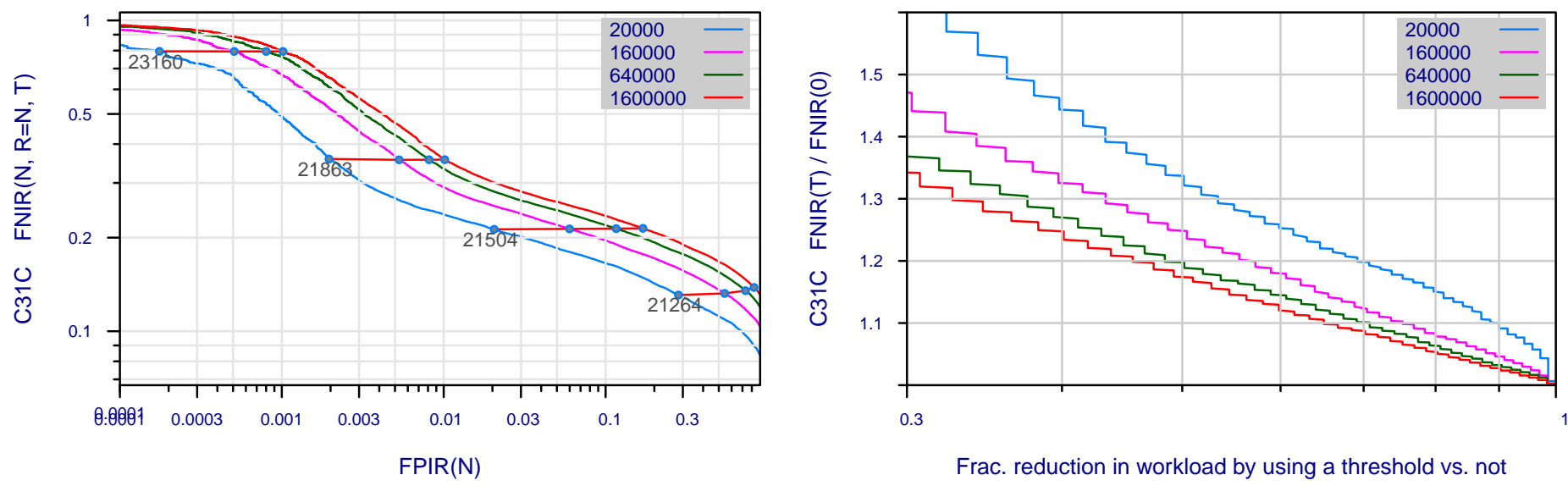

Frac. reduction in workload by using a threshold vs. not
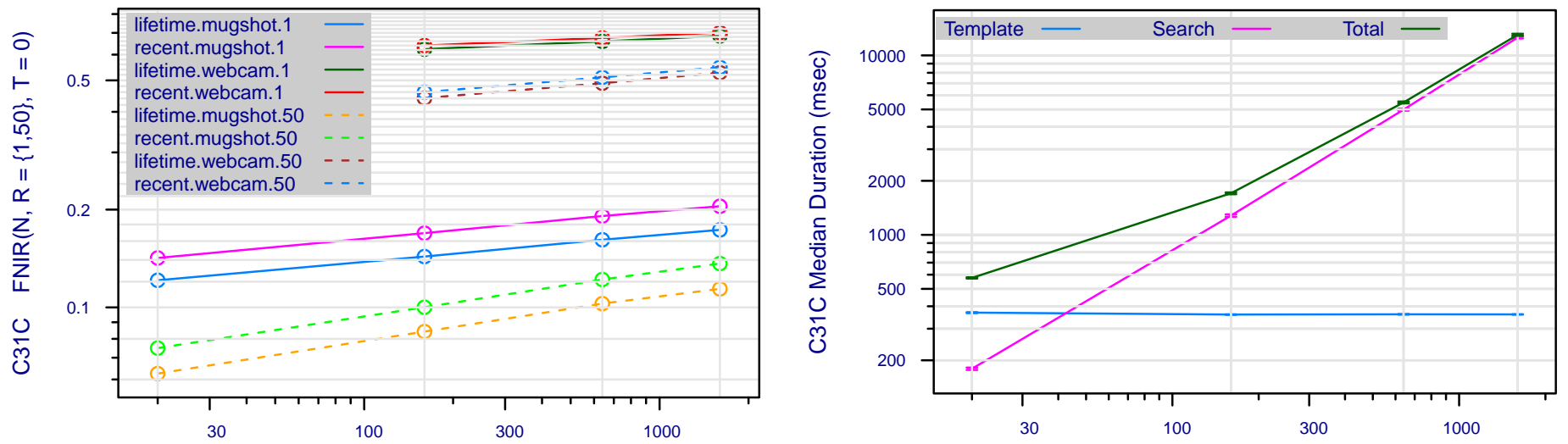

Num. enrolled identities, $\mathrm{N}$ (thousands)

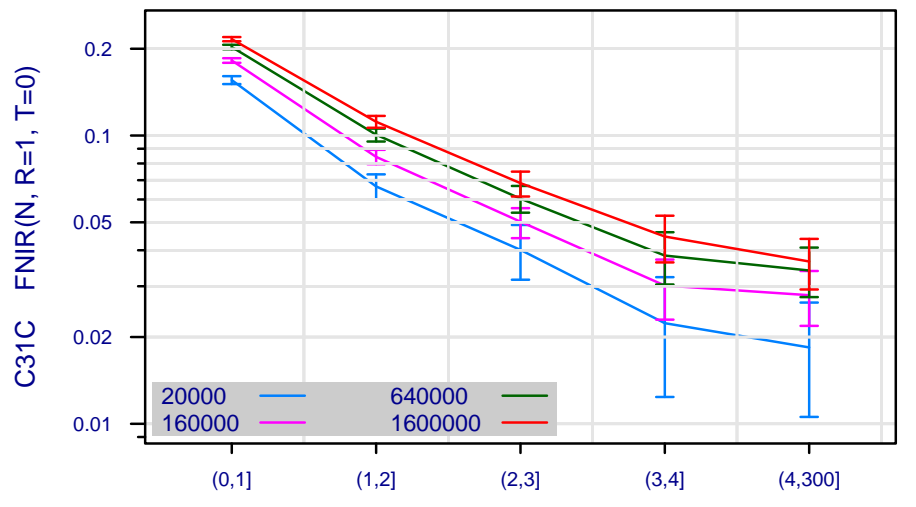

Num. enrolled images per identity

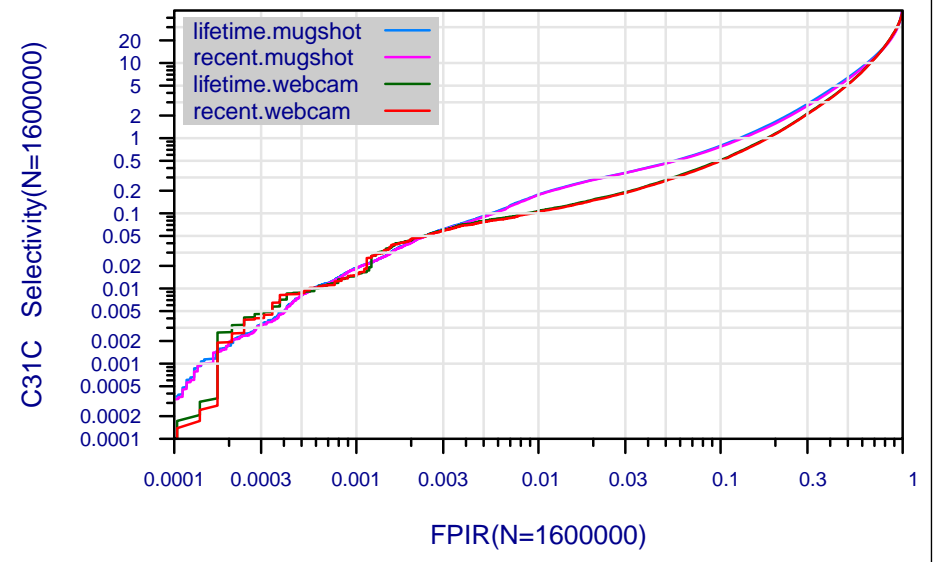

Figure 28: Collected performance reports for algorithm C31C. The figures are described at the beginning of this Appendix.

\begin{tabular}{|c|c|c|c|c|c|c|}
\hline $\begin{array}{l}\mathrm{A}=3 \mathrm{M} / \text { Cogent } \\
\mathrm{G}=\text { Hisign } \\
\mathrm{P}=\text { Zhuhai-Yisheng }\end{array}$ & $\begin{array}{l}B=\text { Cognitec } \\
H=\text { CAS-IA } \\
Q=\text { JunYu }\end{array}$ & $\begin{array}{l}\mathrm{C}=\text { Neurotechnology } \\
\mathrm{I}=\text { CAS-ICT } \\
\mathrm{S}=\text { Decatur }\end{array}$ & $\begin{array}{l}\mathrm{D}=\text { Safran Morpho } \\
\mathrm{J}=\text { Toshiba } \\
\mathrm{T}=\text { Ayonix }\end{array}$ & $\begin{array}{l}\mathrm{E}=\mathrm{NEC} \\
\mathrm{L}=\text { Tsinghua U. II }\end{array}$ & $\begin{array}{l}\mathrm{F}=\text { Tsinghua } \mathrm{U} \\
\mathrm{M}=\mathrm{HP}\end{array}$ & $\begin{array}{l}\text { FNIR(N,R,T,L) “Miss rate" } \\
\operatorname{FPIR}(\mathrm{N}, \mathrm{T}, \mathrm{L}) \text { “False alarm rate" }\end{array}$ \\
\hline
\end{tabular}



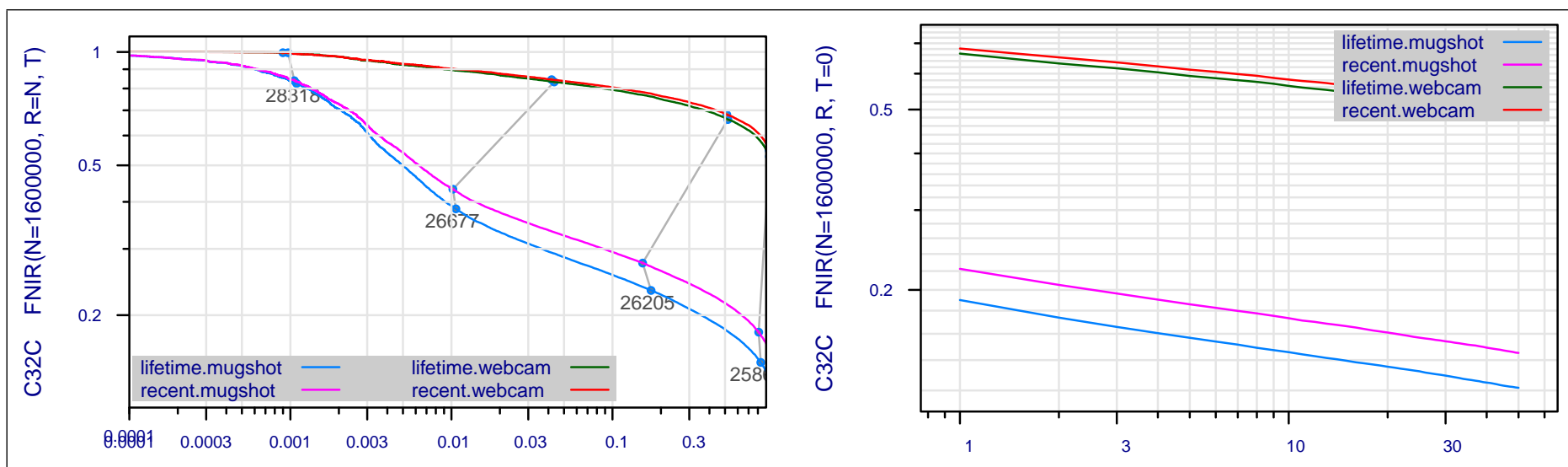

$\operatorname{FPIR}(\mathrm{N}=1600000)$
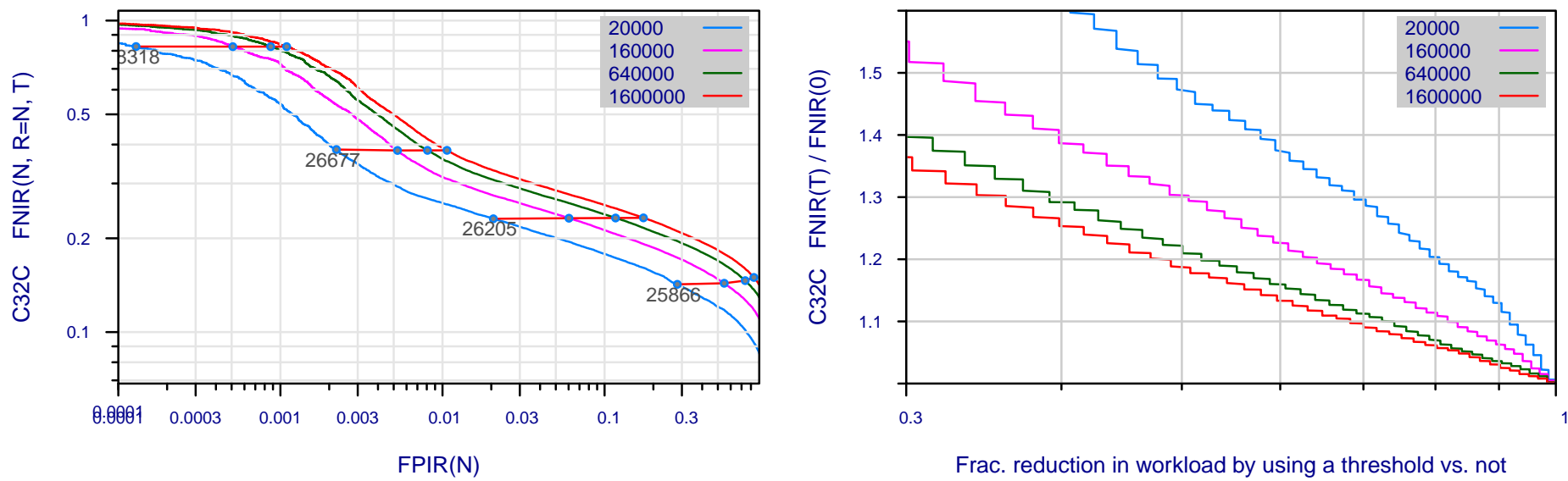

0.3

Frac. reduction in workload by using a threshold vs. not
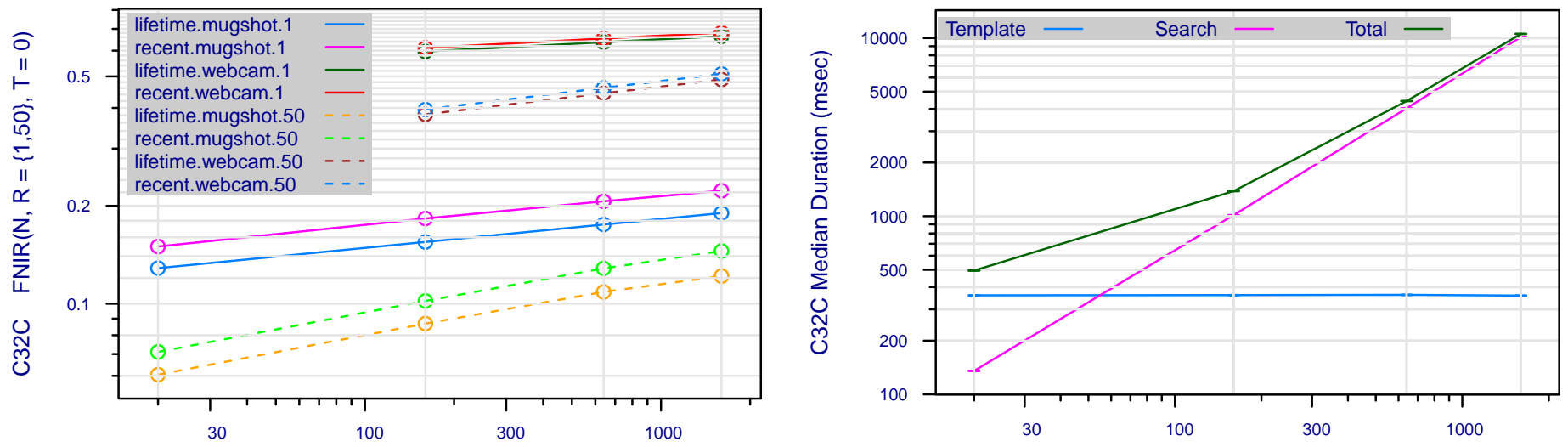

Num. enrolled identities, $\mathrm{N}$ (thousands)

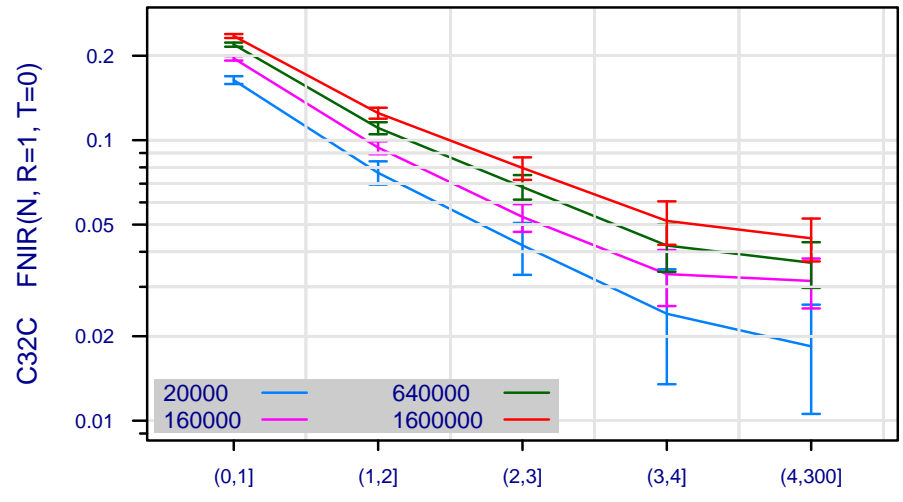

Num. enrolled images per identity

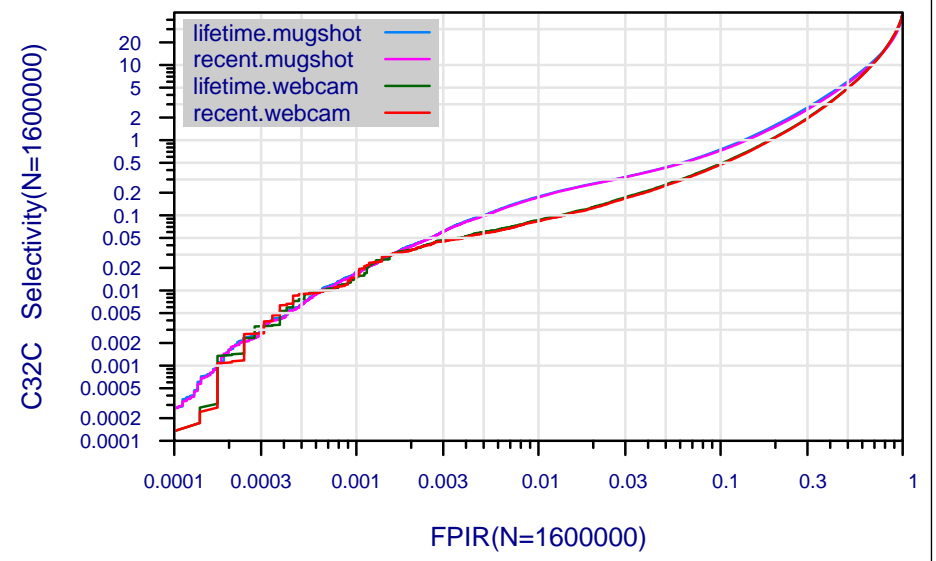

Figure 29: Collected performance reports for algorithm C32C. The figures are described at the beginning of this Appendix.

\begin{tabular}{|c|c|c|c|c|c|c|}
\hline $\begin{array}{l}\mathrm{A}=3 \mathrm{M} / \text { Cogent } \\
\mathrm{G}=\text { Hisign } \\
\mathrm{P}=\text { Zhuhai-Yisheng }\end{array}$ & $\begin{array}{l}B=\text { Cognitec } \\
H=\text { CAS-IA } \\
Q=\text { JunYu }\end{array}$ & $\begin{array}{l}\mathrm{C}=\text { Neurotechnology } \\
\mathrm{I}=\text { CAS-ICT } \\
\mathrm{S}=\text { Decatur }\end{array}$ & $\begin{array}{l}\mathrm{D}=\text { Safran Morpho } \\
\mathrm{J}=\text { Toshiba } \\
\mathrm{T}=\text { Ayonix }\end{array}$ & $\begin{array}{l}\mathrm{E}=\mathrm{NEC} \\
\mathrm{L}=\text { Tsinghua U. II }\end{array}$ & $\begin{array}{l}\mathrm{F}=\text { Tsinghua } \mathrm{U} \\
\mathrm{M}=\mathrm{HP}\end{array}$ & $\begin{array}{l}\text { FNIR(N,R,T,L) “Miss rate" } \\
\operatorname{FPIR}(\mathrm{N}, \mathrm{T}, \mathrm{L}) \text { “False alarm rate" }\end{array}$ \\
\hline
\end{tabular}



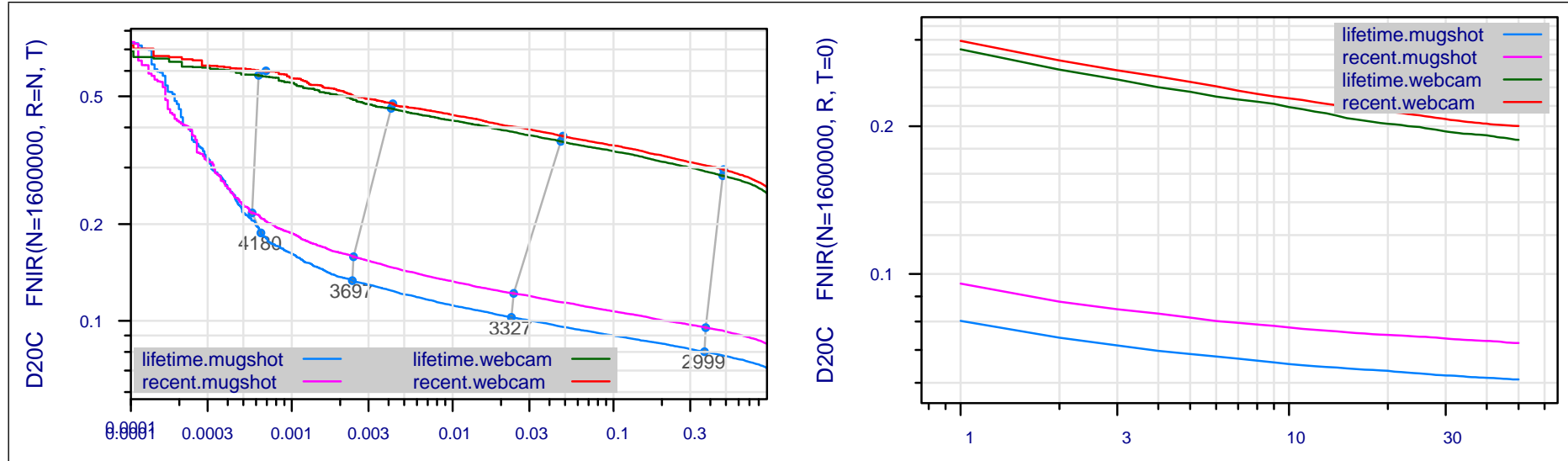

$\operatorname{FPIR}(\mathrm{N}=1600000)$
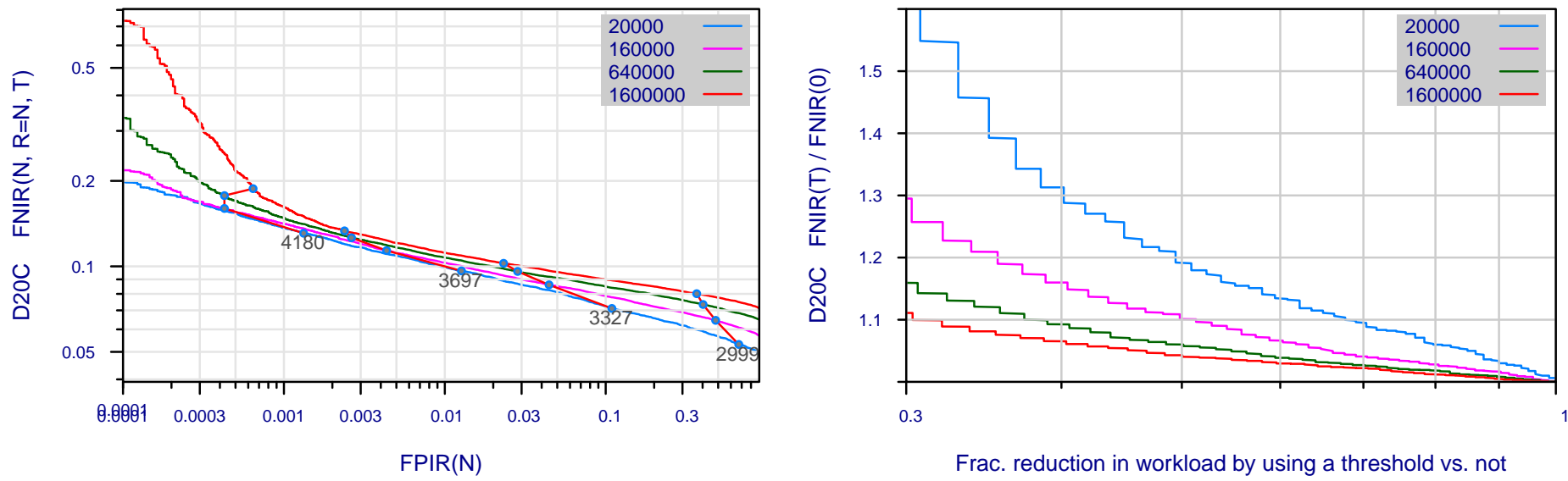

Frac. reduction in workload by using a threshold vs. not
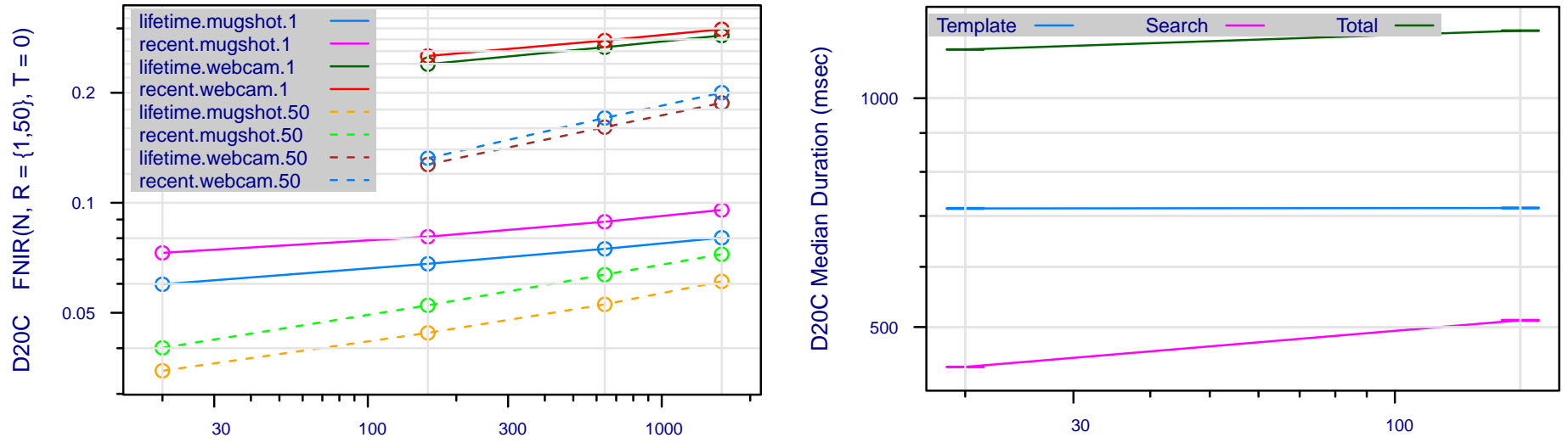

Num. enrolled identities, $\mathrm{N}$ (thousands)

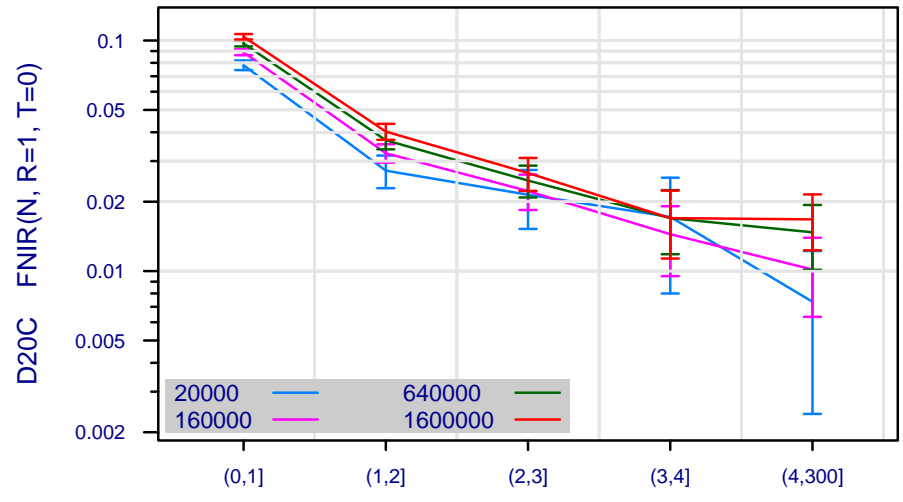

Num. enrolled images per identity

Num. enrolled identities, $\mathrm{N}$ (thousands)

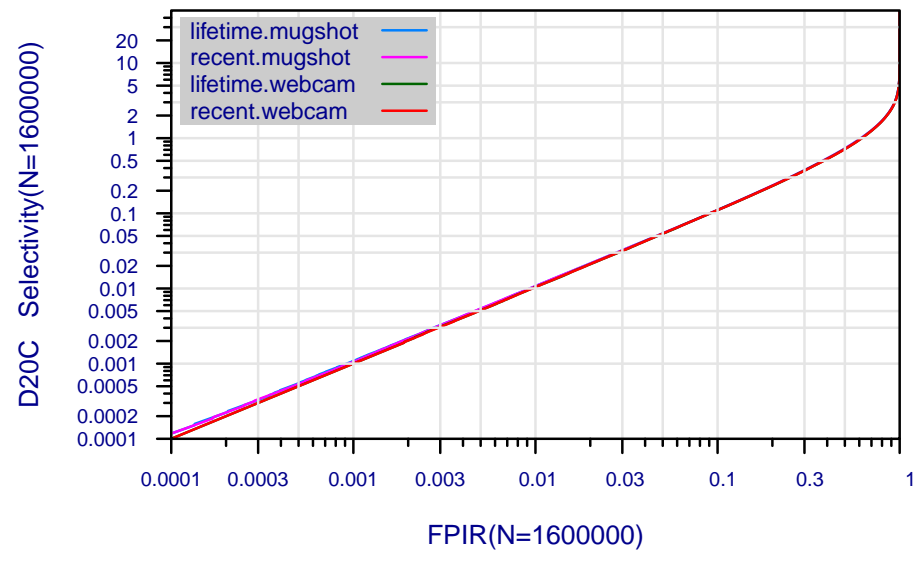

Figure 30: Collected performance reports for algorithm D20C. The figures are described at the beginning of this Appendix.

\begin{tabular}{|c|c|c|c|c|c|c|}
\hline $\begin{array}{l}\mathrm{A}=3 \mathrm{M} / \text { Cogent } \\
\mathrm{G}=\text { Hisign } \\
\mathrm{P}=\text { Zhuhai-Yisheng }\end{array}$ & $\begin{array}{l}B=\text { Cognitec } \\
H=\text { CAS-IA } \\
Q=\text { JunYu }\end{array}$ & $\begin{array}{l}\mathrm{C}=\text { Neurotechnology } \\
\mathrm{I}=\text { CAS-ICT } \\
\mathrm{S}=\text { Decatur }\end{array}$ & $\begin{array}{l}\mathrm{D}=\text { Safran Morpho } \\
\mathrm{J}=\text { Toshiba } \\
\mathrm{T}=\text { Ayonix }\end{array}$ & $\begin{array}{l}\mathrm{E}=\mathrm{NEC} \\
\mathrm{L}=\text { Tsinghua U. II }\end{array}$ & $\begin{array}{l}\mathrm{F}=\text { Tsinghua } \mathrm{U} \\
\mathrm{M}=\mathrm{HP}\end{array}$ & $\begin{array}{l}\text { FNIR(N,R,T,L) “Miss rate" } \\
\operatorname{FPIR}(\mathrm{N}, \mathrm{T}, \mathrm{L}) \text { “False alarm rate" }\end{array}$ \\
\hline
\end{tabular}




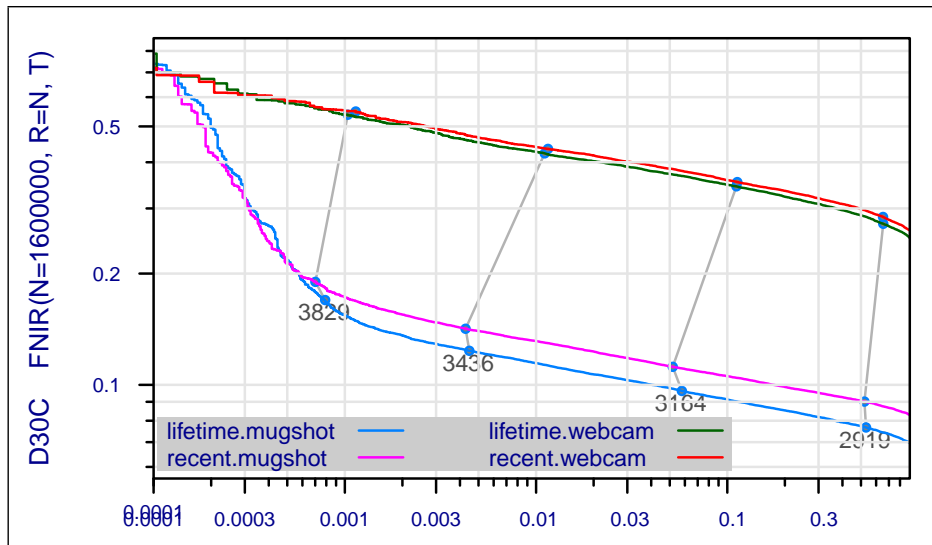

$\operatorname{FPIR}(\mathrm{N}=1600000)$
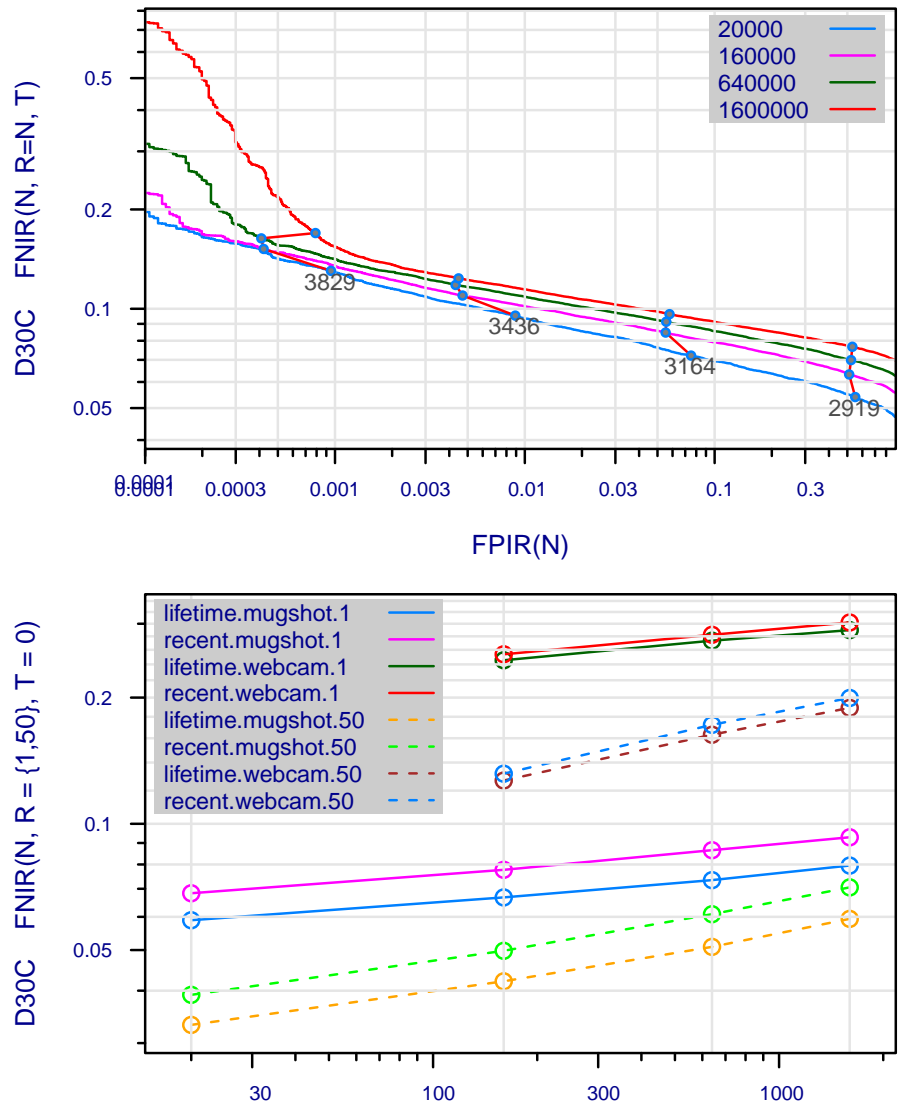

Num. enrolled identities, $\mathrm{N}$ (thousands)

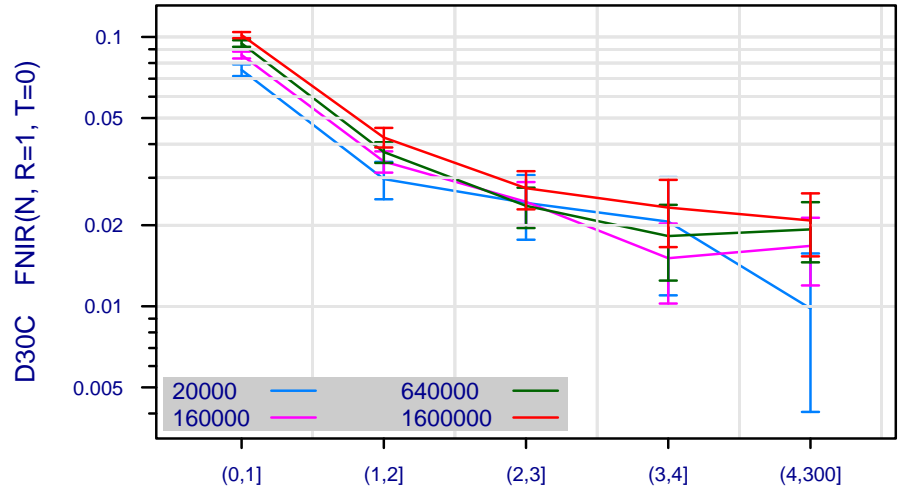

Num. enrolled images per identity

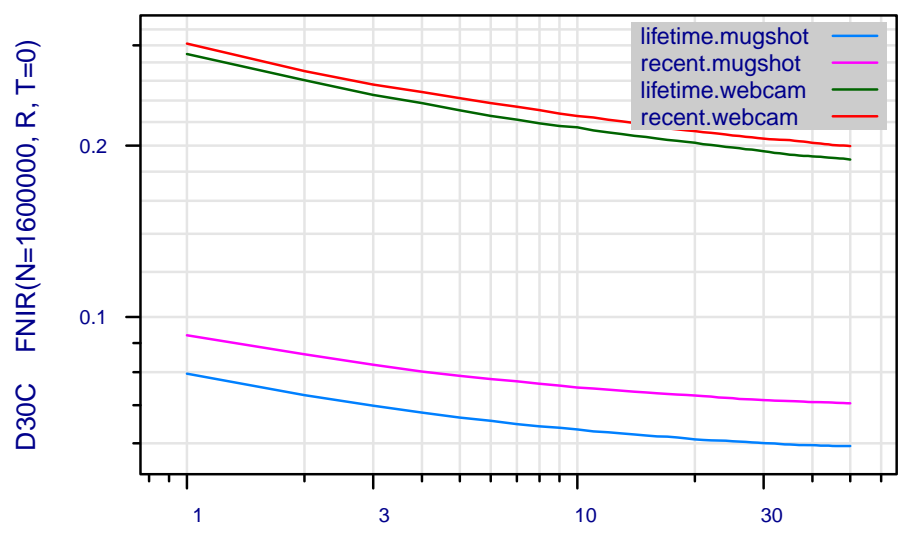

Rank, $\mathrm{R}$

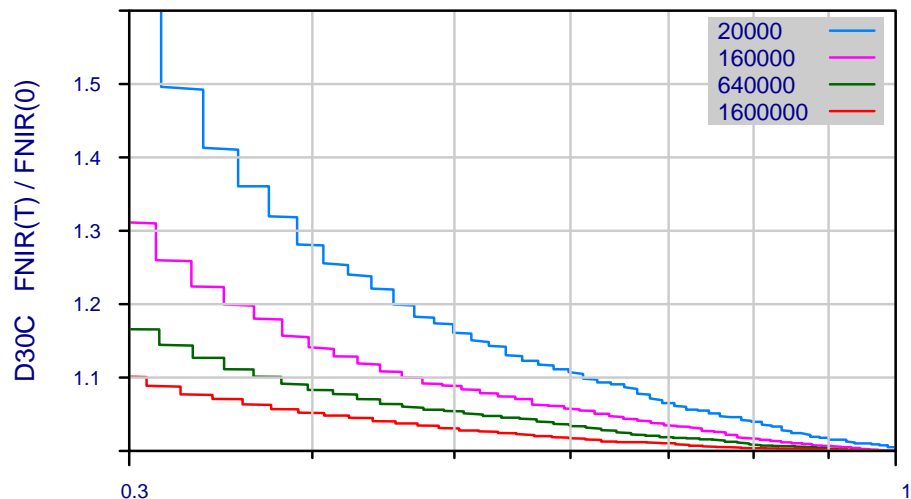

Frac. reduction in workload by using a threshold vs. not

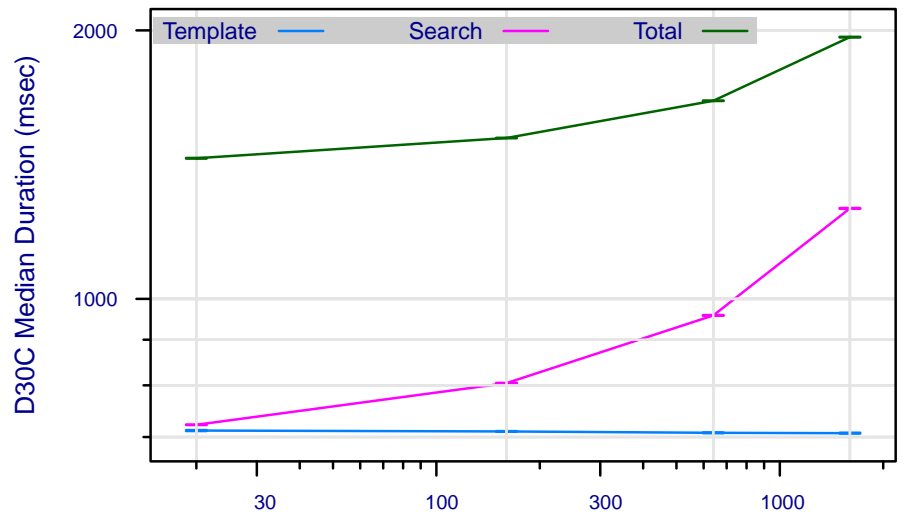

Num. enrolled identities, $\mathrm{N}$ (thousands)

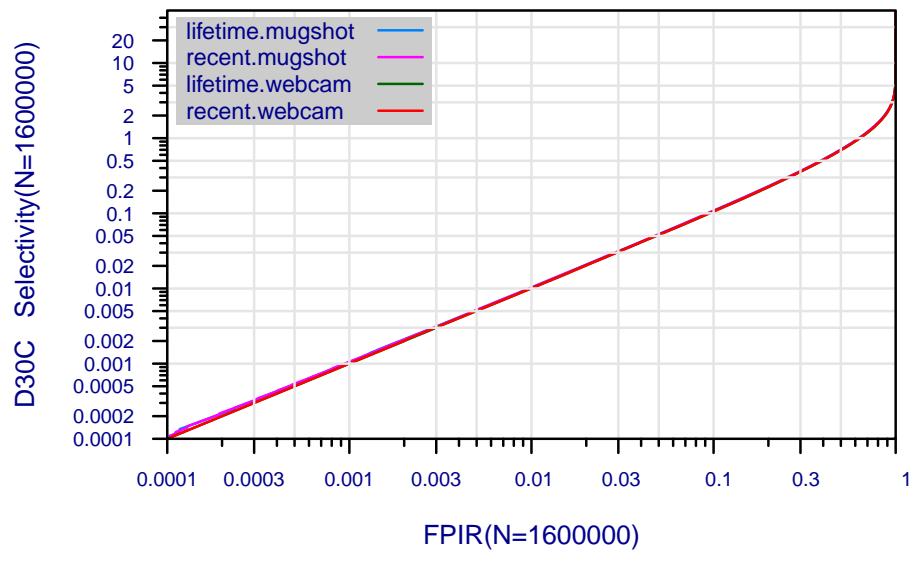

Figure 31: Collected performance reports for algorithm D30C. The figures are described at the beginning of this Appendix.

\begin{tabular}{|c|c|c|c|c|c|c|}
\hline $\begin{array}{l}\mathrm{A}=3 \mathrm{M} / \text { Cogent } \\
\mathrm{G}=\text { Hisign } \\
\mathrm{P}=\text { Zhuhai-Yisheng }\end{array}$ & $\begin{array}{l}\mathrm{B}=\text { Cognitec } \\
\mathrm{H}=\text { CAS-IA } \\
\mathrm{Q}=\text { JunYu }\end{array}$ & $\begin{array}{l}\mathrm{C}=\text { Neurotechnology } \\
\mathrm{I}=\mathrm{CAS}-\mathrm{ICT} \\
\mathrm{S}=\text { Decatur }\end{array}$ & $\begin{array}{l}\mathrm{D}=\text { Safran Morpho } \\
\mathrm{J}=\text { Toshiba } \\
\mathrm{T}=\text { Ayonix }\end{array}$ & $\begin{array}{l}\mathrm{E}=\mathrm{NEC} \\
\mathrm{L}=\text { Tsinghua U. II }\end{array}$ & $\begin{array}{l}\mathrm{F}=\text { Tsinghua } \mathrm{U} . \\
\mathrm{M}=\mathrm{HP}\end{array}$ & $\begin{array}{l}\text { FNIR(N,R,T,L) “Miss rate" } \\
\text { FPIR(N,T,L) “False alarm rate" }\end{array}$ \\
\hline
\end{tabular}



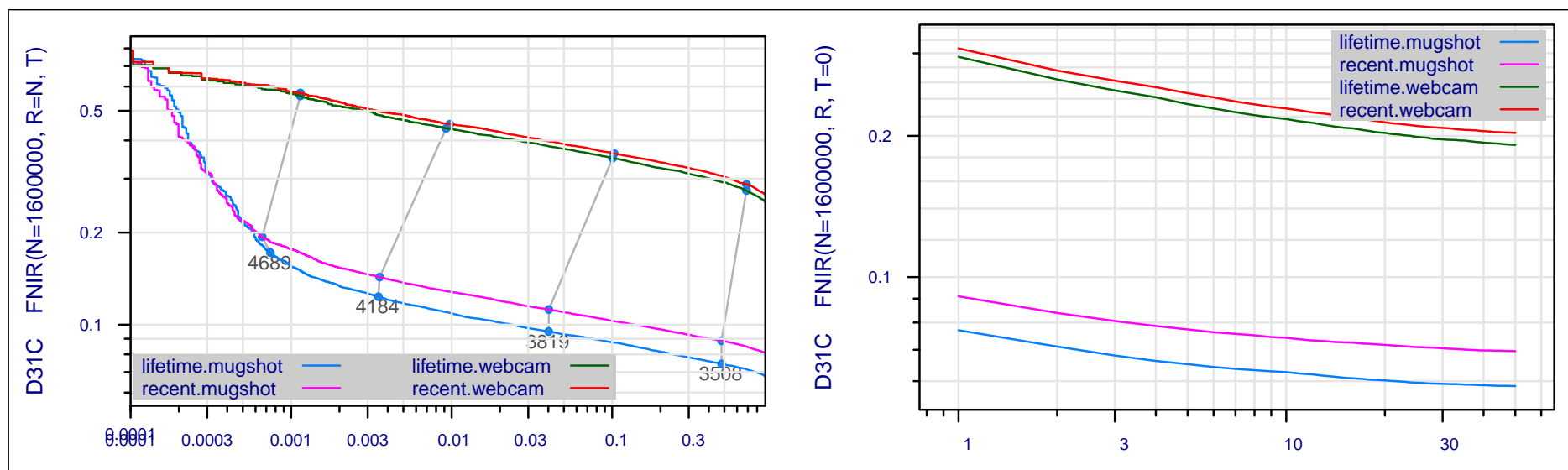

$\operatorname{FPIR}(\mathrm{N}=1600000)$
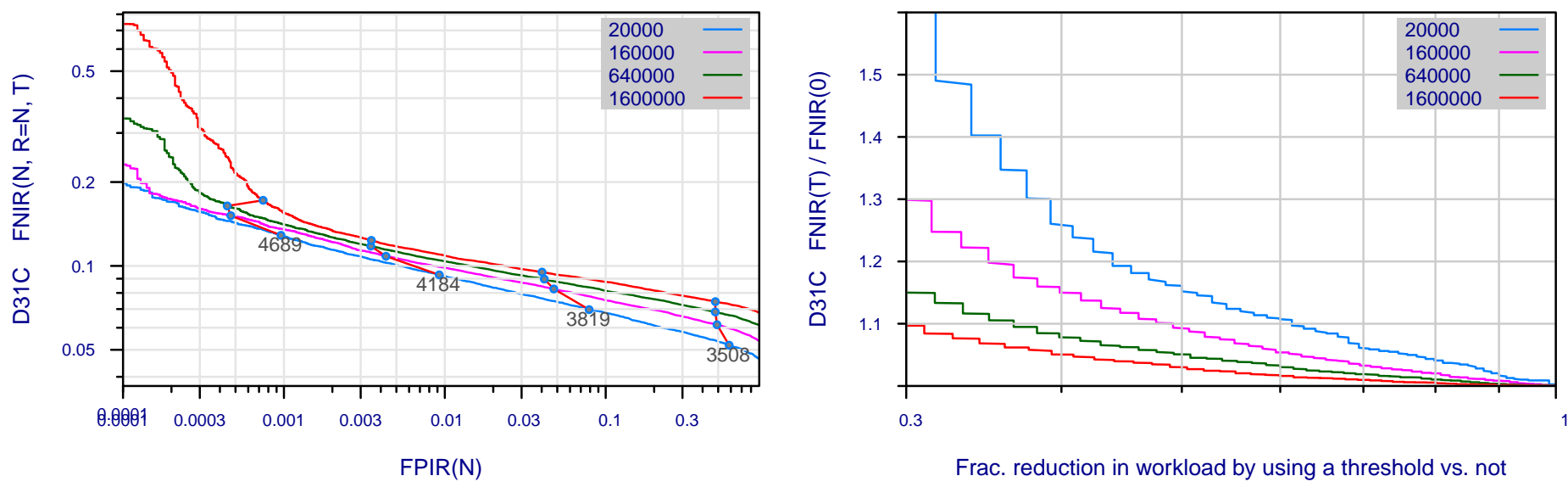

Frac. reduction in workload by using a threshold vs. not
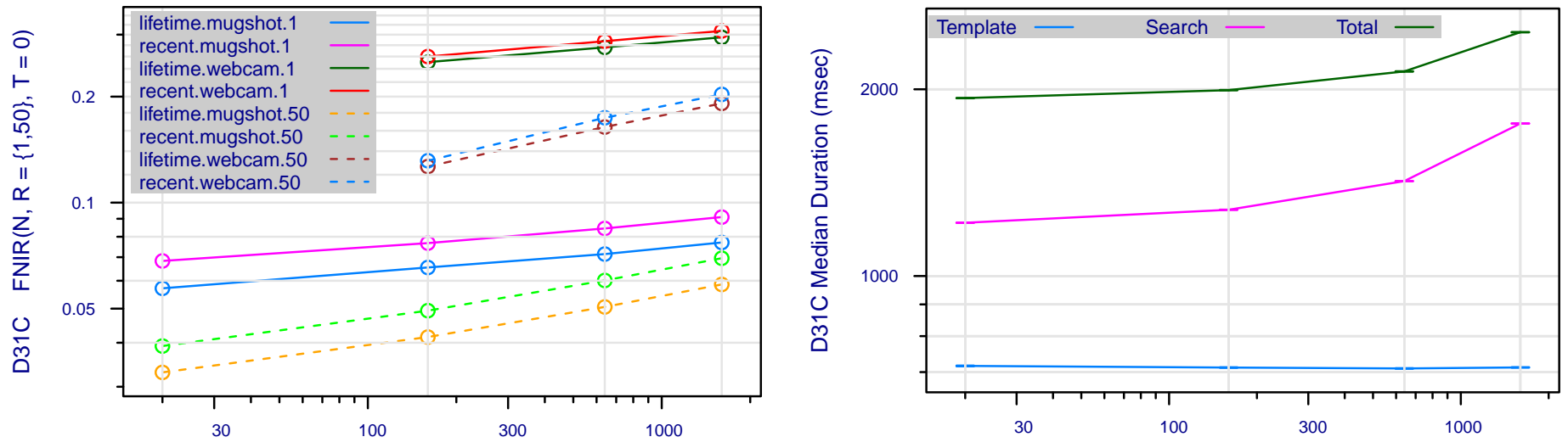

Num. enrolled identities, $\mathrm{N}$ (thousands)

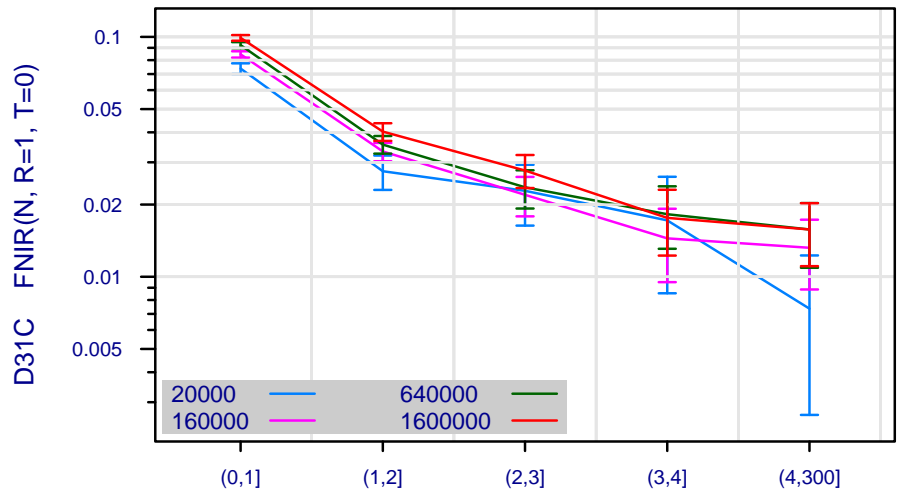

Num. enrolled images per identity

Num. enrolled identities, $\mathrm{N}$ (thousands)

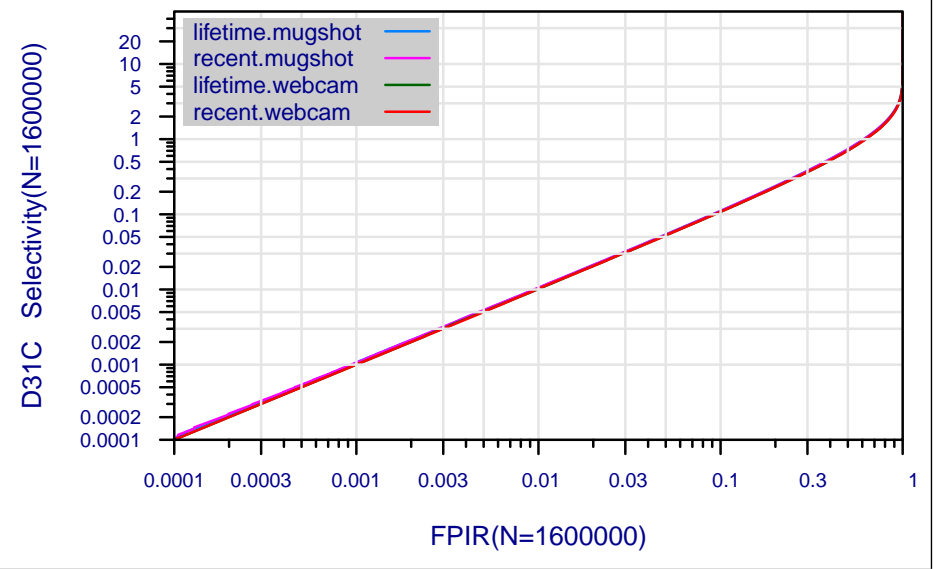

Figure 32: Collected performance reports for algorithm D31C. The figures are described at the beginning of this Appendix.

\begin{tabular}{|c|c|c|c|c|c|c|}
\hline $\begin{array}{l}\mathrm{A}=3 \mathrm{M} / \text { Cogent } \\
\mathrm{G}=\text { Hisign } \\
\mathrm{P}=\text { Zhuhai-Yisheng }\end{array}$ & $\begin{array}{l}B=\text { Cognitec } \\
H=\text { CAS-IA } \\
Q=\text { JunYu }\end{array}$ & $\begin{array}{l}\mathrm{C}=\text { Neurotechnology } \\
\mathrm{I}=\text { CAS-ICT } \\
\mathrm{S}=\text { Decatur }\end{array}$ & $\begin{array}{l}\mathrm{D}=\text { Safran Morpho } \\
\mathrm{J}=\text { Toshiba } \\
\mathrm{T}=\text { Ayonix }\end{array}$ & $\begin{array}{l}\mathrm{E}=\mathrm{NEC} \\
\mathrm{L}=\text { Tsinghua U. II }\end{array}$ & $\begin{array}{l}\mathrm{F}=\text { Tsinghua } \mathrm{U} \\
\mathrm{M}=\mathrm{HP}\end{array}$ & $\begin{array}{l}\text { FNIR(N,R,T,L) “Miss rate" } \\
\operatorname{FPIR}(\mathrm{N}, \mathrm{T}, \mathrm{L}) \text { “False alarm rate" }\end{array}$ \\
\hline
\end{tabular}



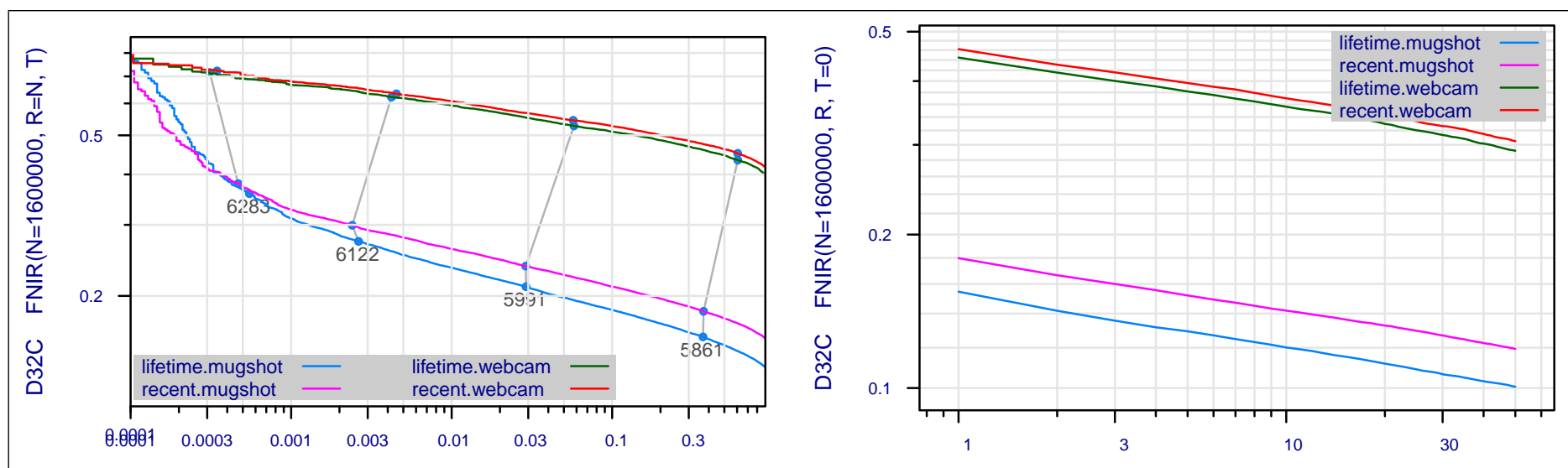

$\operatorname{FPIR}(\mathrm{N}=1600000)$
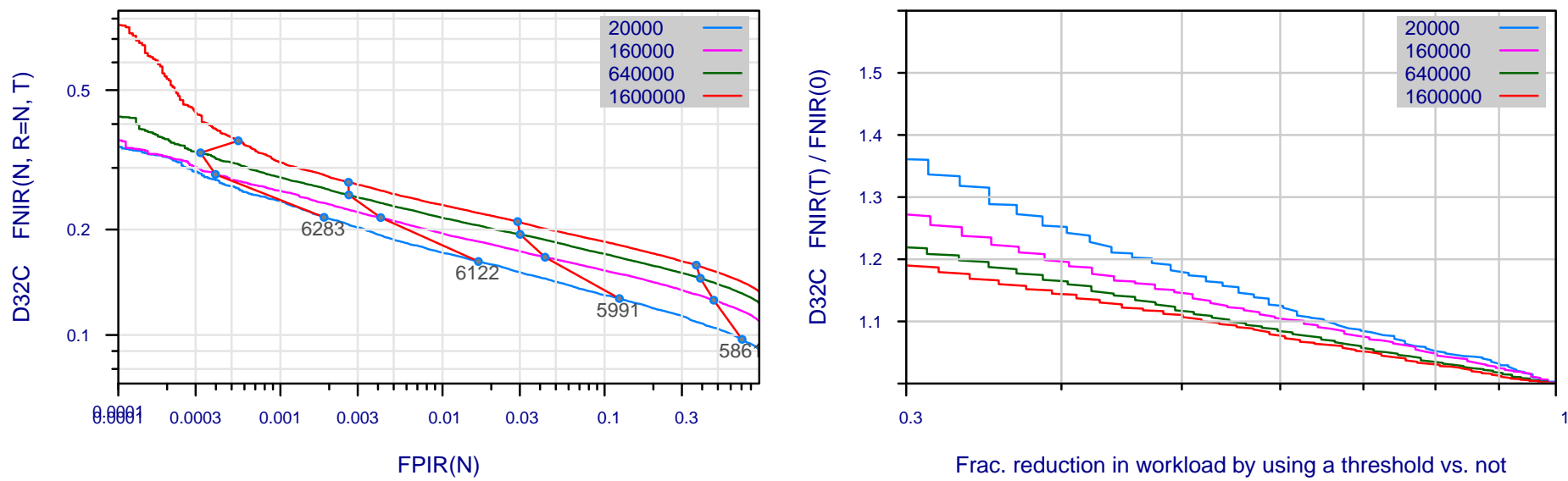

0.3

Frac. reduction in workload by using a threshold vs. not
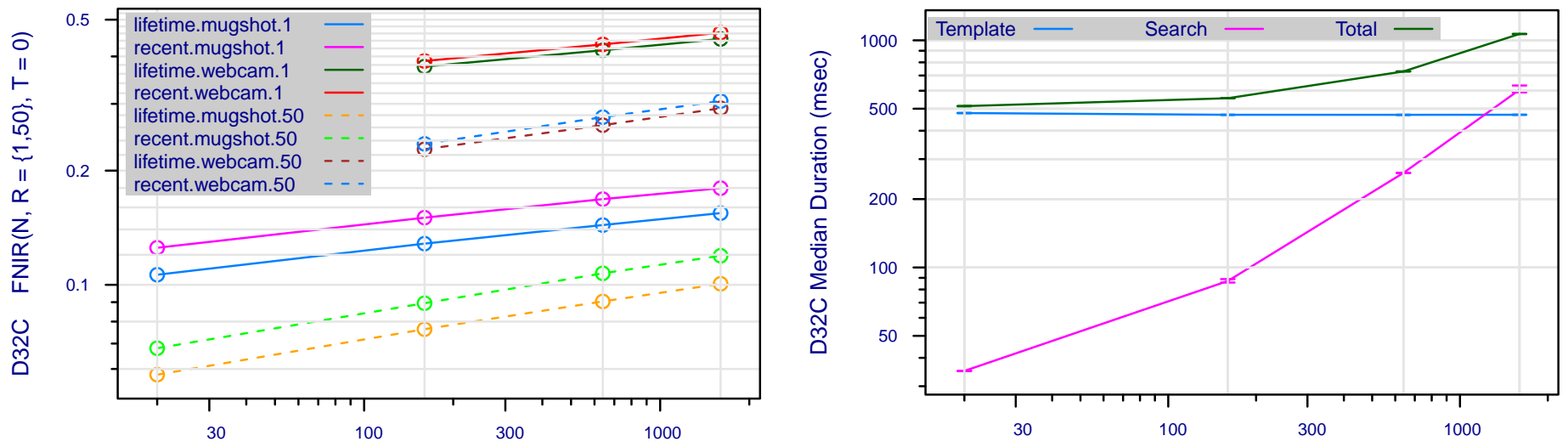

Num. enrolled identities, $\mathrm{N}$ (thousands)

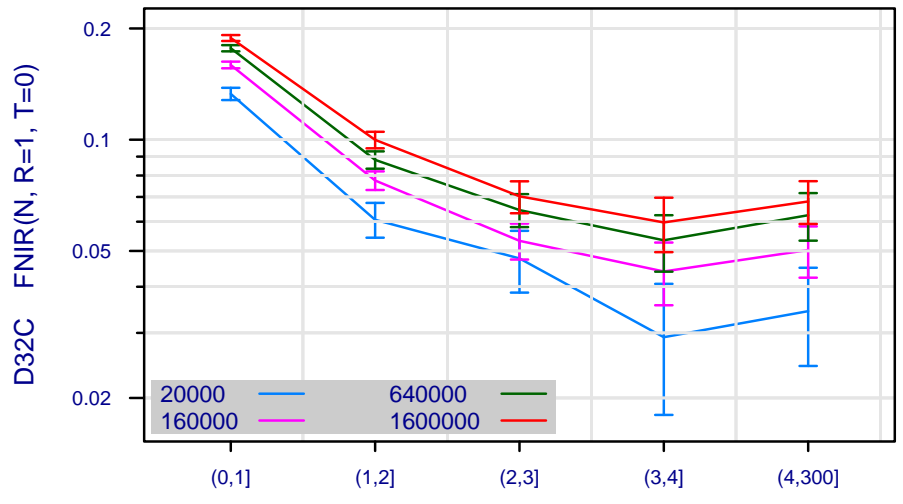

Num. enrolled images per identity

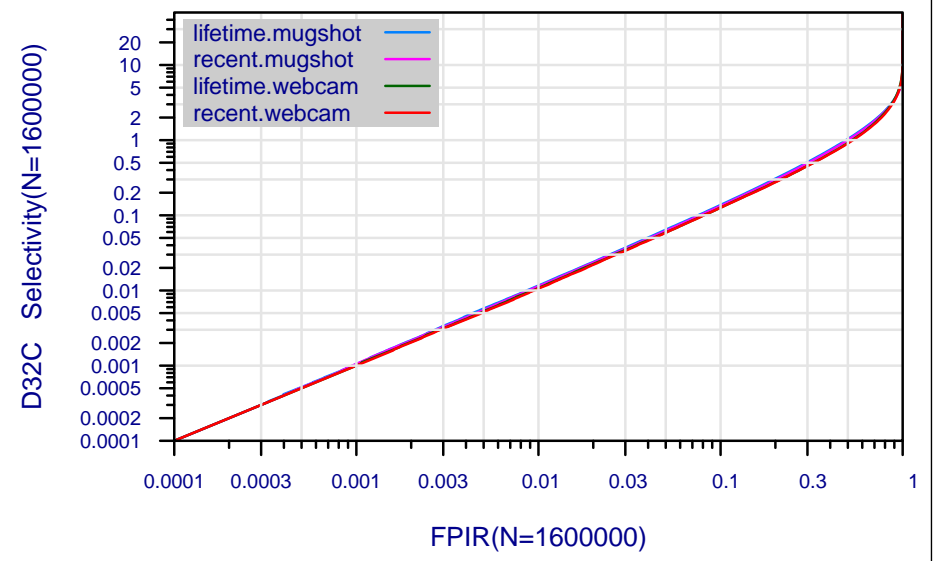

Figure 33: Collected performance reports for algorithm D32C. The figures are described at the beginning of this Appendix.

\begin{tabular}{|c|c|c|c|c|c|c|}
\hline $\begin{array}{l}\mathrm{A}=3 \mathrm{M} / \text { Cogent } \\
\mathrm{G}=\text { Hisign } \\
\mathrm{P}=\text { Zhuhai-Yisheng }\end{array}$ & $\begin{array}{l}B=\text { Cognitec } \\
H=\text { CAS-IA } \\
Q=\text { JunYu }\end{array}$ & $\begin{array}{l}\mathrm{C}=\text { Neurotechnology } \\
\mathrm{I}=\text { CAS-ICT } \\
\mathrm{S}=\text { Decatur }\end{array}$ & $\begin{array}{l}\mathrm{D}=\text { Safran Morpho } \\
\mathrm{J}=\text { Toshiba } \\
\mathrm{T}=\text { Ayonix }\end{array}$ & $\begin{array}{l}\mathrm{E}=\mathrm{NEC} \\
\mathrm{L}=\text { Tsinghua U. II }\end{array}$ & $\begin{array}{l}\mathrm{F}=\text { Tsinghua } \mathrm{U} \\
\mathrm{M}=\mathrm{HP}\end{array}$ & $\begin{array}{l}\text { FNIR(N,R,T,L) “Miss rate" } \\
\operatorname{FPIR}(\mathrm{N}, \mathrm{T}, \mathrm{L}) \text { “False alarm rate" }\end{array}$ \\
\hline
\end{tabular}




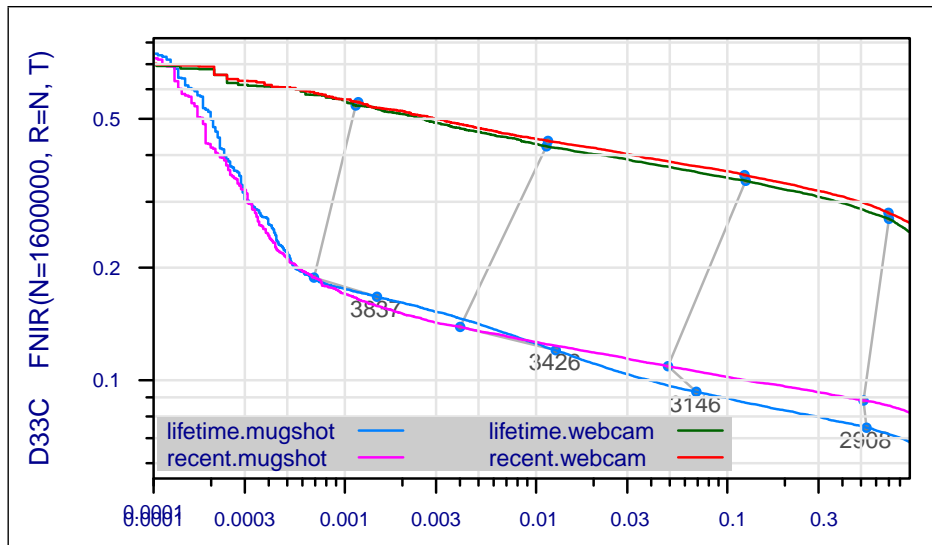

$\operatorname{FPIR}(\mathrm{N}=1600000)$
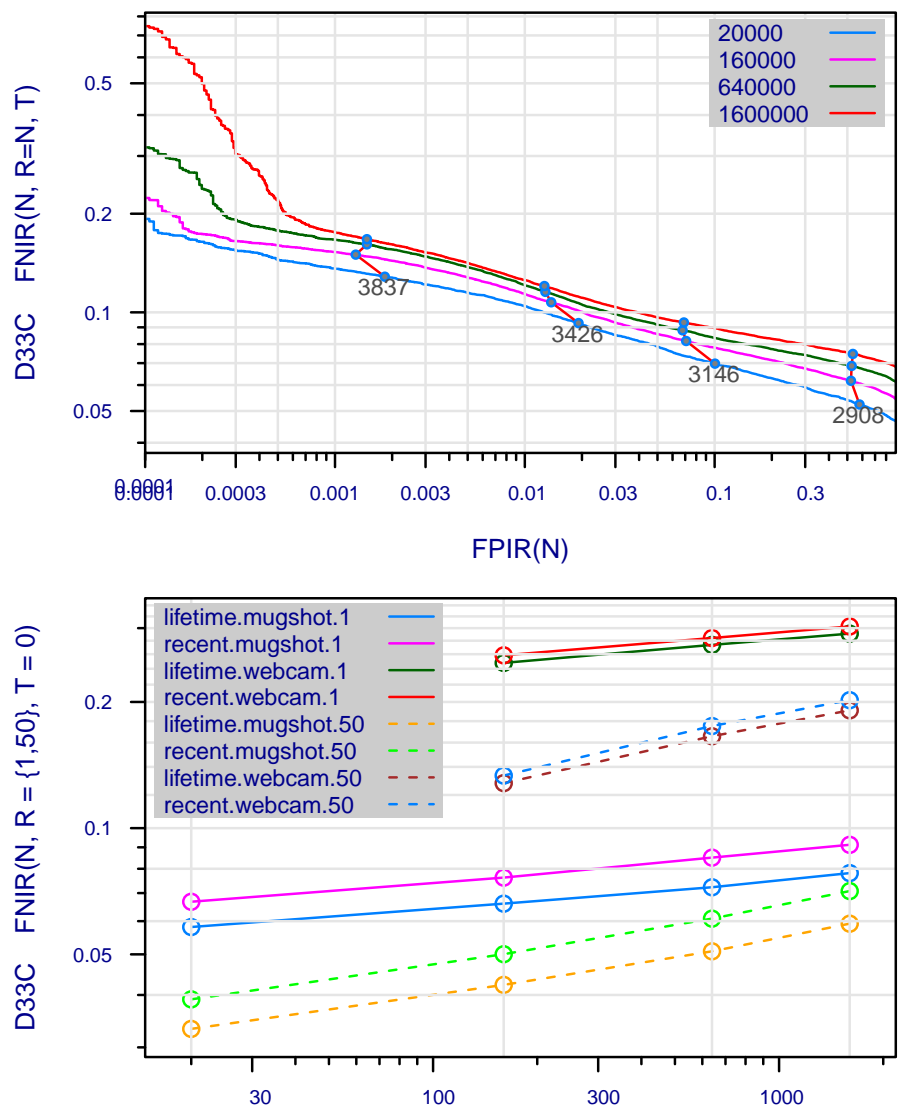

Num. enrolled identities, $\mathrm{N}$ (thousands)

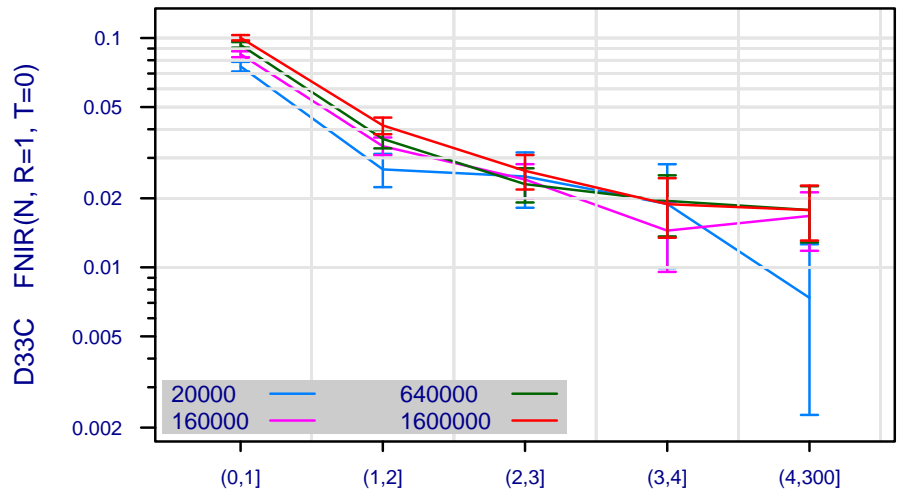

Num. enrolled images per identity

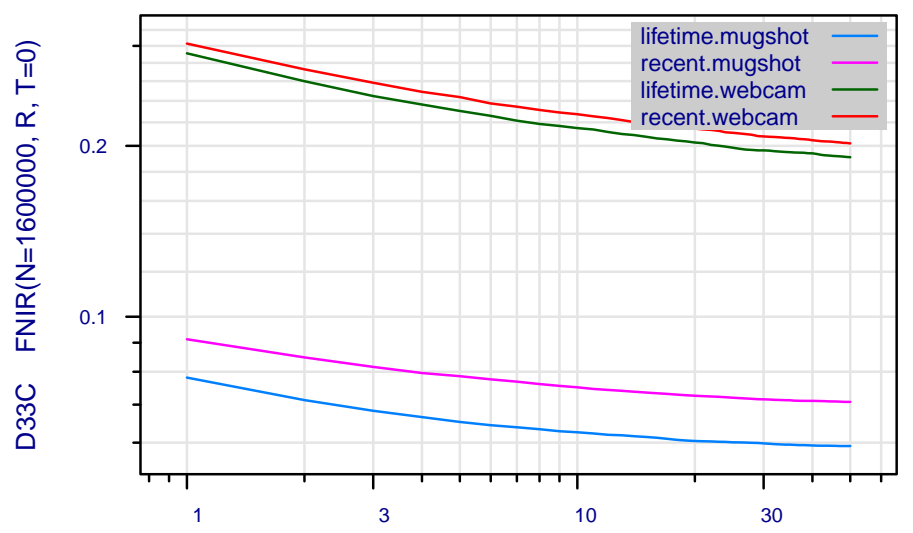

Rank, $\mathrm{R}$

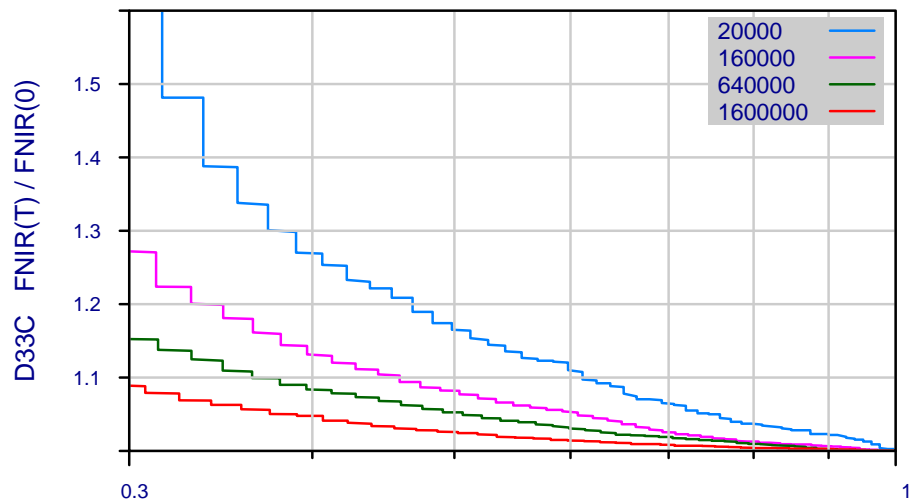

Frac. reduction in workload by using a threshold vs. not

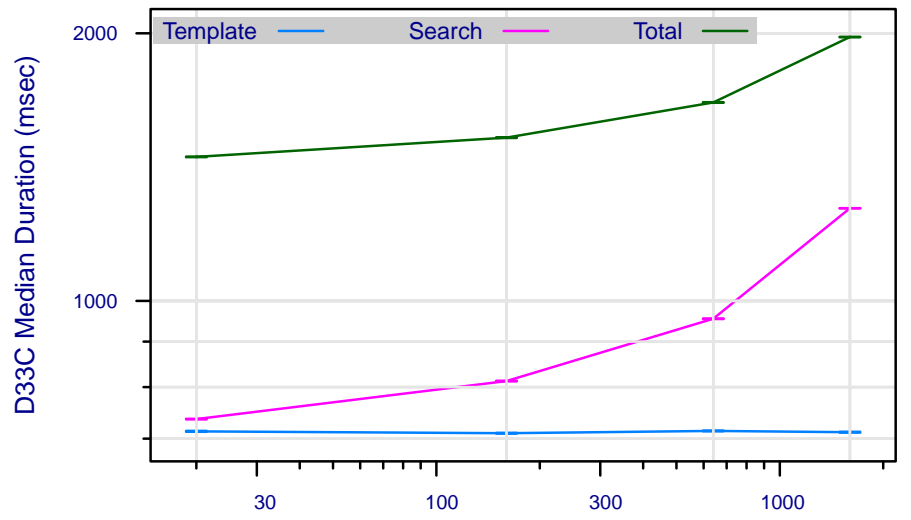

Num. enrolled identities, $\mathrm{N}$ (thousands)

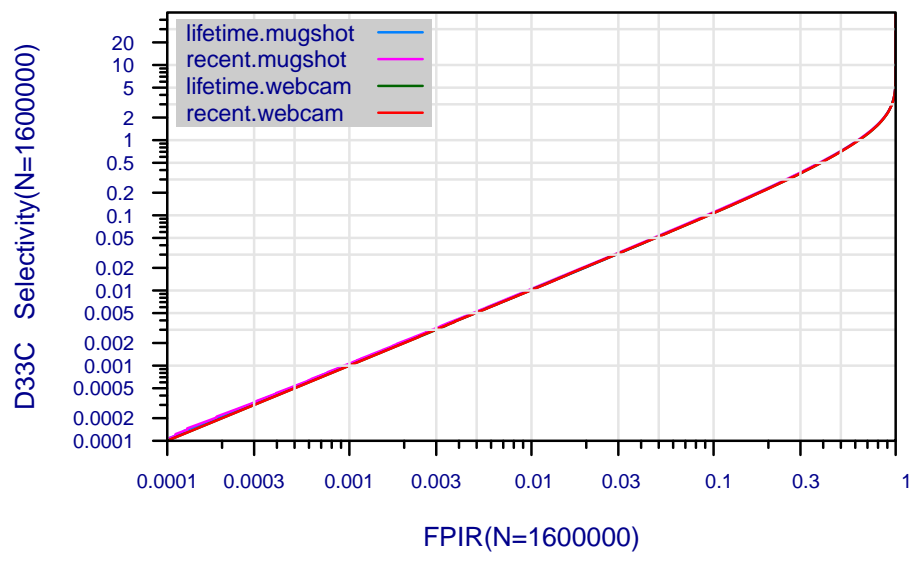

Figure 34: Collected performance reports for algorithm D33C. The figures are described at the beginning of this Appendix.

\begin{tabular}{|c|c|c|c|c|c|c|}
\hline $\begin{array}{l}\mathrm{A}=3 \mathrm{M} / \text { Cogent } \\
\mathrm{G}=\text { Hisign } \\
\mathrm{P}=\text { Zhuhai-Yisheng }\end{array}$ & $\begin{array}{l}B=\text { Cognitec } \\
H=\text { CAS-IA } \\
Q=\text { JunYu }\end{array}$ & $\begin{array}{l}\mathrm{C}=\text { Neurotechnology } \\
\mathrm{I}=\text { CAS-ICT } \\
\mathrm{S}=\text { Decatur }\end{array}$ & $\begin{array}{l}\mathrm{D}=\text { Safran Morpho } \\
\mathrm{J}=\text { Toshiba } \\
\mathrm{T}=\text { Ayonix }\end{array}$ & $\begin{array}{l}\mathrm{E}=\mathrm{NEC} \\
\mathrm{L}=\text { Tsinghua U. II }\end{array}$ & $\begin{array}{l}\mathrm{F}=\text { Tsinghua } \mathrm{U} \\
\mathrm{M}=\mathrm{HP}\end{array}$ & $\begin{array}{l}\text { FNIR(N,R,T,L) “Miss rate" } \\
\operatorname{FPIR}(\mathrm{N}, \mathrm{T}, \mathrm{L}) \text { “False alarm rate" }\end{array}$ \\
\hline
\end{tabular}




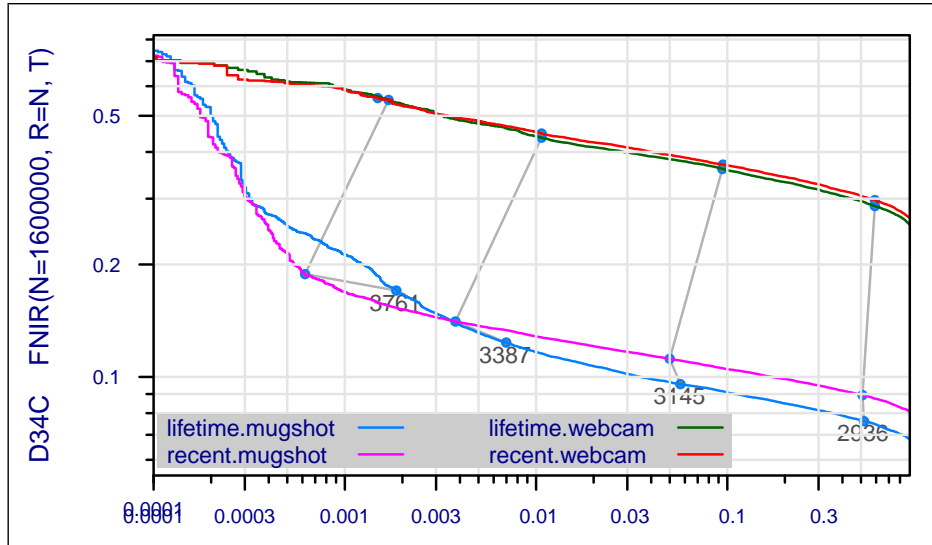

$\operatorname{FPIR}(\mathrm{N}=1600000)$
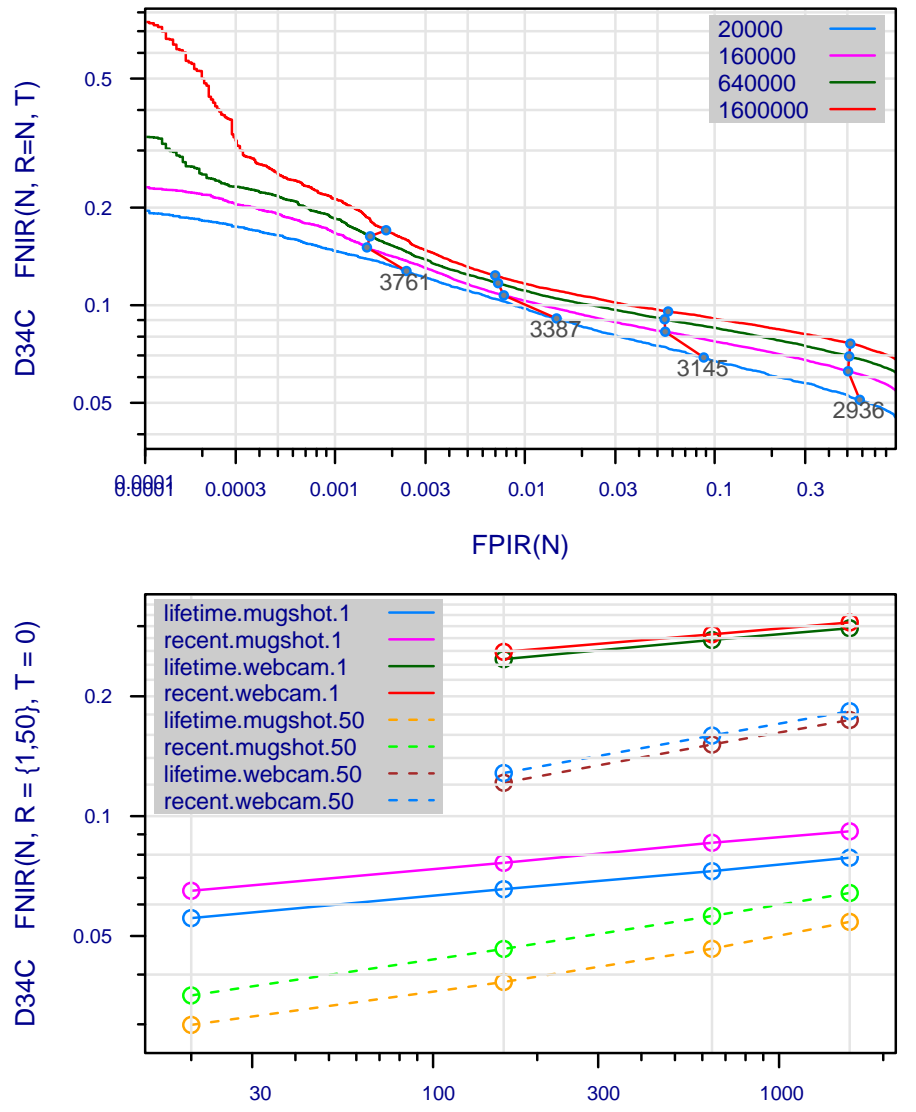

Num. enrolled identities, $\mathrm{N}$ (thousands)

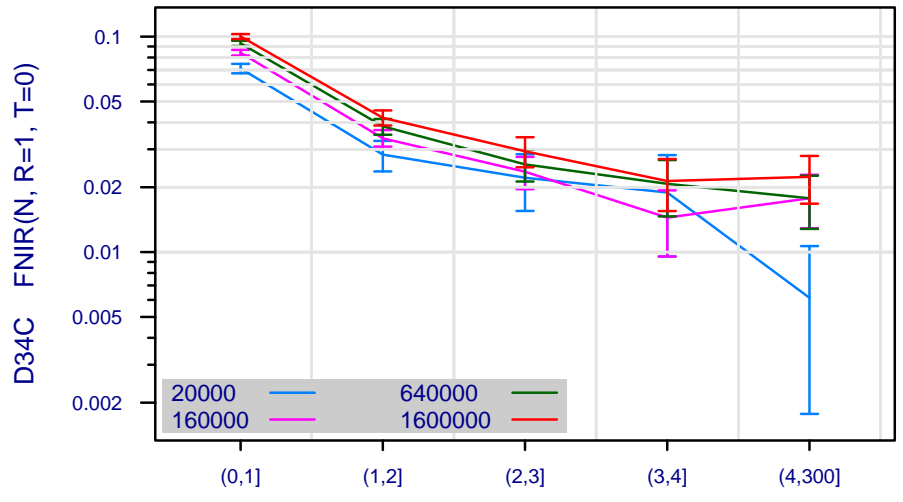

Num. enrolled images per identity

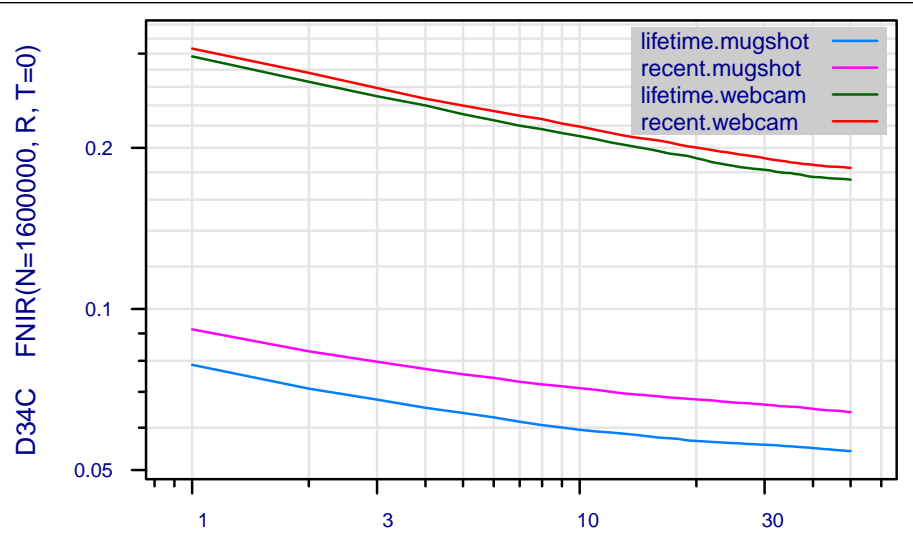

Rank, $\mathrm{R}$

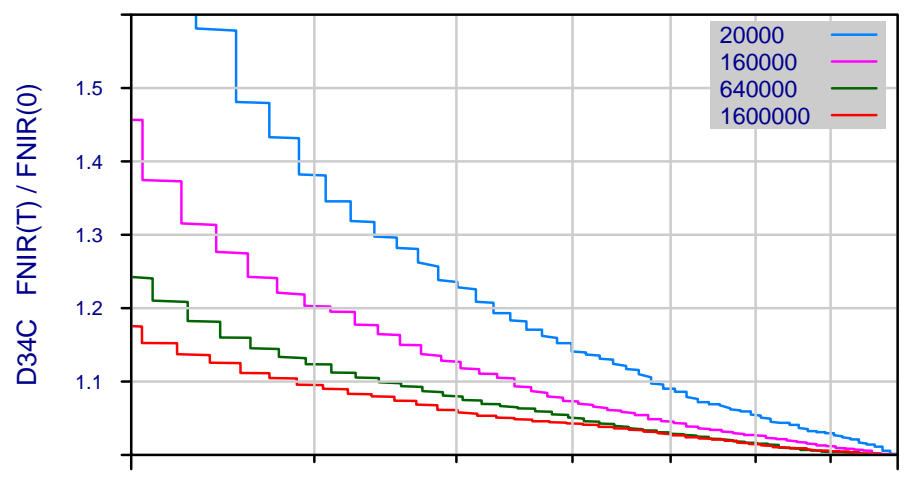

Frac. reduction in workload by using a threshold vs. not

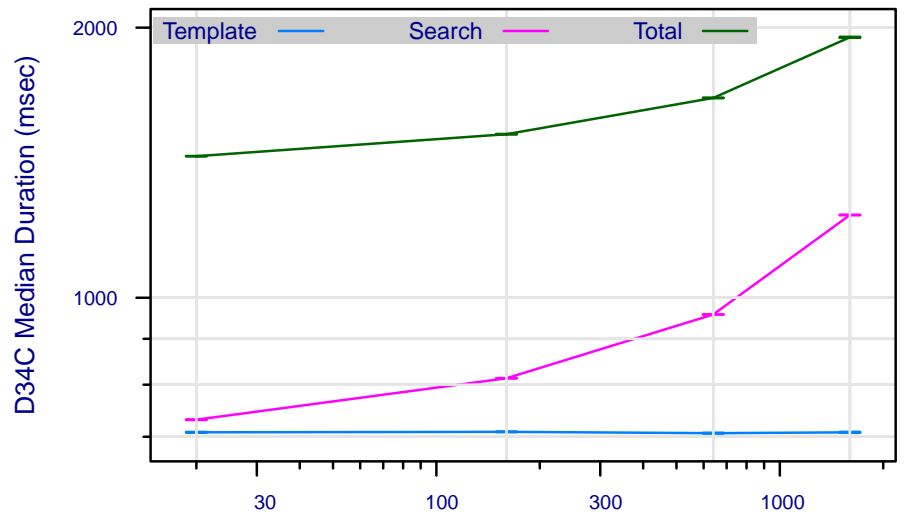

Num. enrolled identities, $\mathrm{N}$ (thousands)

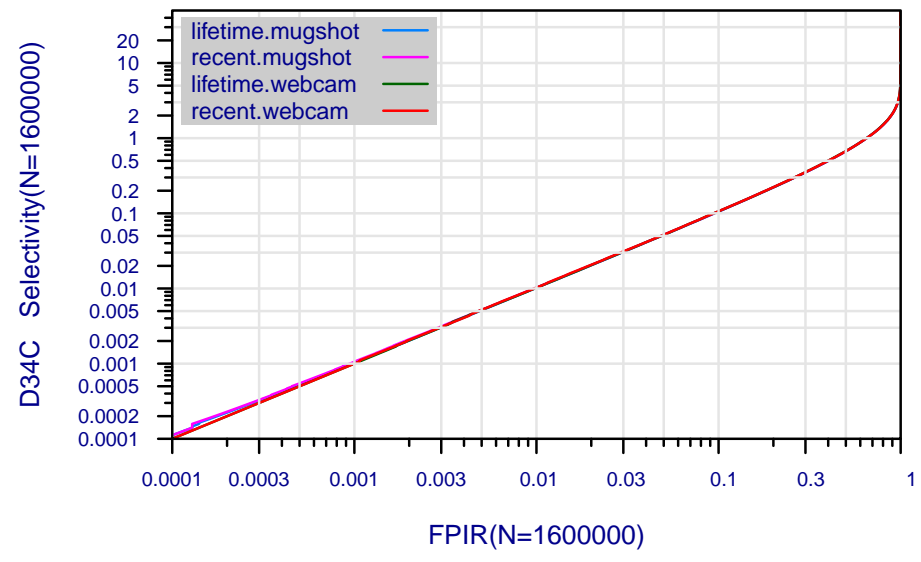

Figure 35: Collected performance reports for algorithm D34C. The figures are described at the beginning of this Appendix.

\begin{tabular}{|c|c|c|c|c|c|c|}
\hline $\begin{array}{l}\mathrm{A}=3 \mathrm{M} / \text { Cogent } \\
\mathrm{G}=\text { Hisign } \\
\mathrm{P}=\text { Zhuhai-Yisheng }\end{array}$ & $\begin{array}{l}B=\text { Cognitec } \\
H=\text { CAS-IA } \\
Q=\text { JunYu }\end{array}$ & $\begin{array}{l}\mathrm{C}=\text { Neurotechnology } \\
\mathrm{I}=\text { CAS-ICT } \\
\mathrm{S}=\text { Decatur }\end{array}$ & $\begin{array}{l}\mathrm{D}=\text { Safran Morpho } \\
\mathrm{J}=\text { Toshiba } \\
\mathrm{T}=\text { Ayonix }\end{array}$ & $\begin{array}{l}\mathrm{E}=\mathrm{NEC} \\
\mathrm{L}=\text { Tsinghua U. II }\end{array}$ & $\begin{array}{l}\mathrm{F}=\text { Tsinghua } \mathrm{U} \\
\mathrm{M}=\mathrm{HP}\end{array}$ & $\begin{array}{l}\text { FNIR(N,R,T,L) “Miss rate" } \\
\operatorname{FPIR}(\mathrm{N}, \mathrm{T}, \mathrm{L}) \text { “False alarm rate" }\end{array}$ \\
\hline
\end{tabular}




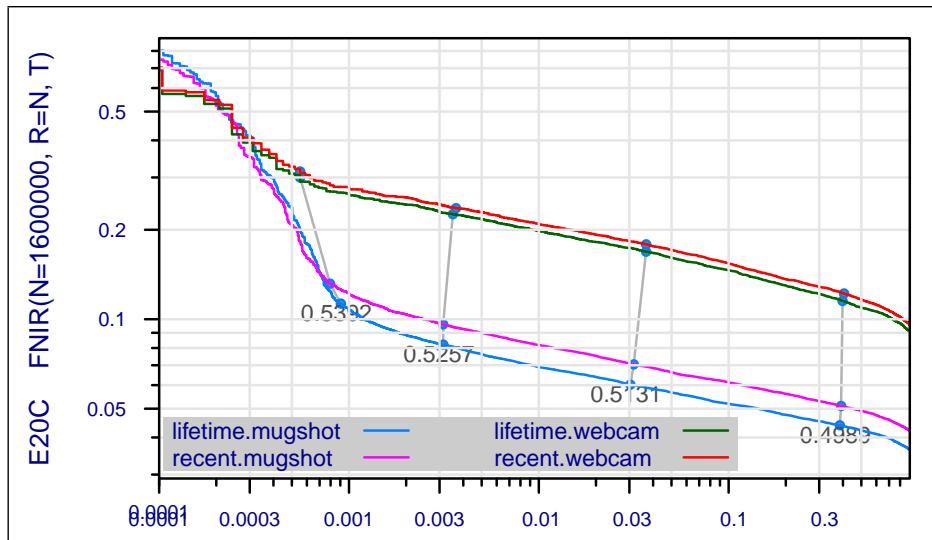

$\operatorname{FPIR}(\mathrm{N}=1600000)$
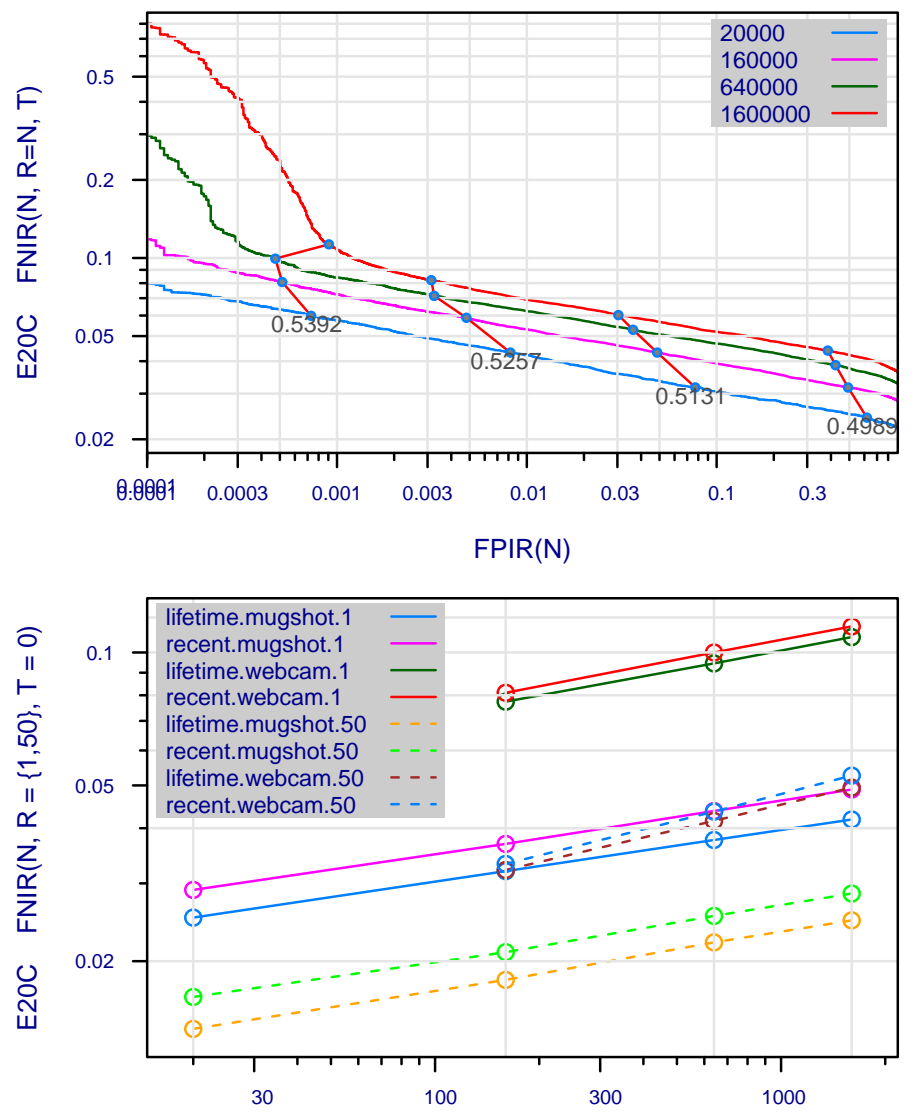

Num. enrolled identities, $\mathrm{N}$ (thousands)

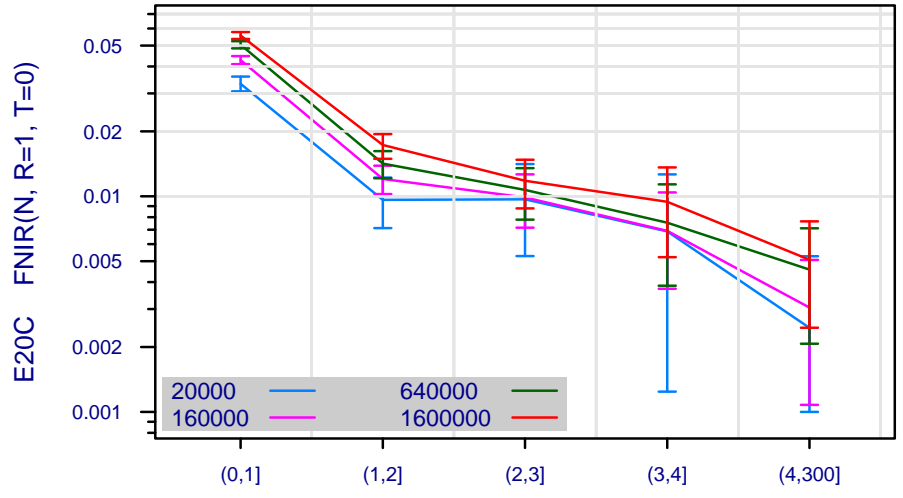

Num. enrolled images per identity

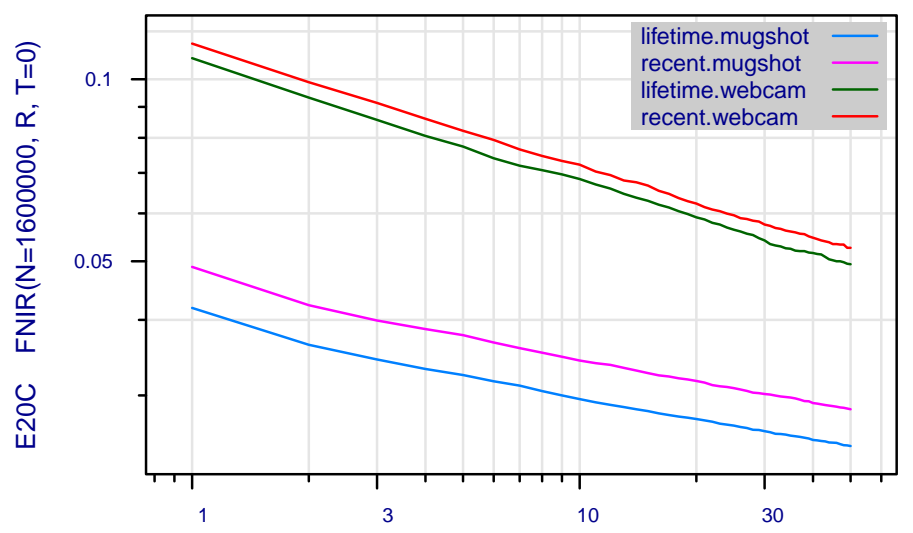

Rank, $\mathrm{R}$

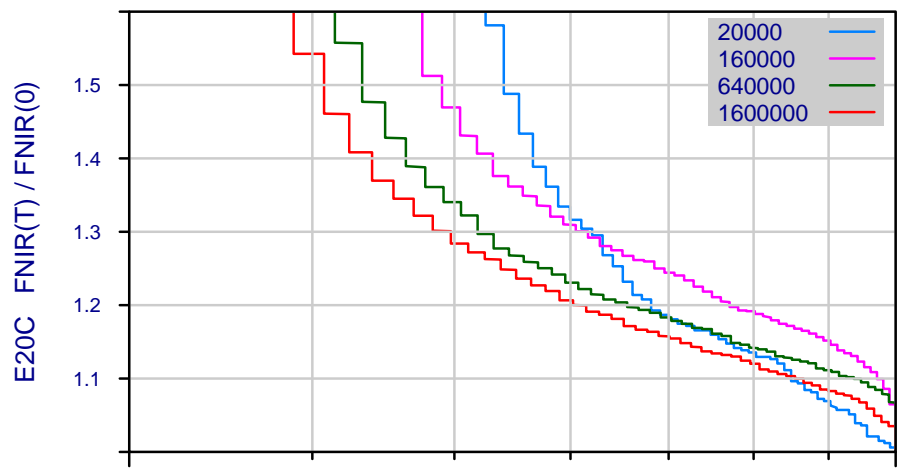

Frac. reduction in workload by using a threshold vs. not

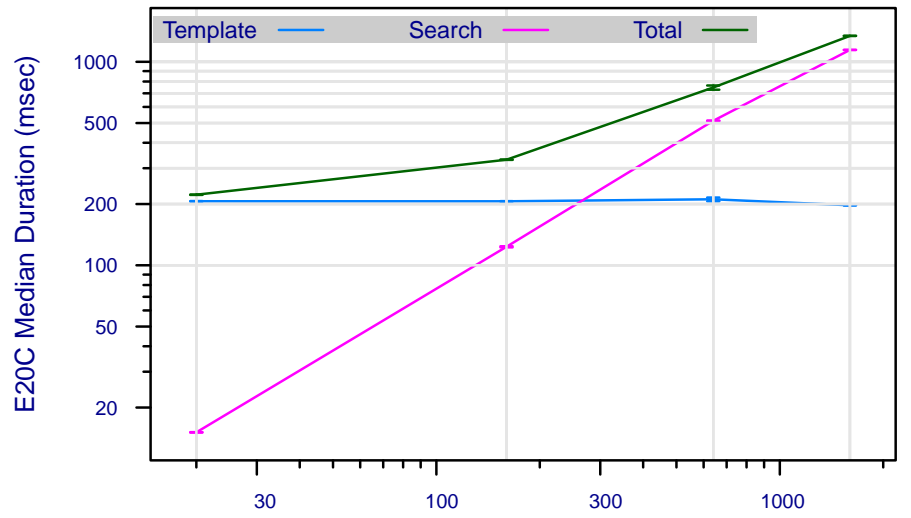

Num. enrolled identities, $\mathrm{N}$ (thousands)

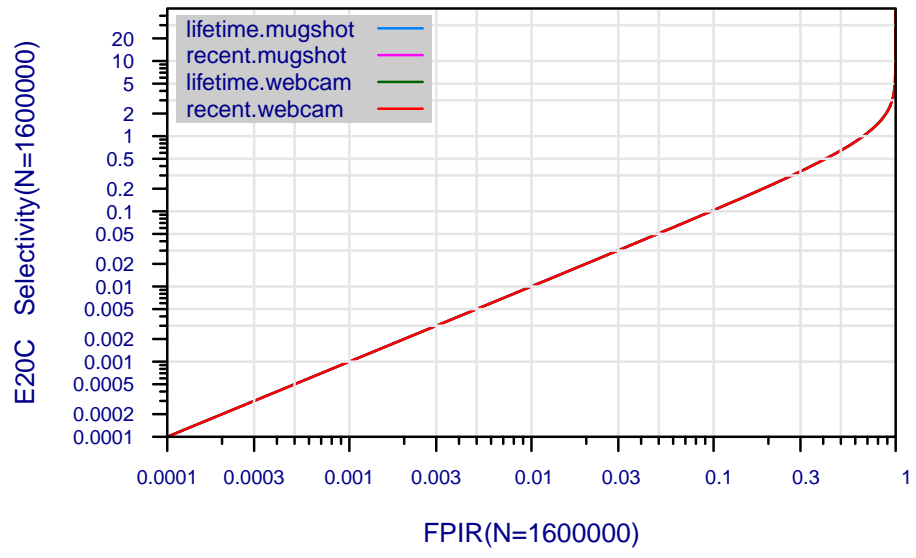

Figure 36: Collected performance reports for algorithm E20C. The figures are described at the beginning of this Appendix.

\begin{tabular}{|c|c|c|c|c|c|c|}
\hline $\begin{array}{l}A=3 \mathrm{M} / \text { Cogent } \\
G=\text { Hisign } \\
P=\text { Zhuhai-Yisheng }\end{array}$ & $\begin{array}{l}\mathrm{B}=\text { Cognitec } \\
\mathrm{H}=\text { CAS-IA } \\
\mathrm{Q}=\text { JunYu }\end{array}$ & $\begin{array}{l}\mathrm{C}=\text { Neurotechnology } \\
\mathrm{I}=\text { CAS-ICT } \\
\mathrm{S}=\text { Decatur }\end{array}$ & $\begin{array}{l}\mathrm{D}=\text { Safran Morpho } \\
\mathrm{J}=\text { Toshiba } \\
\mathrm{T}=\text { Ayonix }\end{array}$ & $\begin{array}{l}\mathrm{E}=\mathrm{NEC} \\
\mathrm{L}=\text { Tsinghua U. II }\end{array}$ & $\begin{array}{l}\mathrm{F}=\text { Tsinghua } \mathrm{U} \\
\mathrm{M}=\mathrm{HP}\end{array}$ & $\begin{array}{l}\text { FNIR(N,R,T,L) "Miss rate" } \\
\text { FPIR(N,T,L) “False alarm rate" }\end{array}$ \\
\hline
\end{tabular}



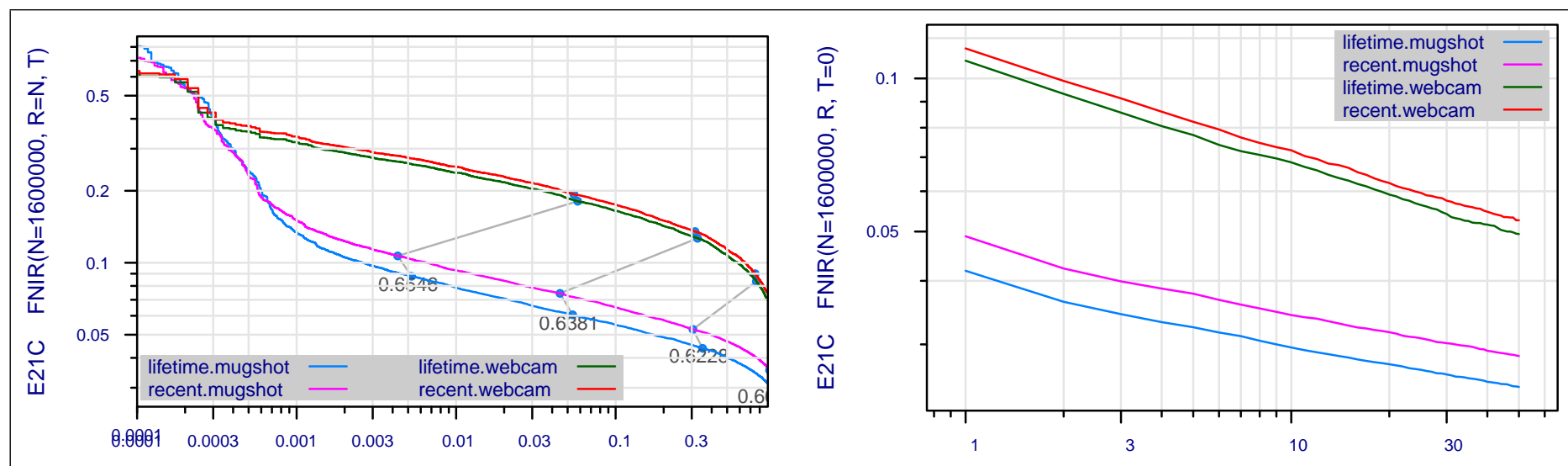

$\operatorname{FPIR}(\mathrm{N}=1600000)$
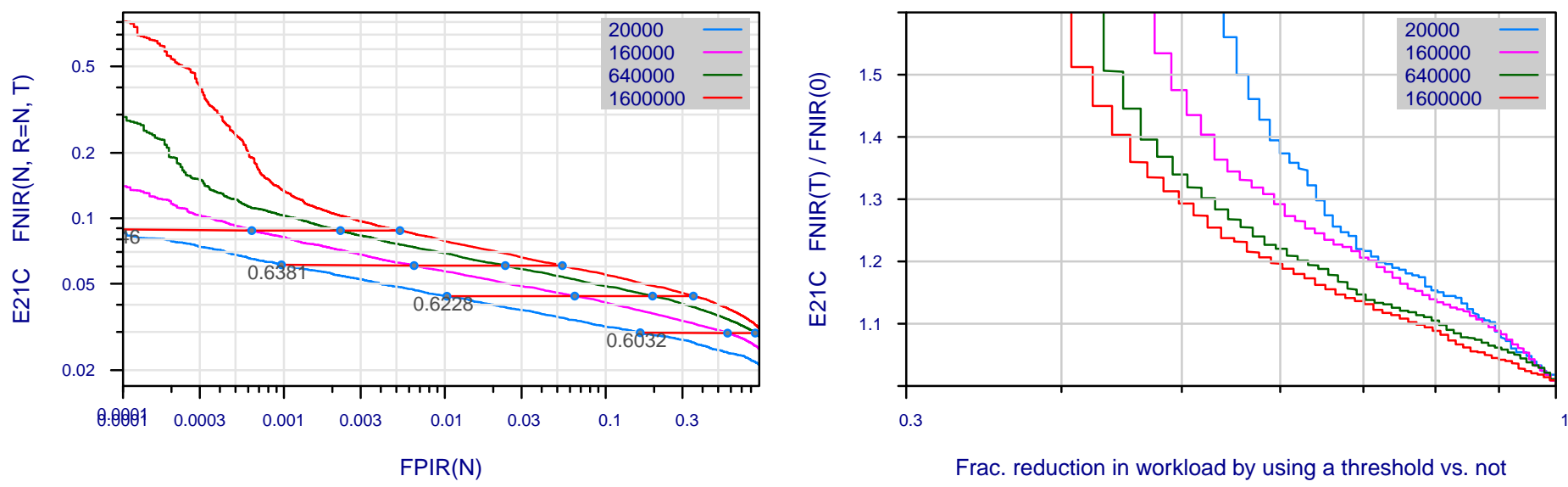

0.3

Frac. reduction in workload by using a threshold vs. not
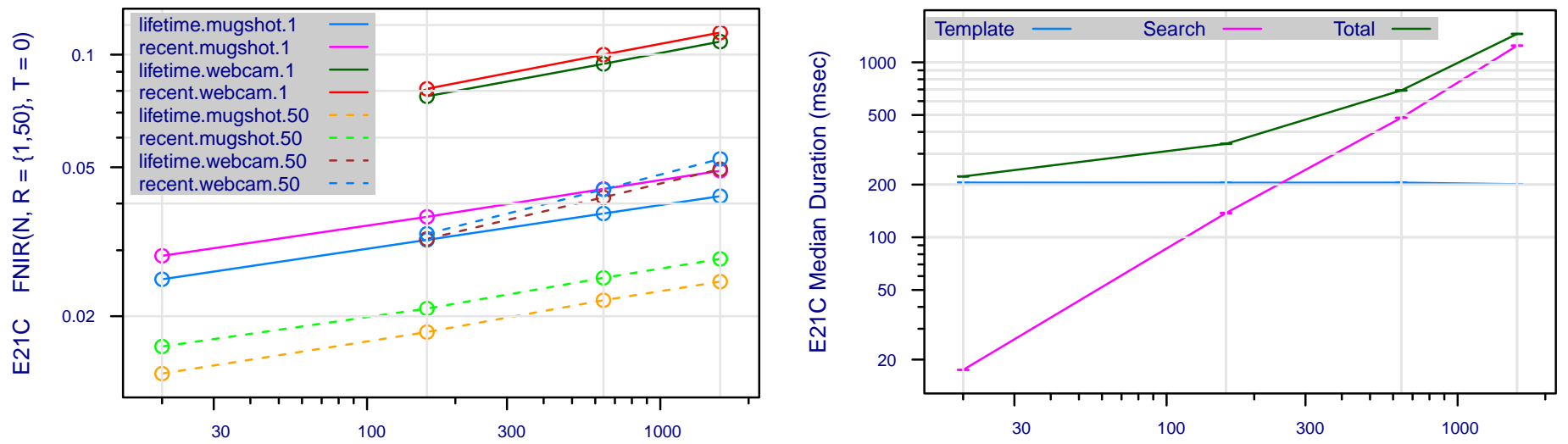

Num. enrolled identities, $\mathrm{N}$ (thousands)

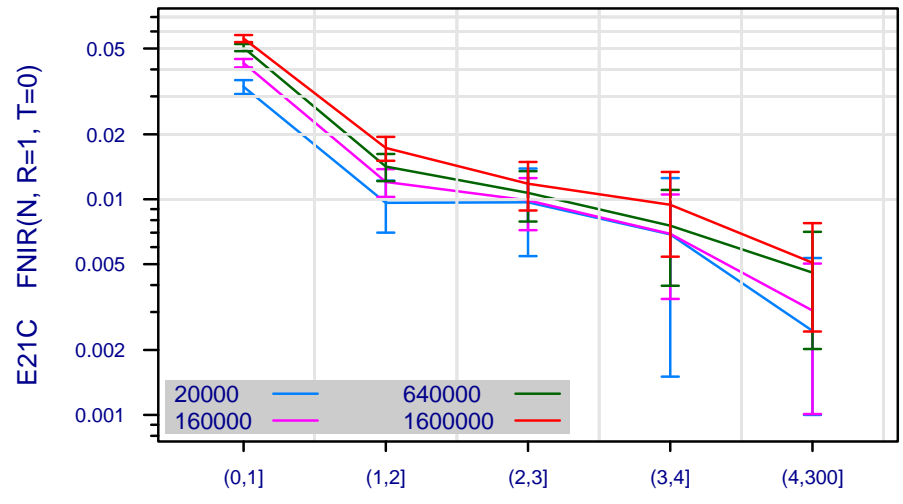

Num. enrolled images per identity

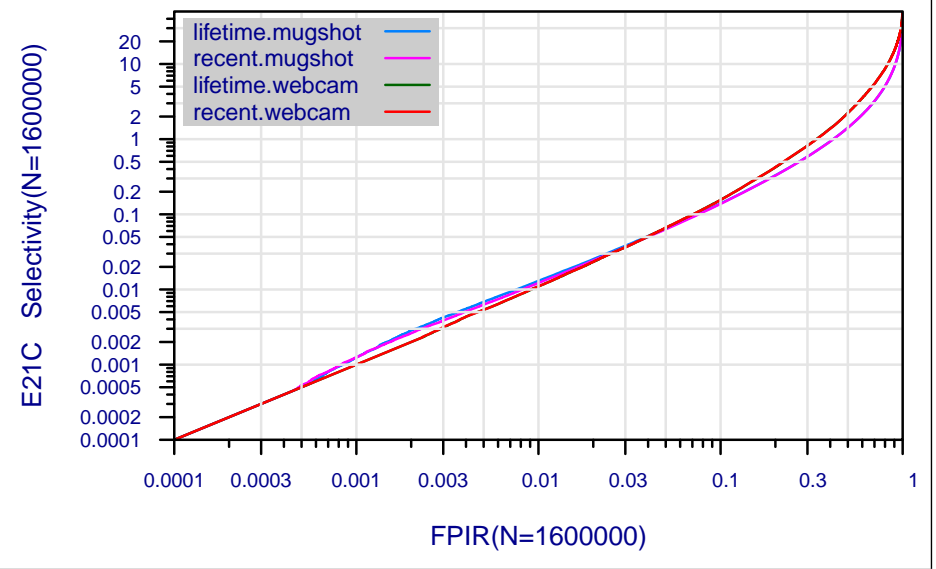

Figure 37: Collected performance reports for algorithm E21C. The figures are described at the beginning of this Appendix.

\begin{tabular}{|c|c|c|c|c|c|c|}
\hline $\begin{array}{l}\mathrm{A}=3 \mathrm{M} / \text { Cogent } \\
\mathrm{G}=\text { Hisign } \\
\mathrm{P}=\text { Zhuhai-Yisheng }\end{array}$ & $\begin{array}{l}B=\text { Cognitec } \\
H=\text { CAS-IA } \\
Q=\text { JunYu }\end{array}$ & $\begin{array}{l}\mathrm{C}=\text { Neurotechnology } \\
\mathrm{I}=\text { CAS-ICT } \\
\mathrm{S}=\text { Decatur }\end{array}$ & $\begin{array}{l}\mathrm{D}=\text { Safran Morpho } \\
\mathrm{J}=\text { Toshiba } \\
\mathrm{T}=\text { Ayonix }\end{array}$ & $\begin{array}{l}\mathrm{E}=\mathrm{NEC} \\
\mathrm{L}=\text { Tsinghua U. II }\end{array}$ & $\begin{array}{l}\mathrm{F}=\text { Tsinghua } \mathrm{U} \\
\mathrm{M}=\mathrm{HP}\end{array}$ & $\begin{array}{l}\text { FNIR(N,R,T,L) “Miss rate" } \\
\operatorname{FPIR}(\mathrm{N}, \mathrm{T}, \mathrm{L}) \text { “False alarm rate" }\end{array}$ \\
\hline
\end{tabular}




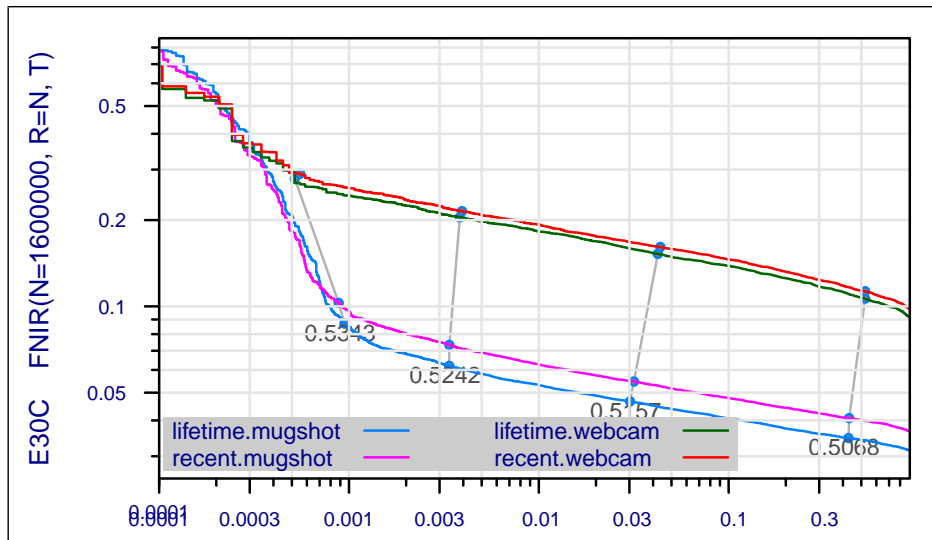

$\operatorname{FPIR}(\mathrm{N}=1600000)$
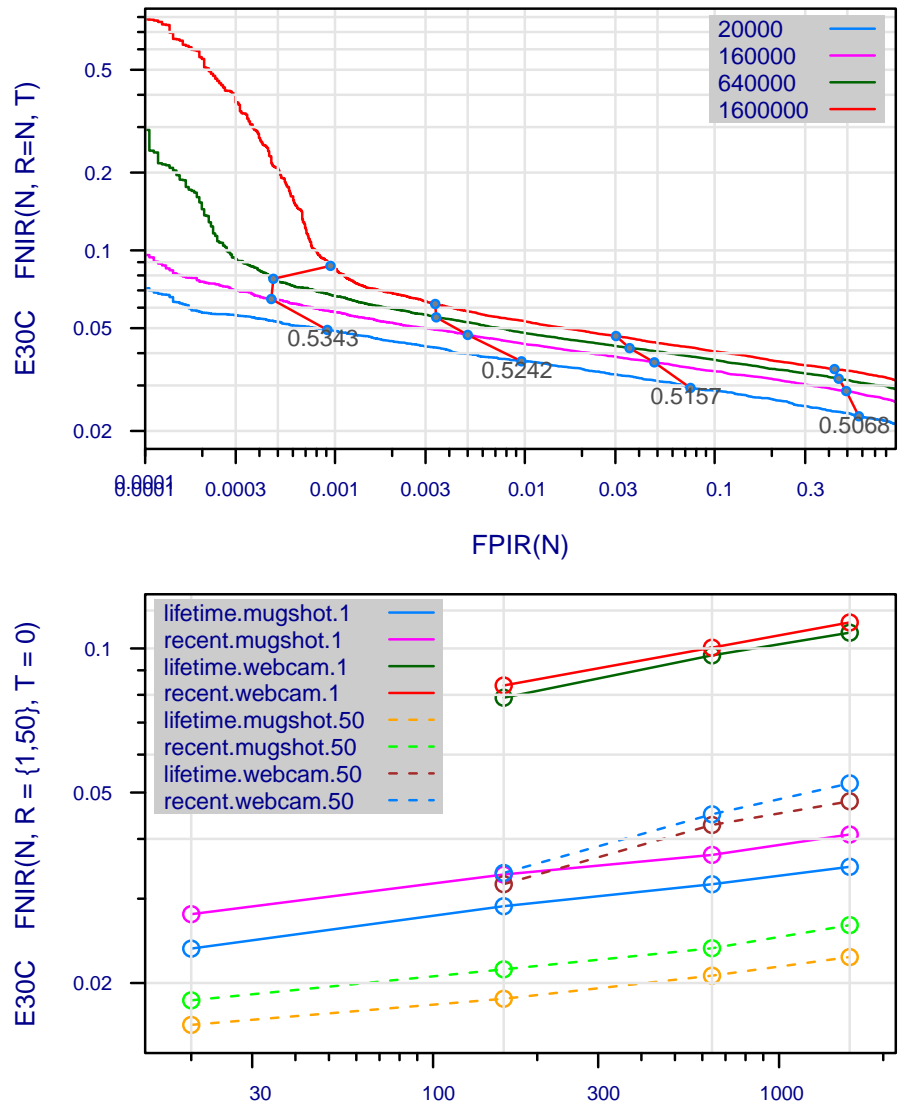

Num. enrolled identities, $\mathrm{N}$ (thousands)

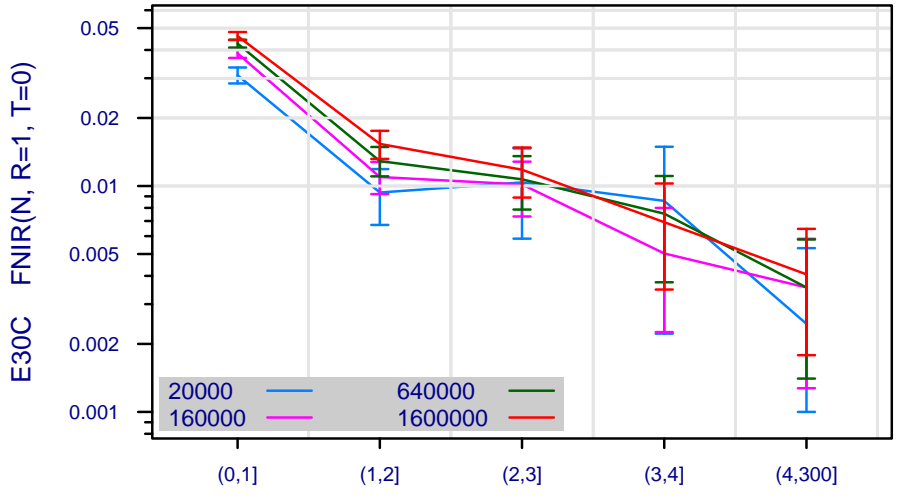

Num. enrolled images per identity

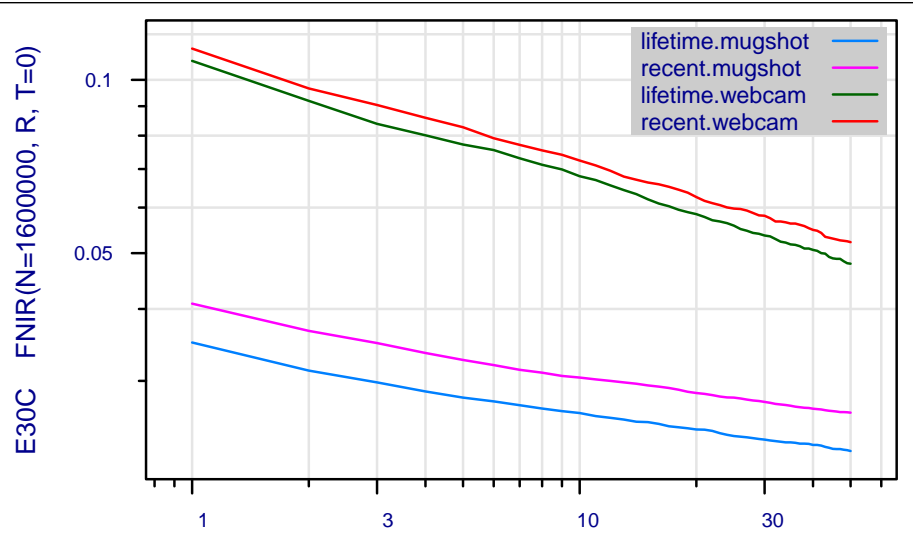

Rank, R

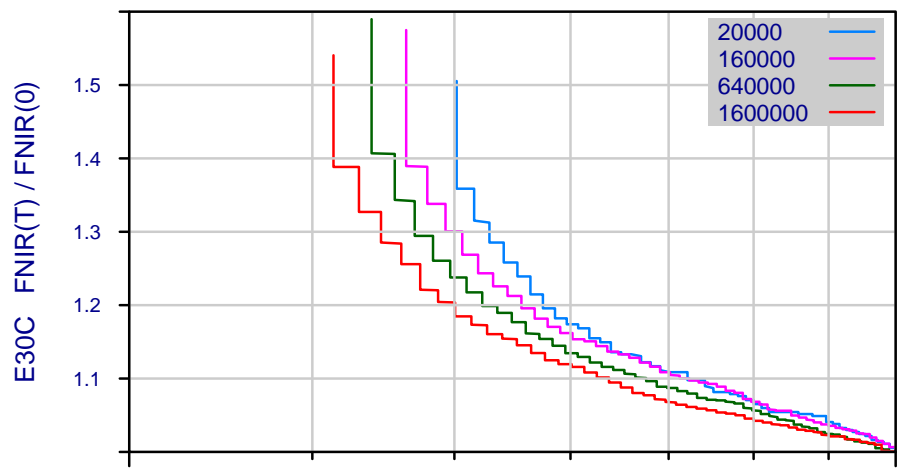

Frac. reduction in workload by using a threshold vs. not

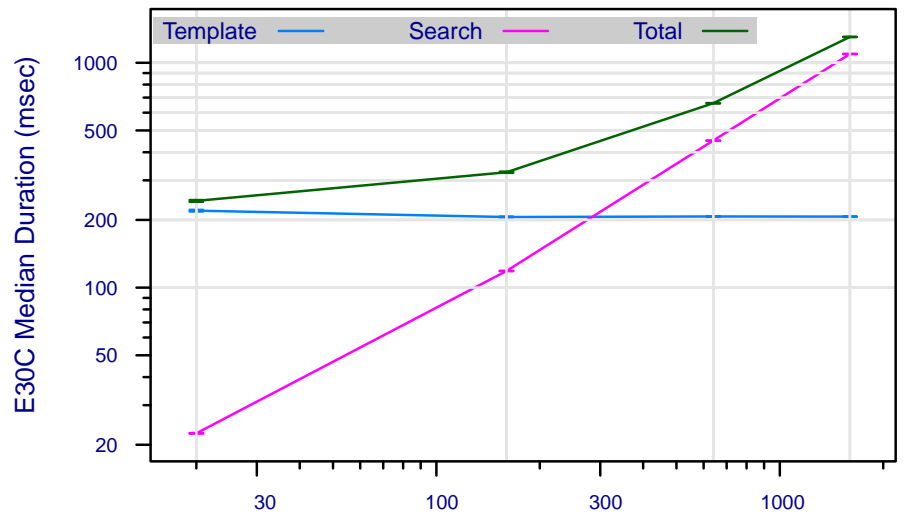

Num. enrolled identities, $\mathrm{N}$ (thousands)

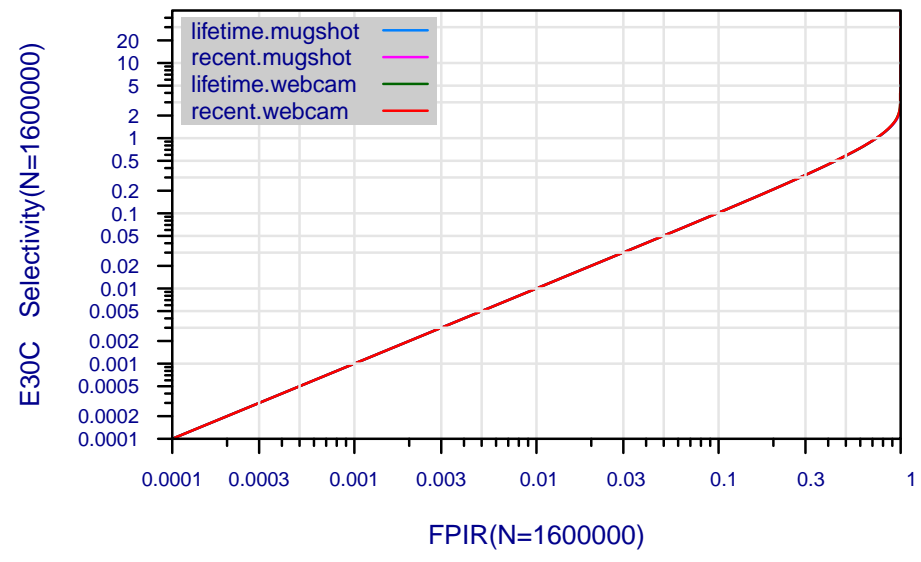

Figure 38: Collected performance reports for algorithm E30C. The figures are described at the beginning of this Appendix.

\begin{tabular}{|c|c|c|c|c|c|c|}
\hline $\begin{array}{l}A=3 \mathrm{M} / \text { Cogent } \\
G=\text { Hisign } \\
P=\text { Zhuhai-Yisheng }\end{array}$ & $\begin{array}{l}\mathrm{B}=\text { Cognitec } \\
\mathrm{H}=\text { CAS-IA } \\
\mathrm{Q}=\text { JunYu }\end{array}$ & $\begin{array}{l}\mathrm{C}=\text { Neurotechnology } \\
\mathrm{I}=\text { CAS-ICT } \\
\mathrm{S}=\text { Decatur }\end{array}$ & $\begin{array}{l}\mathrm{D}=\text { Safran Morpho } \\
\mathrm{J}=\text { Toshiba } \\
\mathrm{T}=\text { Ayonix }\end{array}$ & $\begin{array}{l}\mathrm{E}=\mathrm{NEC} \\
\mathrm{L}=\text { Tsinghua U. II }\end{array}$ & $\begin{array}{l}\mathrm{F}=\text { Tsinghua } \mathrm{U} \\
\mathrm{M}=\mathrm{HP}\end{array}$ & $\begin{array}{l}\text { FNIR(N,R,T,L) "Miss rate" } \\
\text { FPIR(N,T,L) “False alarm rate" }\end{array}$ \\
\hline
\end{tabular}




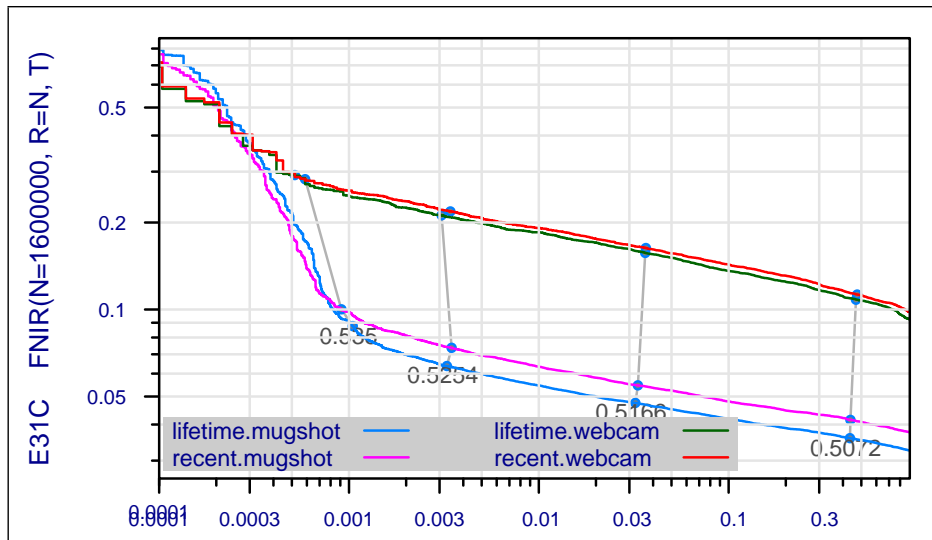

$\operatorname{FPIR}(\mathrm{N}=1600000)$
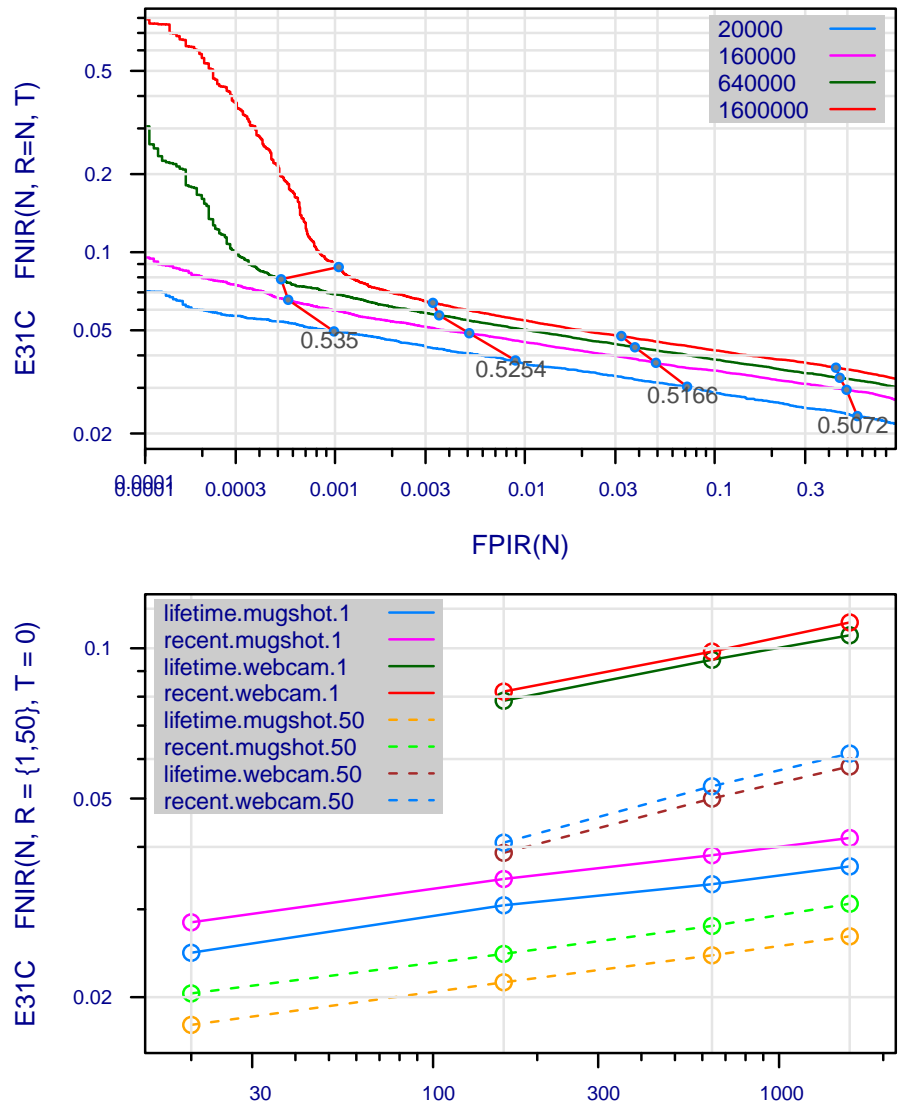

Num. enrolled identities, $\mathrm{N}$ (thousands)

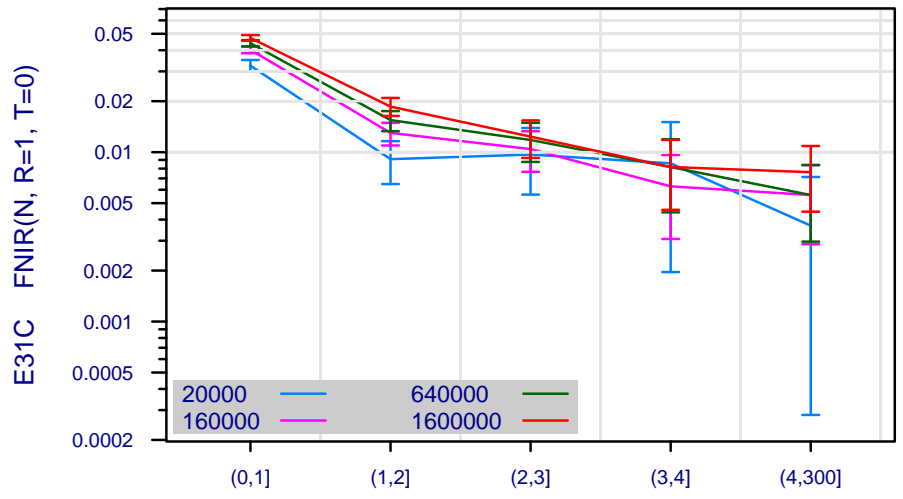

Num. enrolled images per identity

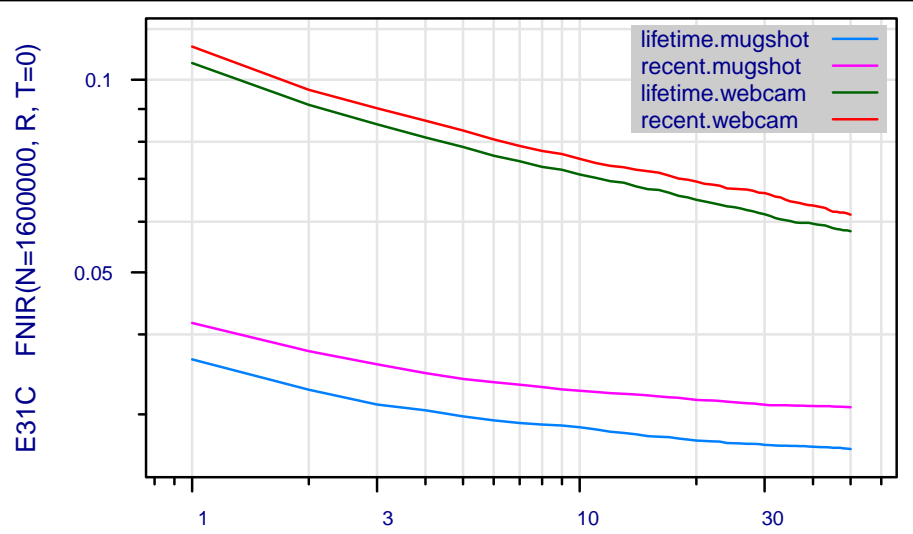

Rank, $\mathrm{R}$

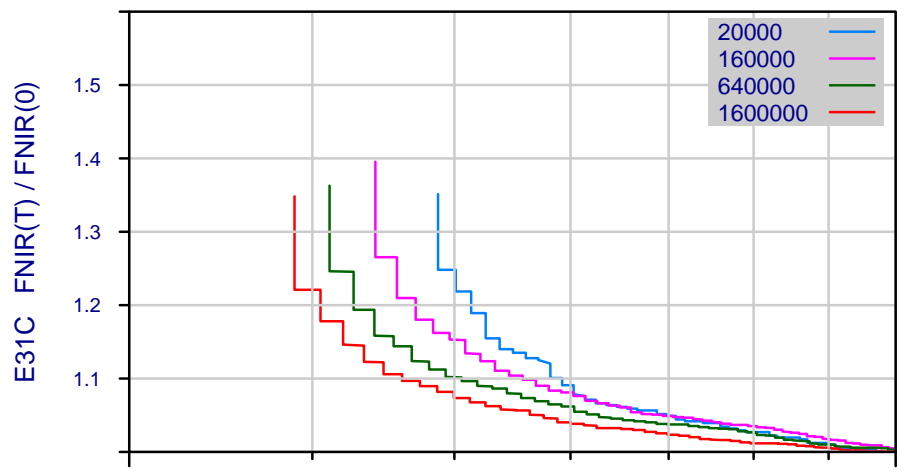

Frac. reduction in workload by using a threshold vs. not

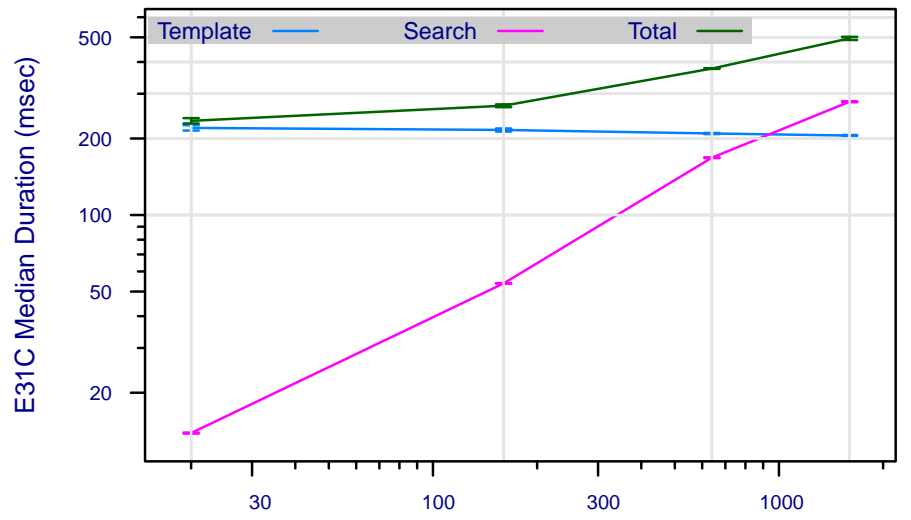

Num. enrolled identities, $\mathrm{N}$ (thousands)

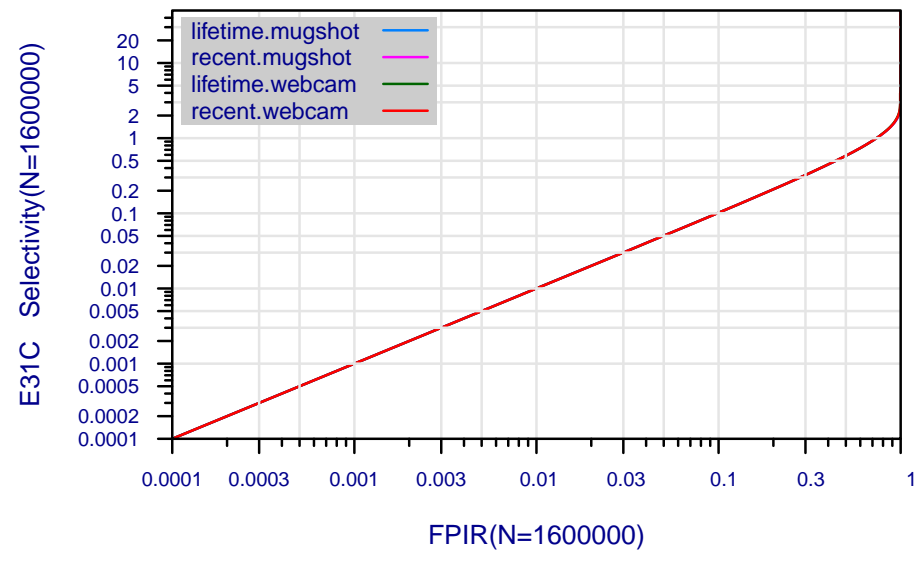

Figure 39: Collected performance reports for algorithm E31C. The figures are described at the beginning of this Appendix.

\begin{tabular}{|c|c|c|c|c|c|c|}
\hline $\begin{array}{l}\mathrm{A}=3 \mathrm{M} / \text { Cogent } \\
\mathrm{G}=\text { Hisign } \\
\mathrm{P}=\text { Zhuhai-Yisheng }\end{array}$ & $\begin{array}{l}B=\text { Cognitec } \\
H=\text { CAS-IA } \\
Q=\text { JunYu }\end{array}$ & $\begin{array}{l}\mathrm{C}=\text { Neurotechnology } \\
\mathrm{I}=\text { CAS-ICT } \\
\mathrm{S}=\text { Decatur }\end{array}$ & $\begin{array}{l}\mathrm{D}=\text { Safran Morpho } \\
\mathrm{J}=\text { Toshiba } \\
\mathrm{T}=\text { Ayonix }\end{array}$ & $\begin{array}{l}\mathrm{E}=\mathrm{NEC} \\
\mathrm{L}=\text { Tsinghua U. II }\end{array}$ & $\begin{array}{l}\mathrm{F}=\text { Tsinghua } \mathrm{U} \\
\mathrm{M}=\mathrm{HP}\end{array}$ & $\begin{array}{l}\text { FNIR(N,R,T,L) "Miss rate" } \\
\text { FPIR(N,T,L) "False alarm rate" }\end{array}$ \\
\hline
\end{tabular}



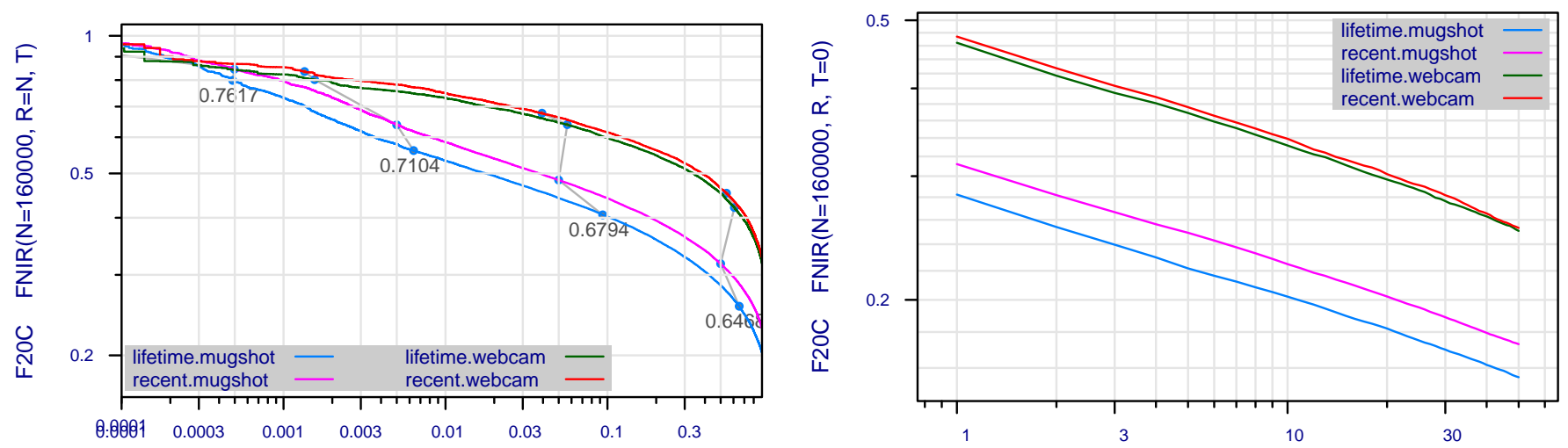

$\operatorname{FPIR}(\mathrm{N}=160000)$
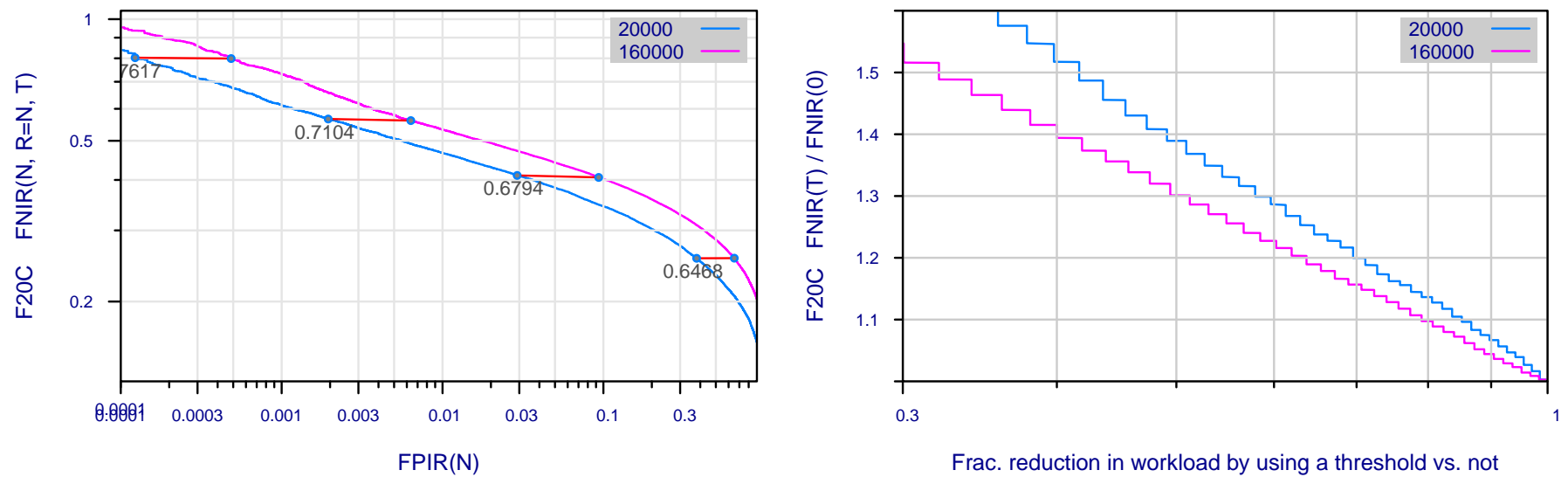

Frac. reduction in workload by using a threshold vs. not
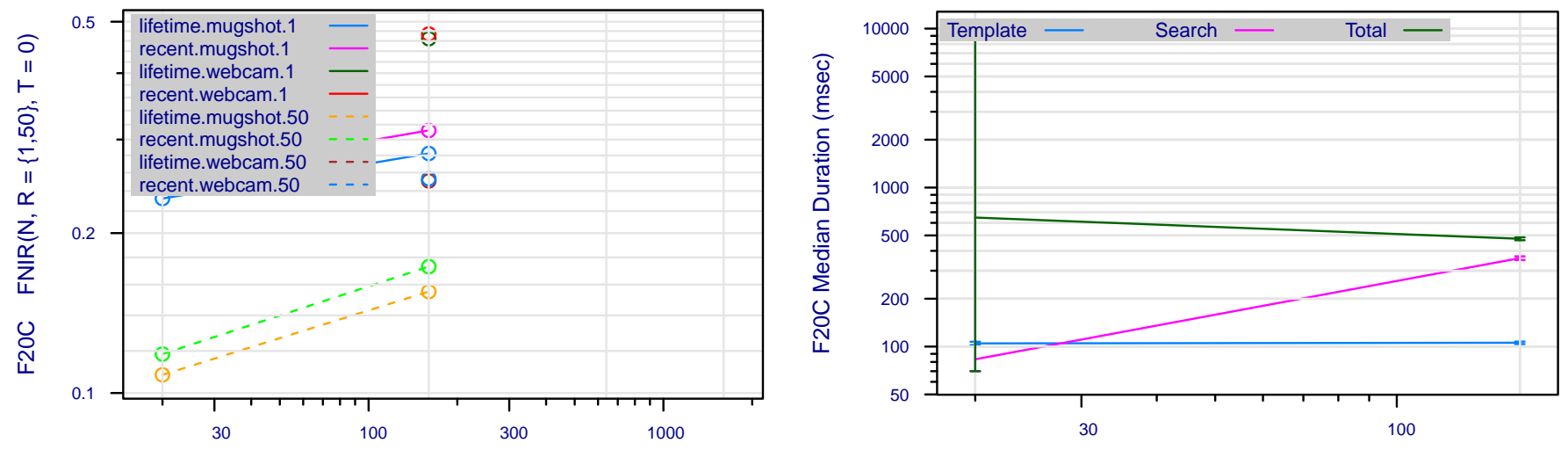

Num. enrolled identities, $\mathrm{N}$ (thousands)

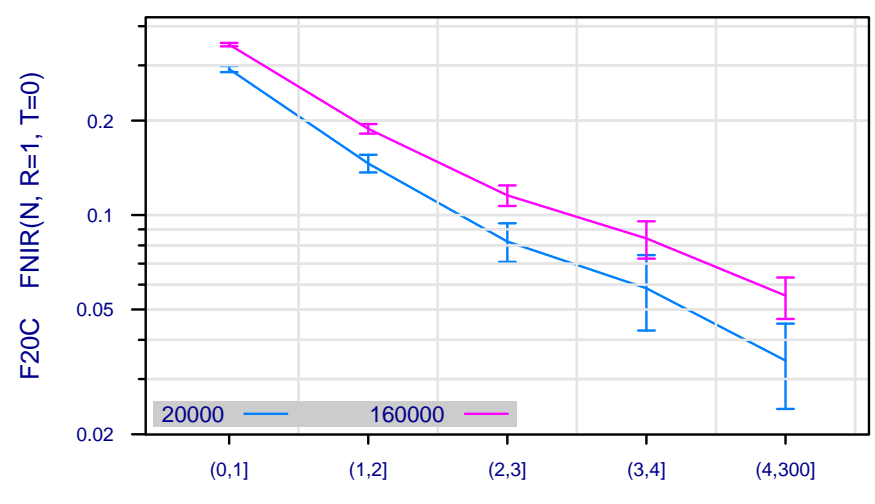

Num. enrolled identities, $\mathrm{N}$ (thousands)

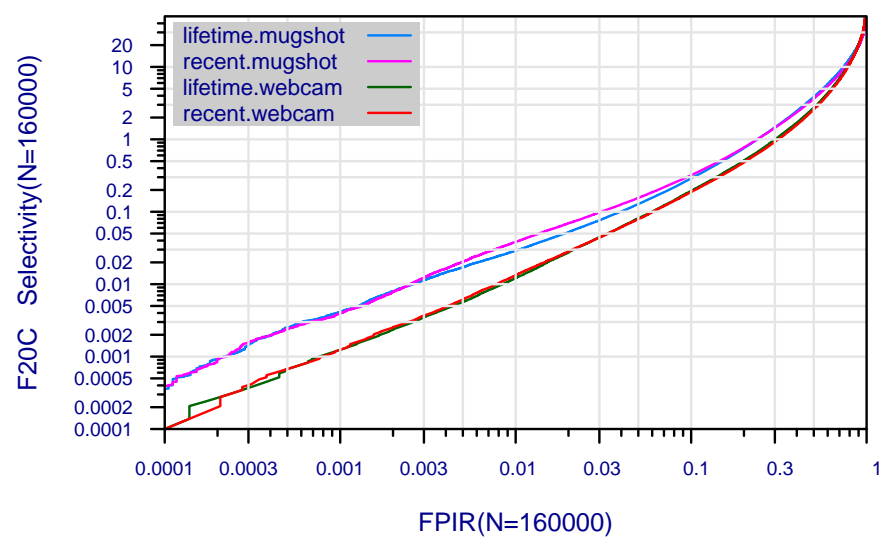

Figure 40: Collected performance reports for algorithm F20C. The figures are described at the beginning of this Appendix.

\begin{tabular}{|c|c|c|c|c|c|c|}
\hline $\begin{array}{l}\mathrm{A}=3 \mathrm{M} / \text { Cogent } \\
\mathrm{G}=\text { Hisign } \\
\mathrm{P}=\text { Zhuhai-Yisheng }\end{array}$ & $\begin{array}{l}B=\text { Cognitec } \\
H=\text { CAS-IA } \\
Q=\text { JunYu }\end{array}$ & $\begin{array}{l}\mathrm{C}=\text { Neurotechnology } \\
\mathrm{I}=\text { CAS-ICT } \\
\mathrm{S}=\text { Decatur }\end{array}$ & $\begin{array}{l}\mathrm{D}=\text { Safran Morpho } \\
\mathrm{J}=\text { Toshiba } \\
\mathrm{T}=\text { Ayonix }\end{array}$ & $\begin{array}{l}\mathrm{E}=\mathrm{NEC} \\
\mathrm{L}=\text { Tsinghua U. II }\end{array}$ & $\begin{array}{l}\mathrm{F}=\text { Tsinghua } \mathrm{U} \\
\mathrm{M}=\mathrm{HP}\end{array}$ & $\begin{array}{l}\text { FNIR(N,R,T,L) "Miss rate" } \\
\text { FPIR(N,T,L) "False alarm rate" }\end{array}$ \\
\hline
\end{tabular}



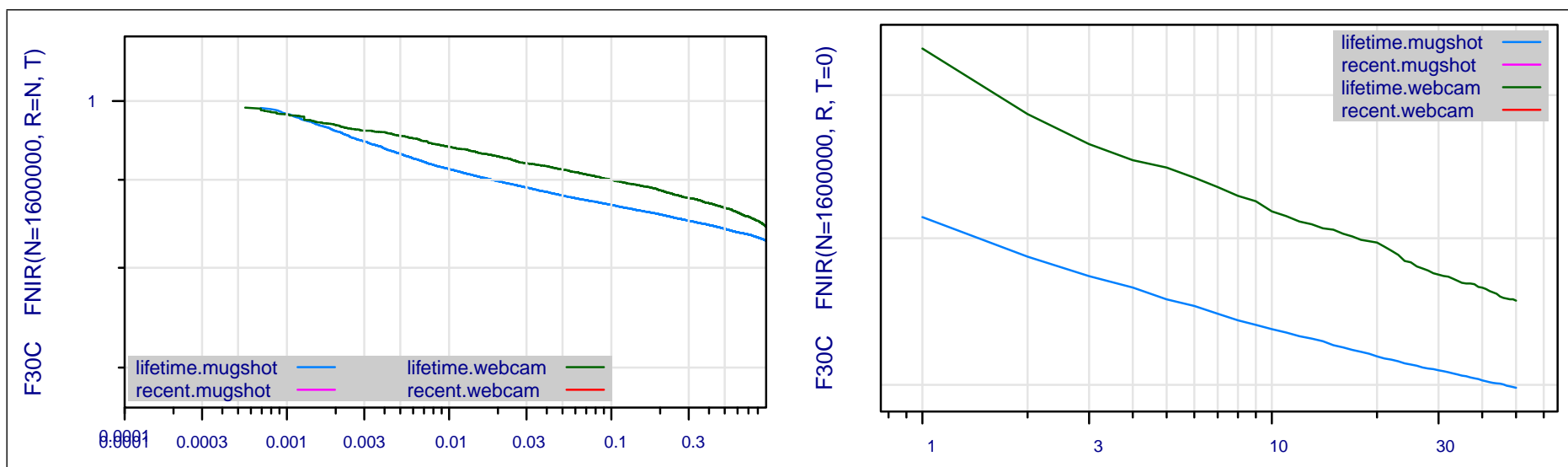

$\operatorname{FPIR}(\mathrm{N}=1600000)$
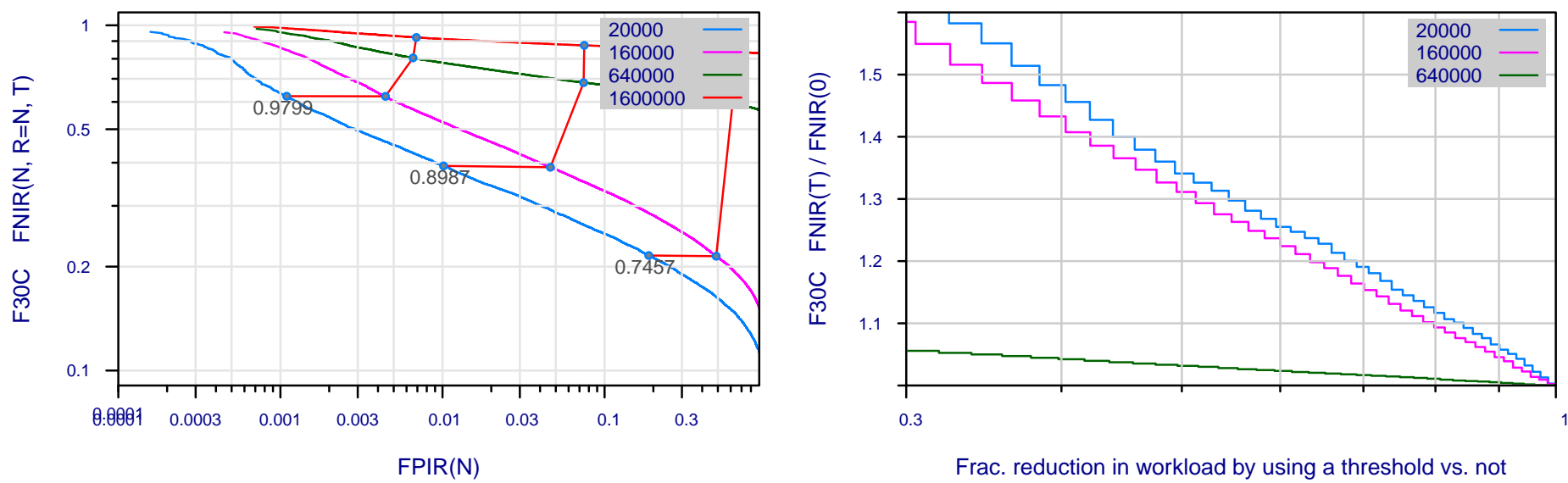

0.3

Frac. reduction in workload by using a threshold vs. not
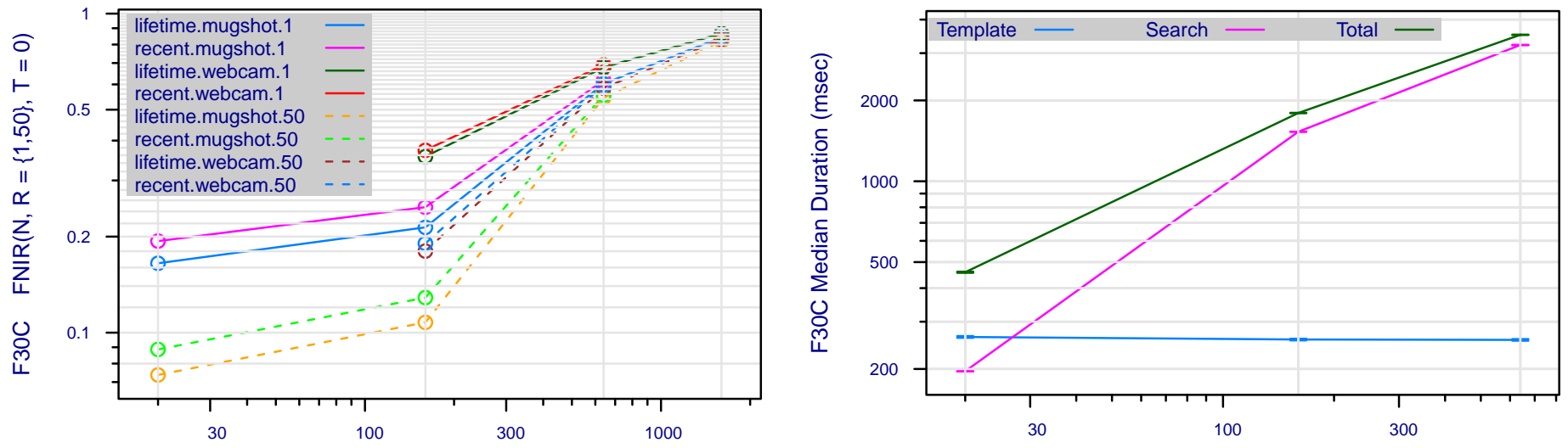

Num. enrolled identities, $\mathrm{N}$ (thousands)

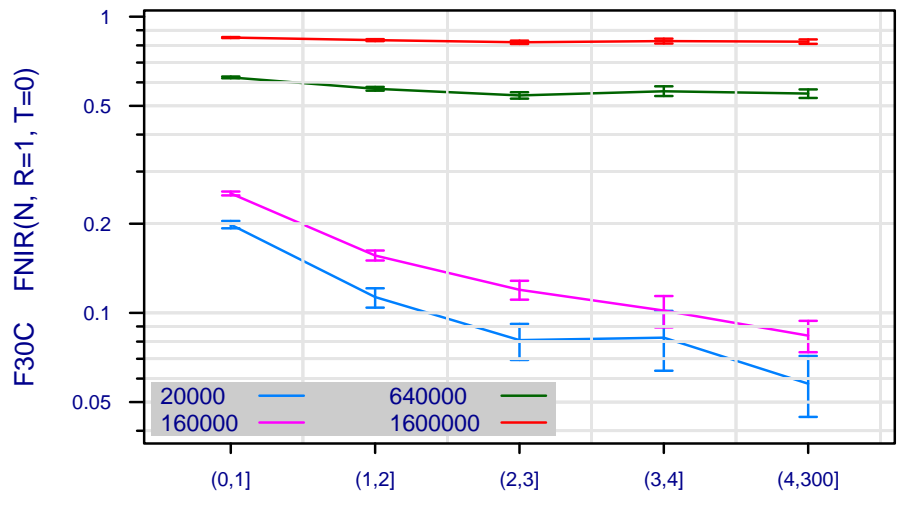

Num. enrolled images per identity

Num. enrolled identities, $\mathrm{N}$ (thousands)

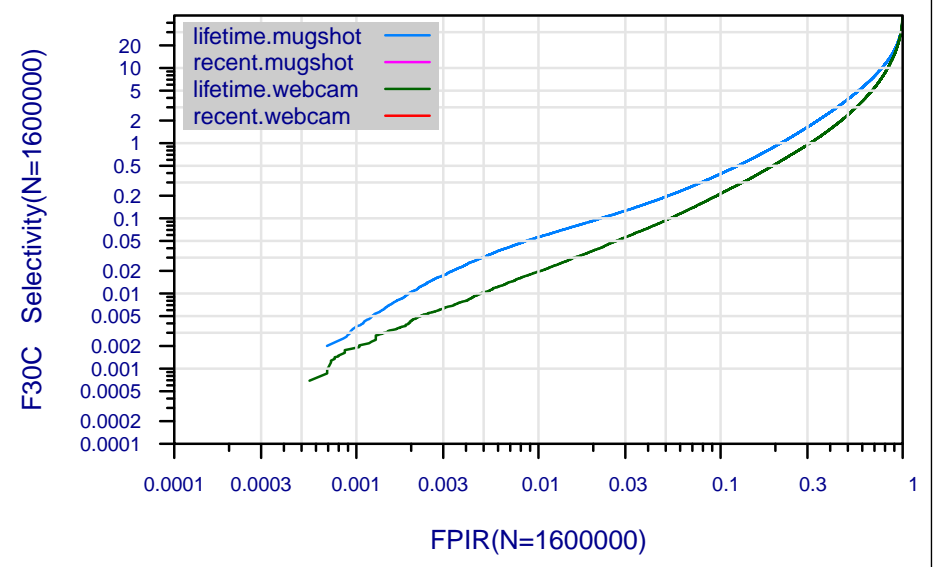

Figure 41: Collected performance reports for algorithm F30C. The figures are described at the beginning of this Appendix.

\begin{tabular}{|c|c|c|c|c|c|c|}
\hline $\begin{array}{l}\mathrm{A}=3 \mathrm{M} / \text { Cogent } \\
\mathrm{G}=\text { Hisign } \\
\mathrm{P}=\text { Zhuhai-Yisheng }\end{array}$ & $\begin{array}{l}B=\text { Cognitec } \\
H=\text { CAS-IA } \\
Q=\text { JunYu }\end{array}$ & $\begin{array}{l}\mathrm{C}=\text { Neurotechnology } \\
\mathrm{I}=\text { CAS-ICT } \\
\mathrm{S}=\text { Decatur }\end{array}$ & $\begin{array}{l}\mathrm{D}=\text { Safran Morpho } \\
\mathrm{J}=\text { Toshiba } \\
\mathrm{T}=\text { Ayonix }\end{array}$ & $\begin{array}{l}\mathrm{E}=\mathrm{NEC} \\
\mathrm{L}=\text { Tsinghua U. II }\end{array}$ & $\begin{array}{l}\mathrm{F}=\text { Tsinghua } \mathrm{U} \\
\mathrm{M}=\mathrm{HP}\end{array}$ & $\begin{array}{l}\text { FNIR(N,R,T,L) "Miss rate" } \\
\text { FPIR(N,T,L) "False alarm rate" }\end{array}$ \\
\hline
\end{tabular}



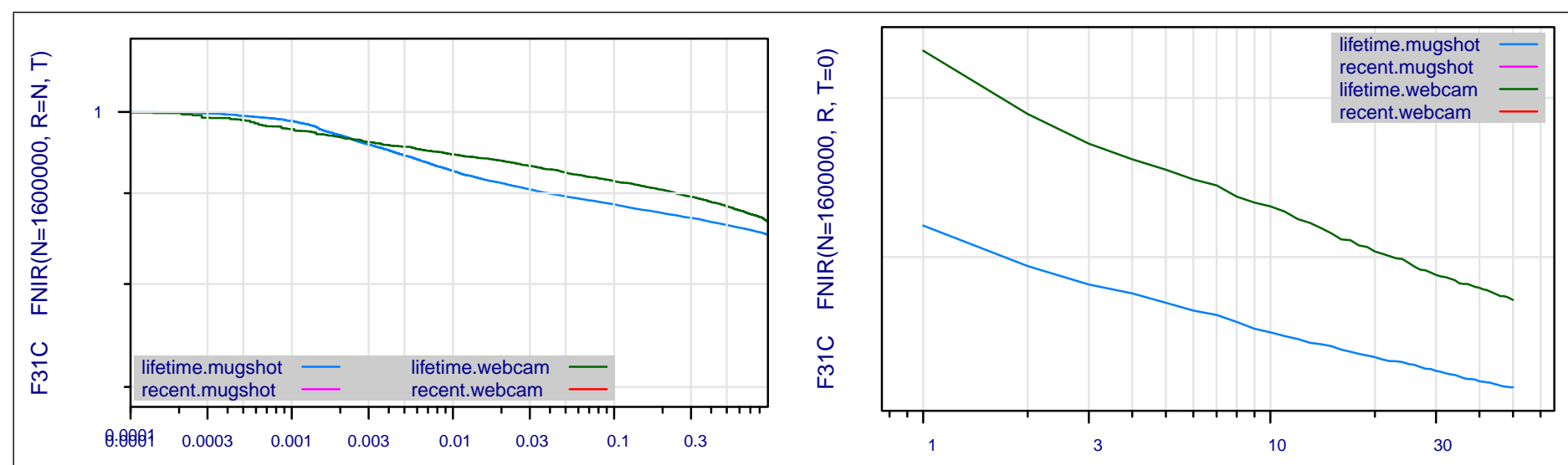

$\operatorname{FPIR}(\mathrm{N}=1600000)$
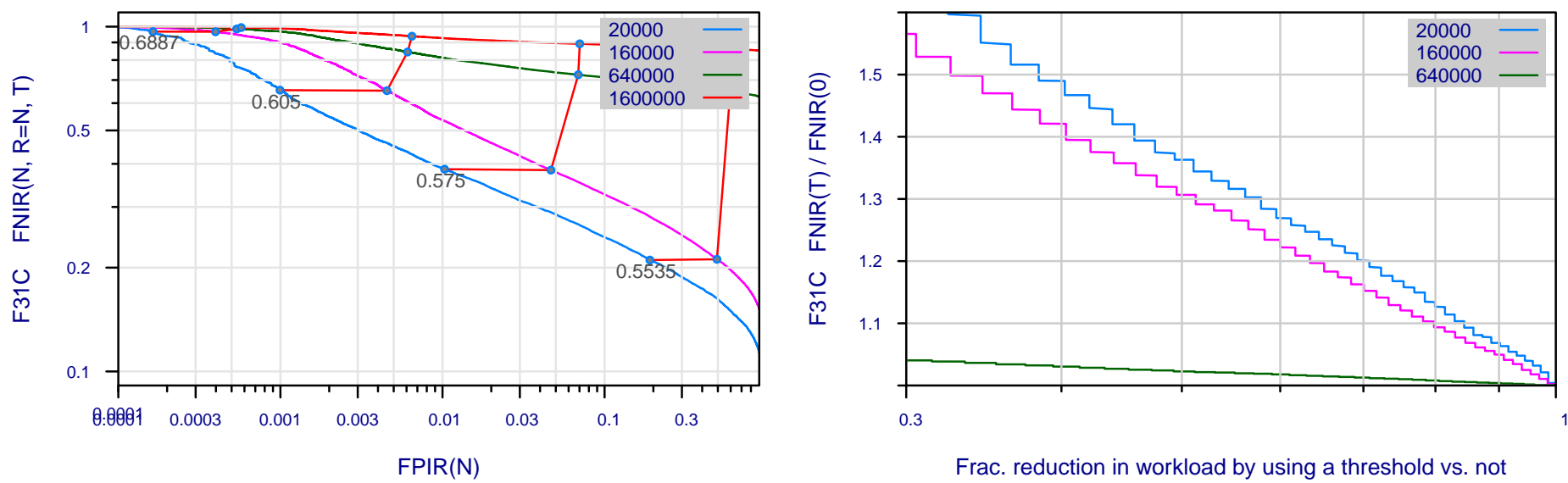

Frac. reduction in workload by using a threshold vs. not
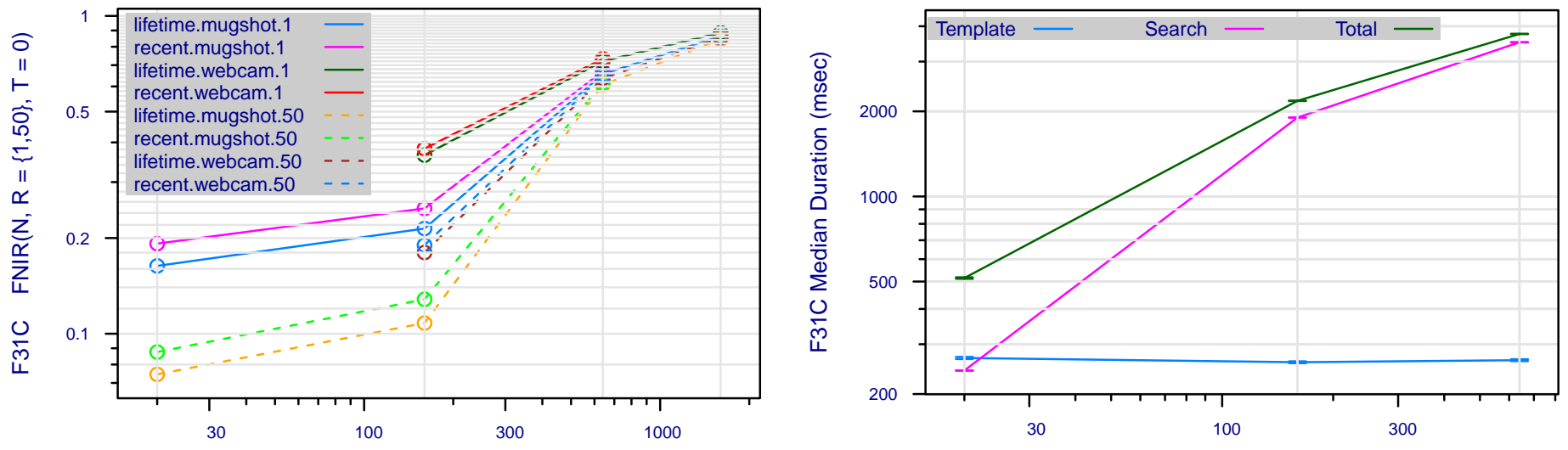

Num. enrolled identities, $\mathrm{N}$ (thousands)

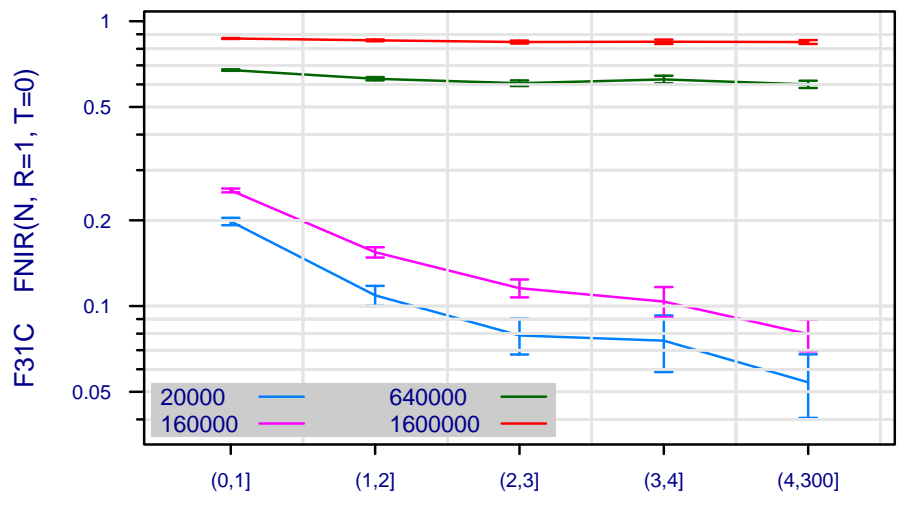

Num. enrolled images per identity

Num. enrolled identities, $\mathrm{N}$ (thousands)

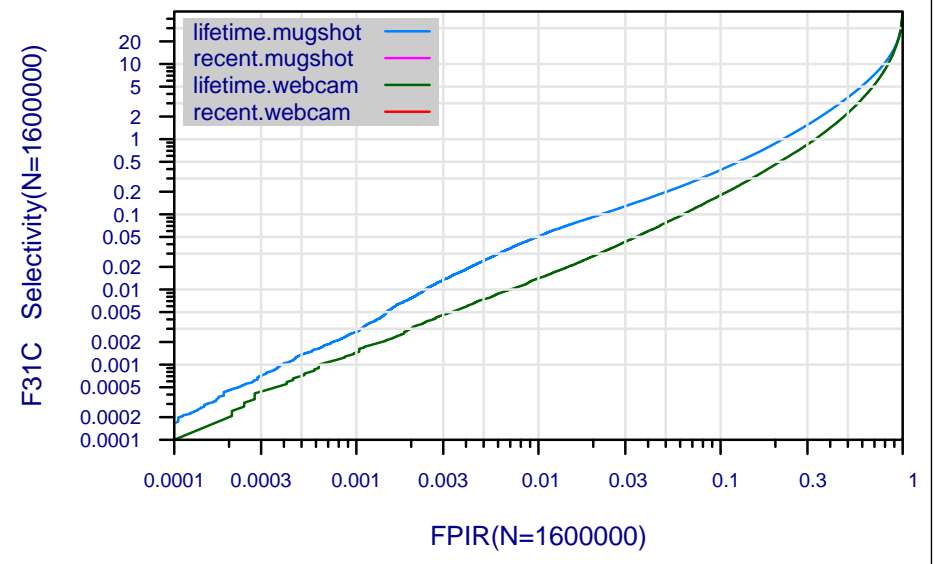

Figure 42: Collected performance reports for algorithm F31C. The figures are described at the beginning of this Appendix.

\begin{tabular}{|c|c|c|c|c|c|c|}
\hline $\begin{array}{l}\mathrm{A}=3 \mathrm{M} / \text { Cogent } \\
\mathrm{G}=\text { Hisign } \\
\mathrm{P}=\text { Zhuhai-Yisheng }\end{array}$ & $\begin{array}{l}B=\text { Cognitec } \\
H=\text { CAS-IA } \\
Q=\text { JunYu }\end{array}$ & $\begin{array}{l}\mathrm{C}=\text { Neurotechnology } \\
\mathrm{I}=\text { CAS-ICT } \\
\mathrm{S}=\text { Decatur }\end{array}$ & $\begin{array}{l}\mathrm{D}=\text { Safran Morpho } \\
\mathrm{J}=\text { Toshiba } \\
\mathrm{T}=\text { Ayonix }\end{array}$ & $\begin{array}{l}\mathrm{E}=\mathrm{NEC} \\
\mathrm{L}=\text { Tsinghua U. II }\end{array}$ & $\begin{array}{l}\mathrm{F}=\text { Tsinghua } \mathrm{U} \\
\mathrm{M}=\mathrm{HP}\end{array}$ & $\begin{array}{l}\text { FNIR(N,R,T,L) "Miss rate" } \\
\text { FPIR(N,T,L) "False alarm rate" }\end{array}$ \\
\hline
\end{tabular}




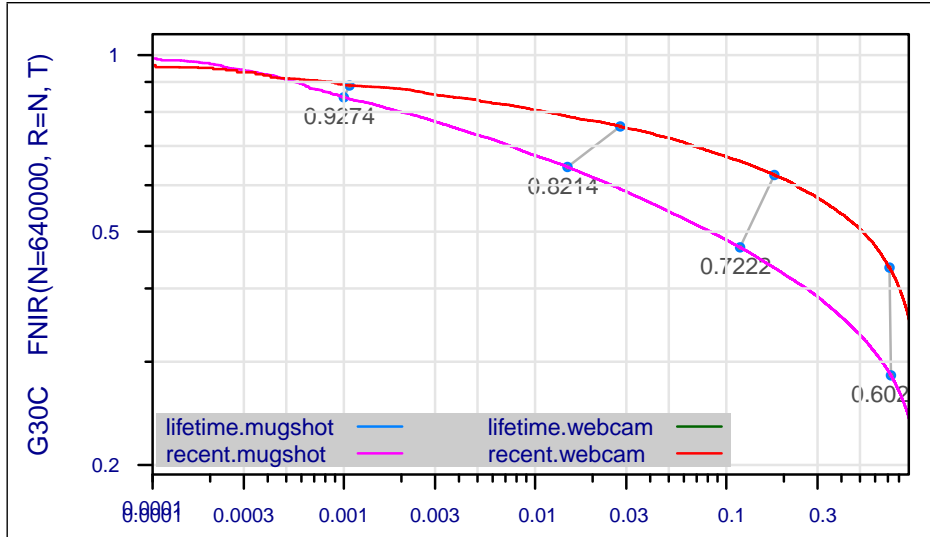

$\operatorname{FPIR}(\mathrm{N}=640000)$
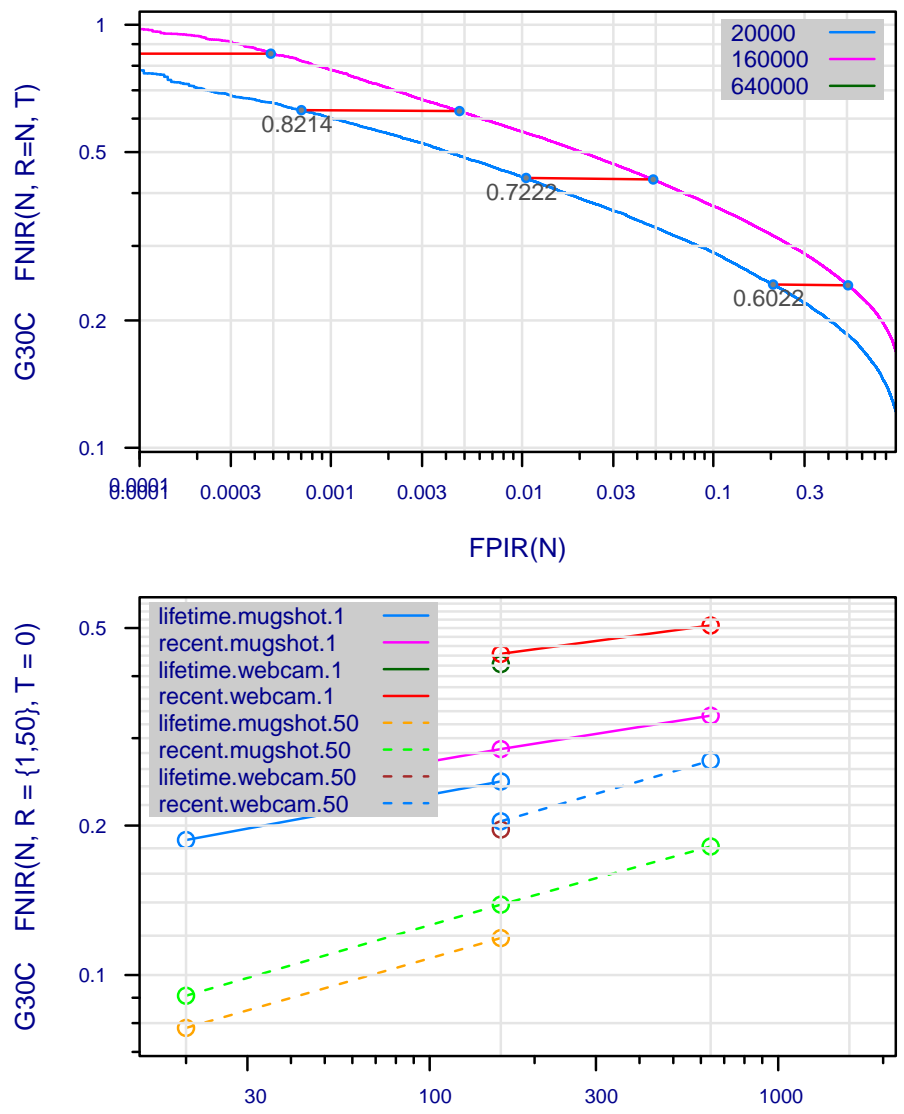

Num. enrolled identities, $\mathrm{N}$ (thousands)

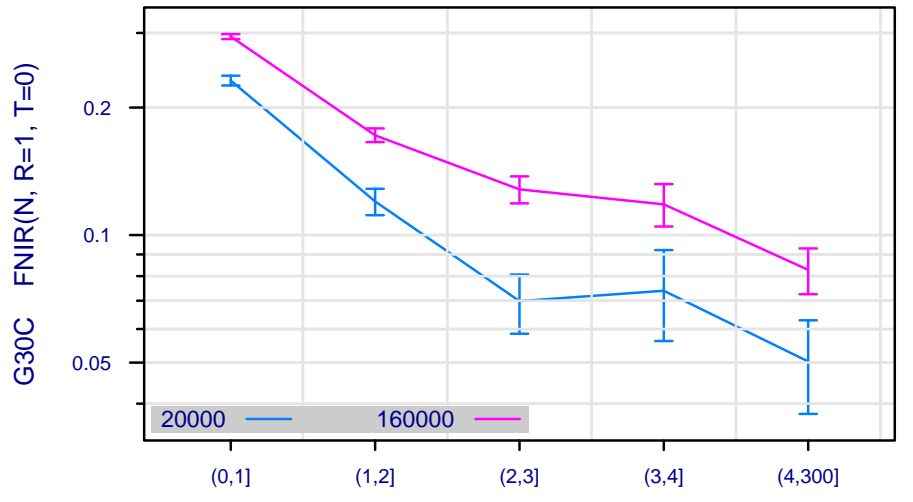

Num. enrolled images per identity

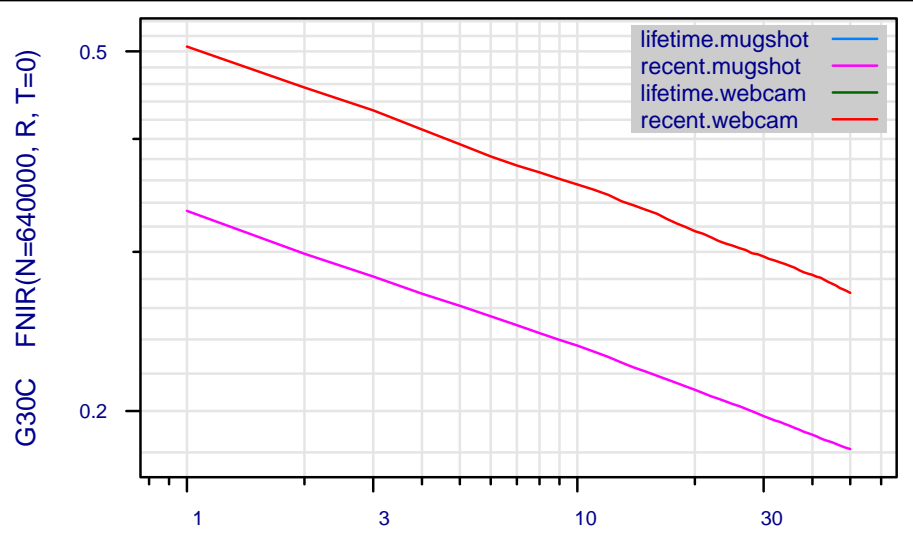

Rank, $\mathrm{R}$

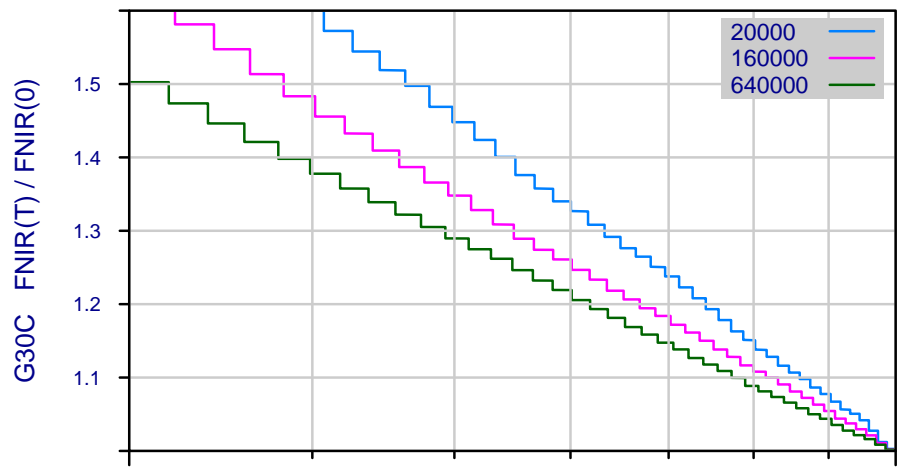

Frac. reduction in workload by using a threshold vs. not

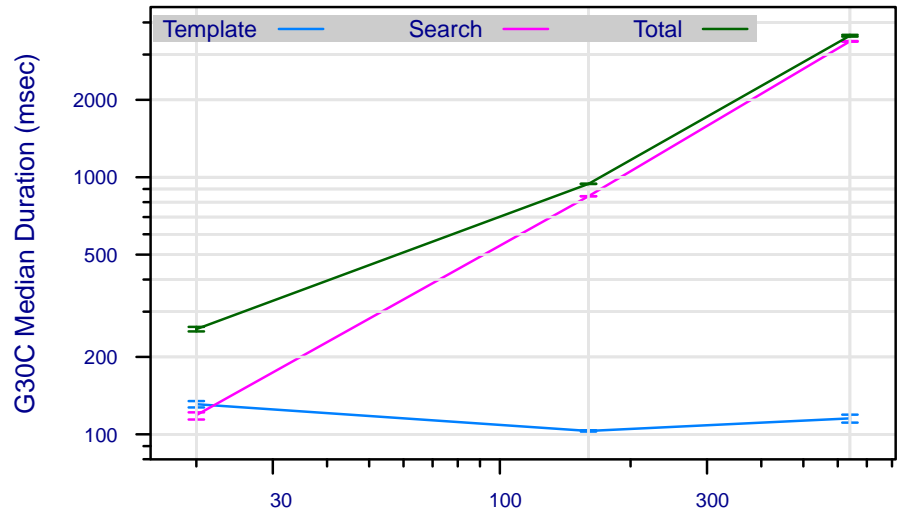

Num. enrolled identities, $\mathrm{N}$ (thousands)

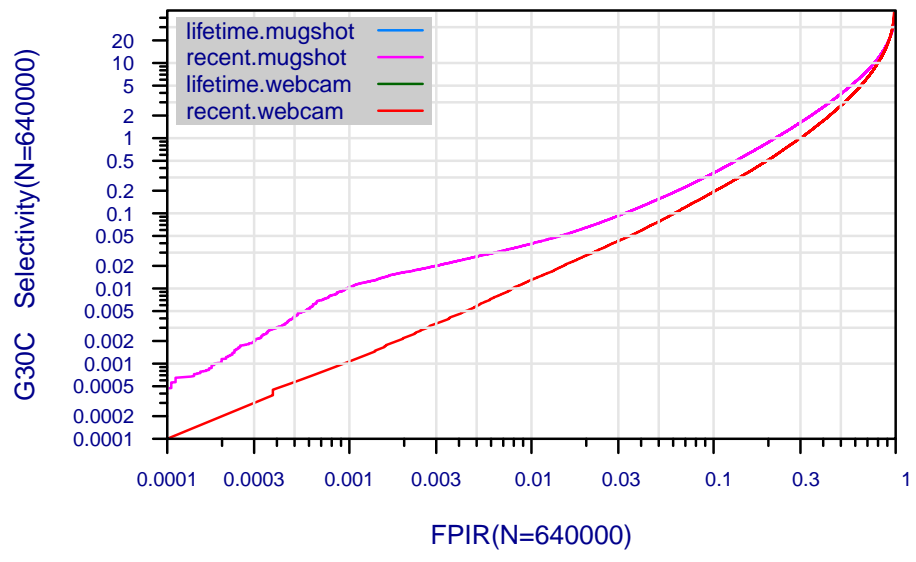

Figure 43: Collected performance reports for algorithm G30C. The figures are described at the beginning of this Appendix.

\begin{tabular}{|c|c|c|c|c|c|c|}
\hline $\begin{array}{l}\mathrm{A}=3 \mathrm{M} / \text { Cogent } \\
\mathrm{G}=\text { Hisign } \\
\mathrm{P}=\text { Zhuhai-Yisheng }\end{array}$ & $\begin{array}{l}\mathrm{B}=\text { Cognitec } \\
\mathrm{H}=\text { CAS-IA } \\
\mathrm{Q}=\text { JunYu }\end{array}$ & $\begin{array}{l}\mathrm{C}=\text { Neurotechnology } \\
\mathrm{I}=\mathrm{CAS}-\mathrm{ICT} \\
\mathrm{S}=\text { Decatur }\end{array}$ & $\begin{array}{l}\mathrm{D}=\text { Safran Morpho } \\
\mathrm{J}=\text { Toshiba } \\
\mathrm{T}=\text { Ayonix }\end{array}$ & $\begin{array}{l}\mathrm{E}=\mathrm{NEC} \\
\mathrm{L}=\text { Tsinghua U. II }\end{array}$ & $\begin{array}{l}\mathrm{F}=\text { Tsinghua } \mathrm{U} . \\
\mathrm{M}=\mathrm{HP}\end{array}$ & $\begin{array}{l}\text { FNIR(N,R,T,L) “Miss rate" } \\
\text { FPIR(N,T,L) “False alarm rate" }\end{array}$ \\
\hline
\end{tabular}



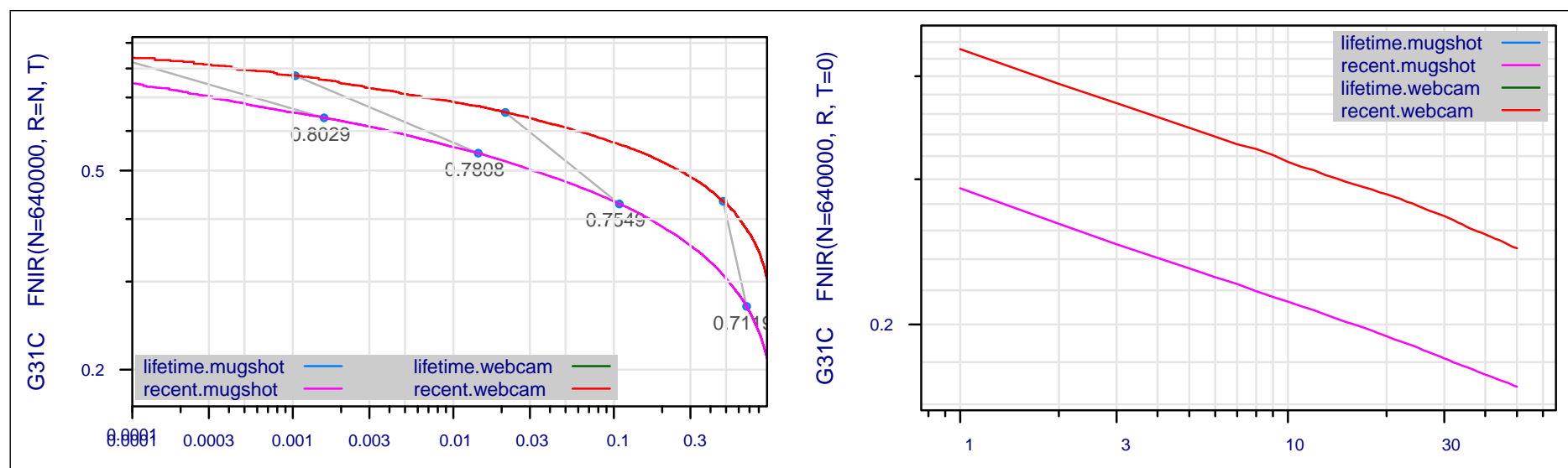

$\operatorname{FPIR}(\mathrm{N}=640000)$
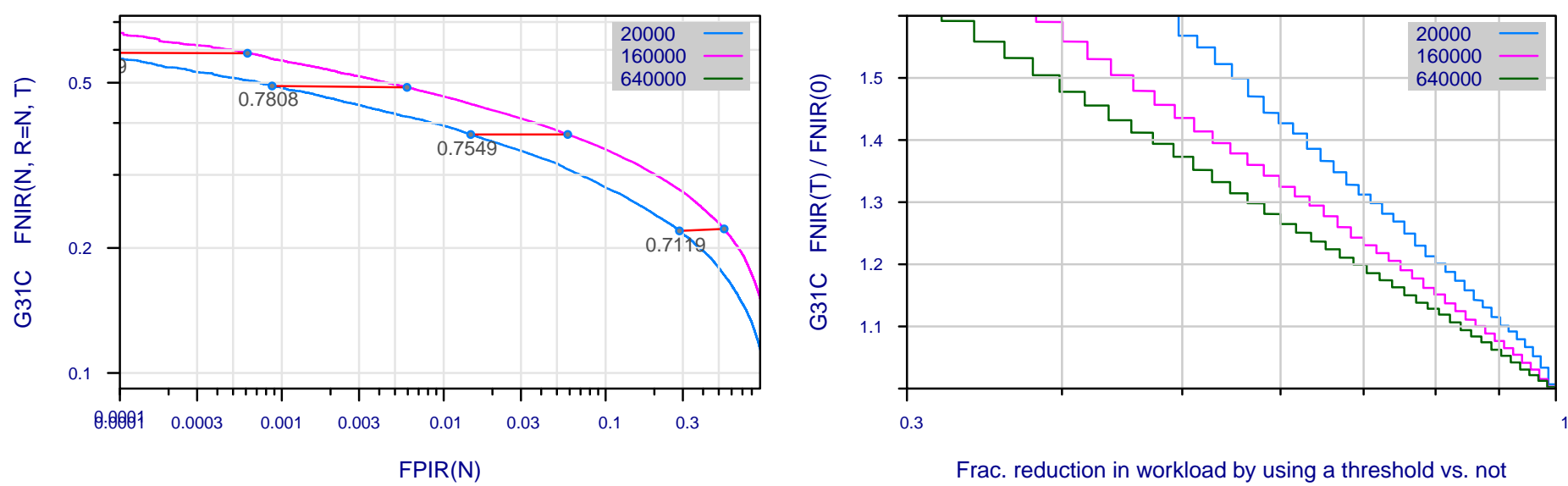

0.3

Frac. reduction in workload by using a threshold vs. not
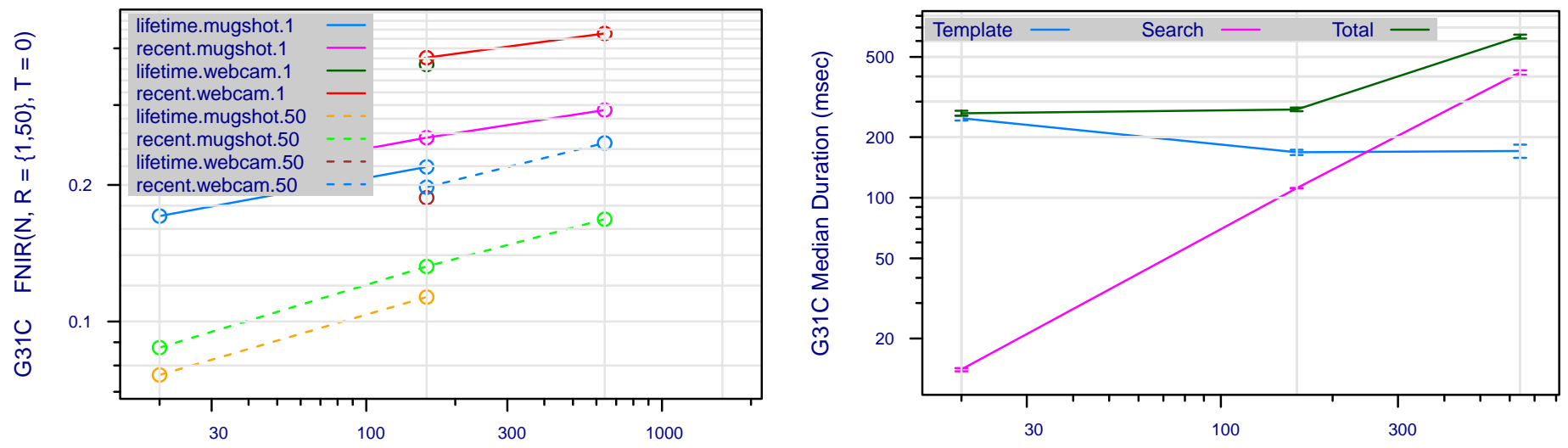

Num. enrolled identities, $\mathrm{N}$ (thousands)

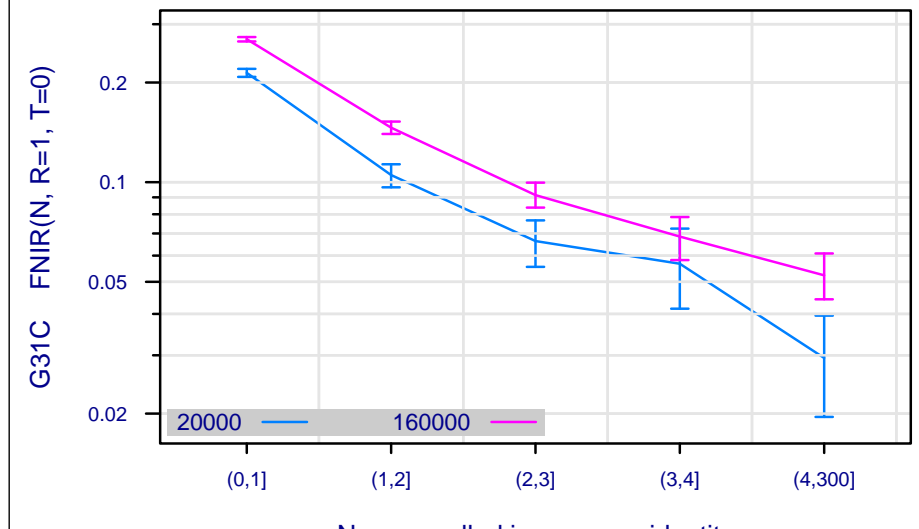

Num. enrolled images per identity

Num. enrolled identities, $\mathrm{N}$ (thousands)

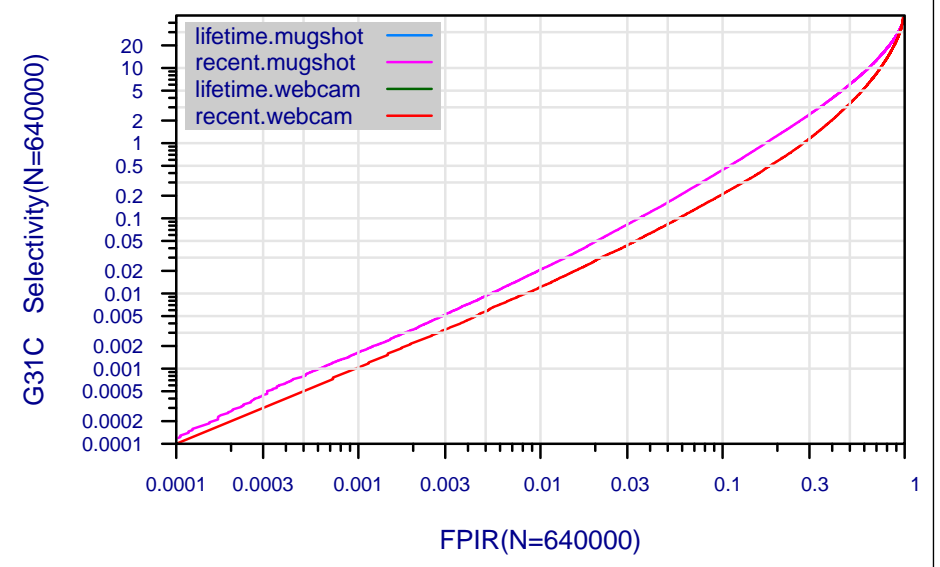

Figure 44: Collected performance reports for algorithm G31C. The figures are described at the beginning of this Appendix.

\begin{tabular}{|c|c|c|c|c|c|c|}
\hline $\begin{array}{l}\mathrm{A}=3 \mathrm{M} / \text { Cogent } \\
\mathrm{G}=\text { Hisign } \\
\mathrm{P}=\text { Zhuhai-Yisheng }\end{array}$ & $\begin{array}{l}\mathrm{B}=\text { Cognitec } \\
\mathrm{H}=\text { CAS-IA } \\
\mathrm{Q}=\text { Jun } \mathrm{Yu}\end{array}$ & $\begin{array}{l}\mathrm{C}=\text { Neurotechnology } \\
\mathrm{I}=\mathrm{CAS}-\mathrm{ICT} \\
\mathrm{S}=\text { Decatur }\end{array}$ & $\begin{array}{l}\mathrm{D}=\text { Safran Morpho } \\
\mathrm{J}=\text { Toshiba } \\
\mathrm{T}=\text { Ayonix }\end{array}$ & $\begin{array}{l}\mathrm{E}=\mathrm{NEC} \\
\mathrm{L}=\text { Tsinghua U. II }\end{array}$ & $\begin{array}{l}\mathrm{F}=\text { Tsinghua } \mathrm{U} . \\
\mathrm{M}=\mathrm{HP}\end{array}$ & $\begin{array}{l}\text { FNIR(N,R,T,L) “Miss rate" } \\
\text { FPIR(N,T,L) “False alarm rate" }\end{array}$ \\
\hline
\end{tabular}



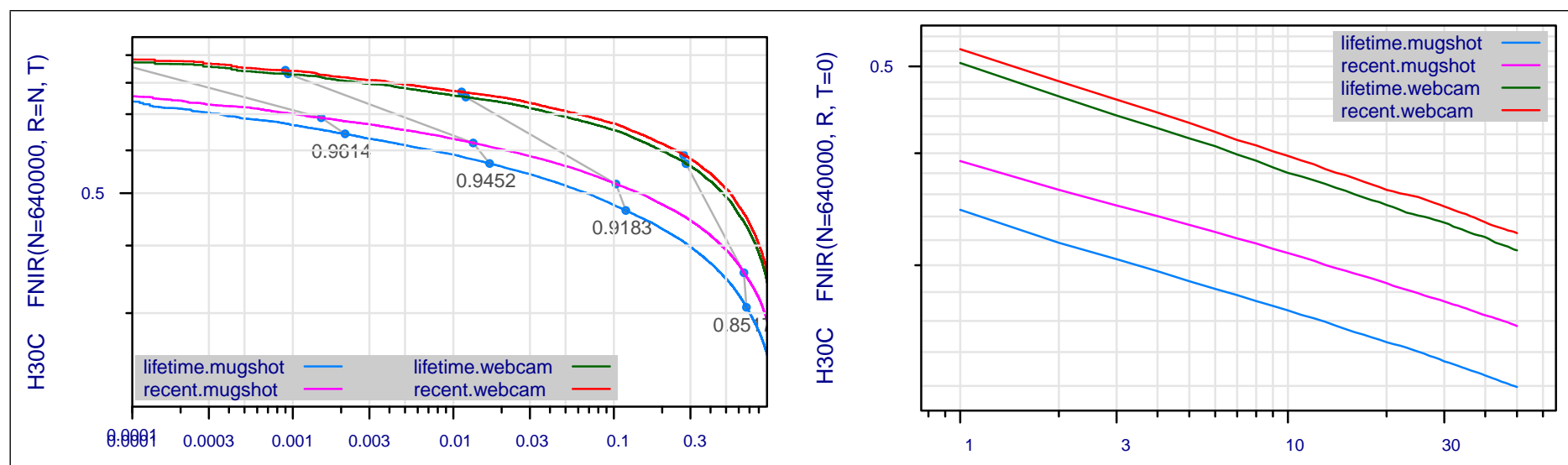

$\operatorname{FPIR}(\mathrm{N}=640000)$
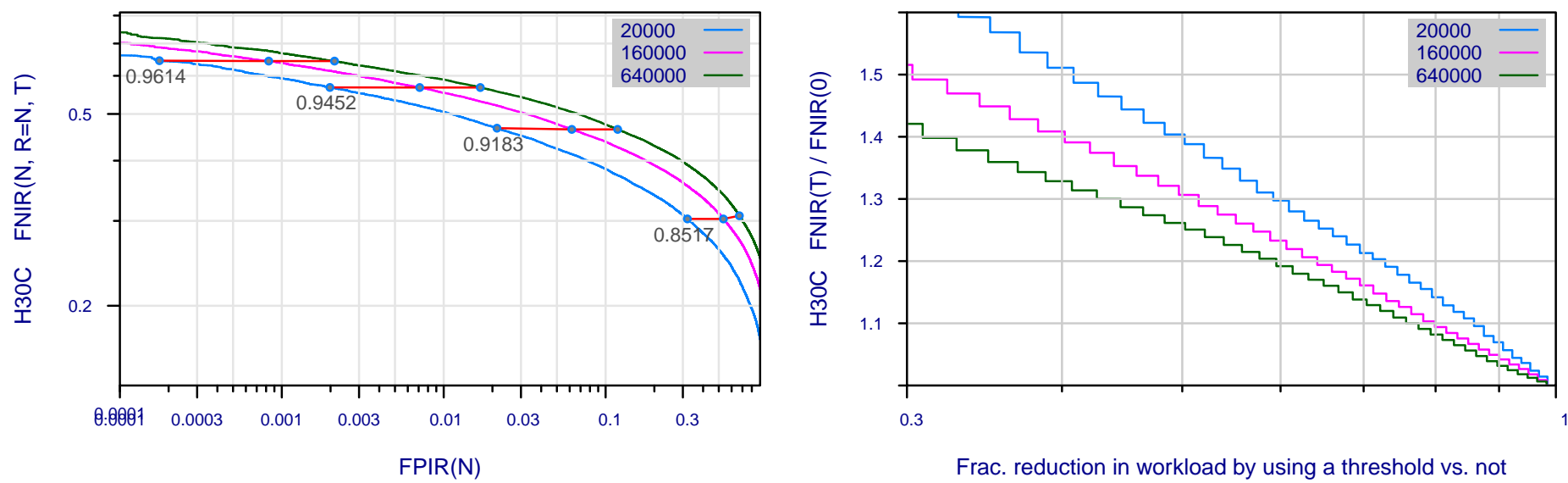

Frac. reduction in workload by using a threshold vs. not
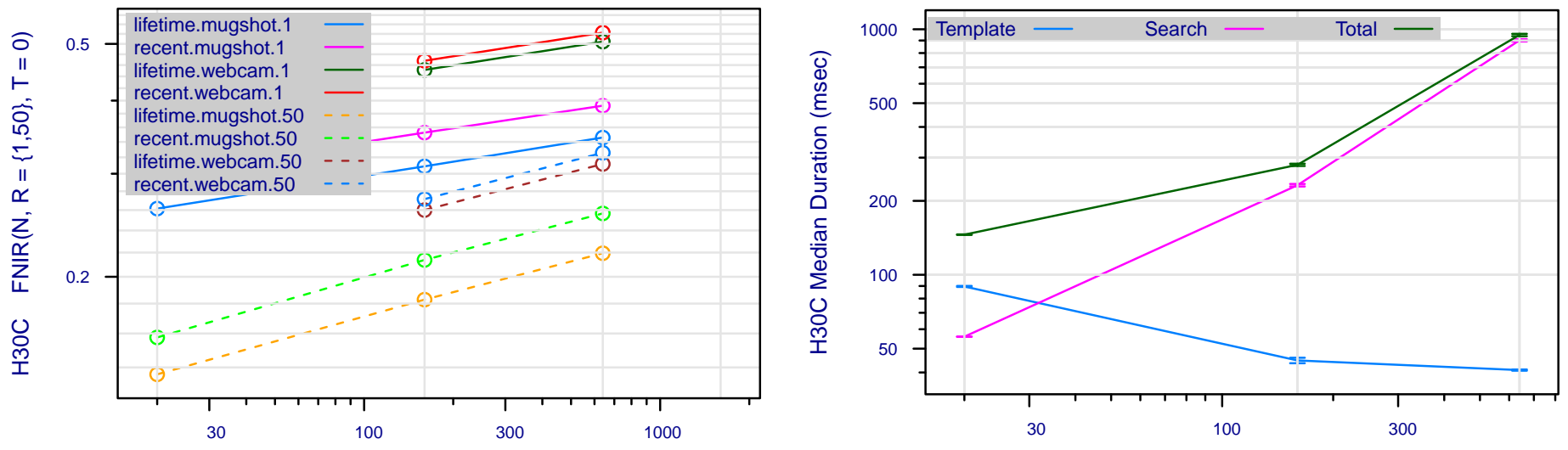

Num. enrolled identities, $\mathrm{N}$ (thousands)

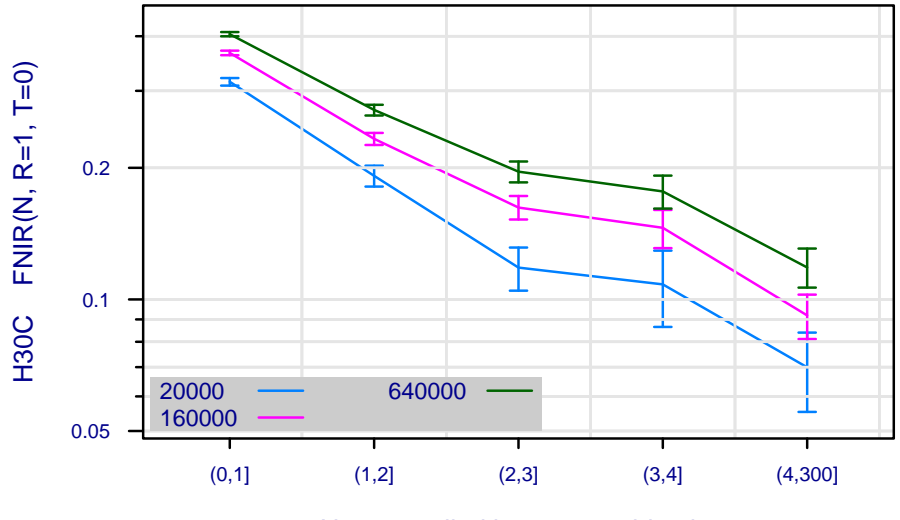

Num. enrolled images per identity

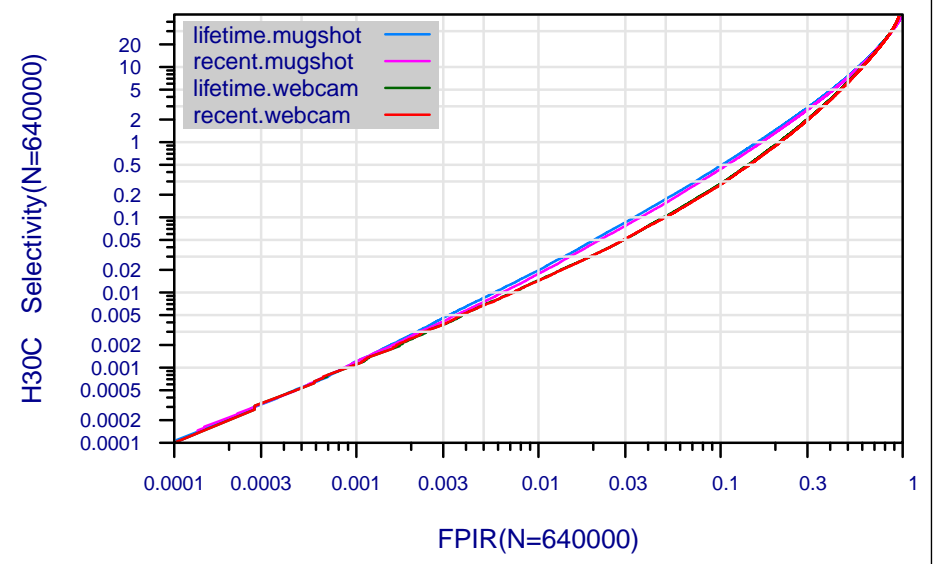

Figure 45: Collected performance reports for algorithm H30C. The figures are described at the beginning of this Appendix.

\begin{tabular}{|c|c|c|c|c|c|c|}
\hline $\begin{array}{l}\mathrm{A}=3 \mathrm{M} / \text { Cogent } \\
\mathrm{G}=\text { Hisign } \\
\mathrm{P}=\text { Zhuhai-Yisheng }\end{array}$ & $\begin{array}{l}B=\text { Cognitec } \\
H=\text { CAS-IA } \\
Q=\text { JunYu }\end{array}$ & $\begin{array}{l}\mathrm{C}=\text { Neurotechnology } \\
\mathrm{I}=\text { CAS-ICT } \\
\mathrm{S}=\text { Decatur }\end{array}$ & $\begin{array}{l}\mathrm{D}=\text { Safran Morpho } \\
\mathrm{J}=\text { Toshiba } \\
\mathrm{T}=\text { Ayonix }\end{array}$ & $\begin{array}{l}\mathrm{E}=\mathrm{NEC} \\
\mathrm{L}=\text { Tsinghua U. II }\end{array}$ & $\begin{array}{l}\mathrm{F}=\text { Tsinghua } \mathrm{U} \\
\mathrm{M}=\mathrm{HP}\end{array}$ & $\begin{array}{l}\text { FNIR(N,R,T,L) “Miss rate" } \\
\operatorname{FPIR}(\mathrm{N}, \mathrm{T}, \mathrm{L}) \text { “False alarm rate" }\end{array}$ \\
\hline
\end{tabular}



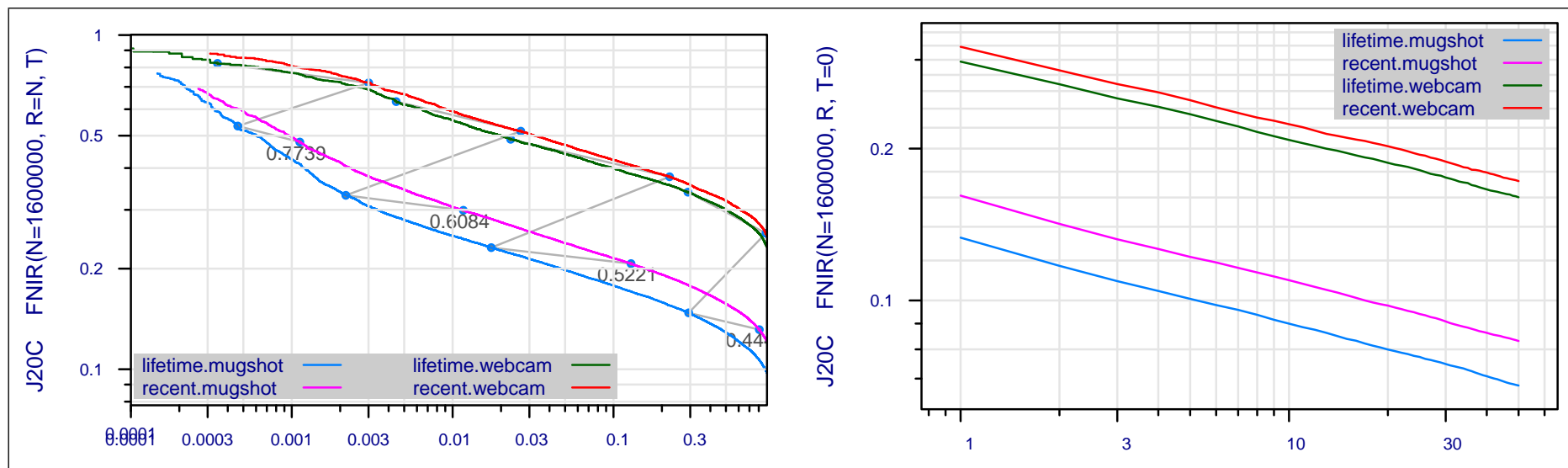

$\operatorname{FPIR}(\mathrm{N}=1600000)$
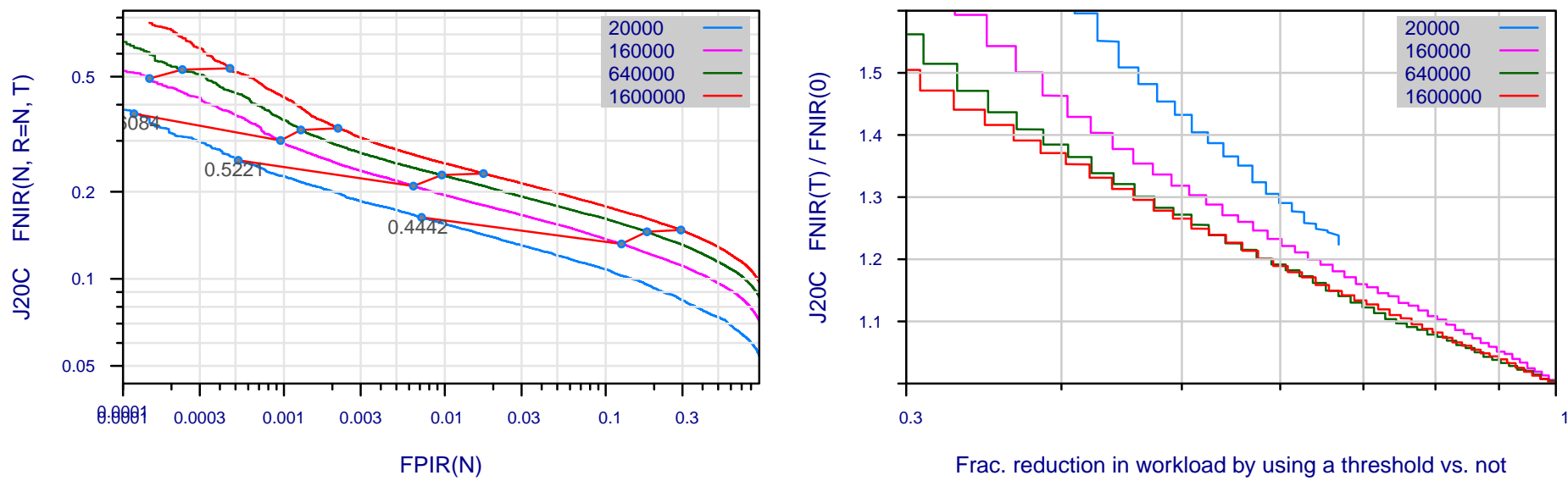

0.3

Frac. reduction in workload by using a threshold vs. not
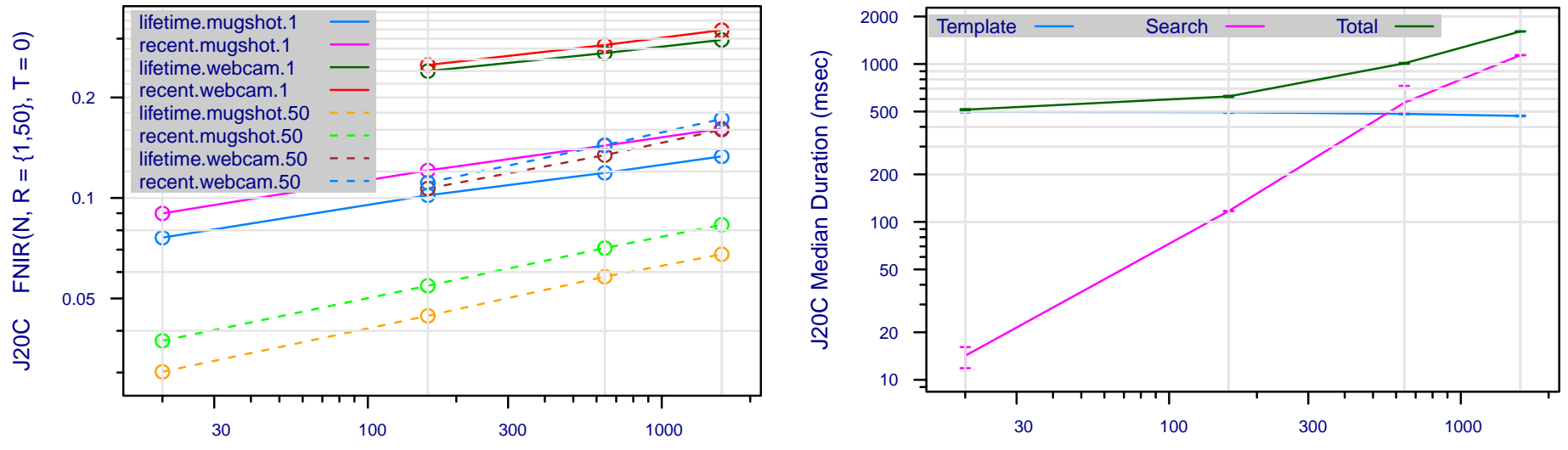

Num. enrolled identities, $\mathrm{N}$ (thousands)

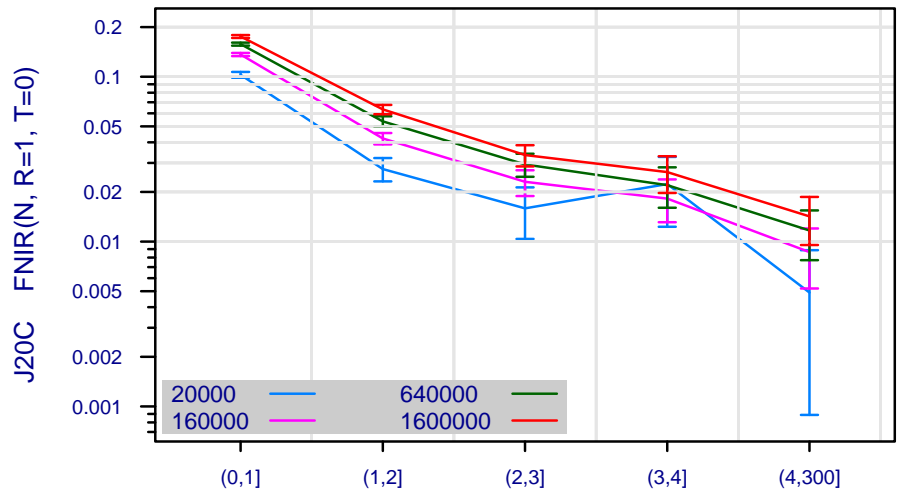

Num. enrolled images per identity

Num. enrolled identities, $\mathrm{N}$ (thousands)

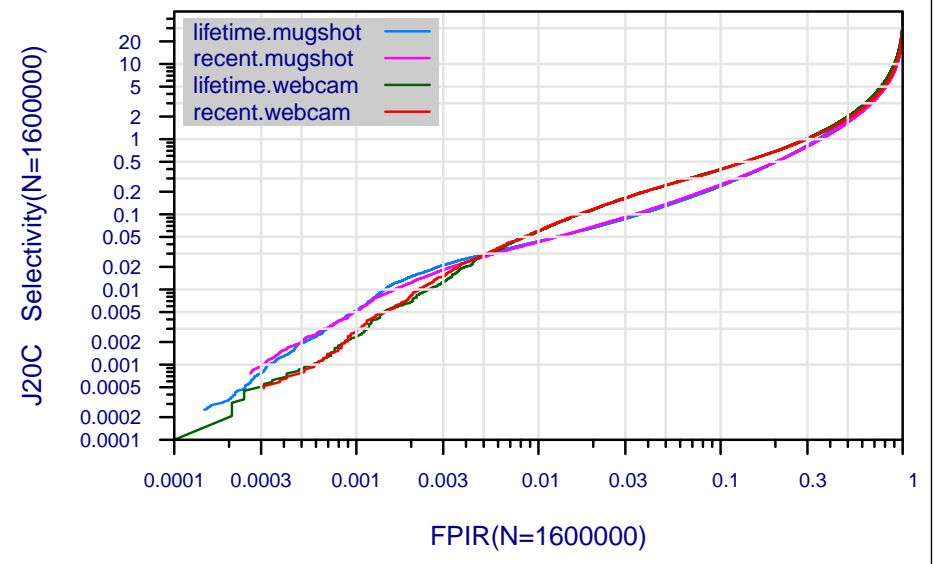

Figure 46: Collected performance reports for algorithm J20C. The figures are described at the beginning of this Appendix.

\begin{tabular}{|c|c|c|c|c|c|c|}
\hline $\begin{array}{l}\mathrm{A}=3 \mathrm{M} / \text { Cogent } \\
\mathrm{G}=\text { Hisign } \\
\mathrm{P}=\text { Zhuhai-Yisheng }\end{array}$ & $\begin{array}{l}B=\text { Cognitec } \\
H=\text { CAS-IA } \\
Q=\text { JunYu }\end{array}$ & $\begin{array}{l}\mathrm{C}=\text { Neurotechnology } \\
\mathrm{I}=\text { CAS-ICT } \\
\mathrm{S}=\text { Decatur }\end{array}$ & $\begin{array}{l}\mathrm{D}=\text { Safran Morpho } \\
\mathrm{J}=\text { Toshiba } \\
\mathrm{T}=\text { Ayonix }\end{array}$ & $\begin{array}{l}\mathrm{E}=\mathrm{NEC} \\
\mathrm{L}=\text { Tsinghua U. II }\end{array}$ & $\begin{array}{l}\mathrm{F}=\text { Tsinghua } \mathrm{U} \\
\mathrm{M}=\mathrm{HP}\end{array}$ & $\begin{array}{l}\text { FNIR(N,R,T,L) “Miss rate" } \\
\operatorname{FPIR}(\mathrm{N}, \mathrm{T}, \mathrm{L}) \text { “False alarm rate" }\end{array}$ \\
\hline
\end{tabular}



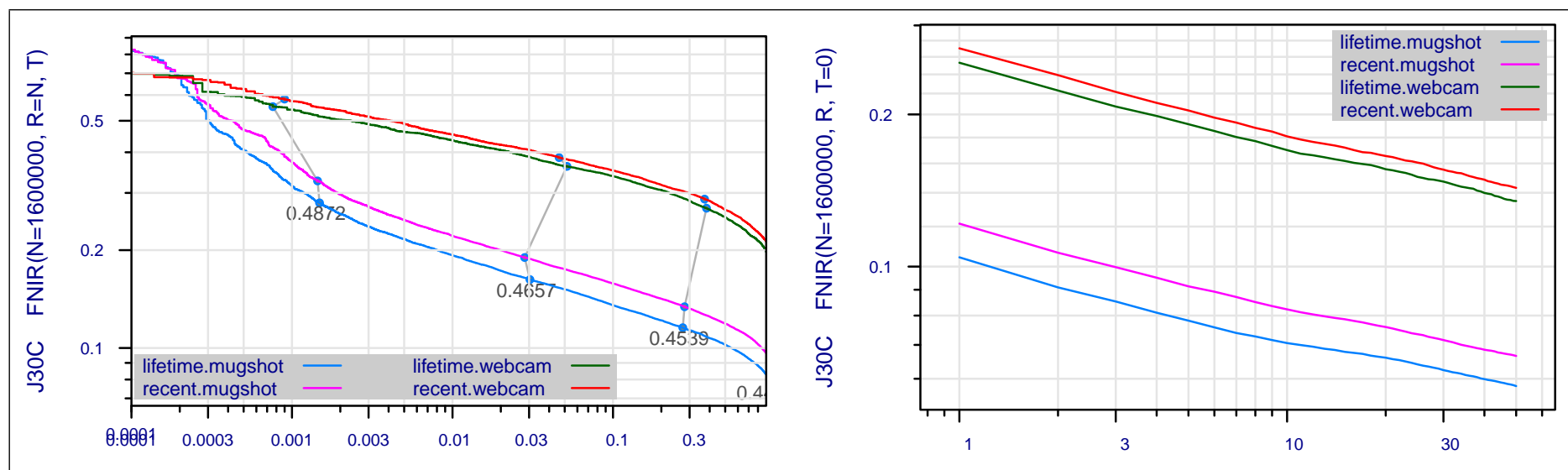

$\operatorname{FPIR}(\mathrm{N}=1600000)$
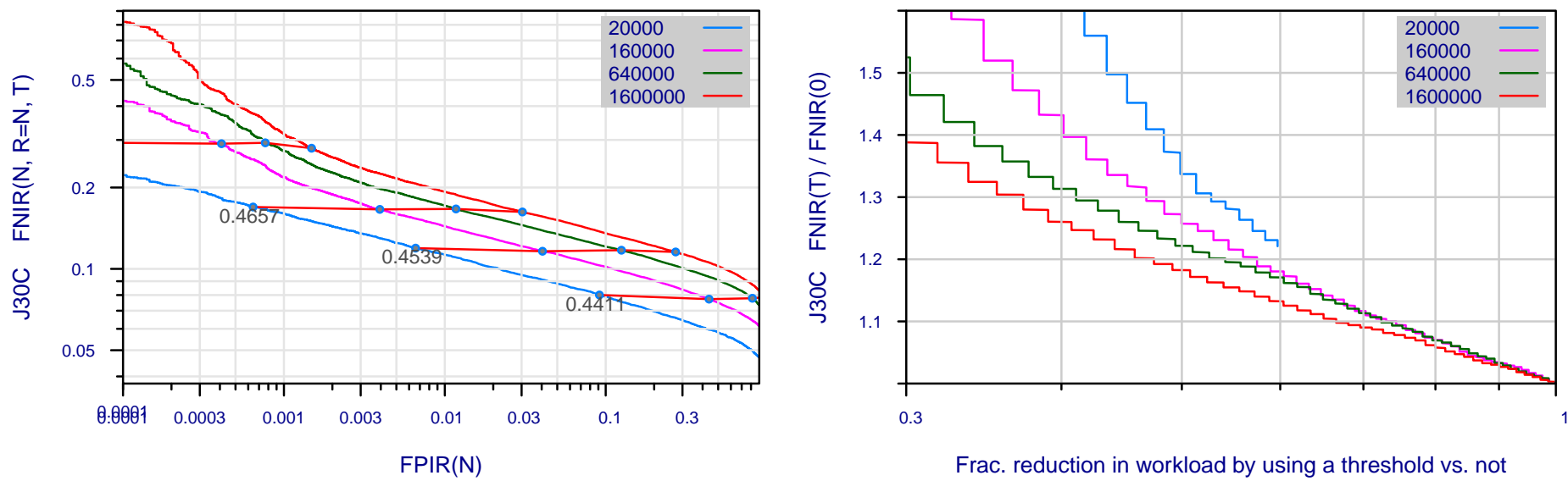

Frac. reduction in workload by using a threshold vs. not
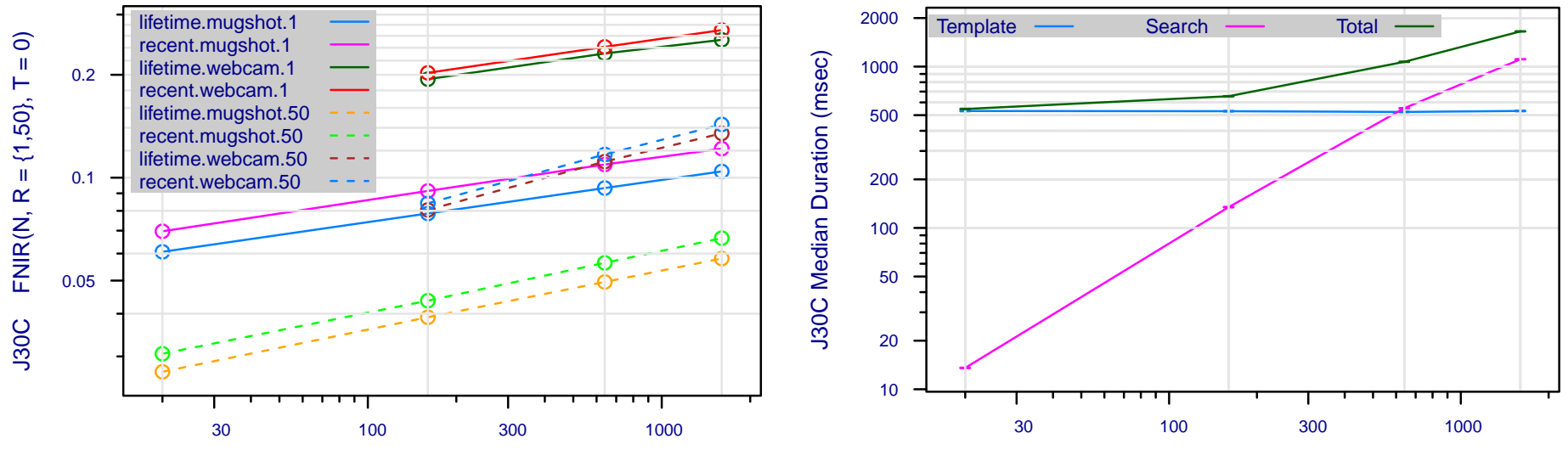

Num. enrolled identities, $\mathrm{N}$ (thousands)

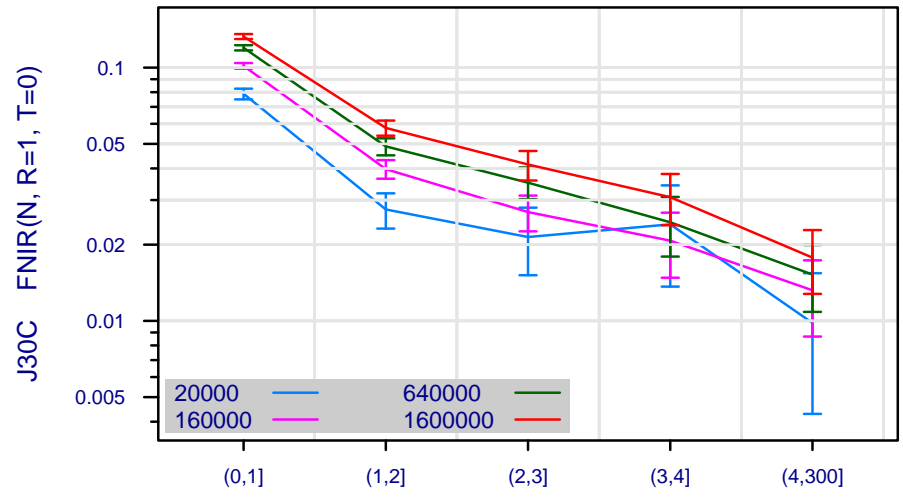

Num. enrolled images per identity

Num. enrolled identities, $\mathrm{N}$ (thousands)

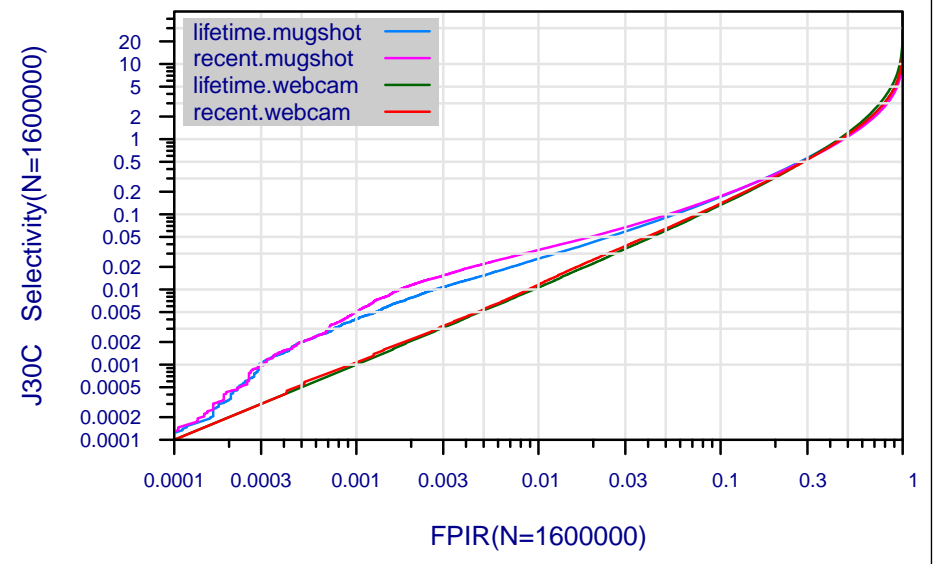

Figure 47: Collected performance reports for algorithm J30C. The figures are described at the beginning of this Appendix.

\begin{tabular}{|c|c|c|c|c|c|c|}
\hline $\begin{array}{l}\mathrm{A}=3 \mathrm{M} / \text { Cogent } \\
\mathrm{G}=\text { Hisign } \\
\mathrm{P}=\text { Zhuhai-Yisheng }\end{array}$ & $\begin{array}{l}B=\text { Cognitec } \\
H=\text { CAS-IA } \\
Q=\text { JunYu }\end{array}$ & $\begin{array}{l}\mathrm{C}=\text { Neurotechnology } \\
\mathrm{I}=\text { CAS-ICT } \\
\mathrm{S}=\text { Decatur }\end{array}$ & $\begin{array}{l}\mathrm{D}=\text { Safran Morpho } \\
\mathrm{J}=\text { Toshiba } \\
\mathrm{T}=\text { Ayonix }\end{array}$ & $\begin{array}{l}\mathrm{E}=\mathrm{NEC} \\
\mathrm{L}=\text { Tsinghua U. II }\end{array}$ & $\begin{array}{l}\mathrm{F}=\text { Tsinghua } \mathrm{U} \\
\mathrm{M}=\mathrm{HP}\end{array}$ & $\begin{array}{l}\text { FNIR(N,R,T,L) “Miss rate" } \\
\operatorname{FPIR}(\mathrm{N}, \mathrm{T}, \mathrm{L}) \text { “False alarm rate" }\end{array}$ \\
\hline
\end{tabular}



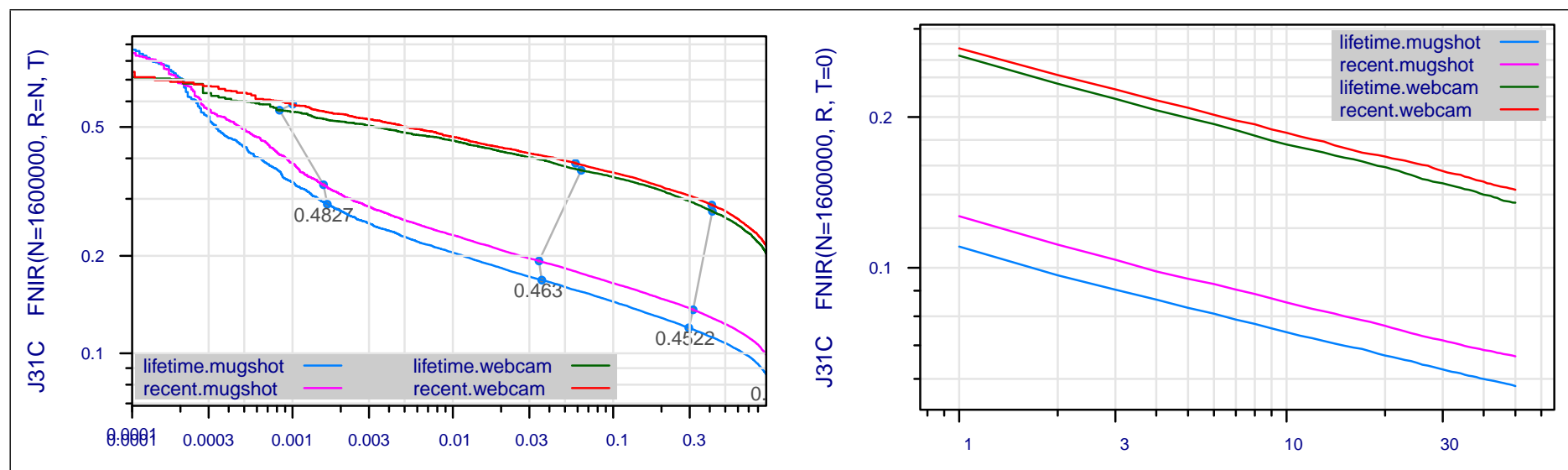

$\operatorname{FPIR}(\mathrm{N}=1600000)$
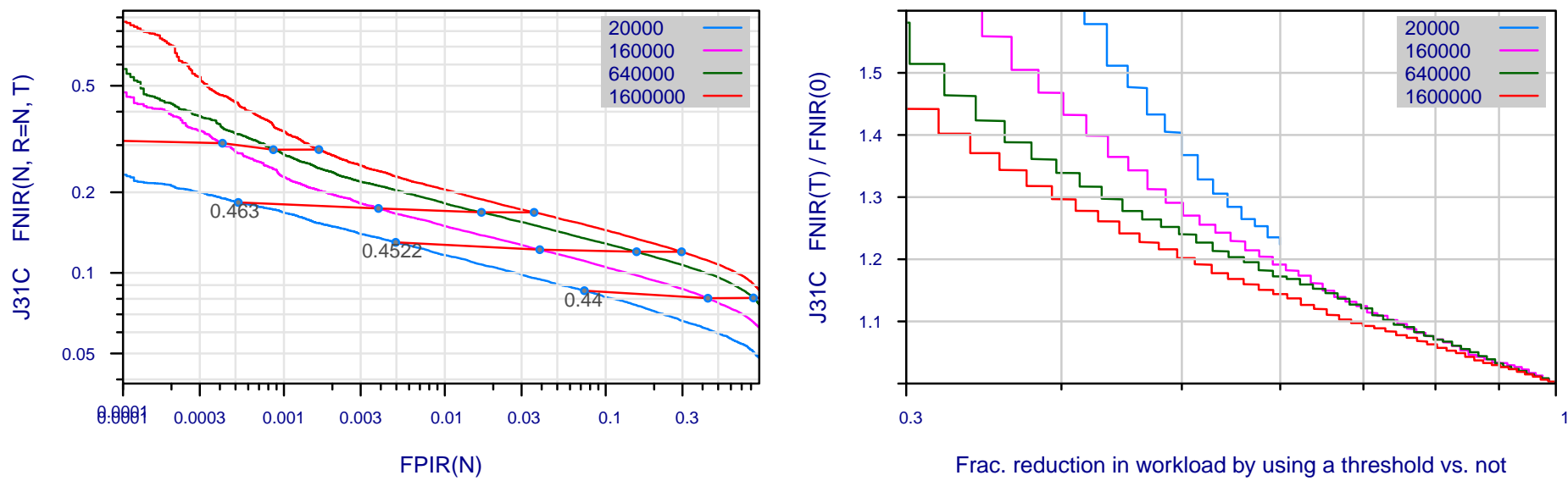

0.3

Frac. reduction in workload by using a threshold vs. not
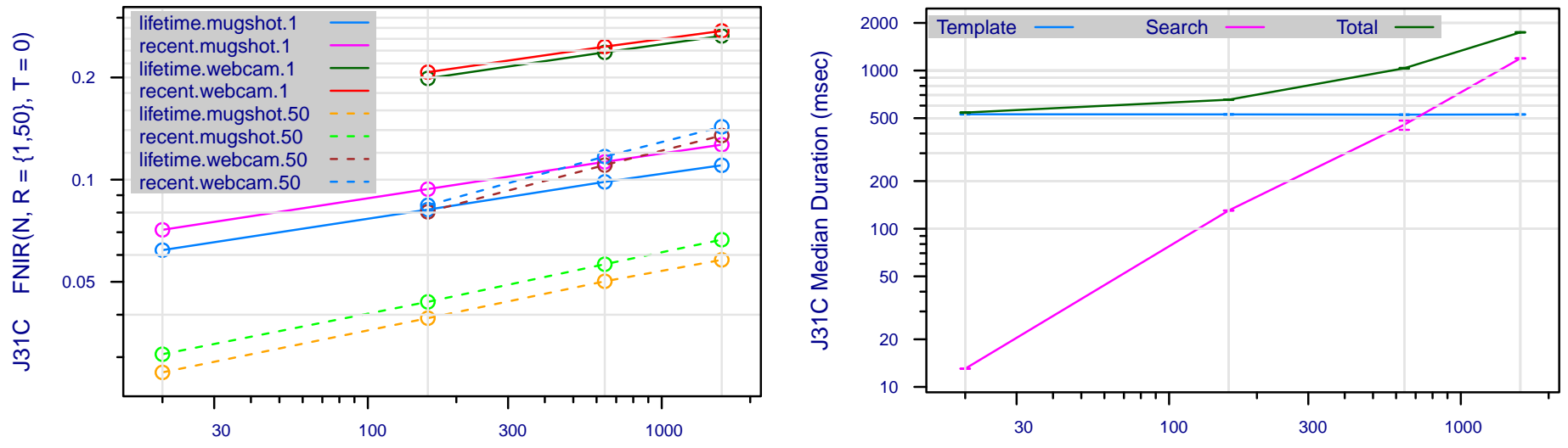

Num. enrolled identities, $\mathrm{N}$ (thousands)

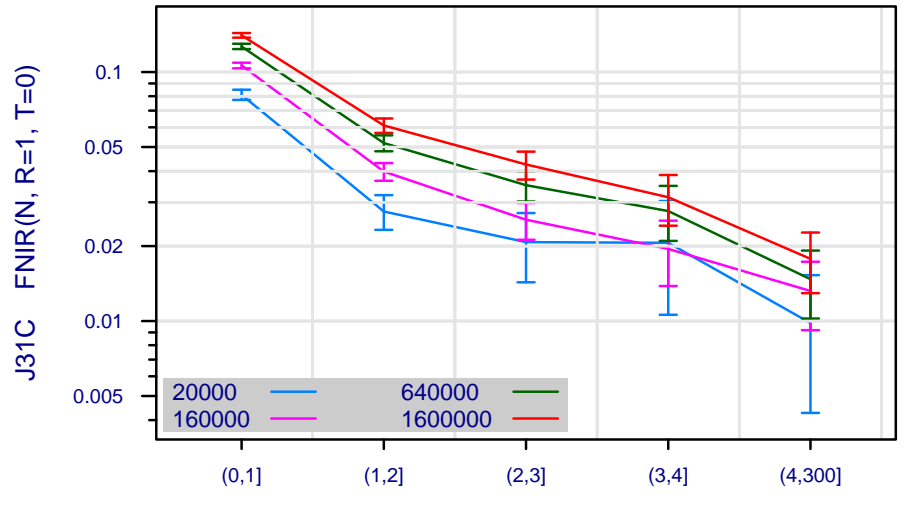

Num. enrolled images per identity

Num. enrolled identities, $\mathrm{N}$ (thousands)

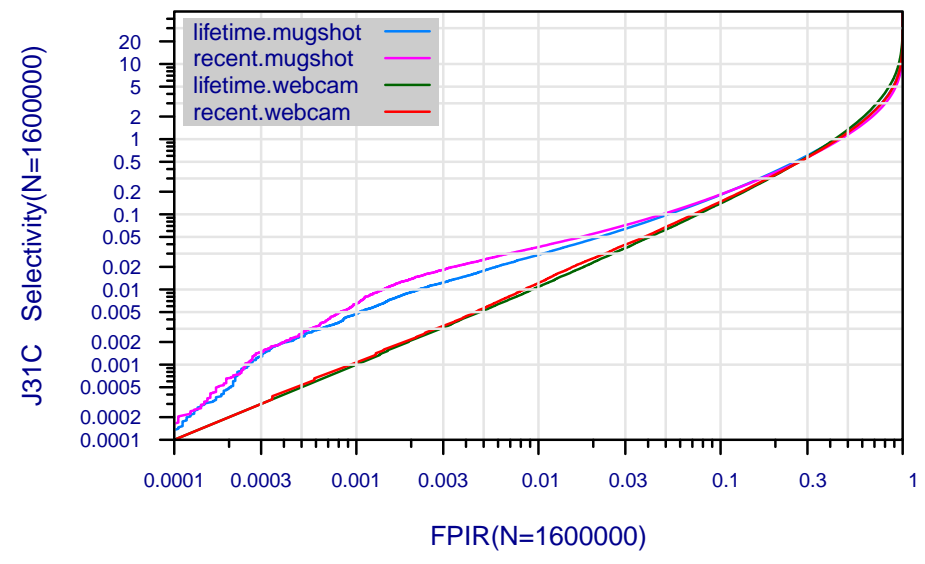

Figure 48: Collected performance reports for algorithm J31C. The figures are described at the beginning of this Appendix.

\begin{tabular}{|c|c|c|c|c|c|c|}
\hline $\begin{array}{l}\mathrm{A}=3 \mathrm{M} / \text { Cogent } \\
\mathrm{G}=\text { Hisign } \\
\mathrm{P}=\text { Zhuhai-Yisheng }\end{array}$ & $\begin{array}{l}B=\text { Cognitec } \\
H=\text { CAS-IA } \\
Q=\text { JunYu }\end{array}$ & $\begin{array}{l}\mathrm{C}=\text { Neurotechnology } \\
\mathrm{I}=\text { CAS-ICT } \\
\mathrm{S}=\text { Decatur }\end{array}$ & $\begin{array}{l}\mathrm{D}=\text { Safran Morpho } \\
\mathrm{J}=\text { Toshiba } \\
\mathrm{T}=\text { Ayonix }\end{array}$ & $\begin{array}{l}\mathrm{E}=\mathrm{NEC} \\
\mathrm{L}=\text { Tsinghua U. II }\end{array}$ & $\begin{array}{l}\mathrm{F}=\text { Tsinghua } \mathrm{U} \\
\mathrm{M}=\mathrm{HP}\end{array}$ & $\begin{array}{l}\text { FNIR(N,R,T,L) “Miss rate" } \\
\operatorname{FPIR}(\mathrm{N}, \mathrm{T}, \mathrm{L}) \text { “False alarm rate" }\end{array}$ \\
\hline
\end{tabular}



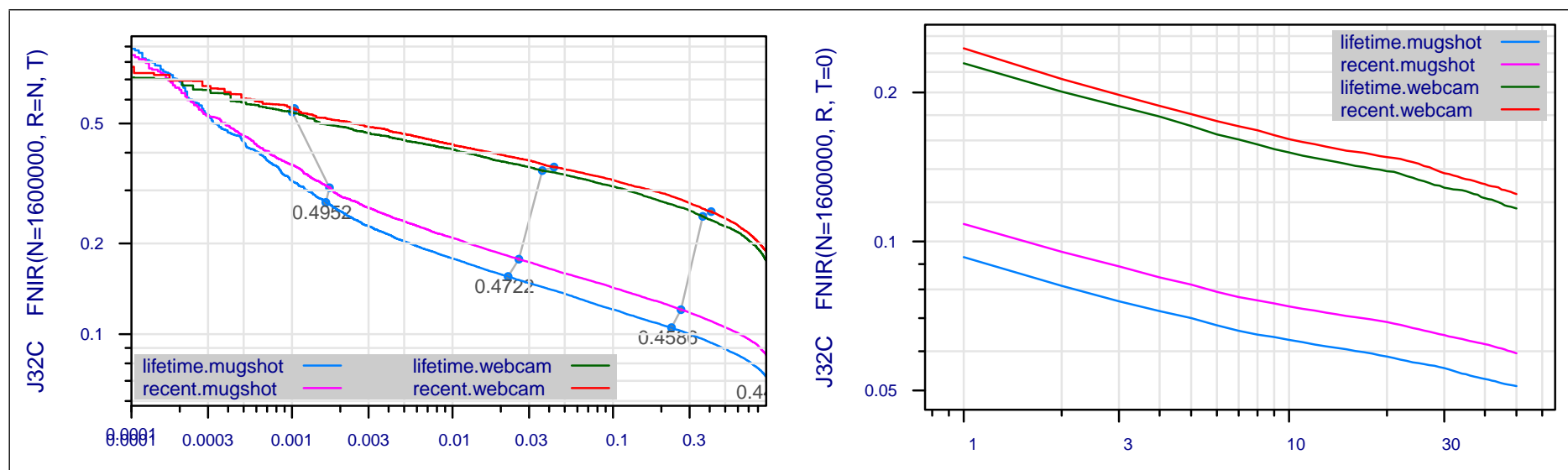

$\operatorname{FPIR}(\mathrm{N}=1600000)$
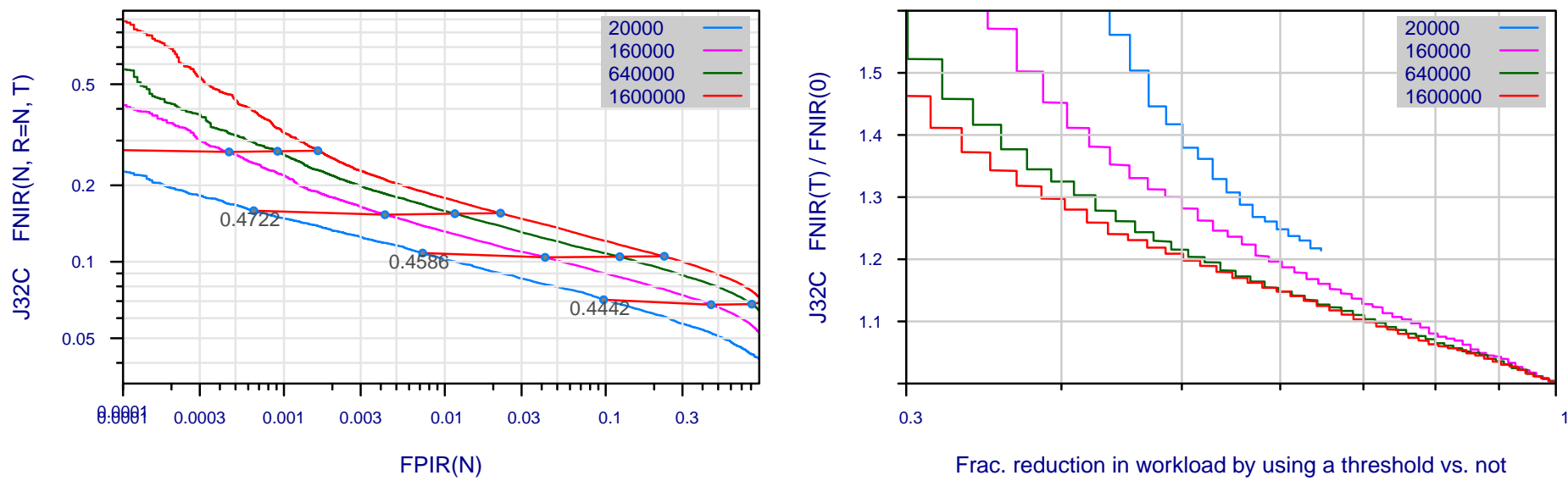

0.3

Frac. reduction in workload by using a threshold vs. not
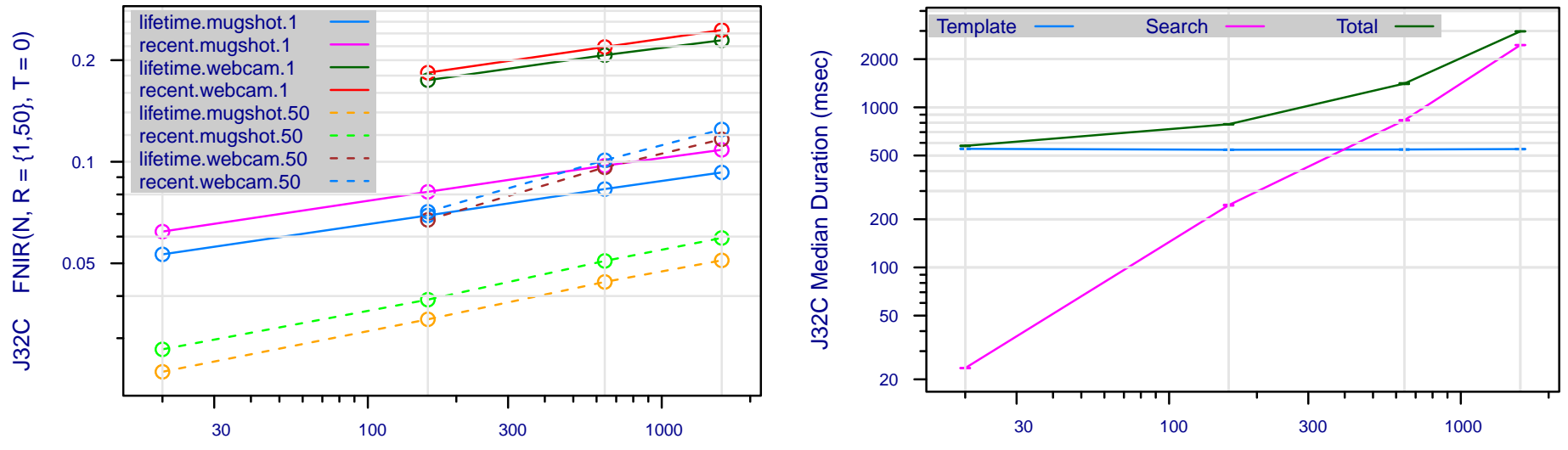

Num. enrolled identities, $\mathrm{N}$ (thousands)

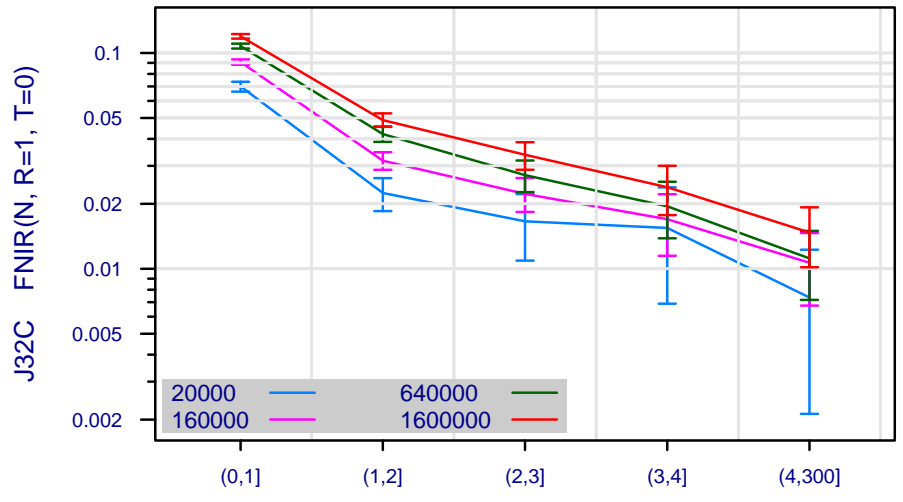

Num. enrolled images per identity

Num. enrolled identities, $\mathrm{N}$ (thousands)

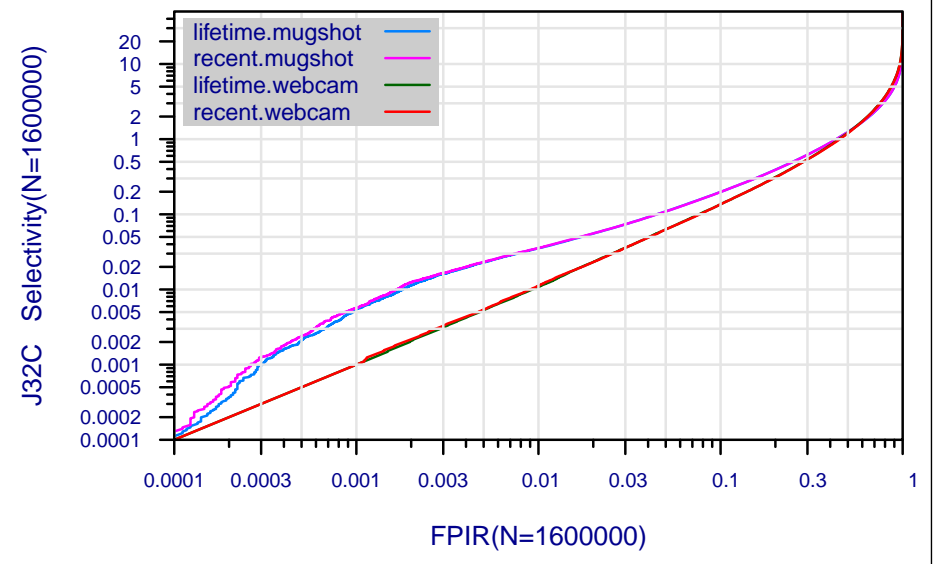

Figure 49: Collected performance reports for algorithm J32C. The figures are described at the beginning of this Appendix.

\begin{tabular}{|c|c|c|c|c|c|c|}
\hline $\begin{array}{l}\mathrm{A}=3 \mathrm{M} / \text { Cogent } \\
\mathrm{G}=\text { Hisign } \\
\mathrm{P}=\text { Zhuhai-Yisheng }\end{array}$ & $\begin{array}{l}B=\text { Cognitec } \\
H=\text { CAS-IA } \\
Q=\text { JunYu }\end{array}$ & $\begin{array}{l}\mathrm{C}=\text { Neurotechnology } \\
\mathrm{I}=\text { CAS-ICT } \\
\mathrm{S}=\text { Decatur }\end{array}$ & $\begin{array}{l}\mathrm{D}=\text { Safran Morpho } \\
\mathrm{J}=\text { Toshiba } \\
\mathrm{T}=\text { Ayonix }\end{array}$ & $\begin{array}{l}\mathrm{E}=\mathrm{NEC} \\
\mathrm{L}=\text { Tsinghua U. II }\end{array}$ & $\begin{array}{l}\mathrm{F}=\text { Tsinghua } \mathrm{U} \\
\mathrm{M}=\mathrm{HP}\end{array}$ & $\begin{array}{l}\text { FNIR(N,R,T,L) “Miss rate" } \\
\operatorname{FPIR}(\mathrm{N}, \mathrm{T}, \mathrm{L}) \text { “False alarm rate" }\end{array}$ \\
\hline
\end{tabular}



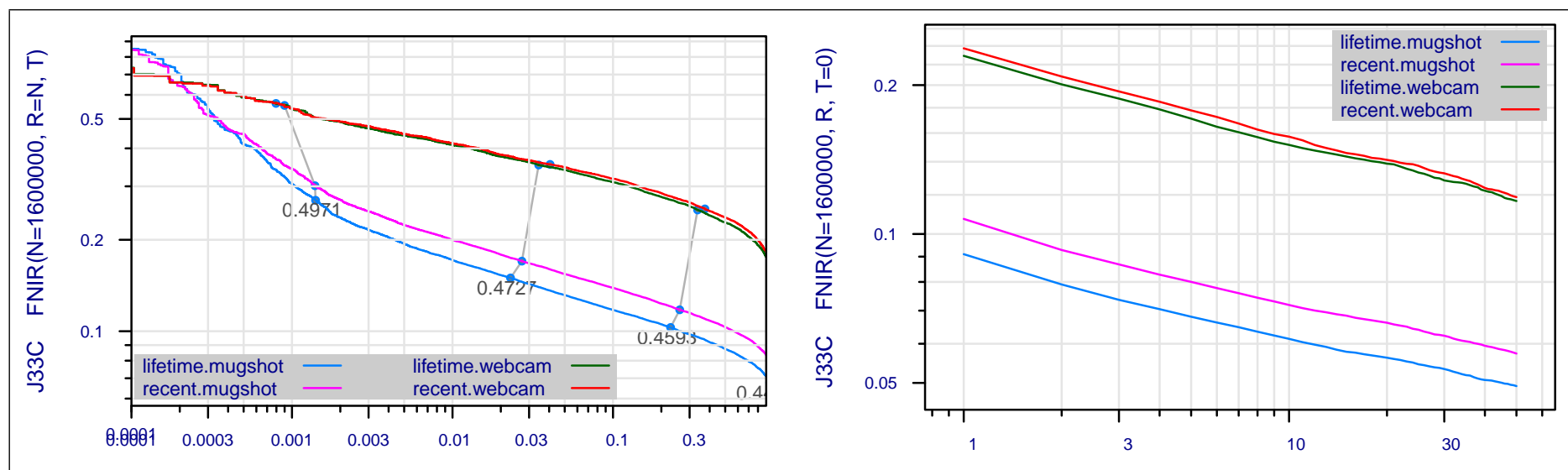

$\operatorname{FPIR}(\mathrm{N}=1600000)$
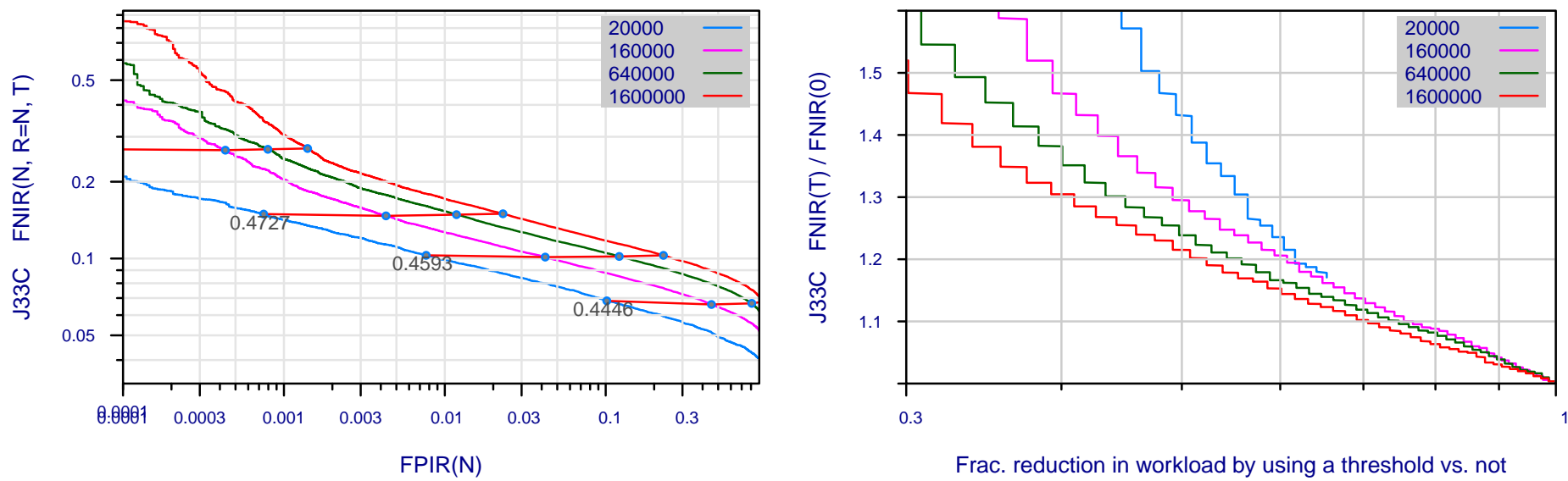

0.3

Frac. reduction in workload by using a threshold vs. not
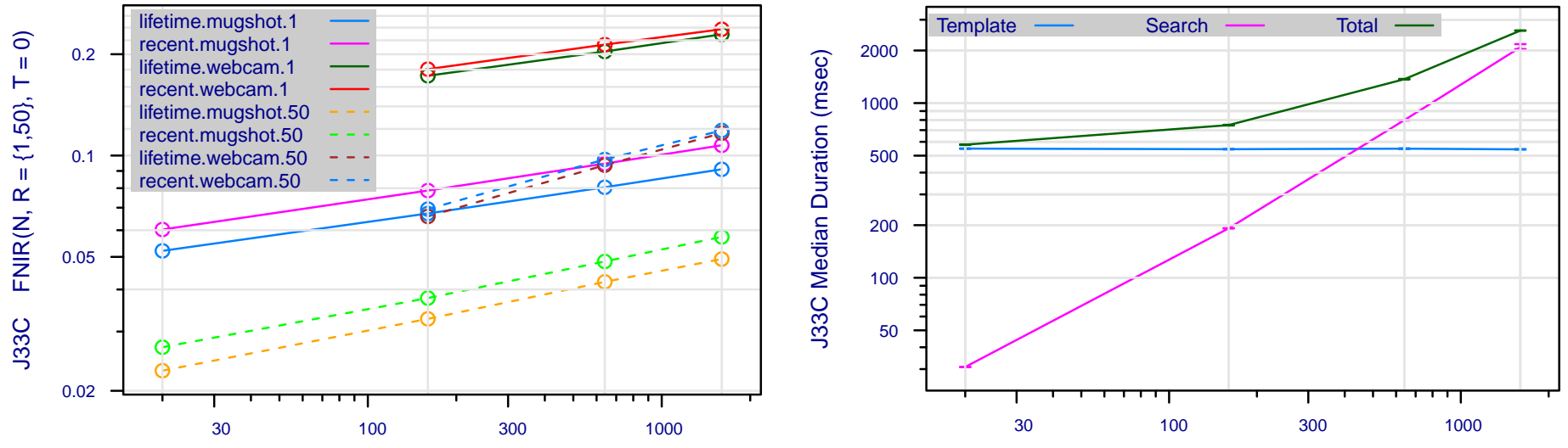

Num. enrolled identities, $\mathrm{N}$ (thousands)

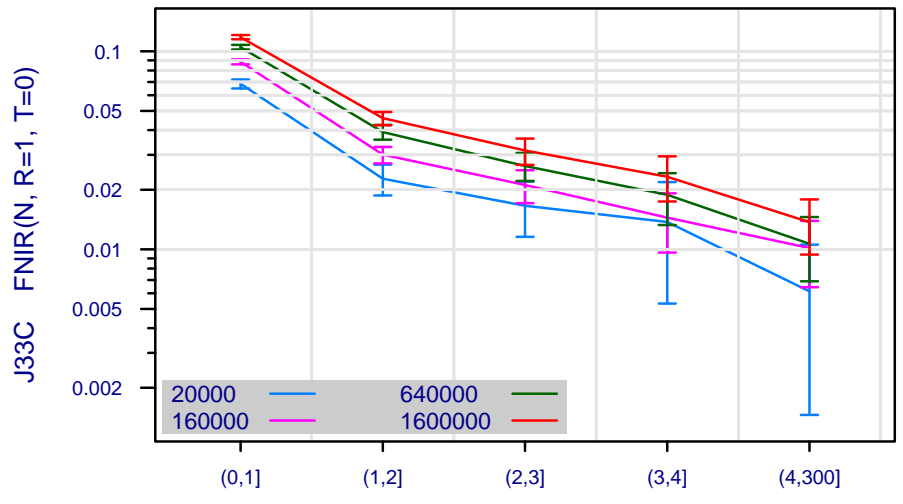

Num. enrolled images per identity

Num. enrolled identities, $\mathrm{N}$ (thousands)

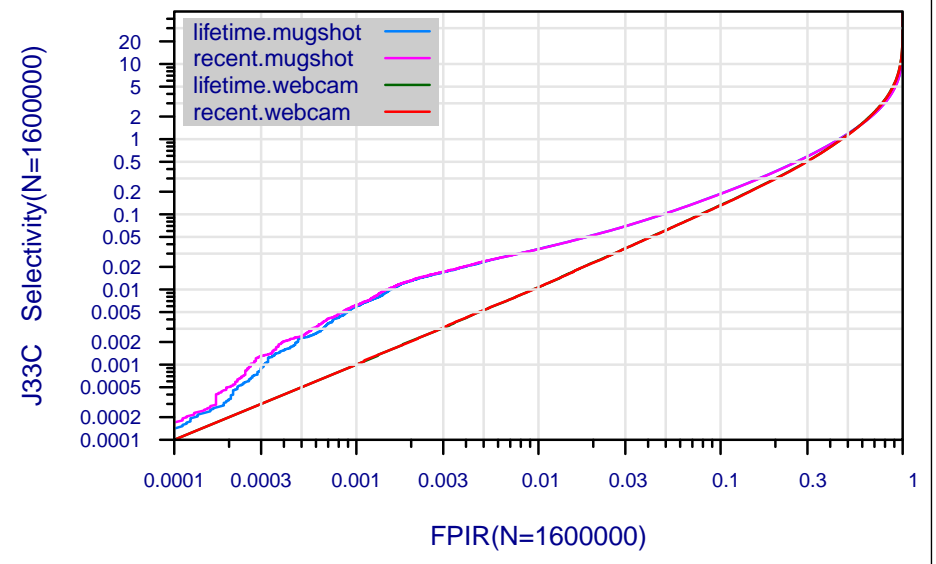

Figure 50: Collected performance reports for algorithm J33C. The figures are described at the beginning of this Appendix.

\begin{tabular}{|c|c|c|c|c|c|c|}
\hline $\begin{array}{l}\mathrm{A}=3 \mathrm{M} / \text { Cogent } \\
\mathrm{G}=\text { Hisign } \\
\mathrm{P}=\text { Zhuhai-Yisheng }\end{array}$ & $\begin{array}{l}B=\text { Cognitec } \\
H=\text { CAS-IA } \\
Q=\text { JunYu }\end{array}$ & $\begin{array}{l}\mathrm{C}=\text { Neurotechnology } \\
\mathrm{I}=\text { CAS-ICT } \\
\mathrm{S}=\text { Decatur }\end{array}$ & $\begin{array}{l}\mathrm{D}=\text { Safran Morpho } \\
\mathrm{J}=\text { Toshiba } \\
\mathrm{T}=\text { Ayonix }\end{array}$ & $\begin{array}{l}\mathrm{E}=\mathrm{NEC} \\
\mathrm{L}=\text { Tsinghua U. II }\end{array}$ & $\begin{array}{l}\mathrm{F}=\text { Tsinghua } \mathrm{U} \\
\mathrm{M}=\mathrm{HP}\end{array}$ & $\begin{array}{l}\text { FNIR(N,R,T,L) “Miss rate" } \\
\operatorname{FPIR}(\mathrm{N}, \mathrm{T}, \mathrm{L}) \text { “False alarm rate" }\end{array}$ \\
\hline
\end{tabular}




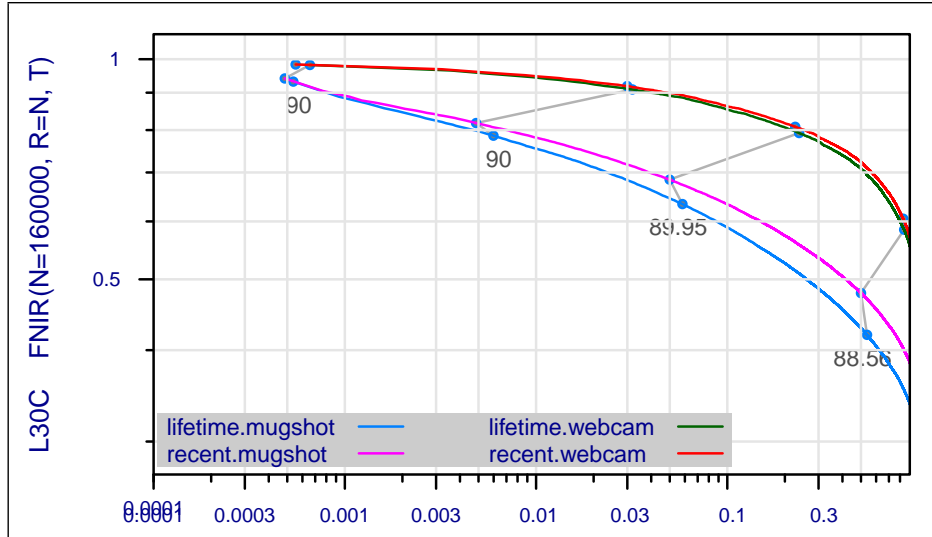

$\operatorname{FPIR}(\mathrm{N}=160000)$
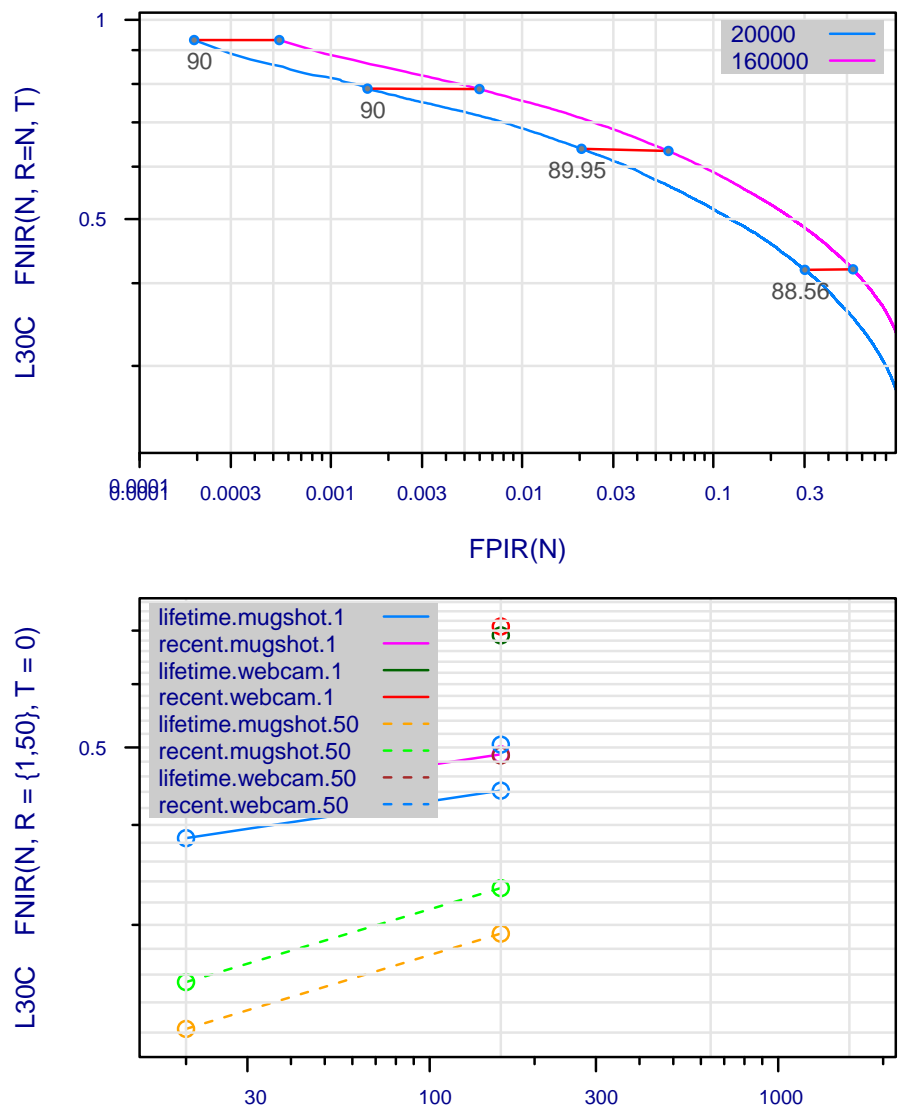

Num. enrolled identities, $\mathrm{N}$ (thousands)

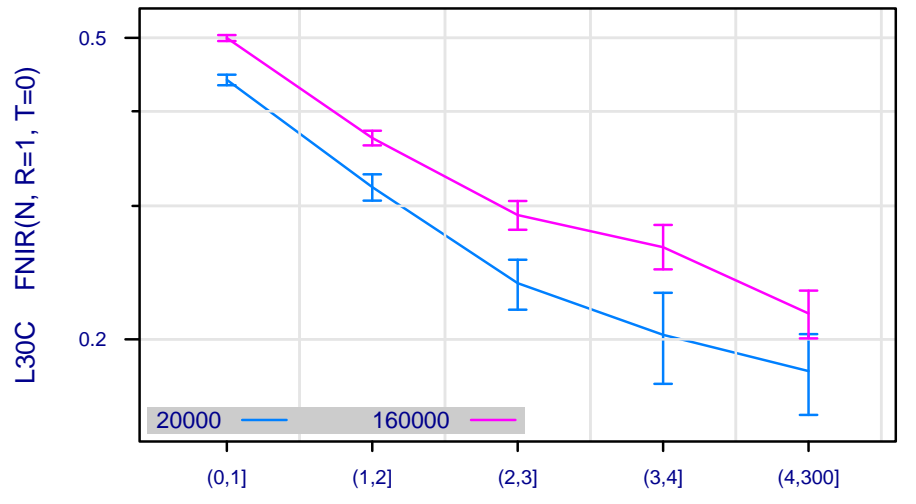

Num. enrolled images per identity

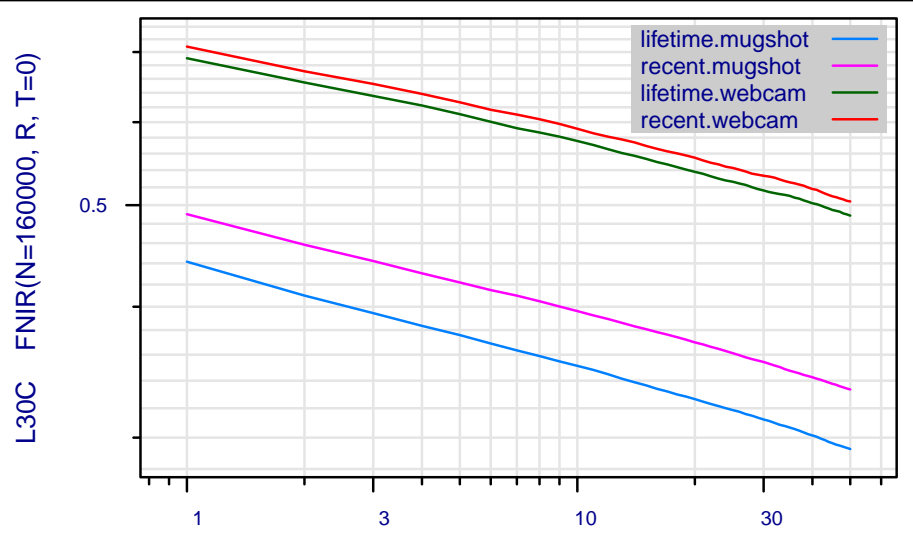

Rank, $\mathrm{R}$

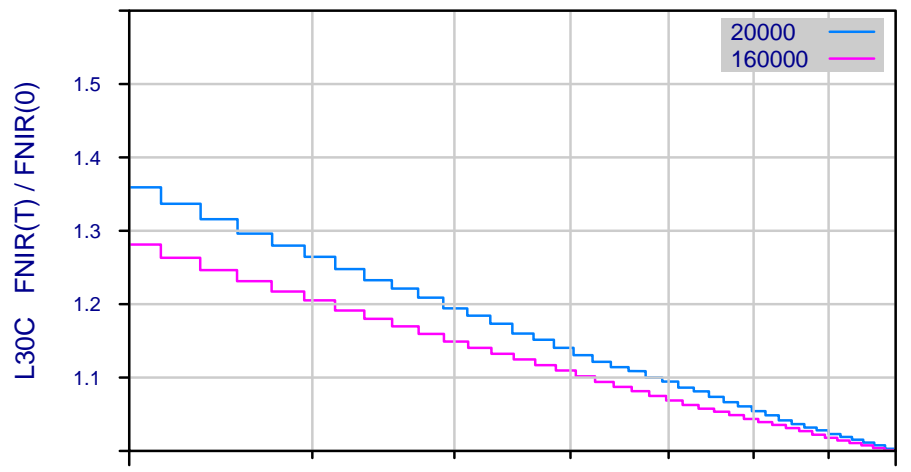

Frac. reduction in workload by using a threshold vs. not

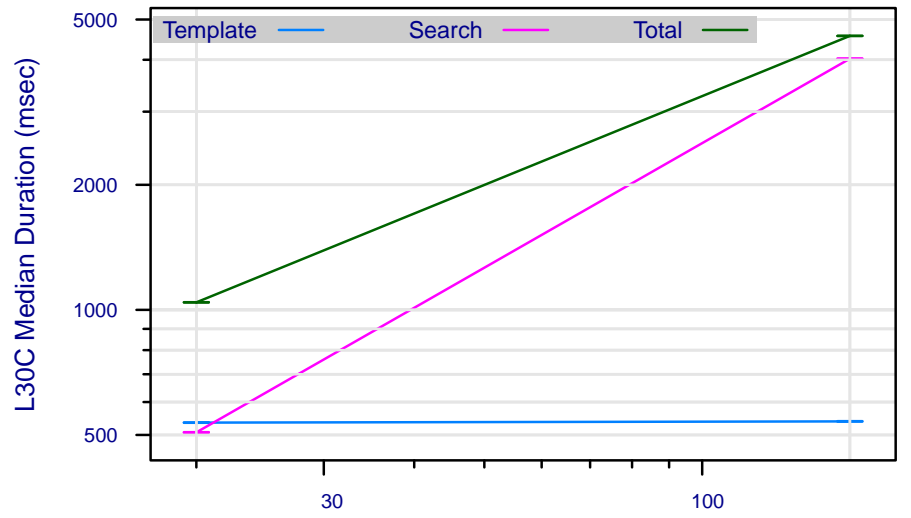

Num. enrolled identities, $\mathrm{N}$ (thousands)

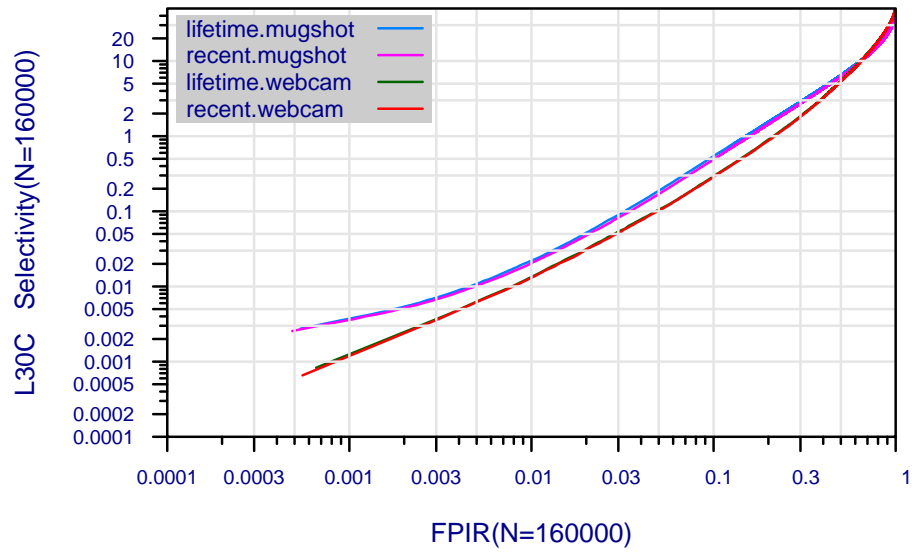

Figure 51: Collected performance reports for algorithm L30C. The figures are described at the beginning of this Appendix.

\begin{tabular}{|c|c|c|c|c|c|c|}
\hline $\begin{array}{l}A=3 \mathrm{M} / \text { Cogent } \\
G=\text { Hisign } \\
P=\text { Zhuhai-Yisheng }\end{array}$ & $\begin{array}{l}\mathrm{B}=\text { Cognitec } \\
\mathrm{H}=\text { CAS-IA } \\
\mathrm{Q}=\text { JunYu }\end{array}$ & $\begin{array}{l}\mathrm{C}=\text { Neurotechnology } \\
\mathrm{I}=\text { CAS-ICT } \\
\mathrm{S}=\text { Decatur }\end{array}$ & $\begin{array}{l}\mathrm{D}=\text { Safran Morpho } \\
\mathrm{J}=\text { Toshiba } \\
\mathrm{T}=\text { Ayonix }\end{array}$ & $\begin{array}{l}\mathrm{E}=\mathrm{NEC} \\
\mathrm{L}=\text { Tsinghua U. II }\end{array}$ & $\begin{array}{l}\mathrm{F}=\text { Tsinghua } \mathrm{U} \\
\mathrm{M}=\mathrm{HP}\end{array}$ & $\begin{array}{l}\text { FNIR(N,R,T,L) "Miss rate" } \\
\text { FPIR(N,T,L) “False alarm rate" }\end{array}$ \\
\hline
\end{tabular}




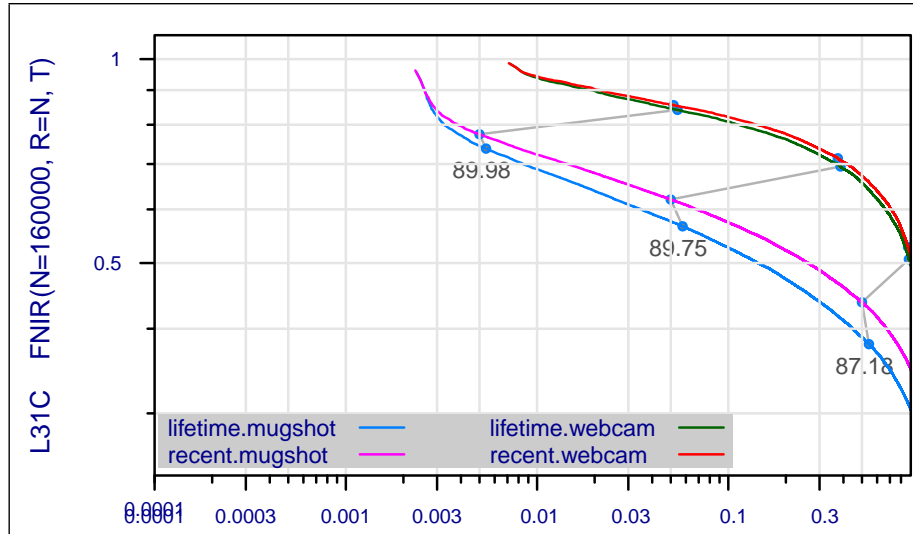

$\operatorname{FPIR}(\mathrm{N}=160000)$
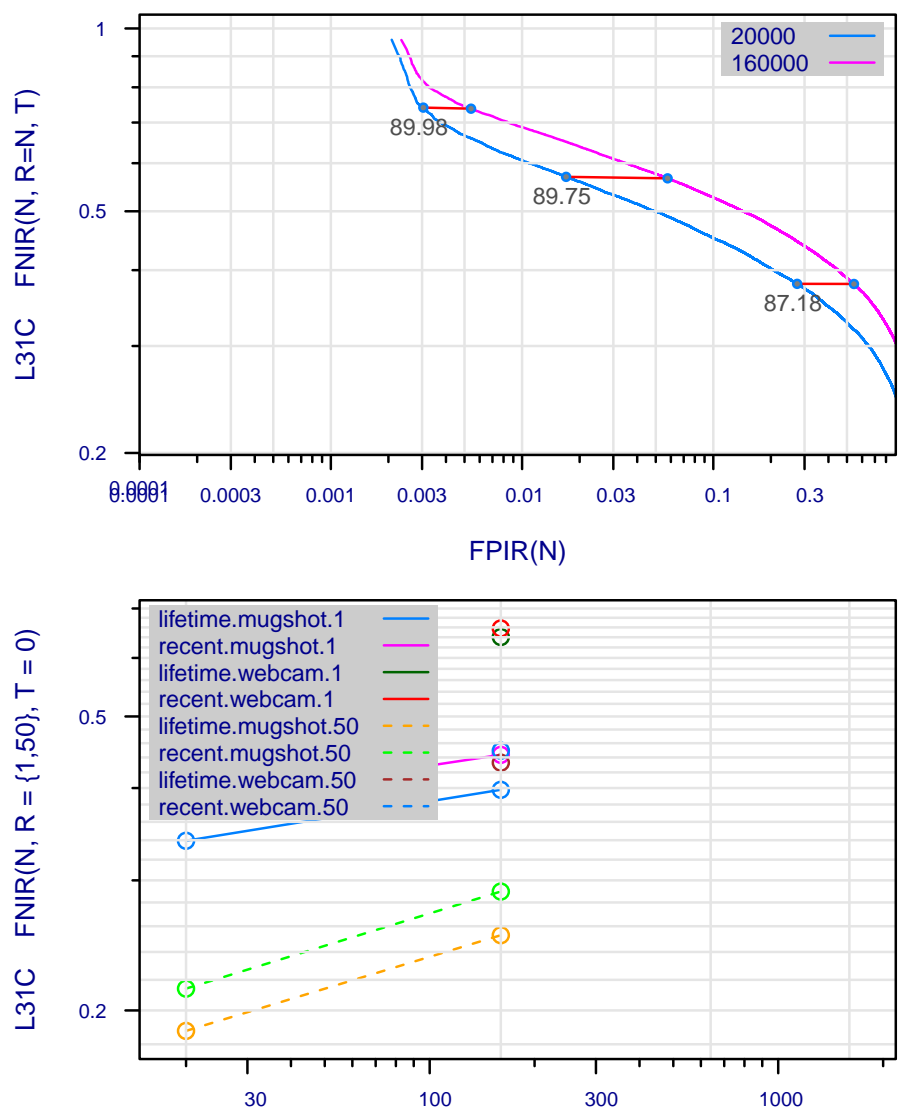

Num. enrolled identities, $\mathrm{N}$ (thousands)

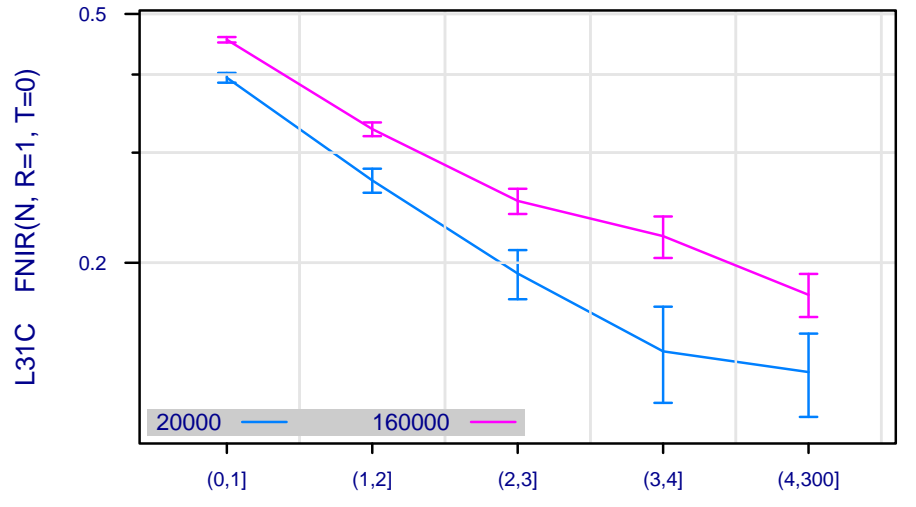

Num. enrolled images per identity

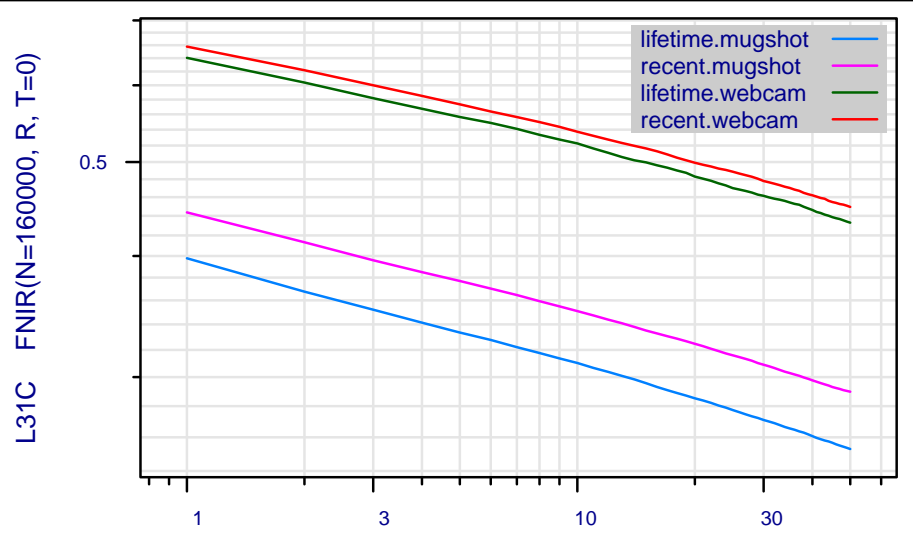

Rank, $\mathrm{R}$

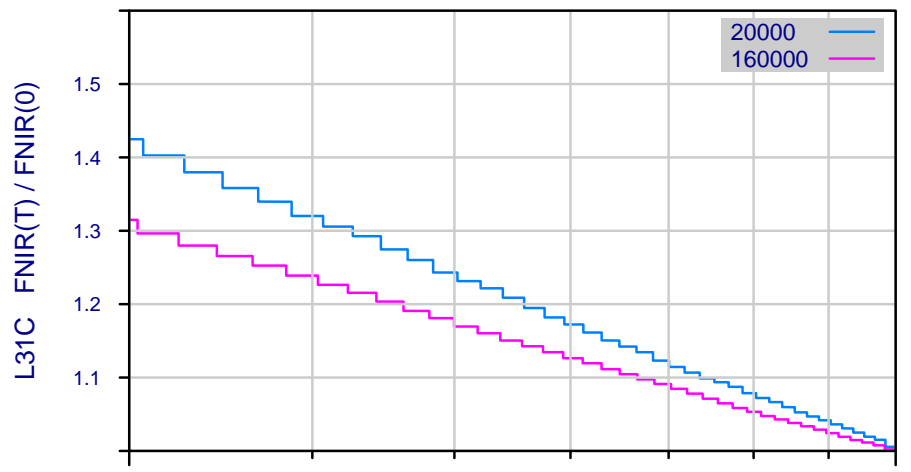

0.3

Frac. reduction in workload by using a threshold vs. not

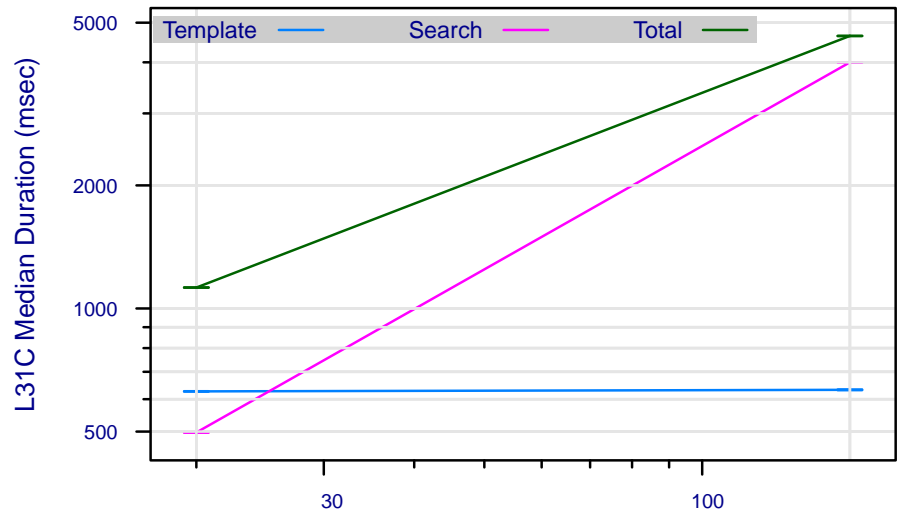

Num. enrolled identities, $\mathrm{N}$ (thousands)

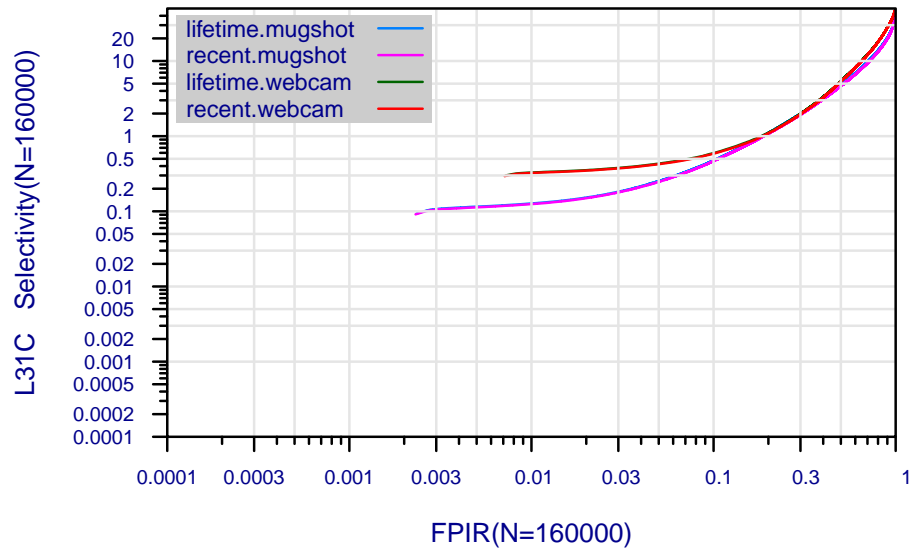

Figure 52: Collected performance reports for algorithm L31C. The figures are described at the beginning of this Appendix.

\begin{tabular}{|c|c|c|c|c|c|c|}
\hline $\begin{array}{l}A=3 \mathrm{M} / \text { Cogent } \\
G=\text { Hisign } \\
P=\text { Zhuhai-Yisheng }\end{array}$ & $\begin{array}{l}\mathrm{B}=\text { Cognitec } \\
\mathrm{H}=\text { CAS-IA } \\
\mathrm{Q}=\text { JunYu }\end{array}$ & $\begin{array}{l}\mathrm{C}=\text { Neurotechnology } \\
\mathrm{I}=\text { CAS-ICT } \\
\mathrm{S}=\text { Decatur }\end{array}$ & $\begin{array}{l}\mathrm{D}=\text { Safran Morpho } \\
\mathrm{J}=\text { Toshiba } \\
\mathrm{T}=\text { Ayonix }\end{array}$ & $\begin{array}{l}\mathrm{E}=\mathrm{NEC} \\
\mathrm{L}=\text { Tsinghua U. II }\end{array}$ & $\begin{array}{l}\mathrm{F}=\text { Tsinghua } \mathrm{U} \\
\mathrm{M}=\mathrm{HP}\end{array}$ & $\begin{array}{l}\text { FNIR(N,R,T,L) "Miss rate" } \\
\text { FPIR(N,T,L) “False alarm rate" }\end{array}$ \\
\hline
\end{tabular}




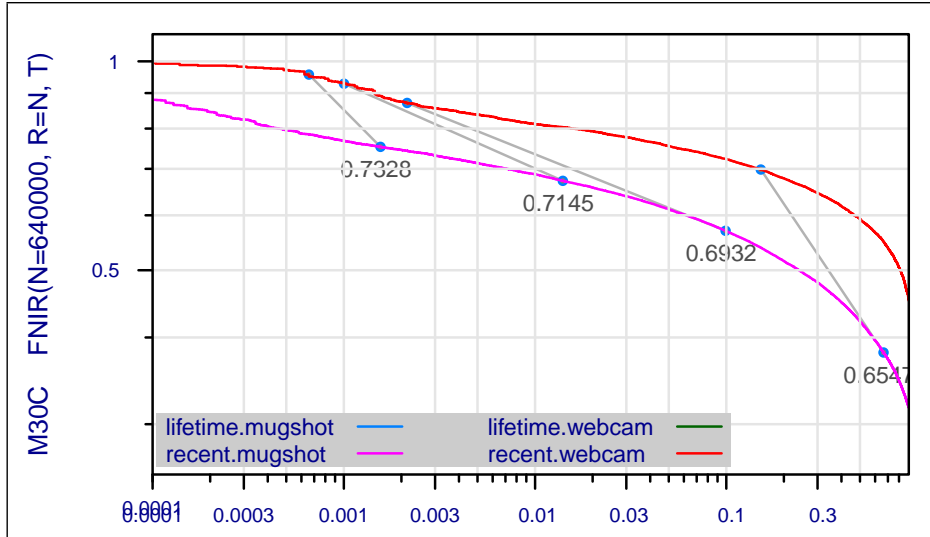

$\operatorname{FPIR}(\mathrm{N}=640000)$
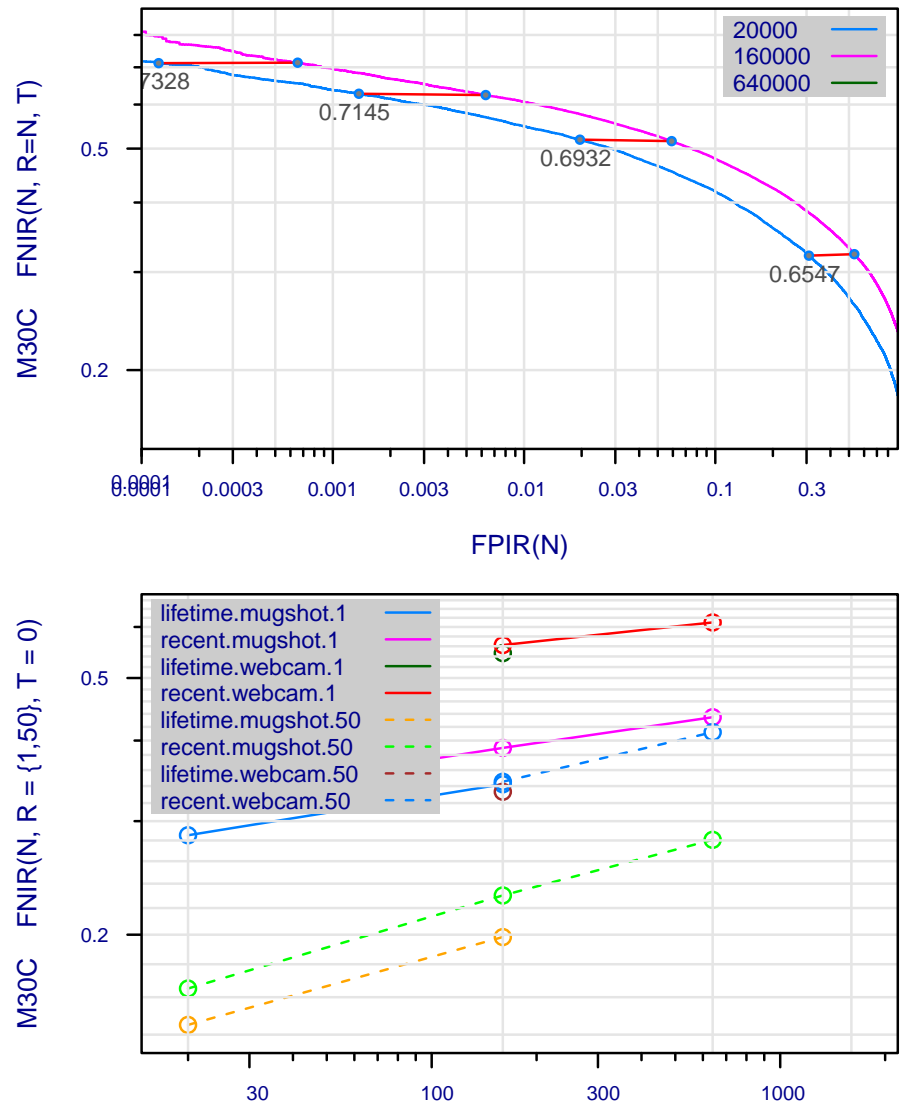

Num. enrolled identities, $\mathrm{N}$ (thousands)

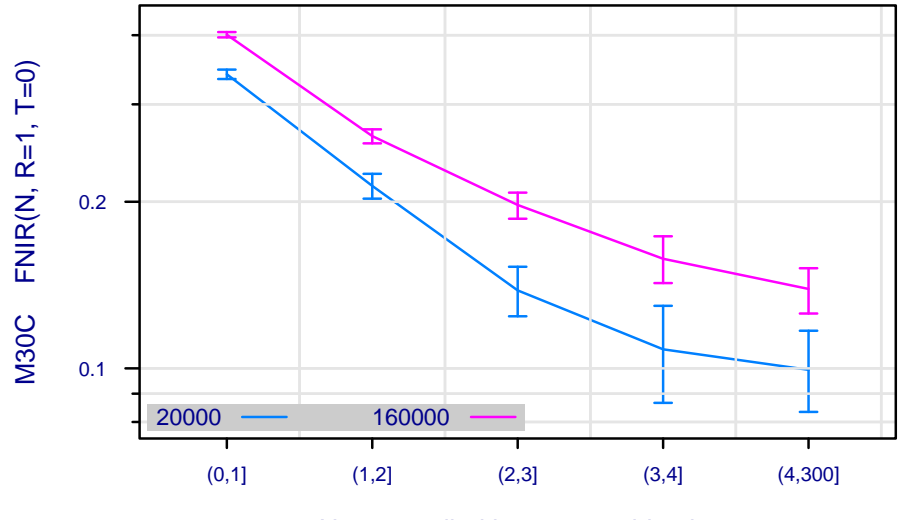

Num. enrolled images per identity

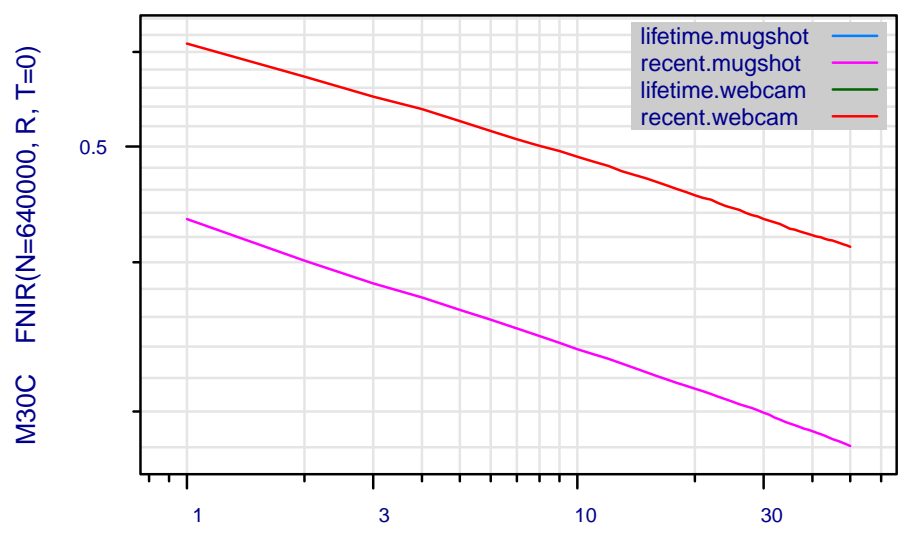

Rank, R

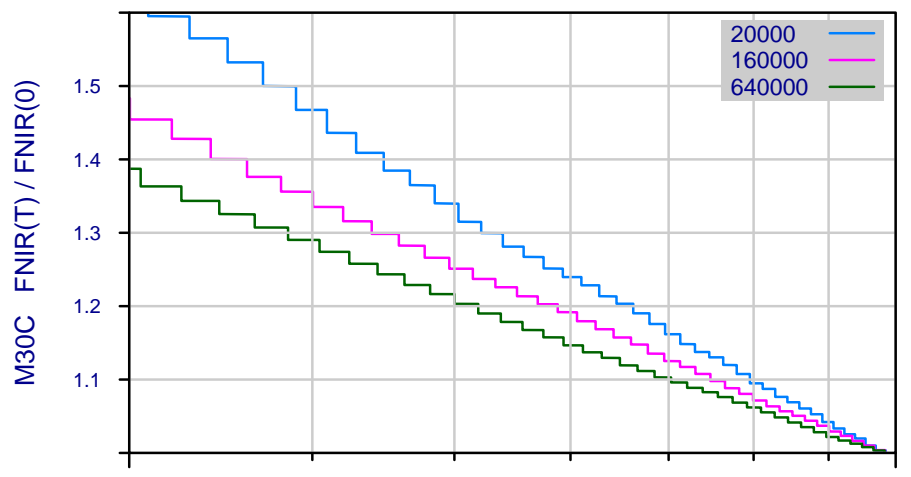

0.3

Frac. reduction in workload by using a threshold vs. not

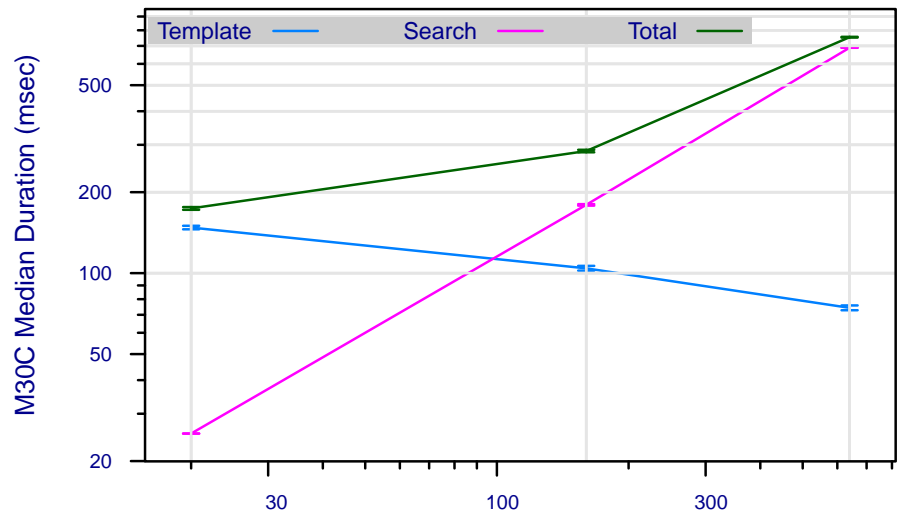

Num. enrolled identities, $\mathrm{N}$ (thousands)

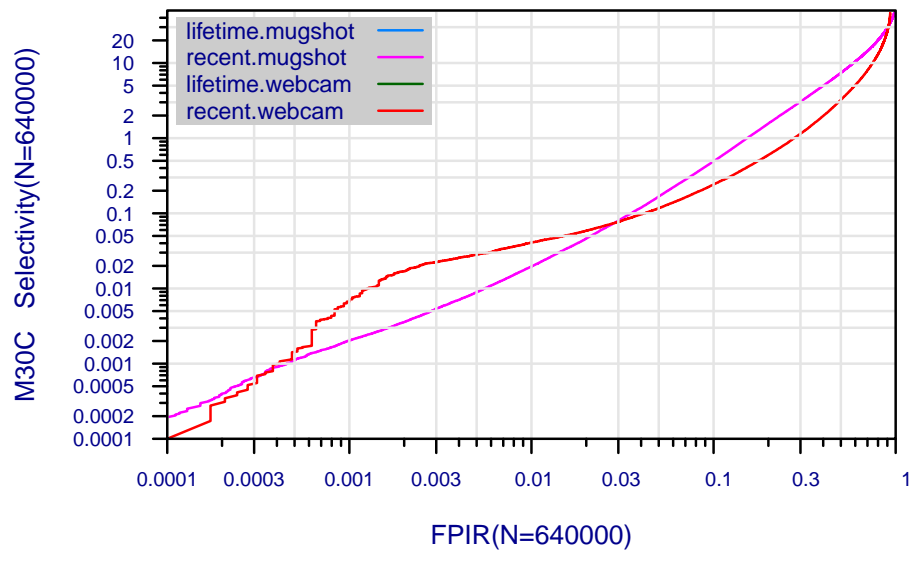

Figure 53: Collected performance reports for algorithm M30C. The figures are described at the beginning of this Appendix.

\begin{tabular}{|c|c|c|c|c|c|c|}
\hline $\begin{array}{l}A=3 \mathrm{M} / \text { Cogent } \\
G=\text { Hisign } \\
P=\text { Zhuhai-Yisheng }\end{array}$ & $\begin{array}{l}\mathrm{B}=\text { Cognitec } \\
\mathrm{H}=\text { CAS-IA } \\
\mathrm{Q}=\text { JunYu }\end{array}$ & $\begin{array}{l}\mathrm{C}=\text { Neurotechnology } \\
\mathrm{I}=\text { CAS-ICT } \\
\mathrm{S}=\text { Decatur }\end{array}$ & $\begin{array}{l}\mathrm{D}=\text { Safran Morpho } \\
\mathrm{J}=\text { Toshiba } \\
\mathrm{T}=\text { Ayonix }\end{array}$ & $\begin{array}{l}\mathrm{E}=\mathrm{NEC} \\
\mathrm{L}=\text { Tsinghua U. II }\end{array}$ & $\begin{array}{l}\mathrm{F}=\text { Tsinghua } \mathrm{U} \\
\mathrm{M}=\mathrm{HP}\end{array}$ & $\begin{array}{l}\text { FNIR(N,R,T,L) "Miss rate" } \\
\text { FPIR(N,T,L) “False alarm rate" }\end{array}$ \\
\hline
\end{tabular}



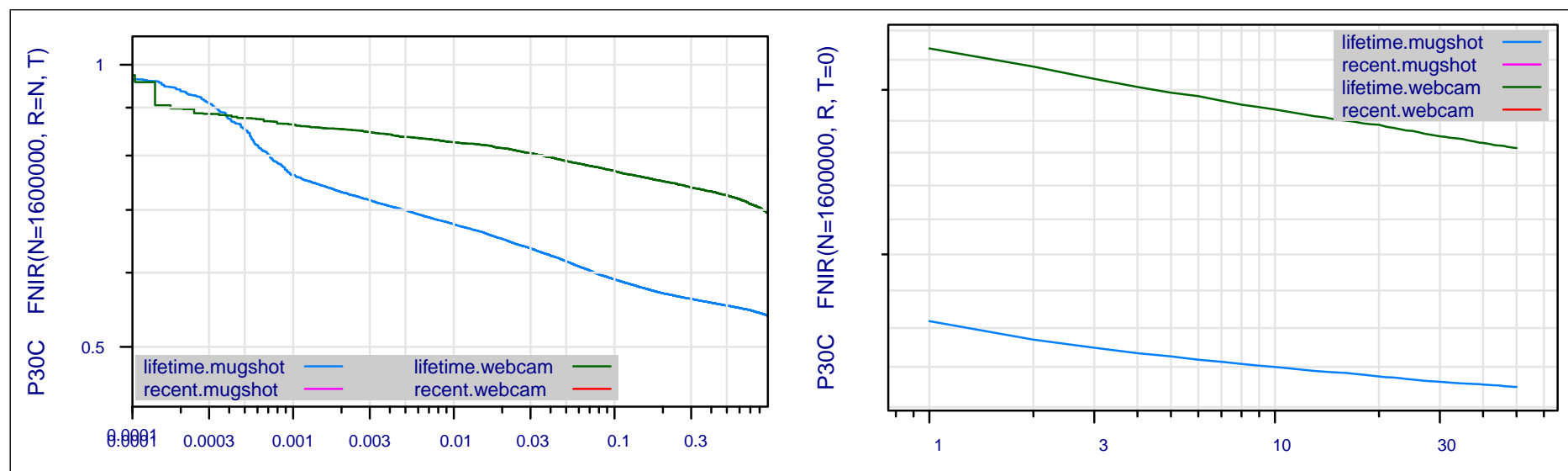

$\operatorname{FPIR}(\mathrm{N}=1600000)$
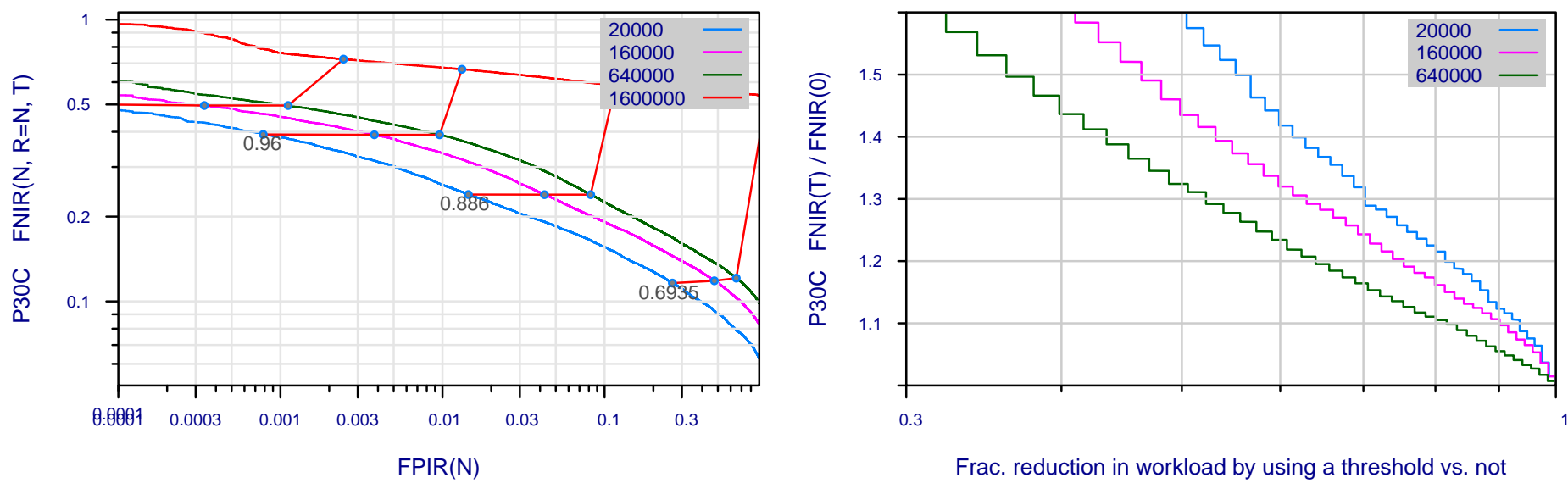

Frac. reduction in workload by using a threshold vs. not
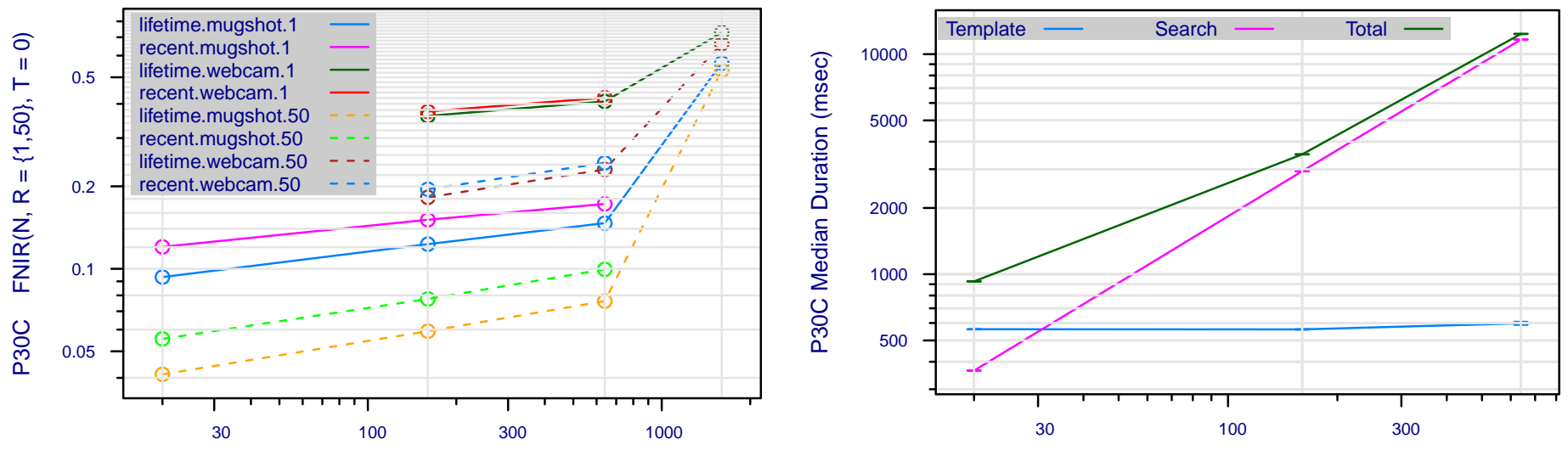

Num. enrolled identities, $\mathrm{N}$ (thousands)

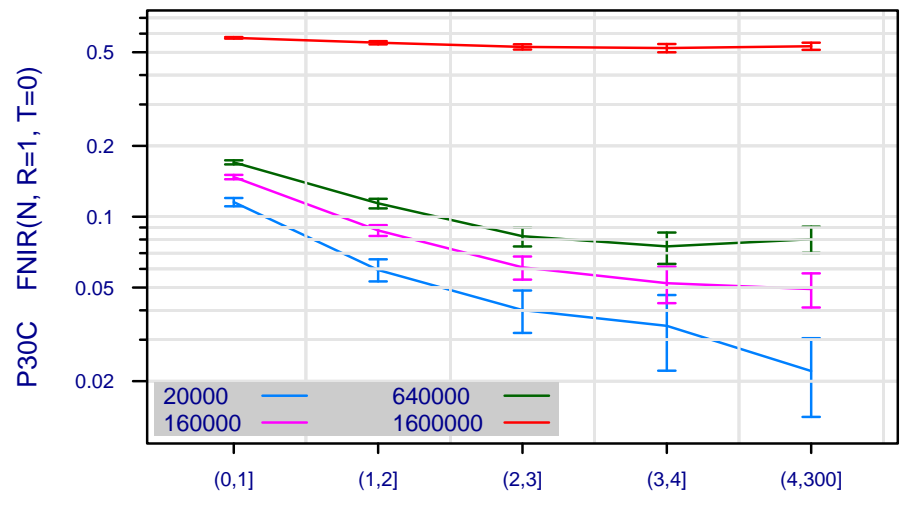

Num. enrolled images per identity

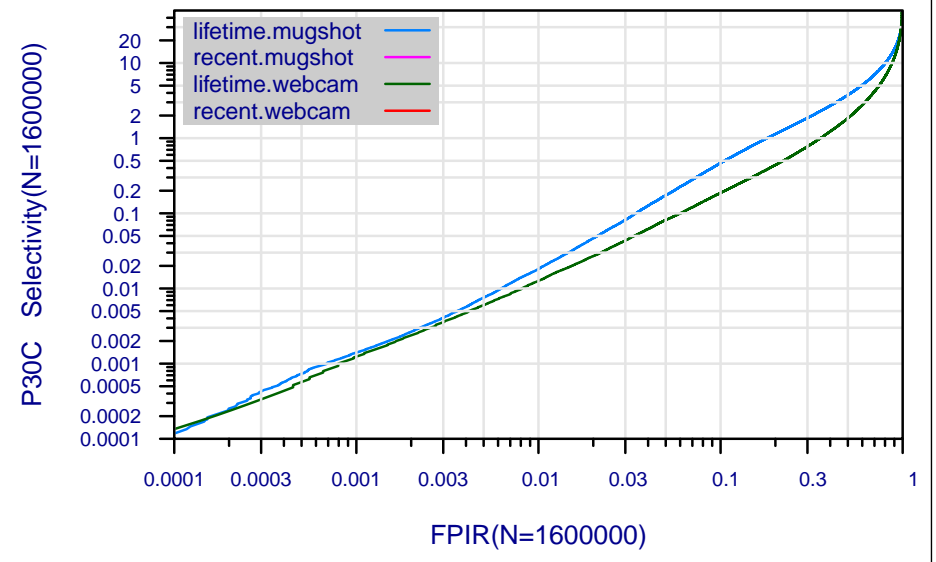

Figure 54: Collected performance reports for algorithm P30C. The figures are described at the beginning of this Appendix.

\begin{tabular}{|c|c|c|c|c|c|c|}
\hline $\begin{array}{l}\mathrm{A}=3 \mathrm{M} / \text { Cogent } \\
\mathrm{G}=\text { Hisign } \\
\mathrm{P}=\text { Zhuhai-Yisheng }\end{array}$ & $\begin{array}{l}B=\text { Cognitec } \\
H=\text { CAS-IA } \\
Q=\text { JunYu }\end{array}$ & $\begin{array}{l}\mathrm{C}=\text { Neurotechnology } \\
\mathrm{I}=\text { CAS-ICT } \\
\mathrm{S}=\text { Decatur }\end{array}$ & $\begin{array}{l}\mathrm{D}=\text { Safran Morpho } \\
\mathrm{J}=\text { Toshiba } \\
\mathrm{T}=\text { Ayonix }\end{array}$ & $\begin{array}{l}\mathrm{E}=\mathrm{NEC} \\
\mathrm{L}=\text { Tsinghua U. II }\end{array}$ & $\begin{array}{l}\mathrm{F}=\text { Tsinghua } \mathrm{U} \\
\mathrm{M}=\mathrm{HP}\end{array}$ & $\begin{array}{l}\text { FNIR(N,R,T,L) “Miss rate" } \\
\operatorname{FPIR}(\mathrm{N}, \mathrm{T}, \mathrm{L}) \text { “False alarm rate" }\end{array}$ \\
\hline
\end{tabular}




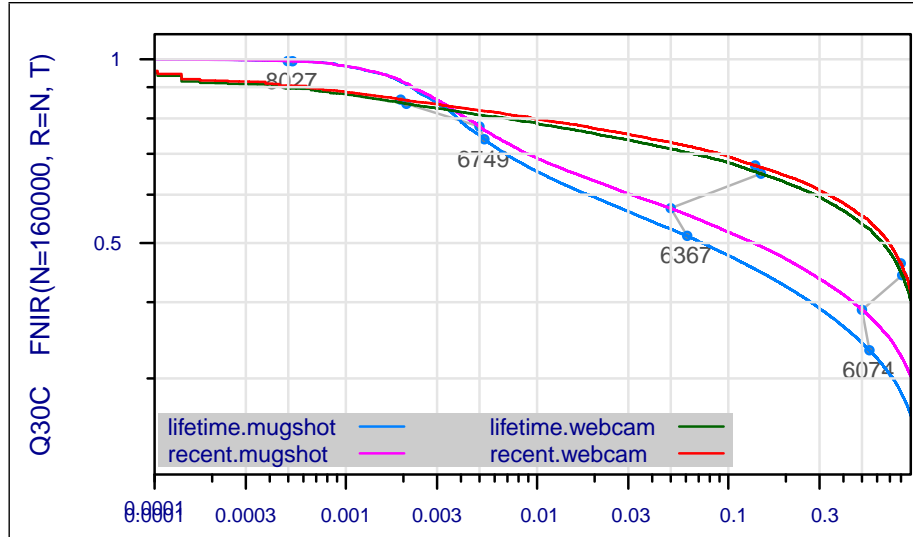

$\operatorname{FPIR}(\mathrm{N}=160000)$
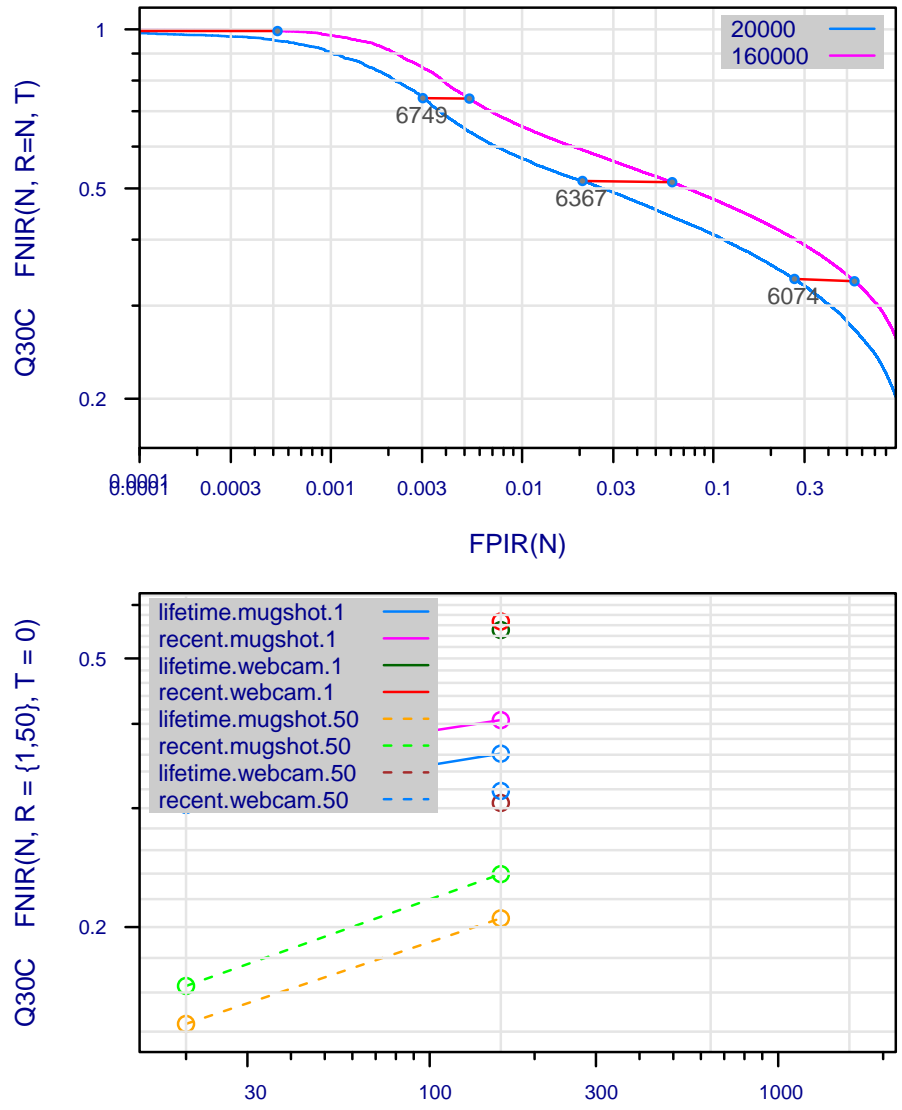

Num. enrolled identities, $\mathrm{N}$ (thousands)

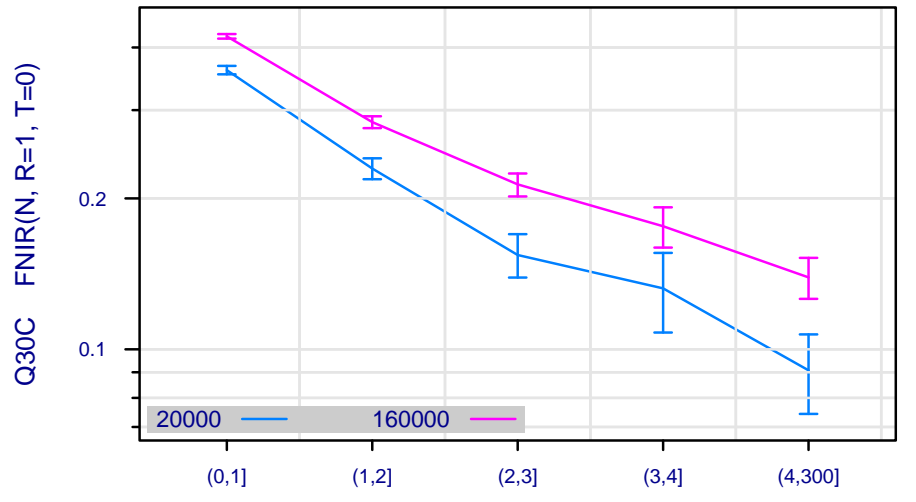

Num. enrolled images per identity

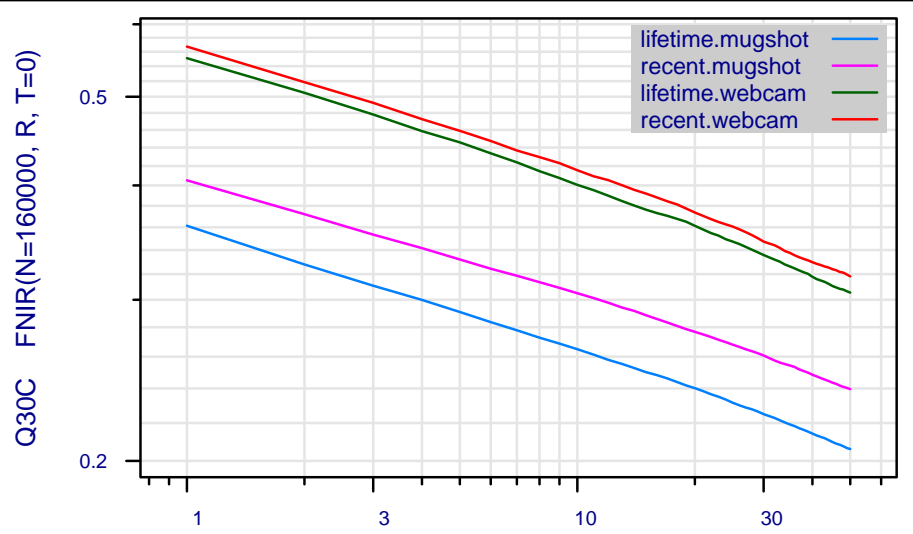

Rank, $\mathrm{R}$

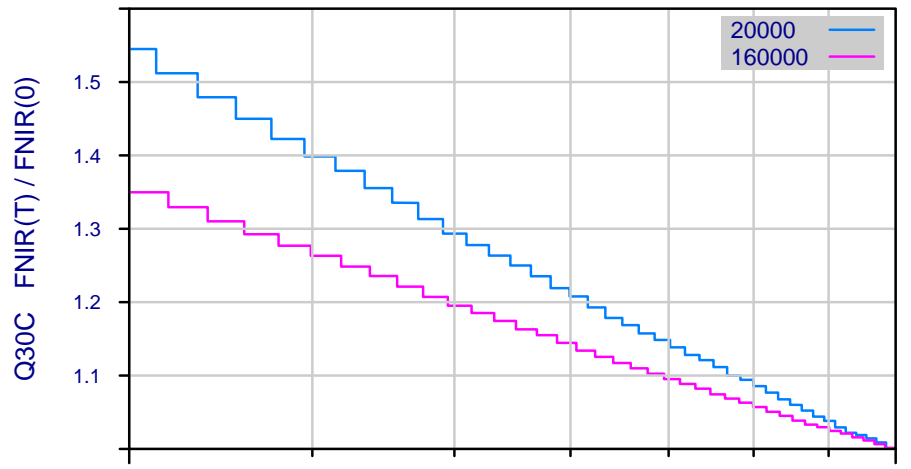

0.3

Frac. reduction in workload by using a threshold vs. not

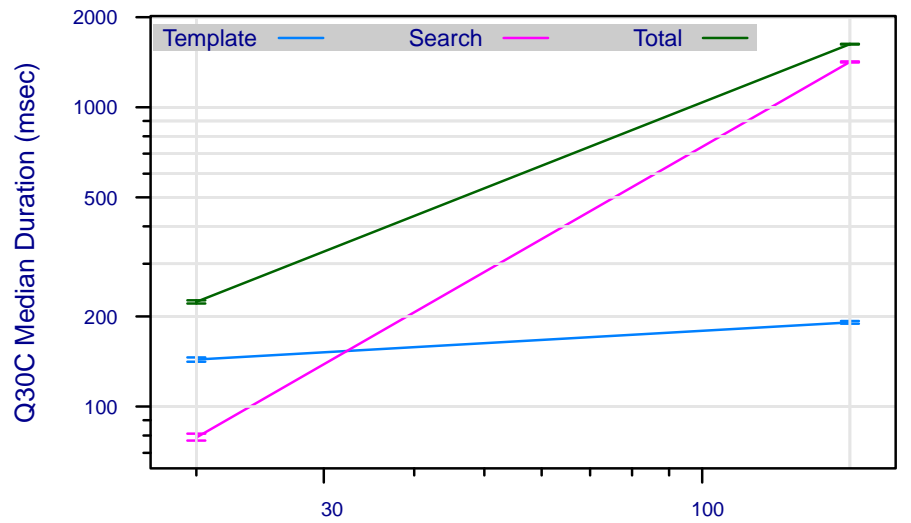

Num. enrolled identities, $\mathrm{N}$ (thousands)

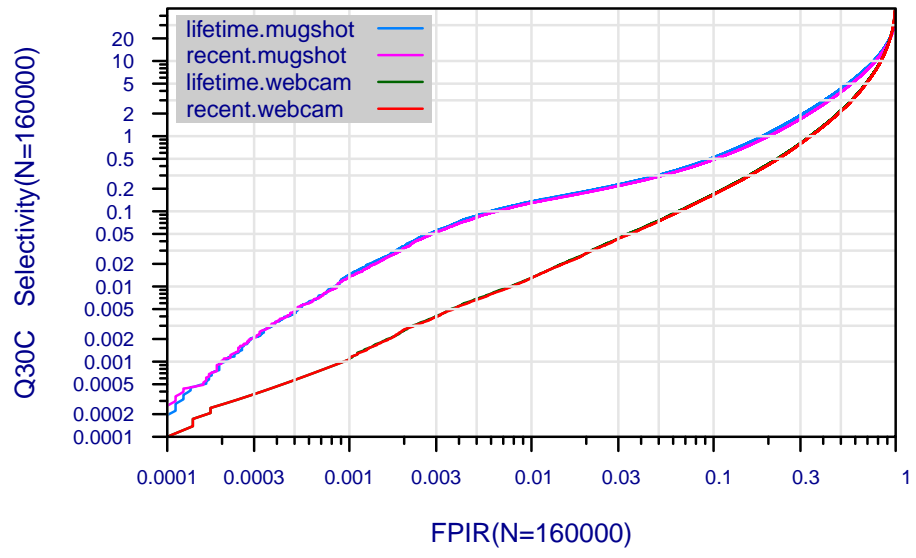

Figure 55: Collected performance reports for algorithm Q30C. The figures are described at the beginning of this Appendix.

\begin{tabular}{|c|c|c|c|c|c|c|}
\hline $\begin{array}{l}\mathrm{A}=3 \mathrm{M} / \text { Cogent } \\
\mathrm{G}=\text { Hisign } \\
\mathrm{P}=\text { Zhuhai-Yisheng }\end{array}$ & $\begin{array}{l}\mathrm{B}=\text { Cognitec } \\
\mathrm{H}=\text { CAS-IA } \\
\mathrm{Q}=\text { JunYu }\end{array}$ & $\begin{array}{l}\mathrm{C}=\text { Neurotechnology } \\
\mathrm{I}=\mathrm{CAS}-\mathrm{ICT} \\
\mathrm{S}=\text { Decatur }\end{array}$ & $\begin{array}{l}\mathrm{D}=\text { Safran Morpho } \\
\mathrm{J}=\text { Toshiba } \\
\mathrm{T}=\text { Ayonix }\end{array}$ & $\begin{array}{l}\mathrm{E}=\mathrm{NEC} \\
\mathrm{L}=\text { Tsinghua U. II }\end{array}$ & $\begin{array}{l}\mathrm{F}=\text { Tsinghua } \mathrm{U} . \\
\mathrm{M}=\mathrm{HP}\end{array}$ & $\begin{array}{l}\text { FNIR(N,R,T,L) “Miss rate" } \\
\text { FPIR(N,T,L) “False alarm rate" }\end{array}$ \\
\hline
\end{tabular}



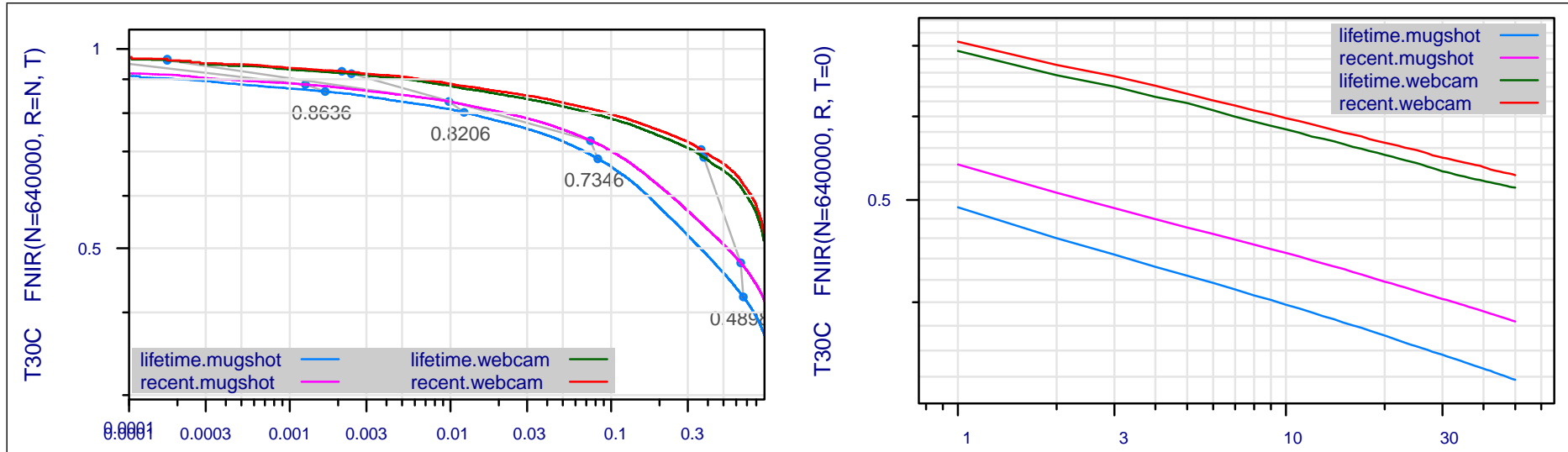

$\operatorname{FPIR}(\mathrm{N}=640000)$
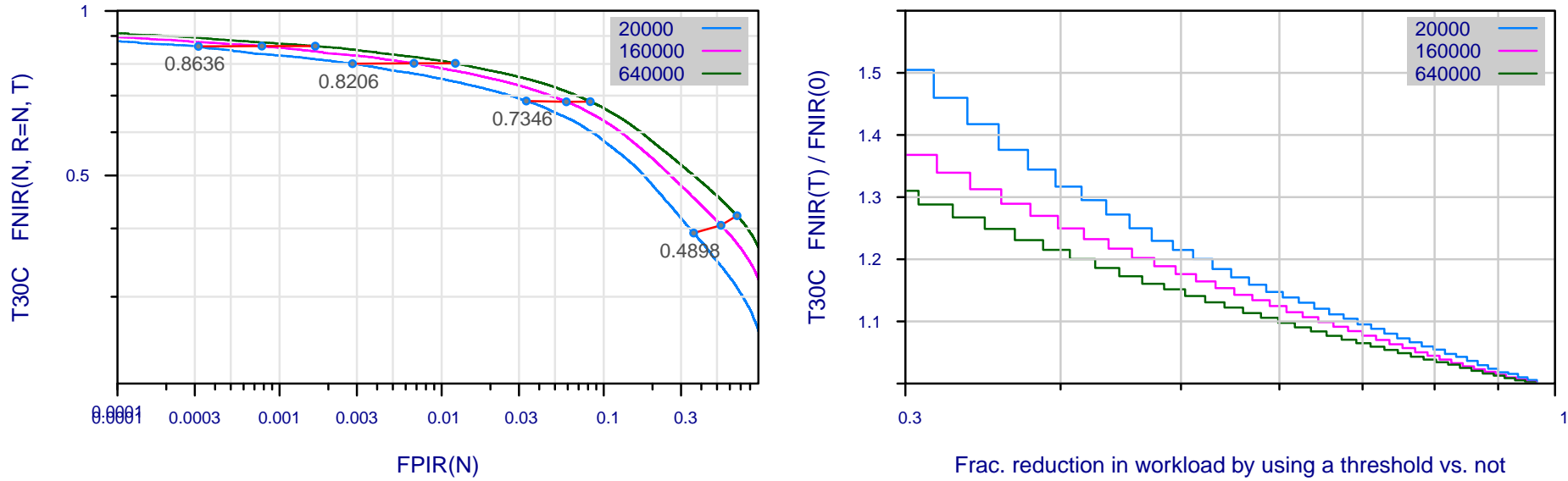

0.3

Frac. reduction in workload by using a threshold vs. not
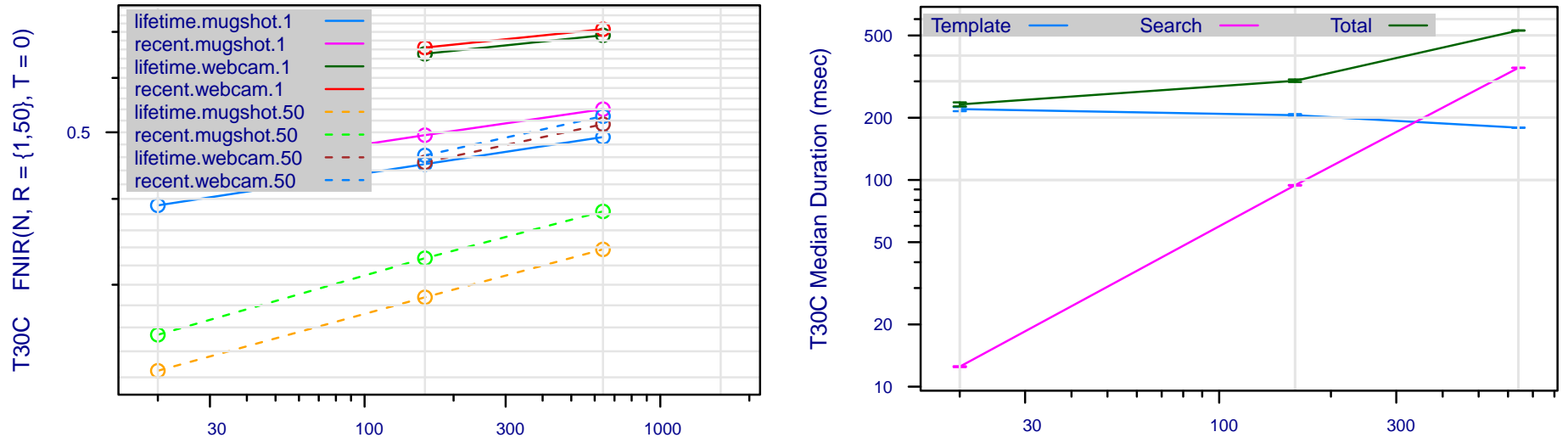

Num. enrolled identities, $\mathrm{N}$ (thousands)

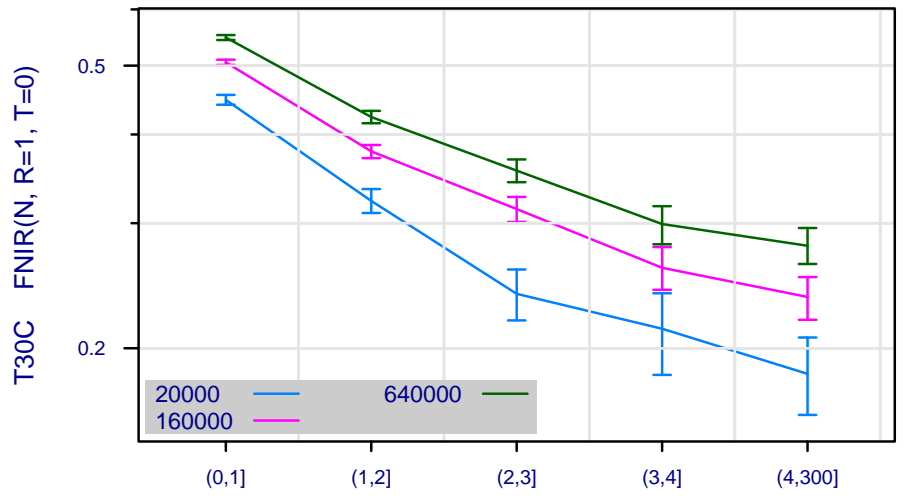

Num. enrolled images per identity

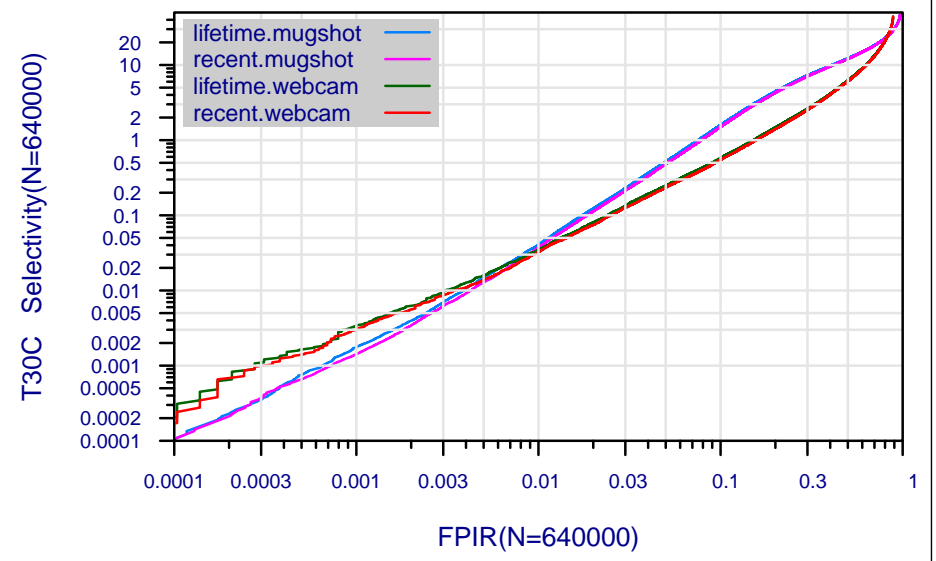

Figure 56: Collected performance reports for algorithm T30C. The figures are described at the beginning of this Appendix.

\begin{tabular}{|c|c|c|c|c|c|c|}
\hline $\begin{array}{l}\mathrm{A}=3 \mathrm{M} / \text { Cogent } \\
\mathrm{G}=\text { Hisign } \\
\mathrm{P}=\text { Zhuhai-Yisheng }\end{array}$ & $\begin{array}{l}B=\text { Cognitec } \\
H=\text { CAS-IA } \\
Q=\text { JunYu }\end{array}$ & $\begin{array}{l}\mathrm{C}=\text { Neurotechnology } \\
\mathrm{I}=\text { CAS-ICT } \\
\mathrm{S}=\text { Decatur }\end{array}$ & $\begin{array}{l}\mathrm{D}=\text { Safran Morpho } \\
\mathrm{J}=\text { Toshiba } \\
\mathrm{T}=\text { Ayonix }\end{array}$ & $\begin{array}{l}\mathrm{E}=\mathrm{NEC} \\
\mathrm{L}=\text { Tsinghua U. II }\end{array}$ & $\begin{array}{l}\mathrm{F}=\text { Tsinghua } \mathrm{U} \\
\mathrm{M}=\mathrm{HP}\end{array}$ & $\begin{array}{l}\text { FNIR(N,R,T,L) "Miss rate" } \\
\text { FPIR(N,T,L) "False alarm rate" }\end{array}$ \\
\hline
\end{tabular}




\section{A Algorithm accuracy by age group}

This section details individual algorithm performance by age group as discussed in section 5.5.

\begin{tabular}{|c|c|c|c|c|c|c|}
\hline $\begin{array}{l}\mathrm{A}=3 \mathrm{M} / \text { Cogent } \\
\mathrm{G}=\text { Hisign } \\
\mathrm{P}=\text { Zhuhai-Yisheng }\end{array}$ & $\begin{array}{l}\mathrm{B}=\text { Cognitec } \\
\mathrm{H}=\mathrm{CAS}-\mathrm{IA} \\
\mathrm{Q}=\text { JunYu }\end{array}$ & $\begin{array}{l}\mathrm{C}=\text { Neurotechnology } \\
\mathrm{I}=\text { CAS-ICT } \\
\mathrm{S}=\text { Decatur }\end{array}$ & $\begin{array}{l}\mathrm{D}=\text { Safran Morpho } \\
\mathrm{J}=\text { Toshiba } \\
\mathrm{T}=\text { Ayonix }\end{array}$ & $\begin{array}{l}\mathrm{E}=\mathrm{NEC} \\
\mathrm{L}=\text { Tsinghua U. II }\end{array}$ & $\begin{array}{l}\mathrm{F}=\text { Tsinghua } \mathrm{U} \\
\mathrm{M}=\mathrm{HP}\end{array}$ & $\begin{array}{l}\text { FNIR(N,R,T,L) "Miss rate" } \\
\text { FPIR(N,T,L) “False alarm rate" }\end{array}$ \\
\hline
\end{tabular}




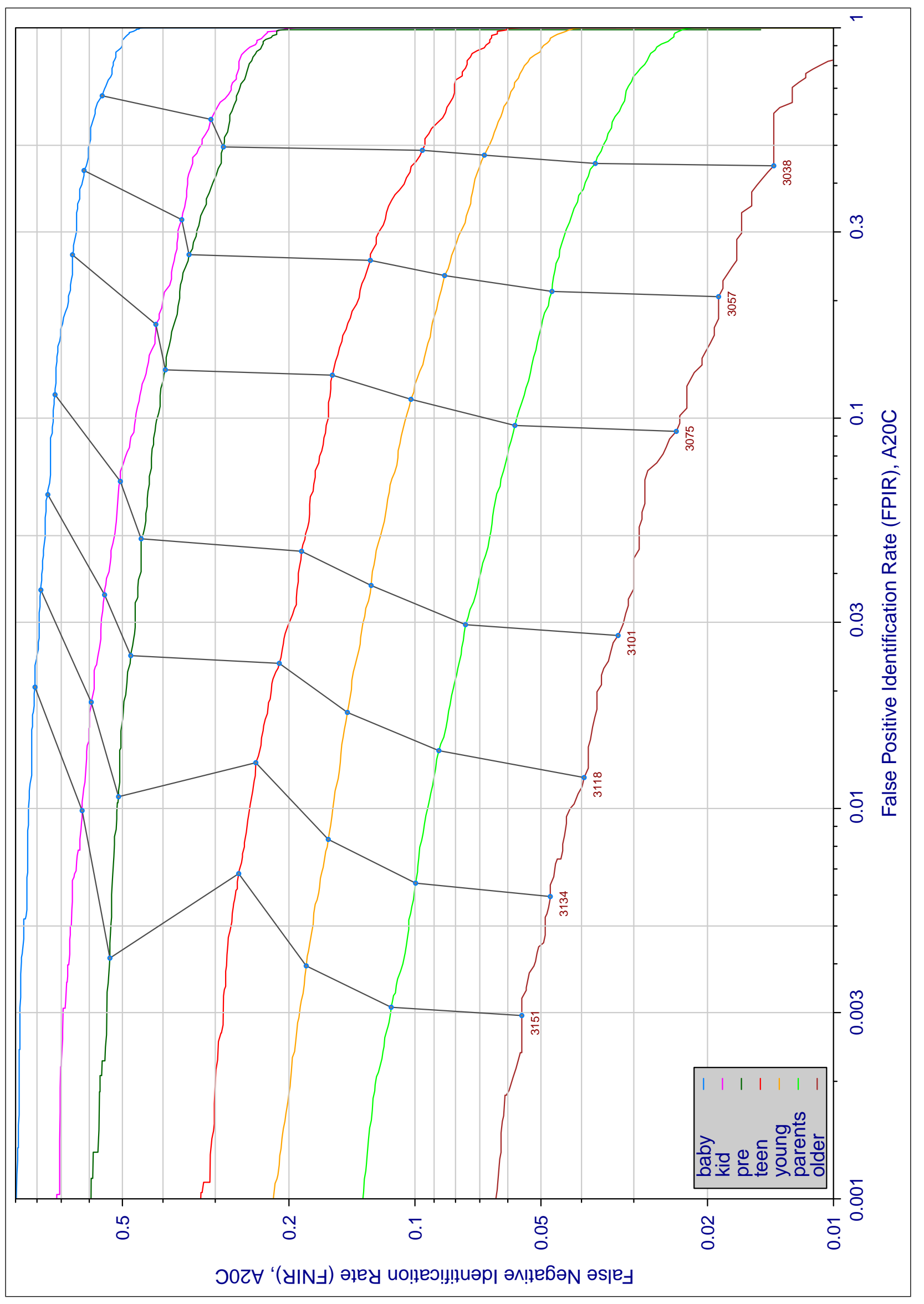

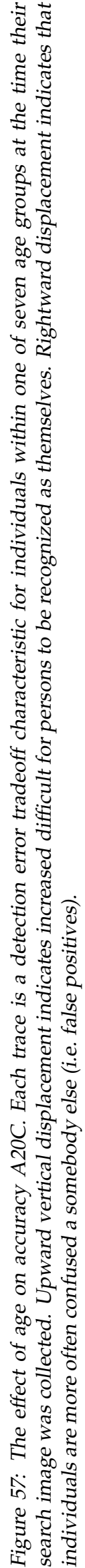

\begin{tabular}{|c|c|c|c|c|c|c|}
\hline $\begin{array}{l}\mathrm{A}=3 \mathrm{M} / \text { Cogent } \\
\mathrm{G}=\text { Hisign } \\
\mathrm{P}=\text { Zhuhai-Yisheng }\end{array}$ & $\begin{array}{l}B=\text { Cognitec } \\
H=C A S-I A \\
Q=\text { JunYu }\end{array}$ & $\begin{array}{l}\mathrm{C}=\text { Neurotechnology } \\
\mathrm{I}=\text { CAS-ICT } \\
\mathrm{S}=\text { Decatur }\end{array}$ & $\begin{array}{l}\mathrm{D}=\text { Safran Morpho } \\
\mathrm{J}=\text { Toshiba } \\
\mathrm{T}=\text { Ayonix }\end{array}$ & $\begin{array}{l}E=N E C \\
L=\text { Tsinghua U. II }\end{array}$ & $\begin{array}{l}\mathrm{F}=\text { Tsinghua } \mathrm{U} \\
\mathrm{M}=\mathrm{HP}\end{array}$ & $\begin{array}{l}\text { FNIR(N,R,T,L) “Miss rate" } \\
\text { FPIR(N,T,L) "False alarm rate" }\end{array}$ \\
\hline
\end{tabular}




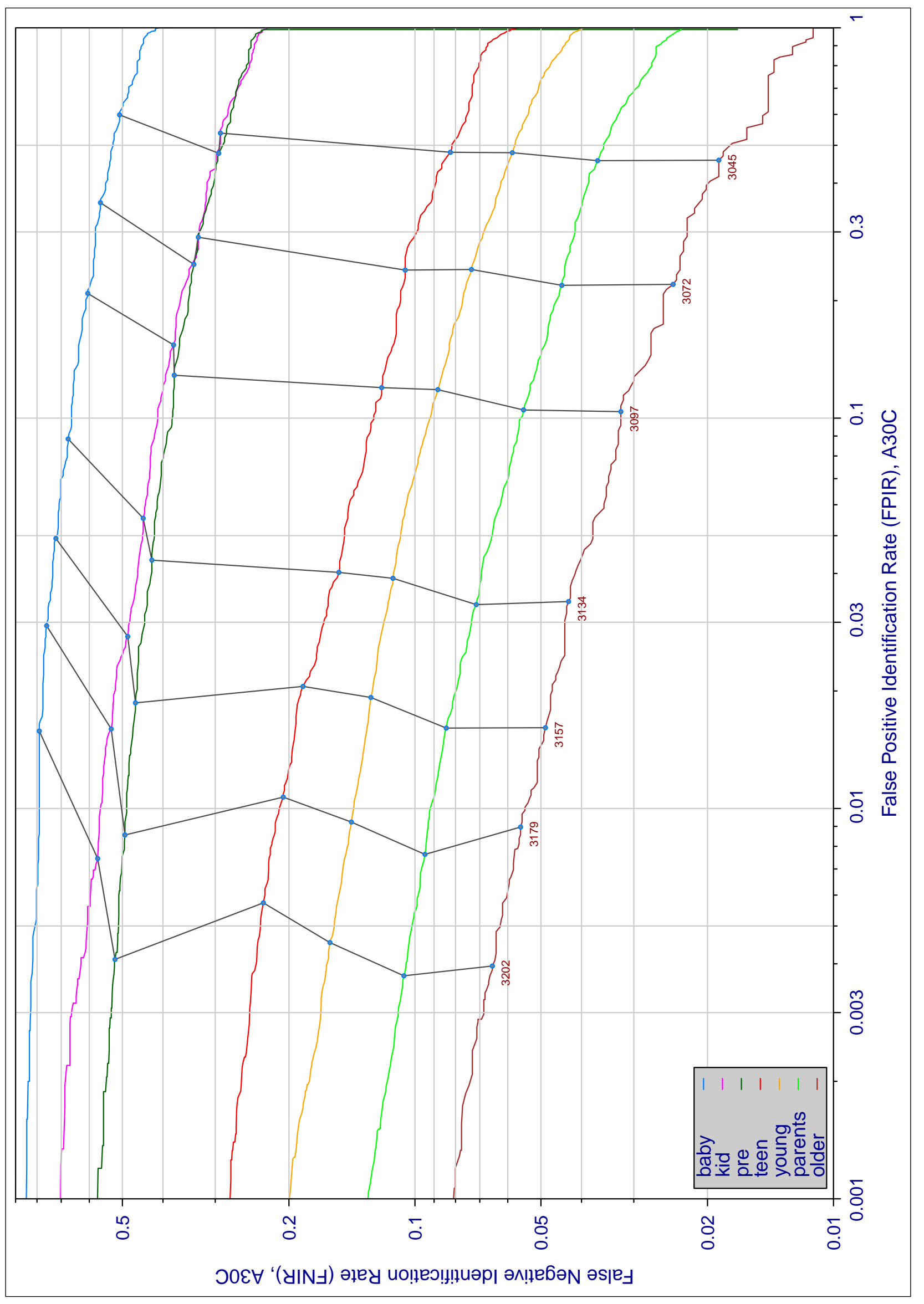

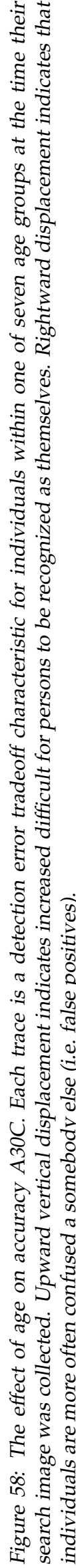

\begin{tabular}{|c|c|c|c|c|c|c|}
\hline $\begin{array}{l}\mathrm{A}=3 \mathrm{M} / \text { Cogent } \\
\mathrm{G}=\text { Hisign } \\
\mathrm{P}=\text { Zhuhai-Yisheng }\end{array}$ & $\begin{array}{l}B=\text { Cognitec } \\
H=C A S-I A \\
Q=\text { JunYu }\end{array}$ & $\begin{array}{l}\mathrm{C}=\text { Neurotechnology } \\
\mathrm{I}=\text { CAS-ICT } \\
\mathrm{S}=\text { Decatur }\end{array}$ & $\begin{array}{l}\mathrm{D}=\text { Safran Morpho } \\
\mathrm{J}=\text { Toshiba } \\
\mathrm{T}=\text { Ayonix }\end{array}$ & $\begin{array}{l}E=N E C \\
L=\text { Tsinghua U. II }\end{array}$ & $\begin{array}{l}\mathrm{F}=\text { Tsinghua } \mathrm{U} \\
\mathrm{M}=\mathrm{HP}\end{array}$ & $\begin{array}{l}\text { FNIR(N,R,T,L) “Miss rate" } \\
\text { FPIR(N,T,L) "False alarm rate" }\end{array}$ \\
\hline
\end{tabular}




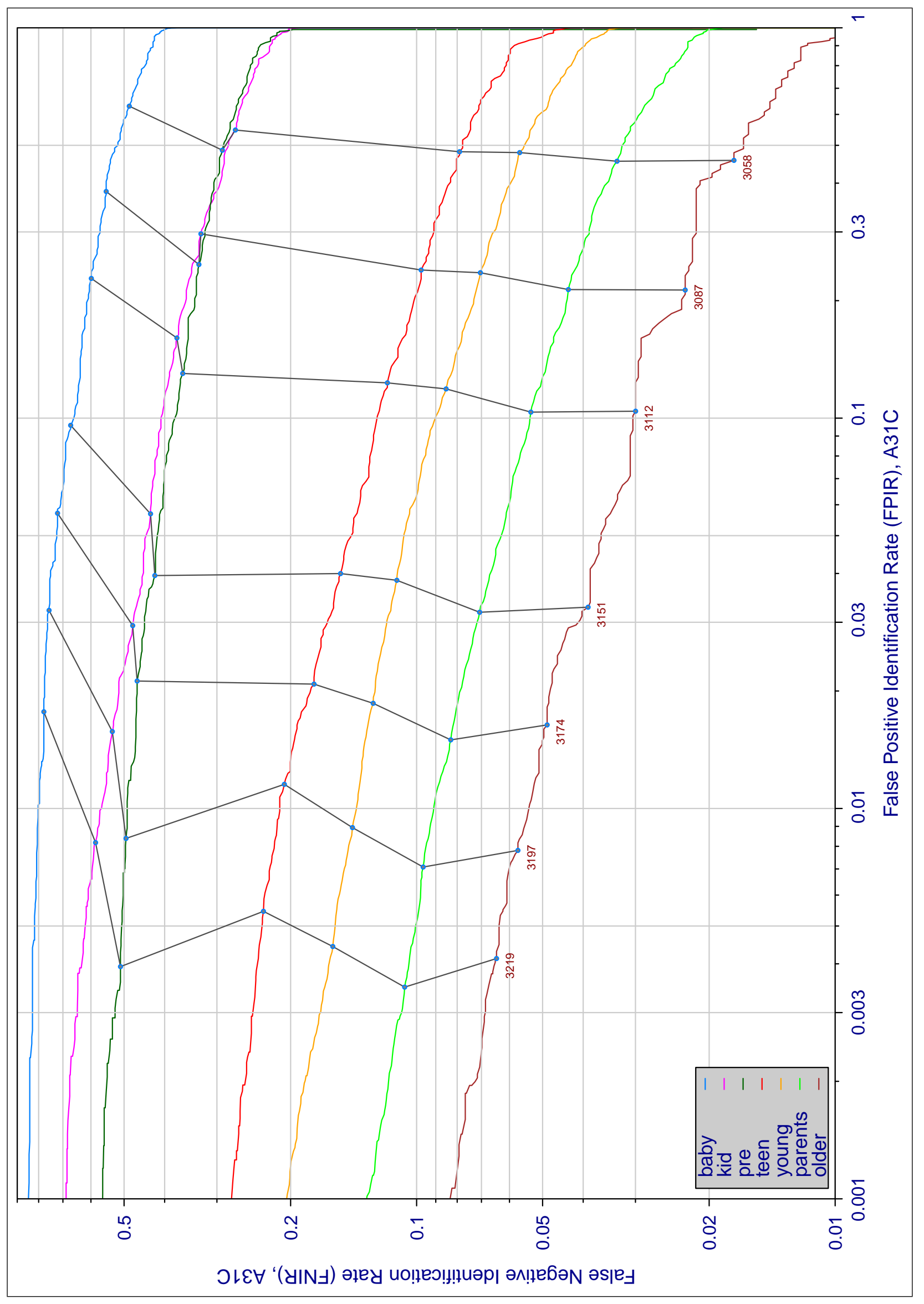

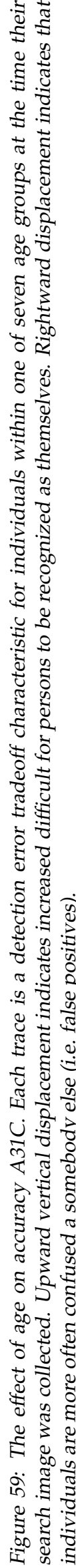

\begin{tabular}{|c|c|c|c|c|c|c|}
\hline $\begin{array}{l}\mathrm{A}=3 \mathrm{M} / \text { Cogent } \\
\mathrm{G}=\text { Hisign } \\
\mathrm{P}=\text { Zhuhai-Yisheng }\end{array}$ & $\begin{array}{l}B=\text { Cognitec } \\
H=C A S-I A \\
Q=\text { JunYu }\end{array}$ & $\begin{array}{l}\mathrm{C}=\text { Neurotechnology } \\
\mathrm{I}=\text { CAS-ICT } \\
\mathrm{S}=\text { Decatur }\end{array}$ & $\begin{array}{l}\mathrm{D}=\text { Safran Morpho } \\
\mathrm{J}=\text { Toshiba } \\
\mathrm{T}=\text { Ayonix }\end{array}$ & $\begin{array}{l}E=N E C \\
L=\text { Tsinghua U. II }\end{array}$ & $\begin{array}{l}\mathrm{F}=\text { Tsinghua } \mathrm{U} \\
\mathrm{M}=\mathrm{HP}\end{array}$ & $\begin{array}{l}\text { FNIR(N,R,T,L) “Miss rate" } \\
\text { FPIR(N,T,L) "False alarm rate" }\end{array}$ \\
\hline
\end{tabular}




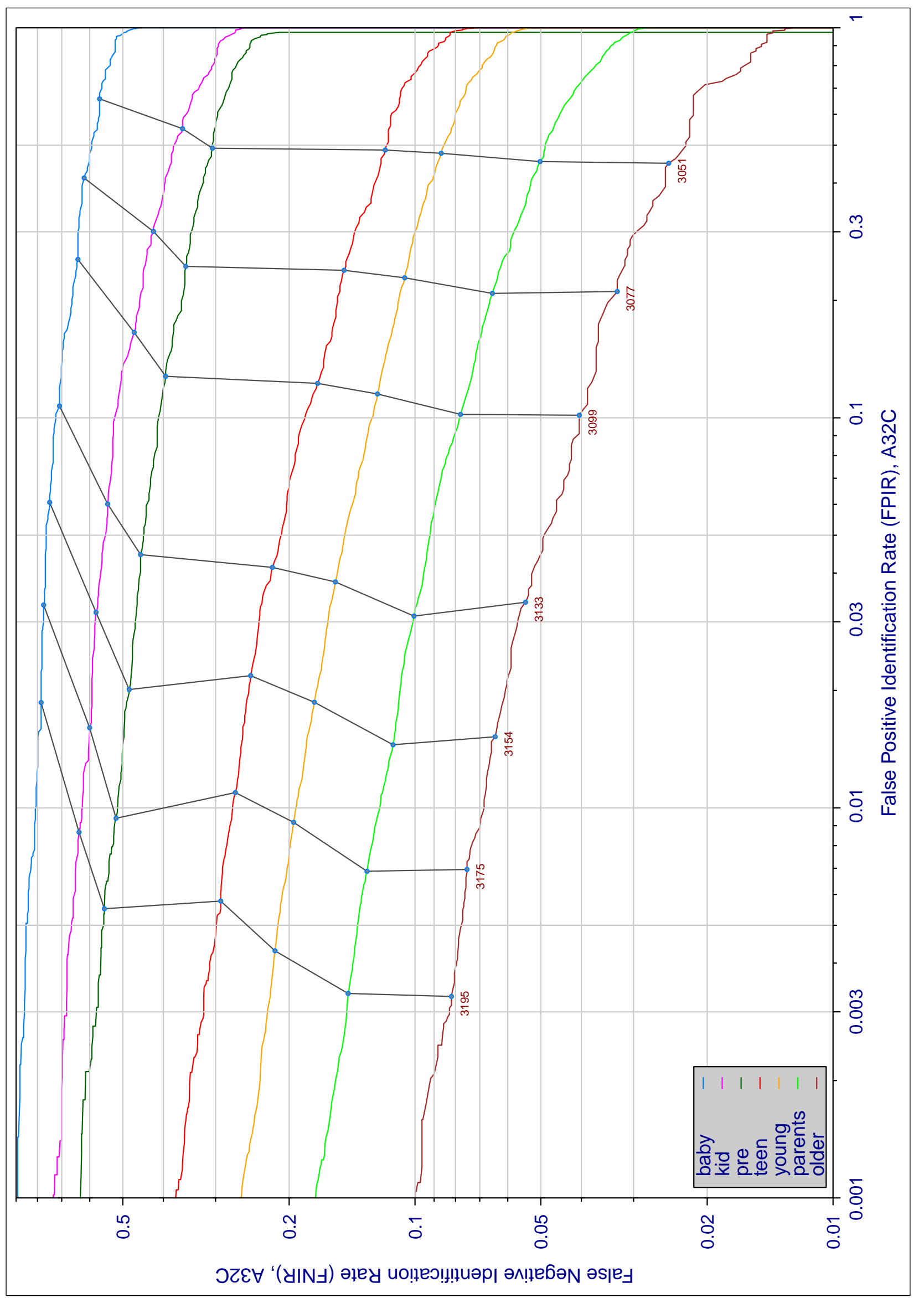

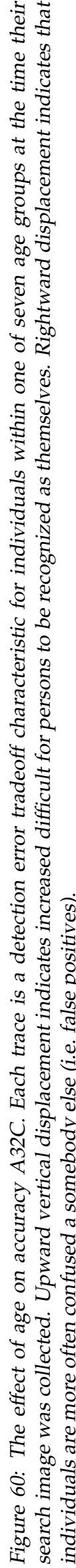

\begin{tabular}{|c|c|c|c|c|c|c|}
\hline $\begin{array}{l}\mathrm{A}=3 \mathrm{M} / \text { Cogent } \\
\mathrm{G}=\text { Hisign } \\
\mathrm{P}=\text { Zhuhai-Yisheng }\end{array}$ & $\begin{array}{l}B=\text { Cognitec } \\
H=C A S-I A \\
Q=\text { JunYu }\end{array}$ & $\begin{array}{l}\mathrm{C}=\text { Neurotechnology } \\
\mathrm{I}=\text { CAS-ICT } \\
\mathrm{S}=\text { Decatur }\end{array}$ & $\begin{array}{l}\mathrm{D}=\text { Safran Morpho } \\
\mathrm{J}=\text { Toshiba } \\
\mathrm{T}=\text { Ayonix }\end{array}$ & $\begin{array}{l}E=N E C \\
L=\text { Tsinghua U. II }\end{array}$ & $\begin{array}{l}\mathrm{F}=\text { Tsinghua } \mathrm{U} \\
\mathrm{M}=\mathrm{HP}\end{array}$ & $\begin{array}{l}\text { FNIR(N,R,T,L) “Miss rate" } \\
\text { FPIR(N,T,L) "False alarm rate" }\end{array}$ \\
\hline
\end{tabular}




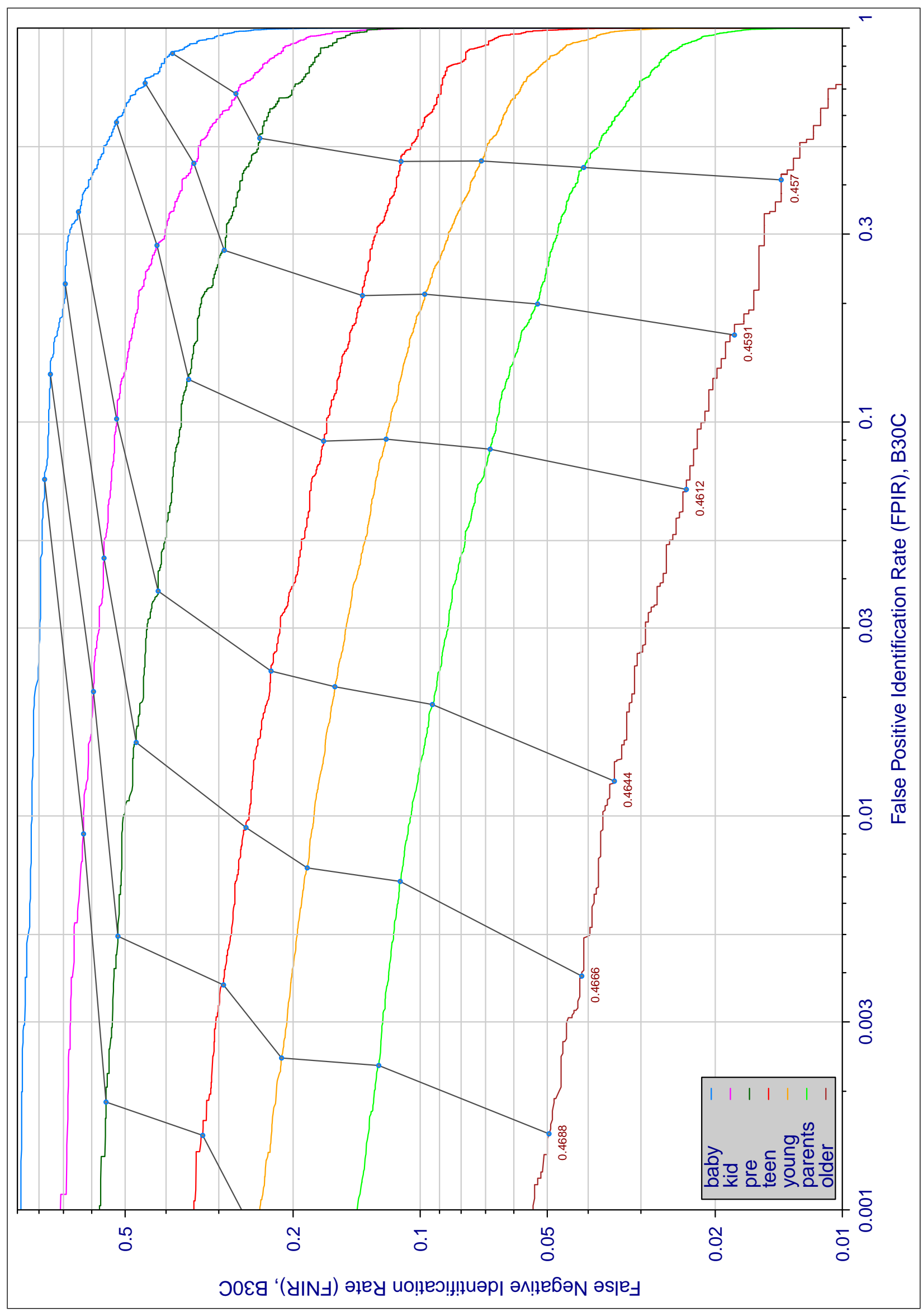

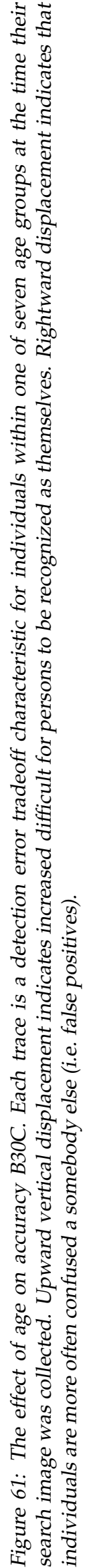

\begin{tabular}{|c|c|c|c|c|c|c|}
\hline $\begin{array}{l}\mathrm{A}=3 \mathrm{M} / \text { Cogent } \\
\mathrm{G}=\text { Hisign } \\
\mathrm{P}=\text { Zhuhai-Yisheng }\end{array}$ & $\begin{array}{l}B=\text { Cognitec } \\
H=C A S-I A \\
Q=\text { JunYu }\end{array}$ & $\begin{array}{l}\mathrm{C}=\text { Neurotechnology } \\
\mathrm{I}=\text { CAS-ICT } \\
\mathrm{S}=\text { Decatur }\end{array}$ & $\begin{array}{l}\mathrm{D}=\text { Safran Morpho } \\
\mathrm{J}=\text { Toshiba } \\
\mathrm{T}=\text { Ayonix }\end{array}$ & $\begin{array}{l}E=N E C \\
L=\text { Tsinghua U. II }\end{array}$ & $\begin{array}{l}\mathrm{F}=\text { Tsinghua } \mathrm{U} \\
\mathrm{M}=\mathrm{HP}\end{array}$ & $\begin{array}{l}\text { FNIR(N,R,T,L) “Miss rate" } \\
\text { FPIR(N,T,L) "False alarm rate" }\end{array}$ \\
\hline
\end{tabular}




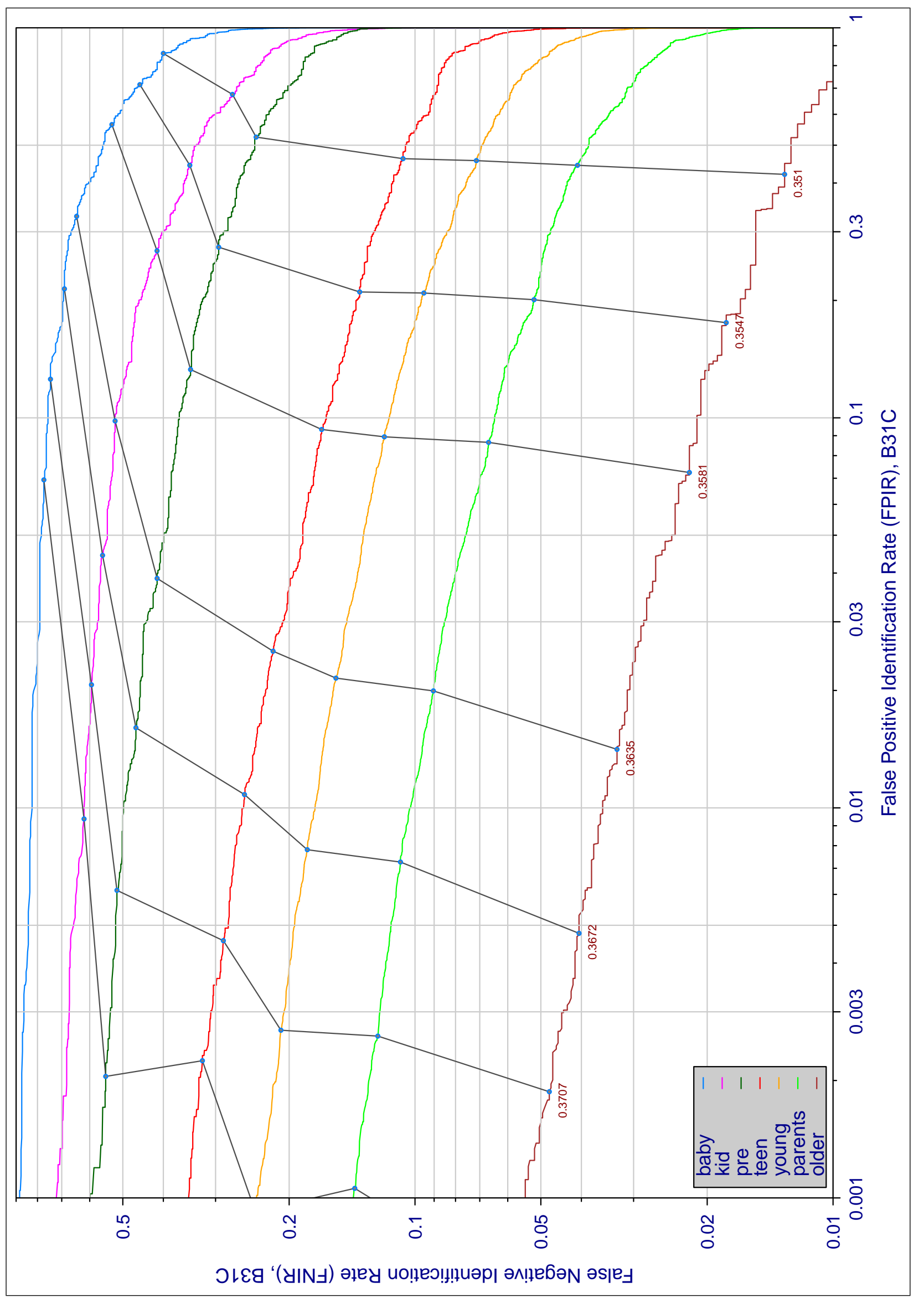

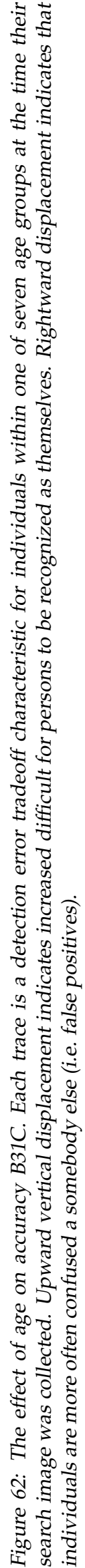

\begin{tabular}{|c|c|c|c|c|c|c|}
\hline $\begin{array}{l}\mathrm{A}=3 \mathrm{M} / \text { Cogent } \\
\mathrm{G}=\text { Hisign } \\
\mathrm{P}=\text { Zhuhai-Yisheng }\end{array}$ & $\begin{array}{l}B=\text { Cognitec } \\
H=C A S-I A \\
Q=\text { JunYu }\end{array}$ & $\begin{array}{l}\mathrm{C}=\text { Neurotechnology } \\
\mathrm{I}=\text { CAS-ICT } \\
\mathrm{S}=\text { Decatur }\end{array}$ & $\begin{array}{l}\mathrm{D}=\text { Safran Morpho } \\
\mathrm{J}=\text { Toshiba } \\
\mathrm{T}=\text { Ayonix }\end{array}$ & $\begin{array}{l}E=N E C \\
L=\text { Tsinghua U. II }\end{array}$ & $\begin{array}{l}\mathrm{F}=\text { Tsinghua } \mathrm{U} \\
\mathrm{M}=\mathrm{HP}\end{array}$ & $\begin{array}{l}\text { FNIR(N,R,T,L) “Miss rate" } \\
\text { FPIR(N,T,L) "False alarm rate" }\end{array}$ \\
\hline
\end{tabular}




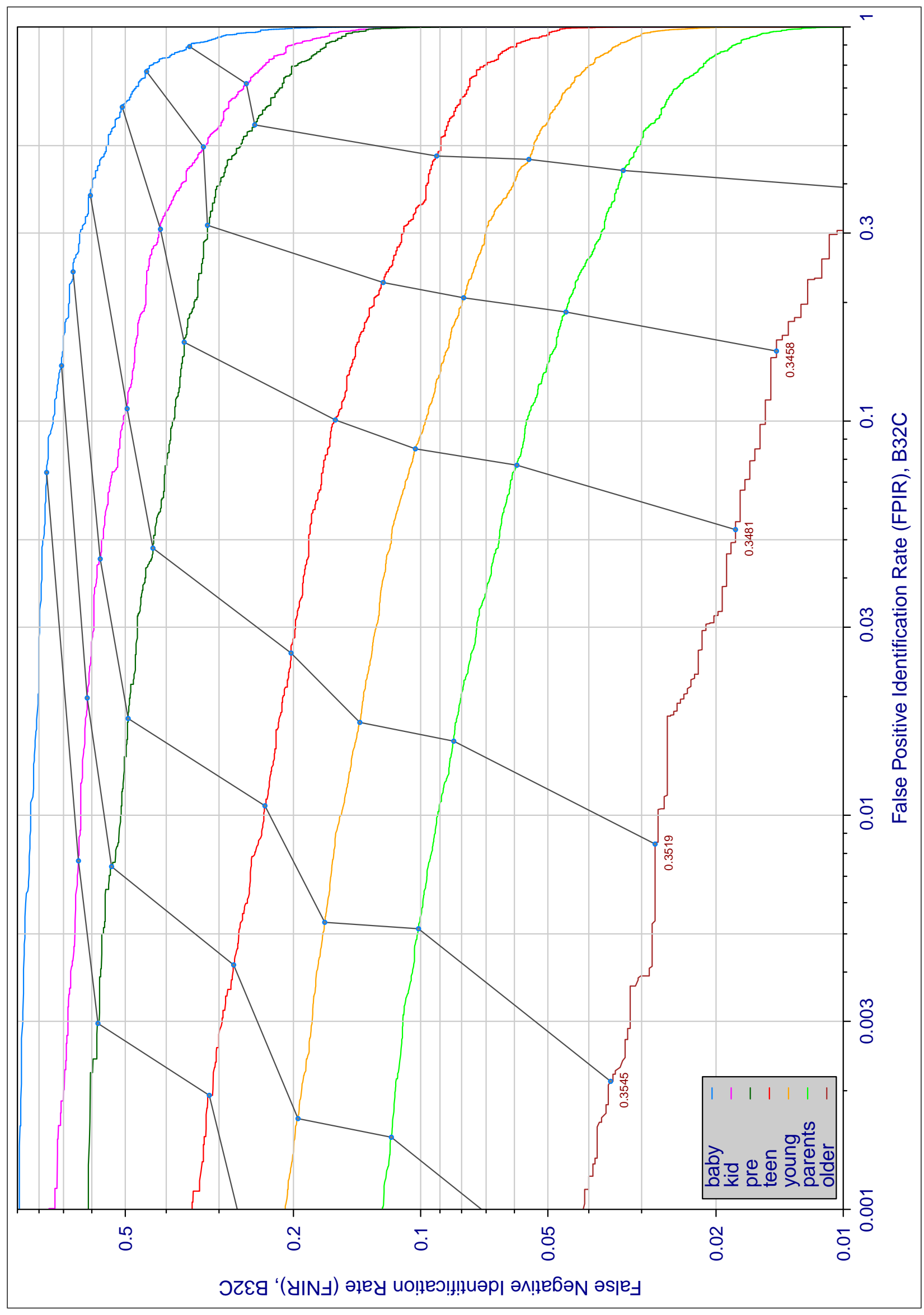

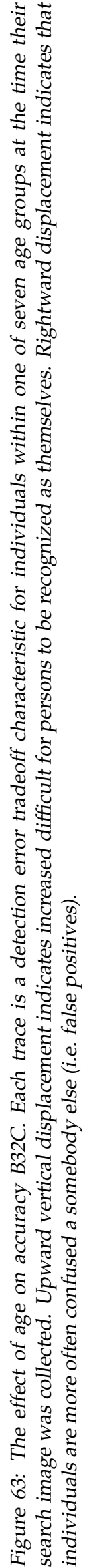

\begin{tabular}{|c|c|c|c|c|c|c|}
\hline $\begin{array}{l}\mathrm{A}=3 \mathrm{M} / \text { Cogent } \\
\mathrm{G}=\text { Hisign } \\
\mathrm{P}=\text { Zhuhai-Yisheng }\end{array}$ & $\begin{array}{l}B=\text { Cognitec } \\
H=C A S-I A \\
Q=\text { JunYu }\end{array}$ & $\begin{array}{l}\mathrm{C}=\text { Neurotechnology } \\
\mathrm{I}=\text { CAS-ICT } \\
\mathrm{S}=\text { Decatur }\end{array}$ & $\begin{array}{l}\mathrm{D}=\text { Safran Morpho } \\
\mathrm{J}=\text { Toshiba } \\
\mathrm{T}=\text { Ayonix }\end{array}$ & $\begin{array}{l}E=N E C \\
L=\text { Tsinghua U. II }\end{array}$ & $\begin{array}{l}\mathrm{F}=\text { Tsinghua } \mathrm{U} \\
\mathrm{M}=\mathrm{HP}\end{array}$ & $\begin{array}{l}\text { FNIR(N,R,T,L) “Miss rate" } \\
\text { FPIR(N,T,L) "False alarm rate" }\end{array}$ \\
\hline
\end{tabular}




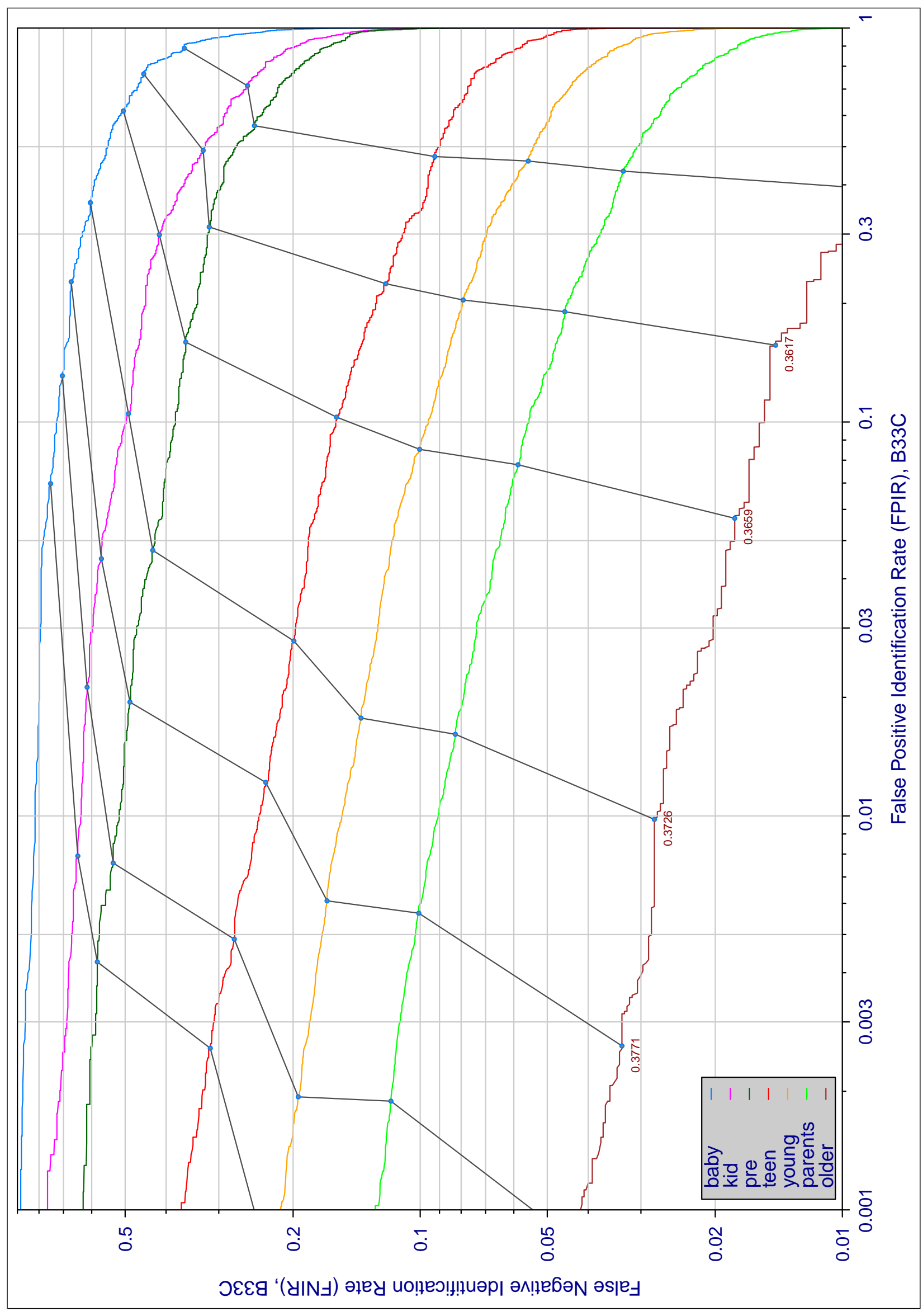

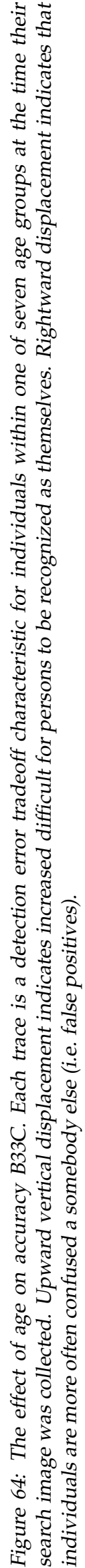

\begin{tabular}{|c|c|c|c|c|c|c|}
\hline $\begin{array}{l}\mathrm{A}=3 \mathrm{M} / \text { Cogent } \\
\mathrm{G}=\text { Hisign } \\
\mathrm{P}=\text { Zhuhai-Yisheng }\end{array}$ & $\begin{array}{l}B=\text { Cognitec } \\
H=C A S-I A \\
Q=\text { JunYu }\end{array}$ & $\begin{array}{l}\mathrm{C}=\text { Neurotechnology } \\
\mathrm{I}=\text { CAS-ICT } \\
\mathrm{S}=\text { Decatur }\end{array}$ & $\begin{array}{l}\mathrm{D}=\text { Safran Morpho } \\
\mathrm{J}=\text { Toshiba } \\
\mathrm{T}=\text { Ayonix }\end{array}$ & $\begin{array}{l}E=N E C \\
L=\text { Tsinghua U. II }\end{array}$ & $\begin{array}{l}\mathrm{F}=\text { Tsinghua } \mathrm{U} \\
\mathrm{M}=\mathrm{HP}\end{array}$ & $\begin{array}{l}\text { FNIR(N,R,T,L) “Miss rate" } \\
\text { FPIR(N,T,L) "False alarm rate" }\end{array}$ \\
\hline
\end{tabular}




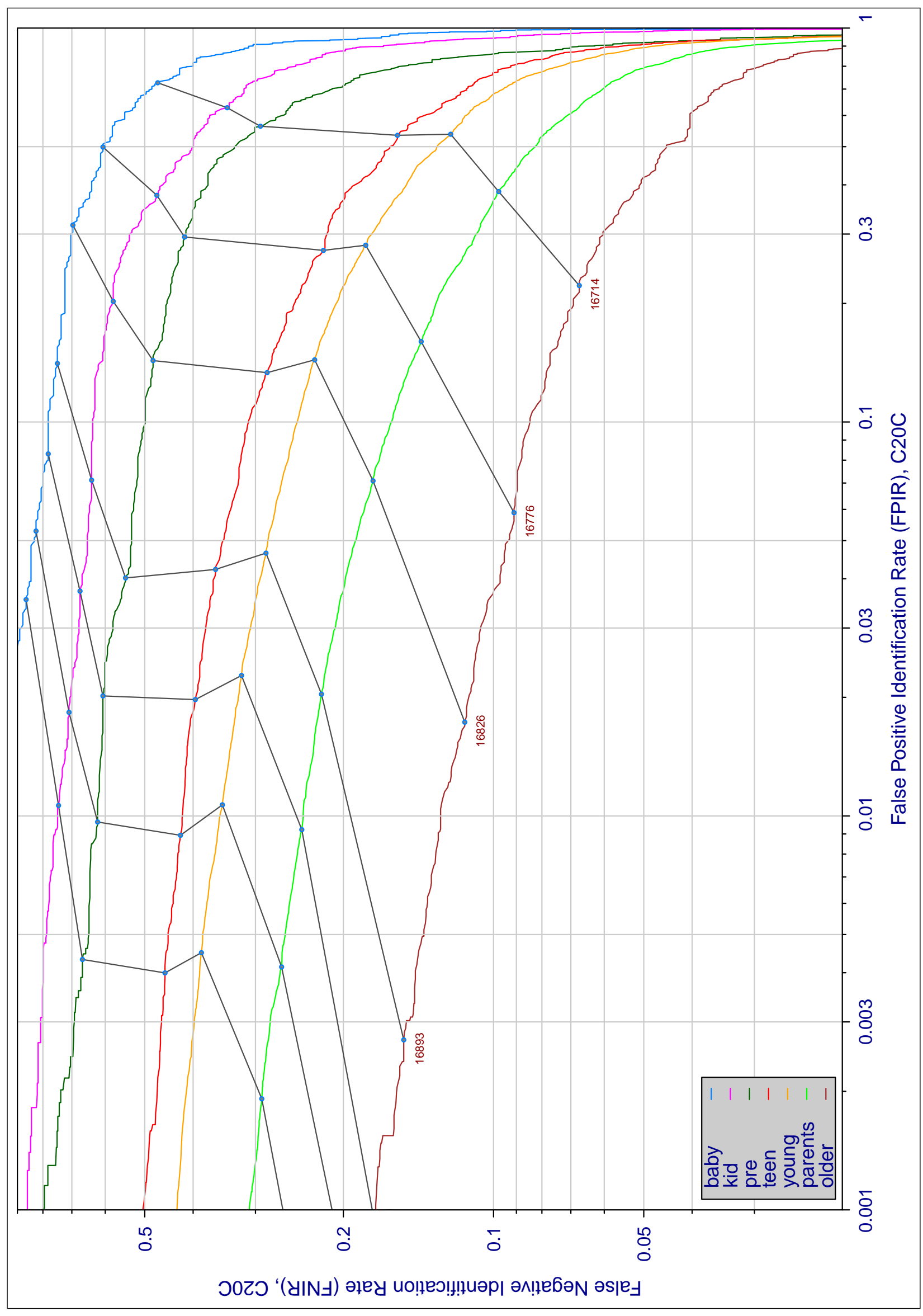

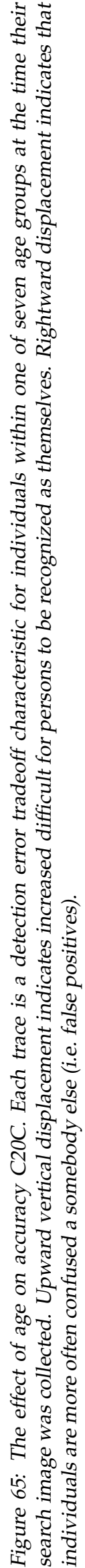

\begin{tabular}{|c|c|c|c|c|c|c|}
\hline $\begin{array}{l}\mathrm{A}=3 \mathrm{M} / \text { Cogent } \\
\mathrm{G}=\text { Hisign } \\
\mathrm{P}=\text { Zhuhai-Yisheng }\end{array}$ & $\begin{array}{l}\mathrm{B}=\text { Cognitec } \\
\mathrm{H}=\mathrm{CAS}-\mathrm{IA} \\
\mathrm{Q}=\text { JunYu }\end{array}$ & $\begin{array}{l}\mathrm{C}=\text { Neurotechnology } \\
\mathrm{I}=\text { CAS-ICT } \\
\mathrm{S}=\text { Decatur }\end{array}$ & $\begin{array}{l}\mathrm{D}=\text { Safran Morpho } \\
\mathrm{J}=\text { Toshiba } \\
\mathrm{T}=\text { Ayonix }\end{array}$ & $\begin{array}{l}E=N E C \\
L=\text { Tsinghua U. II }\end{array}$ & $\begin{array}{l}\mathrm{F}=\text { Tsinghua } \mathrm{U} \\
\mathrm{M}=\mathrm{HP}\end{array}$ & $\begin{array}{l}\text { FNIR(N,R,T,L) “Miss rate" } \\
\text { FPIR(N,T,L) "False alarm rate" }\end{array}$ \\
\hline
\end{tabular}




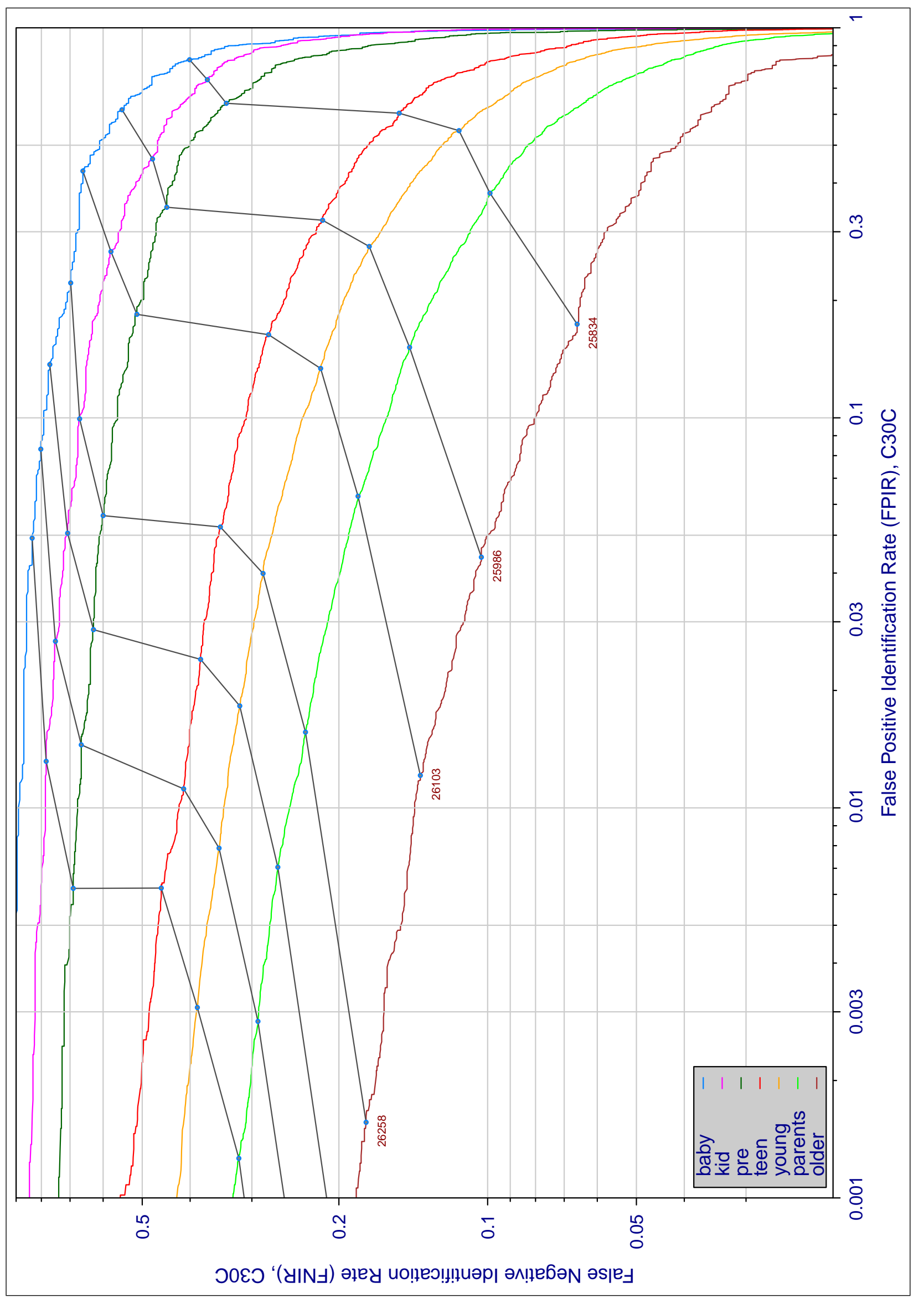

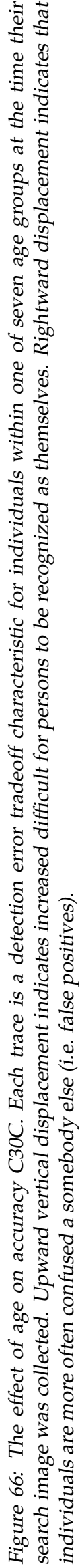

\begin{tabular}{|c|c|c|c|c|c|c|}
\hline $\begin{array}{l}\mathrm{A}=3 \mathrm{M} / \text { Cogent } \\
\mathrm{G}=\text { Hisign } \\
\mathrm{P}=\text { Zhuhai-Yisheng }\end{array}$ & $\begin{array}{l}B=\text { Cognitec } \\
H=C A S-I A \\
Q=\text { JunYu }\end{array}$ & $\begin{array}{l}\mathrm{C}=\text { Neurotechnology } \\
\mathrm{I}=\text { CAS-ICT } \\
\mathrm{S}=\text { Decatur }\end{array}$ & $\begin{array}{l}\mathrm{D}=\text { Safran Morpho } \\
\mathrm{J}=\text { Toshiba } \\
\mathrm{T}=\text { Ayonix }\end{array}$ & $\begin{array}{l}E=N E C \\
L=\text { Tsinghua U. II }\end{array}$ & $\begin{array}{l}\mathrm{F}=\text { Tsinghua } \mathrm{U} \\
\mathrm{M}=\mathrm{HP}\end{array}$ & $\begin{array}{l}\text { FNIR(N,R,T,L) “Miss rate" } \\
\text { FPIR(N,T,L) "False alarm rate" }\end{array}$ \\
\hline
\end{tabular}




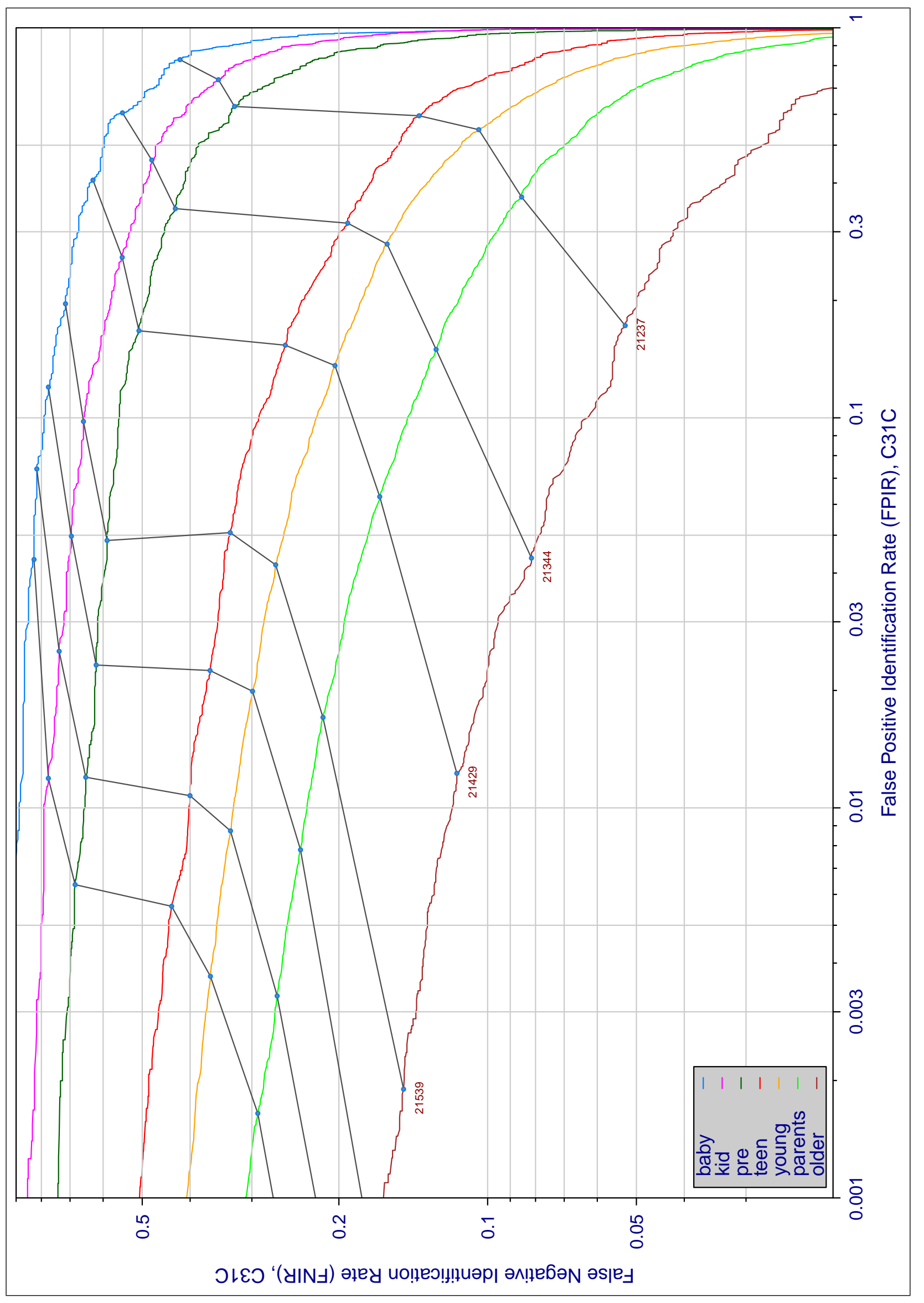

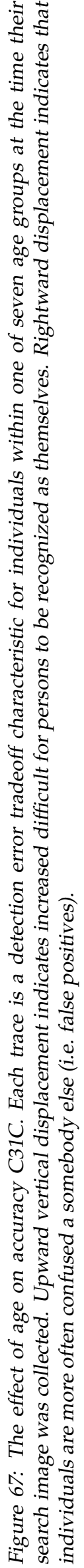

\begin{tabular}{|c|c|c|c|c|c|c|}
\hline $\begin{array}{l}\mathrm{A}=3 \mathrm{M} / \text { Cogent } \\
\mathrm{G}=\text { Hisign } \\
\mathrm{P}=\text { Zhuhai-Yisheng }\end{array}$ & $\begin{array}{l}B=\text { Cognitec } \\
H=C A S-I A \\
Q=\text { JunYu }\end{array}$ & $\begin{array}{l}\mathrm{C}=\text { Neurotechnology } \\
\mathrm{I}=\text { CAS-ICT } \\
\mathrm{S}=\text { Decatur }\end{array}$ & $\begin{array}{l}\mathrm{D}=\text { Safran Morpho } \\
\mathrm{J}=\text { Toshiba } \\
\mathrm{T}=\text { Ayonix }\end{array}$ & $\begin{array}{l}E=N E C \\
L=\text { Tsinghua U. II }\end{array}$ & $\begin{array}{l}\mathrm{F}=\text { Tsinghua } \mathrm{U} \\
\mathrm{M}=\mathrm{HP}\end{array}$ & $\begin{array}{l}\text { FNIR(N,R,T,L) “Miss rate" } \\
\text { FPIR(N,T,L) "False alarm rate" }\end{array}$ \\
\hline
\end{tabular}




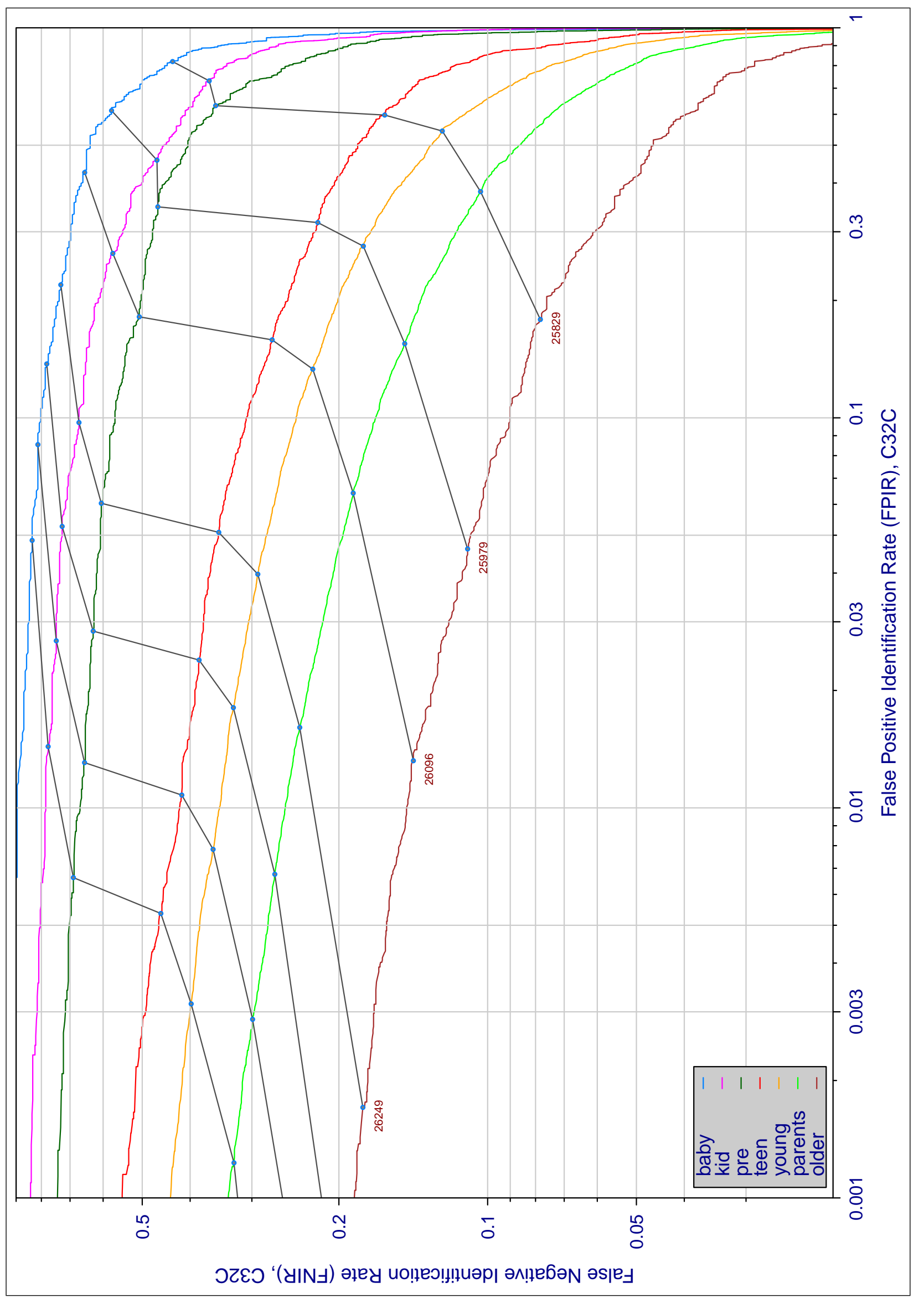

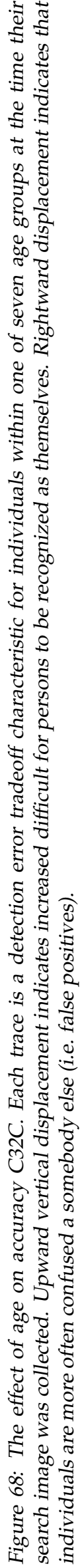

\begin{tabular}{|c|c|c|c|c|c|c|}
\hline $\begin{array}{l}\mathrm{A}=3 \mathrm{M} / \text { Cogent } \\
\mathrm{G}=\text { Hisign } \\
\mathrm{P}=\text { Zhuhai-Yisheng }\end{array}$ & $\begin{array}{l}B=\text { Cognitec } \\
H=C A S-I A \\
Q=\text { JunYu }\end{array}$ & $\begin{array}{l}\mathrm{C}=\text { Neurotechnology } \\
\mathrm{I}=\text { CAS-ICT } \\
\mathrm{S}=\text { Decatur }\end{array}$ & $\begin{array}{l}\mathrm{D}=\text { Safran Morpho } \\
\mathrm{J}=\text { Toshiba } \\
\mathrm{T}=\text { Ayonix }\end{array}$ & $\begin{array}{l}E=N E C \\
L=\text { Tsinghua U. II }\end{array}$ & $\begin{array}{l}\mathrm{F}=\text { Tsinghua } \mathrm{U} \\
\mathrm{M}=\mathrm{HP}\end{array}$ & $\begin{array}{l}\text { FNIR(N,R,T,L) “Miss rate" } \\
\text { FPIR(N,T,L) "False alarm rate" }\end{array}$ \\
\hline
\end{tabular}




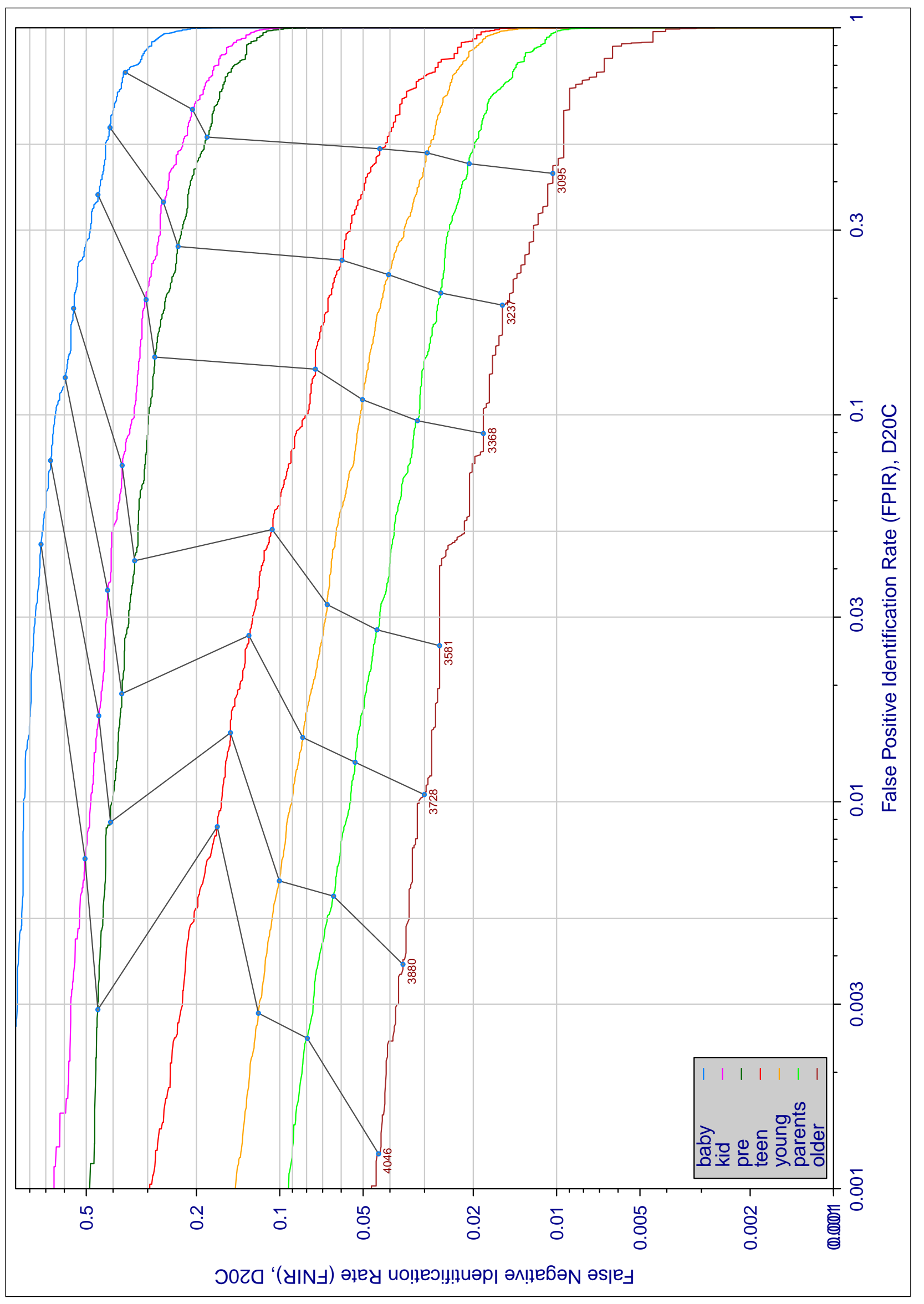

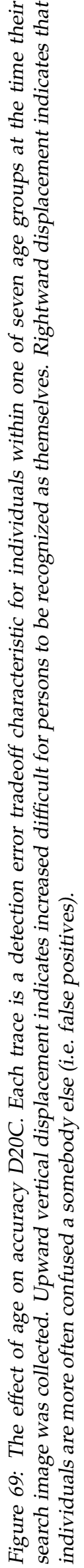

\begin{tabular}{|c|c|c|c|c|c|c|}
\hline $\begin{array}{l}\mathrm{A}=3 \mathrm{M} / \text { Cogent } \\
\mathrm{G}=\text { Hisign } \\
\mathrm{P}=\text { Zhuhai-Yisheng }\end{array}$ & $\begin{array}{l}B=\text { Cognitec } \\
H=C A S-I A \\
Q=\text { JunYu }\end{array}$ & $\begin{array}{l}\mathrm{C}=\text { Neurotechnology } \\
\mathrm{I}=\text { CAS-ICT } \\
\mathrm{S}=\text { Decatur }\end{array}$ & $\begin{array}{l}\mathrm{D}=\text { Safran Morpho } \\
\mathrm{J}=\text { Toshiba } \\
\mathrm{T}=\text { Ayonix }\end{array}$ & $\begin{array}{l}E=N E C \\
L=\text { Tsinghua U. II }\end{array}$ & $\begin{array}{l}\mathrm{F}=\text { Tsinghua } \mathrm{U} \\
\mathrm{M}=\mathrm{HP}\end{array}$ & $\begin{array}{l}\text { FNIR(N,R,T,L) “Miss rate" } \\
\text { FPIR(N,T,L) "False alarm rate" }\end{array}$ \\
\hline
\end{tabular}




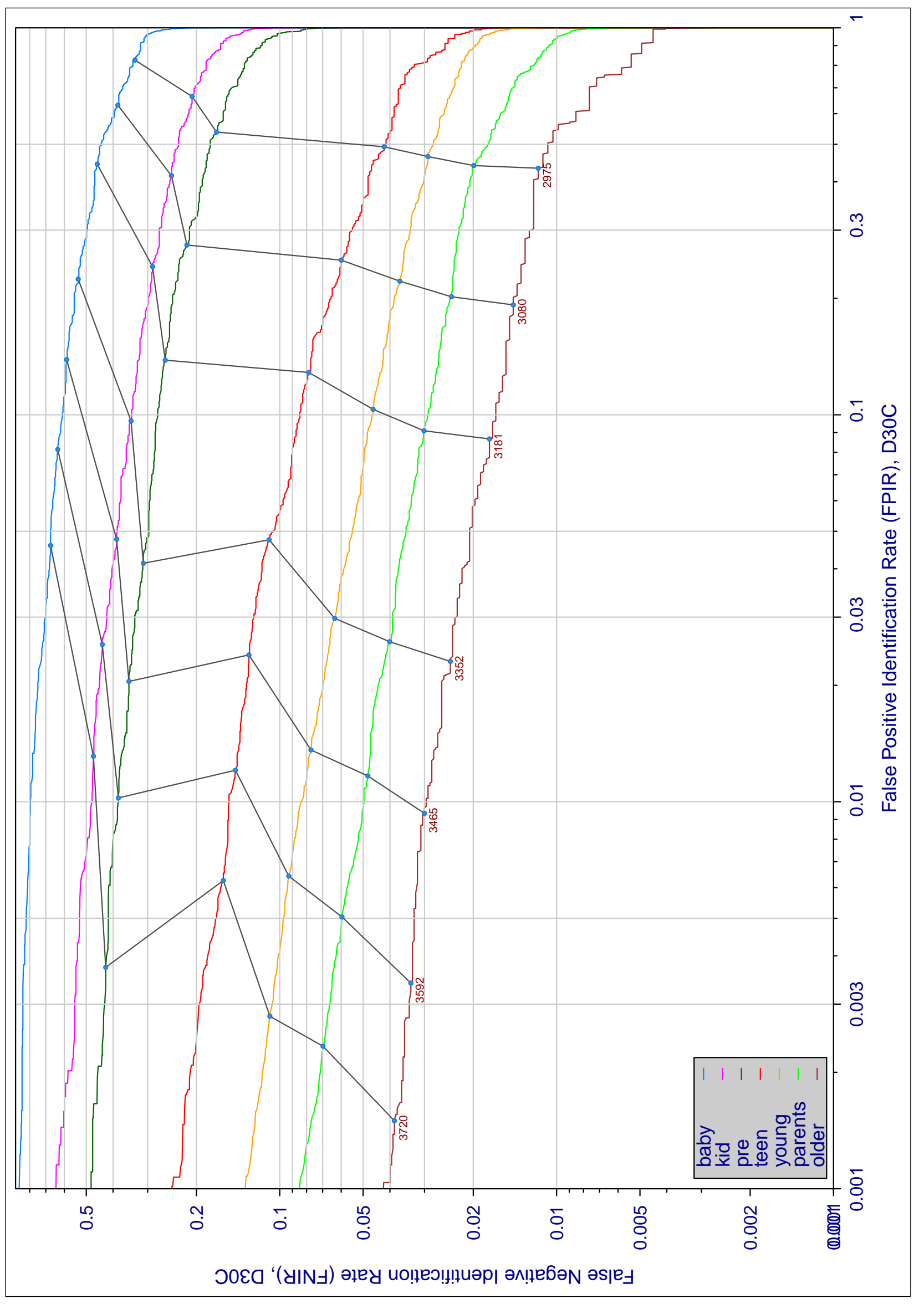

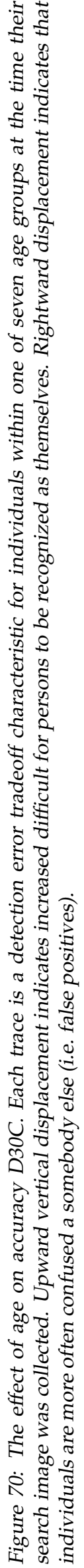

\begin{tabular}{|c|c|c|c|c|c|c|}
\hline $\begin{array}{l}\mathrm{A}=3 \mathrm{M} / \text { Cogent } \\
\mathrm{G}=\text { Hisign } \\
\mathrm{P}=\text { Zhuhai-Yisheng }\end{array}$ & $\begin{array}{l}B=\text { Cognitec } \\
H=C A S-I A \\
Q=\text { JunYu }\end{array}$ & $\begin{array}{l}\mathrm{C}=\text { Neurotechnology } \\
\mathrm{I}=\text { CAS-ICT } \\
\mathrm{S}=\text { Decatur }\end{array}$ & $\begin{array}{l}\mathrm{D}=\text { Safran Morpho } \\
\mathrm{J}=\text { Toshiba } \\
\mathrm{T}=\text { Ayonix }\end{array}$ & $\begin{array}{l}E=N E C \\
L=\text { Tsinghua U. II }\end{array}$ & $\begin{array}{l}\mathrm{F}=\text { Tsinghua } \mathrm{U} \\
\mathrm{M}=\mathrm{HP}\end{array}$ & $\begin{array}{l}\text { FNIR(N,R,T,L) “Miss rate" } \\
\text { FPIR(N,T,L) "False alarm rate" }\end{array}$ \\
\hline
\end{tabular}




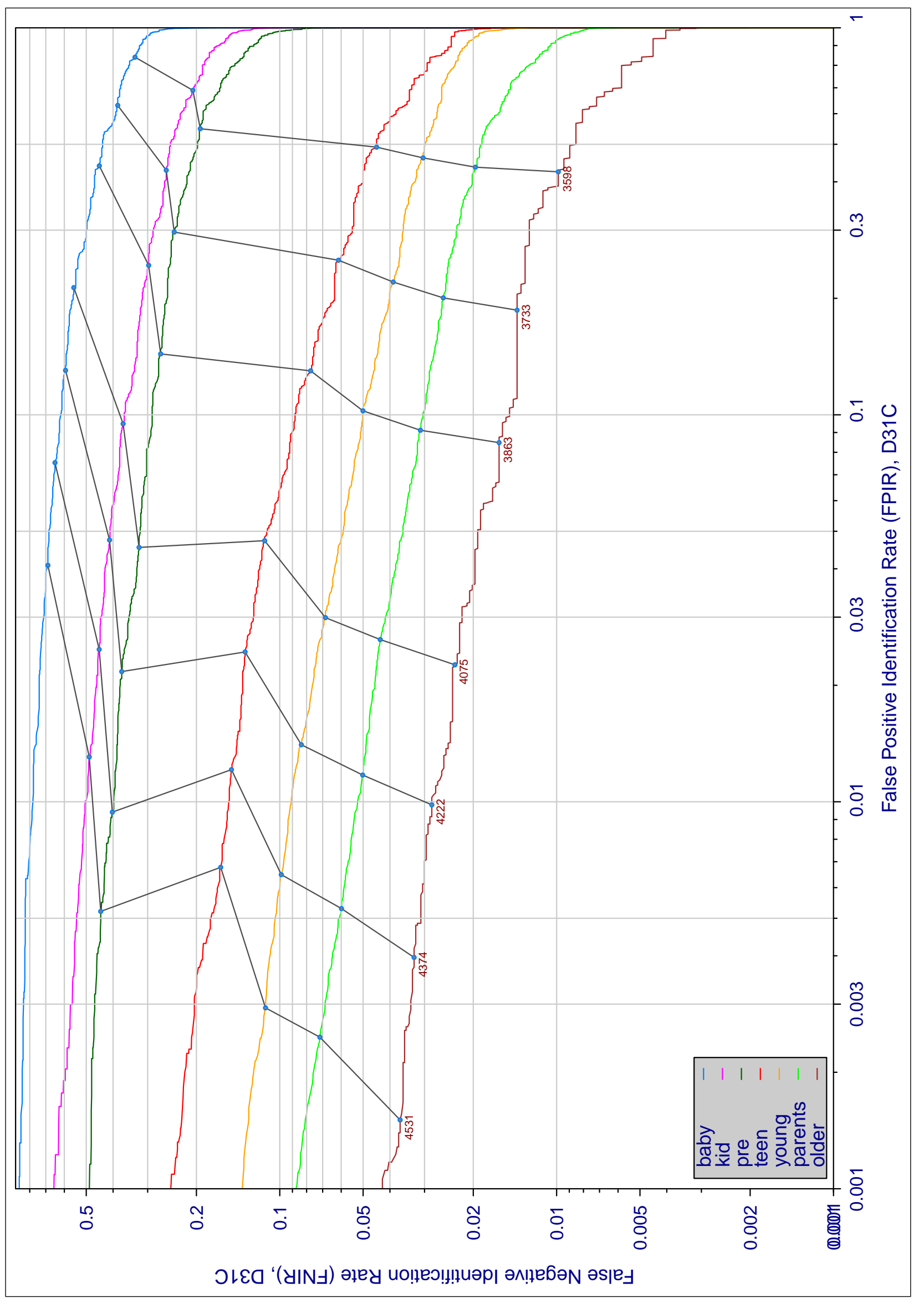

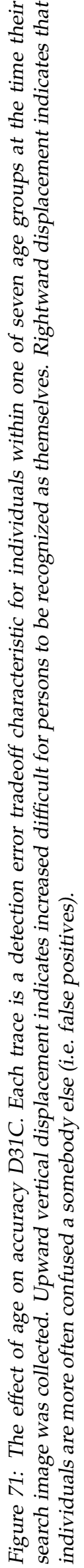

\begin{tabular}{|c|c|c|c|c|c|c|}
\hline $\begin{array}{l}\mathrm{A}=3 \mathrm{M} / \text { Cogent } \\
\mathrm{G}=\text { Hisign } \\
\mathrm{P}=\text { Zhuhai-Yisheng }\end{array}$ & $\begin{array}{l}B=\text { Cognitec } \\
H=C A S-I A \\
Q=\text { JunYu }\end{array}$ & $\begin{array}{l}\mathrm{C}=\text { Neurotechnology } \\
\mathrm{I}=\text { CAS-ICT } \\
\mathrm{S}=\text { Decatur }\end{array}$ & $\begin{array}{l}\mathrm{D}=\text { Safran Morpho } \\
\mathrm{J}=\text { Toshiba } \\
\mathrm{T}=\text { Ayonix }\end{array}$ & $\begin{array}{l}E=N E C \\
L=\text { Tsinghua U. II }\end{array}$ & $\begin{array}{l}\mathrm{F}=\text { Tsinghua } \mathrm{U} \\
\mathrm{M}=\mathrm{HP}\end{array}$ & $\begin{array}{l}\text { FNIR(N,R,T,L) “Miss rate" } \\
\text { FPIR(N,T,L) "False alarm rate" }\end{array}$ \\
\hline
\end{tabular}




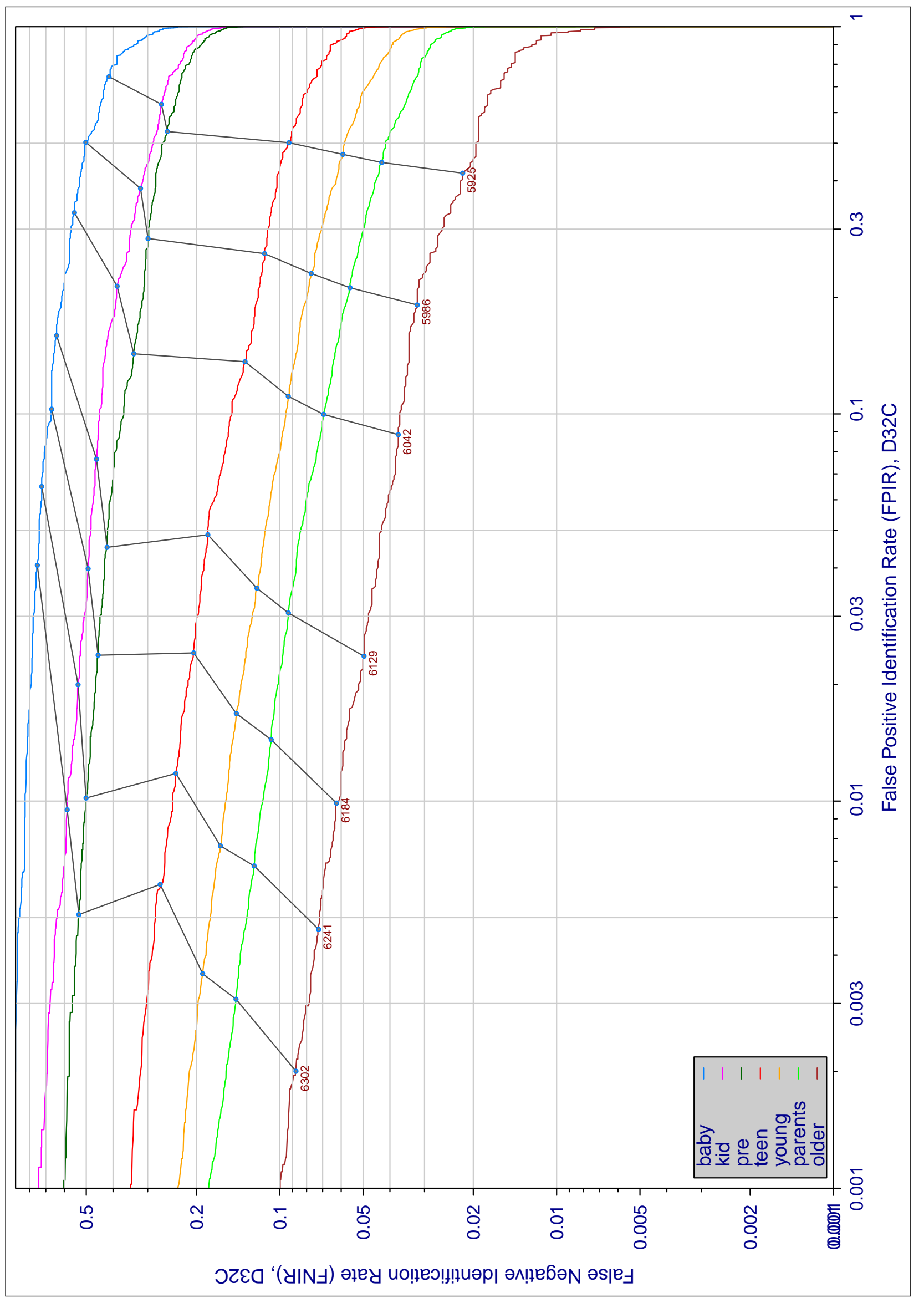

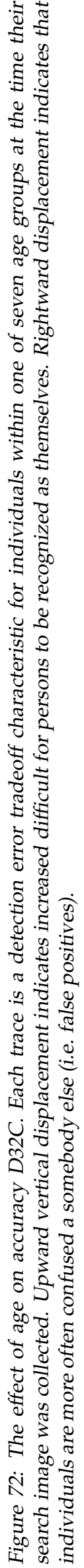

\begin{tabular}{|c|c|c|c|c|c|c|}
\hline $\begin{array}{l}\mathrm{A}=3 \mathrm{M} / \text { Cogent } \\
\mathrm{G}=\text { Hisign } \\
\mathrm{P}=\text { Zhuhai-Yisheng }\end{array}$ & $\begin{array}{l}\mathrm{B}=\text { Cognitec } \\
\mathrm{H}=\mathrm{CAS}-\mathrm{IA} \\
\mathrm{Q}=\text { JunYu }\end{array}$ & $\begin{array}{l}\mathrm{C}=\text { Neurotechnology } \\
\mathrm{I}=\text { CAS-ICT } \\
\mathrm{S}=\text { Decatur }\end{array}$ & $\begin{array}{l}\mathrm{D}=\text { Safran Morpho } \\
\mathrm{J}=\text { Toshiba } \\
\mathrm{T}=\text { Ayonix }\end{array}$ & $\begin{array}{l}\mathrm{E}=\mathrm{NEC} \\
\mathrm{L}=\text { Tsinghua U. II }\end{array}$ & $\begin{array}{l}\mathrm{F}=\text { Tsinghua } \mathrm{U} \\
\mathrm{M}=\mathrm{HP}\end{array}$ & $\begin{array}{l}\text { FNIR(N,R,T,L) “Miss rate" } \\
\text { FPIR(N,T,L) “False alarm rate" }\end{array}$ \\
\hline
\end{tabular}




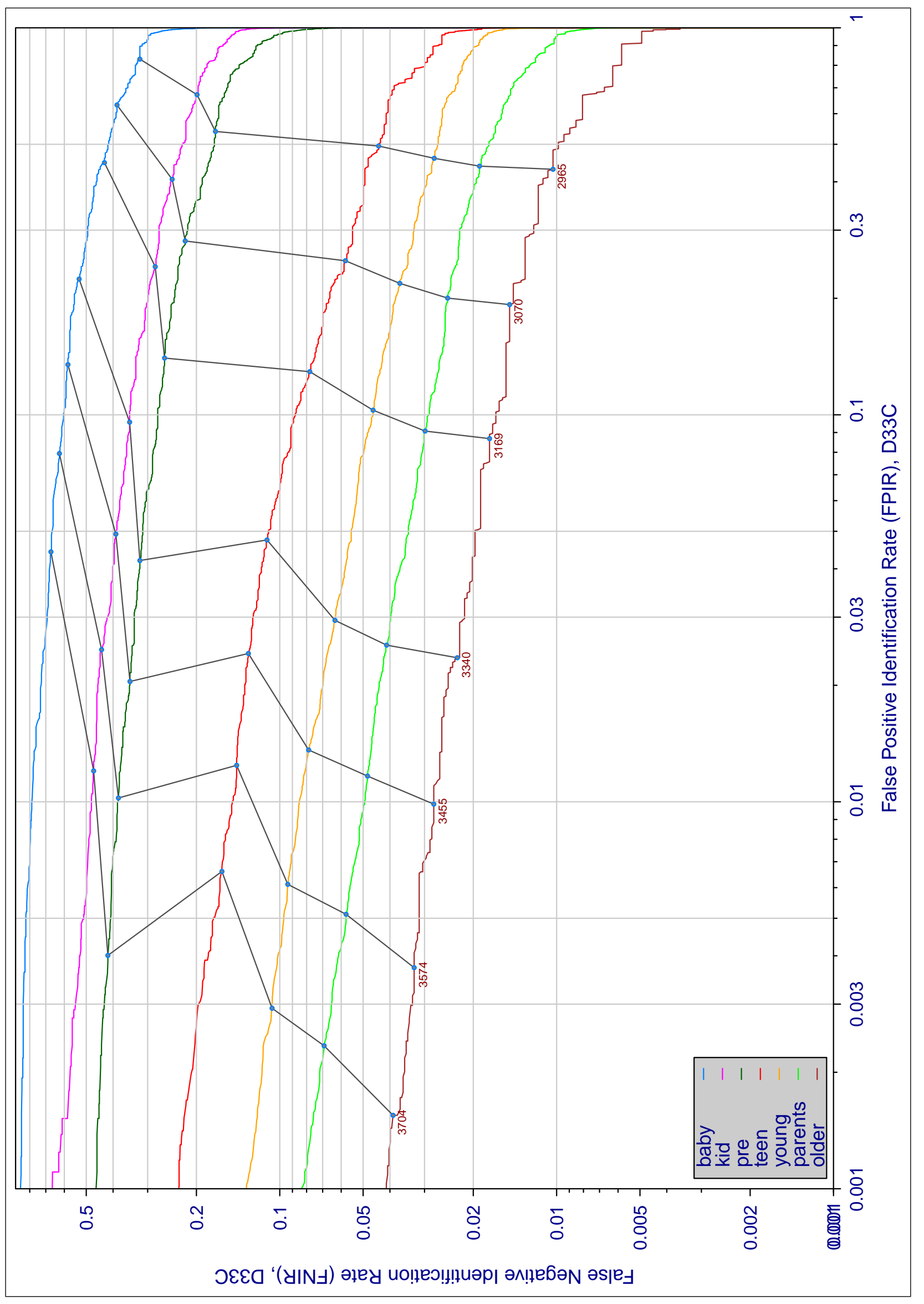

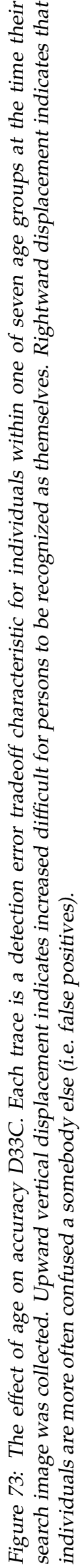

\begin{tabular}{|c|c|c|c|c|c|c|}
\hline $\begin{array}{l}\mathrm{A}=3 \mathrm{M} / \text { Cogent } \\
\mathrm{G}=\text { Hisign } \\
\mathrm{P}=\text { Zhuhai-Yisheng }\end{array}$ & $\begin{array}{l}B=\text { Cognitec } \\
H=C A S-I A \\
Q=\text { JunYu }\end{array}$ & $\begin{array}{l}\mathrm{C}=\text { Neurotechnology } \\
\mathrm{I}=\text { CAS-ICT } \\
\mathrm{S}=\text { Decatur }\end{array}$ & $\begin{array}{l}\mathrm{D}=\text { Safran Morpho } \\
\mathrm{J}=\text { Toshiba } \\
\mathrm{T}=\text { Ayonix }\end{array}$ & $\begin{array}{l}E=N E C \\
L=\text { Tsinghua U. II }\end{array}$ & $\begin{array}{l}\mathrm{F}=\text { Tsinghua } \mathrm{U} \\
\mathrm{M}=\mathrm{HP}\end{array}$ & $\begin{array}{l}\text { FNIR(N,R,T,L) “Miss rate" } \\
\text { FPIR(N,T,L) "False alarm rate" }\end{array}$ \\
\hline
\end{tabular}




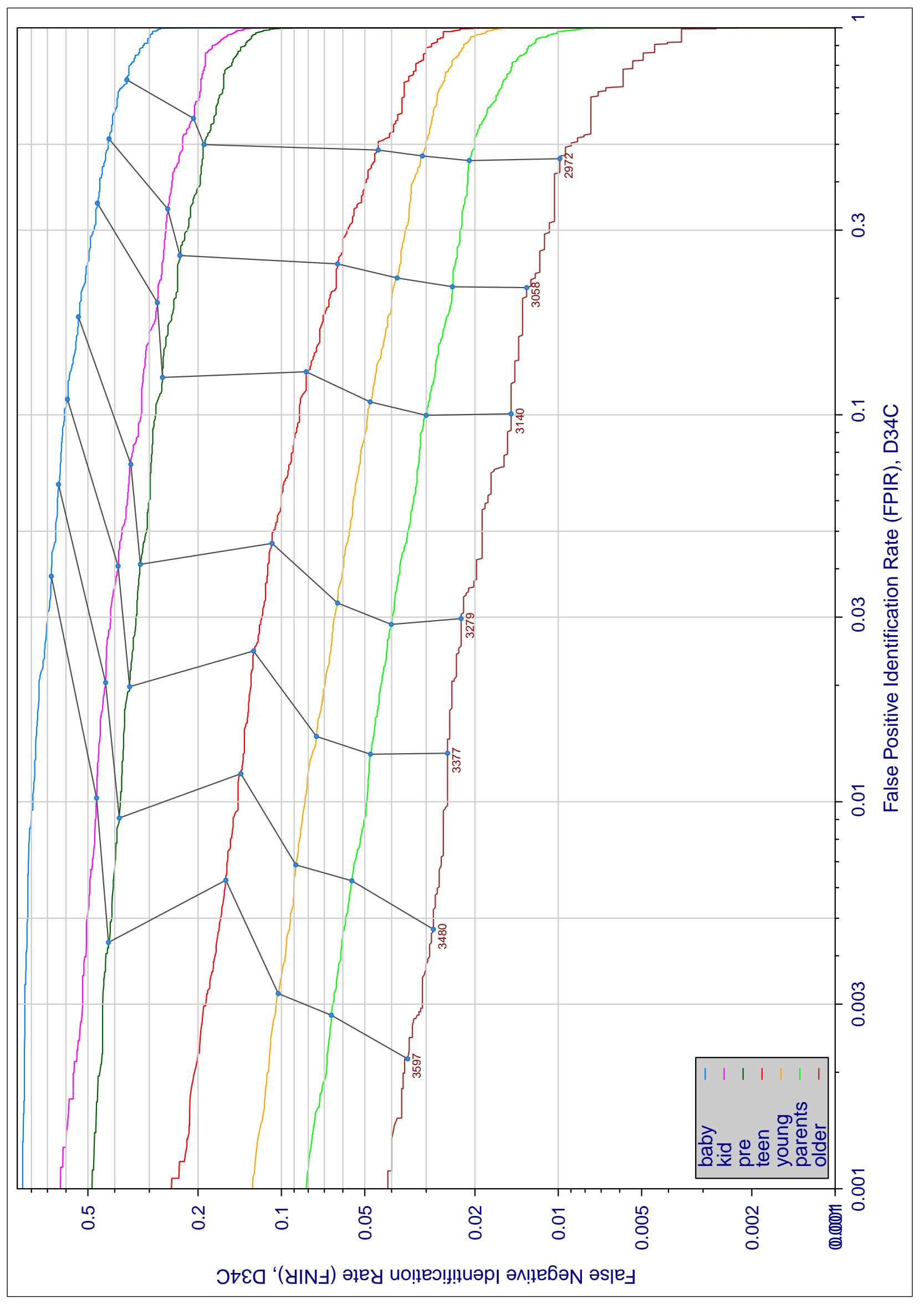

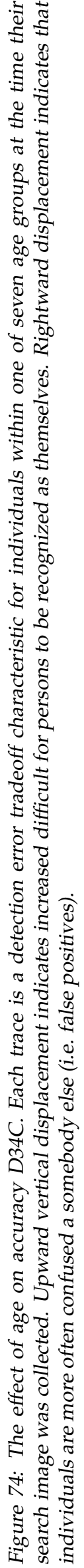

\begin{tabular}{|c|c|c|c|c|c|c|}
\hline $\begin{array}{l}\mathrm{A}=3 \mathrm{M} / \text { Cogent } \\
\mathrm{G}=\text { Hisign } \\
\mathrm{P}=\text { Zhuhai-Yisheng }\end{array}$ & $\begin{array}{l}B=\text { Cognitec } \\
H=C A S-I A \\
Q=\text { JunYu }\end{array}$ & $\begin{array}{l}\mathrm{C}=\text { Neurotechnology } \\
\mathrm{I}=\text { CAS-ICT } \\
\mathrm{S}=\text { Decatur }\end{array}$ & $\begin{array}{l}\mathrm{D}=\text { Safran Morpho } \\
\mathrm{J}=\text { Toshiba } \\
\mathrm{T}=\text { Ayonix }\end{array}$ & $\begin{array}{l}E=N E C \\
L=\text { Tsinghua U. II }\end{array}$ & $\begin{array}{l}\mathrm{F}=\text { Tsinghua } \mathrm{U} \\
\mathrm{M}=\mathrm{HP}\end{array}$ & $\begin{array}{l}\text { FNIR(N,R,T,L) “Miss rate" } \\
\text { FPIR(N,T,L) "False alarm rate" }\end{array}$ \\
\hline
\end{tabular}




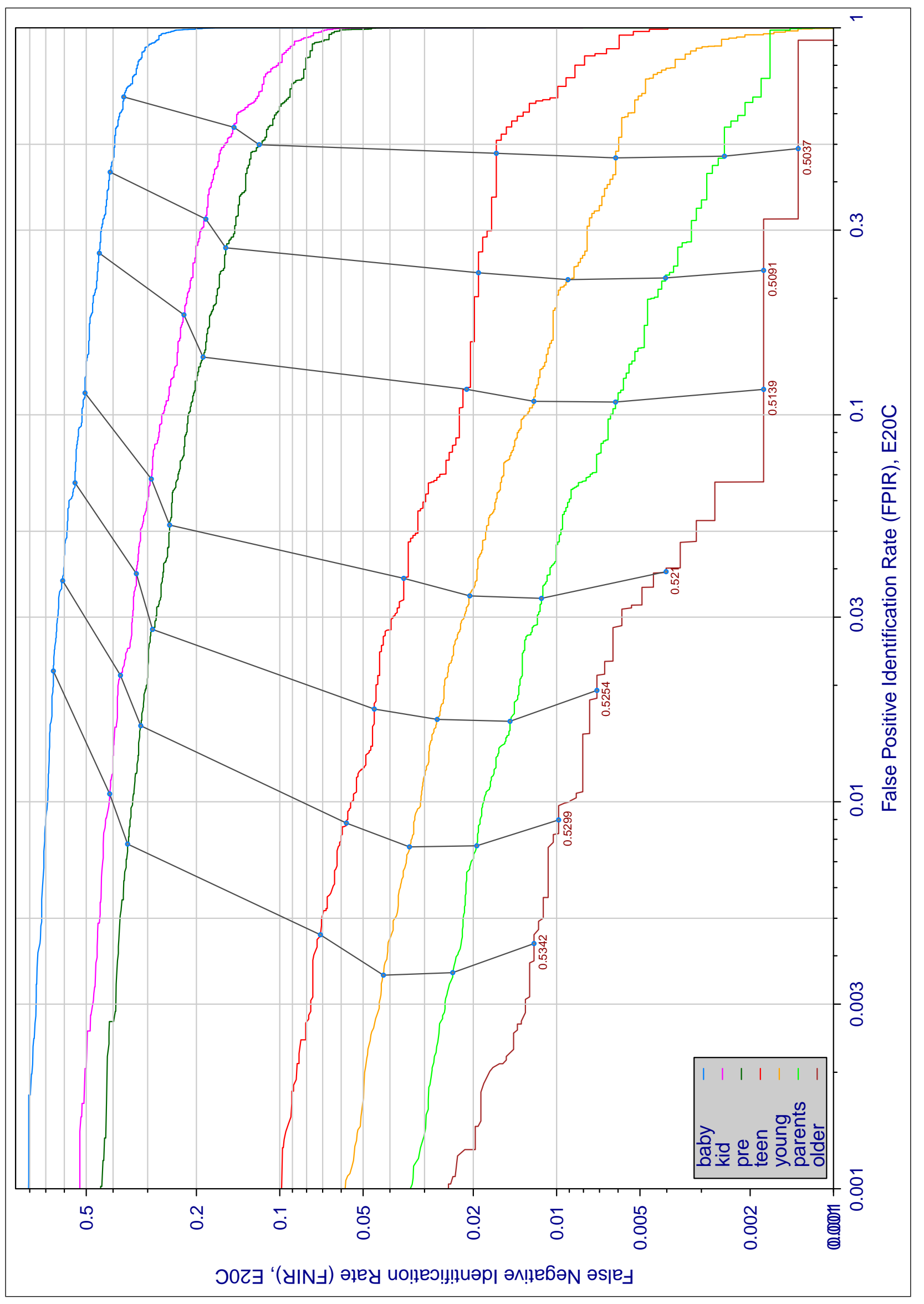

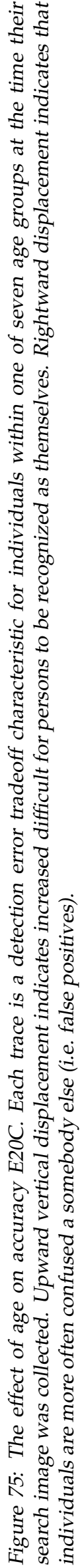

\begin{tabular}{|c|c|c|c|c|c|c|}
\hline $\begin{array}{l}\mathrm{A}=3 \mathrm{M} / \text { Cogent } \\
\mathrm{G}=\text { Hisign } \\
\mathrm{P}=\text { Zhuhai-Yisheng }\end{array}$ & $\begin{array}{l}B=\text { Cognitec } \\
H=C A S-I A \\
Q=\text { JunYu }\end{array}$ & $\begin{array}{l}\mathrm{C}=\text { Neurotechnology } \\
\mathrm{I}=\text { CAS-ICT } \\
\mathrm{S}=\text { Decatur }\end{array}$ & $\begin{array}{l}\mathrm{D}=\text { Safran Morpho } \\
\mathrm{J}=\text { Toshiba } \\
\mathrm{T}=\text { Ayonix }\end{array}$ & $\begin{array}{l}E=N E C \\
L=\text { Tsinghua U. II }\end{array}$ & $\begin{array}{l}\mathrm{F}=\text { Tsinghua } \mathrm{U} \\
\mathrm{M}=\mathrm{HP}\end{array}$ & $\begin{array}{l}\text { FNIR(N,R,T,L) “Miss rate" } \\
\text { FPIR(N,T,L) "False alarm rate" }\end{array}$ \\
\hline
\end{tabular}




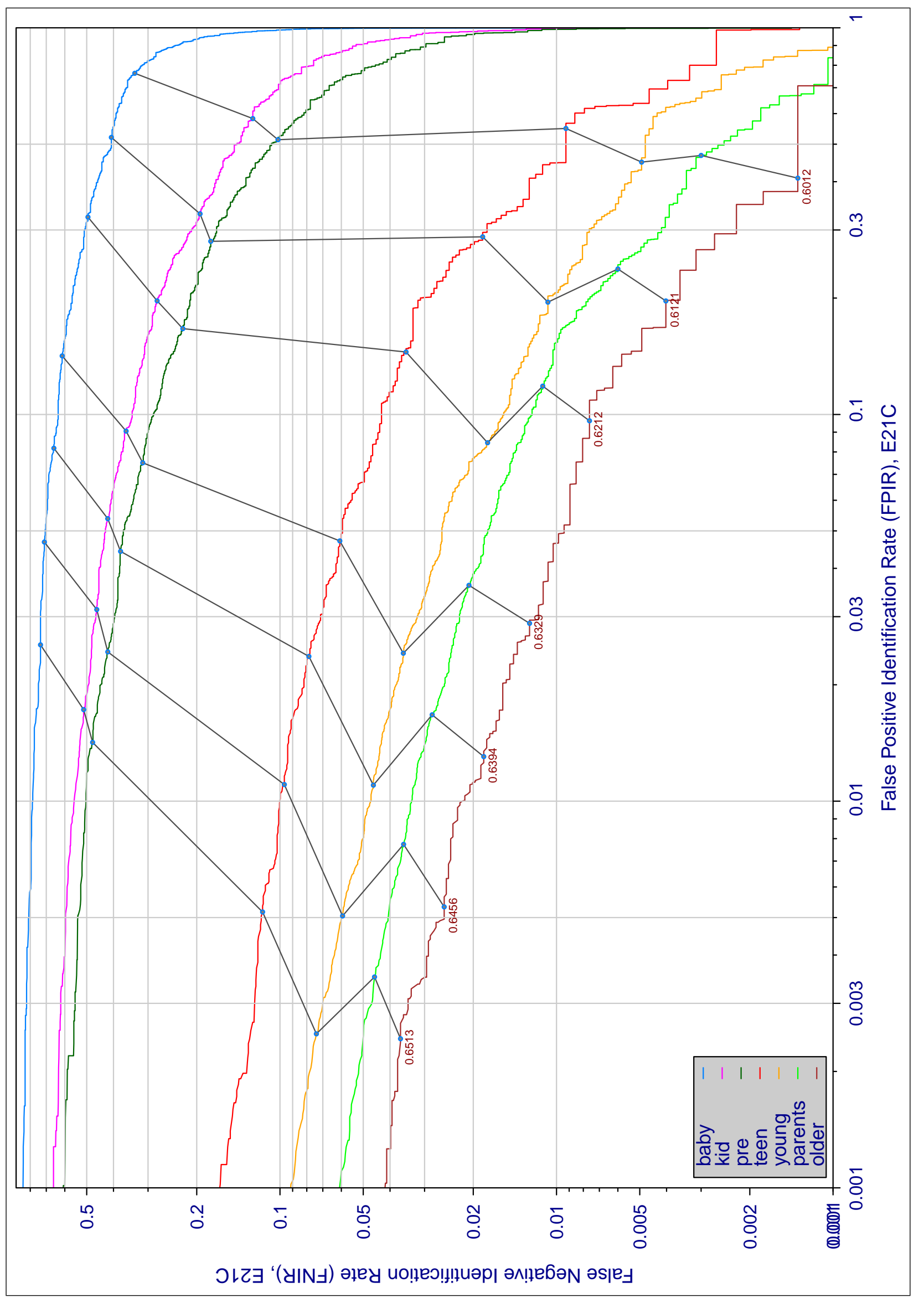

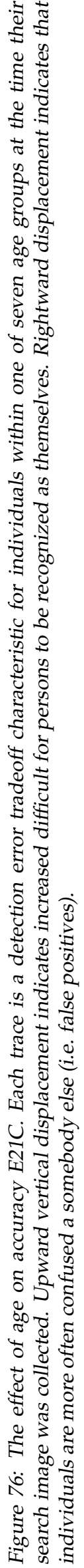

\begin{tabular}{|c|c|c|c|c|c|c|}
\hline $\begin{array}{l}\mathrm{A}=3 \mathrm{M} / \text { Cogent } \\
\mathrm{G}=\text { Hisign } \\
\mathrm{P}=\text { Zhuhai-Yisheng }\end{array}$ & $\begin{array}{l}B=\text { Cognitec } \\
H=C A S-I A \\
Q=\text { JunYu }\end{array}$ & $\begin{array}{l}\mathrm{C}=\text { Neurotechnology } \\
\mathrm{I}=\text { CAS-ICT } \\
\mathrm{S}=\text { Decatur }\end{array}$ & $\begin{array}{l}\mathrm{D}=\text { Safran Morpho } \\
\mathrm{J}=\text { Toshiba } \\
\mathrm{T}=\text { Ayonix }\end{array}$ & $\begin{array}{l}E=N E C \\
L=\text { Tsinghua U. II }\end{array}$ & $\begin{array}{l}\mathrm{F}=\text { Tsinghua } \mathrm{U} \\
\mathrm{M}=\mathrm{HP}\end{array}$ & $\begin{array}{l}\text { FNIR(N,R,T,L) “Miss rate" } \\
\text { FPIR(N,T,L) "False alarm rate" }\end{array}$ \\
\hline
\end{tabular}




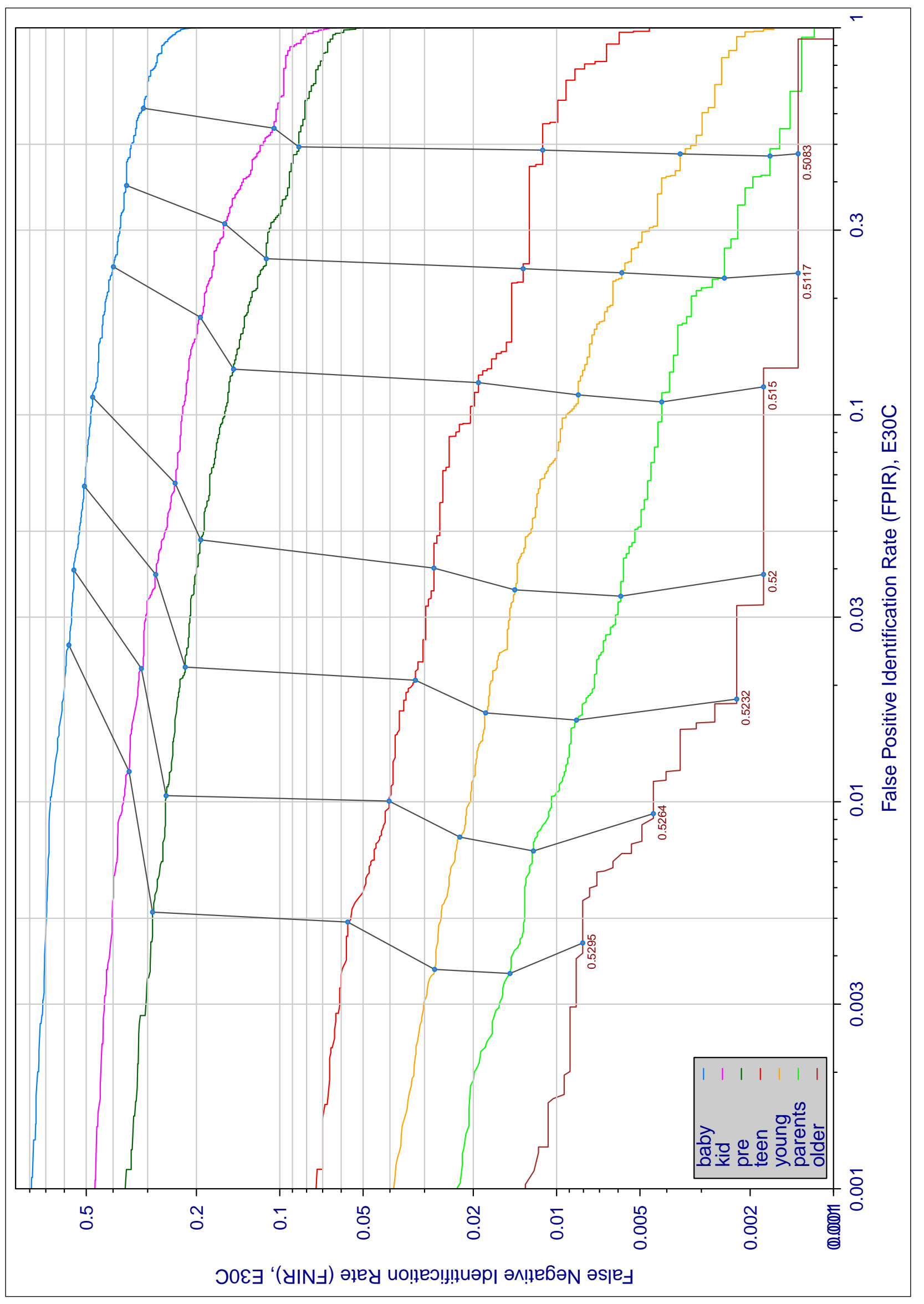

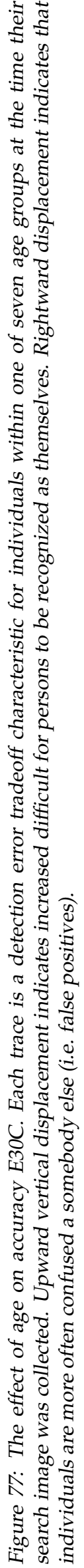

\begin{tabular}{|c|c|c|c|c|c|c|}
\hline $\begin{array}{l}\mathrm{A}=3 \mathrm{M} / \text { Cogent } \\
\mathrm{G}=\text { Hisign } \\
\mathrm{P}=\text { Zhuhai-Yisheng }\end{array}$ & $\begin{array}{l}B=\text { Cognitec } \\
H=C A S-I A \\
Q=\text { JunYu }\end{array}$ & $\begin{array}{l}\mathrm{C}=\text { Neurotechnology } \\
\mathrm{I}=\text { CAS-ICT } \\
\mathrm{S}=\text { Decatur }\end{array}$ & $\begin{array}{l}\mathrm{D}=\text { Safran Morpho } \\
\mathrm{J}=\text { Toshiba } \\
\mathrm{T}=\text { Ayonix }\end{array}$ & $\begin{array}{l}E=N E C \\
L=\text { Tsinghua U. II }\end{array}$ & $\begin{array}{l}\mathrm{F}=\text { Tsinghua } \mathrm{U} \\
\mathrm{M}=\mathrm{HP}\end{array}$ & $\begin{array}{l}\text { FNIR(N,R,T,L) “Miss rate" } \\
\text { FPIR(N,T,L) "False alarm rate" }\end{array}$ \\
\hline
\end{tabular}




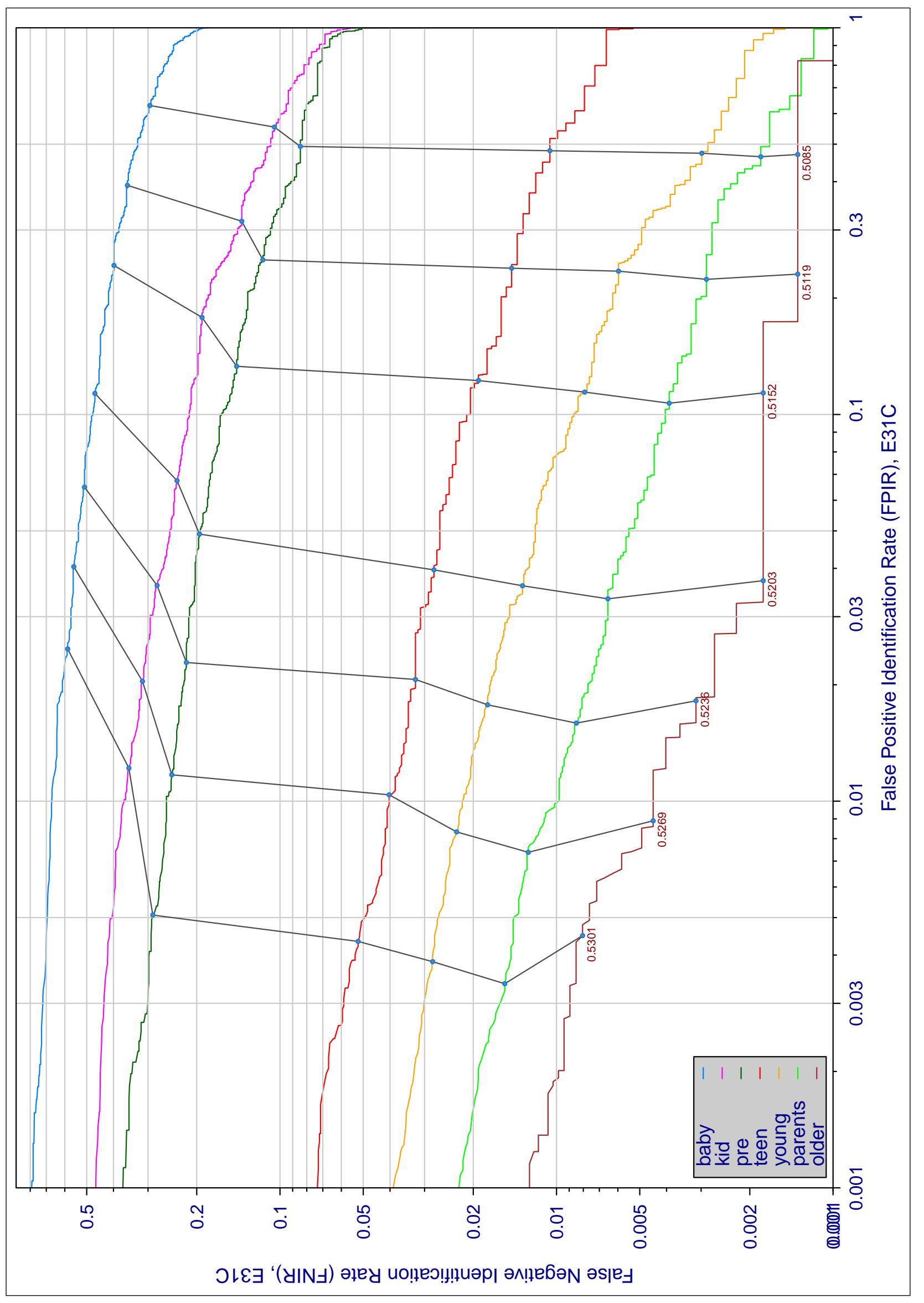

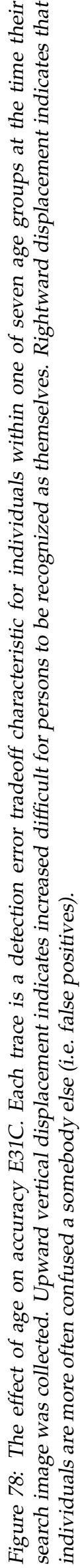

\begin{tabular}{|c|c|c|c|c|c|c|}
\hline $\begin{array}{l}\mathrm{A}=3 \mathrm{M} / \text { Cogent } \\
\mathrm{G}=\text { Hisign } \\
\mathrm{P}=\text { Zhuhai-Yisheng }\end{array}$ & $\begin{array}{l}B=\text { Cognitec } \\
H=C A S-I A \\
Q=\text { JunYu }\end{array}$ & $\begin{array}{l}\mathrm{C}=\text { Neurotechnology } \\
\mathrm{I}=\text { CAS-ICT } \\
\mathrm{S}=\text { Decatur }\end{array}$ & $\begin{array}{l}\mathrm{D}=\text { Safran Morpho } \\
\mathrm{J}=\text { Toshiba } \\
\mathrm{T}=\text { Ayonix }\end{array}$ & $\begin{array}{l}E=N E C \\
L=\text { Tsinghua U. II }\end{array}$ & $\begin{array}{l}\mathrm{F}=\text { Tsinghua } \mathrm{U} \\
\mathrm{M}=\mathrm{HP}\end{array}$ & $\begin{array}{l}\text { FNIR(N,R,T,L) “Miss rate" } \\
\text { FPIR(N,T,L) "False alarm rate" }\end{array}$ \\
\hline
\end{tabular}




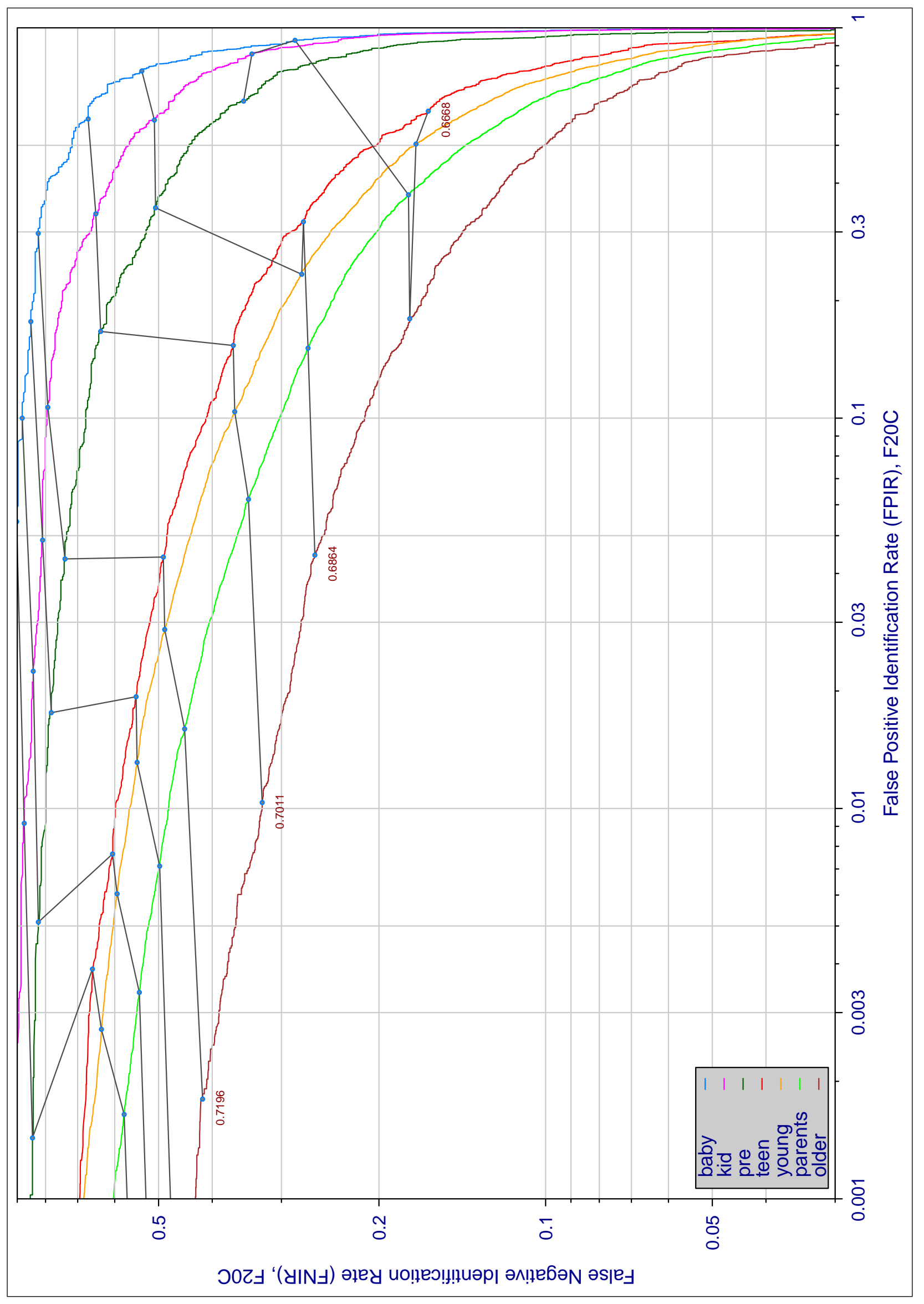

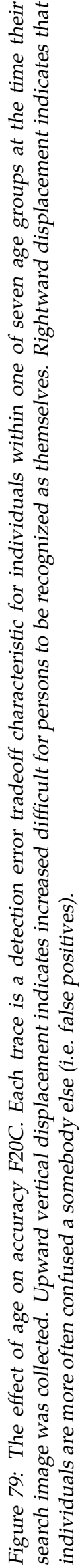

\begin{tabular}{|c|c|c|c|c|c|c|}
\hline $\begin{array}{l}\mathrm{A}=3 \mathrm{M} / \text { Cogent } \\
\mathrm{G}=\text { Hisign } \\
\mathrm{P}=\text { Zhuhai-Yisheng }\end{array}$ & $\begin{array}{l}B=\text { Cognitec } \\
H=C A S-I A \\
Q=\text { JunYu }\end{array}$ & $\begin{array}{l}\mathrm{C}=\text { Neurotechnology } \\
\mathrm{I}=\text { CAS-ICT } \\
\mathrm{S}=\text { Decatur }\end{array}$ & $\begin{array}{l}\mathrm{D}=\text { Safran Morpho } \\
\mathrm{J}=\text { Toshiba } \\
\mathrm{T}=\text { Ayonix }\end{array}$ & $\begin{array}{l}E=N E C \\
L=\text { Tsinghua U. II }\end{array}$ & $\begin{array}{l}\mathrm{F}=\text { Tsinghua } \mathrm{U} \\
\mathrm{M}=\mathrm{HP}\end{array}$ & $\begin{array}{l}\text { FNIR(N,R,T,L) “Miss rate" } \\
\text { FPIR(N,T,L) "False alarm rate" }\end{array}$ \\
\hline
\end{tabular}




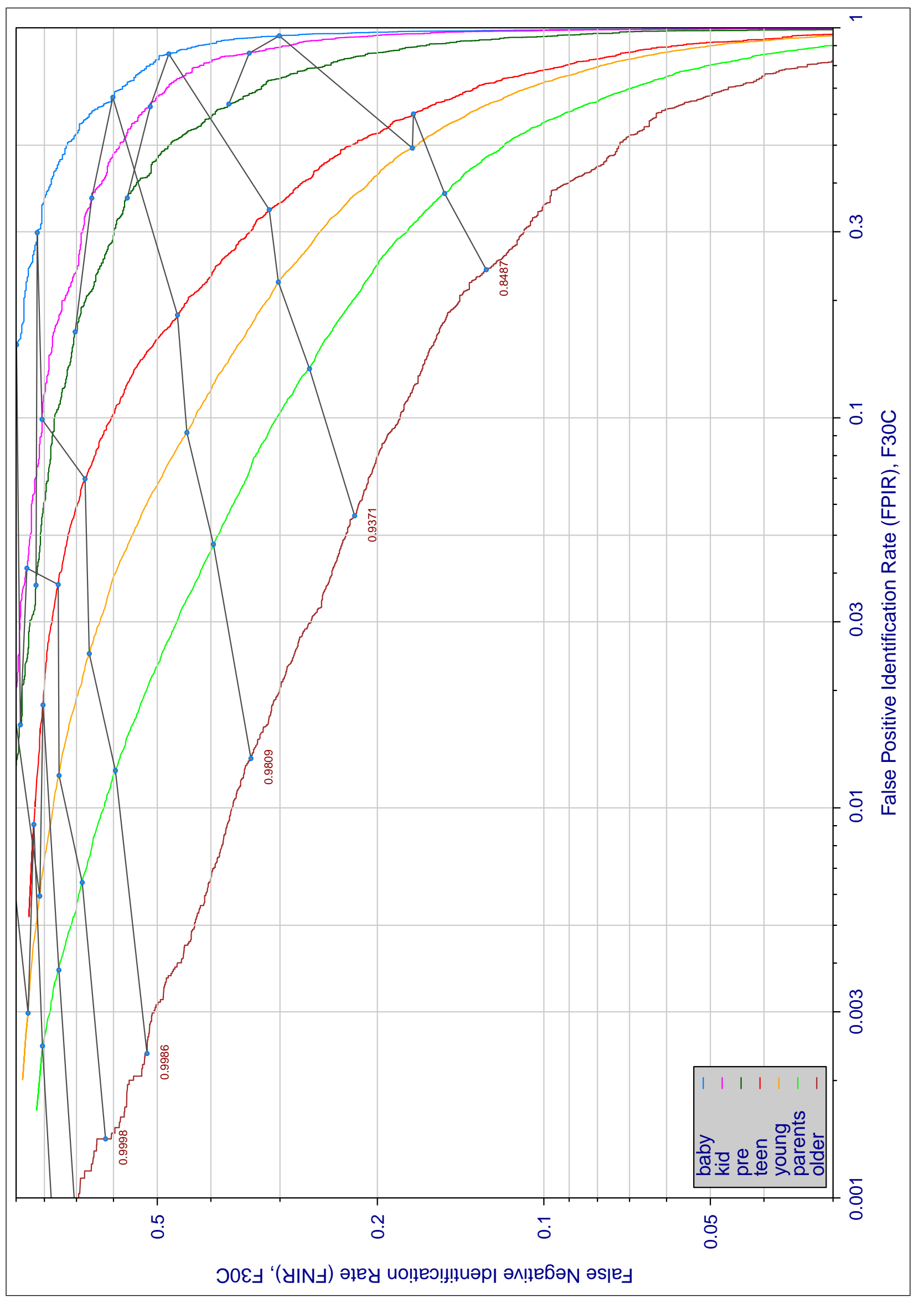

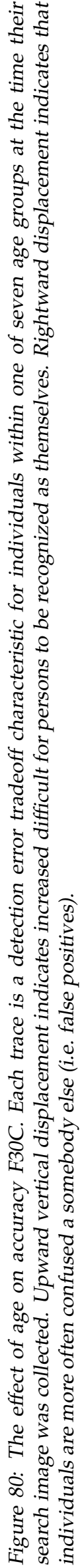

\begin{tabular}{|c|c|c|c|c|c|c|}
\hline $\begin{array}{l}\mathrm{A}=3 \mathrm{M} / \text { Cogent } \\
\mathrm{G}=\text { Hisign } \\
\mathrm{P}=\text { Zhuhai-Yisheng }\end{array}$ & $\begin{array}{l}B=\text { Cognitec } \\
H=C A S-I A \\
Q=\text { JunYu }\end{array}$ & $\begin{array}{l}\mathrm{C}=\text { Neurotechnology } \\
\mathrm{I}=\text { CAS-ICT } \\
\mathrm{S}=\text { Decatur }\end{array}$ & $\begin{array}{l}\mathrm{D}=\text { Safran Morpho } \\
\mathrm{J}=\text { Toshiba } \\
\mathrm{T}=\text { Ayonix }\end{array}$ & $\begin{array}{l}E=N E C \\
L=\text { Tsinghua U. II }\end{array}$ & $\begin{array}{l}\mathrm{F}=\text { Tsinghua } \mathrm{U} \\
\mathrm{M}=\mathrm{HP}\end{array}$ & $\begin{array}{l}\text { FNIR(N,R,T,L) “Miss rate" } \\
\text { FPIR(N,T,L) "False alarm rate" }\end{array}$ \\
\hline
\end{tabular}




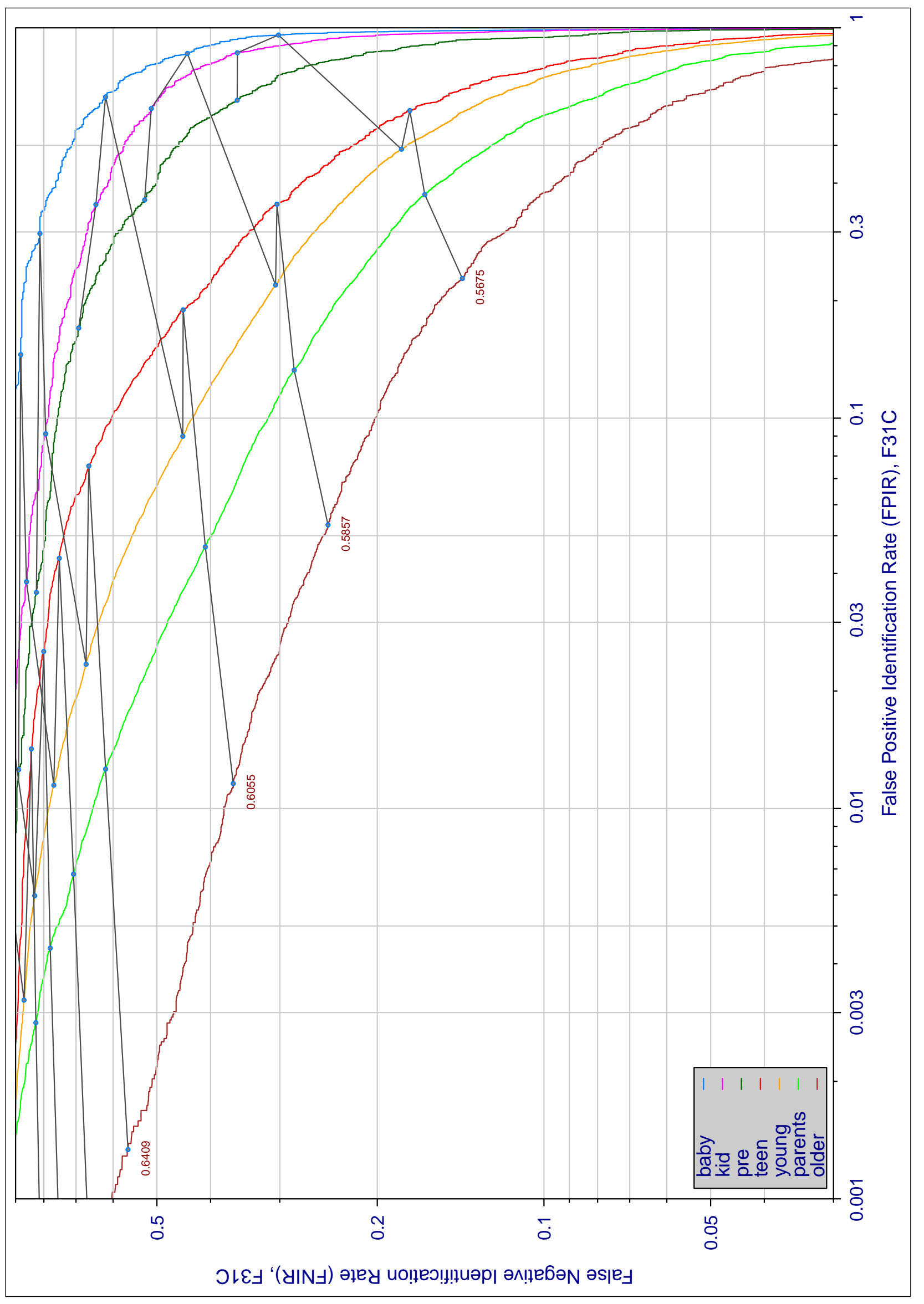

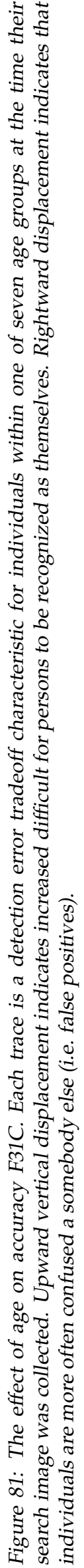

\begin{tabular}{|c|c|c|c|c|c|c|}
\hline $\begin{array}{l}\mathrm{A}=3 \mathrm{M} / \text { Cogent } \\
\mathrm{G}=\text { Hisign } \\
\mathrm{P}=\text { Zhuhai-Yisheng }\end{array}$ & $\begin{array}{l}B=\text { Cognitec } \\
H=C A S-I A \\
Q=\text { JunYu }\end{array}$ & $\begin{array}{l}\mathrm{C}=\text { Neurotechnology } \\
\mathrm{I}=\text { CAS-ICT } \\
\mathrm{S}=\text { Decatur }\end{array}$ & $\begin{array}{l}\mathrm{D}=\text { Safran Morpho } \\
\mathrm{J}=\text { Toshiba } \\
\mathrm{T}=\text { Ayonix }\end{array}$ & $\begin{array}{l}E=N E C \\
L=\text { Tsinghua U. II }\end{array}$ & $\begin{array}{l}\mathrm{F}=\text { Tsinghua } \mathrm{U} \\
\mathrm{M}=\mathrm{HP}\end{array}$ & $\begin{array}{l}\text { FNIR(N,R,T,L) “Miss rate" } \\
\text { FPIR(N,T,L) "False alarm rate" }\end{array}$ \\
\hline
\end{tabular}




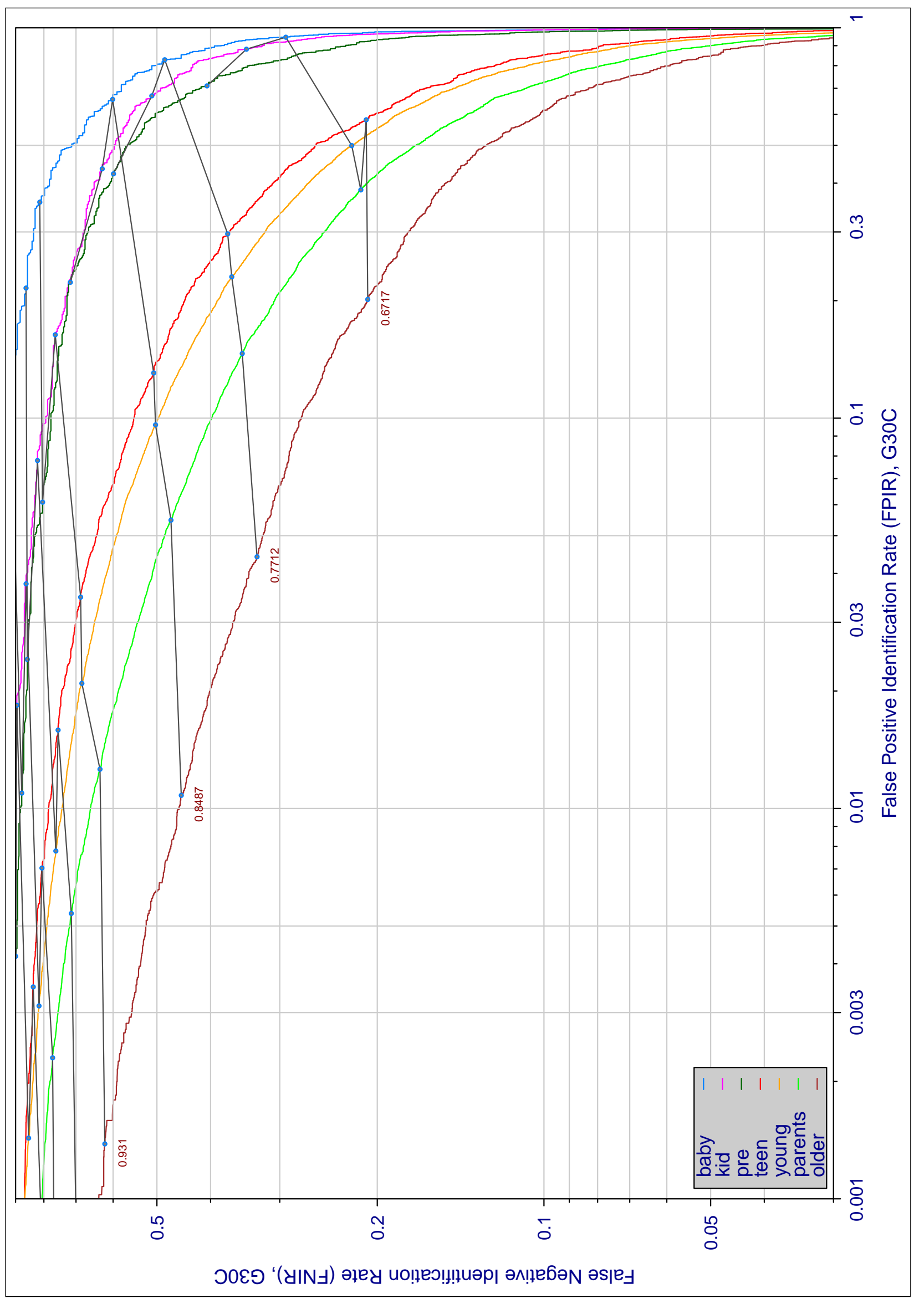

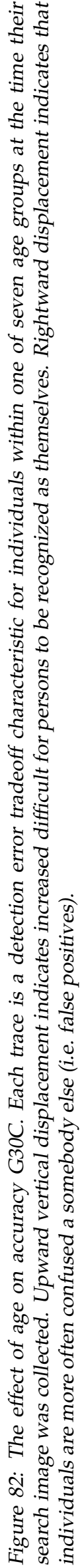

\begin{tabular}{|c|c|c|c|c|c|c|}
\hline $\begin{array}{l}\mathrm{A}=3 \mathrm{M} / \text { Cogent } \\
\mathrm{G}=\text { Hisign } \\
\mathrm{P}=\text { Zhuhai-Yisheng }\end{array}$ & $\begin{array}{l}B=\text { Cognitec } \\
H=C A S-I A \\
Q=\text { JunYu }\end{array}$ & $\begin{array}{l}\mathrm{C}=\text { Neurotechnology } \\
\mathrm{I}=\text { CAS-ICT } \\
\mathrm{S}=\text { Decatur }\end{array}$ & $\begin{array}{l}\mathrm{D}=\text { Safran Morpho } \\
\mathrm{J}=\text { Toshiba } \\
\mathrm{T}=\text { Ayonix }\end{array}$ & $\begin{array}{l}E=N E C \\
L=\text { Tsinghua U. II }\end{array}$ & $\begin{array}{l}\mathrm{F}=\text { Tsinghua } \mathrm{U} \\
\mathrm{M}=\mathrm{HP}\end{array}$ & $\begin{array}{l}\text { FNIR(N,R,T,L) “Miss rate" } \\
\text { FPIR(N,T,L) "False alarm rate" }\end{array}$ \\
\hline
\end{tabular}




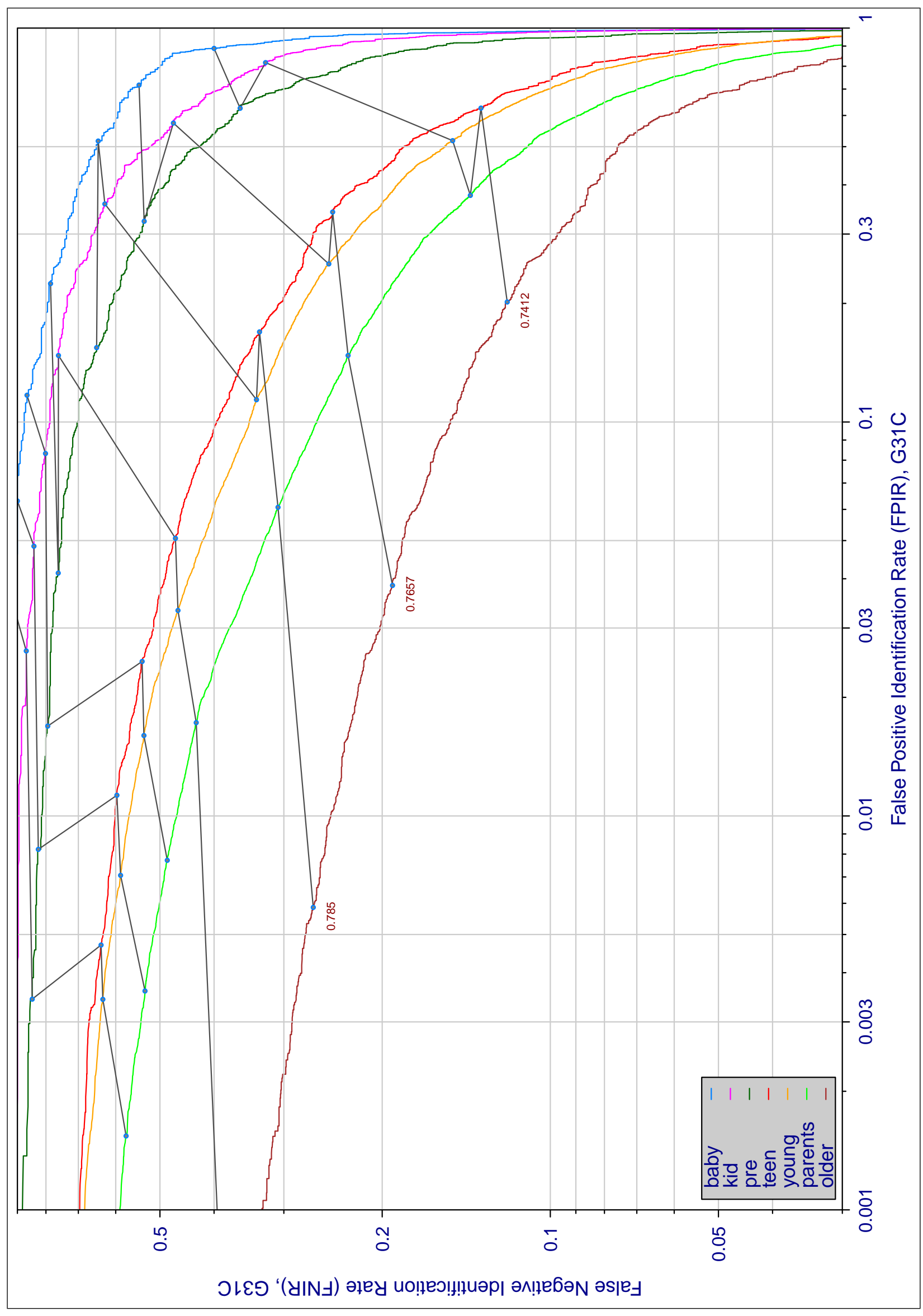

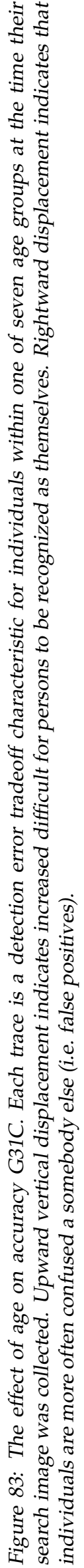

\begin{tabular}{|c|c|c|c|c|c|c|}
\hline $\begin{array}{l}\mathrm{A}=3 \mathrm{M} / \text { Cogent } \\
\mathrm{G}=\text { Hisign } \\
\mathrm{P}=\text { Zhuhai-Yisheng }\end{array}$ & $\begin{array}{l}B=\text { Cognitec } \\
H=C A S-I A \\
Q=\text { JunYu }\end{array}$ & $\begin{array}{l}\mathrm{C}=\text { Neurotechnology } \\
\mathrm{I}=\text { CAS-ICT } \\
\mathrm{S}=\text { Decatur }\end{array}$ & $\begin{array}{l}\mathrm{D}=\text { Safran Morpho } \\
\mathrm{J}=\text { Toshiba } \\
\mathrm{T}=\text { Ayonix }\end{array}$ & $\begin{array}{l}E=N E C \\
L=\text { Tsinghua U. II }\end{array}$ & $\begin{array}{l}\mathrm{F}=\text { Tsinghua } \mathrm{U} \\
\mathrm{M}=\mathrm{HP}\end{array}$ & $\begin{array}{l}\text { FNIR(N,R,T,L) “Miss rate" } \\
\text { FPIR(N,T,L) "False alarm rate" }\end{array}$ \\
\hline
\end{tabular}




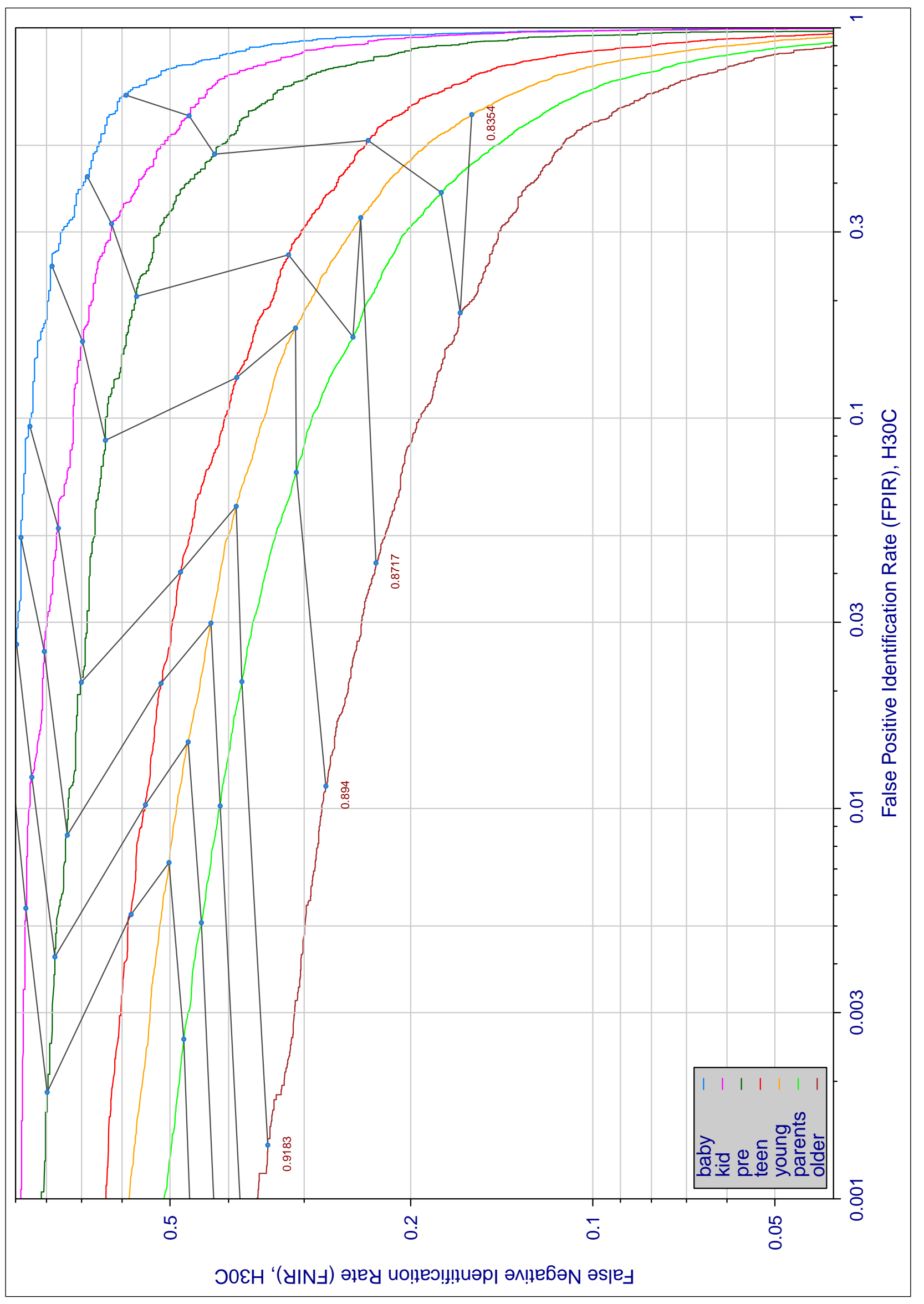

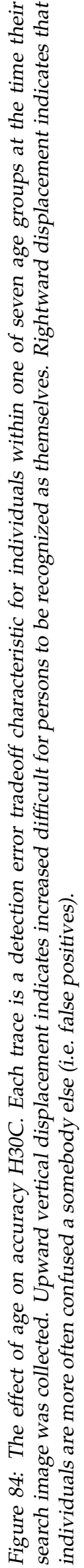

\begin{tabular}{|c|c|c|c|c|c|c|}
\hline $\begin{array}{l}\mathrm{A}=3 \mathrm{M} / \text { Cogent } \\
\mathrm{G}=\text { Hisign } \\
\mathrm{P}=\text { Zhuhai-Yisheng }\end{array}$ & $\begin{array}{l}\mathrm{B}=\text { Cognitec } \\
\mathrm{H}=\mathrm{CAS}-\mathrm{IA} \\
\mathrm{Q}=\text { JunYu }\end{array}$ & $\begin{array}{l}\mathrm{C}=\text { Neurotechnology } \\
\mathrm{I}=\text { CAS-ICT } \\
\mathrm{S}=\text { Decatur }\end{array}$ & $\begin{array}{l}\mathrm{D}=\text { Safran Morpho } \\
\mathrm{J}=\text { Toshiba } \\
\mathrm{T}=\text { Ayonix }\end{array}$ & $\begin{array}{l}E=N E C \\
L=\text { Tsinghua U. II }\end{array}$ & $\begin{array}{l}\mathrm{F}=\text { Tsinghua } \mathrm{U} \\
\mathrm{M}=\mathrm{HP}\end{array}$ & $\begin{array}{l}\text { FNIR(N,R,T,L) “Miss rate" } \\
\text { FPIR(N,T,L) "False alarm rate" }\end{array}$ \\
\hline
\end{tabular}




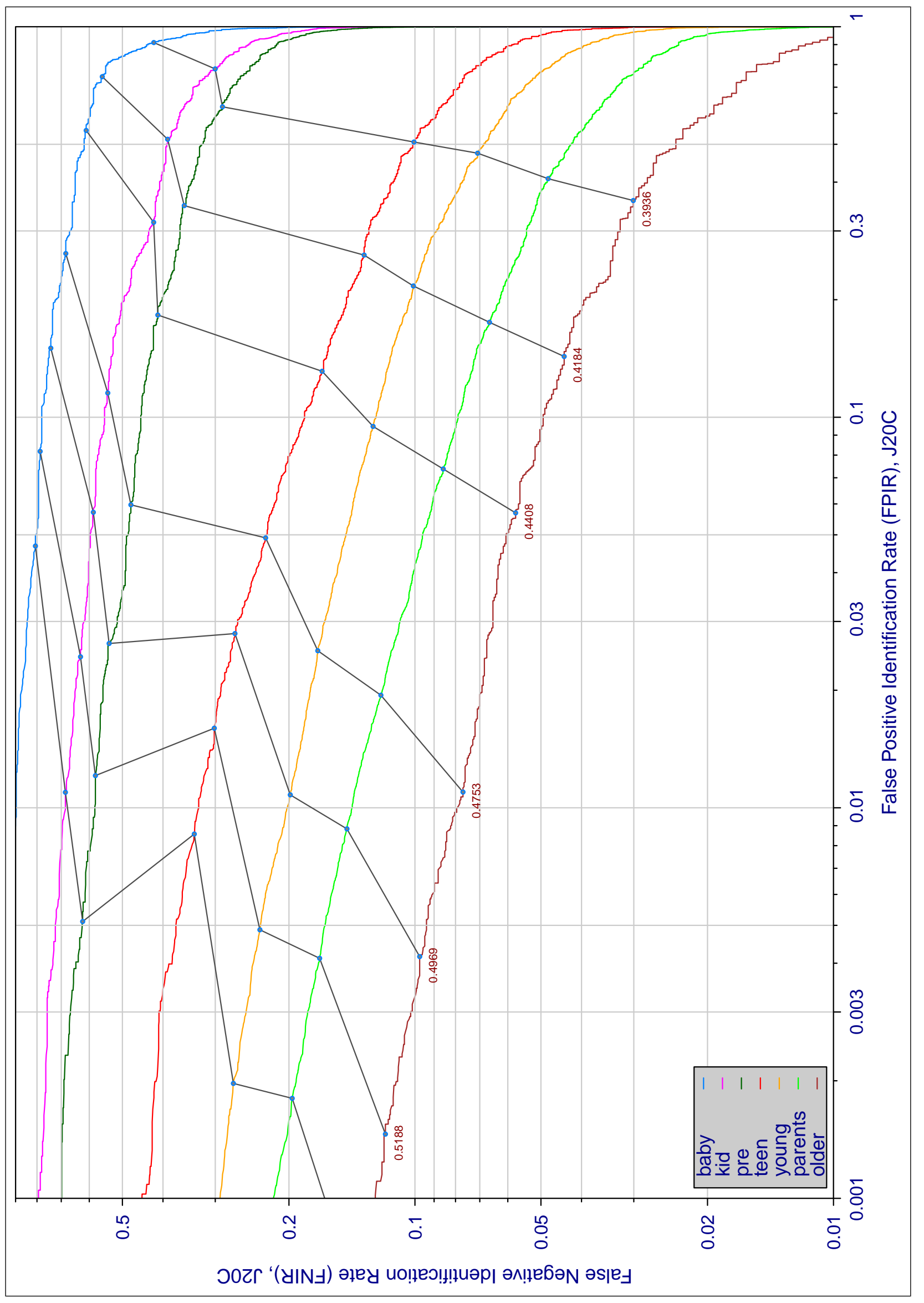

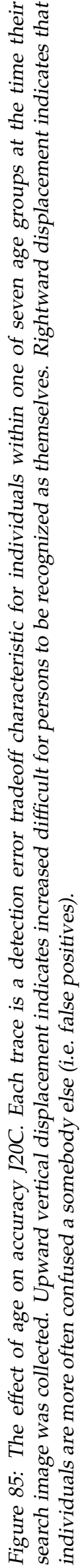

\begin{tabular}{|c|c|c|c|c|c|c|}
\hline $\begin{array}{l}\mathrm{A}=3 \mathrm{M} / \text { Cogent } \\
\mathrm{G}=\text { Hisign } \\
\mathrm{P}=\text { Zhuhai-Yisheng }\end{array}$ & $\begin{array}{l}B=\text { Cognitec } \\
H=C A S-I A \\
Q=\text { JunYu }\end{array}$ & $\begin{array}{l}\mathrm{C}=\text { Neurotechnology } \\
\mathrm{I}=\text { CAS-ICT } \\
\mathrm{S}=\text { Decatur }\end{array}$ & $\begin{array}{l}\mathrm{D}=\text { Safran Morpho } \\
\mathrm{J}=\text { Toshiba } \\
\mathrm{T}=\text { Ayonix }\end{array}$ & $\begin{array}{l}E=N E C \\
L=\text { Tsinghua U. II }\end{array}$ & $\begin{array}{l}\mathrm{F}=\text { Tsinghua } \mathrm{U} \\
\mathrm{M}=\mathrm{HP}\end{array}$ & $\begin{array}{l}\text { FNIR(N,R,T,L) “Miss rate" } \\
\text { FPIR(N,T,L) "False alarm rate" }\end{array}$ \\
\hline
\end{tabular}




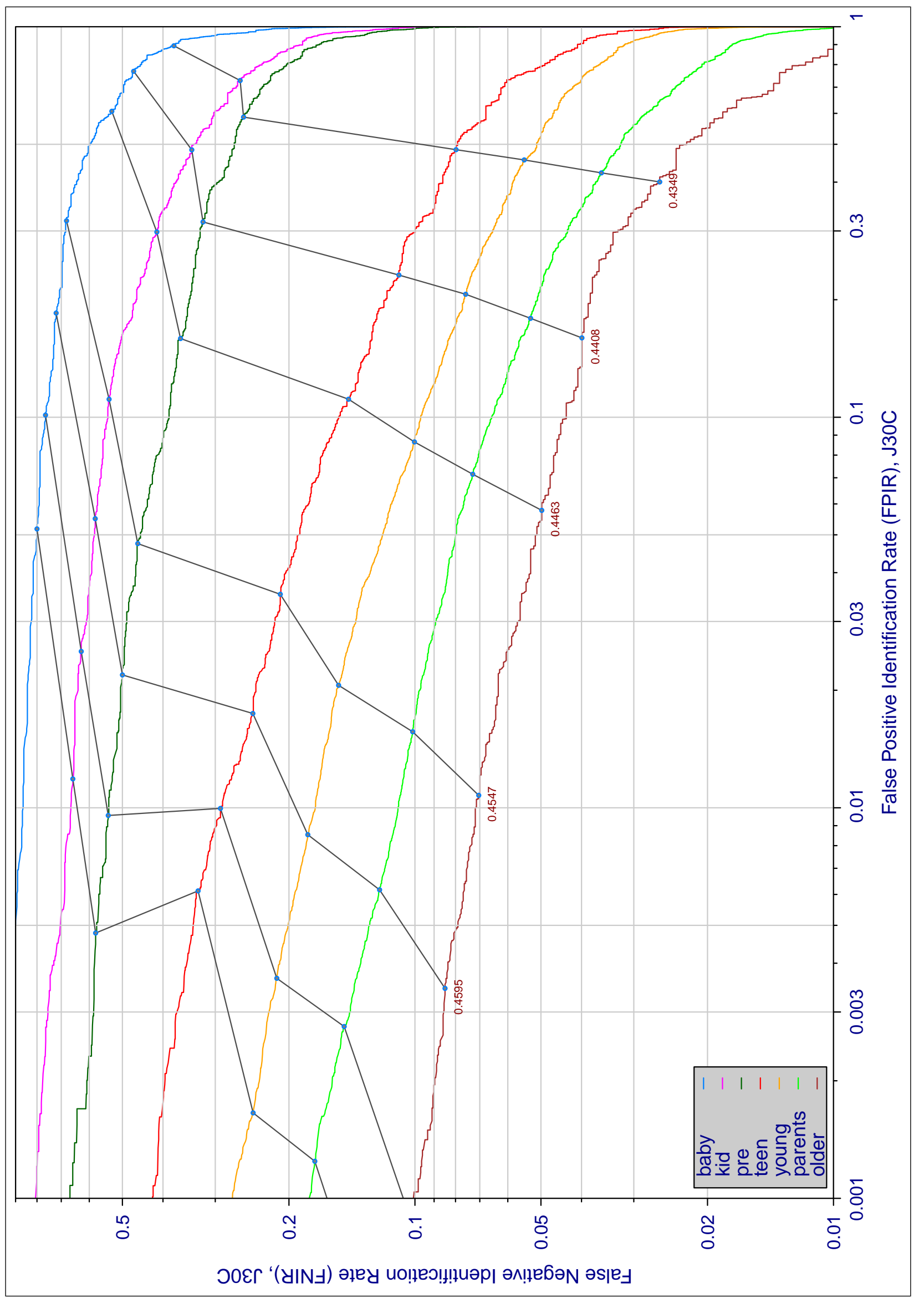

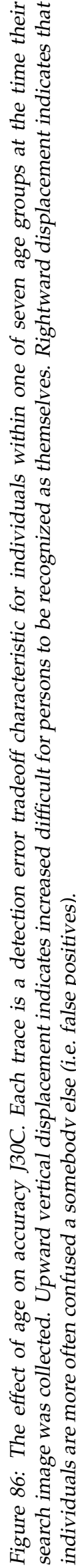

\begin{tabular}{|c|c|c|c|c|c|c|}
\hline $\begin{array}{l}\mathrm{A}=3 \mathrm{M} / \text { Cogent } \\
\mathrm{G}=\text { Hisign } \\
\mathrm{P}=\text { Zhuhai-Yisheng }\end{array}$ & $\begin{array}{l}B=\text { Cognitec } \\
H=C A S-I A \\
Q=\text { JunYu }\end{array}$ & $\begin{array}{l}\mathrm{C}=\text { Neurotechnology } \\
\mathrm{I}=\text { CAS-ICT } \\
\mathrm{S}=\text { Decatur }\end{array}$ & $\begin{array}{l}\mathrm{D}=\text { Safran Morpho } \\
\mathrm{J}=\text { Toshiba } \\
\mathrm{T}=\text { Ayonix }\end{array}$ & $\begin{array}{l}E=N E C \\
L=\text { Tsinghua U. II }\end{array}$ & $\begin{array}{l}\mathrm{F}=\text { Tsinghua } \mathrm{U} \\
\mathrm{M}=\mathrm{HP}\end{array}$ & $\begin{array}{l}\text { FNIR(N,R,T,L) “Miss rate" } \\
\text { FPIR(N,T,L) "False alarm rate" }\end{array}$ \\
\hline
\end{tabular}




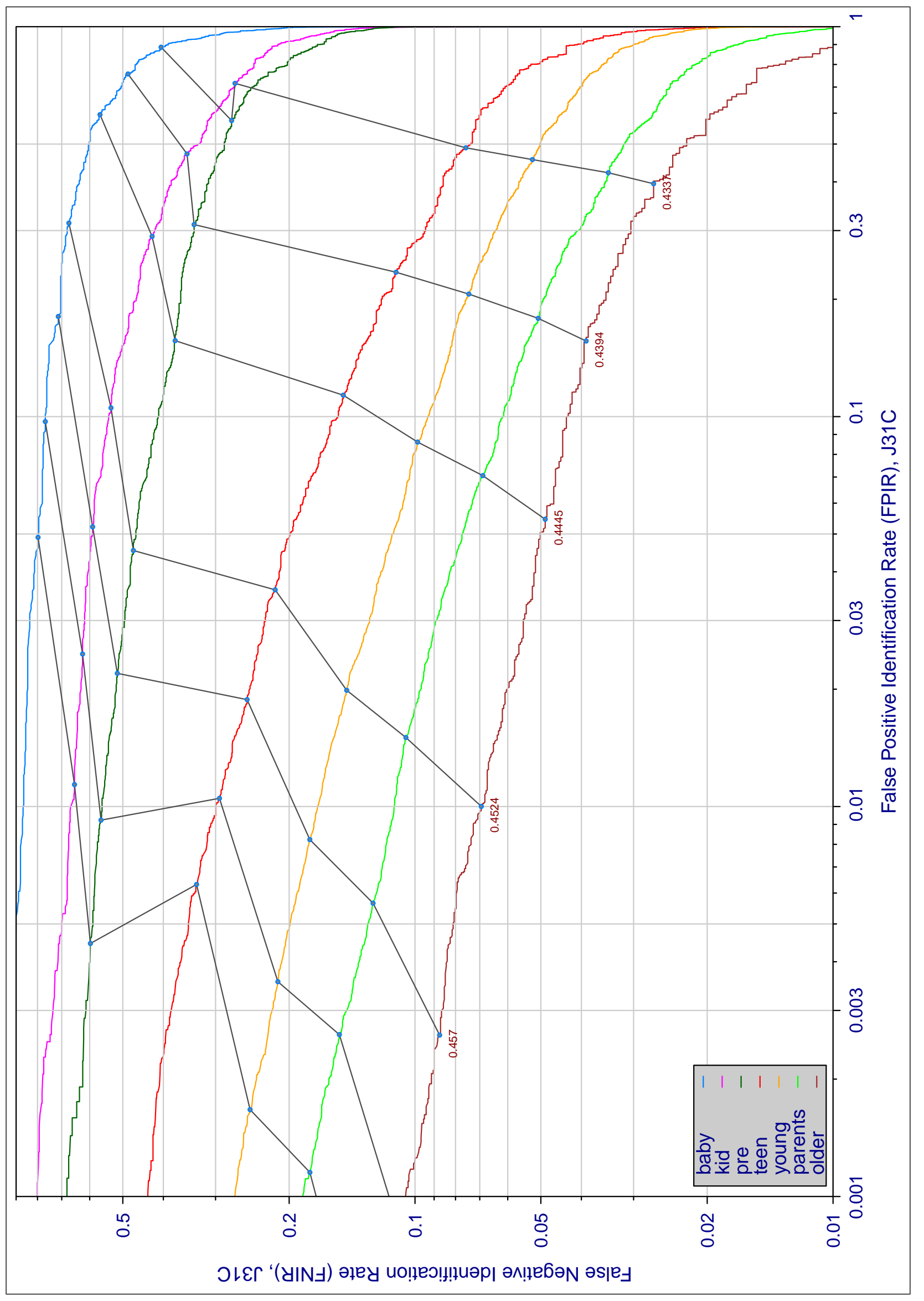

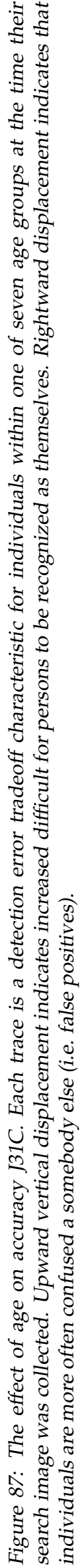

\begin{tabular}{|c|c|c|c|c|c|c|}
\hline $\begin{array}{l}\mathrm{A}=3 \mathrm{M} / \text { Cogent } \\
\mathrm{G}=\text { Hisign } \\
\mathrm{P}=\text { Zhuhai-Yisheng }\end{array}$ & $\begin{array}{l}B=\text { Cognitec } \\
H=C A S-I A \\
Q=\text { JunYu }\end{array}$ & $\begin{array}{l}\mathrm{C}=\text { Neurotechnology } \\
\mathrm{I}=\text { CAS-ICT } \\
\mathrm{S}=\text { Decatur }\end{array}$ & $\begin{array}{l}\mathrm{D}=\text { Safran Morpho } \\
\mathrm{J}=\text { Toshiba } \\
\mathrm{T}=\text { Ayonix }\end{array}$ & $\begin{array}{l}E=N E C \\
L=\text { Tsinghua U. II }\end{array}$ & $\begin{array}{l}\mathrm{F}=\text { Tsinghua } \mathrm{U} \\
\mathrm{M}=\mathrm{HP}\end{array}$ & $\begin{array}{l}\text { FNIR(N,R,T,L) “Miss rate" } \\
\text { FPIR(N,T,L) "False alarm rate" }\end{array}$ \\
\hline
\end{tabular}




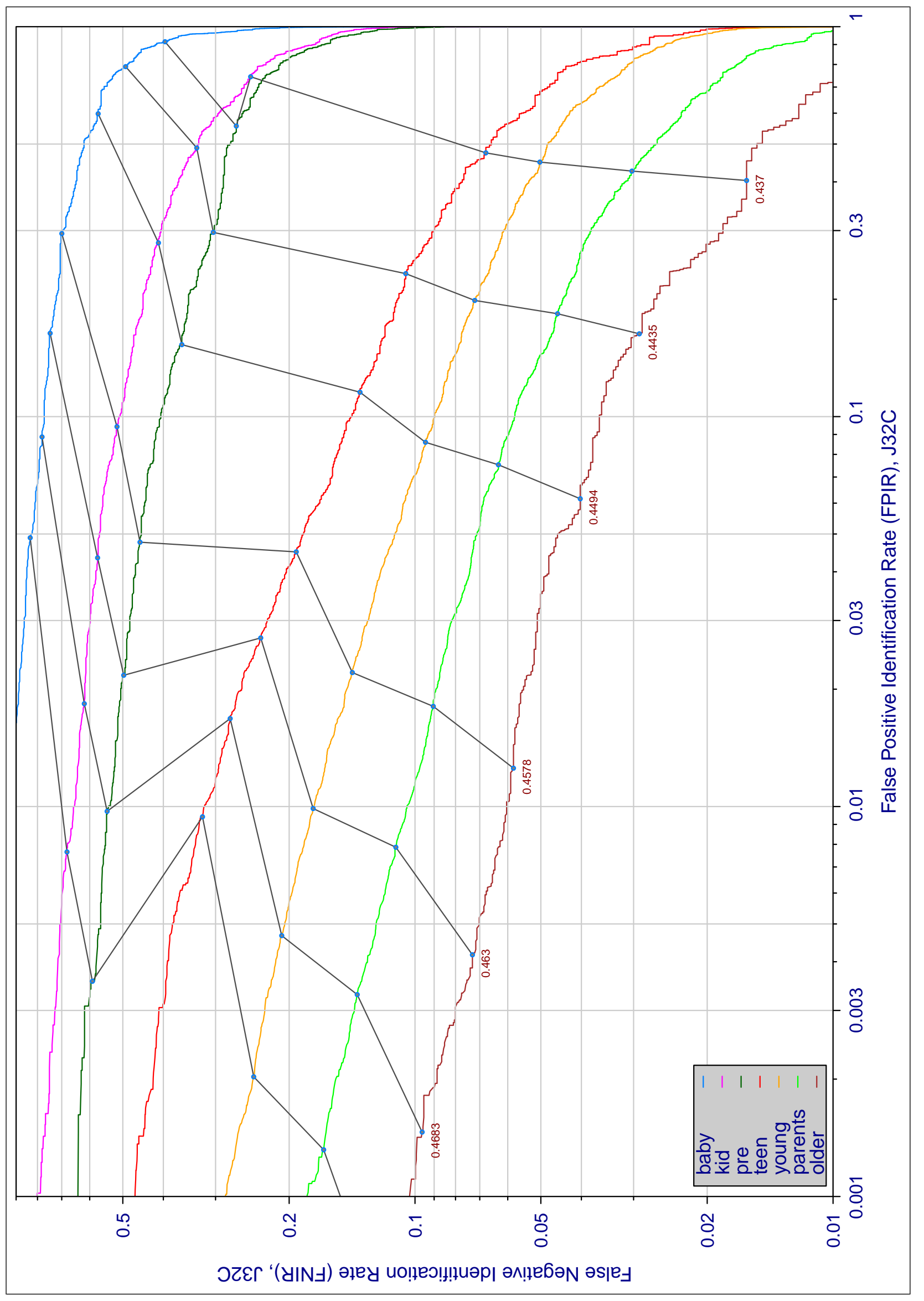

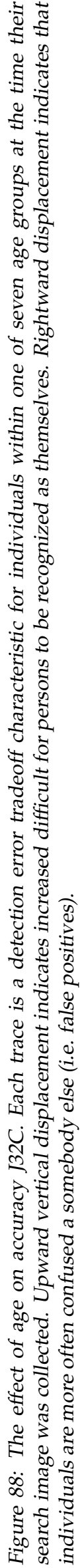

\begin{tabular}{|c|c|c|c|c|c|c|}
\hline $\begin{array}{l}\mathrm{A}=3 \mathrm{M} / \text { Cogent } \\
\mathrm{G}=\text { Hisign } \\
\mathrm{P}=\text { Zhuhai-Yisheng }\end{array}$ & $\begin{array}{l}B=\text { Cognitec } \\
H=C A S-I A \\
Q=\text { JunYu }\end{array}$ & $\begin{array}{l}\mathrm{C}=\text { Neurotechnology } \\
\mathrm{I}=\text { CAS-ICT } \\
\mathrm{S}=\text { Decatur }\end{array}$ & $\begin{array}{l}\mathrm{D}=\text { Safran Morpho } \\
\mathrm{J}=\text { Toshiba } \\
\mathrm{T}=\text { Ayonix }\end{array}$ & $\begin{array}{l}E=N E C \\
L=\text { Tsinghua U. II }\end{array}$ & $\begin{array}{l}\mathrm{F}=\text { Tsinghua } \mathrm{U} \\
\mathrm{M}=\mathrm{HP}\end{array}$ & $\begin{array}{l}\text { FNIR(N,R,T,L) “Miss rate" } \\
\text { FPIR(N,T,L) "False alarm rate" }\end{array}$ \\
\hline
\end{tabular}




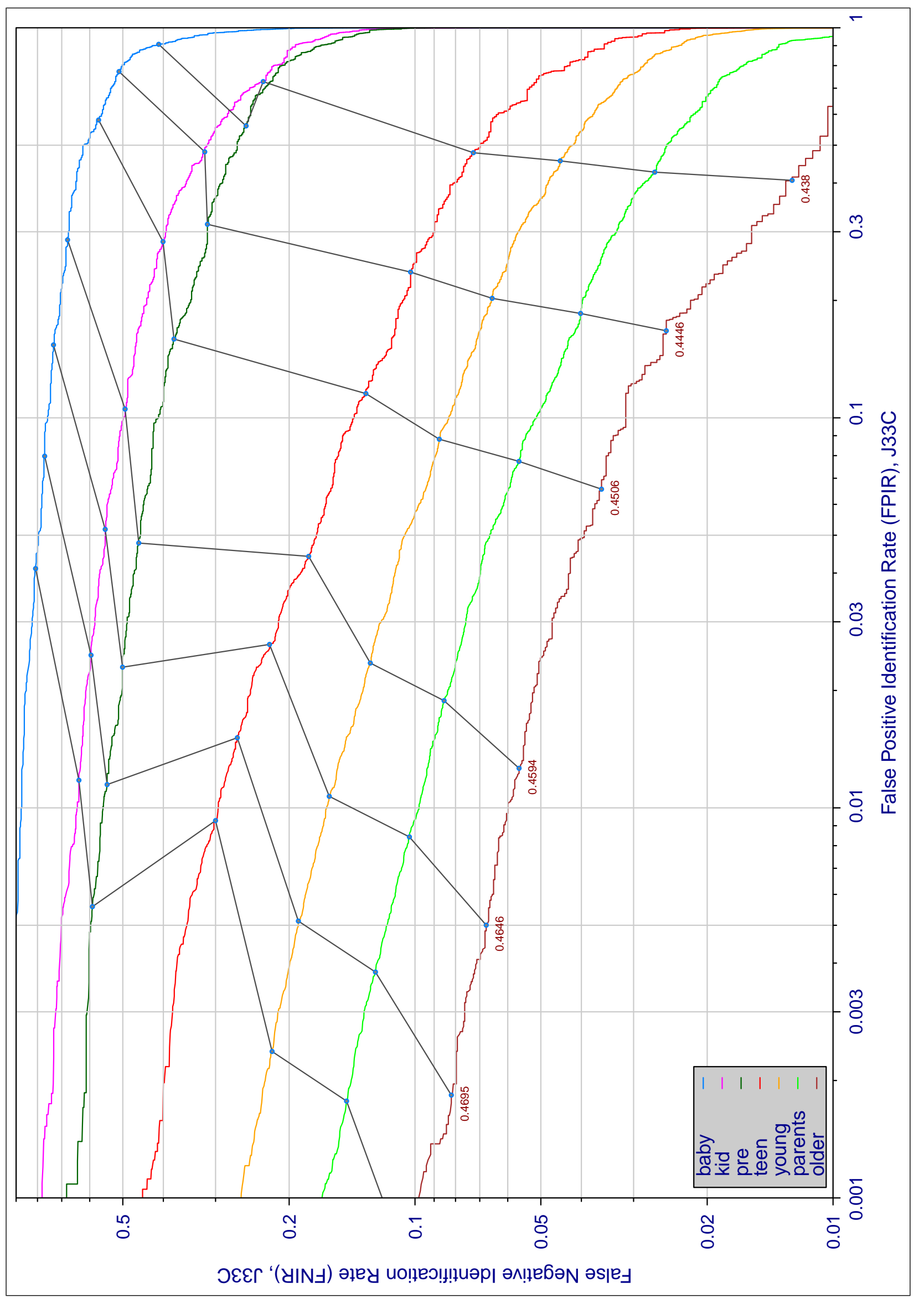

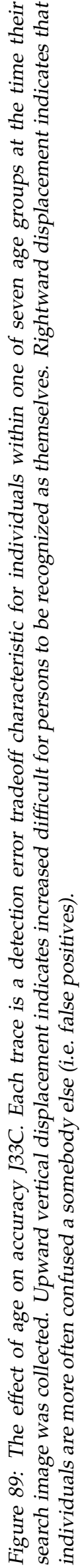

\begin{tabular}{|c|c|c|c|c|c|c|}
\hline $\begin{array}{l}\mathrm{A}=3 \mathrm{M} / \text { Cogent } \\
\mathrm{G}=\text { Hisign } \\
\mathrm{P}=\text { Zhuhai-Yisheng }\end{array}$ & $\begin{array}{l}B=\text { Cognitec } \\
H=C A S-I A \\
Q=\text { JunYu }\end{array}$ & $\begin{array}{l}\mathrm{C}=\text { Neurotechnology } \\
\mathrm{I}=\text { CAS-ICT } \\
\mathrm{S}=\text { Decatur }\end{array}$ & $\begin{array}{l}\mathrm{D}=\text { Safran Morpho } \\
\mathrm{J}=\text { Toshiba } \\
\mathrm{T}=\text { Ayonix }\end{array}$ & $\begin{array}{l}E=N E C \\
L=\text { Tsinghua U. II }\end{array}$ & $\begin{array}{l}\mathrm{F}=\text { Tsinghua } \mathrm{U} \\
\mathrm{M}=\mathrm{HP}\end{array}$ & $\begin{array}{l}\text { FNIR(N,R,T,L) “Miss rate" } \\
\text { FPIR(N,T,L) "False alarm rate" }\end{array}$ \\
\hline
\end{tabular}




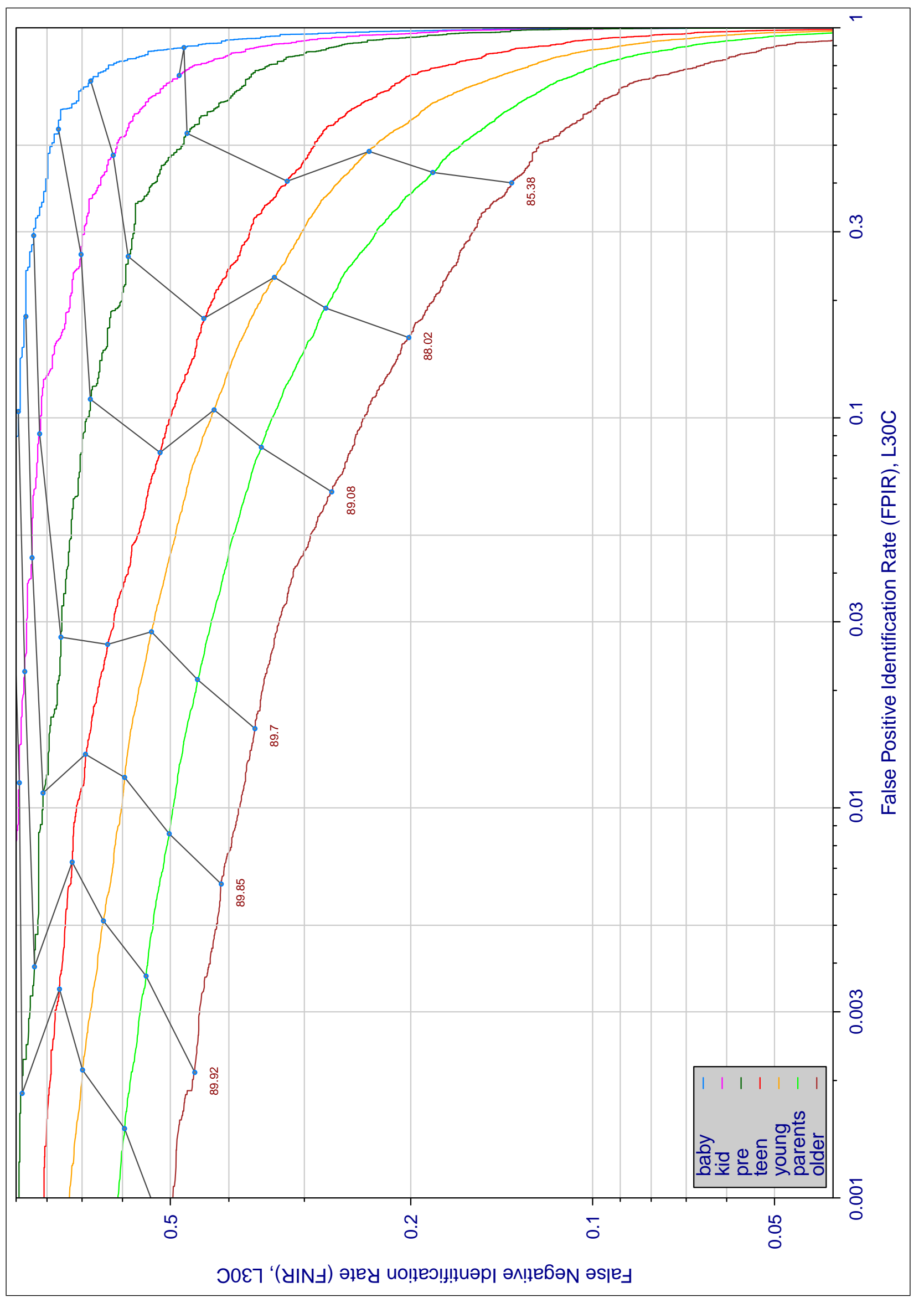

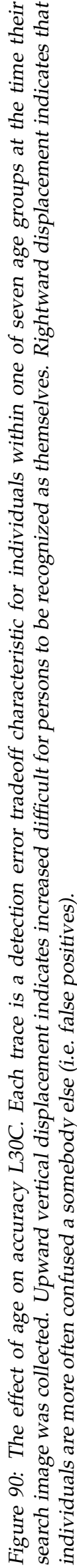

\begin{tabular}{|c|c|c|c|c|c|c|}
\hline $\begin{array}{l}\mathrm{A}=3 \mathrm{M} / \text { Cogent } \\
\mathrm{G}=\text { Hisign } \\
\mathrm{P}=\text { Zhuhai-Yisheng }\end{array}$ & $\begin{array}{l}B=\text { Cognitec } \\
H=C A S-I A \\
Q=\text { JunYu }\end{array}$ & $\begin{array}{l}\mathrm{C}=\text { Neurotechnology } \\
\mathrm{I}=\text { CAS-ICT } \\
\mathrm{S}=\text { Decatur }\end{array}$ & $\begin{array}{l}\mathrm{D}=\text { Safran Morpho } \\
\mathrm{J}=\text { Toshiba } \\
\mathrm{T}=\text { Ayonix }\end{array}$ & $\begin{array}{l}E=N E C \\
L=\text { Tsinghua U. II }\end{array}$ & $\begin{array}{l}\mathrm{F}=\text { Tsinghua } \mathrm{U} \\
\mathrm{M}=\mathrm{HP}\end{array}$ & $\begin{array}{l}\text { FNIR(N,R,T,L) “Miss rate" } \\
\text { FPIR(N,T,L) "False alarm rate" }\end{array}$ \\
\hline
\end{tabular}




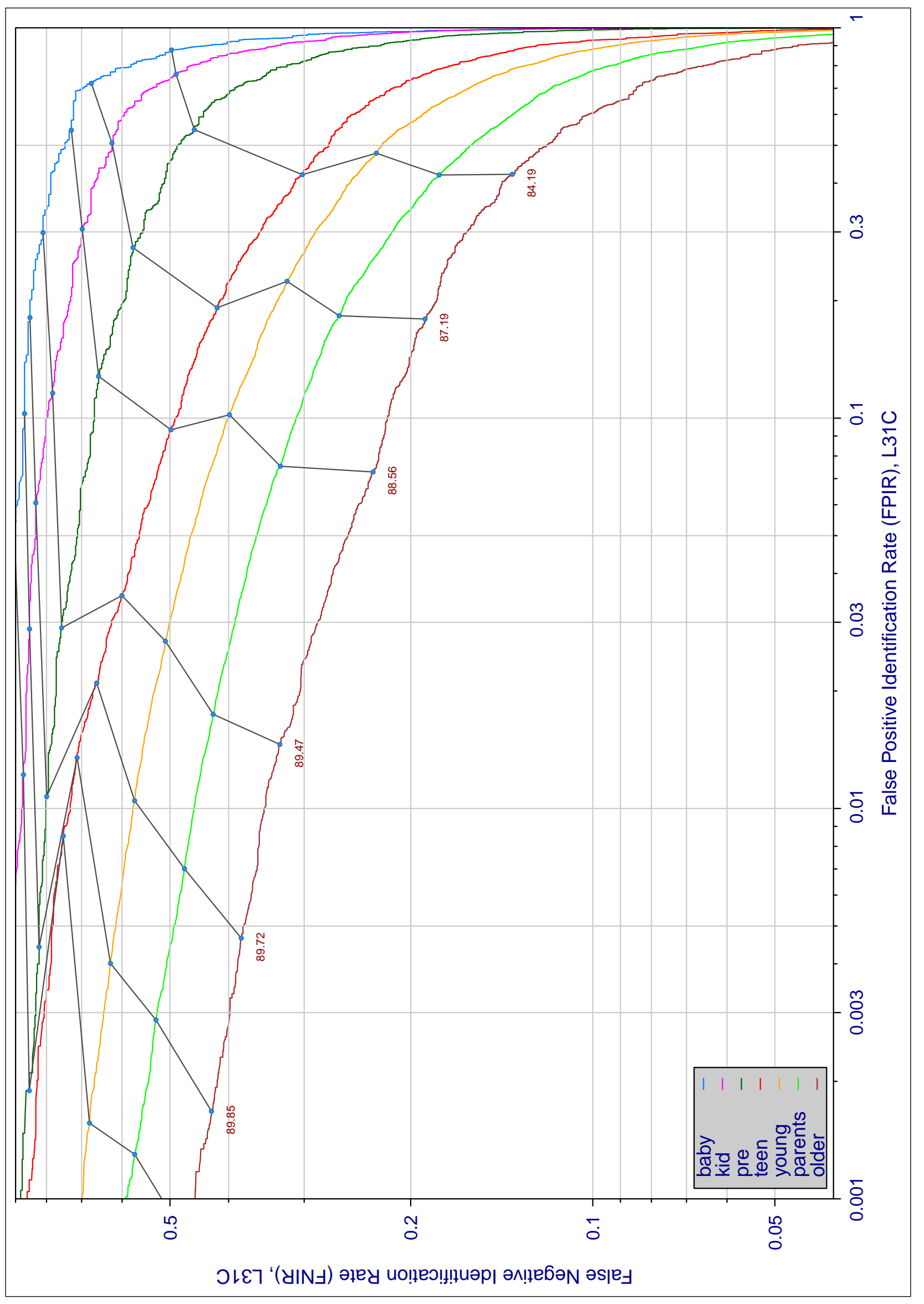

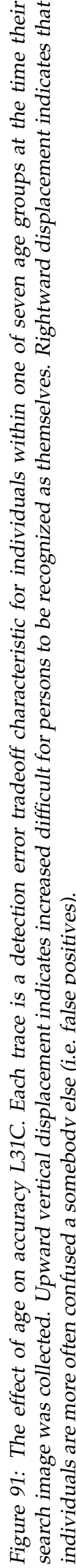

\begin{tabular}{|c|c|c|c|c|c|c|}
\hline $\begin{array}{l}\mathrm{A}=3 \mathrm{M} / \text { Cogent } \\
\mathrm{G}=\text { Hisign } \\
\mathrm{P}=\text { Zhuhai-Yisheng }\end{array}$ & $\begin{array}{l}B=\text { Cognitec } \\
H=C A S-I A \\
Q=\text { JunYu }\end{array}$ & $\begin{array}{l}\mathrm{C}=\text { Neurotechnology } \\
\mathrm{I}=\text { CAS-ICT } \\
\mathrm{S}=\text { Decatur }\end{array}$ & $\begin{array}{l}\mathrm{D}=\text { Safran Morpho } \\
\mathrm{J}=\text { Toshiba } \\
\mathrm{T}=\text { Ayonix }\end{array}$ & $\begin{array}{l}E=N E C \\
L=\text { Tsinghua U. II }\end{array}$ & $\begin{array}{l}\mathrm{F}=\text { Tsinghua } \mathrm{U} \\
\mathrm{M}=\mathrm{HP}\end{array}$ & $\begin{array}{l}\text { FNIR(N,R,T,L) “Miss rate" } \\
\text { FPIR(N,T,L) "False alarm rate" }\end{array}$ \\
\hline
\end{tabular}




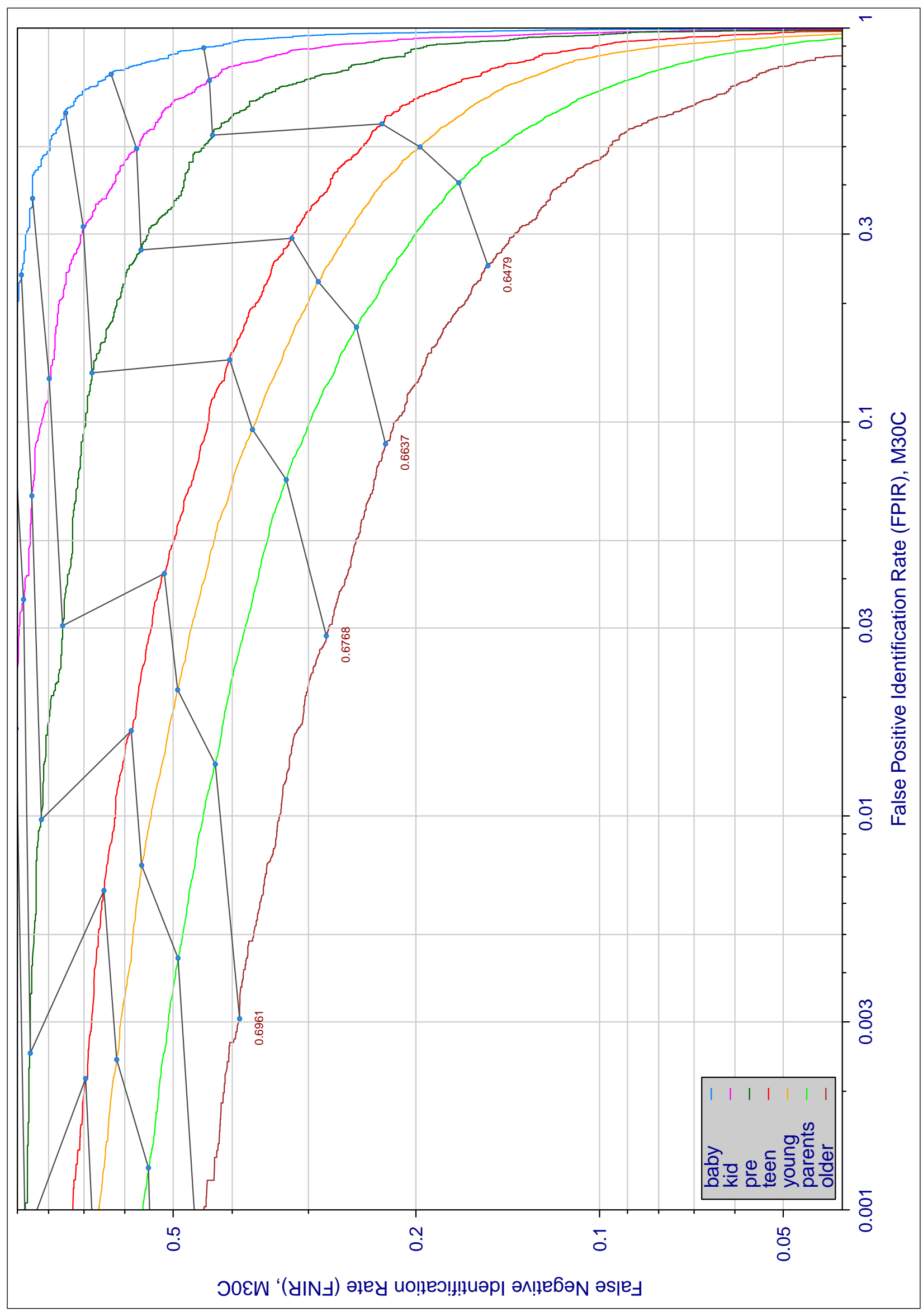

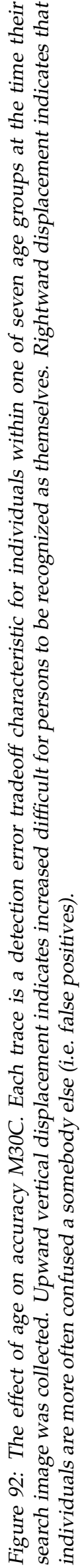

\begin{tabular}{|c|c|c|c|c|c|c|}
\hline $\begin{array}{l}\mathrm{A}=3 \mathrm{M} / \text { Cogent } \\
\mathrm{G}=\text { Hisign } \\
\mathrm{P}=\text { Zhuhai-Yisheng }\end{array}$ & $\begin{array}{l}B=\text { Cognitec } \\
H=C A S-I A \\
Q=\text { JunYu }\end{array}$ & $\begin{array}{l}\mathrm{C}=\text { Neurotechnology } \\
\mathrm{I}=\text { CAS-ICT } \\
\mathrm{S}=\text { Decatur }\end{array}$ & $\begin{array}{l}\mathrm{D}=\text { Safran Morpho } \\
\mathrm{J}=\text { Toshiba } \\
\mathrm{T}=\text { Ayonix }\end{array}$ & $\begin{array}{l}E=N E C \\
L=\text { Tsinghua U. II }\end{array}$ & $\begin{array}{l}\mathrm{F}=\text { Tsinghua } \mathrm{U} \\
\mathrm{M}=\mathrm{HP}\end{array}$ & $\begin{array}{l}\text { FNIR(N,R,T,L) “Miss rate" } \\
\text { FPIR(N,T,L) "False alarm rate" }\end{array}$ \\
\hline
\end{tabular}




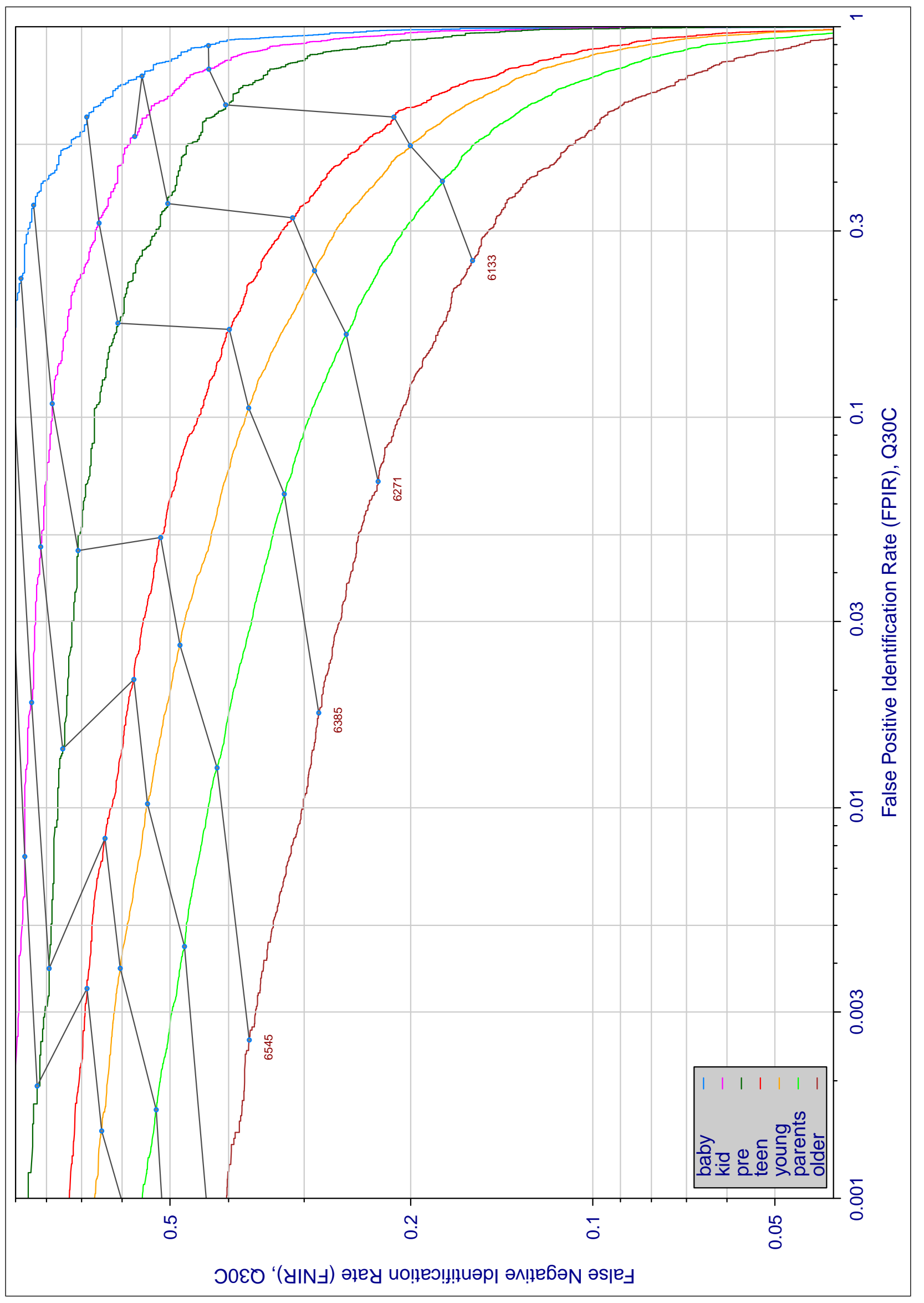

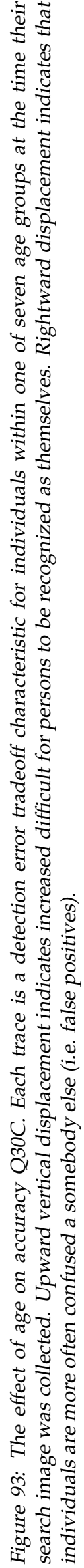

\begin{tabular}{|c|c|c|c|c|c|c|}
\hline $\begin{array}{l}\mathrm{A}=3 \mathrm{M} / \text { Cogent } \\
\mathrm{G}=\text { Hisign } \\
\mathrm{P}=\text { Zhuhai-Yisheng }\end{array}$ & $\begin{array}{l}B=\text { Cognitec } \\
H=C A S-I A \\
Q=\text { JunYu }\end{array}$ & $\begin{array}{l}\mathrm{C}=\text { Neurotechnology } \\
\mathrm{I}=\text { CAS-ICT } \\
\mathrm{S}=\text { Decatur }\end{array}$ & $\begin{array}{l}\mathrm{D}=\text { Safran Morpho } \\
\mathrm{J}=\text { Toshiba } \\
\mathrm{T}=\text { Ayonix }\end{array}$ & $\begin{array}{l}E=N E C \\
L=\text { Tsinghua U. II }\end{array}$ & $\begin{array}{l}\mathrm{F}=\text { Tsinghua } \mathrm{U} \\
\mathrm{M}=\mathrm{HP}\end{array}$ & $\begin{array}{l}\text { FNIR(N,R,T,L) “Miss rate" } \\
\text { FPIR(N,T,L) "False alarm rate" }\end{array}$ \\
\hline
\end{tabular}




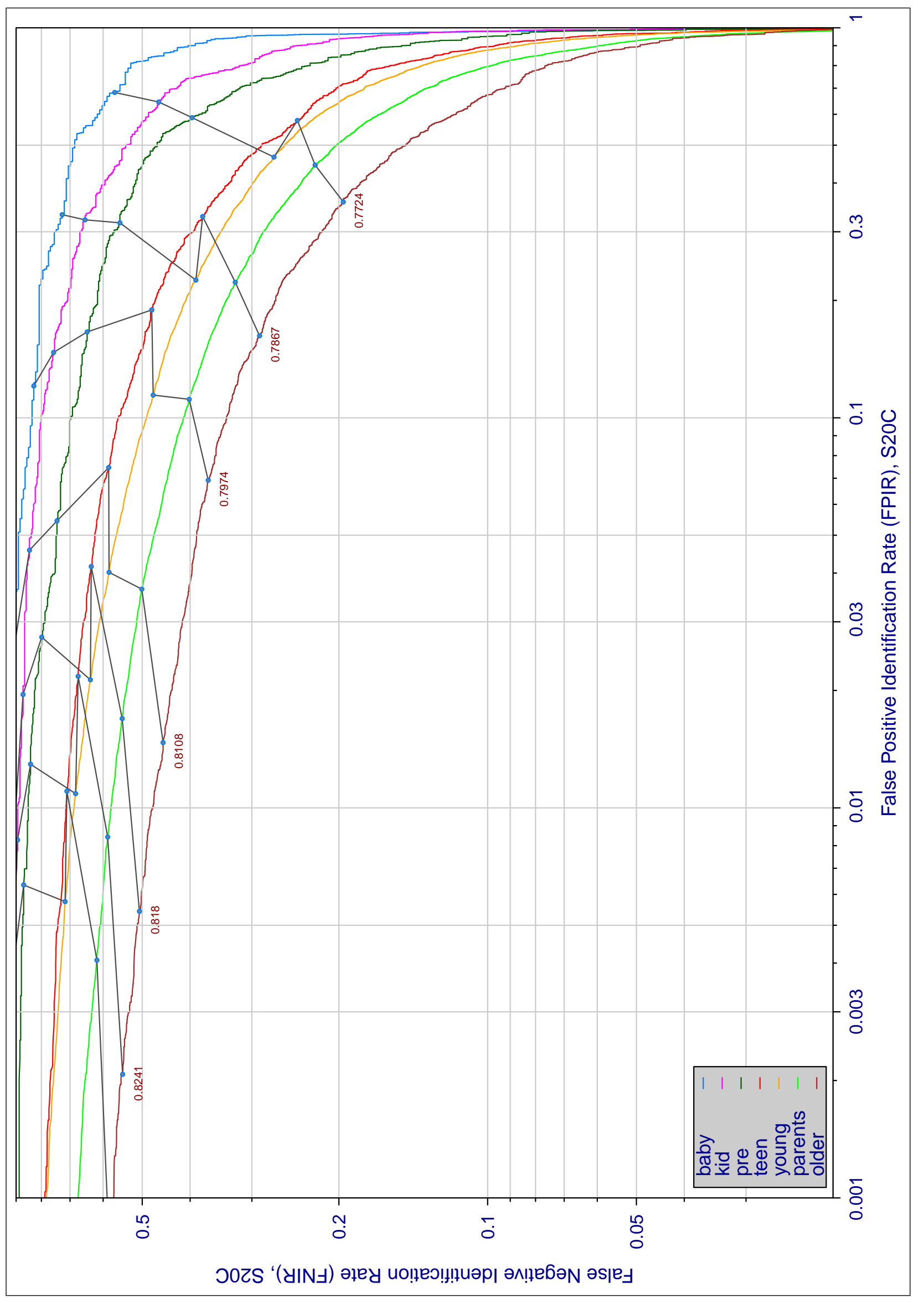

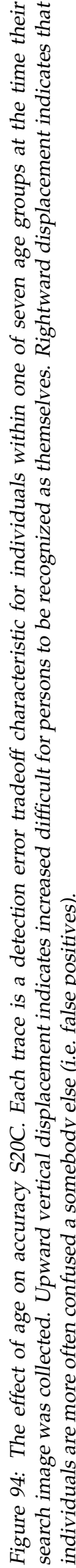

\begin{tabular}{|c|c|c|c|c|c|c|}
\hline $\begin{array}{l}\mathrm{A}=3 \mathrm{M} / \text { Cogent } \\
\mathrm{G}=\text { Hisign } \\
\mathrm{P}=\text { Zhuhai-Yisheng }\end{array}$ & $\begin{array}{l}B=\text { Cognitec } \\
H=C A S-I A \\
Q=\text { JunYu }\end{array}$ & $\begin{array}{l}\mathrm{C}=\text { Neurotechnology } \\
\mathrm{I}=\text { CAS-ICT } \\
\mathrm{S}=\text { Decatur }\end{array}$ & $\begin{array}{l}\mathrm{D}=\text { Safran Morpho } \\
\mathrm{J}=\text { Toshiba } \\
\mathrm{T}=\text { Ayonix }\end{array}$ & $\begin{array}{l}E=N E C \\
L=\text { Tsinghua U. II }\end{array}$ & $\begin{array}{l}\mathrm{F}=\text { Tsinghua } \mathrm{U} \\
\mathrm{M}=\mathrm{HP}\end{array}$ & $\begin{array}{l}\text { FNIR(N,R,T,L) “Miss rate" } \\
\text { FPIR(N,T,L) "False alarm rate" }\end{array}$ \\
\hline
\end{tabular}




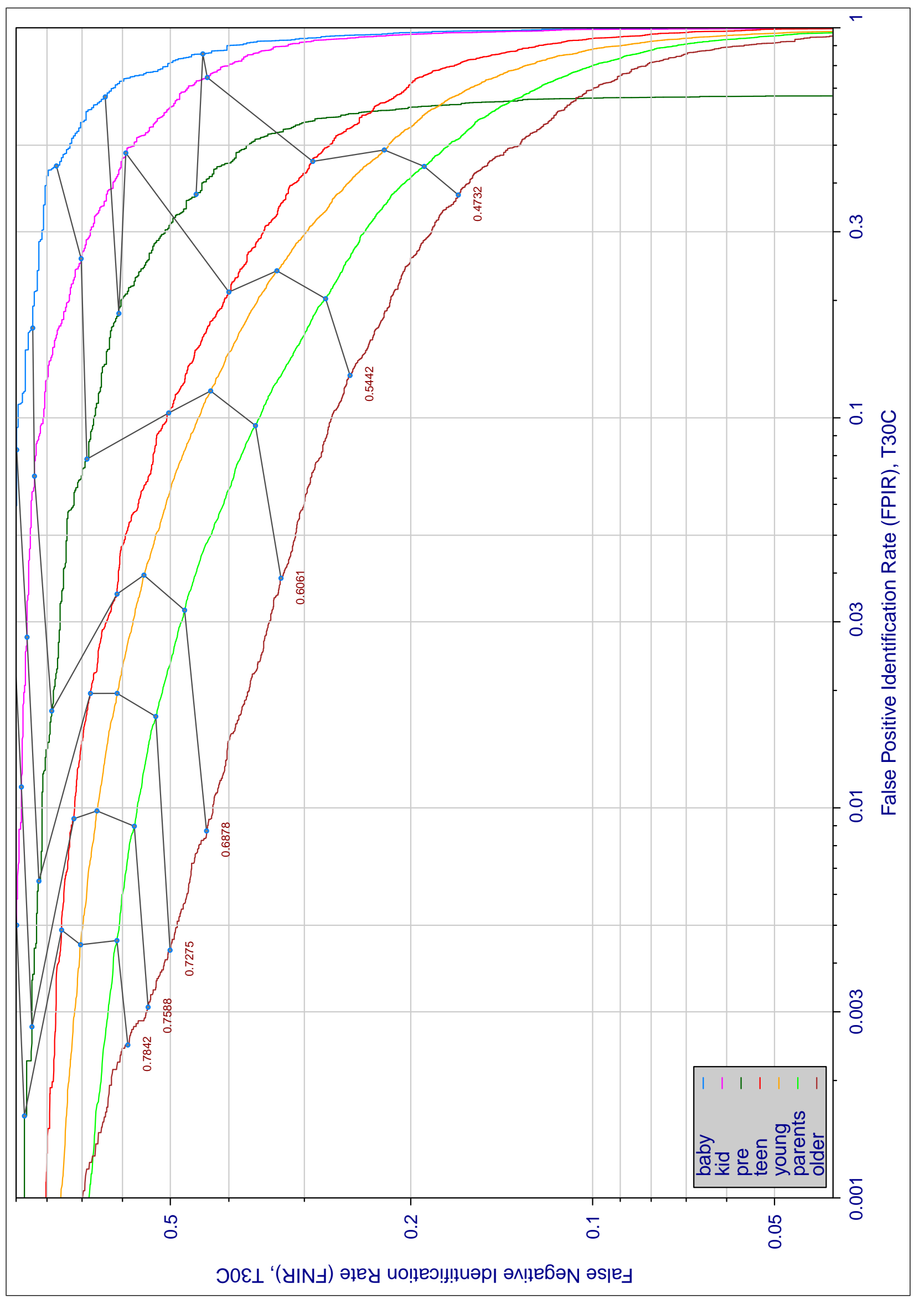

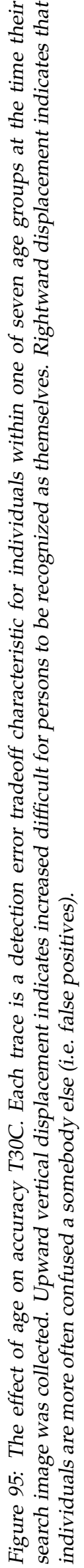

\begin{tabular}{|c|c|c|c|c|c|c|}
\hline $\begin{array}{l}\mathrm{A}=3 \mathrm{M} / \text { Cogent } \\
\mathrm{G}=\text { Hisign } \\
\mathrm{P}=\text { Zhuhai-Yisheng }\end{array}$ & $\begin{array}{l}B=\text { Cognitec } \\
H=C A S-I A \\
Q=\text { JunYu }\end{array}$ & $\begin{array}{l}\mathrm{C}=\text { Neurotechnology } \\
\mathrm{I}=\text { CAS-ICT } \\
\mathrm{S}=\text { Decatur }\end{array}$ & $\begin{array}{l}\mathrm{D}=\text { Safran Morpho } \\
\mathrm{J}=\text { Toshiba } \\
\mathrm{T}=\text { Ayonix }\end{array}$ & $\begin{array}{l}E=N E C \\
L=\text { Tsinghua U. II }\end{array}$ & $\begin{array}{l}\mathrm{F}=\text { Tsinghua } \mathrm{U} \\
\mathrm{M}=\mathrm{HP}\end{array}$ & $\begin{array}{l}\text { FNIR(N,R,T,L) “Miss rate" } \\
\text { FPIR(N,T,L) "False alarm rate" }\end{array}$ \\
\hline
\end{tabular}

